\title{
THE EVOLVING APPRECIATION OF PROJECTS - A STUDY OF STAKEHOLDER DYNAMICS
}

\author{
BY \\ VAN THI LA
}

\begin{abstract}
A thesis
submitted to the Victoria University of Wellington in fulfilment of the requirements for the degree of Doctor of Philosophy
\end{abstract}

Victoria University of Wellington 2021 



\begin{abstract}
Even though many project management techniques and methods have been developed and established, project managers and practitioners still find themselves unprepared and overwhelmed by the complexity and changing nature of stakeholder thinking and behaviour. The literature of stakeholder management and stakeholders in projects indicates that this complexity can be explained by the dynamics, complex interactions, and individual differences of project stakeholders. Thus, stakeholder perception and their social networks are dynamic and influence each other during project implementation. Both of them are also affected by the individual characteristics of stakeholders, which are linked to their past experience and background. However, while studies have focused on stakeholder dynamics as influenced by their social networks, very few of them focus on these dynamics as influenced by both the social networks and individual characteristics of stakeholders. This research addresses this gap by considering both aspects in the analysis of stakeholder dynamics or in other words, stakeholder dynamics at individual levels.
\end{abstract}

The research follows the social constructionist paradigm and uses Vickers' concept of appreciative systems as the theoretical lens. Through this lens, the above dynamics of stakeholder perception are described as the dynamics of their appreciation of projects as influenced by their standards and perceptions of events and ideas. The Repertory Grid Technique was the main data collection and analysis method. Data were collected from 47 interviews with ten participants who were each involved with one of three university accreditation projects, two in Vietnam and one in New Zealand. Data analysis consisted of the principal component analysis of repertory grids to identify the changes in standards and appreciation, the integration of these changes with the participants' perceptions of events and ideas through the lens of Vickers' concept, and the identification of the common themes.

The research found that stakeholders may change the interactions that they focus on and their levels of concern about these interactions during project implementation. The drivers of these dynamics are the changes in the standards and social networks of stakeholders. A framework is proposed to summarise these dynamics and drivers. Overall, the research provides further understanding of the complex dynamics of stakeholders as influenced by their individual characteristics. Based on this understanding, the research identifies empirical strategies for project managers to understand and address stakeholders at individual levels. Finally, the research suggests potential directions for future research of stakeholder dynamics in projects. 


\section{ACKNOWLEDGEMENTS}

I wish to express my deepest gratitude to my two supervisors, Associate Professor Arun Elias and Professor John Brocklesby, who have helped and encouraged me at all stages of my dissertation work. Without your guidance and persistent help, this dissertation would not have been possible. You have patiently listened to me, given me insightful comments and suggestions, and helped me go through the research that at times seems to be overwhelming. You not only provide me with academic support but also always care about my living in Wellington since the first day I came to the city. It is my privilege and honour to have an opportunity to work with you.

I am indebted to the participants of this research in Vietnam and New Zealand, who generously dedicated their time to this study. My thanks also go to the staff in the School of Management, Postgraduate Programme, and Student Learning for providing the best conditions for my studying. I will also never forget my $\mathrm{PhD}$ colleagues and friends who have become a part of my $\mathrm{PhD}$ experience.

All my love and my thanks to my husband, An Xuan Pham and my two kids, Mit and Nhim. Thank you for being with me during the ups and downs of my $\mathrm{PhD}$ study. Thank you for your support and empathy when I cannot give you as much time and attention as I should. Mit and Nhim, I always feel blessed to have you with me throughout this study. Your energy, joy and sense of humour enliven and enrich my life. With you, I know that I am never lonely in whatever journey that I take.

I would like to dedicate this dissertation to my mum, Dan Thi Cao, and my dad, Cuong Viet La. It is thanks to my mum's vision that I, as a girl coming from a small town in Vietnam, have come this far. Mum, since the day that you rewarded me with candy for my good marks in primary school to the day that you urged me to pursue $\mathrm{PhD}$ study, you have always been my ultimate source of motivation. Dad, I know that I am, always and forever, your little daughter. Thank you for your unconditional love and for even now still calling me often to make sure that my children and I are fine in a foreign country. This dissertation is for you, mum and dad. 


\section{TABLE OF CONTENTS}

Chapter 1 Introduction 1

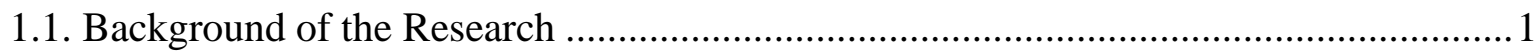

1.2. Research Objective and Research Questions ..........................................................

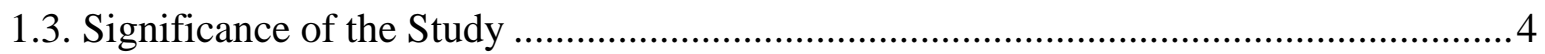

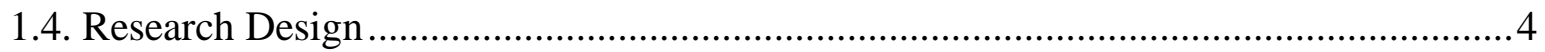

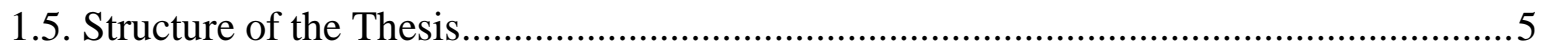

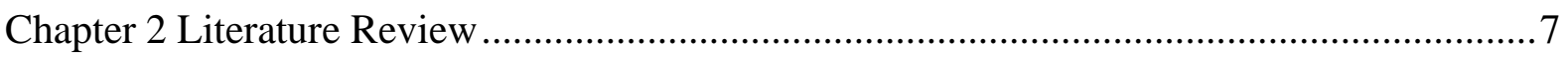

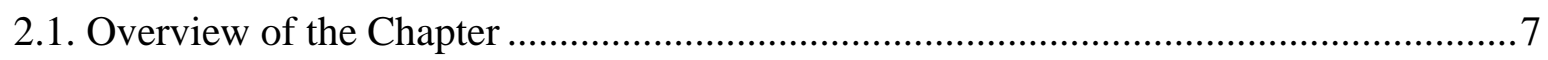

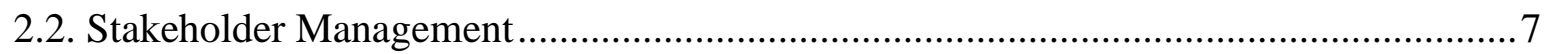

2.2.1. Evolution of Stakeholder Literature ...............................................................

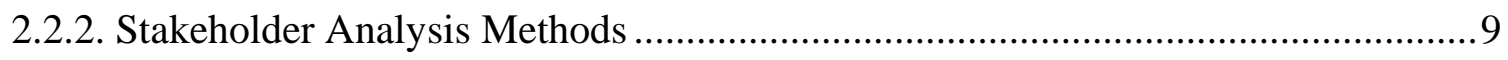

2.2.3. Limitations of Current Stakeholder Analysis Methods. ......................................... 11

2.2.4. Stakeholder Dynamics in Their Dyadic Relationship with Focal Organisations ... 12

2.2.5. Social Networks and Stakeholder Networks .................................................. 13

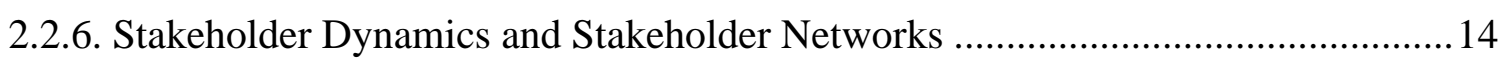

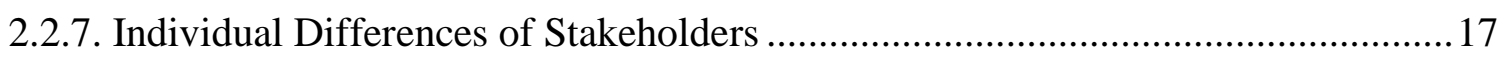

2.2.8. Summary of Stakeholder Literature Review .................................................... 19

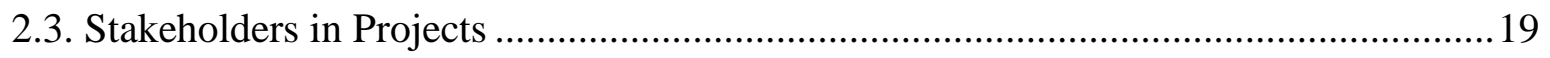

2.3.1. Overview of Projects and Project Stakeholders ................................................. 19

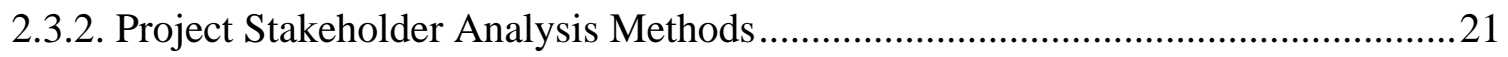

2.3.3. Limitations of Project Stakeholder Analysis Methods ..........................................22

2.3.4. Stakeholder Dynamics in Their Dyadic Relationships with Focal Projects ...........25

2.3.5. Project Stakeholder Networks and the Dynamics of Project Stakeholders ...........26

2.3.6. Individual Differences of Project Stakeholders ................................................28

2.3.7. Summary of the Review of Stakeholders in Projects ...........................................29

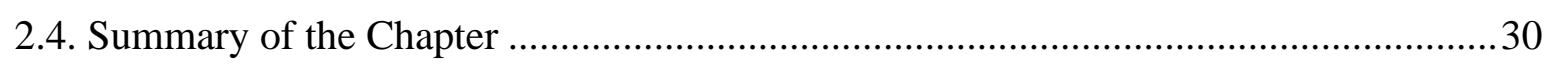

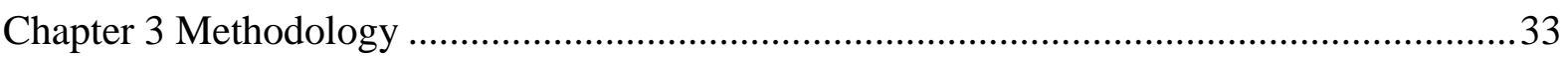




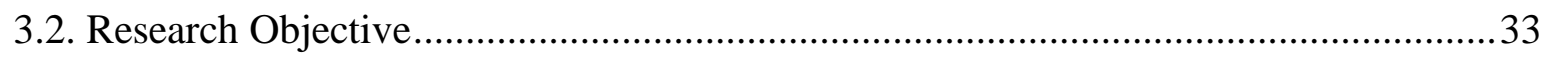

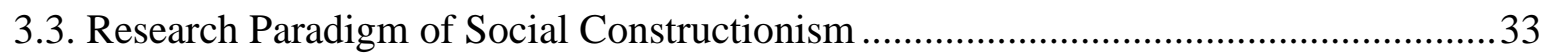

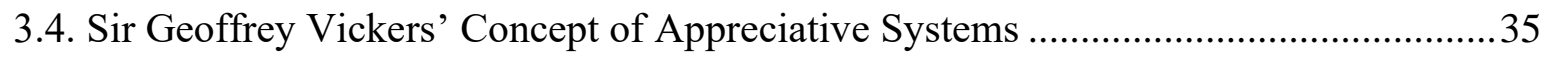

3.4.1. Overview of Vickers' Concept of Appreciative Systems.................................... 35

3.4.2. Academic Appreciation and Empirical Application of Vickers' Concept .............. 38

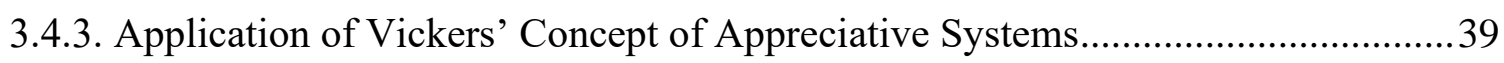

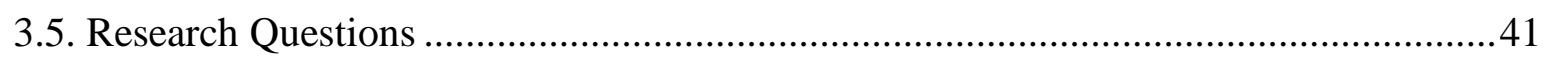

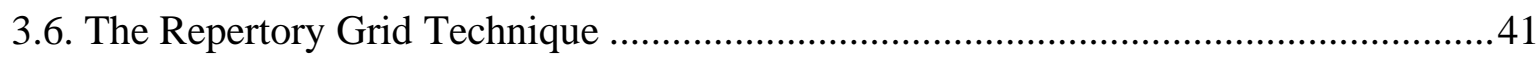

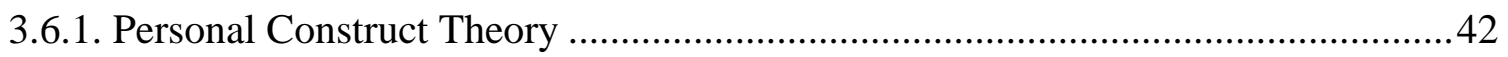

3.6.2. The Repertory Grid Technique and its Application in Project Management ......... 43

3.6.3. Application of the Repertory Grid Technique ................................................... 45

3.7. Case Study Research as Empirical Research Methods ..............................................4

3.7.1. Case Study Research and the Unit of Analysis ................................................... 47

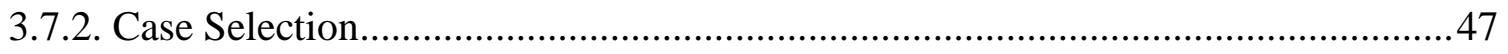

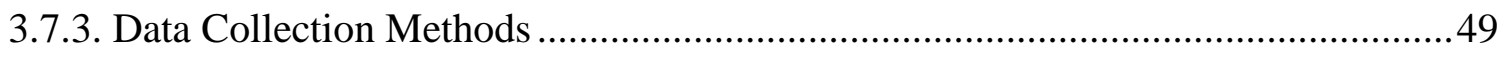

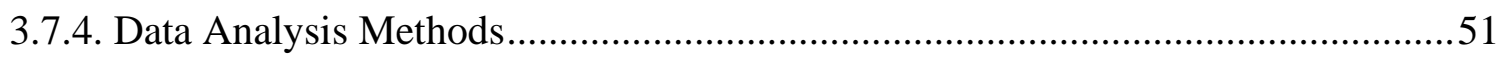

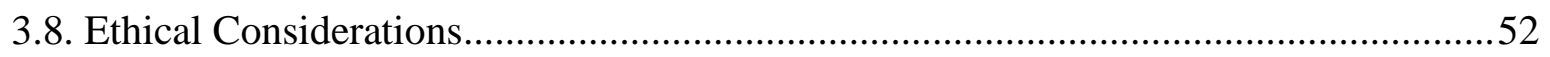

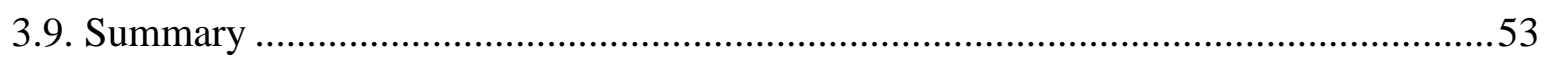

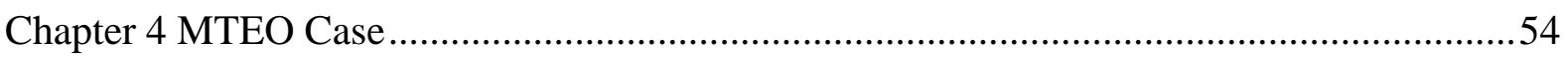

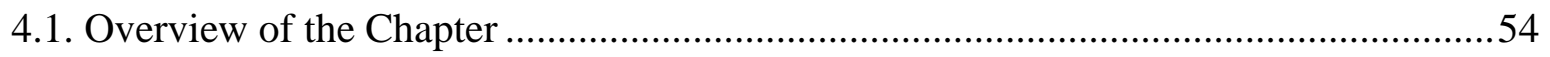

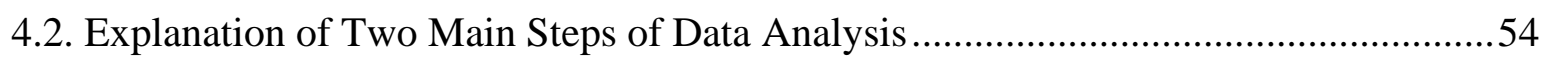

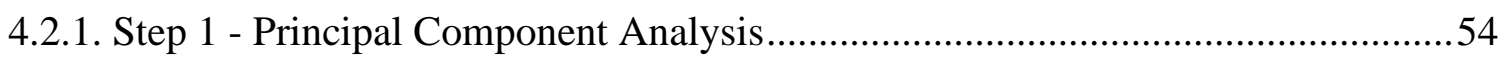

4.2.1.1. Sub-step 1.1: Identifying the Number of Principal Components.....................56

4.2.1.2. Sub-step 1.2: Interpreting the Principal Components....................................57

4.2.1.3. Sub-step 1.3: Identifying the Changes in the Standards of Stakeholders for Projects 
4.2.1.4. Sub-step 1.4: Identifying the Changes in Stakeholders' Appreciation of Projects.

4.2.2. Step 2 - the Application of Vickers' Concept of Appreciative Systems .66

4.3. Overview of the MTEO Accreditation Project .68

4.4. Changes in Thu's Appreciation of the MTEO Project. 69

4.4.1. Participant Background 69

4.4.2. Changes in Thu's Standards for and Appreciation of the MTEO Project 69

4.4.3. Changes in Thu's Appreciation of the MTEO Project through the Lens of Vickers' Concept. .76

4.5. Changes in Nga's Appreciation of the MTEO Project .............................................. 79

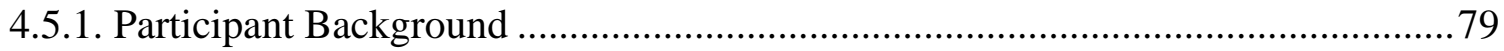

4.5.2. Changes in Nga's Standards for and Appreciation of the MTEO Project .............. 79

4.5.3. Changes in Nga's Appreciation of the MTEO Project through the Lens of Vickers'

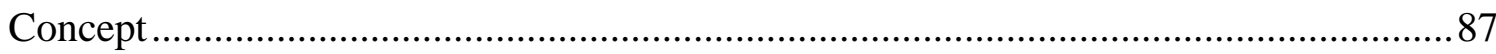

4.6. Changes in Hung' Appreciation of the MTEO Project............................................. 90

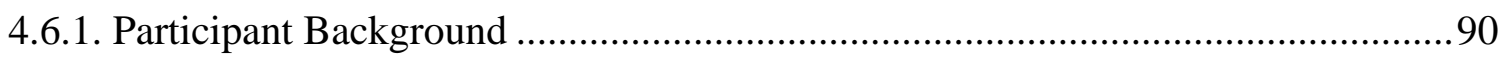

4.6.2. Changes in Hung's Standards for and Appreciation of the MTEO Project ............90

4.6.3. Changes in Hung's Appreciation of the MTEO Project through the Lens of

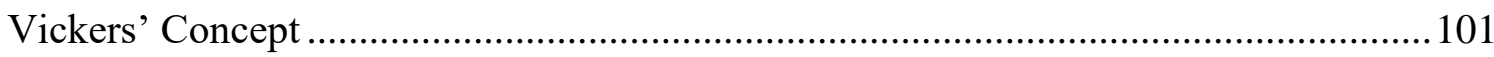

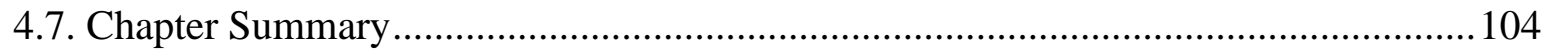

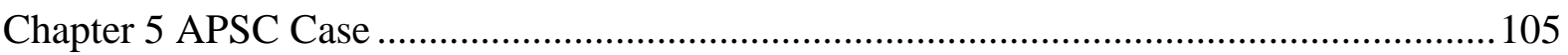

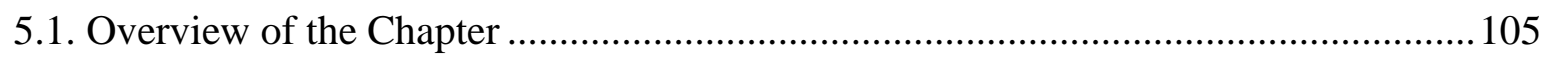

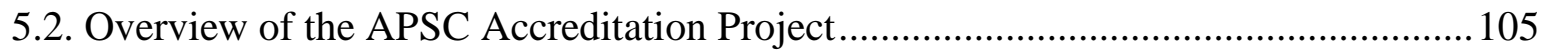

5.3. Changes in Tung's Appreciation of the APSC Project ….......................................... 106

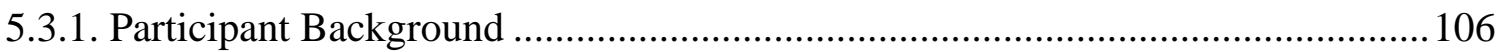

5.3.2. Changes in Tung's Standards for and Appreciation of the APSC Project ............ 106

5.3.3. Changes in Tung's Appreciation of the APSC Project through the Lens of Vickers' Concept. 111

5.4. Changes in Tai's Appreciation of the APSC Project 113 
5.4.2. Changes in Tai's Standards for and Appreciation of the APSC Project

5.4.3. Changes in Tai's Appreciation of the APSC Project through the Lens of Vickers'

Concept.

5.5. Changes in Khiem's Appreciation of the APSC Project

5.5.1. Participant Background

5.5.2. Changes in Khiem's Standards for and Appreciation of the APSC Project.

5.5.3. Changes in Khiem's Appreciation of the APSC Project through the Lens of

Vickers' Concept

5.6. Changes in Rafat's Appreciation of the APSC Project

5.6.1. Participant Background

5.6.2. Changes in Rafat's Standards for and Appreciation of the APSC Project.

5.6.3. Changes in Rafat's Appreciation of the APSC Project through the Lens of Vickers' Concept. 125

5.7. Chapter Summary. 126

Chapter 6 ABCD Case 127

6.1. Overview of the Chapter 127

6.2. Overview of the ABCD Accreditation Project. 127

6.3. Changes in Amish' Appreciation of the ABCD Project 128

6.3.1. Participant Background 128

6.3.2. Changes in Amish's Standards for and Appreciation of the ABCD Project 128

6.3.3. Changes in Amish's Appreciation of the ABCD Project through the Lens of Vickers' Concept 134

6.4. Changes in Nikki's Appreciation of the ABCD Project 140

6.4.1. Participant Background 140

6.4.2. Changes in Nikki's Standards for and Appreciation of the ABCD Project 140

6.4.3. Changes in Nikki's Appreciation of the ABCD Project through the Lens of Vickers' Concept 146

6.5. Changes in Sumy's Appreciation of the ABCD Project 151 
6.5.2. Changes in Sumy's Standards for and Appreciation of the ABCD Project

6.5.3. Changes in Sumy's Appreciation of the ABCD Project through the Lens of

Vickers' Concept

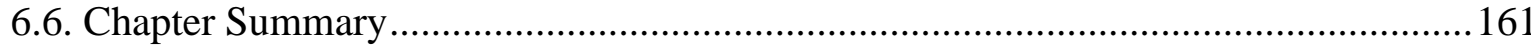

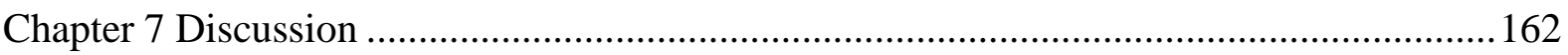

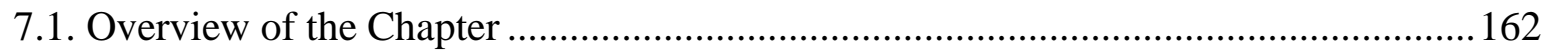

7.2. Nature and Dynamics of the Perceptions of Related Events and Ideas ...................... 162

7.2.1. Stakeholder Networks as Sources of the Events and Ideas ................................ 162

7.2.2. Dynamics of the Perceptions of Related Events and Ideas .................................. 165

7.3. Nature and Dynamics of Standards of Individual Stakeholders ................................. 168

7.3.1. Nature of Individual Stakeholders' Perception of Ideal and Less than Ideal Projects

7.3.2. Nature of Individual Stakeholders' Standards.................................................... 169

7.3.3. Dynamics of Individual Stakeholders' Standards ................................................ 172

7.4. Nature and Dynamics of Appreciation of Individual Stakeholders ............................ 177

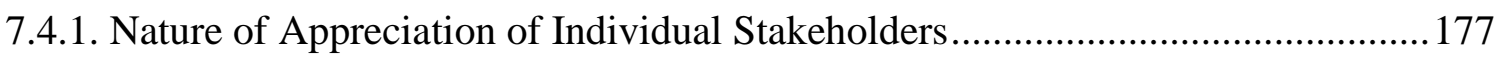

7.4.2. Dynamics of Appreciation by Individual Stakeholders..................................... 180

7.5. Proposed Framework of the Dynamics of Individual Stakeholders' Appreciation .... 183

7.5.1. Framework of the Dynamics of Individual Stakeholders' Appreciation.............. 183

7.5.2. An Example of the Application of the Framework …......................................... 186

7.5.3. Discussion of the Framework as the Main Research Findings ............................ 188

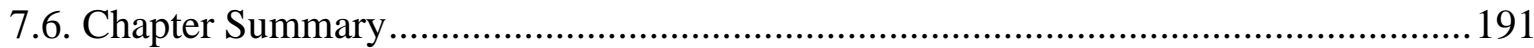

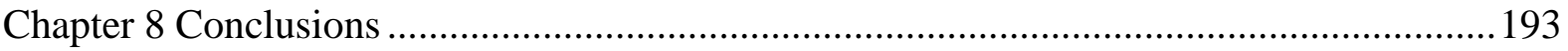

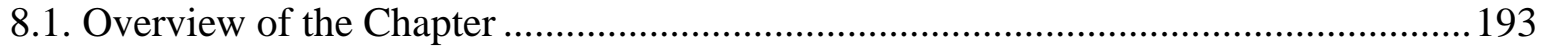

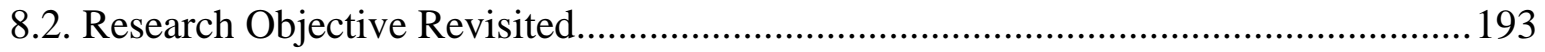

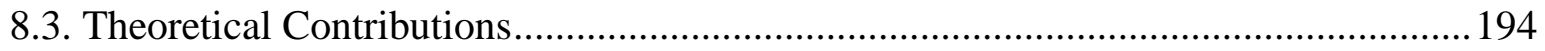

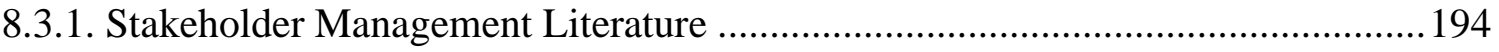




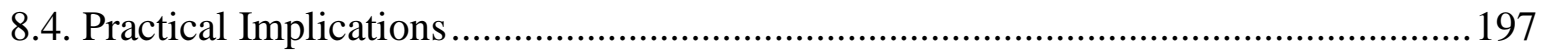

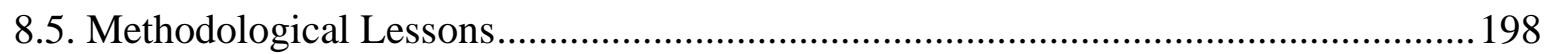

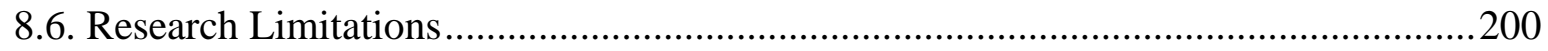

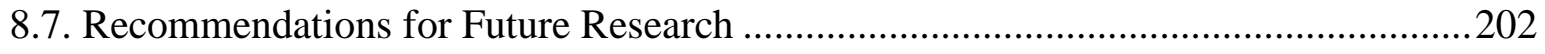

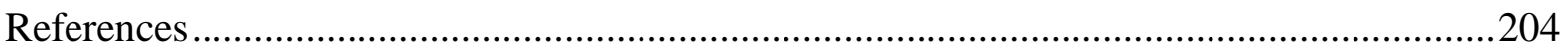

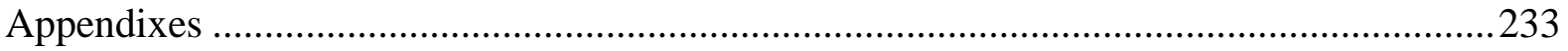

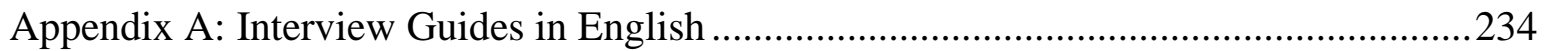

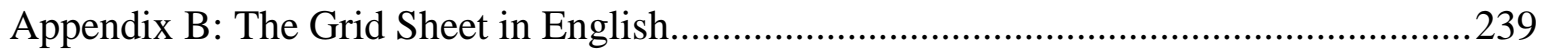

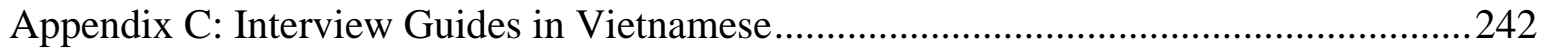

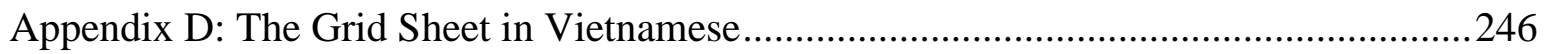

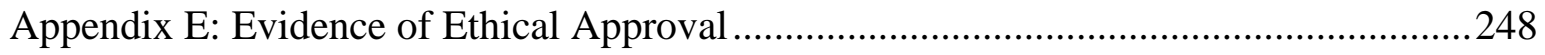

Appendix F: Information Sheets and Consent Forms for Supervisors in English and

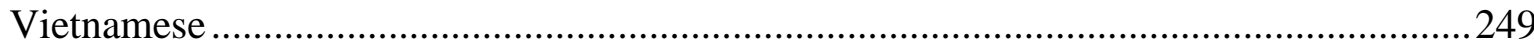

Appendix G: Information Sheets and Consent Forms for Participants in English and Vietnamese

Appendix H: Results of the Principal Component Analysis of Thu's Repertory Grids -

MTEO Case

Appendix I: Results of the Principal Component Analysis of Nga's Repertory Grids -

MTEO Case

Appendix J: Results of the Principal Component Analysis of Hung's Repertory Grids MTEO Case

Appendix K: Results of the Principal Component Analysis of Tung's Repertory Grids APSC Case

Appendix L: Results of the Principal Component Analysis of Tai's Repertory Grids APSC Case

Appendix M: Results of the Principal Component Analysis of Khiem's Repertory Grids APSC Case

Appendix N: Results of the Principal Component Analysis of Rafat's Repertory Grids - 
Appendix O: Results of the Principal Component Analysis of Amish's Repertory Grids ABCD Case

Appendix P: Results of the Principal Component Analysis of Nikki's Repertory Grids ABCD Case.....

Appendix Q: Results of the Principal Component Analysis of Sumy's Repertory Grids ABCD Case 


\section{LIST OF TABLES}

Table 2.1 Examples of project stakeholder analysis methods .23

Table 3.1 Summary of the cases, projects, participants, and number of interviews

Table 4.1 An example of percentage variances explained by principal components (Source:

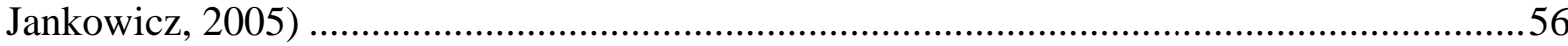

Table 4.2 Percentage of variance for each component (Cmp) (Source: Bezzi, 1999)............57

Table 4.3 An example of construct loadings on components (Sources: self-produced based on

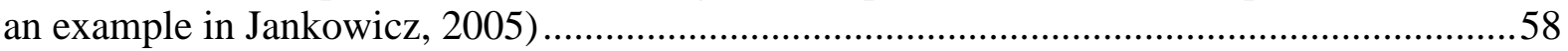

Table 4.4 An example of the loadings of elements on principal components (Sources: selfproduced from an example in Jankowicz, 2005)

Table 4.5 An example of the loadings of a participant's appreciation of the ideal project, the less than ideal project, and the target project on the two components at one interview

Table 4.6 An example of the changes in the loadings

Table 4.7 Constructs in Thu's repertory grids in her four interviews - the MTEO project...... 70

Table 4.8: Percentage variances explained by the two components over the four interviews Thu - MTEO project

Table 4.9 Loadings of Thu's appreciation of the ideal project, the less than ideal project, and the MTEO project on the two components....

Table 4.10 List of constructs - Nga - MTEO case.

Table 4.11 Percentage variances explained by the two components over the four interviews $\mathrm{Nga}$ - MTEO project

Table 4.12 Loadings of Nga's appreciation of the ideal project, the less than ideal project and the MTEO project on the two components over the four interviews.....

Table 4.13 List of constructs - Hung - MTEO case

Table 4.14 Percentage variances explained by three components - Hung - MTEO project...92

Table 4.15 The loadings of Hung's appreciation of the ideal project, the less than ideal project and the MTEO project on the three components over the four interviews 98

Table 5.1 Percentage variances explained by two principal components over the four interviews - Tung - APSC project.

Table 5.2 The loadings of Tung's appreciation of the ideal project, the less than ideal project and the APSC project on the two components 
Table 5.3 Percentage variances explained by each principal component over the four interviews - Tai - APSC project

Table 5.4 Loadings of Tai's appreciation of the ideal project, the less than ideal project and the APSC project on the two components in the four interviews

Table 5.5 Loadings of Khiem's appreciation of the ideal project, the less than ideal project, and the APSC project on the component

Table 5.6 Percentage variances explained by the two components over the two interviews -

Rafat - APSC project

Table 5.7 Loadings of Rafat's appreciation of the ideal project, the less than ideal project, and the APSC project on the two components over the two interviews

Table 6.1 Percentage variances explained by two principal components during eight interviews - Amish - ABCD project

Table 6.2 Loadings of Amish's appreciation of the ideal and less than ideal projects and the $\mathrm{ABCD}$ project on the two principal components over the eight interviews

Table 6.3 Percentage variances explained by two principal components over six interviews -

Nikki - ABCD project

Table 6.4 Loadings of Nikki's appreciation of the ideal and less than ideal projects and the $\mathrm{ABCD}$ project on the two components

Table 6.5 Percentage variances explained by two principal components over the eight interviews - Sumy - ABCD project.

Table 6.6 Loadings of Sumy's appreciation of the ideal project and less than ideal projects and the ABCD project on the two components 


\section{LIST OF FIGURES}

Figure 2.1 An illustration of a stakeholder network. Reprinted from "Moving beyond dyadic ties: a network theory of stakeholder influences" (Rowley, 1997)......................................... 14

Figure 2.2 An approach to describe the complexity of stakeholder thinking and behaviour in projects

Figure 2.3 A description of the complexity of stakeholder thinking and behaviour in projects

Figure 3.1 The dynamics of appreciative systems (Source: Checkland, 2000) .....

Figure 3.2 The dynamics of stakeholder perception of projects according to Vickers' concept

Figure 3.3 An example of a repertory grid (Source: Jankowicz, 2005) ................................43

Figure 3.4 An application of the Repertory Grid Technique ............................................46

Figure 3.5 An illustration of elements for the triadic elicitation.......................................50

Figure 3.6 Two first steps of data analysis for each participant .......................................51

Figure 4.1 Four sub-steps of the principal component analysis using the Rep Plus V1.1R software to identify the changes in the standards and appreciation of each stakeholder

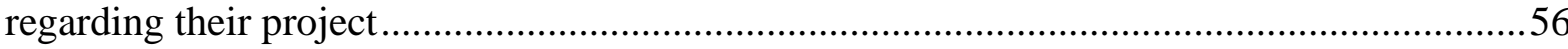

Figure 4.2 An example of the distribution of constructs on a PrinGrid map (Source: Jankowicz, 2005)

Figure 4.3 An example of a PrinGrid map that illustrates the loadings of the appreciation of the ideal project, the less than ideal project, and the target project on the components at one interview

Figure 4.4 An example of changes of elements on PrinGrid maps (Source: Bezzi, 1999) .....64

Figure 4.5 A PrinGrid map which uses the trajectory function to describe the changes in a participant's appreciation of her project over four interviews

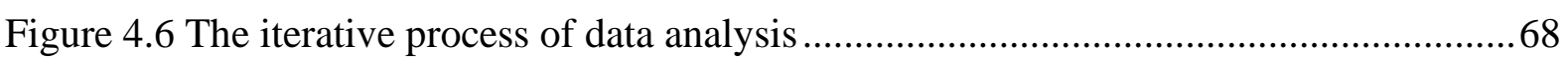

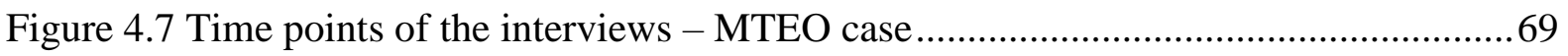

Figure 4.8 PrinGrid map - the loadings of the constructs on the two principal components -

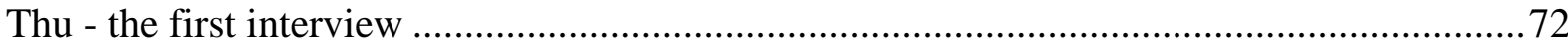

Figure 4.9 Changes in Thu's appreciation of the MTEO project over the four interviews (T1,

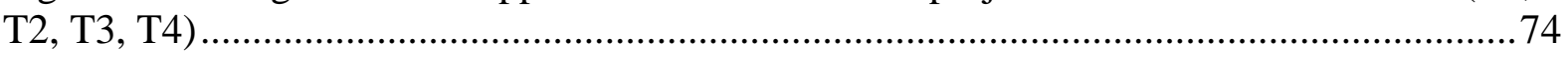

Figure 4.10 Nga's PrinGrid map in the first interview - MTEO case .................................. 82 
Figure 4.11 Nga's PrinGrid map in the third interview - MTEO case

Figure 4.12 Changes in Nga's appreciation of the MTEO project over the four interviews.... 86

Figure 4.13 Hung's PrinGrid map - components 1 and 2 - the first interview - the MTEO project

Figure 4.14 Hung's PrinGrid map - components 1 and 3 - the first interview - the MTEO project

Figure 4.15 PrinGrid map - components 1 and 3 - Hung - the third interview.....

Figure 4.16 Changes in Hung' appreciation of the MTEO project according to component 1 and component 2 over the four interviews....

Figure 4.17 Changes in Hung's appreciation of the MTEO project according to component 1 and component 3 over the four interviews

Figure 5.1 Time points of the interviews - APSC project 106

Figure 5.2 Tung's appreciation of the APSC project at the first interview 109

Figure 5.3 Changes in Tung's appreciation of the APSC project from his second to his fourth interview

Figure 5.4 Changes in Tai's appreciation of the APSC project over the four interviews 115

Figure 5.5 Changes in Khiem's appreciation of the APSC project over the three interviews 120

Figure 5.6 Changes in Rafat's appreciation of the APSC project at FXN University over the two interviews

Figure 6.1 Time points of the interviews

Figure 6.2 Changes in Amish's appreciation of the ABCD project, ideal and less than ideal projects from the first to the fifth interview

Figure 6.3 Changes in Amish's appreciation of the ABCD project, ideal, and less than ideal projects from the sixth to the eighth interview

Figure 6.4 Nikki's appreciation of the $\mathrm{ABCD}$ project, and the ideal and less than ideal projects at the first interview (T1)

Figure 6.5 Nikki's appreciation of the ABCD project, and the ideal and less than ideal projects at the second interview (T2).

Figure 6.6 Nikki's appreciation of the ABCD project, and the ideal and less than ideal projects at the third interview (T3)

Figure 6.7 Changes in Nikki's appreciation of the ABCD project, and the ideal and less than ideal projects from the fourth (T4) to the sixth interview (T8). 
Figure 6.8 Changes in Sumy's appreciation of the ABCD project, and the ideal and less than ideal projects from the first to the third interview

Figure 6.9 Changes in Sumy's appreciation of the ABCD project, and the ideal and less than ideal projects from the fourth to the eighth interview 155

Figure 7.1 Dynamics of stakeholder access to events and ideas. 166

Figure 7.2 Thu's thinking of how the stakeholder interactions in the MTEO project should be at $\mathrm{T} 1$ 168

Figure 7.3 Hung's thinking of how the stakeholder interactions in the MTEO project should be at $\mathrm{T} 1$

Figure 7.4 Thu's primary and secondary standards for stakeholder interactions in the MTEO project at $\mathrm{T} 1$ 170

Figure 7.5 Hung's standards for the stakeholder interactions in the MTEO project at T1 ....171

Figure 7.6 Changes in Nikki's standards for the ABCD project between T1 and T2, while her expectation of the overall stakeholder interactions remained the same.....

Figure 7.7 Changes in Sumy's standards for the ABCD project between T3 and T4, while her expectation of the overall stakeholder interactions changed

Figure 7.8 Hung's levels of concern about the MTEO project at T1

Figure 7.9 Dynamic relationships between individual stakeholders' appreciation of projects and their drivers

Figure 7.10 Dynamics of individual stakeholders' appreciation of projects and their drivers 


\section{Chapter 1 Introduction}

\subsection{Background of the Research}

Projects have become dominant models and indispensable elements in industries from manufacturing, construction, infrastructure to film production and education (Gupta et al., 2019; Winter et al., 2006). A project is "a temporary endeavour undertaken to create a unique product, service, or result" (Project Management Institute, 2017, section 1.2.1, para. 4). Projects involve a wide range of different activities in different industries (Winter et al., 2006). In manufacturing, such activities may include developing new products, innovating existing products, and improving deficient products (Gupta et al., 2019). In services such as education, these activities may include improving research or training quality.

Given the importance and prevalence of projects, many project management techniques and tools have been developed, and many bodies specialising in project management have been established. Project managers and other roles in projects have also become professionally certified and specialised (Hughes et al., 2016). However, despite these developments, project practitioners still feel unprepared and overwhelmed by issues that arise during project implementation (Eskerod, Huemann, \& Savage, 2015). Many of the issues perceived by project practitioners are related to the changing nature and complexity of the thinking and behaviour of groups and individuals involved in projects or project stakeholders (Antony et al., 2019; Catalano et al., 2019; Gupta et al., 2019; Hughes et al., 2016; Mukherjee, 2019).

During project implementation, stakeholders may change their thinking and behaviour in complex and unpredictable ways. They may initially support then resist projects (Antony et al., 2019; Hughes et al., 2016). They may be committed to projects at the beginning, then become uncommitted (Antony et al., 2019). They may at first focus on and pay attention to projects and then lose their focus and attention (Hughes et al., 2016; Mukherjee, 2019). They may get along very well with each other and then have conflicts or do not trust each other (Catalano et al., 2019). Some key stakeholders, such as staff with irreplaceable technical and managerial skills, may decide to leave projects before completion (Hughes et al., 2016). Some other stakeholders, such as customers, may not have the skills and expertise to set project requirements or frequently change the requirements (Mukherjee, 2019).

Based on the stakeholder management literature, this complexity of stakeholder thinking and behaviour can be explained by their dynamics (Pouloudi et al., 2016; Solaimani et al., 2013), complex interaction (Beaulieu \& Pasquero, 2002), and individual differences 
(Johnson et al., 2008). Specifically, it was found that stakeholders interact in complex social networks (Rowley, 1997; Pouloudi et al., 2016). Their interactions in these networks are dynamic and influence each other (Afreen \& Kumar, 2016; Dorobantu et al., 2016). The relationship between these interactions and stakeholder perception is also dynamic (Khurram \& Petit, 2017; Sloan \& Oliver, 2013). Moreover, both stakeholder thinking and behaviour can be affected by stakeholders' individual characteristics, which are linked to their experience and background (Bridoux \& Stoelhorst, 2016; Weitzner \& Deutsch, 2015).

In line with the stakeholder management literature, the literature on stakeholders in projects also highlights the necessity of studying the complexity of the thinking and behaviour of project stakeholders. This complexity, which has been described theoretically by scholars such as Eskerod and Larsen (2018), Kreiner (1995), and Padalkar and Gopinath (2016), is in line with the observations made regarding the dynamics, social networks, and individual differences of project stakeholders. The review of these aspects then indicates that stakeholder perception and interactions in their networks are dynamic and influence each other (Aaltonen et al., 2015; Besson \& Rowe, 2001). The individual characteristics of stakeholders, which are linked to their experience and background, also influence stakeholder thinking and behaviour (Aaltonen, 2011; Tukiainen et al., 2010).

Overall, the complexity of stakeholder thinking and behaviour in projects can be explained by the dynamics of their perception of projects, as influenced by their networks and individual characteristics. While the networks refer to the present interactions of stakeholders, the individual characteristics refer to their past experience and background. However, most of the existing studies only focus on either the dynamics of stakeholders as influenced by their networks (e.g., Aaltonen et al., 2015; Besson \& Rowe, 2001) or the impact of the individual characteristics of stakeholders on their thinking and behaviour (e.g., Aaltonen, 2011; Tukiainen et al., 2010). Very few studies focus on the dynamics of stakeholder perception of projects as influenced by both their individual characteristics and social networks; or in other words, the dynamics of stakeholders at individual levels. Studies on these aspects of stakeholder dynamics will increase our understanding of the complexity of stakeholder thinking and behaviour.

To investigate these dynamics, this research focuses on university accreditation projects. Accreditation in higher education institutions or universities is a process in which governmental, non-governmental, or private bodies evaluate the quality of the institutions or programmes to formally recognise them as having met specific pre-determined minimal criteria of standards (Vlăsceanu et al., 2004). Accreditation helps to boost the reputation of universities and institutions (Blom et al., 2012) and provide guidelines to improve their teaching, curricula, 
infrastructure (Romero, 2008), and strategic management (Elliott \& Goh, 2013). With these benefits, university accreditation projects have become increasingly popular over the last twenty years (AACSB International, 2020; Alani \& Ilusanya, 2008; AMBA, 2020; EFMD Global, 2020; Nguyen et al., 2017).

Accreditation projects involve a wide range of stakeholders with different roles and backgrounds, such as accreditation panels, managerial teams, academic and administrative staff, students, and employers (Vlăsceanu et al., 2004). The thinking and behaviour of these stakeholders are also complex and unpredictable. They may doubt the benefits of accreditation and resist engaging in it (Alghamdi, 2016; Romero, 2008). They may also delay the delivery of documents related to accreditation, question the agenda behind it, or convince university leaders of the need of accreditation (Alghamdi, 2016). Stakeholders may also not understand how to apply the accreditation standards to their programmes and universities (Jenkins, 2011). In some developing countries, along with the mass development of universities, staff may be under-trained and may not support accreditation or any quality assurance systems to avoid new responsibilities (Onsman, 2010). Overall, the complexity and unpredictability of stakeholder thinking and behaviour, combined with their diverse background and experience, make accreditation projects suitable to study the dynamics of stakeholders as influenced by their individual characteristics. The researcher, with nearly ten years work experience at universities, also has good access to collect data from these projects. With the focus on accreditation projects, the research objectives and questions are specified in the next section.

\subsection{Research Objective and Research Questions}

The overall objective of this research is to explore the dynamics of individual stakeholders in projects. The research aims to provide an improved methodological approach for managing individual stakeholders in complex projects. Specifically, the research focuses on the dynamics of stakeholder perception of projects, as influenced by their individual characteristics. These characteristics are linked to the background and experience of stakeholders and reflect their distinctiveness at individual levels. Vickers' concept of appreciative systems (Vickers, 1965/1995) is applied as the theoretical lens, and the Repertory Grid Technique (Kelly, 1955/2001) is applied as the main data collection and analysis method. Through the lens of Vickers' concept, stakeholder perception of projects is described as their appreciation of projects. The research questions, therefore, are specified as:

Research question 1: How do individual stakeholders change their appreciation in projects over time? 
Research question 2: What are the drivers of the changes in individual stakeholders' appreciation in projects?

\subsection{Significance of the Study}

By providing further insights into the dynamics of stakeholders' appreciation of projects and the dynamics of stakeholders as influenced by their individual characteristics, the research makes theoretical and practical contributions to the field.

Theoretically, the research contributes to the stakeholder literature. It deepens our understanding of stakeholder dynamics at individual levels or in other words, the impact of individual characteristics of stakeholders on their dynamics. This enriches the existing literature by improving the prevalent conceptions of the complexity and changing nature of stakeholder thinking and behaviour. The focus on exploring the complexity of specific project contexts means that the research also contributes to the project management literature. It gives more insights into the complexity of the project environment and issues.

The study applies a new approach, namely the combination of Vickers' concept of appreciative systems and the Repertory Grid Technique, to analyse stakeholders. The research shows that Vickers' concept can be applied to explore the dynamics of stakeholders in projects. Moreover, it also shows that the Repertory Grid Technique can be used to collect data for different components of Vickers' concept.

Regarding the practical implications, the research provides project managers with a better understanding of stakeholder dynamics during project implementation. It helps project managers understand how the individual characteristics of stakeholders, which are linked to the past experience and background of stakeholders, interact with the present social networks of stakeholders and affect the changes in stakeholder thinking and perception. It also provides project managers with methods to specifically identify these changes. Based on this, project managers may be better prepared to develop strategies to deal with stakeholders.

\subsection{Research Design}

With the purposes of studying the dynamics of stakeholder perception as influenced by their individual characteristics, this research follows the social constructionist paradigm, which emphasises how individual interactions continuously shape their knowledge of reality. Vickers' concept of appreciative systems (Vickers, 1965/1995) is used as the theoretical lens. Through this lens, the above dynamics of stakeholder perception are described as the dynamics 
of their appreciation of projects as influenced by their standards and perceptions of events and ideas. The Repertory Grid Technique, which was developed from the Personal Construct Theory (Kelly, 1955/2001), was the main data collection and analysis method. Data were collected from 47 interviews with ten stakeholders from three university accreditation projects, two in Vietnam and one in New Zealand, that were conducted every one to two months over periods of four to six months. Data analysis consisted of three steps. In the first step, the principal component analysis of the repertory grids identified the changes in the standards and appreciation of each stakeholder regarding their projects. In the second step, these changes were integrated with stakeholder perceptions of events and ideas through the lens of Vickers' concept. The common themes of these changes were then identified in the final step. Based on these themes, a framework to describe the dynamics of stakeholder perception of projects as influenced by their individual characteristics was proposed.

\subsection{Structure of the Thesis}

The thesis is organised into eight chapters. Chapter one has presented an overview of the research, including the research background, research objective, research questions, research significance, and research design.

Chapter two presents the literature review, which focuses on the literature of stakeholder management and stakeholders in projects. This review identifies that studying the dynamics of stakeholder perception of projects as influenced by their individual characteristics as well as their networks will provide a better understanding of the complexity and changing nature of stakeholder thinking and behaviour in projects. Based on this identification, chapter three presents the research methodology. In this chapter, Vickers' concept of appreciative systems as the main theoretical lens and the Repertory Grid Technique as the main data collection and analysis method are presented. Case study research as the empirical research method is also explained.

Chapter four, five, and six present the results of the data analysis for the three cases: MTEO, APSC, and ABCD, respectively. A detailed explanation of the data analysis methods is also presented in chapter four. The results of the data analysis for each participant are divided into two parts. The first part is the principal component analysis of the repertory grids to identify the changes in the standards and appreciation. The second part is the integration of these changes with the participant perceptions of events and ideas by applying Vickers' concept of appreciative systems. 
In chapter seven, the nature and common themes of the changes in the participant's appreciation, standards, and perceptions of events and ideas are identified. Based on these common themes, a framework to describe the dynamics of stakeholder perception of projects at their individual levels is proposed. Following this proposal, chapter eight presents the research conclusions by first revisiting the research objectives. This is followed by the identification of the theoretical contributions, managerial implications, methodological lessons, and limitations of the research, and finally by the presentation of suggestions for directions for future research. 


\section{Chapter 2 Literature Review}

\subsection{Overview of the Chapter}

The introduction chapter has presented the exploration of the complexity and changing nature of stakeholder thinking and behaviour in projects as the main research interest of this study. This chapter presents an overview of the existing literature on stakeholder management and stakeholders in projects. It identifies and describes key theories and prevalent research approaches in this field in order to highlight the research gap the current study seeks to address, which is presented in the chapter summary.

\subsection{Stakeholder Management}

This section begins with an overview of the evolution of the stakeholder literature, followed by a discussion of existing stakeholder analysis methods and their limitations. Subsequently, the dynamics which refer to the changes over time (Windsor, 2010), the social networks, and the individual differences of stakeholders are reviewed. Based on this review, the most suitable approach to describing the changing nature and complexity of stakeholder thinking and behaviour is selected.

\subsubsection{Evolution of Stakeholder Literature}

The concept of the stakeholder was first introduced in an international memorandum at the Stanford Research Institute in 1963 (Freeman, 1984). In this memorandum, stakeholders were defined as "those groups without whose support the organisation would cease to exist" (Freeman, 1984, p.31). After this introduction, the stakeholder concept was developed in four research areas: corporate planning, systems theory, corporate social responsibility, and organisation theory (Freeman, 1984). Following this development, Freeman (1984), in his influential book Strategic management: a stakeholder approach, defined a stakeholder as "any group or individual who can affect or is affected by the achievement of the firms' objectives" (p. 46). Freeman (1984) also emphasised that stakeholders have legitimate rights to their interests in firms, and firms should pay attention to all stakeholders. He proposed a framework to analyse stakeholders at three levels: rational, process, and transactional. Overall, Freeman (1984) was the first to offer a theoretical approach for the examination of the stakeholder concept (Elias et al., 2000). After Freeman (1984), studies in stakeholder management have followed three inter-related approaches: descriptive, instrumental, and normative (Donaldson $\&$ Preston, 1995). Studies following descriptive approaches focus on describing and explaining 
how organisations deal with stakeholders. Studies following instrumental approaches focus on evaluating the relationship between stakeholder management and organisational objectives such as profit or revenue. Studies following normative approaches focus on the legitimate rights of stakeholders (Donaldson \& Preston, 1995).

Stakeholder studies can also be divided into theoretical discussions and empirical studies. Both types of research focus on the relationship between stakeholder management and company performances (e.g., Campbell et al., 2012; Clifton \& Amran, 2011; Coombs \& Gilley, 2005; De Luque et al., 2008; Henisz et al., 2014; Hillman \& Keim, 2001; Mitchell et al., 2016; Phillips, 2010; Russo \& Perrini, 2010; Schneider, 2002). However, theoretical discussions also focus on the essence of stakeholder theory such as whether it comes from a "socialist worldview" (Freeman \& Phillips, 2002, p.14) or whether creating benefits for shareholders, in the end, will create benefits for all stakeholders (e.g., Freeman et al., 2004; Sundaram \& Inkpen, 2004). Empirical studies, on the other hand, apply the stakeholder concept to develop specific criteria to measure stakeholder benefits and behaviours (e.g., El Akremi et al., 2015; Hall et al., 2015; Harrison \& Wicks, 2012; Kaptein, 2008; Länsiluoto et al., 2013; Tantalo \& Priem, 2014).

Overall, stakeholder theory has been widely applied in different fields such as business ethics, corporate strategy, finance, accounting, management, and marketing (Parmar et al., 2010). It has also been applied in specific areas such as tourism (e.g., Moutinho \& VargasSanchez, 2018), research and development (e.g., Elias, 2016), scenario planning (e.g., Soste et al., 2015), environmental conflict (e.g., Elias, 2012), wine industry (e.g., Marshall et al., 2010), energy policy (e.g., Elias, 2008), wildlife management (e.g., Chase et al., 2002), logistics management (e.g., Knemeyer et al., 2002), manufacturing (e.g., Heugens \& Van Oosterhout, 2002), and public policy (e.g., Leach et al., 2002).

Despite these developments and applications, stakeholder literature still has limitations. One of them is the over-simplification of stakeholder thinking and behaviour (Mainardes et al., 2011). The literature does not explain sufficiently the external environment of stakeholders, the relationship between stakeholders and focal organisations (Ripolles Meliá et al., 2010; Un \& Montoro-Sanchez, 2010), the differences among stakeholders, and the division of stakeholders into groups (Lépineux, 2005). Stakeholder theory is also too ideological as it cannot explain clearly how benefits for all stakeholders are created (Antonacopoulou \& Meric, 2005; Baggio \& Cooper, 2010; Sundaram \& Inkpen, 2004). Scholars, therefore, believe that 
more studies are needed to enrich the literature and help it gain more importance in the general organisation literature (Mitchell, 2012; Pedrini \& Ferri, 2019). In order to gain a better understanding of these limitations, the next section will review stakeholder analysis methods and aspects of stakeholder thinking and behaviour that they have addressed.

\subsubsection{Stakeholder Analysis Methods}

Stakeholder analysis is "a set of (social science) methods and techniques to investigate, for example, the interest, power, resources and perspectives of stakeholders" (Cuppen, 2016, p. 208). Primarily, it seeks to identify the stakeholders, analyse their stakes, and assess their positions (Elias, 2004; Elias et al., 2001). Stakeholder analysis helps organisations understand stakeholders (Brugha \& Varvasovszky, 2000) and select suitable approaches to manage them (Cuppen, 2016). The current development of society and technology and the increasing interconnection of the world make stakeholder thinking and behaviour more complex and make stakeholder analysis more important (Bryson, 2004; Serravalle et al., 2019). A wide range of stakeholder analysis methods have been developed to analyse stakeholder thinking and behaviour (Elias, 2019; Solaimani et al., 2013).

Many stakeholder analysis methods, especially classical ones, focus on the "dyadic" (Rowley, 1997, p. 890) relationship between stakeholders and focal organisations. This dyadic relationship refers to the view that the organisation is at the centre of all stakeholder relationships. Methods focusing on this relationship differentiate stakeholders from focal organisations and consider the interaction between them, rather the interaction among stakeholders (Rowley, 1997). One example of these methods is the stakeholder management framework proposed by Freeman (1984). This framework consists of three levels of analysis which are concerned with the identification of the stakes of stakeholders, the assessment of whether the processes and procedures of organisations fit stakeholders, and the selection of approaches to manage stakeholders. Other analysis methods that focus on the dyadic relationship include the ranking of stakeholders into primary and secondary stakeholders based on their importance to organisations (Clarkson, 1995) or dividing them into the categories of internal, interface, and external based on their functional positions in relation to organisations (Fottler et al., 1989). Mendelow's (1991) attributes of stakeholder power and interest and Mitchell et al.'s (1997) stakeholder salience attributes of power, legitimacy, and urgency also reflect the researchers' focus on the dyadic relationship. They both imply that stakeholders have particular attributes in their relationships with focal organisations and focal organisations 
could classify stakeholders and identify strategies to manage stakeholders based on these attributes.

More recent approaches in stakeholder analysis have moved away from the dyadic relationship model to also account for relationships among stakeholders. One of these methods to identify what to do with stakeholders regarding particular issues was proposed by Vandekerckhove and Dentchev (2005). Their method is based on the comparison of two network maps. The first map focuses on the dyadic relationship and puts focal organisations at the centre of the networks. The second map, however, focuses on between-stakeholder relationships. Another method in this group was proposed by Memon and Wilson (2007) for their analysis of stakeholder perceptions of forests. The method is based on a combination of "multi-layered forest management decision making" and "governance" (Memon \& Wilson, 2007, p.745) concepts. The former accounts for the different political, social, economic, and cultural expectations of stakeholder groups. The latter accounts for the collaboration and interaction between state and non-state actors, which illustrates the belief that stakeholder perceptions are not only influenced by their relationship with the organisation but also by intrastakeholder interactions.

Stakeholder analysis methods also focus on the changes in stakeholder involvement over time or stakeholder dynamics. Mitchell et al.'s (1997) proposed attributes of stakeholder salience described above represent one classical method to capture these changes over time, as the authors emphasised that these attributes are susceptible to changes. More contemporary methods and studies may collect and analyse data about stakeholders over time to identify their changes. One example is the stakeholder-based innovation acceptance web (SIAW) that was developed by Postema et al. (2012) to identify the changes in stakeholder influence and acceptance of an innovation. Another example is the dynamic and interactive approach, which provides a framework to collect and analyse the changes in stakeholder perception (Pouloudi et al., 2016). The concept of systems thinking, which focuses on the interrelated components of a whole (Maani \& Cavana, 2007), is also applied to explore stakeholder dynamics. The concept can be combined with other theoretical concepts to develop frameworks to analyse the dynamics of stakeholder interaction, such as the Rapid Stakeholder and Conflict Assessment framework proposed by Hjorts $\emptyset$ et al. (2005). Methodologies developed from the concept can also be used to analyse stakeholder dynamics. One example is the causal loop models which were used in Elias (2012, 2021), Elias and Davis (2018), and Richardson and Andersen (2010) to analyse the causal effects between different stakeholder issues and interactions. Soft system 
methodology, which differentiates problems in real-world from the conceptual analysis, was also proposed to identify the changes in missions, goals, and activities of organisations and the involvement of stakeholders (Wang et al., 2015).

In summary, a wide range of methods have been developed to address different aspects of stakeholder thinking and behaviour. These methods focus on the dyadic relationship between focal organisations and stakeholders, the relationship among stakeholders, and the dynamics of stakeholders. However, these methods still have limitations. These limitations are discussed in the next section.

\subsubsection{Limitations of Current Stakeholder Analysis Methods.}

Twenty years ago, scholars, such as Beaulieu and Pasquero (2002), have argued that stakeholder analysis methods focus too much on the static interpretation of stakeholder interest, interaction and requirements. More recently, while there has been research on the dynamics of stakeholders, scholars such as Pouloudi et al. (2016), Solaimani et al. (2013) still argue the same thing. They pointed out that many publications still focus on static topics such as defining stakeholder analysis or classifying stakeholders. Little attention is paid to the changes in stakeholders or their requirements.

Stakeholder analysis methods also place too much emphasis on the roles of the so-called focal organisations and little emphasis on the relationships between stakeholders (Beaulieu \& Pasquero, 2002). Many methods assume that focal organisations identify, classify, and rank stakeholders in order to identify ways to manage them. While this approach is not wrong, it is not sufficient because stakeholder management is actually "a process of mutual contribution rather than one of unilateral accommodation" (Beaulieu \& Pasquero, 2002, p.55). The objectives of stakeholder management are not managing stakeholders but taking into consideration the presence and the influence of stakeholders into decision-making processes. Stakeholders, rather than focal organisations, therefore, must be at the core of stakeholder analysis (Beaulieu \& Pasquero, 2002).

The division of stakeholders into groups is another limitation as it cannot capture the heterogeneity of stakeholders (Beaulieu \& Pasquer, 2002; Johnson et al., 2008). For example, dividing stakeholders into groups according to roles cannot capture the differences of individuals taking the same roles. If a new individual takes that role, their position and activities related to that role can change significantly. Stakeholder analysis, therefore, must pay attention to the differences among individual stakeholders (Johnson et al., 2008). 
In summary, current stakeholder analysis methods are limited in terms of their ability to capture the dynamics, complex relationships, and individual differences of stakeholders. As the presentation of existing stakeholder analysis methods reflects, while the methods have focused on the dynamics of stakeholders in relation to their complex relationships with the organisation and other stakeholders (e.g., Elias \& Davis, 2018; Richardson \& Andersen, 2010), they do not explore the changes in light of the stakeholders' individual characteristics. As a result, current approaches are only able to capture a partial picture of the complexity of stakeholder thinking and behaviour. Studies that explore all the aspects of stakeholder dynamics, complex interactions and individual differences will provide greater insights into this complexity. These aspects are reviewed in detail in the next sections.

\subsubsection{Stakeholder Dynamics in Their Dyadic Relationship with Focal Organisations}

Dynamics can be understood as changes over time. The opposite of a dynamic state is a static state which does not include time dimensions (Windsor, 2010). Since the inception of the field, researchers have been concerned about capturing the dynamic nature of stakeholders. For example, when proposing the stakeholder map, Freeman (1984) highlighted that the map depicts stakeholders as static even though stakeholders and their stakes change over time. Despite this early indication, stakeholder dynamics have been overlooked by scholars for a long time (Fassin, 2008). Recently, with the development of theories and methodologies in related fields such as the strategy and organisation literature, stakeholder dynamics have been paid more attention to, and different aspects of stakeholder dynamics have been explored (Windsor, 2010).

Many studies focus on the dynamics of the dyadic relationship between stakeholders and focal organisations. For example, studies found that the composition of stakeholders is dynamic. New stakeholders may appear while old stakeholders are forced out of context. (Pouloudi \& Whitley, 1997). The extent to which stakeholders are involved in the decisionmaking processes of focal organisations can also shift between different forms: informative, consultative, and decisional (Green \& Hunton-Clarke, 2003). The thinking and behaviour of stakeholders and focal organisations are not only dynamic but can also influence each other. Regarding the influence of stakeholder thinking on their behaviour, Agle et al. (1999) found that manager perception of stakeholder salience impacts how they prioritise stakeholders. Savage et al. (2004) demonstrated that stakeholder perception of the creditability of organisational leaders and the legitimacy of organisational activities affect their support to focal organisations in crises. Meanwhile, regarding the impact of stakeholder behaviour on 
their thinking, Eesley and Lenox (2006) found that the legitimacy and urgency of the requests of stakeholders affects manager perception of stakeholder salience.

In summary, the thinking and behaviour of stakeholders and focal organisations in their dyadic relationship are dynamic and influence each other. In other words, one change in the behaviour and activities of stakeholders or focal organisations can lead to changes in their thinking or perception, and these changes in the thinking or perception can lead to other changes in other activities and behaviour. These dynamics together can explain the complexity and changing nature of stakeholder thinking and behaviour. However, as suggested in the discussion of the limitations of current stakeholder analysis methods (section 2.2.3), stakeholders can have complex relationships with each other, which cannot be captured by their dyadic relationship with focal organisations. These complex relationships are discussed in the next section of social networks and stakeholder networks.

\subsubsection{Social Networks and Stakeholder Networks}

A social network is a set of socially relevant nodes that are connected by one or more relations (Scott \& Carrington, 2014). These nodes are called network members or units and are most commonly persons or organisations (Scott \& Carrington, 2014). Research began to employ social networks to describe the shape and characteristics of social structures in the 1930s (Scott $\&$ Carrington, 2014). Social network studies emerged in the 1970s when researchers considered network analysis to be a useful approach to studying social connections among organisations (Wasserman \& Galaskiewicz, 1994). During the 1990s, social network analysis was further developed and applied in many different fields (Carrington et al., 2005). One important issue in studying social networks is identifying network boundaries or, in other words, actors and activities that belong to networks (Laumann et al., 1989; Marsden, 2005). The characteristics, participation, and social connections of actors can be used to identify these boundaries (Laumann et al., 1989).

Social network approaches have been proposed as one of nine lenses to study and describe organisations and stakeholders (Ramirez, 1999). Following these approaches, organisations are conceptualised as sets of multilateral relationships among stakeholders (Freeman \& Evan, 1990). These relationships create complex networks of interaction, interests, and power. Some of them are formal, visible, and direct; some are subtle and indirect (Pouloudi $\&$ Whitley, 1997). In these networks, a stakeholder can both be a member of a variety of groups (Key, 1999) and have a subset of stakeholders (Fassin, 2008) at the same time. The 
relationships in these subsets can affect stakeholders more than the relationships with other direct stakeholders of focal organisations (Fassin, 2008). Formal and informal relationships are also equally important in stakeholder networks (Ackermann \& Eden, 2011). The boundaries of stakeholder networks, therefore, can be blurred. Distant stakeholders, such as media and environmental groups, may have a great influence on organisations, while more direct stakeholders, such as employees or suppliers, only influence them slightly (Phillips, 2004). An illustration of stakeholder networks is presented below.

Figure 2.1 An illustration of a stakeholder network. Reprinted from "Moving beyond dyadic ties: a network theory of stakeholder influences" (Rowley, 1997)

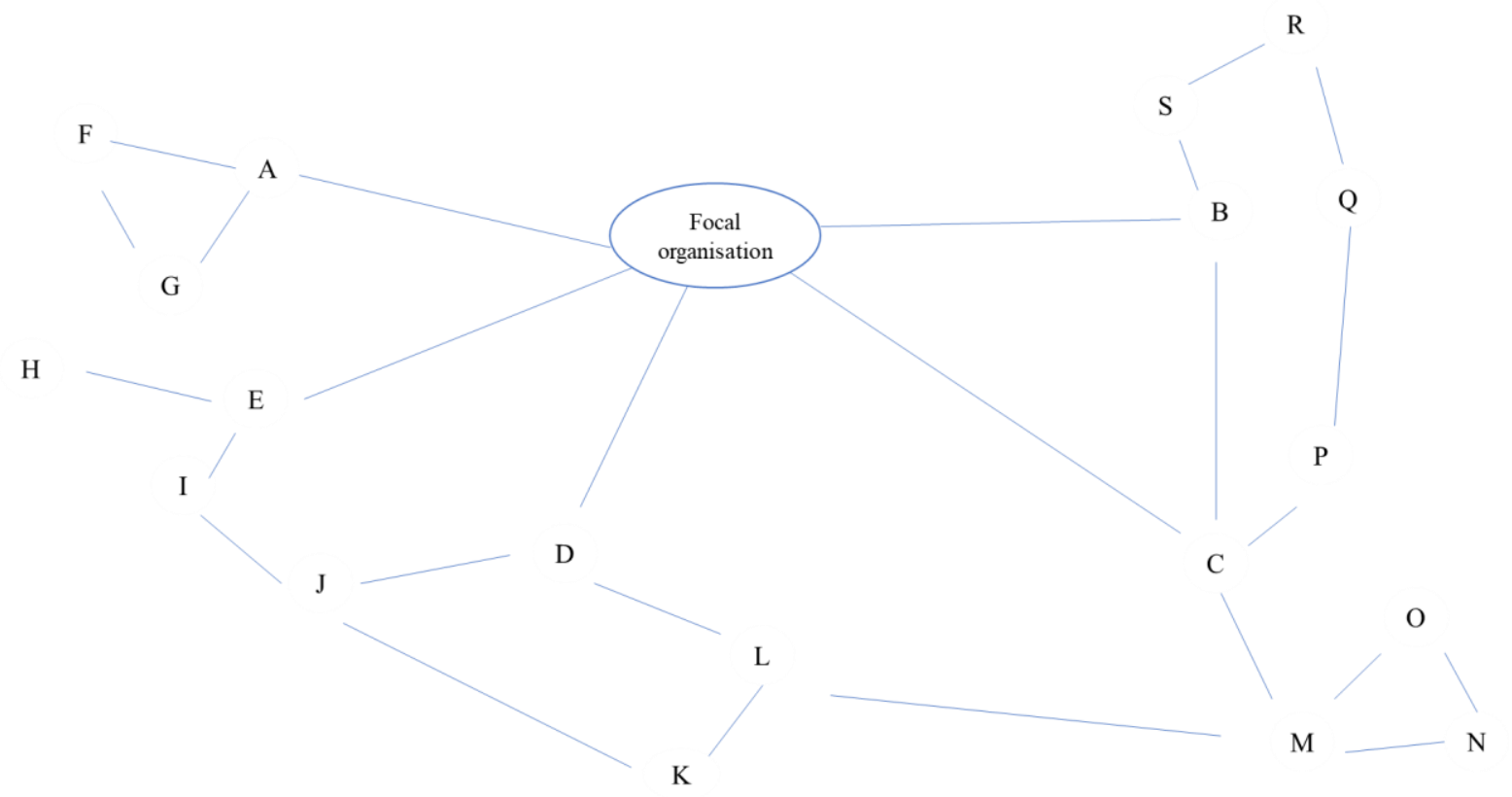

Given the complex relationships of stakeholders in their networks, scholars (e.g., Baron, 1995; Post et al., 2002; Rowley, 1997) have emphasised the importance of analysing and dealing with stakeholders under the consideration of their networks. Along with this emphasis, many studies of stakeholder dynamics pay attention to stakeholder networks. These studies are reviewed in the next section.

\subsubsection{Stakeholder Dynamics and Stakeholder Networks}

Various studies have investigated the dynamics of stakeholders with regard to their social networks. In these networks, stakeholders may choose to act independently, act together, or oppose each other and create dynamic and turbulent environments for organisations (Frost, 1995). Along with these actions, the roles, perspectives, and alliances of stakeholders may change (Pouloudi et al., 2016). The dynamics of stakeholder interaction in their networks are 
critical to making significant changes to organisations (Lamberg et al., 2008). In special situations, such as over different phases of public-private partnership (South et al., 2015), during the transformation of universities to be more "entrepreneurial" (Miller et al., 2014, p. 268), or during crises (Engelbrecht and Thomas, 2017), stakeholder interaction in the networks also change substantially.

The interactions of stakeholders within their networks are also dynamically related to each other. Put simply, an action of a stakeholder can lead to a series of actions of other stakeholders (Dorobantu et al., 2016). In a more complex example, stakeholder interactions, which are described by their positions in their networks, are found to affect their behaviour (Mahon et al., 2004) or their abilities to communicate with other stakeholders (Ziervogel \& Downing, 2004). Stakeholder interaction in the networks can also impact activities of focal organisations, such as focal organisations' implementation of sustainable practices (Afreen \& Kumar, 2016; Martini \& Buffa, 2015), or their decisions to keep or not keep their promises to stakeholders (Crilly et al., 2012). Stakeholder networks also affect the functional positions that stakeholders can have in relation to organisations (Pouloudi et al., 2016) or their levels of competition and cooperation (Akpinar \& Vincze, 2016).

Stakeholder perception and interactions in their networks can also influence each other. Such interactions have been found to influence stakeholder thinking and perception include the institutional environment (Khurram \& Petit, 2017), the alliance of stakeholders (Vallaster \& Von Wallpach, 2013), the positions of stakeholders in their networks (Pajunen, 2006). These interactions also include the interaction between stake-seekers whom organisations benefit indirectly and stake-watchers who supervise whether organisations provide benefits to stakeholders (Fassin, 2010). The interactions also include "emotional laden events" (Sloan \& Oliver, 2013, p.1845) which refer to critical turning points of the partnerships between stakeholders and can trigger intensive feelings in stakeholders. Stakeholder thinking and perception which is influenced by these interactions includes the trust between stakeholders (Sloan \& Oliver, 2013) and stakeholder positions on the online branding of organisations (Vallaster and Von Wallpach, 2013). This thinking and perception also includes organisations' perception of different aspects of stakeholders such as stakeholder influence on the survival of organisations (Pajunen, 2006), stakeholder salience or which stakeholders that organisations should pay attention to (Fassin, 2010), and the relationship between different attributes of this salience (Khurram and Petit, 2017). Stakeholder thinking and perception itself can also affect stakeholder interactions in their networks. For example, in a study on actions of individual 
stakeholders in groups, Rowley and Moldoveanu (2003) noted that the appreciation of stakeholders of their identities associated with their groups affects their likelihood to act to influence organisations.

The interrelationship between stakeholder thinking and their networks can also be described in detail. For example, the pre-existing beliefs of stakeholders about an organisation influence the ways in which they react to organisations. Shareholder perception of these beliefs and reactions, in turn, affects their valuation of organisations (Dorobantu et al., 2016). In another example, Friedman and Miles (2002) described stakeholder interaction as the institutional and contingent factors and stakeholder perception as the sets of ideas held by stakeholders or organisations and their material interests. The changes of these interactions and perception can lead to the changes in the relationships between stakeholders and focal organisations, which are described by two aspects: the compatibility of material interests and the forms of the contracts between stakeholders and focal organisations. Finally, Orlikowski and Gash (1994) have shown that stakeholders' frames of reference affect their actions. Stakeholders' frames of reference are implicit guidelines that organise and shape stakeholders' interpretations of organisations' events and phenomena. Stakeholders' frames of reference are also dynamic and change according to different social contexts (Orlikowski \& Gash,1994).

In summary, stakeholder dynamics should be studied not only with regard to their dyadic relationship with focal organisations but also their networks. Stakeholders have complex interactions in their networks. In these networks, stakeholder interactions are dynamic and influence each other. There are also dynamic relationships between the stakeholder perception and interaction in these networks. In other words, one interaction of stakeholders in their networks can change stakeholder thinking and perception. These changes, in turn, can lead to changes in other interactions of the stakeholders. The dynamics of stakeholder perception in their networks, therefore, can explain the dynamics of stakeholder interactions in the networks. Overall, the complexity of stakeholder thinking and behaviour can be explained by the dynamics of stakeholder perception, as influenced by their networks. However, as suggested in the discussion of the limitations of stakeholder analysis methods (section 2.2.3), these dynamics are not enough to explain the complexity of stakeholder thinking and behaviour because they do not capture the differences among individual stakeholders. These differences and their impacts on stakeholder thinking and behaviour are reviewed in the next section. 


\subsubsection{Individual Differences of Stakeholders}

Studies have shown that individual stakeholders are different in many aspects. First, an individual stakeholder can occupy several roles or belong to more than one stakeholder group at the same time (Jansson, 2005; Post et al., 2002; Pouloudi et al., 2016) and therefore can have different or conflicting objectives and priorities (Pouloudi \& Whitley, 1997). For example, a manager can be a representative of a social or industrial association. An employee can be an environmental activist or a consumer of environmentally damaging products (Winn, 2001). They, therefore, can have a hidden agenda, make it difficult to predict their thinking and behaviour. For example, they may be less likely to take action with the whole groups. Instead, they may try to affect other members in taking actions according to their hidden agendas (Rowley \& Moldoveanu, 2003).

Individual stakeholders can also have diverse characteristics that are products of their personal background and experience. These characteristics are found to affect their thinking and behaviour. For example, individuals in the same community group can have different interests and needs based on their ages, gender, classes, castes, ethnicities, and religions (Hjorts $\varnothing$ et al., 2005). Individual stakeholders' identities and values can also be different and affect their judgment and behaviour (Pouloudi et al., 2016; Rowley \& Moldoveanu, 2003). For example, CEOs' values affect what they found important, which affects their perception of stakeholder salience (Agle et al., 1999). The social identity of stakeholders, which refers to their beliefs of their images regarding their roles, positions and relationships (Rowley \& Moldoveanu, 2003), affects their thinking and behaviour in complex ways. Individual stakeholders may tend to affiliate with ones who do not have similar identities and become hostile to ones who have similar identities. Stakeholders with similar identities may also choose to behave differently to make themselves unique (Rowley \& Moldoveanu, 2003). While values and identities are important, studying them is not easy because they can be implicit or hidden (Pouloudi et al., 2016).

Other more specific individual characteristics of stakeholders have also been found to affect their thinking and behaviour. The characteristics related to top managers include their formal education, work experience, and tenure (Carter, 2006), skills of implementation (Crilly et al., 2012), identity awareness (York et al., 2016), and interest in activities that benefit a broad scope of stakeholders (Coombs \& Gilley, 2005). The characteristics related to general stakeholders include their skills (Jansson, 2005), their priorities (Wolfe \& Putler, 2002), psychological dispositions (VonWerder, 2011), care about fairness (Bridoux \& Stoelhorst, 
2014), "relational models" (Bridoux \& Stoelhorst, 2016, p. 230), concerns about relationships (Weitzner and Deutsch, 2015), and concerns about privacy (Fedorowicz et al., 2010). These characteristics reflect the experience and background of stakeholders. For example, identity awareness refers to the alignment between the "commercial and ecological identities" (York et al., 2016, p. 12) of stakeholders while "relational models" refers to stakeholders' expectations of their own actions and other stakeholder actions. They can think of their interests, be altruistic, or want equality (Bridoux \& Stoelhorst, 2016). Similarly, the characteristic "concerns about relationships" refers to stakeholders' concerns about whether the relationship follows particular values, needs to be maintained, or needs to be rewarded or punished (Weitzner \& Deutsch, 2015). These characteristics reflect only a small selection of possible individual experiences that may affect a stakeholder's decisions, which highlights the importance to consider the background of stakeholders.

The characteristics mentioned in the above paragraph were found to affect different thinking and behaviours of stakeholders. The types of affected thinking and behaviours that have been explored previously include the policies of focal organisations (Crilly et al., 2012), their "reputation management" (Carter, 2006, p. 1145) strategies, engagement in social and environmental activities (York et al., 2016), view of activities that benefit a broad scope of stakeholders and of people engaging in those activities (Coombs \& Gilley, 2005), and approaches to manage stakeholders (Bridoux \& Stoelhorst, 2014). With regard to stakeholders, the types of thinking and behaviours that have been documented include their thinking and behaviours in regard to focal organisations, such as their levels of commitment to the survival of organisations (Jansson, 2005), what they want from focal organisations (Wolfe \& Putler, 2002), their view about data sharing programmes (Fedorowicz et al., 2010), and their contributions to the social activities of focal organisations (Bridoux \& Stoelhorst, 2016). Stakeholder thinking and behaviours regarding other stakeholders have also been studied and include their opportunistic behaviours to each other (Von Werder, 2011), and their prioritisation and attention to particular claims (Weitzner \& Deutsch, 2015).

Overall, the review of the individual differences of stakeholders identifies that a variety of stakeholder characteristics impact their thinking and behaviour. This variety indicates that dividing stakeholders into groups according to a few particular characteristics would mean that these impacts cannot be captured. In addition, many of these characteristics are linked to stakeholder experience and background. The impacts of these individual characteristics, combined with the dynamics of their thinking and behaviour in their networks, as identified in 
sections 2.2.4 and 2.2.6, therefore, can explain the complexity of stakeholder thinking and behaviours. Given the dynamic relationship between stakeholder perception and interaction in their networks, the dynamics of stakeholder perception, as influenced their networks and individual characteristics or in other words, the dynamics of stakeholders at individual levels can explain the complexity of stakeholder thinking and behaviour. This is also the approach to describe the complexity of stakeholder thinking and behaviour.

\subsubsection{Summary of Stakeholder Literature Review}

In summary, this review of stakeholder literature highlights that within the evolution of stakeholder literature, the prevalent stakeholder analysis methods used are still not able to fully explain the complexity of stakeholder thinking and behaviour. Specifically, the methods have not addressed the dynamics, social networks, and individual differences of stakeholders simultaneously. The review of stakeholder dynamics in their dyadic relationships with focal organisations then identifies the complexity of stakeholder thinking and behaviour can be described as the dynamic relationship between the thinking and behaviour of stakeholders and focal organisations. The review of stakeholder dynamics in their networks then shows that this complexity can be described as the dynamic relationship between stakeholder perception and their networks. Meanwhile, the review of individual differences of stakeholders shows that the impacts of different characteristics of stakeholders on their thinking and behaviour can also explain this complexity. Overall, this review indicates that the complexity of stakeholder thinking and behaviour can be explained by the dynamics of stakeholder perception, as influenced by their networks and individual characteristics or in other words, the dynamics of stakeholder perception at individual levels. Because this study focuses specifically on project stakeholders, the literature of stakeholders in projects is reviewed in the next section.

\subsection{Stakeholders in Projects}

Following the review of the stakeholder literature, this section begins with an overview of projects and project stakeholders. It then continues with a review of stakeholder analysis methods in projects and their limitations. Finally, the dynamics of project stakeholders in their dyadic relationship with focal projects, their dynamics in their networks, and their individual differences are reviewed.

\subsubsection{Overview of Projects and Project Stakeholders}

The field of project management was developed from the need of construction and defence industries to plan, control, and manage complex series of tasks such as building hospitals or 
bridges during the 1940s and 1950s (Morris, 1994). Projects are often established to perform specific tasks, and when the tasks are completed, they cease. Project tasks are often not routine but unique, novel, and have some uncertainty and risk (Turner et al., 2010). Projects, therefore, are different from permanent organisations in terms of history, planning, learning, and adaptation (Kreiner, 1995). Although projects are temporary, they are not "lonely" (Engwall, 2003 , p. 790) but open with regard to both time and space. In terms of time, project activities are affected by the experience that project members gained from their past activities, policies that governments and parent organisations issued before the project commencement, events that happen during the project implementation, and expectations that project members have for the project completion. In terms of space, projects are affected by norms, values, routines, and procedures of parent organisations (Engwall, 2003). The procedures of projects can be in line with those of parent organisations or specially customised (Engwall, 2003; Modig, 2007). Stjerne and Svejenova (2016) call these the levels of "attachment" and "detachment" (p.7) of projects and parent organisations.

Project management research became interested in stakeholder thinking in the 1980s through the works of researchers such as Cleland (1986) (Aaltonen et al., 2015). Since then, stakeholder theory has been widely applied in studies of projects (Littau et al., 2010). While the early studies focused on the application of different terminologies of stakeholders in the context of projects, the later studies focused on exploring the complexity of project environment (Littau et al., 2010). Project stakeholders are defined as "people, groups or organisations that could impact or be impacted by a decision, activity or outcome of projects" (Project Management Institute, 2013, p. 391). Stakeholders are expected to provide contributions to project implementation (Eskerod, Huemann \& Savage, 2015). Such contributions range from financial investments to the provision of expertise or supportive behaviour. Examples of supportive behaviour are offering ideas and compliance of stakeholders, and their acceptance of project outputs (Eskerod, Huemann \& Savage, 2015). However, during project implementation, stakeholders may not be committed to projects (Antony et al., 2019; Catalano et al., 2019) or may not have enough skills or abilities to make the expected contributions (Hughes et al., 2016; Mukherjee, 2019). Similarly, while the success or failure of projects depends on stakeholder judgments, these judgments are complex. In many cases, stakeholders may regard projects as unsuccessful, even if they are completed within the original time, budget, and scope (Bourne \& Walker, 2005). 
Project stakeholder analysis helps to build "correct" (Aaltonen, 2011, p.1) pictures of the stakeholder environment (Aaltonen, 2011). These pictures help project managers understand and address opportunities and problems in projects (Andersen et al., 2009). Understanding stakeholders is also challenging because they have free will on whether to provide their contributions, and this free will depends on their expectations, objectives, and interests (Bourne, 2008). These expectations, objectives, and interests are not only different among stakeholders but also susceptible to changes. Stakeholders, therefore, may not act as expected (Aaltonen \& Kujala, 2010; Yang et al., 2014).

In summary, as the review of the literature on stakeholders in projects highlights, the time and space contexts of projects are complex and temporary. Stakeholder analysis, which explores stakeholder thinking and behaviour, provides more understanding of these contexts. The existing stakeholder analysis methods in projects and the aspects of stakeholder involvement that they address will be reviewed in the next section.

\subsubsection{Project Stakeholder Analysis Methods}

Similar to the general stakeholder analysis methods that have been presented in section 2.2.2, a wide range of methods have been developed to analyse stakeholder thinking and behaviour in projects. Many methods analyse stakeholders in their dyadic relationship with focal projects. One example is the Q methodology (Cuppen et al., 2016; Wolsink \& Breukers, 2010), which was designed to identify stakeholder perception and concerns. This method begins by eliciting statements that reflect stakeholder opinions about projects. These statements are then ranked and arranged into tables. The principal component analysis of the tables identifies the factors that describe the main opinions of stakeholders. In addition to Q methodology, visualisation support methods, such as 3D models (Lange \& Hehl-Lange, 2005), are also used to elicit information from stakeholders. Another method which focuses on identifying the underlying concepts of stakeholders' thinking and opinions was also presented in McKenna and Metcalfe (2013). On the same theme, Metcalfe and Sastrowardoyo (2013) applied Toulmin's model of arguments to analyse and explore the conceptual or philosophical arguments of different groups of stakeholders.

Project stakeholder analysis methods also focus on the relationships among stakeholders and their social networks. These methods include frameworks and concepts that analyse stakeholders based on criteria related to stakeholder networks. One example is the Analytical Network Process which is based on identifying and rating a list of criteria to analyse 
stakeholder influence on projects (Aragonés-Beltrán et al., 2017). The criteria of "social skills" or "external” (Aragonés-Beltrán et al., 2017, p.5) refer to the relationships among stakeholders. Another example is Van Offenbeek and Vos' (2016) framework to prioritise stakeholder issues based on the legitimacy of stakeholders and how they can affect each other. Aaltonen and Kujala's (2016) concept of "stakeholder landscape" (p.1538) is another example as it involves relationships among stakeholders to describe stakeholder environment. Yang's (2014) proposal of using "rationalism" (p.840) perspectives to identify and prioritise stakeholders also focuses on these relationships. These perspectives refer to the logical and rational identification of stakeholder networks in general and the application of social networks analysis in particular (Yang, 2014). Following these perspectives, social network analysis attributes, such as network centrality and density, have been used to identify different aspects of stakeholders such as their concern, risk, and salience (Lim et al., 2010; Mok et al., 2017, Yang et al., 2016).

Some project stakeholder analysis methods also focus on stakeholder dynamics. These dynamics can be explored by interviewing and collecting data on the changes in stakeholders, such as their positions and motivation, over time (Spangenberg et al., 2018). Frameworks and concepts can also be used in these interviews. Examples are the stakeholder impact index (Olander, 2007), the Stakeholder Circle Model (Bourne, 2008), and the social mapping framework (Hazelton et al., 2013). These frameworks and concepts specifically focus on the changes in the impact of stakeholders on projects (Olander, 2007), the membership and influence of stakeholders (Bourne, 2008) and their power and risks (Hazelton et al., 2013). Frameworks have also been developed to capture the dynamics of stakeholders. One example is the Value, Information, and Process Framework, which describes the dynamic interaction among stakeholders in three domains: value, information, and business processes (Solaimani et al., 2013). System dynamics in system thinking are also applied to analyse the dynamics of different aspects of stakeholder involvement, including their participation processes (Elias \& Zwikael, 2007), issues (Elias, 2017; Yang \& Yeh, 2014), involvement (Elias, 2019), and interaction (Elias, 2016).

In summary, similar to general stakeholder analysis methods, stakeholder analysis methods in projects have addressed the dyadic relationship between stakeholders and focal projects, the relationships among stakeholders in their networks, and the dynamics of stakeholders. Examples of the methods that have been discussed in this section are summarised in Table 2.1 as follows. 
Table 2.1 Examples of project stakeholder analysis methods

\begin{tabular}{|c|c|c|}
\hline Focus of methods & Descriptions of Methods & Authors \\
\hline \multirow[t]{2}{*}{ Dyadic relationship } & Q methodology & $\begin{array}{l}\text { Wolsink and Breukers (2010), } \\
\text { Cuppen et al. (2016) }\end{array}$ \\
\hline & $\begin{array}{l}\text { Visualisation support methods } \\
\text { (e.g., 3D models) }\end{array}$ & Lange \& Hehl-Lange (2005) \\
\hline \multirow[t]{6}{*}{ Stakeholder networks } & Analytical Network Process & Aragonés-Beltrán et al. (2017) \\
\hline & $\begin{array}{l}\text { Framework based on the } \\
\text { commonality and dependence } \\
\text { of issues }\end{array}$ & van Offenbeek and Vos (2016) \\
\hline & Stakeholder landscape & Aaltonen and Kujala (2016) \\
\hline & Social network analysis & $\begin{array}{l}\text { Lim et al. (2010), Mok et al. } \\
\text { (2017), and Yang et al. (2016) }\end{array}$ \\
\hline & $\begin{array}{l}\text { Index to evaluate stakeholder } \\
\text { impact }\end{array}$ & Olander (2007) \\
\hline & Stakeholder Circle Model & Bourne (2008) \\
\hline \multirow[t]{3}{*}{ Stakeholder dynamics } & Repeated interviews & Spangenberg et al. (2018) \\
\hline & $\begin{array}{l}\text { Value, Information and } \\
\text { Process Framework }\end{array}$ & Solaimani et al. (2013) \\
\hline & System dynamics & $\begin{array}{l}\text { Elias and Zwikael }(2007) \text {, } \\
\text { Elias (2017), Elias }(2019) \text {, } \\
\text { Elias (2016) }\end{array}$ \\
\hline
\end{tabular}

\subsubsection{Limitations of Project Stakeholder Analysis Methods}

Similar to general stakeholder analysis methods, the stakeholder analysis methods in projects are limited in their capacity to capture the full complexity of stakeholder thinking and behaviour. Researchers have used different terms to describe this complexity. For example, Kreiner (1995) used the term "drifting environment" to describe that while stakeholder thinking and behaviour are susceptible to changes, in many cases, the reasons for these changes are not related to other stakeholders of projects but in the wider project context. Projects do not interact with a set of external stakeholders, but a set of stakeholders who are situated in their individual social context. Stakeholder thinking and behaviour, therefore, can be affected by the dynamics of their individual social context. For example, the commitment of parent organisations or the community and government support to projects may change due to reasons beyond project managers' understanding. A project, therefore, operates within a context characterised by a "bewildering complexity of relationships that not necessarily centre around the project itself" 
(Kreiner, 1995, p. 341). The ways in which feedback from stakeholders is understood are also complex (Kreiner, 1995). Project managers can try to seek and understand stakeholder feedback, but it is not always clear what can be learnt from it. Project managers and stakeholders can understand the feedback differently and, consequently, respond differently. In other words, the complexity does not lie in how much information about stakeholders project managers can receive but how they make sense of that information (Kreiner, 1995).

Although Kreiner (1995) emphasised the complexity of stakeholder thinking and behaviour more than twenty years ago, current projects are still characterised by the fact that stakeholder expectations are not sufficiently considered or met (Eskerod, Huemann \& Savage, 2015). Specifically, while much research has focused on the structure complexity and uncertainty of projects, less research has focused on their dynamics and social and political complexity (Geraldi et al., 2011). The social and political complexity refers to the complexity created by the differences between individuals, such as their personality, aspirations, interests, mental models and values (Geraldi et al., 2011). Acknowledging this situation, Eskerod and Larsen (2018) proposed the concept of "shadow of context" to describe how projects are affected by their past, present, and future. Because project managers cannot know everything about the past, present, and future of stakeholders, only stakeholders really know their positions regarding projects. Profound and holistic approaches, rather than reductionist approaches, should therefore be used to study stakeholders. Eskerod and Larsen (2018) called these approaches "shadow of context" (p. 161) approaches. In comparison to reductionist approaches, these approaches are more complex because they require project managers to deal with the complexity of the motives and circumstances that influence stakeholder behaviour and focus on both sides: projects and stakeholders.

"Shadow of context" approaches reflect the "non-deterministic" (Padalkar \& Gopinath, 2016, p.1305) perspective that Padalkar and Gopinath (2016) have proposed for studies in project management. Their proposal emerged from a review of research themes in project management over six decades between 1960 and 2015. This review found that many studies focused on empiricist and deterministic perspectives. Even though these studies have helped to improve project implementation, they are not suitable to study complex project management issues such as project risks and strategies. Following other studies that have explored the complexity and uncertainty of phenomena in projects (e.g., Azim et al., 2010; Thamhain, 2013; Perminova et al., 2008), Padalkar and Gopinath (2016) proposed that more studies of the nondeterministic perspective of projects are needed. 
In summary, only a small subset of the current project management literature addresses the complexity of stakeholder thinking and behaviour. This complexity can be described with reference to stakeholder dynamics as well as their social networks and individual differences. Thus, studies that focus on all of these aspects will provide a deeper understanding of stakeholder thinking and behaviour in projects. Following this indication and similar to the literature of stakeholder management, the dynamics of project stakeholder in their dyadic relationships, the dynamics of project stakeholders in their networks and the individual differences of stakeholders will be reviewed in the following sections.

\subsubsection{Stakeholder Dynamics in Their Dyadic Relationships with Focal Projects}

Dynamism is one inherent characteristic of projects (Collyer \& Warren, 2009). Yet it is an area currently still under-researched (Jepsen \& Eskerod, 2009; Mok et al., 2015). Not many existing studies, frameworks, and tools focus on the dynamic state of projects compared to the static state (Aaltonen et al., 2015). These studies, frameworks, and tools, especially the ones focusing on the dyadic relationships between stakeholders and focal projects, are reviewed in this section.

Studies have examined the dynamics of the dyadic relationship between stakeholders and focal projects. Of interest so far were the dynamics of the interaction between stakeholders and focal projects (Boonstra, 2006), stakeholder influence on focal projects (Olander and Landin, 2005), and the communication modes of stakeholders with focal projects (Turkulainen et al., 2015). Studies have also shown that there are dynamic relationships between the perception and interaction of stakeholders and focal projects. For example, Gällstedt (2003) found that critical incidents, such as the loss of valuable resources or the changes in the preferences of project owners, affect stakeholder perception of the working conditions in

projects as well as their motivation and levels of stress. Boonstra et al.'s (2008) research demonstrated that through the use of strategic activities, project managers can not only change their own interests and power but also those of other stakeholders and thereby increase stakeholder interest in and support to projects. Examples of these activities are having experts explain the project merits or inviting stakeholders to participate in projects. On the same theme, Gattiker and Carter (2010) found that project champions who advocate environmental projects and policies can use different tactics to influence other stakeholders of the projects. These tactics can change the commitment of these stakeholders to the projects. In another example, Liu and Chiu (2016) found that pre-project partnering, which refers to project managers' activities to involve stakeholders before project commencement, can make stakeholders clearer 
about their roles and affect project managers' perception of project risks. Similarly, Eskerod and Vaagaasar (2014) found that the changes in focal projects' strategies to manage stakeholders can lead to the changes in stakeholders' trust in focal projects.

Overall, research has shown that the thinking and behaviour of stakeholders and focal projects in their dyadic relationship is dynamic and influence each other. These dynamics and influence can explain the complexity of stakeholder thinking and behaviour in projects. This observation is in line with the outcome of the review of the stakeholder dynamics in their dyadic relationship with focal organisations in section 2.2.4. However, similar to the review in section 2.2.4, with the focus on the dyadic relationship, it seems that these dynamics are not enough to explain the complexity of the thinking and behaviour of project stakeholders because project stakeholders may have complex relationships with each other. These complex relationships and the dynamics related to them are reviewed in the next section.

\subsubsection{Project Stakeholder Networks and the Dynamics of Project Stakeholders}

Dyadic approaches still dominate project stakeholder research (Eskerod, Huemann, \& Savage, 2015; Missonier \& Loufrani-Fedida, 2014; Papadopoulos \& Merali, 2008). However, these approaches place projects at the centre of stakeholder networks and are limited in their description of the relationship between projects and stakeholders. Projects may not be at the centre of stakeholder attention, and each project stakeholder can have their own social network and set of stakeholders. These sets of stakeholders may affect the stakeholders more than other stakeholders who are directly linked to the projects. Stakeholders may also relate to and influence each other more than the project team and may form coalitions that a dyadic analysis would not be able to capture. Many scholars, therefore, have proposed social network approaches to study not only the networks of project stakeholders but also their dynamics (Mok et al., 2015; Yang et al., 2011).

The complex interaction of project stakeholders in their networks and their dynamics have been described in many empirical studies. For example, Mead (2001) found that the communication of project team members is so complex that they should be described by social network analysis attributes, such as network centrality or the shapes of vectors in networks. In another study, Papadopoulos and Merali (2009) found that the dynamics of stakeholder interaction in lean projects can be described as the interaction between the local and global stakeholder networks. The local networks are the ones inside a project and represent the interaction of actors that implement the project. The global networks are the networks outside 
a project and represent the interaction of actors that provide the project with resources such as money, expertise, and political support. These two networks can interact with each other and use the project as a space to negotiate and achieve their diverse interests (Papadopoulos and Merali, 2009).

There are also dynamic relationships between different stakeholder interactions in their networks. These relationships can be described in simple ways, such as the relationships between the engagement of different stakeholders in projects (Beringer et al., 2012; Zwikael et al., 2012) or the impact of stakeholder communication on their participation in change management processes (Butt et al., 2016). These relationships can also be described through the lens of stakeholder networks. For example, both the positions of focal projects and the power and responses of stakeholders in the networks are susceptible to changes and these changes can lead to changes in focal projects' strategies to deal with stakeholder networks (Aaltonen \& Sivonen, 2009). In another example, online communication networks of project stakeholders, which can be divided into different stable clusters, affect the way in which stakeholders influence projects. Stakeholders in large clusters often deploy their power directly by manipulation, and stakeholders in smaller clusters often form alliances with more powerful groups (Williams et al., 2015).

Research has particularly shown that there are dynamic relationships between stakeholder perception and interaction in their networks. For example, general stakeholder interaction was found to affect the alignment between stakeholder and project interests (Missonier \& Loufrani-Fedida, 2014), stakeholders stances and expectation of projects (Pan, 2005), and stakeholder perception of project visions (Besson \& Rowe, 2001). Moreover, interactions, which have been described as changes in contextual conditions such as political, institutional, and social situations (Aaltonen et al., 2015, Cuppen et al., 2015, Elias et al., 2004), affect stakeholder thinking. This thinking includes stakeholder opposition to focal projects (Aaltonen et al., 2015), stakeholder thinking about what projects should do to achieve particular ends (Cuppen et al., 2015), and stakeholder positions and interests (Elias et al., 2004).

Studies have also described the impact of stakeholder interaction in their networks on stakeholder perception in detail. For example, Aaltonen et al. (2008) found that stakeholder activities in their networks, such as withholding the resources that they provide, creating coalitions with other stakeholders, or influencing the resources which are provided by other stakeholders, affect project managers' perception of stakeholder salience. Eskerod, Huemann, 
and Ringhofer (2015) also highlighted how stakeholder inclusiveness can make projects lose the focus on important stakeholders even though it can make some stakeholders more engaged and satisfied. Stakeholder inclusiveness means that projects not only care about and involve some key stakeholders such as suppliers, customers, or employees but also care about and involve wider groups of stakeholders such as labour unions, or public media. Stakeholder inclusiveness can also raise stakeholders' expectations, and because projects often cannot satisfy stakeholders as much as it is expected, the included stakeholders will be disappointed.

In summary, project stakeholders engage in complex and dynamic networks of interaction. In these networks, there are dynamic relationships between stakeholder perception and interaction. This suggests that the dynamics of stakeholder perception, as influenced by their networks, can explain the complexity of stakeholder thinking and behaviour. This observation aligns with the outcomes of the review of stakeholder dynamics, as influenced by their social networks, presented in section 2.2.6. Moreover, as suggested in the discussion of the limitations of project stakeholder analysis methods in section 2.3.3, these dynamics are not enough to explain the complexity of stakeholder thinking and behaviour because they do not account for the differences among individual stakeholders. These differences and their impacts on the thinking and behaviour of project stakeholders are reviewed in the next section.

\subsubsection{Individual Differences of Project Stakeholders}

Similar to scholars in stakeholder management, scholars in project management have argued that stakeholder analysis will provide better results if stakeholder categories are broken down into smaller categories or at individual levels (Eskerod, Huemann, \& Savage, 2015). These arguments are also in line with Engwall's (2003) argument to study projects in light of their history and future because the experiences, characteristics, and aspirations of individual stakeholders can provide implications about these aspects of projects.

Among the characteristics that have been found to affect stakeholder interactions are the beliefs of project managers about whether they can analyse or change the environment (Aaltonen, 2011) and their emotional intelligence, cognitive flexibility, and systems thinking (Mazur et al., 2014). Emotional intelligence refers to the ability of project managers to understand and express their own emotions and understand others' emotions. Cognitive flexibility refers to their ability to analyse situations from different viewpoints, and systems thinking refers to their ability to see things as a whole (Mazur et al., 2014). Stakeholder interactions that are affected by these characteristics include the relationship between projects 
and stakeholders (Mazur et al., 2014) and the analysis methods that project managers use and the preparation and planning that they have for this analysis (Aaltonen, 2011).

Other individual characteristics of stakeholders found to affect their perception include their work experience and functional positions (Tuuli \& Rowlinson, 2010), their ages and roles in projects (Ojiako et al. 2014), and the cultural ways in which project managers learn and evaluate information (Tukiainen et al., 2010). These kinds of characteristics were shown to affect stakeholders' sense of autonomy in their decision making and confidence in their ability to make a difference in projects as well as their personal connection to projects they are involved in (Tuuli \& Rowlinson, 2010). These characteristics can also affect the information that stakeholders can receive during project implementation, their judgment of project outcomes and project risks, and their ability to articulate this judgment (Ojiako et al. 2014). These characteristics also affect project managers' thinking about unexpected events and what to do with them (Tukiainen et al., 2010).

In summary, many project stakeholders' characteristics that are the products of their professional and educational background and experience affect their thinking and behaviour. This aligns with the conclusions drawn from the review of differences of individual stakeholders in section 2.2.7. This impact of individual characteristics of stakeholders, combined with the results of the reviews in section 2.3.4 and 2.3.5, indicate that the complexity of the thinking and behaviour of project stakeholders can be explained by the dynamics of stakeholder perception, as influenced by their networks and individual characteristics or in other words, the dynamics of project stakeholders at individual levels.

\subsubsection{Summary of the Review of Stakeholders in Projects}

Overall, the review of stakeholders in projects shows that the time and space contexts of projects are complex and temporary. However, the current stakeholder analysis methods still have limitations in addressing the complexity of the thinking and behaviour of project stakeholders. This complexity can be described as the dynamics, social networks, and individual differences of stakeholders. The review of these aspects thus identifies that the dynamics of stakeholder perception, as influenced by their networks and individual characteristics or in other words, the dynamics of stakeholder perception at individual levels can explain the complexity of their thinking and behaviour. This identification aligns with the results of the review of the stakeholder literature in section 2.2. Based on this alignment, a research gap for the research can be identified. This gap is presented along with the overall summary of the chapter in the next section. 


\subsection{Summary of the Chapter}

This chapter has explored the existing literature on stakeholder management and stakeholders in projects in order to identify the relevant concepts and current knowledge related to the complexity and changing nature of stakeholder thinking and behaviour in projects. The review has identified that both the stakeholder and project management literature have the same gaps when it comes to describing this complexity. Thus, in both fields, a wide range of methods have been developed to examine stakeholder thinking and behaviour (e.g., Elias, 2016; Solaimani et al., 2013). These methods have not been able to capture the various aspects affecting the complexity at once (Kreiner, 1995; Padalkar \& Gopinath, 2016). Specifically, very few methods focus on stakeholder dynamics (Pouloudi et al., 2016; Solaimani et al., 2013), their complex interaction in their social networks (Beaulieu \& Pasquero, 2002), and their differences at individual levels (Johnson et al., 2008) simultaneously. The combination of these aspects could provide an approach for the description of the complexity of stakeholder thinking and behaviour in projects. Figure 2.2 below illustrates the combined approach adopted in this study.

Figure 2.2 An approach to describe the complexity of stakeholder thinking and behaviour in projects

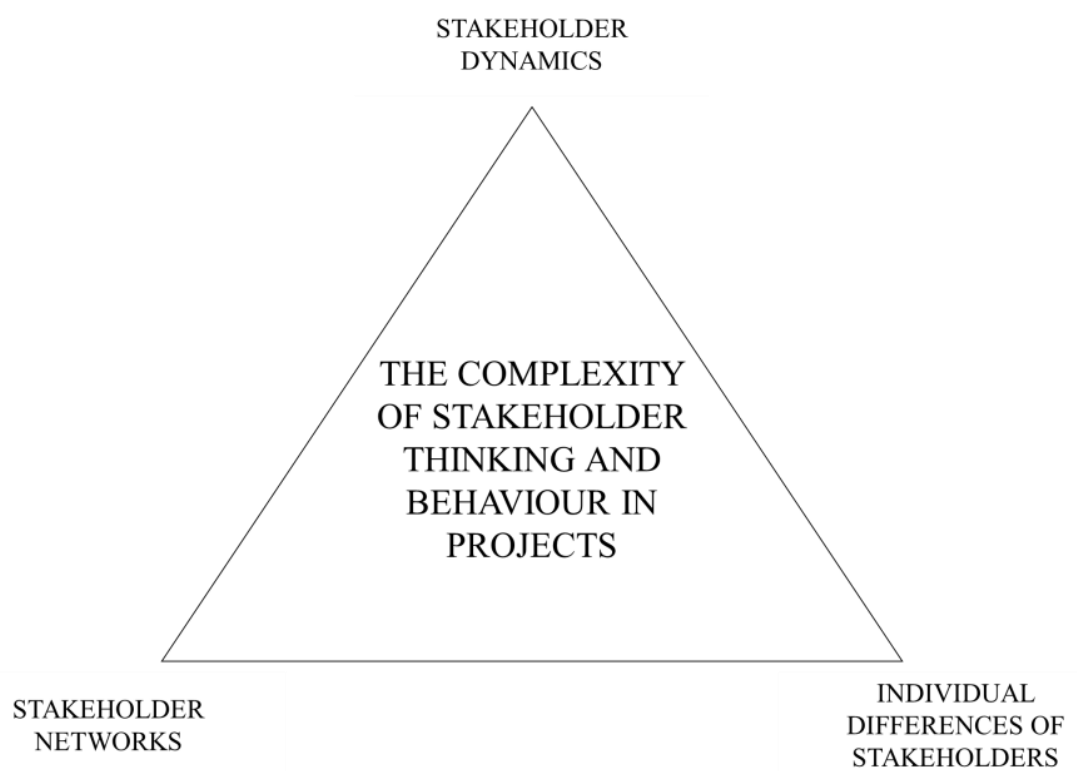

The review of these aspects in detail identified a specific description of this complexity. The results of the review and the description that is adopted for this study are summarised in Figure 2.3 as follows. 


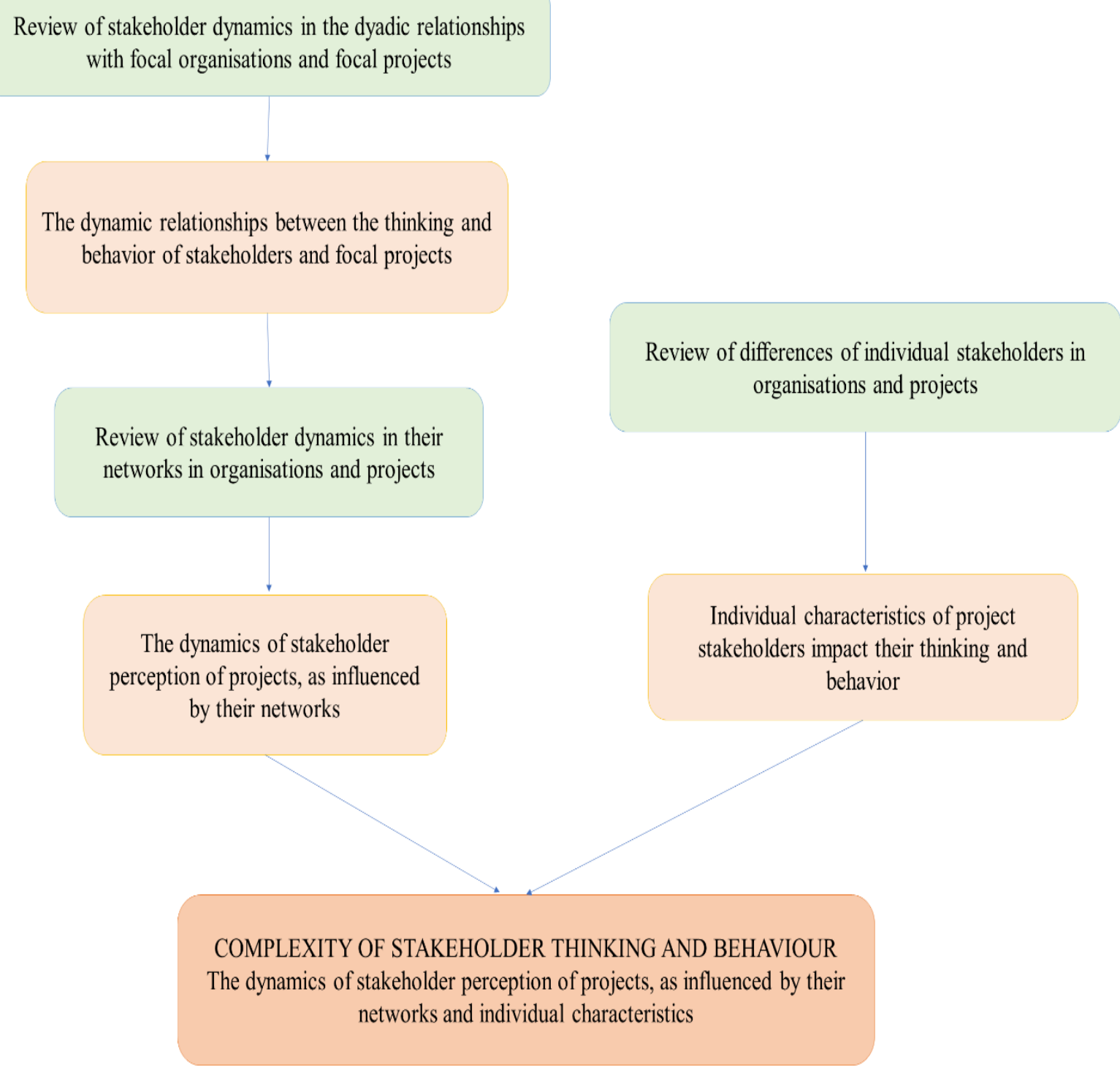

As illustrated in the above figure, the review of stakeholder dynamics in their dyadic relationship with focal organisations (section 2.2.4) and focal projects (section 2.3.4) identified that the complexity of stakeholder thinking and behaviour can be described as the dynamic relationship between the thinking and behaviour of stakeholders and focal projects. The review of stakeholder dynamics in their networks in organisations (section 2.2.6) and projects (section 2.3.5) then identified that this complexity can be described as the dynamics of stakeholder perception, as influenced by their networks. Meanwhile, the review of the individual differences of stakeholders in general (section 2.2.7) and project stakeholders in particular (section 2.3.6) identified that different characteristics of stakeholders affect their thinking and behaviour. Overall, the review highlighted that the complexity of stakeholder thinking and behaviour in projects can be explained by the dynamics of stakeholder perception of projects, 
as influenced by their networks and individual characteristics or the dynamics of stakeholder perception of projects at individual levels. The review also discussed studies on the dynamics of stakeholder perception in their networks (e.g., Aaltonen et al., 2015; Elias et al., 2004) as well as studies on the impact of the individual characteristics of stakeholders on their thinking and behaviour in projects (e.g., Ojiako et al. 2014; Tukiainen et al., 2010). However, very few studies exist that focus on the dynamics of stakeholder perception, as influenced by both their networks and individual characteristics. The dynamics of stakeholder perception at individual levels or in other words, the dynamics of stakeholder perception as influenced by their individual characteristics, therefore, is the gap that the research addresses. The next chapter presents the research objectives, questions, and the research methodology that were developed specifically to address this gap. 


\section{Chapter 3 Methodology}

\subsection{Overview of the Chapter}

The literature review has identified the research gap that this research aims to address. The chapter starts by presenting the research objective, which was informed by this gap. Social constructionism as the research paradigm and Vickers' concept of appreciative systems as the theoretical lens are then discussed, which is followed by a presentation of the research questions. The chapter continues by explaining the Repertory Grid Technique, which was chosen to be the main data collection and analysis approach. Finally, the case study method and ethical considerations of the research are described.

\subsection{Research Objective}

Based on the research gap that has been presented in chapter two, the overall objective of this research is to explore the dynamics of individual stakeholders in projects. Specifically, the research focuses on the dynamics of stakeholder perception of projects, as influenced by their social networks and individual characteristics. The research aims to provide an improved methodological approach for managing individual stakeholders in complex projects.

\subsection{Research Paradigm of Social Constructionism}

Identifying a research paradigm is important in deciding on research methods (Guba \& Lincoln, 1994). Research paradigms consist of research epistemology, research ontology, and research methodology (Guba \& Lincoln, 1994; Saunders, 2007). Each of these constructs is concerned with different sets of questions. Research ontology is concerned with questions about what is real, such as 'What is the form or nature of reality?' 'What can be known about reality?' 'Does a “real” world really exist?' (Guba \& Lincoln, 1994). Research epistemology, on the other hand, is concerned with questions about the relationship between the knowers or would-be knowers and what can be known. For instance, such questions may examine if a "real" reality is assumed, the knowers must be value-free to discover how things really are and how things really work (Guba \& Lincoln, 1994; Saunders, 2007). Research methodology is concerned with questions about how the inquirers or would-be knowers get to know what they believe they can know. The specific questions asked here depend on the answers given to the ontology and epistemology questions (Guba \& Lincoln, 1994). 
Scholars have presented different ways to divide research paradigms. Thus, research paradigms have been divided into positivism, realism, and interpretivism (Saunders, 2007); positivists, postpositivist, constructionist, and transformative (Wiersma, 2009); positivism, postpositivism, critical theory, and constructivism (Guba \& Lincoln, 1994), and positivist, interpretivist, and critical research (Cavana et al., 2001). Among these paradigms, social constructionism can be understood as the opposite of positivism (Easterby-Smith et al., 2008). Positivism is based on the assumption that that the social world exists externally. Its properties, therefore, are measured through objective methods, rather than being inferred subjectively through people's sensation, reflection, and intuition. According to positivism, there is no real knowledge, but only the knowledge that is based on observed facts (Easterby-Smith et al., 2008). Social constructionism, meanwhile, says that our knowledge of reality depends on how we perceive it. People's perception of reality, in turn, depends on their relationships and how they interact with others. Through these interactions, people negotiate their interpretations of reality (Gergen, 1985). This does not mean that there is no independently existing reality or that the knowledge which people have created so far is wrong. It means that reality is "something", but that people rely on some "tradition of sense-making" (Gergen, 2015, p.5) to perceive this "something." This tradition is developed when people interact with each other and becomes established knowledge of reality for future generations (Berger \& Luckmann, 1991). This tradition of meaning creation becomes so commonplace that people forget that it is human creation (Gergen, 2009).

Social constructionism also describes the ways in which people construe their knowledge of reality. Mythology, theology, and modern sciences are examples of disciplines that transmit knowledge of reality between people (Berger \& Luckmann, 1991). Continuing and cohesive communication or input from individuals with power also contributes to the construction of knowledge (Berger \& Luckmann, 1991; Gergen, 1985). The language that people use (Berger \& Luckmann, 1991), including their utterances and other spontaneous communication (Shotter, 1997), also reflects the construction of knowledge. In other words, people construe their perception of reality moment by moment when engaging in different social interactions. The tasks of social scientists, therefore, should be to study the constructions and meanings that people place upon their interactions and experiences (Easterby-Smith et al., 2008).

The social constructionist paradigm aligns with the objectives of the current research as it conceptualises people's perceptions of reality to be constructed through interactions. Thus, 
social constructionism is able to identify specific interactive events, both in the present and the past, to explain changes in stakeholders' perceptions of projects. These changes can then be studied from their subjective perspectives. The study will also draw on Vickers' concept of appreciative systems, a concept related to the paradigm, which is described in the sections below.

\subsection{Sir Geoffrey Vickers' Concept of Appreciative Systems}

\subsubsection{Overview of Vickers' Concept of Appreciative Systems}

Vickers academic career started in 1955; the year he retired. Before that, he had a successful career in the army, law, and civil services (Vickers, 1965/1995). With this experience, much of Vickers' academic work focused on macro issues such as government policy and human history (Checkland \& Casar, 1986). The concept of appreciative systems is one of his most prominent works (Vickers, 1987).

The core idea of Vickers' concept of appreciative systems is that people evaluate and decide activities of themselves and other people not by goals or what they want to achieve but by comparing the activities with their standards (Vickers, 1965/1995). For example, when an individual decides what they should do with an apple, they do not look at the apple but look at what they can do with it. They can eat it, sell it, or cook it. These activities become their standards for activities with the apple. Based on these standards, they decide their activities or evaluate other people's activities with the apple (Vickers, 1965/1995). In another similar example, the daily activities of a salesperson are not decided directly by his sales goal but by what he thinks he should do every day to obtain the goal. For example, he must make a certain number of phone calls to customers or must learn information about products (Vickers, 1965/1995).

Vickers (1965/1995) calls the process in which people evaluate activities according to standards and respond with actions the "process of regulation" (p.50). He describes it as human regulation and differentiates it from physical regulation, which is created by equipment or machines such as the regulation of a ship's course control system. Both regulations involve the assessment and monitoring of situations according to standards. However, because human regulation involves unspecific and changing standards, it is more complex than the physical one (Vickers, 1965/1995). In some cases, however, human regulation may be more predictable than physical regulation because people want their behaviour to meet others' expectations (Vickers, 1970). 
Vickers (1987) proposed the concept of appreciative systems and their dynamics to describe how individuals evaluate activities according to standards. Individuals' appreciative systems reflect the way that they understand the world. Specifically, individuals develop their standards by "abstracting" (Vickers,1970, p.100) regularities from experiences. With the standards, they are interested in and pay attention to particular aspects of the world (Vickers, 1970). The world that people live in, therefore, is an "appreciated world" (Vickers, 1970, p.97). This appreciated world organises people's further experience, mediates their communication, and guides their actions (Vickers, 1970). Appreciative systems are not only the means but also the goals of people's communication. When people communicate with each other, they exchange and test the accuracy of different elements of their appreciative systems (Vickers, 1970).

The appreciative systems consist of three elements: value judgments, reality judgments, and instrumental judgments (Vickers, 1965/1995). Value judgments or standards are "judgments about the significance of the facts to the appreciator or to the body for whole the appreciation is made" (Vickers, 1965/1995, p.54). In other words, they are the perception of what is good or bad, acceptable or unacceptable. They are affected by the actual process of appreciation and neither right nor wrong (Vickers, 1995). Two types of value judgments or standards are "commitments" (Vickers, 1965/1995, p.122) and "enjoyments." (p.123). Commitments are what individuals are committed and obliged to follow. Enjoyments are their preferences. For example, when people choose food for dinners, they follow their commitments if they have diet food because of their health problems. They follow their enjoyments if they choose between beef and pork, both of which they are allowed to eat (Vickers, 1965/1995).

Reality judgments are "judgments about what the state will be or might be on various hypotheses as well as judgments of what it is and has been. They may thus be actual or hypothetical, past, present or future" (Vickers, 1965/1995, p.54). Reality judgments include not only appreciation but also predictions of facts and unobservable things such as people's opinions. Prediction, therefore, is a critical skill to make reality judgments, especially in changing situations. People with more experience are likely to have learned to make better predictions (Vickers, 1965/1995). Finally, instrumental judgments answer the question 'What are we going to do?' (Vickers, 1965/1995, p.103) to reduce the differences between reality and value judgments. For instance, an individual has a value judgment or standard that each person should consume a particular amount of vegetables per day. Based on their observation of food consumption in a family, they may have a reality judgment that the family does not eat as many 
vegetables as they should. The individual may then have instrumental judgments that the family should be more informed about the benefits of vegetables or the ways to cook vegetables. Individuals must be innovative to answer the question related to instrumental judgments effectively. It is also difficult to see whether an instrumental judgment is right or wrong because we will never know the ultimate outcomes of a solution (Vickers, 1965/1995).

The dynamics of appreciative systems are described as the dynamic relationships among these three judgments. These dynamics are illustrated in Figure 3.1 below.

Figure 3.1 The dynamics of appreciative systems (Source: Checkland, 2000)

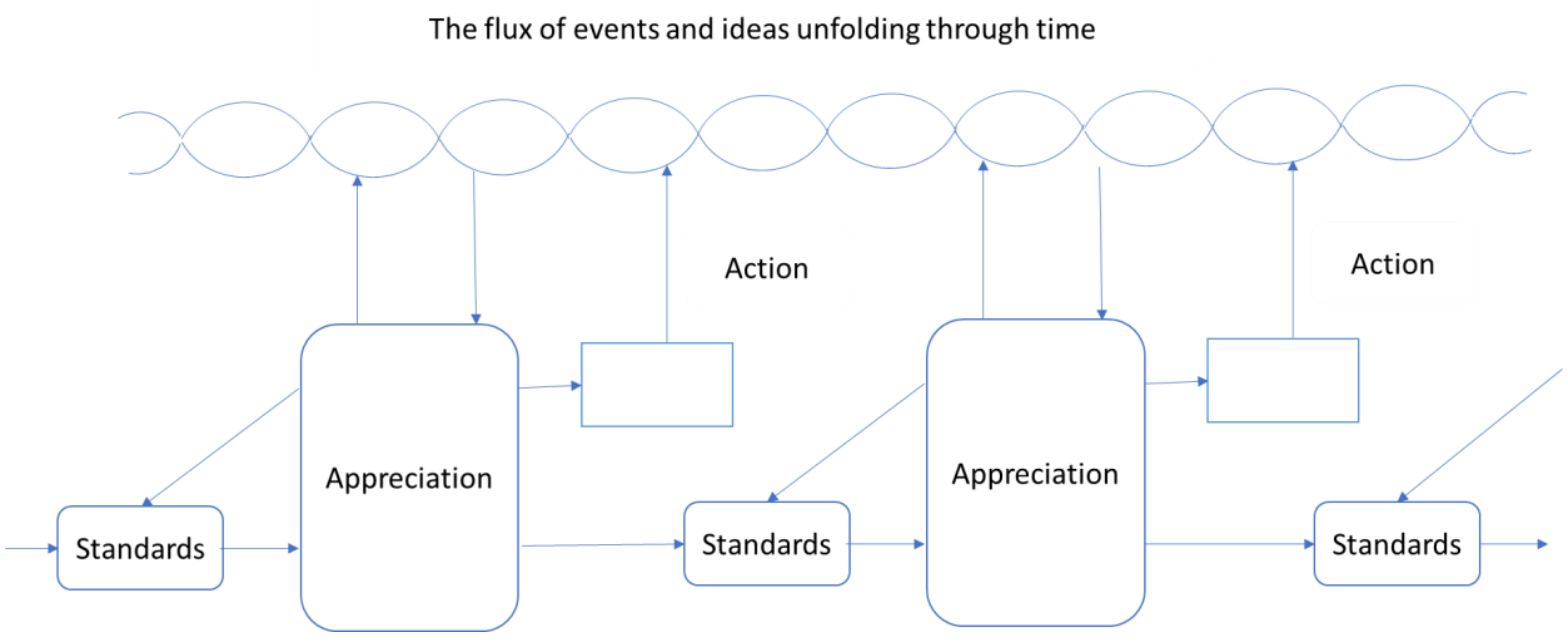

As illustrated in the figure, from experience, individuals have standards for and appreciation of situations. When individuals engage in interactions, they will learn "events and ideas" related to the situations. While the events refer to the facts that people are aware of and the information that they receive, the ideas refer to the abstract ideas, or the appreciative systems of other people (Vickers, 1965/1995). These events and ideas may affect individuals' standards, appreciation, and instrumental judgments regarding situations. With these new judgments, individuals can learn other events and ideas when engaging in other interactions. These events and ideas, in turn, can affect their judgments. The dynamics of appreciative systems of individuals, therefore, can be understood as processes of matches and mismatches between what individuals expect and what really happens (Vickers, 1987). These processes do not always produce direct solutions or actions. In many cases, they only change people's evaluation of situations, and people's actions are not necessarily connected to this evaluation.

In summary, the concept of appreciative systems describes the systemic, dynamic and ongoing ways in which individuals change their perception when interacting with others from the past to the present. The concept, therefore, can be applied to study the changes in individual stakeholders' perception of projects, as influenced by their social networks and individual 
characteristics. The following section further justifies the selection of Vickers' concept of appreciative systems and discusses the academic appreciation and empirical application of it.

\subsubsection{Academic Appreciation and Empirical Application of Vickers' Concept}

There are three reasons why Vickers' concept of appreciative systems was chosen from many social constructionist concepts and theories to study the changes in individual stakeholders' perception of projects.

The first reason is that Vickers' concept both captures the notion of social constructionism and provides a systemic approach to study changes in individual thinking. Examples of other social constructionist concepts and theories are: "schemata" (Fielder, 1982, p.1001); "mental models" (Senge, 1997, p.50); "metaphors" (Morgan, 1980, p.6); "frames" (Davies \& Mabin, 2001, p.858); “sensemaking” (Weick et al., 2005, p.409), and Personal Construct Theory (Kelly, 1955/2001). These concepts and theories describe thought as the interplay between what is in someone's mind and the stimulus they receive from the external environment. However, these concepts differ slightly in the way they conceptualise the two aspects. Thus, according to the concept of schemata, thought is influenced by 'permanent memory structures' (Fielder, 1982, p.1001) and information received from external interaction (Fielder, 1982). The concept of sensemaking regards thought to be the product of someone's abstract ideas that are influenced by their impressions from external interaction (Weick et al., 2005). Vickers' concept has a similar conception of what thought is as it describes an individual's appreciative system as the result of the interplay among their standards, appreciation, instrumental judgments, and the fluxes of events and ideas. The three former components refer to what individuals have in their minds, and the latter refers to the information that individuals receive from the external environment. However, in comparison to other concepts and theories, Vickers' concept provides a more systemic view of the dynamics of individual thinking, which has been illustrated in Figure 3.1 above.

Vickers' concept has attracted attention from scholars, especially systems analysts. One of the main criticisms of Vickers' concept is it lacks detail (Brocklesby, 2007) and that it is not linked to other academic theories (Checkland \& Casar, 1986) and, as a result, is less well known. However, despite these limitations, scholars highly appreciate the concept as it makes a distinctive contribution to philosophy (Bluden, 1994; Williams, 2005) and thus deserves more serious and critical attention. Checkland (1994) specifically proposes it as the theoretical underpinning of soft systems methodology (as cited in Brocklesby, 2007, p.157). 
Vickers' concept has also been applied in empirical studies. For example, in policymaking, Cohen-Blankshtain and Nijkamp (2004) apply the concept to study policy-makers' perceptions of information communication technology (ICT) in cities. The value, reality, and instrumental judgments are used to describe perceptions of the ideal city, ICT and the relevance of ICT policies to cities, respectively. In another example, Blackmore (2005) applies the concept to describe decision-makers' standards for and appreciation of environmental issues. Based on this application, the author proposes that Vickers" work is "full of insights" (Blackmore, 2005, p.15) and relevant to studies of behaviour in organisations. Moreover, Regev, Hayard, and Wegmann (2011) apply the concept to explore how Apple products set new standards for related products in the market. Their study shows that the concept can be used as a theoretical framework for modelling and analysing the service systems and the cocreation of product value. However, even though Vickers' concept has been used in a number of empirical studies, its application in the field of project management is still limited. This research addresses this gap by providing an empirical application of the concept

In summary, with its systemic and dynamic nature, Vickers' concept of appreciative systems provides a systematic approach to study the dynamics of individual stakeholders' perception and thinking of projects, as influenced by their individual characteristics and social networks. This application is also in line with the calls of existing scholars for more empirical applications of the concept. The application of the concept in this research is described below.

\subsubsection{Application of Vickers' Concept of Appreciative Systems}

As mentioned above, the objective of this research is to explore the dynamics of individual stakeholders in projects. Specifically, the research focuses on the dynamics of stakeholder perception and thinking of projects as influenced by their social networks and individual characteristics and proposes to apply Vickers' concept as a lens to describe these dynamics. According to Vickers' concept, stakeholders' individual characteristics that are linked to their experience and background are described as their standards. The present social networks of stakeholders are described as their perceptions of related events and ideas. The stakeholder perception and thinking is described as their appreciation. The instrumental judgments are not included in this application because of three reasons. The first reason is that instrumental judgments are defined as the differences between standards and reality judgments. Information about instrumental judgments, therefore, is implied in standards and reality judgments. Secondly, while instrumental judgments refer to stakeholder thinking of what projects should do, not all stakeholders have the power to act according to these judgments. Individual 
stakeholders, therefore, may find instrumental judgments irrelevant and do not want to repeat what they have implied in their standards and appreciation. The third reason is that instrumental judgments are related to stakeholder actions, but these actions are not the focus of this research. Without instrumental judgments and actions, the dynamics of stakeholder perception of projects, as influenced by their social networks and at their individual levels, are described in Figure 3.2 below.

Figure 3.2 The dynamics of stakeholder perception of projects according to Vickers' concept

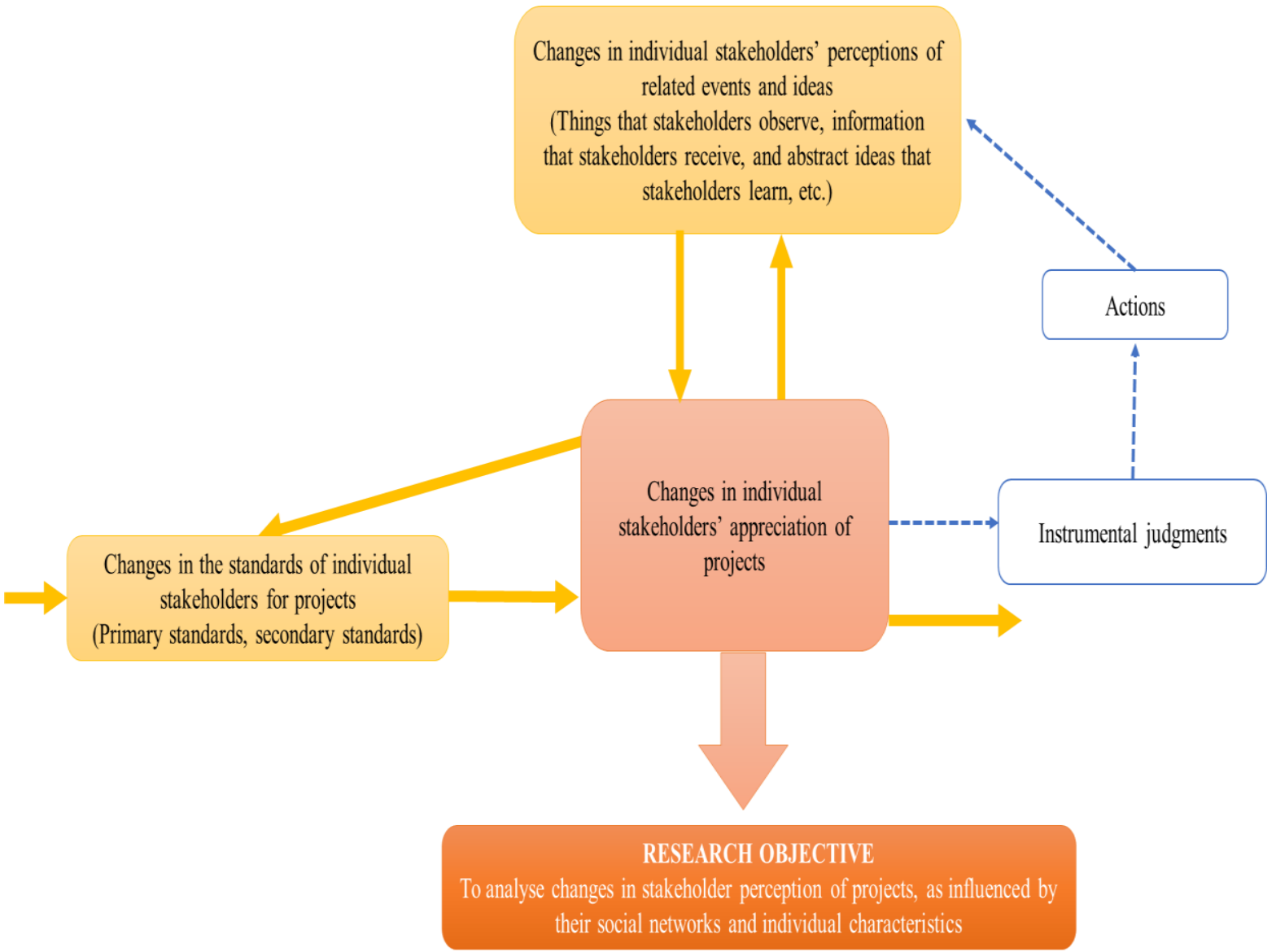

The above figure describes the dynamics of individual stakeholders' appreciative systems regarding their projects. The figure consists of solid and broken lines. The solid lines describe the dynamic relationship between the standards, appreciation, and perceptions of events and ideas and are the focus of this research. The broken lines describe the dynamic relationship related to the instrumental judgments and actions and are not the focus of this research. Following the solid lines, the standards consist of commitments and enjoyments and are named primary and secondary standards, respectively. Primary standards are important and decide whether projects are good or bad. Secondary standards are not important but refer to aspects that should be considered in projects. During project implementation, individual 
stakeholders may learn events and ideas related to projects, and these events and ideas may change their standards for and appreciation of projects. Overall, the dynamics of stakeholder perception and thinking of projects, as influenced by their social networks and individual characteristics, can be described as the dynamics of their appreciation of projects, as influenced by their perceptions of related events and ideas and their standards. This conceptualisation of the dynamics of stakeholder perceptions of projects informs the specific research questions the current study seeks to address, which will be presented in the following section.

\subsection{Research Questions}

In the discussion above established that the research objective is to analyse the dynamics of stakeholder perception and thinking of projects as influenced by their social networks and individual characteristics and proposed the use Vickers' concept of appreciative systems for this purpose. Based on the conceptualisation of dynamics of stakeholder perceptions using Vickers' concept as illustrated in Figure 3.2 above, the research objective will be addressed through the following research questions:

Research question 1: How do individual stakeholders change their appreciation in projects over time?

Research question 2: What are the drivers of the changes in stakeholders' appreciation of projects?

The application of Vickers' concept also provides guidance for collecting and analysing the empirical data. As Vickers noted that collecting and analysing data on the standards and appreciation can be challenging (as cited in Regev et al., 2011, Conclusions and Future Work section), a reliable method, which is the Repertory Grid Technique, was selected for the data collection and analysis. An overview of the technique and its application are presented in the next section.

\subsection{The Repertory Grid Technique}

This section begins with Personal Construct Theory, the theoretical background of the Repertory Grid Technique, and then continues with the overview and application of the technique, specifically in relation to the field of project management. The section ends with a description of the application of the Repertory Grid Technique in this research. 


\subsubsection{Personal Construct Theory}

Personal Construct Theory is the theoretical background of the Repertory Grid Technique. George Kelly, a clinical psychologist and educator, first introduced the theory in his book The Psychology of Personal Constructs in 1955. The philosophical foundation of the theory is “constructive alternativism" (Kelly, 1955/2001, p.15). Constructive alternativism means that there are different alternative ways to understand and interpret reality. People develop, test, and revise their hypotheses about reality from their experience. Through this process of testing and revision, people develop their understanding of the world (Kelly, 1955/2001).

Kelly (1955/2001) proposed the concept of "dichotomous constructs" (p.33) and systems of constructs to describe how people's experiences form and affect their hypotheses of the world. Specifically, dichotomous constructs are created from contrasting objects and events. For example, we only know what a good teacher means when we are aware of the opposite, the bad teacher. Similarly, we only know what a pleasant person is like when we know the opposite, the unpleasant person (Kelly, 1955/2001). Systems of these constructs reflect people's thinking of objects and situations.

These constructs are validated or invalidated based on people's experiences. A construct is validated when the initial prediction based on it matches what people experience. A construct is invalidated when the initial prediction does not match what people experience. When constructs are invalidated, the whole construct systems may be rearranged to accommodate the change. Thus, validation and invalidation affect not only the constructs that the prediction is based on but also other related constructs. In addition to validation and invalidation of existing constructs, new constructs can also be formed. Favourable conditions for the formation of new constructs include using new elements, using experimentation, and providing validating data (Kelly, 1955/2001). Unfavourable conditions include using threats, using old materials, and not using experimentation. Overall, people's experiences do not only depend on things happening to them but also on how they construe and re-construe what happens to them (Kelly, 1955/2001).

The above discussion shows that Personal Construct Theory is compatible with Vickers' concept as both emphasise the subjective views of the world and the impacts of experience on these views. Following this alignment, the Repertory Grid Technique, which is based on Personal Construct Theory, is used to collect and analyse data on the standards, 
perceptions of events and ideas, and the appreciation of individual stakeholders. Next, an overview of the technique and its application in project management are presented.

\subsubsection{The Repertory Grid Technique and its Application in Project Management}

The Repertory Grid Technique is a technique used to elicit and analyse repertory grids which are ways to describe people's viewpoints of the world or some smaller parts of it (Jankowicz, 2005). A repertory grid consists of four main components: topics, constructs, elements, and the links between constructs and elements. Among these components, constructs describe individual thinking about the topics of the grids. Elements are examples or instances of the topics. For example, suppose the topic of a grid is the skills and abilities of salespersons, the elements will be different salespersons, and the constructs are skills and abilities such as communication and knowledge of products. The elements and constructs can be linked to each other in different ways, such as rankings or ratings. These links provide pictures of what people wish to say about each element of the topics (Jankowicz, 2005). An example of a repertory grid that describes a store manager's thinking of skills and abilities of his salespersons is illustrated in Figure 3.3 below.

Figure 3.3 An example of a repertory grid (Source: Jankowicz, 2005)

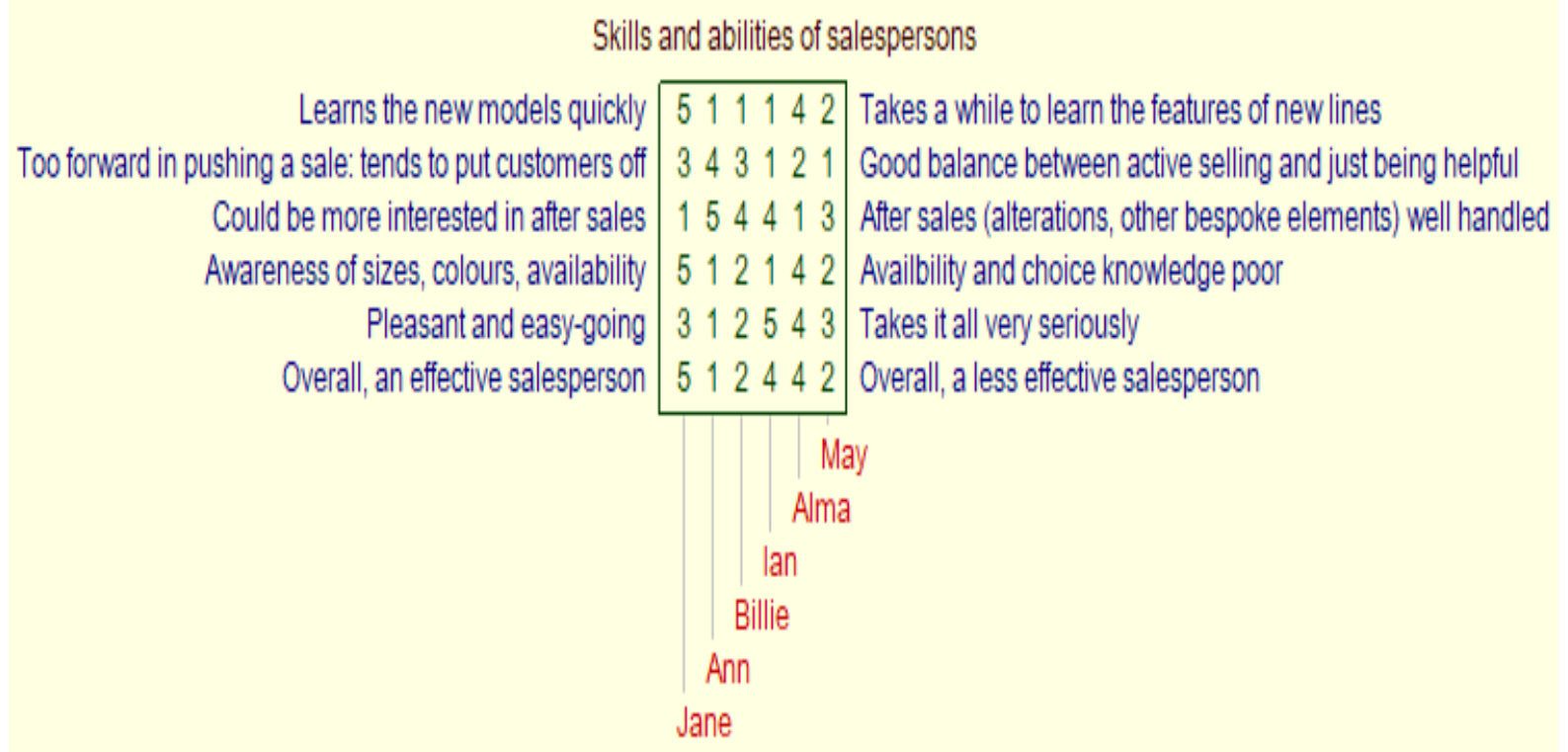

The application of the Repertory Grid Technique in interviews consists of three steps (Curtis et al., 2008; Jankowicz, 2005). The first step takes place before data collection and involves researchers getting to know the topics and choosing the elements and constructs to include in the grids. The second step takes place during interviews and involves the elicitation of the elements and constructs if they were not chosen before in the first step. The most 
common elicitation method is triadic elicitation. In this elicitation, researchers present different sets of three elements and ask interviewees to identify the similarities and differences among them (Curtis et al., 2008). This process of elicitation is illustrated in a study by Napier et al. (2009), which focuses on identifying the skills of successful managers in information technology projects. These researchers used eight elements, namely the ideal and incompetent project managers and six other project managers that the interviewees had worked with. The researchers then used different sets of three elements to ask the interviewees: "With regard to the skills of successful information technology project managers, how are two of these project managers the same and yet different from the third?" The elicitation was done until no new constructs emerged. After elements and constructs are identified, they will be linked to each other by dichotomising, ranking, or rating. Dichotomising means that elements are placed on one of the two poles of constructs, ranking means that elements are ranked on continuous scales, and rating means that elements are rated on each construct. While each way of linking has advantages and disadvantages (Tan \& Hunter, 2002), rating is argued to be the best (Curtis et al., 2008). Finally, the third step takes place after interviews and involves a review of the whole grids by the interviewees to ensure that the grids describe their opinions precisely (Curtis et al., 2008).

Once created, repertory grids can be analysed in different ways. The analysis basically focuses on the relationships between constructs and elements and can be qualitative or quantitative. Qualitative analysis is based on the content of the grids, such as the labels of elements and constructs. Quantitative analysis is based on the ratings or rankings of the grids. Examples of quantitative analysis approaches are the cluster and correlation analysis of constructs and elements (Curtis et al., 2008). Repertory grids of different people can also be compared to each other. However, such a comparison is not a straightforward process because constructs may have different meanings to different people, even if they are expressed similarly (Kelly, 1955/2001). In other words, the best approach to analyse repertory grids is at individual levels (Reger, 1990). Thus, the current research will apply the principal component analysis to identify the changes in the repertory grids of each individual over time. The principal component analysis will be introduced briefly in the data analysis method section of this chapter and explained in more detail in chapter four of the data analysis and findings.

As any methodological approach, the Repertory Grid Technique has a number of advantages and disadvantages. In terms of advantages, the elicitation of constructs from the interviewees minimises the biases of using constructs that were pre-identified by the 
interviewers (Curtis et al., 2008; Reger, 1990). The principal component analysis of the technique also allows identifying the changes in the content and ratings of the grids over time (Tan \& Hunter, 2002). In terms of disadvantages, the technique has been described as boring, time-consuming, and as a cognitive burden for interviewees (Curtis et al., 2008). However, as the advantages outweigh the disadvantages, the technique is widely applied in different fields.

In project management, the technique has been used as both data collection and analysis methods. For example, it has been used to interview and collect data on factors that impact company efforts to do projects (Wagner et al., 2015), characteristics of good project managers (Medina \& Francis, 2015), skills of project managers (Napier et al., 2009), project success criteria (Pankratz et al., 2014), and successful and unsuccessful project responses to unexpected events (Geraldi et al., 2010). Downward and upward laddering techniques, which refer to using questions to clarify the meanings of the constructs, have also been used (e.g., Pankratz et al., 2014; Wagner et al., 2015). Analysis methods, such as content analysis or categorisation of constructs (e.g., Medina \& Francis, 2015; Wagner et al., 2015) and construct comparison (e.g., Geraldi et al., 2010), have also been applied.

Along with the widespread application of the technique, computer software has been developed to support different aspects of the analysis. Examples of this software are IDIOGRID, inGridX and GRIDSTAT (Fransella et al., 2004). Among this software, Rep Plus V1.1R, which was originally developed by Shaw and Gaines in 1988, is probably the most comprehensive and user-friendly software (Sewell et al., 1992). It has been used widely and can now be downloaded for free for personal, academic, and commercial uses from the website: https://pages.cpsc.ucalgary.ca/ gaines/repplus/ (University of Calgary, 2018). This software was selected to construct and analyse the grids in this research.

The above review shows that the Repertory Grid Technique can be applied to collect and analyse data on the changes in the standards, perceptions of events and ideas, and appreciation of individual stakeholders regarding their projects. This application is explained in greater detail in the next section.

\subsubsection{Application of the Repertory Grid Technique}

The application of the Repertory Grid Technique to collect and analyse empirical data, along with the research objective and theoretical framework, are summarised in Figure 3.4 below. 


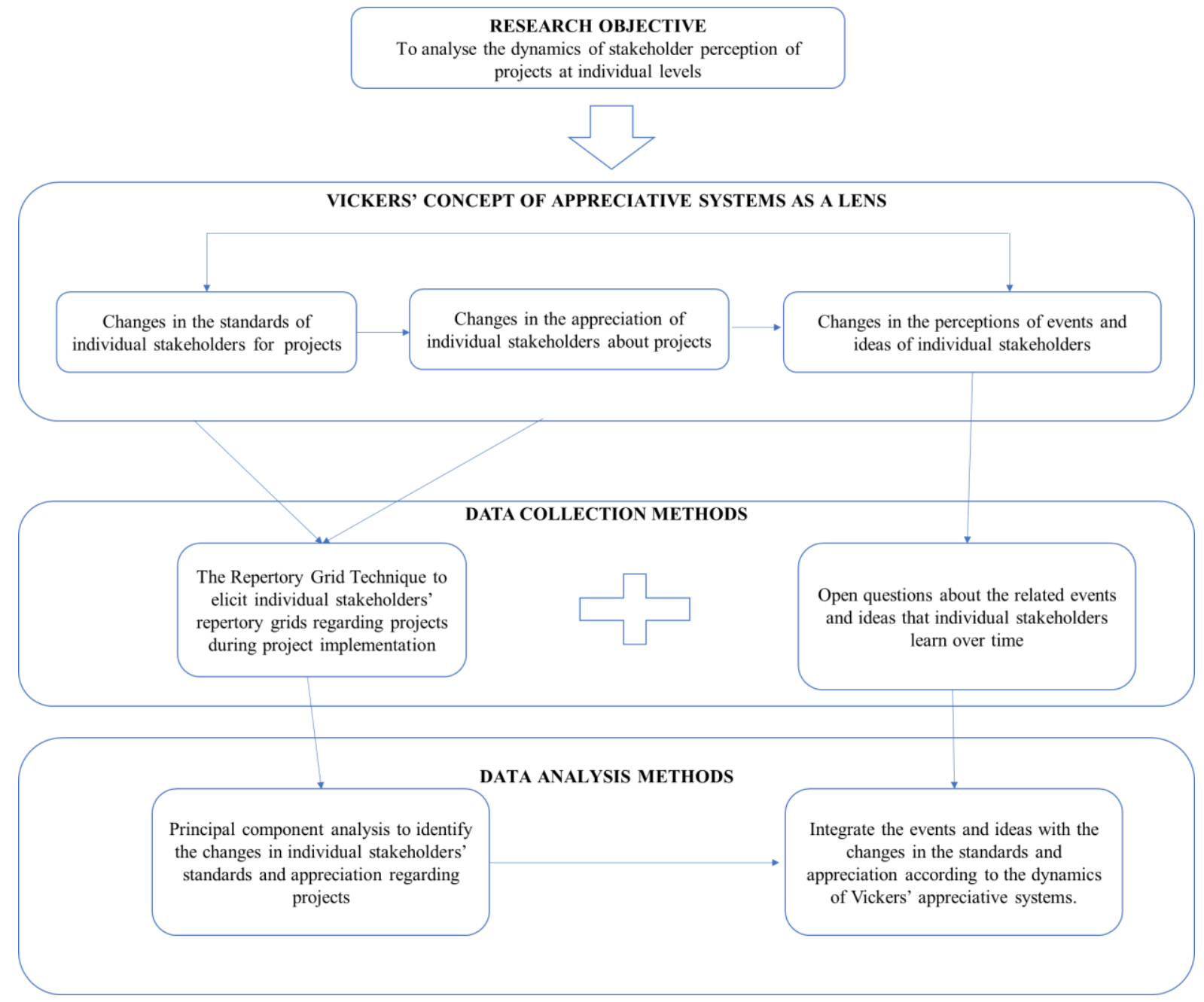

As illustrated in the figure, the two upper layers present the research objective and the application of Vickers' concept. The two lower layers present how the Repertory Grid Technique, combined with open questions, are applied to collect and analyse the empirical data. Specifically, the technique is used to elicit repertory grids that describe individual stakeholders' thinking about projects at different time points. The elements of these grids are the ideal, less than ideal, target projects, and three other projects. The principal component analysis of these grids helps to identify the standards for and appreciation of individual stakeholders about projects at different time points. Based on this, the changes in the standards and appreciation are identified. Meanwhile, open questions are used to elicit information about the events and ideas related to the projects that individual stakeholders learn between these time points. These events and ideas are then combined with the changes in the standards and appreciation by applying Vickers' concept. The results of this integration are stories about the 
changes in the appreciation of each individual stakeholder about their project, as influenced by their standards and perceptions of events and ideas.

In summary, the Repertory Grid Technique can be combined with open questions to collect and analyse data on the changes in the standards, perceptions of events and ideas, and appreciation of individual stakeholders regarding their projects. The empirical research methods chosen in line of these considerations are identified in the following section.

\subsection{Case Study Research as Empirical Research Methods}

\subsubsection{Case Study Research and the Unit of Analysis}

This study adopted a case study approach because it is suitable for the social constructionist paradigm (Easterby-Smith et al., 2008) as it allows for an exploration of the "How" and "Why" research questions (Yin, 2018). This research specifically focused on identifying how and why the appreciation of individual stakeholders about their projects changes in the contemporary and real-world context. Case study research involves detailed and in-depth descriptions of these changes as well as the reasons for these changes. The unit of analysis or the case was a group of at least two individual stakeholders within a university accreditation project during a period of the project implementation. This case allowed the comparison of the changes in individual stakeholders' appreciation of the same project and, therefore, provided insights into these changes.

\subsubsection{Case Selection}

The cases were selected according to three criteria. First, the characteristics of the cases must align with the research questions to ensure that the data collected could answer the research questions (Yin, 2018). Second, even though the number of cases does not affect the analytical generalisation of research results, single-case studies are often criticised for their ability to repeat the same empirical research in other cases. Evidence from multiple cases is also more compelling and robust (Herriott \& Firestone, 1983). Having more than two cases, therefore, was the goal of this research (Yin, 2018). Finally, while multiple cases are favourable, the number of cases must be balanced against pragmatic considerations such as the time or money available for data collection (Eisenhardt, 1989; Yin, 2018).

Based on these criteria, three cases were selected in three accreditation projects. The first project was to renew a programme accreditation at a New Zealand university. The two other projects were located at a university in Vietnam. One of the projects focused on securing national accreditation for the university while the other focused on attaining an international 
accreditation for a specific programme. These cases were selected out of convenience because the researcher was a PhD student in New Zealand and a university lecturer in Vietnam and had professional contacts with the members of these accreditation projects. Considering that the projects took place in two different countries with very different cultures, it could be argued that the cases are not comparable. However, since the research questions are not concerned with concepts that are culturally embedded, it was decided that cultural differences would not impact the research results.

University accreditation projects can also be considered suitable for this research. Accreditation projects involve the self-evaluation of universities or programmes, as well as visits and examinations by members of the accreditation bodies (Vlăsceanu et al., 2004). The projects involve a wide range of stakeholders with diverse background and experience, such as panel members, academic and administrative staff, employers, and students. These stakeholders interact with each other to assess whether the universities or programmes meet the accreditation standards (Vlăsceanu et al., 2004). Accreditation projects, therefore, provide suitable conditions for new information and events to occur and influence stakeholder perceptions. The wide range of stakeholders also provides suitable conditions to examine how the individual characteristics of stakeholders affect their perceptions.

Once the cases were identified, the time points of the interviews could be selected. These times points were the same for all stakeholders of the same project to allow for a comparison of the changes in their perception. More than two time points were chosen per project in order to be able to capture the changes (Ferrer \& Grimm, 2012). The specific number of time points and the intervals between them depended on the speed of change of each case. The more rapid the changes, the larger the number of time points, and the smaller the time intervals between them were. When the changes were slow, the number of time points was smaller, and the time intervals were larger (Ferrer \& Grimm, 2012). To identify the speed of change in each case, the researcher first contacted the project manager to get information about the project plan. Based on these plans, the researcher identified the data collection periods and estimated the involvement of stakeholders during these periods. Based on this estimation, the time points of the interviews and the specific participants were identified. Overall, ten individual stakeholders in the three cases were interviewed over periods of four to six months. Most of the participants were interviewed between four and eight times, and the time interval among the interviews was about one month. Only three participants had fewer than four interviews. The total number of the interviews, therefore, was 47 . The cases, the projects, the 
participants, and the number of interviews for each participant are summarised in Table 3.1 as follows.

Table 3.1 Summary of the cases, projects, participants, and number of interviews

\begin{tabular}{|c|c|c|c|c|}
\hline No. & Cases & $\begin{array}{l}\text { Projects - Data collection } \\
\text { periods }\end{array}$ & Participants & $\begin{array}{l}\text { Number of } \\
\text { interviews }\end{array}$ \\
\hline \multirow[t]{3}{*}{1} & \multirow{3}{*}{$\begin{array}{l}\text { The } \\
\text { ABCD } \\
\text { case }\end{array}$} & \multirow{3}{*}{$\begin{array}{l}\text { An international } \\
\text { accreditation project in } \\
\text { New Zealand - six months }\end{array}$} & 1. The project manager & 8 \\
\hline & & & 2: The data analyst. & 8 \\
\hline & & & $\begin{array}{l}\text { 3: The project } \\
\text { administrator }\end{array}$ & 6 \\
\hline \multirow[t]{3}{*}{2} & \multirow{3}{*}{$\begin{array}{l}\text { The } \\
\text { MTEO } \\
\text { case }\end{array}$} & \multirow{3}{*}{$\begin{array}{l}\text { A Vietnamese national } \\
\text { domestic accreditation } \\
\text { project - four months }\end{array}$} & 4: The project manager & 4 \\
\hline & & & 5: A project member & 4 \\
\hline & & & $\begin{array}{l}\text { 6: A representative of an } \\
\text { employer }\end{array}$ & 4 \\
\hline \multirow[t]{4}{*}{3} & \multirow{4}{*}{$\begin{array}{l}\text { The } \\
\text { APSC } \\
\text { case }\end{array}$} & \multirow{4}{*}{$\begin{array}{l}\text { An international } \\
\text { accreditation project in } \\
\text { Vietnam - four months }\end{array}$} & 7: The project manager & 4 \\
\hline & & & $\begin{array}{l}\text { 8: The leader of the faculty } \\
\text { team }\end{array}$ & 4 \\
\hline & & & $\begin{array}{l}\text { 9: A project member in a } \\
\text { similar accreditation } \\
\text { project at another } \\
\text { university }\end{array}$ & 3 \\
\hline & & & $\begin{array}{l}\text { 10: The regional chair of } \\
\text { the accreditation body }\end{array}$ & 2 \\
\hline & & Total & 10 & 47 \\
\hline
\end{tabular}

\subsubsection{Data Collection Methods}

For each participant, the interviews were divided into the first and the follow-up interviews. In the first interviews, the Repertory Grid Technique was used to elicit the repertory grids, which describe the participant's thinking about their projects. In the follow-up interviews, open questions were used to get information about the changes in the repertory grids and the events and ideas related to the projects. The first interviews often lasted from one and a half to two hours, and the follow-up interviews often lasted from thirty to forty minutes.

\section{The first interview}

Triadic elicitation was used to create the repertory grids that describe the participants' thinking about their projects. In this elicitation, six elements were used. They were the ideal and less 
than ideal projects, the target accreditation projects, and three other projects that the participants found relevant to their perception of the target projects. An illustration of these elements is presented in Figure 3.5 below.

\section{Figure 3.5 An illustration of elements for the triadic elicitation}
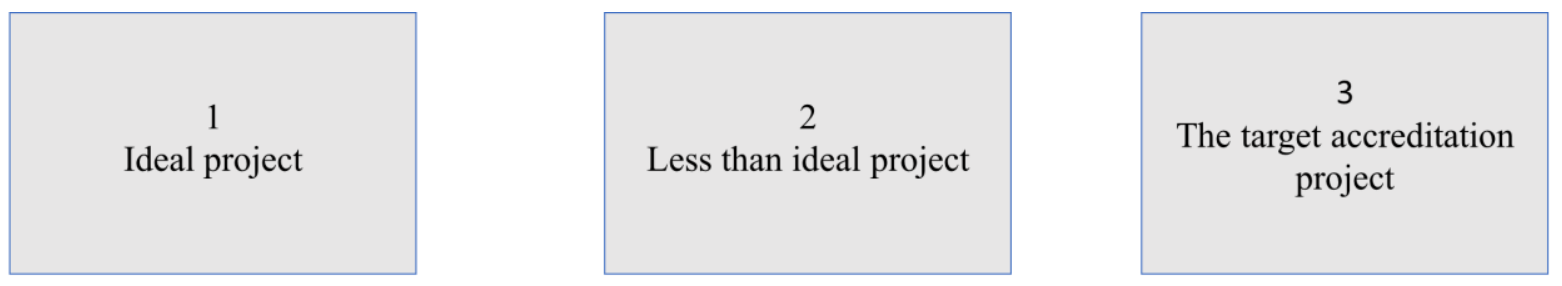

\section{4}

One project that the participant found relevant to the target project
5

One project that the participant found relevant to the target project
6

One project that the participant found relevant to the target project

Different sets of three elements were used for the elicitation until no new answers came up. The elicitation question was "Which two of these are the same in some ways, and different from the third." Laddering questions were also used to clarify the meanings of the answers. Examples of these questions were "What do you mean by...?" and "Can you give me an example of...?" The answers were then written down on the Grid Sheets. A sample of the Grid Sheets and its translation in Vietnamese are exhibited in Appendix B and D, respectively.

After the elicitation of the constructs, the participants rated the elements. A 5-point scale was used for the New Zealand case. After the data collection of this first case, the 7-point scale was selected for the two cases in Vietnam because it could capture the changes in the participants' perception better. After the ratings, the participants reviewed the grids to ensure their accuracy. This review helped to reduce the researcher bias in collecting data from the participants (Cavana et al., 2001).

\section{The follow-up interviews}

In the follow-up interviews, the participants changed the grids to capture the changes in their thinking about the projects. Specifically, at the beginning of the interviews, the researcher first asked the participants about their general thinking about the target projects to avoid biases 
caused by the grids. The participants then went through the content of the grids. They were allowed to change the content of the constructs, add new constructs, remove existing constructs, and change the ratings of the elements. For any change, the participants were asked to explain the reasons. These explanations provided information about their perceptions of related events and ideas.

In summary, the interviews collected data on the changes in the repertory grids of the participants and their perceptions of the related events and ideas. The detailed interview guides and their translation in Vietnamese are presented in Appendix A and C, respectively. The next section continues with an overview of the data analysis methods.

\subsubsection{Data Analysis Methods}

The data analysis consisted of three steps. The first two steps were to analyse data on each participant, as illustrated in Figure 3.6 below.

Figure 3.6 Two first steps of data analysis for each participant

The repertory grids

Step 1: Principal component analysis of the repertory grids at different time points

Changes in the standards and appreciation of each participant regarding their project
The events and ideas related to the project

Step 2: Integration of the events and ideas with the changes in the standards and appreciation 
In the figure above, the yellow ovals represent the collected data, the blue rectangles represent the analysis steps, and the green ovals represent the analysis outcomes. The first step of the analysis was the principal component analysis of the repertory grids using the Rep Plus V1.1R software. This analysis identified changes in the standards and appreciation of the participants regarding their target projects. In the second step, these changes were integrated with the events and ideas. The results of this integration were stories of the changes in the participants' appreciation, as influenced by their standards and perceptions of related events and ideas. For the purpose of presentation, a detailed explanation of these steps will be presented in chapter four of the data analysis and findings.

Following the above two steps, the third step identified the common themes of the dynamics of the participants' appreciation, standards, and perceptions of events and ideas. Based on these themes, a framework was proposed to describe the dynamics of individual stakeholders' appreciation of projects as influenced by their individual characteristics. These common themes and the proposed framework will be presented in chapter seven.

\subsection{Ethical Considerations}

Because this study was a $\mathrm{PhD}$ research done at Victoria University of Wellington, it adhered to the university's ethical guidelines and had to be granted ethical approval from the university human ethics committee before data collection could begin. Evidence of the ethical approval (ethical approval number: 0000025965) is exhibited in Appendix E. The research also followed the ethical guidelines related to the Treaty of Waitangi, which protects the integrity, respect, concern, interest, and wellbeing of Maori individuals and the community in New Zealand. The following paragraphs identify areas of the data collection and analysis processes that required ethical considerations and then describe the measures that were implemented in order to ensure compliance with ethical guidelines

In terms of informed consent, the participants were given information about the research, the researchers, and what they were expected to do. It was also explained to them how their identities would be kept confidential and to whom their identities would be revealed. Moreover, they were informed about their rights in the interviews. Thus, they could choose not to answer questions, withdraw from the research, refuse to be recorded, or ask any question at any time. The project leaders were also given information about the involvement of the participants in the research. The participants could only participate in the research with the 
written consents of the leaders. These forms and other related ethical forms are exhibited in Appendix F and G.

According to the guidelines of the University, the hard copies of the data were stored securely in a locked filing cabinet and their electronic copies, the audio recordings, and other electronic materials were saved on the university servers with password protection. The data analysis was only done on the personal laptop of the researcher and her desktop computer at the university office. The data were only revealed to the researcher and her two supervisors and were not revealed to other parties. The identities of the participants were also protected. In the research report, they were referred to by pseudonyms. In the event that any publishable papers are produced from the research, all sensitive information related to the participants and their projects will be considered carefully to protect participants' and projects' anonymity. The data will be used for this research only and will be destroyed within two years after the completion of the thesis.

\subsection{Summary}

This chapter has presented the research methodology. The data collection for this study involved two to eight interviews with ten individual stakeholders working on the three different projects over a period of four to six months. The Repertory Grid Technique and Vickers' concept of appreciative systems were applied to collect and then analyse data from these participants. The detailed explanation of the data analysis methods and the results of the data analysis of the first case will be presented in the next chapter. 


\section{Chapter 4 MTEO Case}

\subsection{Overview of the Chapter}

Chapter three has presented an overview of the two main steps of the data analysis. This chapter explains these steps in detail. Following this explanation, the results of the data analysis in the MTEO case are presented. These results include the project overview and the changes in the appreciation of three participants in the case, as influenced by their standards and perceptions of events and ideas.

\subsection{Explanation of Two Main Steps of Data Analysis}

The data analysis for each participant involved two main steps. The first step was the principal component analysis of the repertory grids to identify the changes in the standards and appreciation of the participants regarding their projects. The second step was to integrate these changes with the participant perceptions of related events and ideas by applying Vickers' concept of appreciative systems. The results of this step were the changes in the participants' appreciation of their projects, as influenced by their standards and perceptions of events and ideas.

\subsubsection{Step 1 - Principal Component Analysis}

The principal component analysis of repertory grids is a data reduction method that identifies the patterns of ratings and the attributes of these patterns to the total variability of the ratings in the grids (Jankowicz, 2005). In a repertory grid, each row, which reflects the ratings of elements on each construct, can have different variability. The principal component analysis identifies the extent to which the ratings in each row are similar and, in that way, identifies the distinctive patterns of the variability of the ratings. The analysis also identifies the attributes of these distinctive patterns to the total variability of the ratings in grids (Jankowicz, 2005). This analysis is described as an iterative process (Jankowicz, 2005). Once the pattern which accounts for the largest amount of variability is identified, it will be subtracted from the original grids and set aside. The next pattern is then identified likewise, and this process continues until all the variability of the ratings in the grids has been accounted for. These distinctive patterns of variability are called principal components (Jankowicz, 2005). The number of principal components of a grid, therefore, depends on the variability of its ratings. If constructs are different in nature, their ratings of elements are likely to be different, and they can be explained by a high number of principal components. If constructs are similar, their ratings of elements 
are likely to be similar, and they can be explained by a low number of principal components (Jankowicz, 2005).

Experts in repertory grids, such as Fransella et al. (2004) and Jankowicz (2005), recommended using specialised software to analyse repertory grids in general and do the principal component analysis of the grids in particular. The Rep Plus V1.1R software is specialised software to analyse repertory grids (Fransella et al., 2004). The principal component analysis performed by the software is based on the principal component analysis method introduced by Slater $(1976,1977)$. This method uses a non-statistical, distance-based and geometric model and algorithms to conceptualise grid data. Following this method, the Rep Plus V1.1R software treats a grid as a geometric configuration. In this configuration, the constructs form the axes of $\mathrm{n}$-dimensional space, and the elements are represented by points located in that space determined by their ratings on the constructs. The software then rotates the configuration to lower its dimensionality as much as possible so that it may be plotted with principal components as axes in 2 or 3 dimensions (Gaines \& Shaw, 2018b). The command to perform this principal component analysis is described in detail in the manual of the software (Gaines \& Shaw, 2018b).

Researchers, such as Jankowicz (2005) and Bezzi (1999), has introduced guidelines for and examples of using the Rep Plus V1.1R software or its previous version to perform the principal component analysis of repertory grids. Based on these guidelines and examples, the principal component analysis using the Rep Plus V1.1R software in this research involved four sub-steps. These sub-steps are summarised in Figure 4.1 as follows. 
Figure 4.1 Four sub-steps of the principal component analysis using the Rep Plus V1.1R software to identify the changes in the standards and appreciation of each stakeholder regarding their project

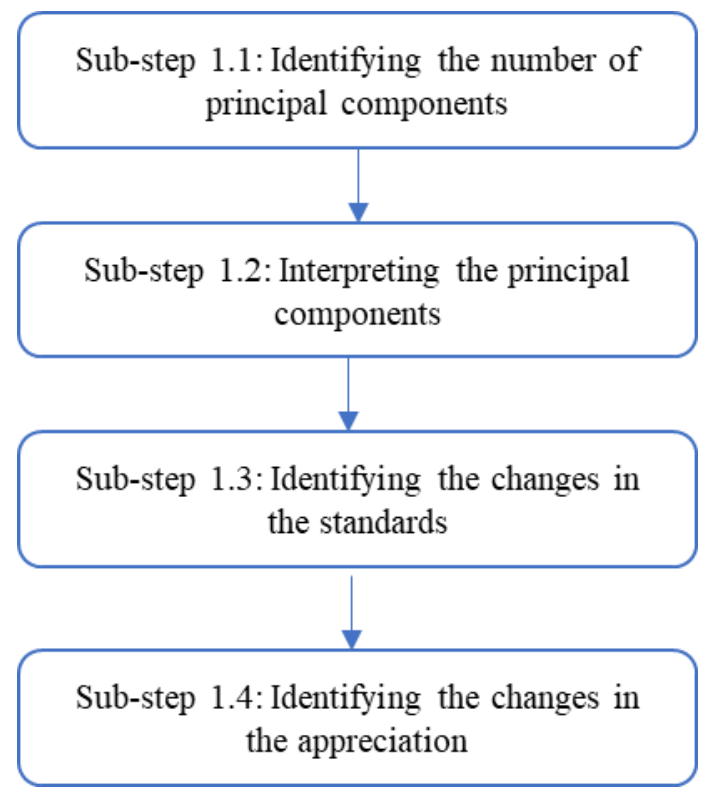

As illustrated in the figure, for each participant, the first sub-step was to identify the number of the principal components of their repertory grids at different time points. The second sub-step was to interpret the meanings of the components. Based on these meanings, the third sub-step identified the participant standards for the project and their changes across time. The final sub-step identified the changes in the participant's appreciation of the project.

4.2.1.1. Sub-step 1.1: Identifying the Number of Principal Components. In technical terms, the number of the principal components is identified based on the percentage variance explained by each component (Jankowicz, 2005). For example, section 3.6.2 features a grid that describes the characteristics of six salespersons (Figure 3.3) (Jankowicz, 2005). Jankowicz (2005) has used the Rep Plus V1.1R software to perform the principal component analysis of the grid. The analysis has produced the percentage variances explained by principal components, which are presented in Table 4.1 as follows.

Table 4.1 An example of percentage variances explained by principal components (Source: Jankowicz, 2005)

\begin{tabular}{|l|c|c|c|c|c|}
\hline $\begin{array}{l}\text { Principal } \\
\text { Components }\end{array}$ & Component 1 & Component 2 & Component 3 & Component 4 & Component 5 \\
\hline $\begin{array}{l}\text { Percentage } \\
\text { variances (\%) }\end{array}$ & 70.99 & 23.61 & 4.46 & 0.52 & 0.42 \\
\hline
\end{tabular}

In this example, even though five components were identified to account for the total variability of the grids' ratings, the variability that components 3, 4 and 5 accounted for was 
tiny. Because of this, only the first two components were chosen (Jankowicz, 2005). Jankowicz (2005) mentioned the rule to select principal components is that they must explain at least $80 \%$ of the variability of the ratings of the grids, or their cumulative variance must be higher than $80 \%$. In the above table, the cumulative percentage variance of the two first components is $94.6 \%(70.99 \%+23.61 \%)$. Similar to Jankowicz (2005), Bezzi (1999) used a principal component analysis command in a previous version of the Rep Plus V1.1R software to perform the principal component analysis of the repertory grids in his research. These grids describe students' perception of different subjects at the beginning and the end of a trimester. One example of the percentages of variance explained by the principal components in Bezzi's (1999) research is presented in Table 4.2 as follows.

Table 4.2 Percentage of variance for each component (Cmp) (Source: Bezzi, 1999)

\begin{tabular}{|c|c|c|c|c|}
\hline Cmp 1 & Cmp 2 & Cmp 3 & Cmp 4 & Cmp5 \\
\hline 68.34 & 26.40 & 3.21 & 1.95 & 0.10 \\
\hline
\end{tabular}

Bezzi (1999) also selected the components so that their cumulative variance was higher than 80\%. In line with Jankowicz's (2005) and Bezzi's (1999) examples, this research used the principal component analysis command of the Rep Plus V1.1R software to identify the percentages of variance explained by the principal components of the grids and select the components. The number of the selected principal components for most participants in this research was often two. Between them, the first component often accounted for a large percentage (from $70 \%$ to $80 \%$ ), and the second component often accounted for a much smaller percentage of the participant appreciation (from 5\% to 20\%). The data only featured one case with one principal component and one case with three.

4.2.1.2. Sub-step 1.2: Interpreting the Principal Components. Principal components are interpreted based on the extents to which constructs explain them. These extents are called construct loadings on components (Fransella et al., 2004). The higher the loadings are, the more constructs characterise components (Bezzi, 1999). An example of construct loadings on components in the above grid of salespersons is presented in Table 4.2 as follows. 
Table 4.3 An example of construct loadings on components (Sources: self-produced based on an example in Jankowicz, 2005)

\begin{tabular}{|c|c|c|c|l|}
\hline Constructs & Component 1 & Component 2 & Component 3 & Constructs in detail \\
\hline C1 & 1.872 & -0.513 & 0.093 & $\begin{array}{l}\text { Learns the new models quickly - } \\
\text { Takes a while to learn the } \\
\text { features of new lines }\end{array}$ \\
\hline C2 & -0.258 & -1.209 & -0.532 & $\begin{array}{l}\text { Too forward in pushing a sale: } \\
\text { tends to put customers off - } \\
\text { Good balance between active } \\
\text { selling and just being helpful }\end{array}$ \\
\hline C3 & -1.832 & 0.112 & -0.338 & $\begin{array}{l}\text { Could be more interested in } \\
\text { after-sales - After-sales well } \\
\text { handled }\end{array}$ \\
\hline C4 & 1.722 & -0.608 & 0.062 & $\begin{array}{l}\text { Awareness of sizes, colours, } \\
\text { availability - Availability and } \\
\text { choice knowledge poor }\end{array}$ \\
\hline C5 & 0.78 & 1.36 & -0.133 & $\begin{array}{l}\text { Pleasant and easy-going - Takes } \\
\text { it all very seriously }\end{array}$ \\
\hline C6 & 1.518 & 0.553 & -0.614 & $\begin{array}{l}\text { Overall, an effective salesperson } \\
\text { - Overall, a less effective person }\end{array}$ \\
\hline
\end{tabular}

In the above table, the first column presents the construct numbers. The second, third and fourth columns present the construct loadings on the components. The fifth column describes the constructs in detail. The loadings can also be graphically illustrated on a twodimensional PrinGrid map (Jankowicz, 2005). The PrinGrid map for the above example of salespersons is presented in Figure 4.2 as follows. 
Figure 4.2 An example of the distribution of constructs on a PrinGrid map (Source: Jankowicz, 2005)

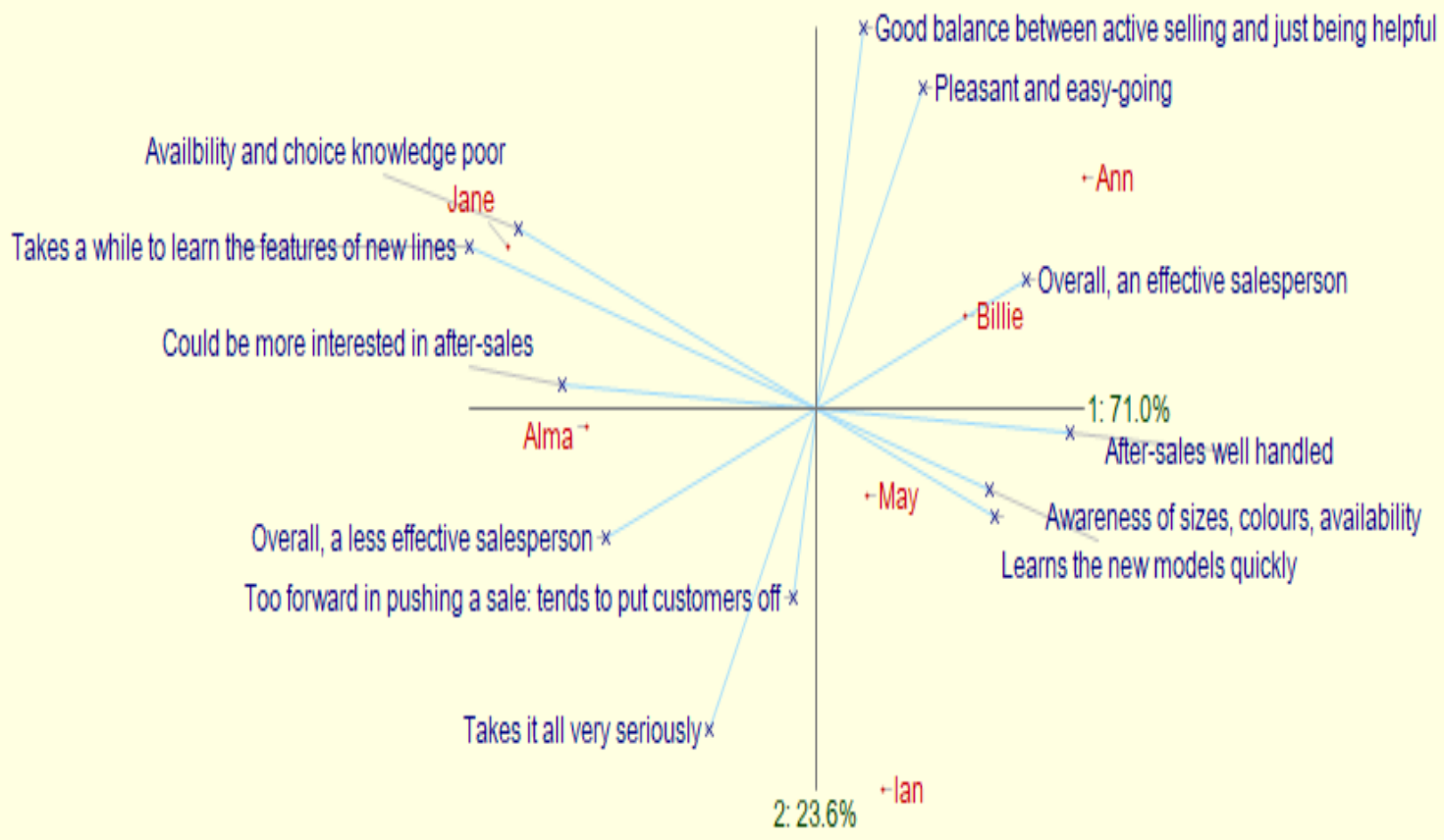

The horizontal axes on PrinGrid maps represent the first components, and vertical axes represent the second components. These two axes are at right angles to each other to illustrate that the two components have maximally distinct patterns of variability. Constructs are plotted as straight lines whose angles with respect to each axis reflect the extents to which components represent constructs. Angles between any two construct lines reflect the extents to which ratings of elements according to those constructs are correlated. The smaller the angles, the greater the similarity of the constructs' ratings (Jankowicz, 2005). For example, as shown in Table 4.2, C1 (refers to whether the salespersons can learn new models quickly) and C4 (refers to the awareness of sizes, colours, and availability) have large loadings on component 1 . On the PrinGrid map, the lines describing these constructs are close to each other. They are also close to the horizontal axis. These two constructs, therefore, are correlated with each other and can be explained by component 1 .

Two aspects of the loadings are considered to interpret the components. They are the values and the "signs" of the loadings. The values of the loadings refer to whether the loadings are large or small. For example, Table 4.2 shows that constructs $\mathrm{C} 1, \mathrm{C} 3, \mathrm{C} 4$, and $\mathrm{C} 6$ have their largest loadings on the first component and constructs C2 and C5 have their largest loadings on the second component. On the map in Figure 4.2, lines representing constructs C1, C3, C4 and C6 are closer to the horizontal axis and lines representing constructs C2 and C5 are closer 
to the vertical axis. The first component, therefore, is interpreted based on the meanings of $\mathrm{C}$, C3, C4 and C6. The second component is interpreted based on the meanings of C2 and C5 (Jankowicz, 2005).

The signs of the loadings refer to how contrary poles of constructs explain contrary poles of components. Each construct has two contrary poles which refer to their positive and negative meaning. Because principal components are interpreted based on these constructs, they also have contrary poles. If the same positive or negative poles of constructs explain the meaning of one pole of the component, the constructs have the same signs of loadings on the component. If the positive pole of one construct and the negative pole of another construct explain the meaning of one pole of the component or vice versa, the constructs have opposite signs of loadings. The signs of loadings are more visually illustrated on the PrinGrid maps. For example, the above map (Figure 4.2) shows that the loadings of the constructs on the two components have the same signs. All the positive poles of $\mathrm{C} 1, \mathrm{C} 3, \mathrm{C} 4, \mathrm{C} 6$, including 'good awareness of sizes and colours' and 'good after-sales', explain one pole of the first component. The situation is similar for the second component. The two components, therefore, are interpreted as the composite meaning of the constructs with large loadings on them. Component 1 is interpreted as technical knowledge, and component 2 is interpreted as personal style of salespersons (Jankowicz, 2005).

For most participants in this research, many of their constructs had considerably large loadings on the first components, and only a few of them had considerably large loadings on the second components. The loadings on the first components often had the same signs, and the loadings on the second components were often divided into two groups with opposite signs. The first components, therefore, were often interpreted as the composite meaning of many constructs and the second components were often interpreted as the comparison between two groups of constructs. Even though the PrinGrid maps are better than tables of loadings in illustrating the values and the signs of loadings, in this thesis, because of space limits, only the loading tables are presented in the Appendixes. The PrinGrid maps, featuring the distributions of constructs, are not presented in either the main text or the appendixes.

\subsubsection{Sub-step 1.3: Identifying the Changes in the Standards of Stakeholders for}

Projects. The changes in stakeholder standards for their projects were identified by comparing the standards over different time points. According to Vickers' concept, stakeholder standards were the aspects of projects that stakeholders focus on and the importance of those aspects. 
The standards, therefore, were identified by analysing the stakeholder appreciation of the target projects, as well as the ideal and the less ideal projects. According to principal component analysis, this appreciation, since they are captured as elements in the repertory grids, can be described by its loadings on the principal components. The loadings of elements on principal components signify the extent to which elements are explained by components (Fransella et al., 2004). An example of the loadings of the above grid of salespersons is presented in Table 4.3 below.

Table 4.4 An example of the loadings of elements on principal components (Sources: selfproduced from an example in Jankowicz, 2005)

\begin{tabular}{|l|r|r|}
\hline Elements & Principal Component 1 & Principal Component 2 \\
\hline E1 - Jane & 2.211 & -0.681 \\
\hline E2 - Ann & -1.924 & -0.974 \\
\hline E3 - Billie & -1.07 & -0.392 \\
\hline E4 - Ian & -0.487 & 1.597 \\
\hline E5 - Alma & 1.642 & 0.079 \\
\hline E6 - May & -0.372 & 0.372 \\
\hline
\end{tabular}

In the above table, the first column presents the salespersons as the elements. The second and third columns present the loadings of the elements on the components. These loadings indicate how the principal components explain the characteristics of the elements or salespersons. For example, element E2, Ann, has large loadings on both component 1 and 2 (1.924 and -0.974 , respectively). Meanwhile, element E5 (Alma) only has a large loading on component 1 (1.642). The PrinGrid maps also illustrate these loadings. The position of each element with respect to each axis is exactly like the position of a point on a graph and illustrates the loadings. The distance between any two elements reflects their differences in ratings. If two elements are close to each other, they tend to have similar ratings. If two elements are far apart, they tend to have different ratings (Jankowicz, 2005). For example, on the PrinGrid map in Figure 4.2, the position of Ann, which is at the far corner of the upper-right quadrant, shows that both her technical knowledge and personal styles are good. Meanwhile, the position of Alma, which is very close to the horizontal axis, shows that her technical knowledge is good, and her personal style is moderate. This is in line with the loadings listed in Table 4.3 (Jankowicz, 2005).

As explained in section 3.4.3., standards can be primary or secondary. Primary standards are important to projects and decide whether projects are good or bad. Secondary standards are not important to projects but refer to noticeable aspects of projects. In this 
research, the primary standards of stakeholders were identified based on the principal components that explained not only stakeholder appreciation of the target projects but also their appreciation of the ideal and less than ideal projects. Meanwhile, the secondary standards were identified based on the principal components that only explained the stakeholders' appreciation of the target projects. More specifically, the primary and secondary standards were identified by the loadings of the participant appreciation of the target, the ideal, and the less than ideal projects on the principal components. One example of the loadings used in this research is presented below.

Table 4.5 An example of the loadings of a participant's appreciation of the ideal project, the less than ideal project, and the target project on the two components at one interview

\begin{tabular}{|l|l|l|}
\hline Component & 1 & 2 \\
\hline Ideal project & -3.837 & -0.612 \\
\hline Less than ideal project & 6.241 & -0.456 \\
\hline Target project & -1.557 & -1.108 \\
\hline
\end{tabular}

In the above example, the participant's appreciation of the target project has loadings on both components. These components, therefore, represent the participant standards for the project. However, while the appreciation of the ideal and less than ideal projects has very large loadings on the first component, the loadings on the second component are very small. The loadings on the first component also have opposite signs. The first component, therefore, can differentiate the appreciation of the ideal project from the appreciation of the less than ideal project and represents the primary standards of the participant for the target project. Meanwhile, the second component cannot differentiate the appreciation of the ideal from the appreciation of the less than ideal project and only indicates the aspects of the project that the participant pays attention to. The second component, therefore, represents the participant's secondary standards for the project. The PrinGrid maps can also illustrate the loadings of elements on components. A PrinGrid map that illustrates the loadings in the above table is presented in Figure 4.3 as follows. 
Figure 4.3 An example of a PrinGrid map that illustrates the loadings of the appreciation of the ideal project, the less than ideal project, and the target project on the components at one interview

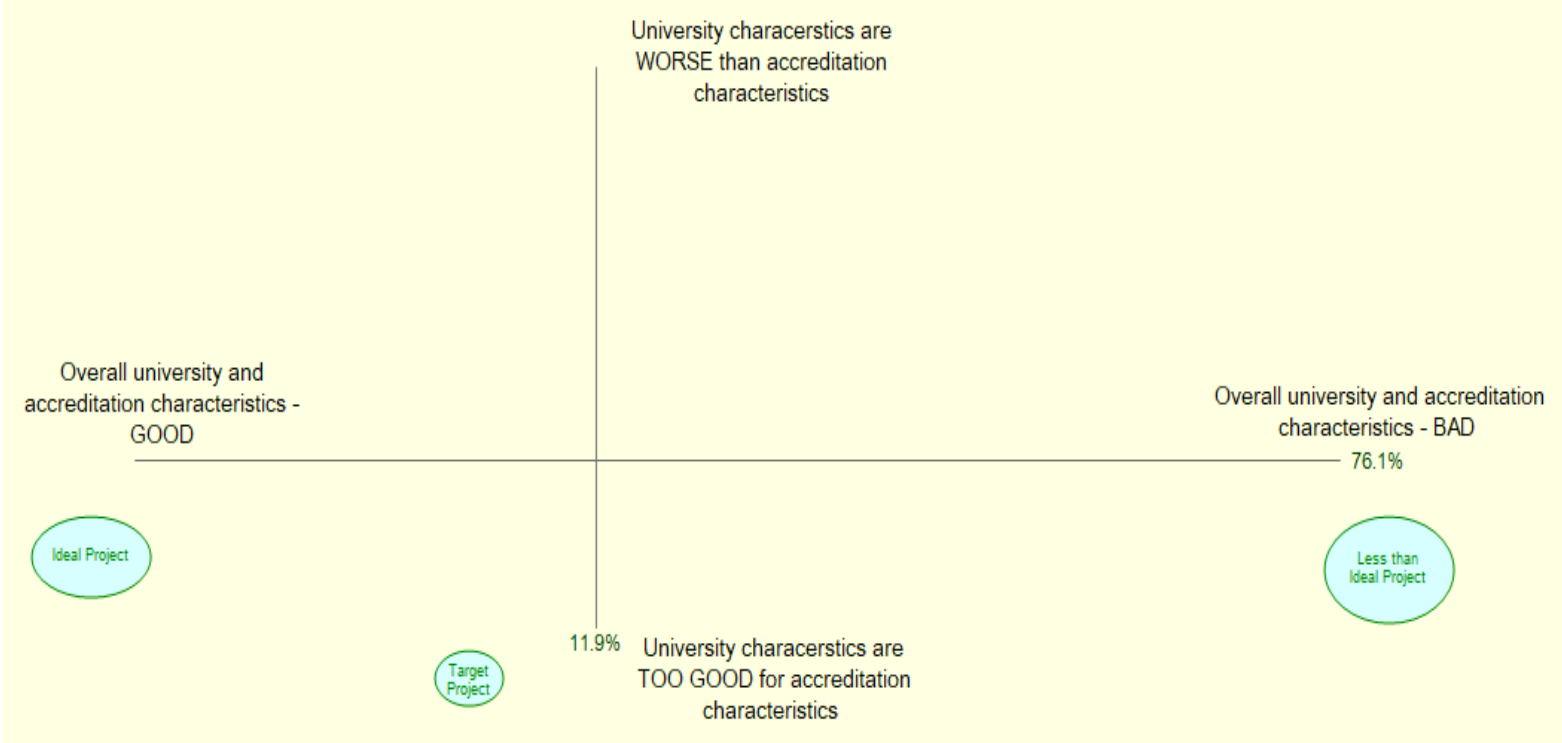

On the above map, the positions of the ideal and the less than ideal project regarding the horizontal axis, which refers to the first component, are opposite. Their positions regarding the vertical axis, which refers to the second component are very similar. This indicates that while the first component can differentiate the ideal project from the less than ideal project, the second component cannot. This is in line with the loadings in Table 4.4. This example also reflects the situation of most participants in this research. The first principal components often represented their primary standards, and the second components often represented their secondary standards. There was only one case in which both components represented the participant's primary standards for the project.

\subsubsection{Sub-step 1.4: Identifying the Changes in Stakeholders' Appreciation of}

Projects. As explained above, stakeholder appreciation of their target projects, as elements, can be interpreted based on its loadings on the principal components. Therefore, the changes in appreciation could be identified by changes in the loadings and in the interpretation of the components. This section focuses on changes in the loadings of the elements as the interpretation of components has already been explained in section 4.2.1.2. These changes were identified based on the loading tables and illustrated by the PrinGrid maps. An example of the map is presented in Figure 4.4 below, which is taken from Bezzi (1999). This map describes changes in student perception of science subjects based on data collected at two separate times points (A and $\mathrm{B})$. 


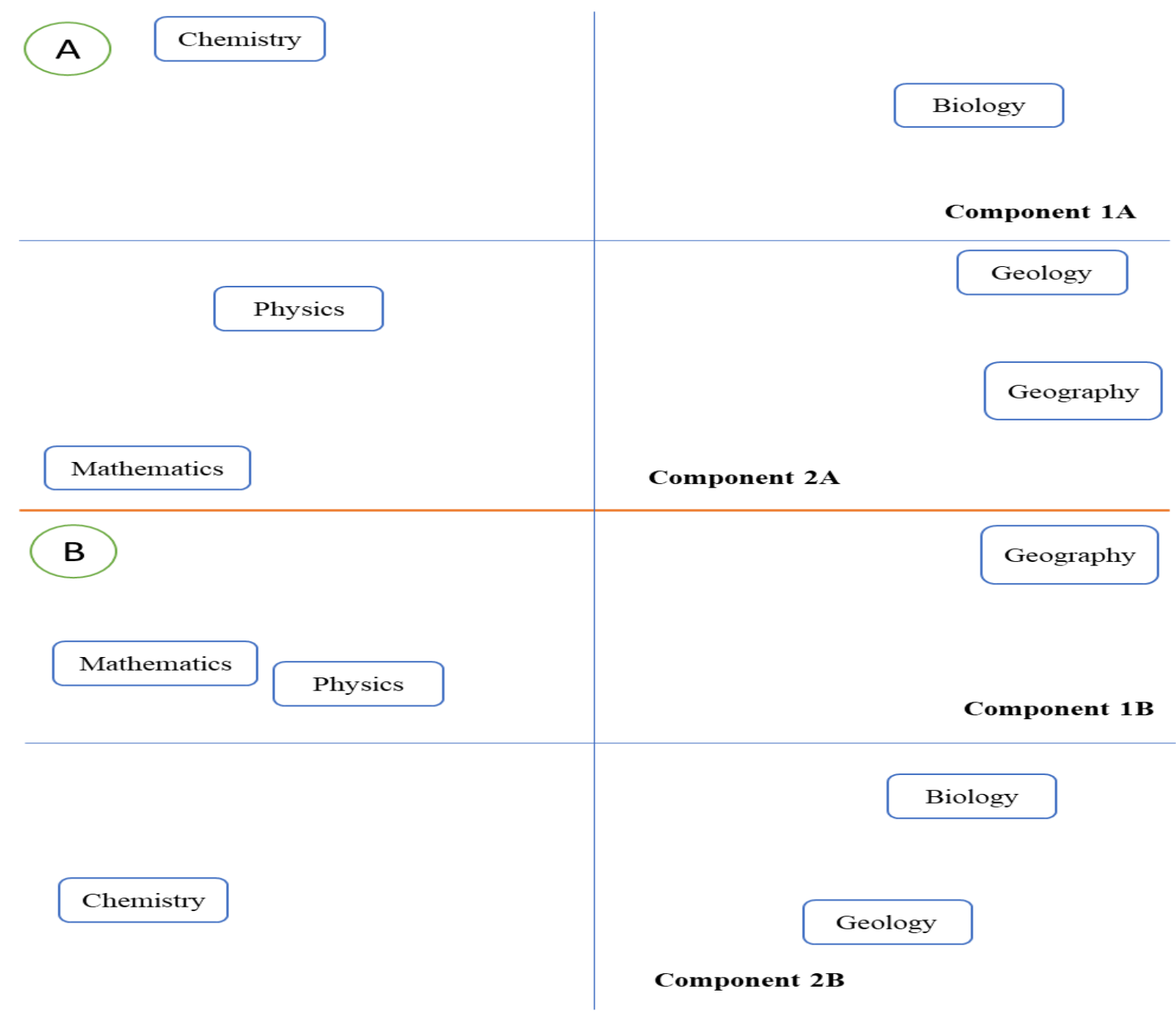

The map shows the positions of the elements related to each other change between the two time points. For example, while biology and geology are in different quadrants of map A, they are in the same quadrant in map B. In this example, changes in the loadings of the elements on the components were significant and could be illustrated by simply putting two PrinGrid maps next to each other. In this research, however, changes were minor, and therefore, were identified and triangulated by two steps of analysis. First, the changes were identified by the loading numbers in the loading tables. Changes were identified in different ways for the components. Thus, changes in the components for the primary standards were determined based on the gaps between the loadings of the appreciation of the target projects and the ideal projects. These gaps reflect the distance between the participant appreciation of the target projects and the ideal projects. Changes in the component representing the secondary standards 
were identified based on the loadings of the appreciation of the target projects. An example of the loadings and their changes are presented and highlighted in Table 4.5 below.

Table 4.6 An example of the changes in the loadings

\begin{tabular}{|l|l|l|l|l|l|l|l|l|}
\hline Interview & \multicolumn{2}{|l}{$T 1$} & \multicolumn{2}{l}{$T 2$} & $T 3$ & \multicolumn{2}{l|}{$T 4$} \\
\hline Component & 1 & 2 & 1 & 2 & 1 & 2 & 1 & 2 \\
\hline Ideal project & -3.837 & -0.612 & -3.798 & -0.576 & -3.688 & -0.92 & -3.921 & -1.18 \\
\hline $\begin{array}{l}\text { Less than ideal } \\
\text { project }\end{array}$ & 6.241 & -0.456 & 6.272 & -0.445 & 6.356 & -0.579 & 6.146 & -0.78 \\
\hline Target project & -1.557 & -1.108 & -1.696 & -1.056 & -2.072 & -0.37 & -2.139 & -0.268 \\
\hline Gap & 2.28 & & 2.102 & & 1.616 & & 1.782 & \\
\hline
\end{tabular}

Second, if the interpretation of the components showed no or minor changes over the time points, the trajectory function was used to triangulate and describe the appreciation changes on the same PrinGrid maps. The appreciation was described at different points, labelled T1, T2, T3, and so on, and the arrows that connect the resulting points illustrate the appreciation changes (Gaines \& Shaw, 2018abcd). An example of a PrinGrid map that features the trajectory function is presented in Figure 4.5 below:

Figure 4.5 A PrinGrid map which uses the trajectory function to describe the changes in a participant's appreciation of her project over four interviews

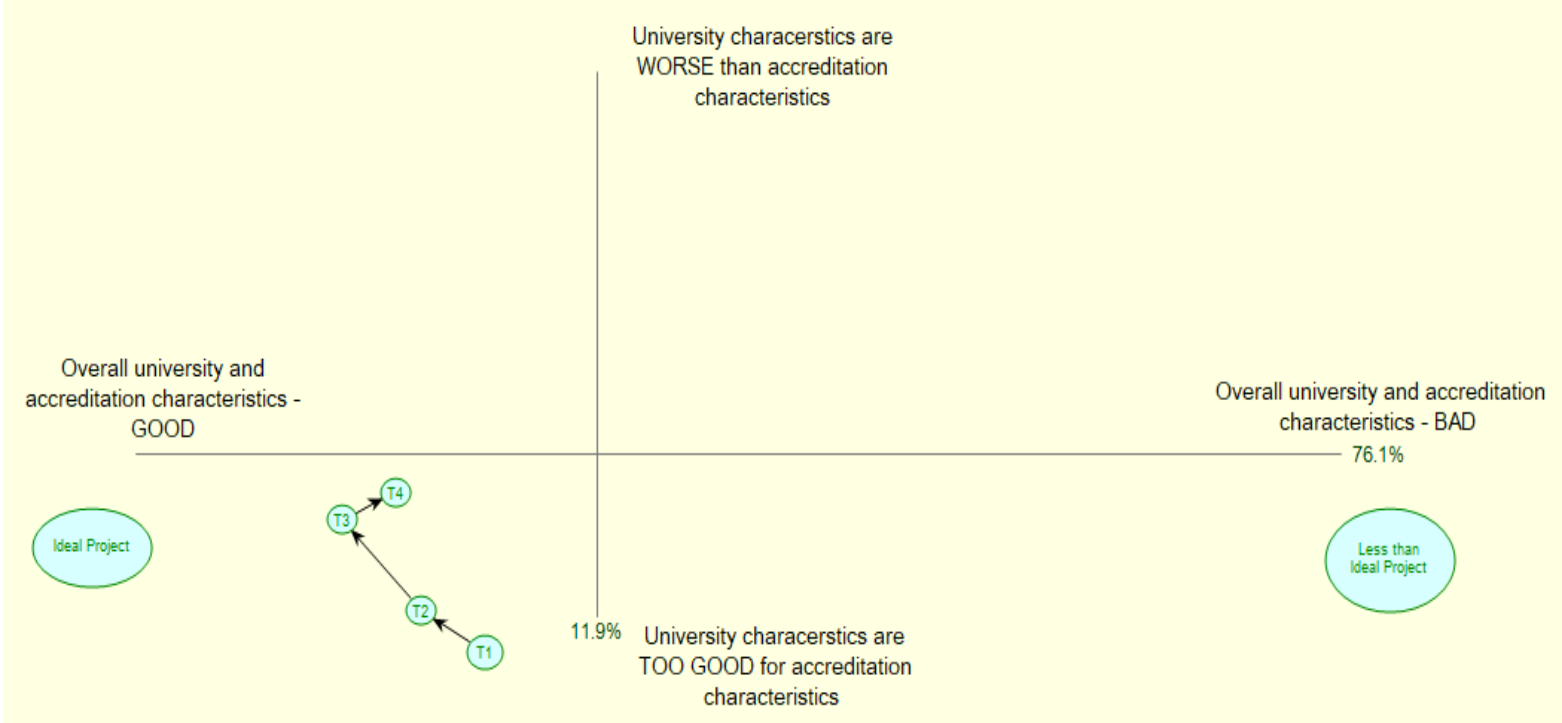

If the interpretation of the components had major changes and these changes affected the presentation of the appreciation at different time points on the same maps, the appreciation was presented on different maps. Therefore, the number of PrinGrid maps differed between the participants as the appreciation at different time points for some participants could be illustrated 
on one map, while for others two or more maps were needed. The maps also captured the appreciation of the ideal and less than ideal projects, which helped to illustrate the primary and secondary standards for the target projects and the distance between the appreciation of the target projects and the ideal projects.

In this research, the two terms "interview" and "time point" are used interchangeably to describe the timing of the changes in stakeholder perception and thinking. While most of the participants had interviews at all the intervals throughout their cases, a few participants did not. Because of this, the term "interview" did not describe precisely the time of the changes in some cases. For example, for one participant, the time point of their fourth interview was not T4 but T6. Because of this, the two terms "interview" and "time point" are used deliberately to describe precisely when the changes in the perception occurred.

In summary, this section has explained how principal component analysis served to identify the changes in the standards and appreciation of stakeholders regarding their projects. The next section explains the application of Vickers' concept of appreciative systems.

\subsubsection{Step 2 - the Application of Vickers' Concept of Appreciative Systems}

Step two consisted of the integration of the events and ideas that stakeholders learned with the changes in their standards and appreciation through the application of the dynamics of Vickers' appreciative systems. These dynamics have been illustrated by Figure 3.1 and explained in section 3.4.1 in the methodology chapter. According to the dynamics of Vickers' appreciative systems, with their experience, each individual stakeholder has standards for their project at each time point. These standards consist of primary standards, which decide whether the project is good or bad, and secondary standards, which refer to noticeable aspects of the project (Vickers, 1984). Along with these standards, the individual stakeholder also has an appreciation of the project. This appreciation reflects the stakeholder's prediction and hypotheses regarding the project (Vickers, 1965/1995). The stakeholder then engages in different interactions and learns events and ideas which may affect their standards and appreciation regarding the project (Vickers, 1965/1995). Equipped with these new standards and appreciation, the individual stakeholder can learn other events and ideas that, in turn, affect their standards for and appreciation of the project, resulting in the dynamics of stakeholders' appreciation of projects.

Based on these dynamics, the participants' perceptions of events or ideas were integrated with the changes in their standards and appreciation. This integration was done by following three steps of grouping. First, the events and ideas were grouped according to their 
impacts on the standards or appreciation. Second, the events and ideas were grouped by the components that explain the standards or appreciation. Finally, for each component, the events and ideas were grouped by their positive or negative impacts. These groupings together generated descriptions of the changes in each participant's appreciation of their project, as influenced by their standards and perceptions of events and ideas.

These descriptions provided rich and precise pictures of the changes in the participants' appreciation and standards regarding their projects. In step 1 of principal component analysis, these changes had been identified based on the contents and ratings of the constructs in the grids. In this step, the descriptions of the changes according to Vickers' concept added further information about their context and helped to triangulate them. The whole interview process, such as the responses or emotion of the participants, were also considered to achieve accurate interpretations of the changes (Jankowicz, 2005). The analysis results were also sent to the participants for feedback to ensure that they reflect their thinking accurately (Jankowicz, 2005). Overall, the analysis was an iterative process that helped reduce the researcher bias in data analysis (Cavana et al., 2001). This process can be illustrated in Figure 4.6 below. Following this process, the principal components were interpreted in a flexible way. In some cases, they were interpreted based on all the constructs with loadings on them. In other cases, they were interpreted based on only the constructs with significantly large loadings on them. This flexibility is in line with B. Gaines's (personal communication, July 25, 2019) emphasis that the ultimate objective of the analysis of repertory grids is to describe the changes in the interviewees' thinking accurately. 


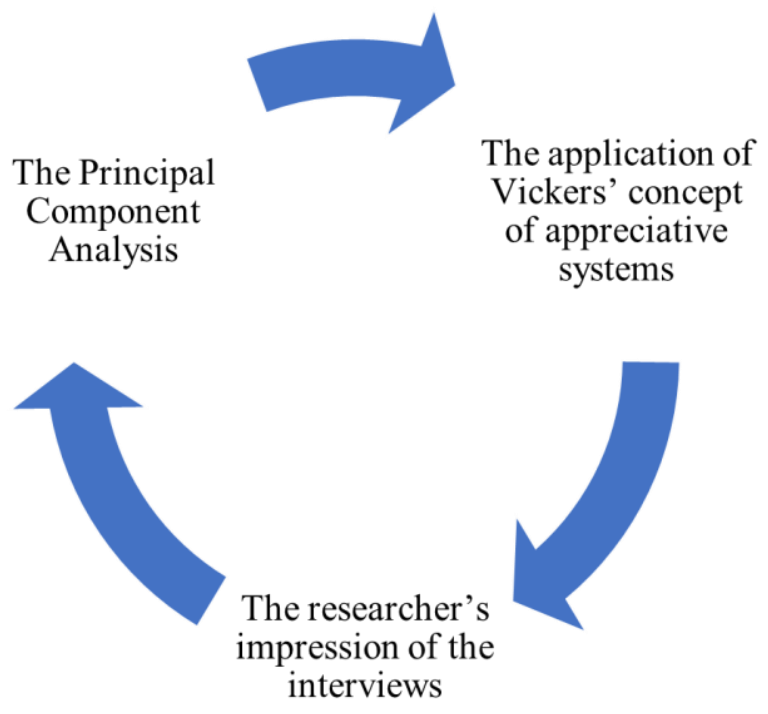

In summary, this section has explained how principal component analysis of the Repertory Grid Technique and Vickers' concept of appreciative systems were applied to identify the changes in the stakeholders' appreciation of their projects, as influenced by their standards and perceptions of events and ideas. These are the approaches that were used in the analysis of the three cases. The data analysis and findings of the MTEO case will be described in the following section.

\subsection{Overview of the MTEO Accreditation Project}

The MTEO was a compulsory national domestic accreditation for universities in Vietnam and was issued and managed by the Ministry of Education. As a private university, FXN University had started a project to obtain this accreditation two years before the data collection for this thesis started. However, the project did not make much progress because many of the accreditation standards at that time were not suitable for private universities. Things changed about one year after that when the Ministry revised the standards and set a deadline, which prompted FXN University into action and prioritised this project. Thus, one month before the first interviews for this thesis were to be conducted, the university had submitted its self-study report, and during the data collection period, it prepared and organised the visits of the accreditation panel.

The MTEO accreditation involved many internal and external stakeholders of FXN University. The internal stakeholders included the Vice-Rector of Teaching, Learning and 
Research as the top leader, the Quality Assurance Department as the core team, the representatives of other departments, and the current students. The external stakeholders included the accreditation panel, the alumni, and the employers. Along with these stakeholders, three participants were selected in this research. They were Thu, the project manager; Nga, a member of the International Collaboration Department; and Hung, the representative of an employer. These participants were interviewed at the same four time points. According to the project manager's perception, these time points were: before the preliminary visit of the panel (T1), after the preliminary visit and before the official visit of the panel (T2), after the official visit of the panel (T3), and after the official result report of the accreditation (T4). These time points are illustrated in Figure 4.7 below.

Figure 4.7 Time points of the interviews - MTEO case

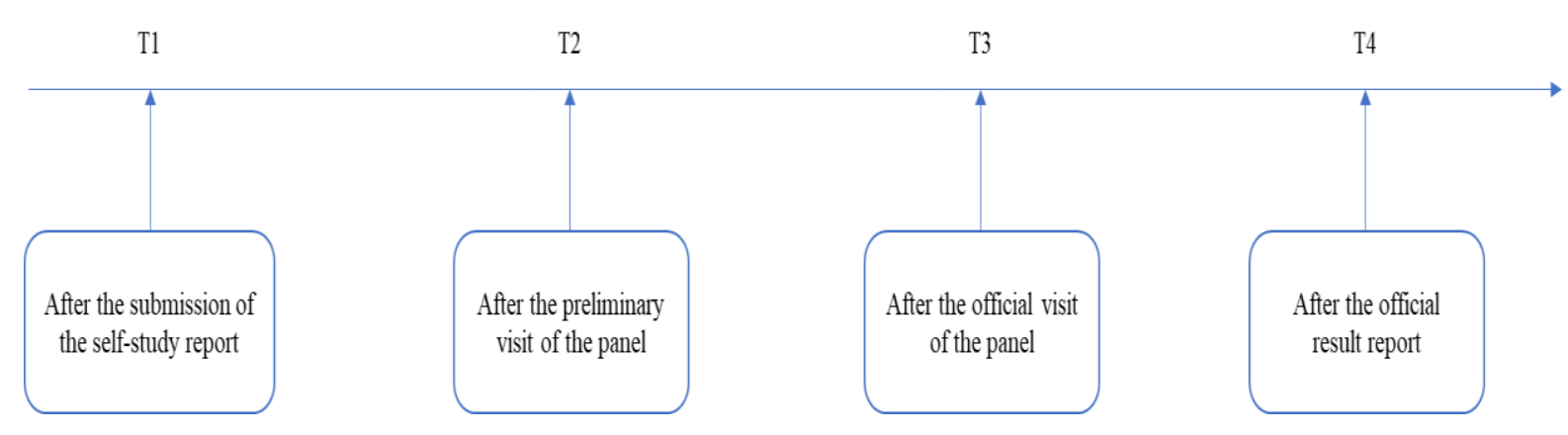

\subsection{Changes in Thu's Appreciation of the MTEO Project}

\subsubsection{Participant Background}

Thu was the Head of Quality Assurance and was the manager of the MTEO project. Thu had rich experience in quality assurance, including four years outside FXN University and ten years inside the university. As the project manager, Thu reported directly to university leaders and was responsible for coordinating all the activities of the project as well as liaising with the panel members.

\subsubsection{Changes in Thu's Standards for and Appreciation of the MTEO Project}

Number of principal components

Thu identified twenty-six constructs in the first interview, and these constructs remained the same in the interviews after that. The constructs are presented in Table 4.7 as follows. 
Table 4.7 Constructs in Thu's repertory grids in her four interviews - the MTEO project

\begin{tabular}{|c|c|c|}
\hline No & Constructs & Opposites \\
\hline $\mathrm{C} 1$ & Projects are important to universities & Projects are not important to universities \\
\hline $\mathrm{C} 2$ & Projects require a lot of resources to collect hard-copy evidence documents & Projects do not require a lot of resources to collect hard-copy evidence documents \\
\hline $\mathrm{C} 3$ & Project implementation procedures are not methodical & Project implementation procedures are methodical \\
\hline $\mathrm{C} 4$ & Bad document management systems & Good document management systems \\
\hline $\mathrm{C} 5$ & Accreditation standards are not issued in a timely way. & Accreditation standards are issued in a timely way \\
\hline C6 & University leaders care about projects & University leaders do not care about projects \\
\hline $\mathrm{C} 7$ & Universities have upgraded their activities & Universities do not upgrade their activities \\
\hline $\mathrm{C} 8$ & Universities invest in accreditation training for staff & Universities do not invest in accreditation training for staff \\
\hline C9 & Staff and faculty support projects & Staff and faculty do not support projects \\
\hline $\mathrm{C} 10$ & Accreditation standards are well-recognised & Accreditation standards are not well-recognised \\
\hline $\mathrm{C} 11$ & Academic curricula meet accreditation standards & Academic curricula do not meet accreditation standards \\
\hline $\mathrm{C} 12$ & Not enough staff. Staff are not trained adequately & Enough staff. Staff are trained adequately \\
\hline $\mathrm{C} 13$ & Accreditation standards restrain the operation of universities & Accreditation standards do not restrain the operation of universities \\
\hline $\mathrm{C} 14$ & Accreditation panels are rigid & Accreditation panels are flexible \\
\hline $\mathrm{C} 15$ & Good quantity of evidence documents & Bad quantity of evidence documents \\
\hline $\mathrm{C} 16$ & Many reference materials & Few reference materials \\
\hline $\mathrm{C} 17$ & Universities are willing to invest in obtaining accreditation & Universities are not willing to invest in obtaining accreditation \\
\hline $\mathrm{C} 18$ & $\begin{array}{l}\text { Accreditation panels have strict requirements for self-study reports and evidence } \\
\text { documents }\end{array}$ & $\begin{array}{l}\text { Accreditation panels do not have strict requirements for self-study reports and } \\
\text { evidence documents }\end{array}$ \\
\hline $\mathrm{C} 19$ & Writing self-study reports is very difficult & Writing self-study reports is easy \\
\hline $\mathrm{C} 20$ & Accreditation panels provide many consultancies and many suggestions & Accreditation panels do not provide consultancy and suggestions \\
\hline $\mathrm{C} 21$ & Accreditation panels examine many activities of universities & Accreditation panels do not examine many activities of universities \\
\hline $\mathrm{C} 22$ & A lot of resources are invested in accreditation projects & Not many resources are invested in accreditation projects \\
\hline $\mathrm{C} 23$ & Projects involve key people in all departments & Projects do not involve key people in all departments \\
\hline $\mathrm{C} 24$ & Bad project planning leads to waste of resources & Good project planning \\
\hline $\mathrm{C} 25$ & Bad project results & Good project results \\
\hline $\mathrm{C} 26$ & Universities' characteristics cannot be adjusted to meet accreditation standards & Universities' characteristics can be adjusted to meet accreditation standards \\
\hline
\end{tabular}


The principal component analysis identified that two principal components could explain Thu's appreciation of the MTEO project over the four interviews (see Appendix H). These components respectively explained from $75 \%$ to $77 \%$ and from $10 \%$ to $13 \%$ of Thu's appreciation of the project. Altogether, they explained from $87 \%$ to $88 \%$ of Thu's appreciation. The percentage variances explained by these two components are presented in Table 4.6 below. Table 4.8: Percentage variances explained by the two components over the four interviews Thu - MTEO project

\begin{tabular}{|l|l|l|l|l|l|l|l|l|}
\hline Interview & T1 & T2 & T3 & T4 \\
\hline Component & 1 & 2 & 1 & 2 & 1 & 2 & 1 & 2 \\
\hline Variance \% & 75.65 & 13.51 & 75.83 & 13.28 & 77.12 & 11.36 & 76.74 & 10.79 \\
\hline Cumulative variance \% & 89.17 & 89.11 & 88.48 & 87.77 & \\
\hline
\end{tabular}

\section{Interpretation of the principal components}

The loadings of the constructs on the components are presented in Appendix H. Because these loadings did not change much over the four interviews, only the PrinGrid map for the first interview is presented in Figure 4.8 as follows for the purpose of illustration. 
Figure 4.8 PrinGrid map - the loadings of the constructs on the two principal components - Thu - the first interview

C2 Projects do not require a lot t f resources to collect hard-copy evidence documents

C18. Accreditation panels have strict requirements for sel-study reports and evidence documents

C16. Many reference materials C5. Accreditation standards are issued in a timely way

C20. Acreditation panels provide much consultancy and many suggestions

C14. Accreditition panels are flexible

C13. Accreditation standards do notrestrain the operation of universities

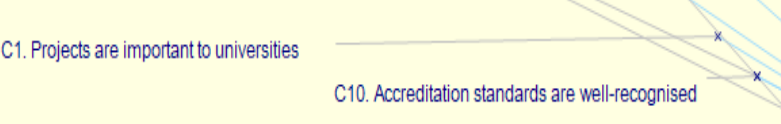

C21. Accreditation panels examine many activities of universities

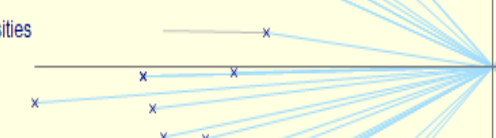

An ideal accreditation project

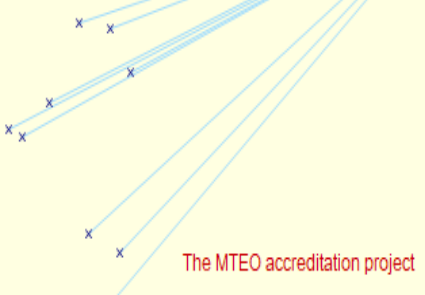

C11. Academic curricular do not meet accreditation standards
C19. Writing self-study reports is very difficult

C21. Universities' characteristics cannot be adjusted to meet accreditation standards

C8. Universities do not invest in accreditation training for staff

C7. Universities do not upgrade their activities

C3. Project implementation procedures are not methodical

C4. Bad document management systems

C6. University leaders do not care about projects

C23. Projects do not involve key people in departments

C24. Bad projectplanning C15. Bad quantity of evidence documents Component 1

${ }^{-17.75 .7 \%} \mathrm{C} 17$. Universities are not willing to invest to obtain accreditation

C25. Bad project results

Aless than ideal accreditation project

C22. Not many resources are invested in accreditation projects

C9. Staff and faculty do not support projects

Component 2 
As shown on the above PrinGrid map, all the constructs had their largest loadings on the first component in the first interview. These constructs could be divided into two groups. The first group consisted of constructs referring to Thu's appreciation of the professionalism of the accreditation processes. These constructs specifically referred to the importance, reputation, benefits, requirements, and reference materials of the accreditation and the flexibility, strictness, consultancy, and suggestions of the panel (C1, C2, C5, C10, C13, C14, C16, C18, $\mathrm{C} 20, \mathrm{C} 21)$. On the map, these constructs are the ones with the labels presented on the left of the map. The second group consisted of constructs related to Thu's appreciation of the university characteristics and activities regarding the accreditation. These constructs specifically referred to the academic curricula, the leadership and investment regarding the accreditation, the support of the related departments, the number of project members, and the project management procedures (C3, C4, C6, C7, C8, C9, C11, C12, C15, C17, C19, C22, C23, $\mathrm{C} 24, \mathrm{C} 25, \mathrm{C} 26)$. On the map, these constructs are the ones with the labels presented on the right of the map. Based on these two groups, the first component could be interpreted as Thu's appreciation of the professionalism of the accreditation processes and the university characteristics and activities regarding the accreditation. Because the constructs' loadings on the first component did not change much over the four interviews, its interpretation was basically the same in these interviews.

Over the four interviews, all the constructs also had some loadings on the second component. These constructs could be divided into two groups with opposite loading signs. The first group consisted of constructs with labels presented on the left of the map. These constructs were related to Thu's appreciation of the professionalism of the accreditation processes, such as the timeliness of the issue of the new standards and the requirements for hard-copy evidence documents $(\mathrm{C} 2, \mathrm{C} 5, \mathrm{C} 13, \mathrm{C} 14)$. The second group consisted of constructs with labels presented on the right of the map. These constructs were related to Thu's appreciation of the university characteristics and activities regarding the accreditation, which included the investment in accreditation training and the number and competence of the project members $(\mathrm{C} 8, \mathrm{C} 11, \mathrm{C} 12)$. The positions of the two groups on the map indicate that one polar of the second component referred to the positive meaning of one group and the negative meaning of the other group. The second component, therefore, could be interpreted as Thu's appreciation of the gap between the professionalism of the accreditation processes and the university characteristics and activities. As the constructs' loadings on the second component did not change much in the other interviews, its interpretation in these interviews was the same as it was in the first interview. 
In summary, Thu's appreciation of the MTEO project over the four interviews could be explained by two components. They referred to Thu's overall appreciation of and comparison between the professionalism of the accreditation processes as well as the university characteristics and activities regarding the accreditation.

Changes in Thu's standards for and appreciation of the MTEO project

This section presents the changes in Thu's standards for and appreciation of the MTEO project. These changes were identified based on the changes in the loadings of Thu's appreciation of the MTEO project, the ideal, and the less than ideal projects on the two components. The loadings and the PrinGrid map, which illustrates these changes, are presented below in Table 4.7 and Figure 4.8, respectively.

Table 4.9 Loadings of Thu's appreciation of the ideal project, the less than ideal project, and the MTEO project on the two components

\begin{tabular}{|l|l|l|l|l|l|l|l|l|}
\hline Interview & \multicolumn{2}{l}{ T1 } & \multicolumn{2}{l|}{$\mathrm{T} 2$} & \multicolumn{2}{l|}{ T3 } & \multicolumn{2}{l|}{ T4 } \\
\hline Component & 1 & 2 & 1 & 2 & 1 & 2 & 1 & 2 \\
\hline Ideal project & -3.837 & -0.612 & -3.798 & -0.576 & -3.688 & -0.92 & -3.921 & -1.18 \\
\hline $\begin{array}{l}\text { Less than ideal } \\
\text { project }\end{array}$ & 6.241 & -0.456 & 6.272 & -0.445 & 6.356 & -0.579 & 6.146 & -0.78 \\
\hline MTEO project & -1.557 & -1.108 & -1.696 & -1.056 & -2.072 & -0.37 & -2.139 & -0.268 \\
\hline Gap & 2.28 & & 2.102 & & 1.616 & & 1.782 & \\
\hline
\end{tabular}

Figure 4.9 Changes in Thu's appreciation of the MTEO project over the four interviews (T1, $T 2, T 3, T 4)$

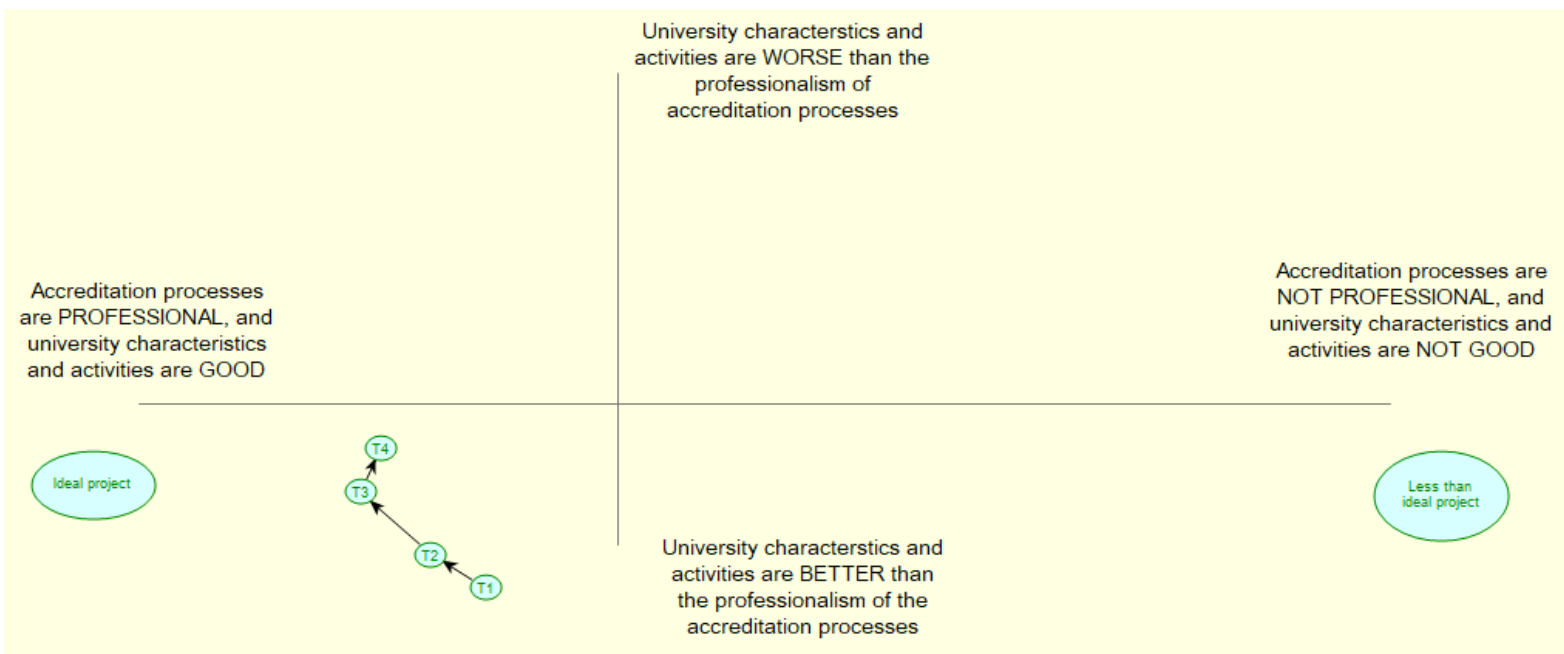

The above table shows that Thu's appreciation of the MTEO project had loadings on the two components over the four interviews. These two components, therefore, represented 
her standards for the project. With regard to Thu's appreciation of the ideal and less than ideal projects, it can be seen that the largest loading was placed on the first component and a small loading on the second. The loadings on the first component also had opposite signs. This indicated that the first component could differentiate Thu's appreciation of the ideal project from her appreciation of the less than ideal project. This can also be seen from the opposite positions of these two projects regarding the horizontal axis of the PrinGrid map. The first component, therefore, represented Thu's primary standards, and the second component represented her secondary standard for the MTEO project. Based on the interpretation of the first component, Thu's primary standards were that the accreditation processes must be professional, and the university characteristics and activities regarding the accreditation must be good. Based on the interpretation of the second component, Thu's secondary standard was that the professionalism of the accreditation processes must match the university characteristics and activities. Because the interpretation of these components remained unchanged over the four interviews, Thu's primary and secondary standards for the project were also unchanged.

The table and the map also show that while Thu generally had a good appreciation of the MTEO project, her appreciation changed over the four interviews. On the PrinGrid map, all the four points (T1), (T2), (T3), and (T4) are in the lower-left quadrant. This indicates that over the four interviews, Thu generally thought that the accreditation processes were professional and the university characteristics and activities regarding the accreditation were good (component 1). However, she also thought that the university characteristics and activities were better than the professionalism of the accreditation processes (component 2). Over the four interviews, regarding component 1 , the gap between the loadings of Thu's appreciation of the MTEO project and her appreciation of the ideal project changed. Thus, this gap decreased from 2.28 to 2.102 from $\mathrm{T} 1$ to $\mathrm{T} 2$, then continued decreasing to 1.616 at $\mathrm{T} 3$, and finally increased slightly to 1.782 at $\mathrm{T} 4$. This pattern is also captured on the map by the three arrows T1T2, T2T3, and T3T4. These changes indicated that except from T3 to T4, Thu's appreciation of the university and accreditation characteristics improved (component 1). The changes in the vertical sides of the arrows indicate that over the four time points, Thu's appreciation of the professionalism of the accreditation processes also improved, in comparison to the inherently good university characteristics and activities (component 2).

In summary, the principal component analysis shows that over the four interviews, Thu's standards for the MTEO project were unchanged. Along with these standards, except for the last interview, Thu's appreciation of the university and accreditation characteristics 
improved. She also thought that the professionalism of the accreditation better matched the inherently good characteristics of the university at all the four interviews.

\subsubsection{Changes in Thu's Appreciation of the MTEO Project through the Lens of Vickers'}

\section{Concept}

The above section has identified the changes in Thu's standards for and appreciation of the MTEO project. This section uses these findings to describe the changes in Thu's appreciation of the project, as influenced by her standards and perceptions of events and ideas, by applying Vickers' concept of appreciative systems.

At the first interview (T1), with her experience, Thu's primary standards for the project were that the accreditation processes must be professional, and the university characteristics and activities must be good. Her secondary standard was that the university characteristics and activities must match the professionalism of the accreditation. Following these standards, Thu had an appreciation that the overall characteristics of the university and the accreditation were good (the primary standards). Thu also thought that the professionalism of the accreditation did not match the characteristics and activities of the university (the secondary standard):

We have submitted the self-study report, we have done what we need to do, we know it is not easy, but now we are quite confident in what we have done.

From T1 to T2, as the project manager, Thu was in charge of preparing and organising the preliminary visit of the panel. During this visit, she had a meeting with the panel and the university's board of management. She saw that the panel provided the university with useful constructive feedback, consultancy, and suggestions:

The consultancy of the panel was very, very $O K$. Before the preliminarily visit, I did not think they could provide such a lot of consultancy and suggestions.

Thu also saw that the panel was more flexible than she had expected:

I had thought that the panel would follow governmental rules strictly, but they were quite open, they focused on the quality of the training. They could skip, not really skip, but they could understand the complexity of the governmental procedures. We have opened a new campus without the official licenses from the government, but they did not question it.

However, Thu also learned that the panel had strict requirements for the self-study report, the evidence documents, and the logistics of their official visit. These learnings together made her more confident in the professionalism of the panel as well as the accreditation processes. 
Besides the professionalism of the accreditation processes, Thu also learned things related to the characteristics and activities of the university. On the positive side, she saw that the university leaders and the related staff members were "more aware of the project's importance." The university leaders "delegated more power" to her and "supervised the project more closely." However, on the negative side, Thu became "less confident" in the evidence documents that the university had prepared because the panel required more of them. Thu also noticed some problems in the project implementation:

The ways in which we have done this project so far are reversed. We should have collected the evidence documents first and written the report later. Instead, we have written the report first and collected the evidence documents later. This way of working is now creating difficulties for us in collecting the evidence documents.

Overall, Thu's appreciation of the professionalism of the accreditation processes and the university characteristics improved (the primary standards). She also thought that the professionalism of the accreditation better matched the university characteristics and activities (the secondary standard).

From T2 to T3, Thu was in charge of preparing and organising the official visit of the panel. During this visit, she realised that the project was more important. She also saw that, while the panel was flexible and open, they continued having strict requirements for the selfstudy report and evidence documents. These learnings reinforced Thu's good appreciation of the panel:

I have observed other accreditation panels in other universities, but this panel is better.

Thu also realised that the accreditation considered more activities of the university than she had expected:

The guidelines of the accreditation have indicated many activities. But when we met the panel, they explained, and we understand that the standards are very broad.

These realisations together made Thu's appreciation of the professionalism of the accreditation processes improve.

In addition to the accreditation, Thu saw that the top university leaders and the related departments were more aware of the importance of the accreditation and supported the project team more closely: 
At this final phase of the project, people collaborated very closely.

However, Thu learned that the project's human resources were not good:

The panel just said that the human resources are not enough. We thought that the human resources are enough according to the regulations, but they are not.

This suggests that these learnings impacted Thu's appreciation of the university characteristics and activities in both positive and negative ways. Altogether, Thu's appreciation of the overall characteristics of the university and the accreditation improved (the primary standards). She also thought that the professionalism of the accreditation processes better matched the university characteristics and activities (the secondary standard).

Between the third to the fourth interview (from T3 and T4), Thu was aware that the project team did not do many activities as the official visit of the panel was over. The only thing that affected Thu's appreciation of the project during this time was the official result report from the panel. In this report, the panel gave the university lower marks than what the university had given itself. Thu generally agreed with the marks of the panel because the university had deliberately increased its self-assessment marks:

This is common. When we self-assess, we always boost the marks a little bit.

Thu also noticed that the panel recommended more accreditation training for the project team:

The recommendations make me realise that we have had much less training than other universities.

Overall, the report made Thu's appreciation of the university characteristics and activities worsen (the primary standards). In line with this appreciation, Thu also thought that the professionalism of the accreditation processes had improved and better matched the university characteristics and activities (the secondary standard).

In summary, with her initial standards and appreciation regarding the MTEO project, Thu learned things related to the project over the four interviews. While these things did not change her standards, they changed her appreciation of the project. Except for the last interview in which Thu's appreciation of the overall project worsened, in the other interviews, her appreciation improved. Along with these changes, Thu also thought that the professionalism of the accreditation better matched the university characteristics and activities. 


\subsection{Changes in Nga's Appreciation of the MTEO Project}

\subsubsection{Participant Background}

Nga was a member of the International Collaboration Department of FXN University. Her educational background includes a bachelor's degree from Vietnam and an MBA from the United States. Before her current role, Nga has worked in different positions at FXN University for six years. Nga task within the project was to collect evidence documents related to the international collaboration of the university.

\subsubsection{Changes in Nga's Standards for and Appreciation of the MTEO Project}

Number of principal components

Nga identified twenty-five constructs about the MTEO project in the first interview. These constructs did not change until the third interview when she added two more new constructs: C26 and C27. The constructs then remained unchanged for the fourth interview. Nga's constructs over the four interviews are presented in Table 4.10 as follows. 
Table 4.10 List of constructs - Nga-MTEO case

\begin{tabular}{|c|c|c|}
\hline No & Constructs & Opposite constructs \\
\hline 1 & Adequate investment in projects & Inadequate investment in projects \\
\hline 2 & Competent project members & Incompetent project members \\
\hline 3 & There is consultancy from experienced experts & No consultancy from experts \\
\hline 4 & Projects make real changes to organisations & Projects do not make real changes to organisations \\
\hline 5 & Projects are not done for real & Project are done for real \\
\hline 6 & Projects cannot involve related people & Projects involve related people \\
\hline 7 & Projects use new project management tools & Projects do not use new project management tools \\
\hline 8 & Projects create new business opportunities for organisations in long-term & Projects do not create new business opportunities for organisations \\
\hline 9 & Projects improve people's professional knowledge & Projects do not improve people's professional knowledge \\
\hline 10 & Projects have been piloted & Projects have not been piloted \\
\hline 11 & Good project results & Bad project results \\
\hline 12 & Project implementers' motivation is good and adequate & Project implementers' motivation is inadequate \\
\hline 13 & Project implementers do not have enough power & Project implementers have enough power \\
\hline 14 & Leaders are determined and have ongoing monitoring & Leaders do not have ongoing monitoring \\
\hline 15 & Projects are musts & Projects are not musts \\
\hline 16 & Projects' requirements are not complex & Projects' requirements are complex \\
\hline 17 & Projects involve many people and are risky & Projects are not related to many people and not risky \\
\hline 18 & A lot of changes in human resources & Not many changes in human resources \\
\hline 19 & Bad document management systems and bad organisational operation & Good document management systems and organisational operation \\
\hline 20 & Organisations are united, innovated, and easy to change & Organisations are not united and difficult to change \\
\hline 21 & Project results can be obtained fast & Project results cannot be obtained fast \\
\hline 22 & Projects follow trends and benefit organisations & Projects do not follow trends and do not benefit organisations \\
\hline 23 & Projects focus on ongoing changes & Projects do not focus on ongoing changes \\
\hline 24 & Projects focus on innovation for organisations & Projects do not focus on innovation for organisations \\
\hline 25 & The motivation of related people is good & The motivation of related people is not good \\
\hline 26 & Accreditation has strict requirements for data analysis & Accreditation's requirements for data analysis are not strict \\
\hline 27 & Suitable project implementation procedures & Unsuitable project implementation procedures \\
\hline
\end{tabular}


The principal component analysis identified two principal components to describe Nga's appreciation of the MTEO project over the four interviews (see Appendix I). These components explained about $80 \%$ and $9 \%$ of her appreciation, respectively. The percentage variance explained by each component at the four time points is presented in Table 4.8 below.

Table 4.11 Percentage variances explained by the two components over the four interviews Nga-MTEO project

\begin{tabular}{|l|l|l|l|l|l|l|l|l|}
\hline Interview & T1 & T2 & T3 & \multicolumn{3}{l|}{ T4 } \\
\hline Component & 1 & 2 & 1 & 2 & 1 & 2 & 1 & 2 \\
\hline Variance \% & 83.95 & 9.74 & 83.89 & 9.69 & 82.89 & 9.51 & 82.54 & 9.48 \\
\hline Cumulative variance \% & \multicolumn{3}{|c|}{93.69} & \multicolumn{3}{|c|}{93.59} & \multicolumn{3}{|c|}{92.4} & & 92.02 \\
\hline
\end{tabular}

\section{Interpretation of the principal components}

The table of loadings in Appendix I shows that the loadings of the constructs on the two components were basically unchanged over the four interviews, regardless of the minor changes caused by the addition of the two new constructs at the third interview. For the purpose of illustration, the loadings of the constructs on the principal components in the first and third interviews are presented on the PrinGrid maps in Figure 4.10 and 4.11, respectively, as follows. 
Figure 4.10 Nga's PrinGrid map in the first interview - MTEO case

C24. Projects focus on innovation for organisations

C22. Projects followtrends and benefit organisations

C12. Project implementers' motivation is good and adequate

C14. Leaders are determined and have ongoing monitoring

C9. Projects improve people's professional knowedge

C2 Competent project members

C23. Projects focus on ongoing changes

C3. There is consultancy from experienced experts

C20. Organisations are united, innovated, and easy to change Projects use new project management tools

\section{The MTEO accreditation project} C4. Projects makes real changes to organisations $x \times x$

C25. The motivation of related people is good

C19. Good documentation systems

C1. Adequate investment in projects

C6. Projects can involve related people

C21. Project results can be obtained fast

C10. Projects have been piloted

C8. Projects create new business opportunities for organisations in long-term

\section{C15. Projects are musts}

An ideal accreditation projecl

13. Project implementers have enough power

C11. Good project results

5. Projects are done for real

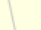

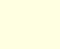


Figure 4.11 Nga's PrinGrid map in the third interview-MTEO case

C24. Projects focus on inoovation for organisations
C22. Projects follow trends and benefit organisations
13. Project implementers' motivation is good and adequate

C14. Leaders are determined and have ongoing monitoring $\quad$ C5. Projects are done for real

C9. Projects improve people's professional knowledge

C2. Competent project members

C23. Projects focus on ongoing changes C7. Projects use new project management tools

The MTEO accreditation project

C20. Organisations are united, innovated, and easy to change C3. There is consultancy from experienced experts

C21. Project results can be obtained fast

C21. Project results can be obtained fast
C10. Projects are piloted

C8. Projects create new business opportunities for organisations in long-term C4. Projects make real changes to organisations

$\int_{x}^{x} \times 19$ Good documentation systems C2. The motivation of related people is good C13. Project implementers have enough power C11. Good project results C15. Projects are musts

C6. Project can involve related people

Anideal accreditation project

C26. The accreditation has strict requirements for data analyis

C27. Suitable project implementation procedures

C18. There are not many changes in human resources

16. Projects' requirements are not complex $x$

C17. Projects are not risky

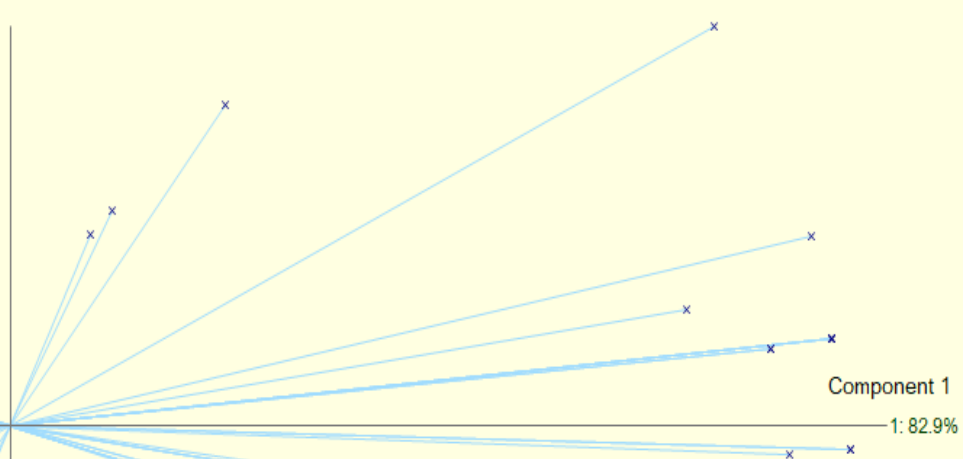

A less than ideal accreditation project 
As shown in Figure 4.10, at the first interview, twenty-two out of twenty-five constructs in Nga's repertory grids had their largest loadings on the first component. These constructs are presented as a bunch of constructs that are close to the horizontal axis of the PrinGrid map. These constructs could be divided into two groups. The first group consisted of constructs related to the accreditation benefits to the university. These constructs referred to whether the accreditation benefited the university, whether the accreditation had a good reputation, whether the accreditation was really done and promoted innovation, and whether the accreditation results could be obtained fast $(\mathrm{C} 4, \mathrm{C} 5, \mathrm{C} 8, \mathrm{C} 9, \mathrm{C} 15, \mathrm{C} 21, \mathrm{C} 22, \mathrm{C} 23, \mathrm{C} 24)$. The second group consisted of constructs related to the efficiency of the project implementation. These constructs referred to the university's leadership and investment, the commitment and support of the project members and related departments, the university's document management systems, and the project results $(\mathrm{C} 1, \mathrm{C} 2, \mathrm{C} 3, \mathrm{C} 6, \mathrm{C} 7, \mathrm{C} 10, \mathrm{C} 11, \mathrm{C} 12, \mathrm{C} 13, \mathrm{C} 14, \mathrm{C} 19, \mathrm{C} 20, \mathrm{C} 25)$. The first component, therefore, could be interpreted as Nga's appreciation of the effectiveness and efficiency of the accreditation project. The term 'effectiveness' here referred to whether the accreditation project benefited the university, while 'efficiency' referred to whether the project was done in an efficient way. As the loadings did not change much in the second interview, its interpretation was basically the same as it was in the first interview.

The two new constructs that were introduced in the third interview also had their largest loadings on the first component. In figure 4.11, the labels of these constructs are presented in the bottom left quadrant of the PrinGrid map. These constructs were the strictness of the accreditation standards (C26) and the steps of the accreditation project implementation (C27). Based on the meanings of these constructs, at T3, the first component could basically be interpreted in the same way as it was at T1 and T2. The only change was that the notions of the effectiveness and efficiency of the project now included more distinctive characteristics of the accreditation project. As the constructs' loadings on the first component did not change much in the fourth interview, its interpretation was also the same as it was at T3.

As shown in the two figures and the table of loadings, the same three constructs had the largest loadings on component 2 over the four interviews. On the PrinGrid maps, these constructs are presented in the bottom-left quadrant and close to the vertical axes. These constructs were the complexity of the project requirements (C16), the risk of the project $(\mathrm{C} 17)$ and the changes in the university's human resources (C18). Because these constructs were related to the concept of project complexity, the second component could be interpreted as $N g a$ 's appreciation of the project complexity. 
In summary, over the four interviews, Nga's appreciation of the MTEO project could be explained by two components, namely her appreciation of the effectiveness and efficiency as well as the complexity of the project. With the introduction of two new constructs in interview three, the component regarding the project effectiveness and efficiency included more distinctive characteristics of the accreditation projects.

\section{Changes in Nga's standards for and appreciation of the MTEO project}

This section presents the changes in Nga's standards and appreciation regarding the MTEO project. These changes were identified based on the changes in the loadings of Nga's appreciation of the MTEO project and the ideal and the less than ideal projects on the two components. Because the changes in the interpretation of the first component in the third interview do not affect the presentation of Nga's appreciation for all four interviews in one PrinGrid map, a PrinGrid map is used to illustrate the changes in her appreciation. In this map, presented in Figure 4.9 below, the changes in the interpretation of the first component are shown in brackets. The loadings are given in Table 4.9.

Table 4.12 Loadings of Nga's appreciation of the ideal project, the less than ideal project and the MTEO project on the two components over the four interviews

\begin{tabular}{|l|l|l|l|l|l|l|l|l|}
\hline Interview & \multicolumn{2}{l}{ T1 } & \multicolumn{2}{l}{ T2 } & \multicolumn{2}{l|}{ T3 } & \multicolumn{2}{l|}{ T4 } \\
\hline Component & 1 & 2 & 1 & 2 & 1 & 2 & 1 & 2 \\
\hline Ideal project & -2.701 & -1.878 & -2.728 & -1.857 & -2.663 & -2.016 & -2.576 & -2.068 \\
\hline $\begin{array}{l}\text { Less than ideal } \\
\text { project }\end{array}$ & 7.165 & -0.73 & 7.141 & -0.741 & 7.562 & -0.675 & 7.619 & -0.66 \\
\hline MTEO project & -1.495 & 0.635 & -1.38 & 0.576 & -1.524 & 0.826 & -1.594 & 0.856 \\
\hline Gap & 1.206 & 2.513 & 1.348 & 2.433 & 1.139 & 2.842 & 0.982 & 2.924 \\
\hline
\end{tabular}




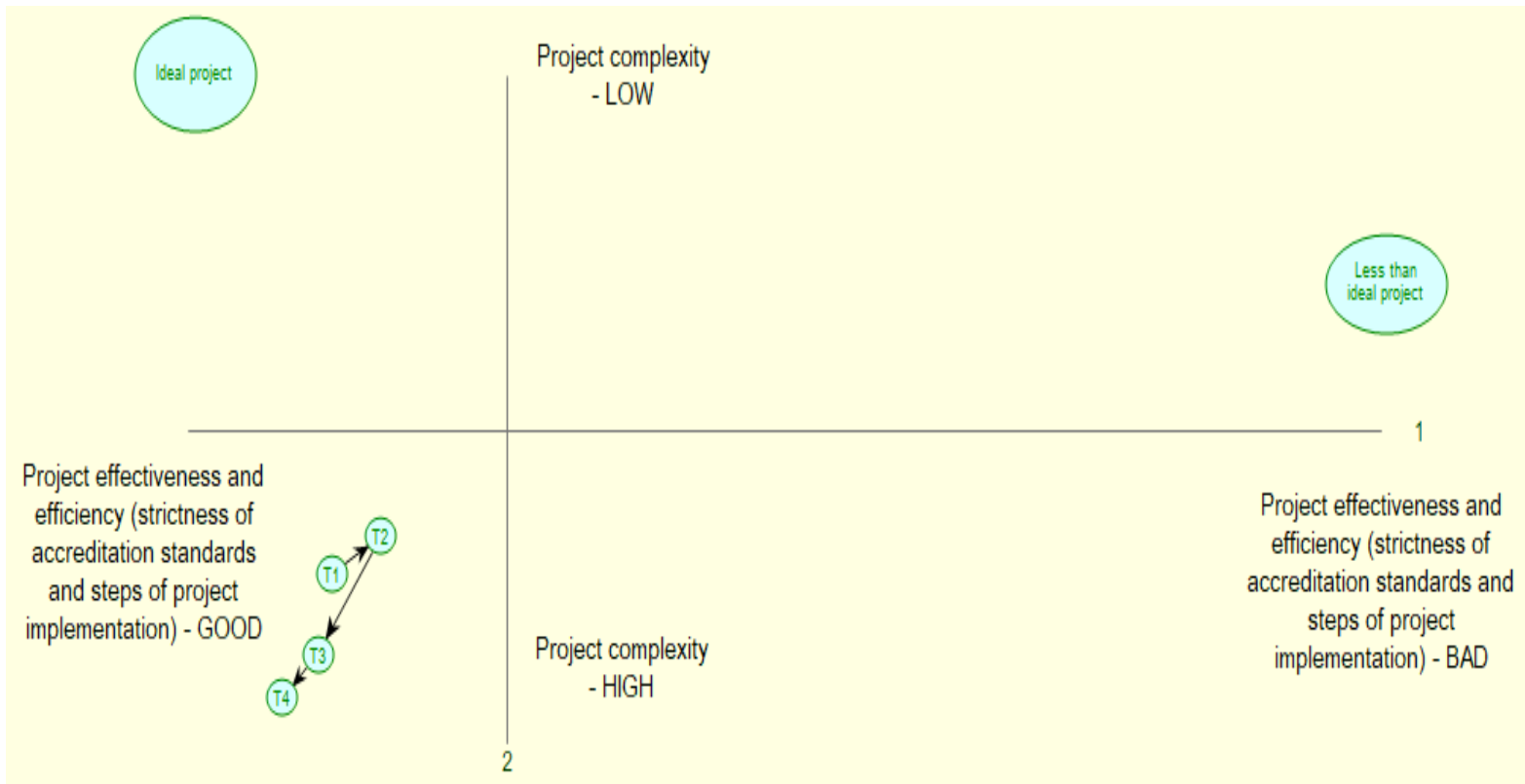

Because over the four interviews, Nga's appreciation of the ideal and less than ideal projects had large loadings on the two components, both components represented her primary standards for the MTEO project. On the PrinGrid map, this is represented by the position of the ideal project in the upper-left quadrant. The interpretation of the components suggests Nga's primary standards be that the accreditation must benefit the university, the project must be done in an efficient way (component 1), and that the project must not be complex (component 2). Because of the minor change in the interpretation of the first component at the third interview, Nga's primary standards included more distinctive characteristics of the MTEO accreditation for interviews three and four.

The table and the map also show that Nga changed her appreciation of the MTEO project over the four time points. At T1, while the gap between the loadings of Nga's appreciation of the MTEO project and her appreciation of the ideal project on the first component was small (1.206), this gap for the second component was large (2.513). This is captured by the point T1 on the map. These gaps indicated that even though Nga generally thought that the accreditation benefited the university and that the project implementation was efficient, she also thought that the project was complex. From T1 to T2, the above gap of loadings regarding the first component increased from 1.206 to 1.348. Meanwhile, the gap regarding the second component decreased from 2.513 to 2.433 . These changes, which are illustrated by arrow T1T2, indicated that between T1 to T2, Nga's appreciation of the effectiveness, efficiency, and complexity of the project diminished. In the interviews after that, 
Nga's appreciation changed in a different direction. These changes are illustrated by the arrows labelled T2T3 and T3T4. Thus, it seemed that Nga considered the project to be increasingly effective and efficient but also more complex over time.

In summary, the principal component analysis shows that Nga's standards for and appreciation of the MOET project changed over the timespan covered by the four interviews. From the third interview, her standards included more distinctive characteristics of accreditation projects. Her appreciation changed in both positive and negative ways. Except for the second interview, when she thought that the project effectiveness, efficiency and complexity had decreased, she generally thought that these aspects of the project had increased.

\subsubsection{Changes in Nga's Appreciation of the MTEO Project through the Lens of Vickers'}

\section{Concept}

The above section has identified the changes in Nga's standards for and appreciation of the MTEO project. This section uses these findings to describe the changes in her appreciation of the project, as influenced by her standards and perceptions of events and ideas, by applying Vickers' concept of appreciative systems.

At the first interview (T1), Nga has just started working on the MTEO project and was not very familiar with all aspects of the project yet. However, with her experience and position at the university, Nga's primary standards were that the project must be effective and efficient but not complex. Following these standards, at T1, Nga had an appreciation that the MTEO project was generally effective and efficient (the first primary standards), but that it was also complex (the second primary standard).

Between the first to the second interview (from T1 to T2), Nga had become more involved in the project and was aware that the panel made a preliminary visit to the university. Nga saw that the motivation of the related people in the university improved:

\section{People now begin understanding that the project is not Thu [the project manager]'s project, Thu just represents the project. Everyone must participate in the project. Only Thu cannot do it.}

However, Nga also learned from her direct supervisor about the panel's negative comments about the international collaboration of the university. These comments made Nga's confidence in the university's investment in the project and the competence of the project members decrease:

Now when I am involved more in the project and get to know the negative comments, I was less confident in the project. 
Overall, from T1 to T2, Nga thought that the project effectiveness and efficiency worsened (the first primary standards). However, as Nga saw that the related departments supported the project more, she thought that the project complexity also decreased (the second primary standard).

Between the second to the third interview (from T2 to T3), Nga had been involved in the official visit of the panel to the university. She attended one direct interview with the panel and was asked to collect extra evidence documents twice. These interactions enabled Nga to understand more about the strict requirements of the accreditation and the steps needed for a successful implementation of accreditation projects. Based on these experiences and ideas, Nga developed two new standards for the project effectiveness and efficiency. Thus, at T3 Nga thought that the accreditation must have strict requirements to benefit the university and that the implementation must follow suitable steps to be efficient, such as "the evidence documents must be collected first, and the report [was] written later." During this time, Nga also learned from the project team and the panel that the project could get the results very fast after the visit:

After one week, we can have the general results. The panel is working hard, days and nights, very fast, they will report very fast. I like that.

These learnings made Nga believe that the accreditation would benefit the university more.

Moreover, Nga also saw that the investment and motivation of the university were better:

Now we do not mind spending anything on this accreditation. It must be successful. If not, it will be a problem for our student recruitment.

Nga understood that the involvement of the related departments and staff was "compulsory" as the top leaders required them "not to go anywhere and be present within 15 minutes of being asked." Nga also could see that when these departments and staff were more involved, they understood more the importance of the project and supported it more:

They realised by themselves. One Department Head said that the project affects everybody. If one person fails, it will be a problem for everyone.

Nga also saw that the project team began to use some management techniques:

Now they have a plan. It is like a work breakdown structure or a checklist. People who complete their tasks must fill out some forms.

However, along with these positive activities, Nga learned about the panel's negative feedback on the university's document management systems: 
One Department Head posted on his Facebook that the panel said our university is good at everything, except the document management systems.

Nga also realised that the project had not followed suitable steps in the implementation process. The project team had written the report first and collected the evidence documents afterwards. These new ideas impacted Nga's appreciation of the project effectiveness and efficiency in both positive and negative ways. Overall, Nga thought that the project effectiveness and efficiency had improved (the first primary standards)

Along with the project effectiveness and efficiency, Nga also realised that the scope of the project was big:

There are too many standards in the accreditation. It has, I think, about twenty-five standards, uncountable, no one in this university can be missed.

When searching for and collecting the extra evidence documents for the project, Nga saw that the changes in the university's human resources had created more challenges to the project:

Not only the people who prepared the documents have left the university, but also the people related to those documents have also quit their jobs.

Nga, therefore, thought that the project was more complex (the second primary standard) at this stage.

From T3 to T4, the panel visit was over. Nga was not involved in the project anymore. However, she still received the result report from the project team and learned about the marks that the panel gave the university. Nga thought that the marks were "generally good", and some were "even better than expected". She gave an example that "the panel criticised the international collaboration a lot but still give us four." From her daily work, Nga learned that the university issued policies to improve its operation according to the accreditation standards. Nga also learned that the Quality Assurance Department recruited "a new experienced member." This information made Nga think that the university's investment in the project and the project effectiveness and efficiency had increased (the first primary standards). The new policies and recruitment also made Nga think that the university would actually follow the accreditation standards rather than merely fulfilling the requirements as a formality. Nga, therefore, thought that the complexity of the project had also increased (the second primary standard).

In summary, over the four interviews, with her primary standards for the project effectiveness, efficiency and complexity, Nga learned different things related to the MTEO 
project. These learnings changed not only her standards but also her appreciation of the project. From the third interview, Nga had become aware of more standards related to the distinctive characteristics of accreditation projects. Overall, she thought that the project effectiveness, efficiency and complexity had increased in all interviews, but the second one.

\subsection{Changes in Hung' Appreciation of the MTEO Project}

\subsubsection{Participant Background}

Hung was an employer representative who participated in the interview with the panel. He was a vice-director of a branch of a big insurance company in Vietnam. His branch provided onthe-job training for business students at FXN university and recruited the university graduates. Hung had rich experience in real estate and insurance and had collaborated with the university before the MTEO project. From this collaboration, Hung had a close relationship with some university staff members, especially the members of the Student Services Department.

\subsubsection{Changes in Hung's Standards for and Appreciation of the MTEO Project}

Number of components

In the first interview (T1), Hung identified seventeen constructs regarding the MTEO project, which did not change in the remaining interviews. These constructs are presented in Table 4.13 as follows. 
Table 4.13 List of constructs - Hung - MTEO case

\begin{tabular}{|r|l|l|}
\hline No. & Constructs & Opposites \\
\hline 1 & Projects benefit organisations & Projects do not benefit organisations \\
\hline 2 & Projects benefit community and society & Projects do not benefit community and society \\
\hline 3 & Projects are assessed fairly, accurately and objectively & Projects are not assessed fairly, accurately, and objectively \\
\hline 4 & Project results can be biased & Project results are not biased \\
\hline 5 & Projects are not too competitive and easy to do & Projects are competitive and not easy to do \\
\hline 6 & Experience of project members is limited & Experienced project members \\
\hline 7 & Project analysis and planning is not good & Project analysis and planning is good \\
\hline 8 & Bad project results & Good project results \\
\hline 9 & Difficulties in projects' human resources & No difficulty in projects' human resources \\
\hline 10 & Projects' work culture is effective & Projects' work culture is not effective \\
\hline 11 & Key people for projects are competent & Key people for projects are incompetent \\
\hline 12 & Projects are supported by governmental policies & Projects are not supported by governmental policies \\
\hline 13 & Unprofessional organisational structure and leadership & Professional organisational structure and leadership \\
\hline 14 & Bad information sharing in projects & Good information sharing in projects \\
\hline 15 & Clear and objective project results & Project results are not clear and objective \\
\hline 16 & Organisations have a lot of experience & Organisations have no experience \\
\hline 17 & Projects are very important for organisations' existence & Projects are not important for organisations' existence \\
\hline
\end{tabular}


Principal component analysis of Hung's repertory grids showed that his appreciation of the MTEO project over the four interviews could be explained by three components. These components explained from $58 \%$ to $65 \%, 15 \%$ to $16 \%$, and $9 \%$ to $15 \%$ of Hung's appreciation, respectively. Together they explained nearly $90 \%$ of his appreciation. The percentage variance for each component is presented in Table 4.10 as follows.

Table 4.14 Percentage variances explained by three components - Hung - MTEO project

\begin{tabular}{|c|c|c|c|c|c|c|}
\hline Interview & \multicolumn{3}{|l|}{$T 1$} & \multicolumn{3}{|l|}{$T 2$} \\
\hline Component & 1 & 2 & 3 & 1 & 2 & 3 \\
\hline Variance $\%$ & 58.24 & 16.56 & 14.91 & 64.96 & 16.04 & 9.58 \\
\hline Cumulative variance $\%$ & 58.24 & 74.8 & 89.7 & 64.96 & 81 & 90.58 \\
\hline Interview & \multicolumn{3}{|l|}{ T3 } & \multicolumn{3}{|l|}{$T 4$} \\
\hline Component & 1 & 2 & 3 & 1 & 2 & 3 \\
\hline Variance $\%$ & 62.35 & 16.09 & 10.45 & 65.8 & 15.64 & 11.2 \\
\hline Cumulative variance $\%$ & 62.35 & 78.44 & 88.88 & 65.8 & 81.44 & 92.63 \\
\hline
\end{tabular}

Interpretation of the components

The table of loadings in Appendix $\mathbf{J}$ shows that there was no significant change in the loadings of the constructs on the components in the first and second interviews. However, there were changes in the loadings on components 1 and 3 in the third interview. Because of this, three PrinGrid maps will be presented in this section to illustrate the loadings of the constructs on the components over the interviews. The first map (Figure 4.13) illustrates the loadings on components 1 and 2 at the first interview, and the two other maps (Figures 4.14 and 4.15) illustrate the loadings on components 1 and 3 at the first and third interviews, respectively. 
Figure 4.13 Hung's PrinGrid map - components 1 and 2 - the first interview - the MTEO project

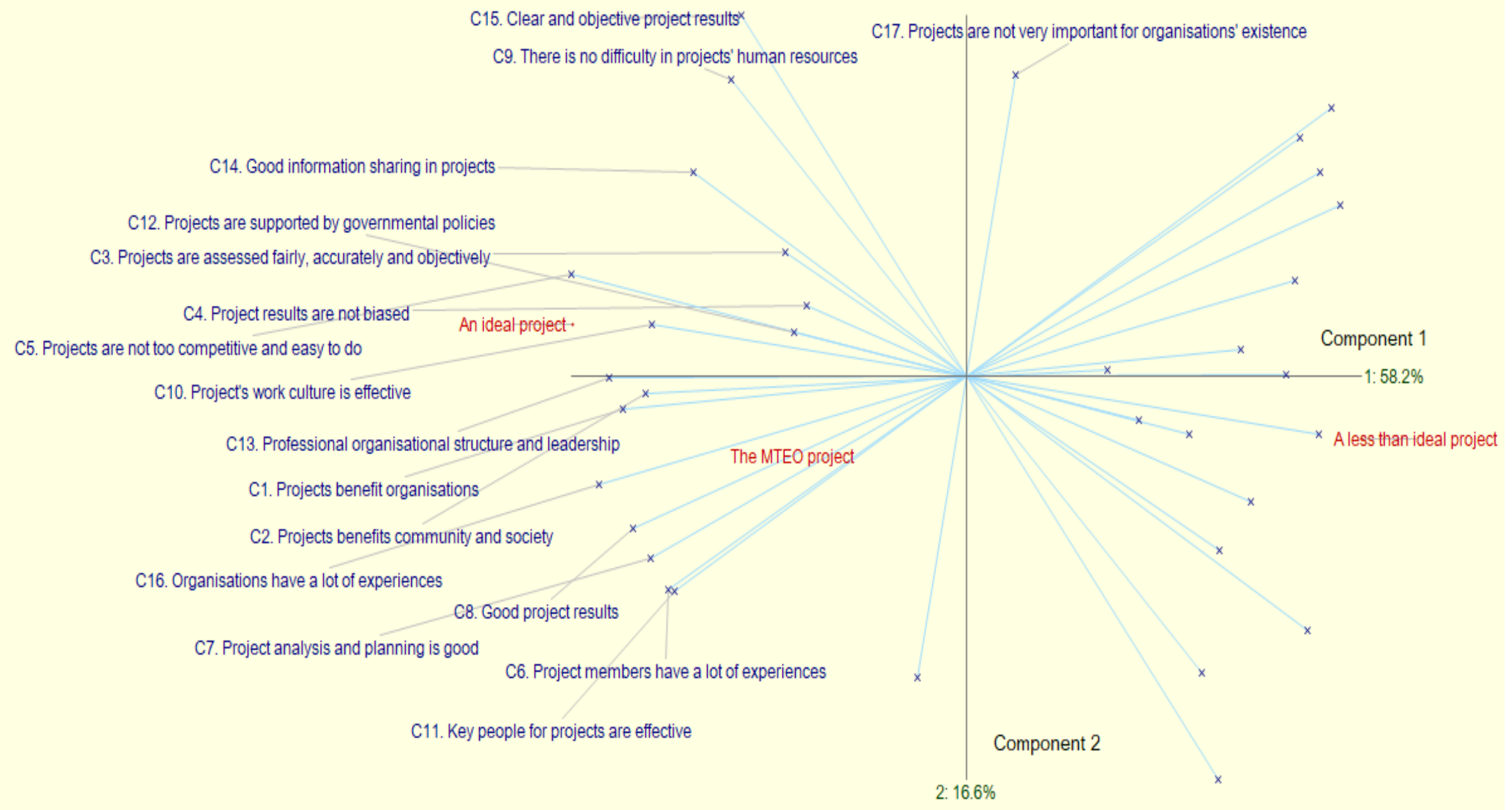


Figure 4.14 Hung's PrinGrid map - components 1 and 3 - the first interview - the MTEO project

C3. Projects are assessed fairly, accurately and objectively

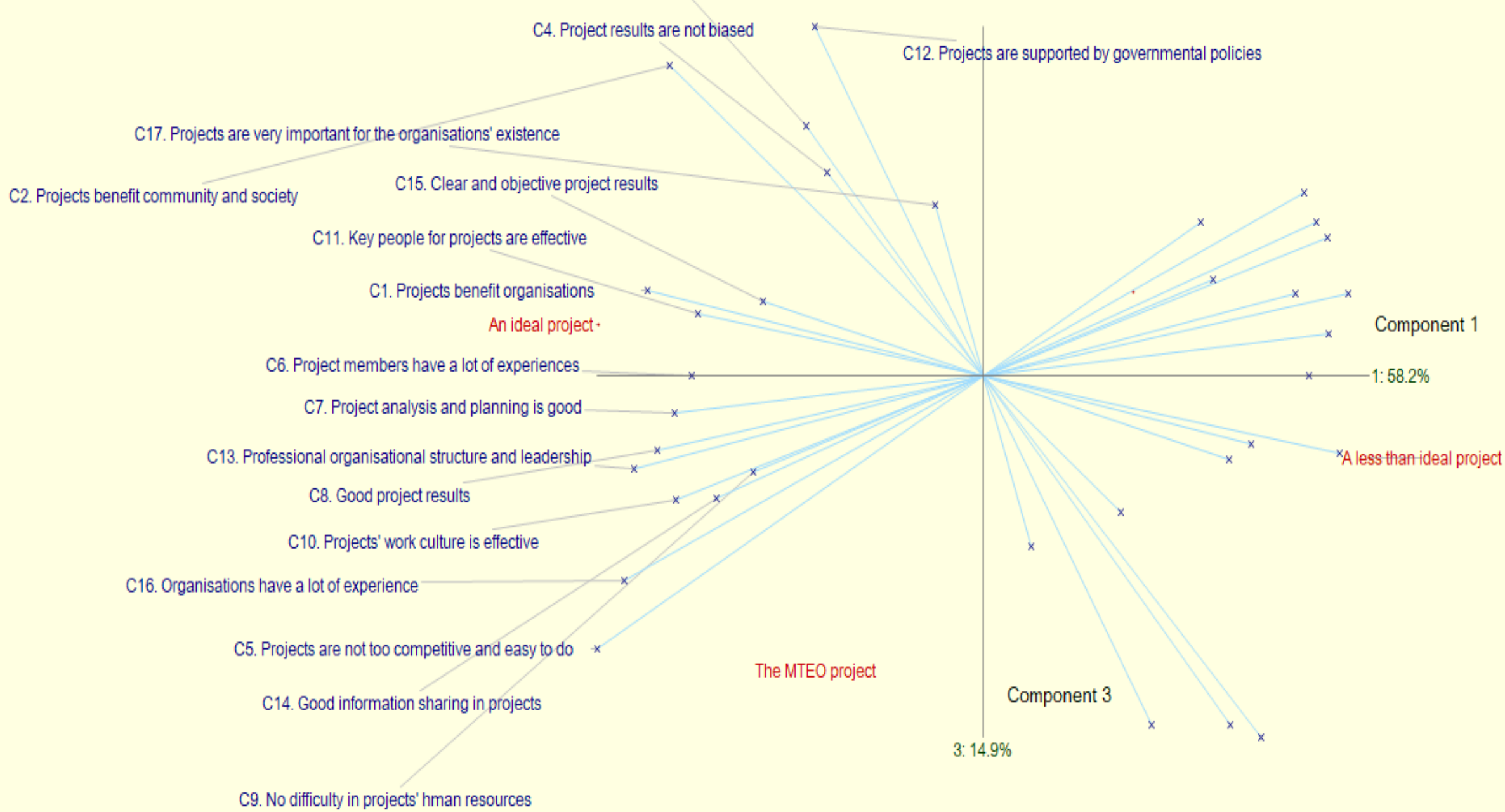


Figure 4.15 PrinGrid map - components 1 and 3 - Hung - the third interview

C3. Projects are assessed fairly, accurately and objectively

C4. Project results are not biased

C2. Projects benefit community and society

C12. Projects are supported by governmental policies

C11. Key people for projects are effective

C1. Projects benefit organisations

C6. Project members have a lot of experiences

C8. Good project results

C17. Projects are very important for organisations' existence

A less than ideal project

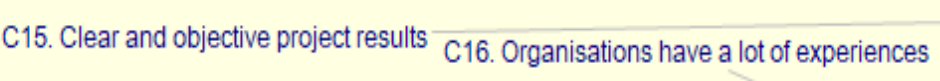
C13. Professional organisational structure and leadership

$-x x^{x} x$

C10. Projects' work culture is effective

C14. Good information sharing in projects

C5. Projects are not too competitive and easy to do

C9. No difficulty in projects' human resources

The MTEO project

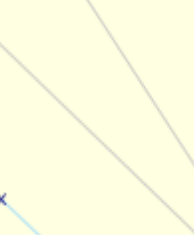


As shown in the table of loading values (Appendix J), at the first interview, all seventeen constructs, except for constructs $\mathrm{C} 12$ and $\mathrm{C} 17$, had their largest or relatively large loadings on the first component. In Figure 4.13, these constructs are the bunch of constructs which is close to the horizontal axis. Even though construct C12 is in this group, the table of loading values shows that the construct actually had a large loading on the third component. Because the map is two-dimensional, it does not illustrate the position of this construct accurately. The constructs with large loadings on the first component, therefore, referred to the project benefits $(\mathrm{C} 1, \mathrm{C} 2, \mathrm{C} 3, \mathrm{C} 4, \mathrm{C} 15)$, the ease of the project implementation (C5), the experience of the university $(\mathrm{C} 6, \mathrm{C} 11, \mathrm{C} 16)$, the project implementation $(\mathrm{C} 7, \mathrm{C} 9, \mathrm{C} 10, \mathrm{C} 13$, $\mathrm{C} 14)$, and the project results (C8). Because the experience of the university affects how easy it is for the university to complete the project, the first component was interpreted as Hung's appreciation of the benefits, the ease, and the implementation of the project. In the second interview, because the loadings of the constructs on the first component did not change much, its interpretation was the same as it was in the first interview. However, at the third interview, there were considerable changes in the loadings of the constructs on the component. In this interview, construct $\mathrm{C} 12$, which referred to the government support of the project, also had a large loading on the first component. Both the PrinGrip map in Figure 4.15 and the table of loading values in Appendix $\mathbf{J}$ show this. The construct is very close to the horizontal axis on the map, and its loading value is high (1.277). The interpretation of the first component, therefore, also included the government support at the third interview. Because the loadings of the constructs on the first component did not change much at the fourth interview, its interpretation was also the same as it was in the third interview.

The table of loading values also shows that the constructs' loadings on the second component did not change much over the four interviews. At the first interview, three constructs had their largest loadings on this component. On the map in Figure 4.13, these constructs are close to the vertical axis. They referred to the project importance (C17), the objectivity of project results (C15) and the sufficiency of human resources (C9). The loadings of the first constructs had the opposite signs to the loadings of the second and third constructs. Therefore, the second component was interpreted as Hung's appreciation of whether the project's human resources were sufficient to carry out the project, given its importance. In the last three interviews, even though some of these loadings were lower than they were for the first, they were still considerably large. The signs of the loadings also did not change. The 
interpretation of the second component, therefore, could be considered unchanged over the four interviews.

While the loadings of the constructs on the second component were unchanged, the loadings of the constructs on the third component changed for the third interview. At the first interview, three constructs had their largest loadings on this component. These constructs were the objectivity of the results (C3, C4) and the government support (C12). In Figure 4.14, these three constructs are close to the vertical axis. Even though the construct C17, which refers to the project's importance, is also close to the vertical axis, the value of the loading of this construct was not high (0.71). The construct had its larger loading on component 2. Because the PrinGrid map is two-dimensional, it does not present the position of this construct accurately. The map also shows that the loadings of the three constructs with large loadings on component 3 had the same signs. In other words, one polar of the component refers to the positive meanings of all three constructs and vice versa. The third component, therefore, could be interpreted as Hung's appreciation of the objectivity and the government support of the project in the first interview. As shown in the table of loading values, the loadings of the constructs on the component did not change much in the second interview. The interpretation of the component, therefore, can be considered unchanged in this interview. At T3, however, only C3 and C4 had large loadings on the component, while C12 had very small loadings (0.387). These changes can be seen in Figure 14.15. While constructs C3 and C4 are still close to the vertical axis, construct $\mathrm{C} 12$ is not close to the axis anymore. The third component, therefore, can be interpreted as Hung's appreciation of the objectivity of the project results for T3. This interpretation was then kept the same in T4 because the loadings of the constructs on this component did not change much in the fourth interview.

In summary, over the four interviews, Hung's appreciation of the MTEO project could be explained by three components. These components respectively referred to the benefits, ease, and implementation of the project; the sufficiency of the human resources to carry out the project, given its importance; and the objectivity and the government support of the project. While the second component could be considered as unchanged, the first and third components underwent minor changes from the third interview onwards.

\section{Changes in Hung's standards for and appreciation of the MTEO project}

This section presents the changes in Hung's standards for and appreciation of the MTEO project. These changes were identified based on the changes in the loadings of Hung's 
appreciation of the MTEO project, the ideal, and the less than ideal projects on the three components. Two PrinGrid maps are used to illustrate these changes. The first map (Figure 4.10) illustrates the changes according to component 1 and component 2 , and the second map (Figure 4.11) illustrates the changes according to component 1 and component 3. Even though the interpretation of component 1 and component 3 had minor changes at T3 and T4, these changes do not affect the illustration of the changes in Hung's appreciation over the four interviews in the same PrinGrid maps. In the maps, the changes in the interpretation of the components are presented in brackets. The loadings are presented in Table 4.11 as follows.

Table 4.15 The loadings of Hung's appreciation of the ideal project, the less than ideal project and the MTEO project on the three components over the four interviews

\begin{tabular}{|l|l|l|l|l|l|l|}
\hline Interview & \multicolumn{3}{|c|}{$T 1$} & \multicolumn{3}{c|}{$T 2$} \\
\hline Component & 1 & 2 & 3 & 1 & 2 & 3 \\
\hline Ideal project & -3.917 & 0.423 & 0.421 & -3.625 & 0.315 & 0.646 \\
\hline Less than ideal project & 3.93 & -0.512 & -0.671 & 4.184 & -0.407 & -0.772 \\
\hline MTEO project & -1.06 & -0.296 & -2.294 & -2.44 & 0.081 & -0.777 \\
\hline Gap & 2.857 & & & 1.185 & \multicolumn{3}{c|}{$T$} \\
\hline Interview & \multicolumn{7}{|c|}{$T 3$} & 2 & 3 & 1 & 2 & 3 \\
\hline Component & 1 & 2 & -3.767 & 0.584 & 0.536 \\
\hline Ideal project & -3.647 & 0.612 & 0.56 & -3.767 & -0.962 \\
\hline Less than ideal project & 4.113 & -0.628 & -0.863 & 3.974 & -0.635 & -1.821 \\
\hline MTEO project & -2.298 & -0.568 & -1.798 & -2.572 & -0.723 & \\
\hline Gap & 1.349 & & & 1.195 & & \\
\hline
\end{tabular}


Figure 4.16 Changes in Hung' appreciation of the MTEO project according to component 1 and component 2 over the four interviews

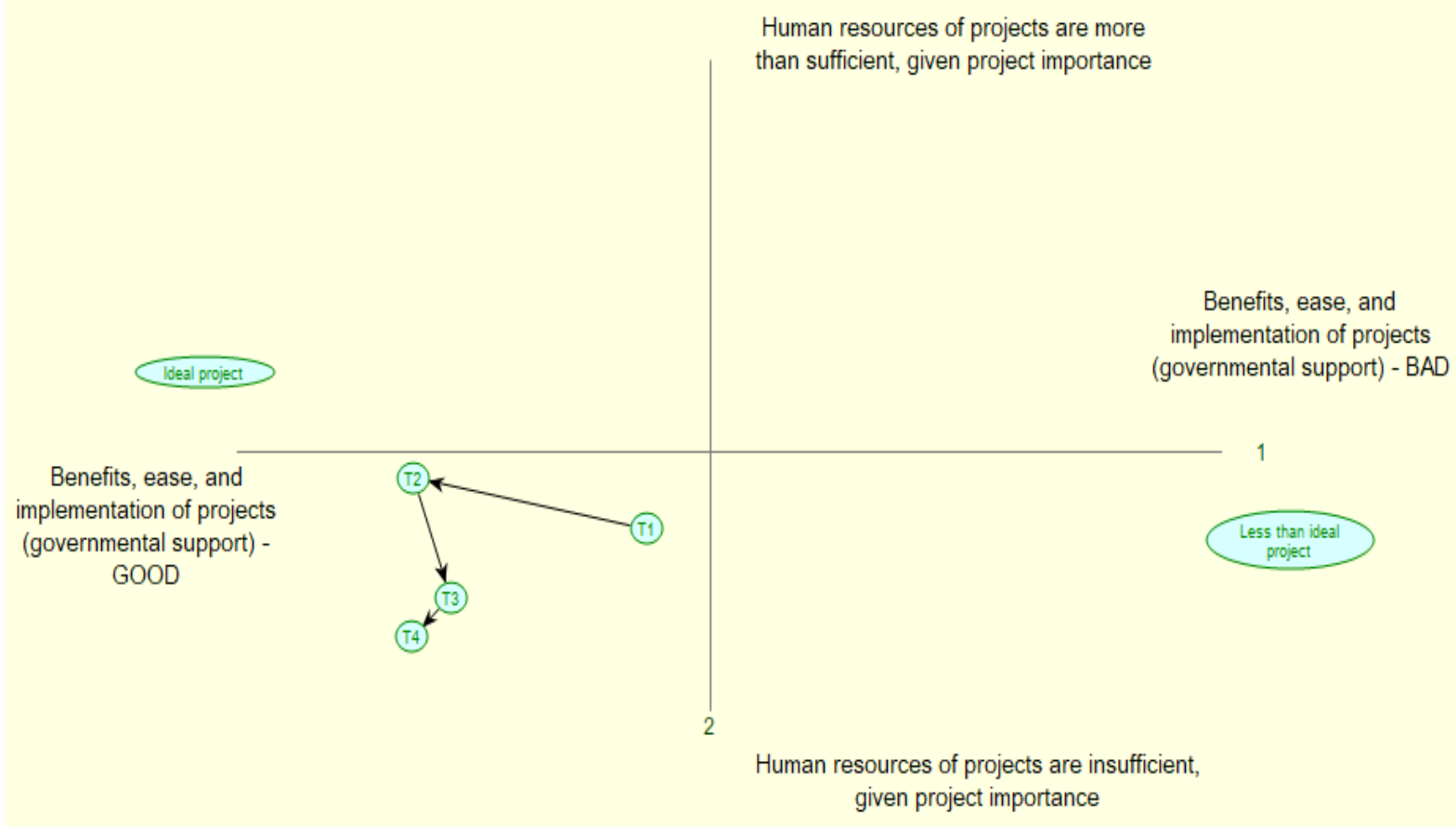

Figure 4.17 Changes in Hung's appreciation of the MTEO project according to component 1 and component 3 over the four interviews

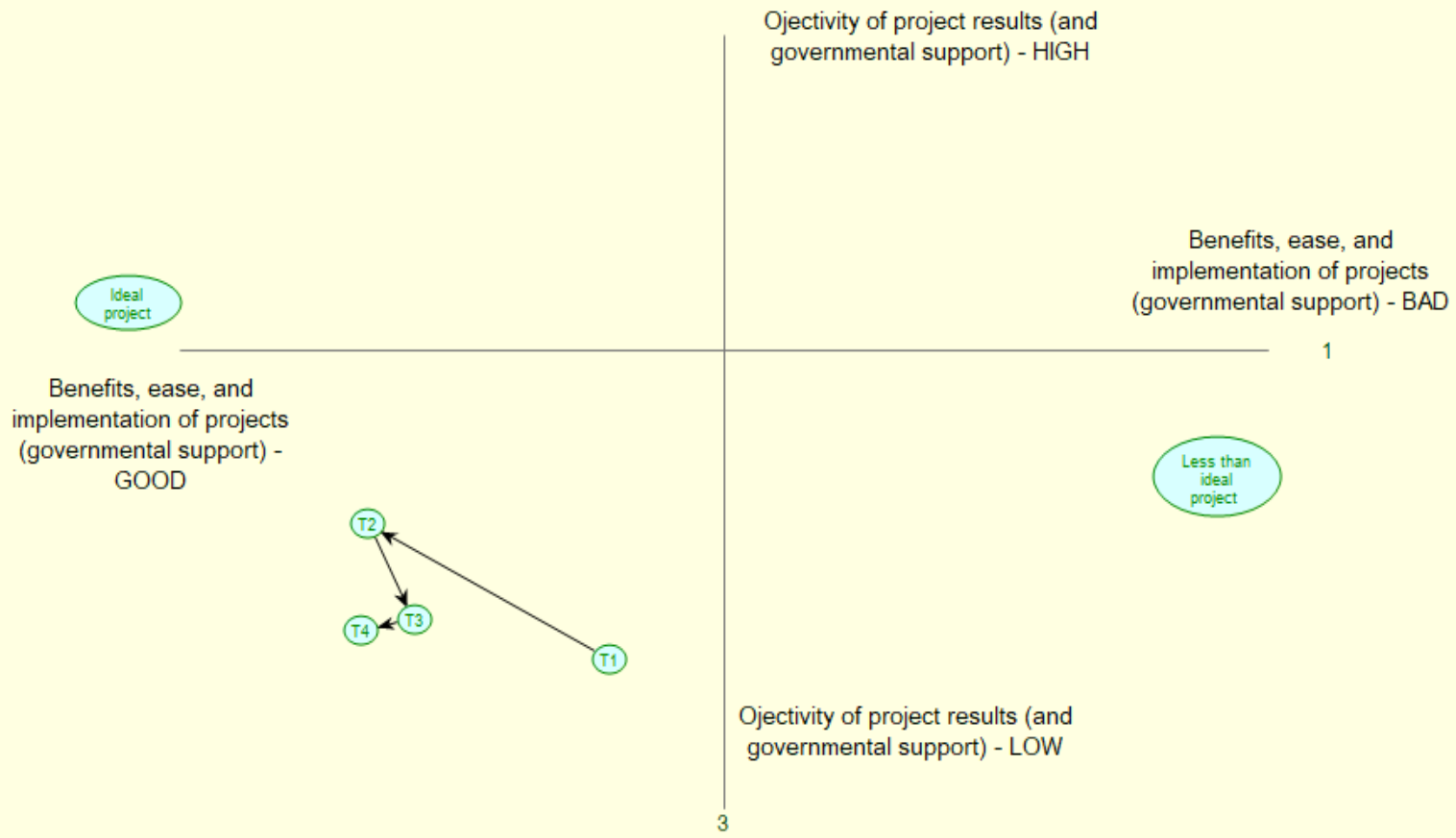

Because Hung's appreciation of the MTEO project had loadings on the three components over the four interviews, these components represented his standards for the 
project. Among these components, Hung's appreciation of the ideal and less than ideal projects had its largest loadings on the first component and very small loadings on the second and third components. The loadings on the first component also had opposite signs. The first component, therefore, represented Hung's primary standards and the second and third components represented his secondary standards for the MTEO project. The positions of the ideal and less than ideal projects on the maps also indicate this. Their positions are opposite regarding the horizontal axes and do not differ much in relation to the vertical axes.

At T1, following the interpretation of the first component, Hung's primary standards were that the project must benefit the university and community, the project must be easy to the university, and the project implementation must be good. Following the interpretation of the second component, one of Hung's secondary standards was that the human resources must be sufficient to carry out the project, given its importance. Meanwhile, following the interpretation of the third component, Hung's other secondary standards were that the objectivity of the results and the government support must be moderate. Because the interpretation of the second component did not change over the four interviews, the standard related to this component also remained unchanged. Because the interpretation of the first and third component had minor changes at $\mathrm{T} 3$, the standards related to these components also changed. At T3 and T4, the primary standards included the government support and the secondary standard related to the third component only referred to the objectivity of the project results.

According to the table and the maps, Hung's appreciation of the MTEO project changed over the four interviews. Specifically, the gap between the loadings of Hung's appreciation of the MTEO project and his appreciation of the ideal project on component 1 changed. It decreased from 2.857 to 1.185 between $\mathrm{T} 1$ and $\mathrm{T} 2$, increased to 1.349 at $\mathrm{T} 3$, and decreased again to 1.195 at T4. These changes are illustrated by arrows T1T2, T2T3, and T3T4. They indicate that between $\mathrm{T} 1$ to $\mathrm{T} 2$ and $\mathrm{T} 3$ to $\mathrm{T} 4$, Hung was aware that the project benefits, the project's ease and the project implementation improved. Between T2 and T3, Hung changed his assessment in that he considered those aspects of the project to be worse. The changes in Hung's appreciation regarding component 2 showed that, in general, he thought that the project's human resources were insufficient to carry out the project, given its importance. He thought that the challenge for the project's human resources had decreased at T2 and increased at T3 and T4. The changes in Hung's appreciation regarding the third component were also similar. While from $\mathrm{T} 1$ to $\mathrm{T} 2$, his appreciation of the project objectivity and the support of 
governmental policies to the project improved, from T2 to T3 and from T3 to T4, his appreciation of the project objectivity diminished.

In summary, over the four interviews, while Hung's standards for the project had minor changes, his appreciation of the project changed in both positive and negative ways. The changes in his appreciation focused on the benefits, ease, and implementation of the project; the sufficiency of the human resources to carry out the project, given its importance; and the objectivity of the project results and government support.

\subsubsection{Changes in Hung's Appreciation of the MTEO Project through the Lens of Vickers' Concept}

Based on the identification of the changes in Hung's standards for and appreciation of the MTEO project, the changes in his appreciation of the project, as influenced by his standards and perceptions of events and ideas, can be described by applying Vickers' concept of appreciative systems as follows.

At the first interview (T1), Hung received an email invitation to participate in the interviews with the panel. With his experience, Hung's primary standards for the project were that the project must benefit the university and community, the university must be able to do the project easily, and the project implementation must be good. Hung's secondary standard was that the project's human resources must be sufficient to carry out the project, given its importance and the objectivity of the project results and the governmental support to the project must be moderate. At this time, Hung only learned about the name of the project and the time of the interviews. Hung thought that this project was similar to "other projects" that he had supported at the university. He thought that the project benefited the university somewhat, that in general the university could do the project easily, and that the project implementation was good (the primary standards). However, Hung also thought that the project was important, and the project's human resources were insufficient to carry out it (secondary standard 1). The project was also not very objective and not supported by governmental policies (secondary standards 2).

From T1 to T2, a member of the Student Services Department persuaded Hung to participate in the interview with the panel. When Hung agreed, the member sent him the interview guidelines. The guidelines provided a detailed explanation of the purposes of the project and the potential interview questions. This information made Hung think that the project was "more professional" and the university was "committed" to it. Based on the fact 
that he received more information, Hung also assumed that information sharing processes applied by the project team were "similarly good." As a member of the Department of Student Services let Hung know about the project leaders, he also had a better appreciation of the leadership:

Now I know that Student Services Head is involved, and the university's Board of management is in charge of the whole project. So, I think the project's leadership is better

Altogether, Hung had a better impression of the project implementation (the primary standards) at T2. The guidelines also caused Hung to believe that the project was more important. However, with a better appreciation of the project implementation, he thought that the project was less challenging for the project's human resources, compared to his assessment at T1 (secondary standard 1):

Now I have read the guidelines, I can see that the accreditation is important to the university, and they seem to be worried, that is why they prepare everything very carefully.

The additional information about the project and the potential interview questions in the guidelines also made Hung think that the project "was more supported by the governmental policies" and was more objective (secondary standards 2).

From T2 to T3, as an employer representative, Hung attended an interview with the panel. At the interview, he learned that the accreditation was a national accreditation, and, therefore, government support was critical to it. This support, therefore, was not his secondary standard anymore but became his primary standard for the project. Subsequently, a university staff member asked him and other employer interviewees to provide "only good answers" about the university, and even though most of the interviewees claimed that they only said the truth, in the end, they all "avoided saying bad things about the university." Hung also learned from his friend, an alumnus of the university, that the situation was similar in her interview. These incidents made Hung realise that the project benefits to the university were less than he expected:

As all people tried to avoid mentioning the really bad things, the university will not learn much from this.

While Hung's perceptions about the benefits of the project diminished, his views on the project implementation had improved: 
After participating in the interviews, I see that the university knows what they are doing, everyone knows their tasks, they show that they have done this kind of activities many times before.

Overall, things that Hung learned impacted his appreciation of the project benefits and implementation in both positive and negative ways. As a result of these impacts, Hung's appreciation of these aspects of the project worsened (the primary standards).

In the interview, the panel also let Hung know that the project would "affect what the Ministry allow[ed] the university to do." This made Hung more aware of the project importance. Because of this, Hung thought that the project was more challenging for the project's human resources than it was at T2 (secondary standard 1). Meanwhile, things he observed in the panel interviews made Hung think that "everything seems to be arranged in advance." He specifically believed that the alumni and the staff groups provided less objective answers than his employer group:

\section{I heard from the panel that the staff members answered very well. So, I think we [the employers] provided the truest answers.}

Hung, therefore, thought that the project objectivity decreased (secondary standard 2).

In the time between T3 and T4, even though Hung was not officially involved in the project anymore, a member of the Department informally let him know about the good project result. Hung, therefore, thought that the project management in general and the project planning and analysis, in particular, "must be better to get those results" (the primary standards). However, based on what he had experienced in the interviews with the panel, Hung thought that the project was less objective at T4 compared to T3 (secondary standard 2). Along with this appreciation of the project's objectivity, Hung also thought that the project was more difficult and challenging for the human resources (secondary standard 1).

In summary, over the four interviews, with his initial standards, Hung learned different things related to the MTEO project, and these learnings changed both his standards and appreciation regarding the project. The changes in Hung's appreciation focused on the benefits, level of difficulty, and implementation of the project; the sufficiency of the project's human resources to carry out the project, given its importance; and the objectivity and the government support of the project. 


\subsection{Chapter Summary}

This chapter has described the changes in the appreciation of three participants in the MTEO case about the project, as influenced by their standards and perceptions of events and ideas. Following the same presentation format, the next chapter will present the data analysis and findings of the APSC case. 


\section{Chapter 5 APSC Case}

\subsection{Overview of the Chapter}

This chapter presents the data analysis and findings of the APSC case using the same steps and presentation format established in chapter four. The chapter begins with an overview of the project. After that, it presents the changes in the four participants' appreciation of the project. These changes are presented along with the changes in their standards and perceptions of related events and ideas.

\subsection{Overview of the APSC Accreditation Project}

APSC is the name of a program accreditation certificate. It was initially developed and popularised in the United States and then introduced to other countries. FXN University was one of the first universities in Vietnam that applied for this accreditation. The application process was initiated two years before the data collection for this thesis started. After one year, the university had submitted the self-study report. However, because of limitations in teaching and research, the report had not been approved, and FXN University was given more time to improve its activities. The data for the current thesis were collected when the university focused on improving its teaching and research, organised its last external assessment of student learning outcomes, and prepared for the re-submission of the self-study report.

During the data collection period, the APSC project involved internal and external stakeholders. The internal stakeholders included the project manager, members of the Research and Development Department, and the faculty team. The external stakeholders included representatives of the accreditation body and members of APSC accreditation projects at other Vietnamese universities. Data collection for this thesis involved interviews with four of these internal and external stakeholders at the same four time points. The participants were: Tung, the project manager; Tai, the leader of the faculty team; Khiem, a member of the APSC accreditation project at another Vietnamese university (NUE University); and Rafat, the regional chair of the accreditation body. Due to logistical reasons, only the first two participants had four interviews, while Khiem and Rafat had only three and two, respectively. After discussing the time line of the project with the project manager, the four time points for the interviews were set as follows: before the last external assessment of student learning outcomes (T1), after the external assessment (T2), before the new trimester (T3) and after the review 
meeting of the trimester (T4). Figure 5.1 below illustrates the different time points and lists the interviews that were conducted at each stage.

Figure 5.1 Time points of the interviews - APSC project

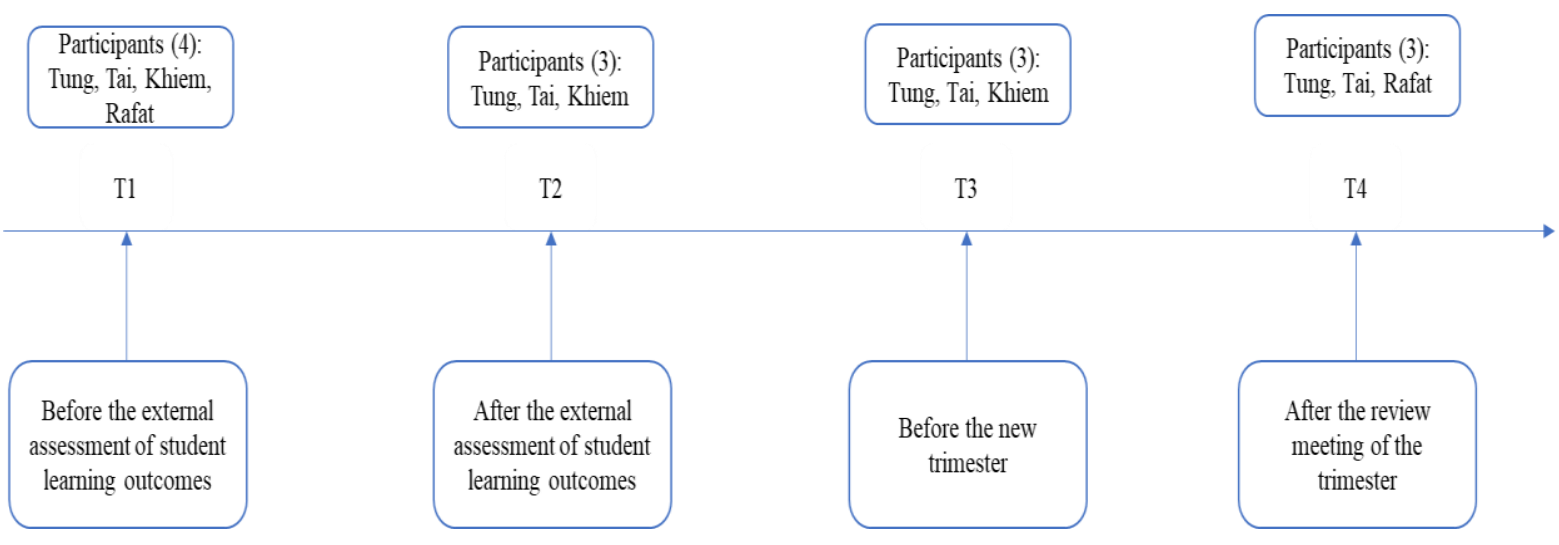

\subsection{Changes in Tung's Appreciation of the APSC Project}

\subsubsection{Participant Background}

Tung was the project manager. He started his career as a lecturer and researcher in a research institute and had managed educational centres before joining FXN University. At the time of the interviews, Tung was one of four vice-rectors of the university and managed its campus in Ho Chi Minh city.

\subsubsection{Changes in Tung's Standards for and Appreciation of the APSC Project}

Number of components

Tung was interviewed four times. At the first interview, he identified twenty-two constructs regarding the APSC project. At the second interview, he added two more new constructs and thus increased the total number of constructs to twenty-four. These constructs were kept the same in the third and fourth interviews. The principal component analysis (see Appendix K) identified that over the four interviews, two principal components could explain $90 \%$ of Tung's appreciation of the APSC project. The components explained $83 \%$ to $88 \%$ and $5 \%$ to $7 \%$ of the appreciation, respectively. The percentage variances explained by the components are presented in Table 5.1 below. 
Table 5.1 Percentage variances explained by two principal components over the four interviews - Tung - APSC project

\begin{tabular}{|c|c|c|c|c|c|c|c|c|}
\hline Interview & \multicolumn{2}{|l|}{$T 1$} & \multicolumn{2}{|l|}{$T 2$} & \multicolumn{2}{|l|}{ T3 } & \multicolumn{2}{|l|}{$T 4$} \\
\hline Component & 1 & 2 & 1 & 2 & 1 & 2 & 1 & 2 \\
\hline Variance $\%$ & 88.28 & 5.42 & 83.69 & 8.56 & 83.84 & 7.95 & 84.95 & 7.13 \\
\hline Cumulative variance $\%$ & & 93.7 & & 92.25 & & 91.79 & & 92.08 \\
\hline
\end{tabular}

Interpretation of the components

The construct loadings on the components show that the additions of the two new constructs resulted in minor changes to the interpretation of the two components at the second interview. The interpretation was then unchanged in the remaining interviews.

At the first interview (T1), all twenty-two constructs had their largest loadings with the same signs on the first component. These constructs could be divided into three groups. The first group consisted of constructs related to the transparency of the accreditation processes. These constructs specifically referred to the specification and strictness of the accreditation standards and to the standardisation of the accreditation project implementation $(\mathrm{C} 7, \mathrm{C} 10, \mathrm{C} 16$, $\mathrm{C} 17, \mathrm{C} 19, \mathrm{C} 20)$. The second group consisted of constructs related to the programme quality. These constructs referred to the programme characteristics, faculty qualifications, research activities and student quality (C3, C4, C5, C6, C13, C15). The third group consisted of constructs related to the university efforts to do the project. These constructs referred to the pressure, determination, motivation, activities, and resources of the university and the project progress $(\mathrm{C} 1, \mathrm{C} 2, \mathrm{C} 8, \mathrm{C} 9, \mathrm{C} 11, \mathrm{C} 12, \mathrm{C} 14, \mathrm{C} 18, \mathrm{C} 21, \mathrm{C} 22)$. Because the programme quality is also affected by the university efforts, the first component could be interpreted as the transparency of the accreditation processes, and the university efforts to do the project. From the second interview onwards, the two added constructs, which referred to the faculty qualifications (C23, C24), also had considerably large loadings on the first component. The first component, therefore, could be interpreted in the same way as it was in the first interview, except including the faculty qualifications.

The interpretation of the second component underwent similar changes. At the first interview, three constructs had considerably large loadings on it. These constructs referred to the research activities (C5), the project progress (C9), and the strictness of the accreditation standards for the programme quality (C16). Because the loadings of the former two constructs had opposite signs to the loadings of the latter construct, the second component could be interpreted as the gap between the research activities and the accreditation standards. From 
the second interview (T2) onwards, in addition to the three constructs mentioned above, the two new constructs also had their largest loadings on the second component. Because the loadings of the new constructs $(\mathrm{C} 23, \mathrm{C} 24)$ had the same signs as the loadings of the construct which referred to the research activities (C5), the second component could be interpreted as the gap between the research activities and faculty qualifications and the accreditation standards. In other words, starting from the second interview, Tung's concern included not only the research but also whether the faculty qualifications met the accreditation standards.

In summary, for all four interviews, Tung's appreciation of the APSC project could be explained by two components. The first component referred to his appreciation of the transparency of the accreditation processes and the university efforts to do the project. The second component referred to his comparison between the research activities and faculty qualifications of the university and the accreditation standards.

\section{Changes in Tung's standards for and appreciation of the APSC project}

This section presents the changes in Tung's standards for and appreciation of the APSC project over the four interviews. These changes were identified by the changes in the loadings of Tung's appreciation of the APSC project and the ideal and less than ideal projects on the two components (Table 5.2). Because the interpretation of the two components changed since the second interview, the changes in Tung's appreciation over the four time points are illustrated by two PrinGrid maps. The first map (Figure 5.2) illustrates his appreciation at the first interview and the second map (Figure 5.3) illustrates his appreciation in the remaining interviews.

Table 5.2 The loadings of Tung's appreciation of the ideal project, the less than ideal project and the APSC project on the two components

\begin{tabular}{|l|l|l|l|l|l|l|l|l|}
\hline Interview & \multicolumn{2}{l}{ T1 } & \multicolumn{2}{l}{ T2 } & T3 & \multicolumn{2}{l|}{ 44 } \\
\hline Component & 1 & 2 & 1 & 2 & 1 & 2 & 1 & 2 \\
\hline Ideal project & -2.174 & 0.174 & -2.33 & 0.174 & -2.297 & -0.013 & -2.275 & -0.275 \\
\hline $\begin{array}{l}\text { Less than ideal } \\
\text { project }\end{array}$ & 7.114 & -0.174 & 7.345 & -0.191 & 7.374 & -0.175 & 7.396 & 0.105 \\
\hline APSC project & -0.73 & 1.574 & -0.752 & 1.869 & -0.9 & 1.849 & -1.009 & -1.553 \\
\hline Gap & 1.444 & & 1.578 & & 1.397 & & 1.266 & \\
\hline
\end{tabular}


Figure 5.2 Tung's appreciation of the APSC project at the first interview

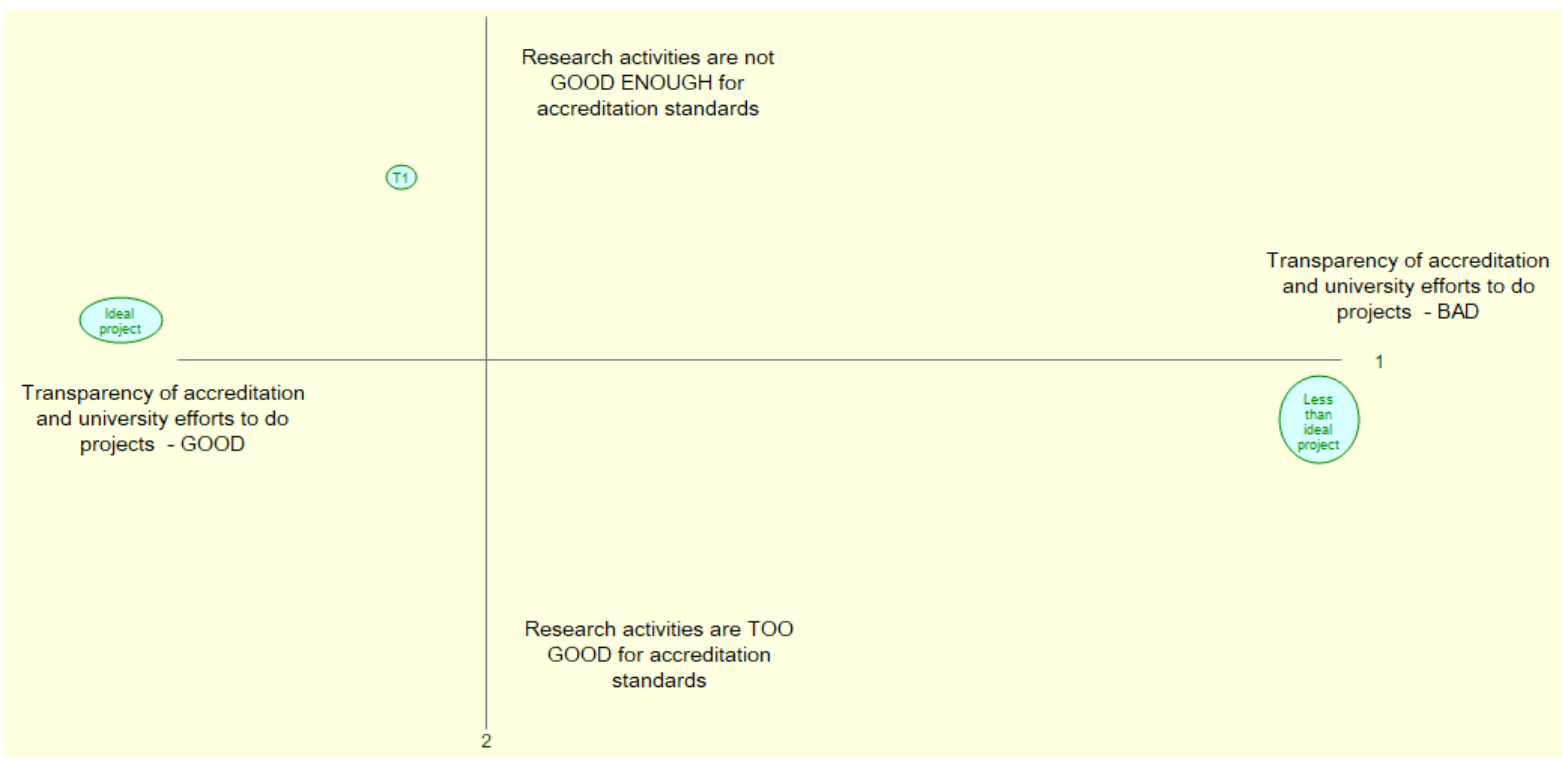

Figure 5.3 Changes in Tung's appreciation of the APSC project from his second to his fourth interview

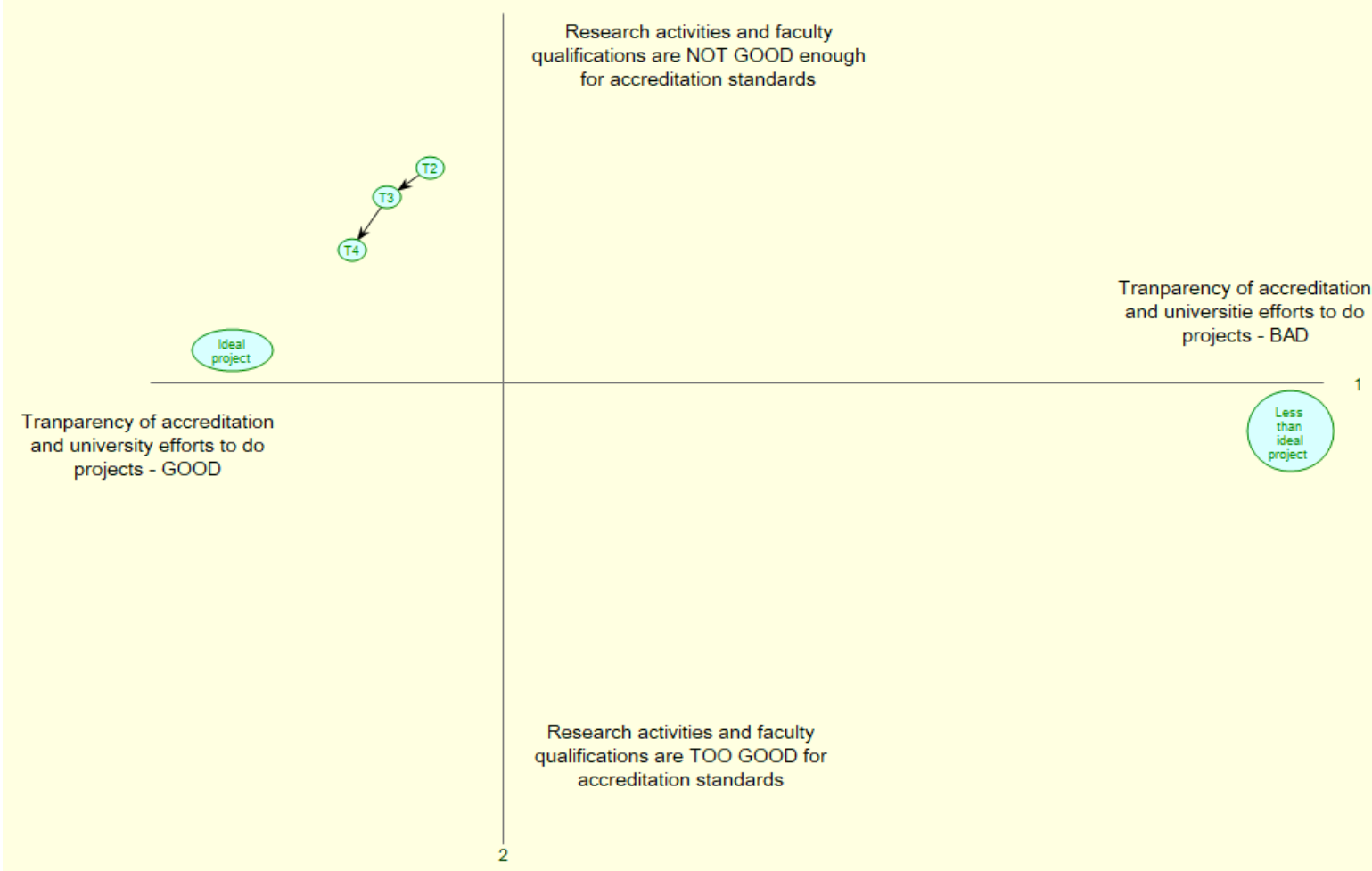

The table shows that Tung's appreciation of the APSC project had loadings on the two components over the four interviews. These components, therefore, represented his standards for the project. A comparison of these components shows that Tung's appreciation of the ideal 
and less than ideal projects had its largest loadings on the first component and very small loadings on the second component. The loadings on the first component also had opposite signs. This indicates that the first component could differentiate Tung's appreciation of the ideal project from the less than ideal project. The second component, meanwhile, only represented his concern about the project. The positions of the ideal and less than ideal projects on the PrinGrid maps also indicate this. They are opposite regarding the horizontal axes and do not differ much regarding the vertical axes. Therefore, the first component represented Tung's primary standards, and the second component represented his secondary standard for the project. Based on the interpretation of the components, at the first interview, Tung's primary standards were that the accreditation must be transparent, and the university efforts must be good. His secondary standard was that the research activities must match the accreditation standards. From the second interview onward, following the additions of the two new constructs, Tung's primary standards included the efforts in improving the faculty qualifications. He also adopted a new secondary standard, namely that the research activities and faculty qualifications of the programme must match the accreditation standards.

The table and the PrinGid maps also show the changes in Tung's appreciation of the APSC project over the four interviews. On the maps, all the four points (T1), (T2), (T3) and (T4) are in the upper left quadrants. This indicates that over the four interviews, Tung thought that the accreditation processes were transparent, and the university efforts were generally good. However, he believed that the research activities were not good enough for the accreditation standards. From T1 to T2, the gap between the loadings of Tung's appreciation of the APSC project and the ideal project on the first component increased from 1.444 to 1.578. Meanwhile, the loadings of his appreciation of the APSC project on the second component increased from 1.574 to 1.869 . These changes, along with the changes in the interpretation of the components, indicated that Tung considered the transparency of the accreditation processes and the university efforts to be generally worse and the gap between the research activities and faculty qualifications of the programme and the accreditation standards to be bigger at T2 compared to before. The changes in Tung's appreciation of the APSC project in the remaining interviews are illustrated by the two arrows T2T3 and T3T4. These arrows show that his appreciation became more positive. He thought that the transparency of the accreditation processes and the university efforts had improved. The gap between the research and faculty qualifications of the university and the accreditation standards had also decreased at T4. 
In summary, the principal component analysis identifies the changes in both Tung's standards for and appreciation of the APSC project over the four interviews. In the second interview, Tung developed new standards related to the faculty qualifications. Along with these standards, he thought that the transparency of the accreditation processes and the university efforts had worsened. He also thought that the gap between the research and faculty qualifications of the university and the accreditation standards had widened. His appreciation of these aspects of the project then became more positive in the remaining interviews.

\subsubsection{Changes in Tung's Appreciation of the APSC Project through the Lens of Vickers'}

\section{Concept}

The above section has identified the changes in Tung's standards and appreciation regarding the APSC project. This section uses these findings to describe the changes in his appreciation of the project, as influenced by his standards and perceptions of related events and ideas, by applying Vickers' concept of appreciative systems.

At the first interview (T1), with his experience, Tung's primary standards for the APSC project were that the accreditation processes must be transparent, and the university efforts to do the project must be good. His secondary standard was that the research activities of the programme must match the accreditation standards. Following these standards, Tung thought that the accreditation processes were "rigorous" and made the implementers "trust" the accreditation. He also regarded the overall efforts made by the university to do the project as good (the primary standards). However, the research activities of the university did not match the accreditation standards (the secondary standard).

From T1 to T2, as the vice-rector, Tung was involved in an accreditation panel visit at the university for a different accreditation. In this visit, he learned about the comments of the panel on the limitations of the faculty qualifications. This reminded Tung of similar comments from previous accreditations of the university:

Not only this accreditation but also other previous accreditations have shown this problem. This accreditation is the most recent one, and it shows the problem very strongly.

This event caused Tung to add the faculty qualifications to both his primary and secondary standards.

Based on the modified standards, Tung thought that the faculty qualifications were a problem of the project. Except for this concern, Tung learned things that positively affected his 
appreciation of the project. He saw that related departments, such as the Accounting Department, supported the project more:

Previously, without me, when Ngoc [the project administrator] applied for any payment from Accounting Department, I must intervene, but this time I don't have to. So, it [the support] is better.

Tung guessed that the reasons for this improvement were that the university had been applying for many accreditations. The related departments, therefore, were "getting used to support the accreditation processes." In addition, Tung received information from the Research and Development Department about the improvements in teaching and faculty qualifications. Tung himself also had positive contacts with the regional chair of the accreditation body:

He [the regional chair] has asked me to organise some events. We will have opportunities to boost our image. The accreditation body will understand us more and support us more.

Despite these positive learnings, the concern about the faculty qualifications caused Tung's overall appreciation of the university efforts to diminish (the primary standards). He also thought that the research and faculty qualifications of the programme matched the accreditation standards less (the secondary standard).

Between the second to the third interview (from T2 to T3), Tung saw that the university leaders and the Research and Development Head paid more attention to and asked more about the project progress:

The Research and Development Head has just sent me an email asking about this project. He did not ask anything in the last six months and has just sent an email two days ago. The Vice-Rector of Research and Teaching also asked me about this project.

Tung also learned from the Research and Development Department that the university had some new lecturers with good qualifications, which resulted in "some improvements, about $20 \%$ to $30 \%$ of the faculty qualifications." However, as time passed, Tung was concerned that the deadline of the accreditation was close, and the university activities were not enough to make the project progress. Tung also felt that the representatives of the accreditation body did not pay as much attention to him at this stage compared to T2:

It has been taking too long, and it will not have any meaning anymore. Even the accreditation body will take us lightly. It is not good. 
Despite these problems, with the generally more positive nature of his learnings, Tung thought that the university efforts had improved (the primary standards). The research activities and faculty qualifications also better matched the accreditation standards (the secondary standard).

Between the third to the fourth interview (from T3 to T4), Tung attended the review meeting of the trimester. In this meeting, he learned from the report of the Research and Development Department that the faculty qualifications, teaching, and research activities were "getting better." Tung also learned that the Head of the Department had contacted the representatives of the accreditation body and the body had approved that the university could "re-submit its self-study report in September." The university would also send people to the annual international conference of the accreditation body. This information made Tung think that the university efforts to do the project had increased (the primary standards). He also considered the research activities and faculty qualifications of the university to better match the accreditation standards (the secondary standard).

In summary, over the four interviews, with his initial standards for and appreciation of the APSC project, Tung learned different things related to the project by the second interview. Based on these learnings, Tung added new standards for the faculty qualifications to the ones already established. These new standards remained unchanged for all subsequent interviews. In his second interview, Tung also thought that the university efforts had decreased and that the gap between the research activities and the faculty qualifications and the accreditation standards had widened. In the other interviews, his appreciation of these aspects of the project improved.

\subsection{Changes in Tai's Appreciation of the APSC Project}

\subsubsection{Participant Background}

Tai was a lecturer in economics and finance at FXN University and the leader of the faculty team of the programme. Before his academic career, Tai had worked for many years in the banking industry. In the APSC project, Tai was responsible for assigning lecturers to classes, promoting research activities, and recruiting new faculty members.

\subsubsection{Changes in Tai's Standards for and Appreciation of the APSC Project}

Number of the principal components

Tai was interviewed four times. In the first interview, he identified twelve constructs, and these constructs did not change in the remaining interviews. The principal component analysis of Tai's repertory grids (see Appendix L) identified that for all the four interviews, two principal 
components could explain from $93 \%$ to $96 \%$ of his appreciation of the APSC project. The two components explained $84 \%$ to $89 \%$ and $6 \%$ to $9 \%$ of the appreciation, respectively. The percentage variances explained by the components are described in Table 5.3 as follows.

Table 5.3 Percentage variances explained by each principal component over the four interviews - Tai - APSC project

\begin{tabular}{|c|c|c|c|c|c|c|c|c|}
\hline Interview & \multicolumn{2}{|l|}{$T 1$} & \multicolumn{2}{|l|}{$T 2$} & \multicolumn{2}{|l|}{$T 3$} & \multicolumn{2}{|l|}{$T 4$} \\
\hline Component & 1 & 2 & 1 & 2 & 1 & 2 & 1 & 2 \\
\hline Variance $\%$ & 84.46 & 8.61 & 86.89 & 7.81 & 85.98 & 8.21 & 89.66 & 6.5 \\
\hline Cumulative variance $\%$ & & 93.07 & & 94.7 & & 94.18 & & 96.16 \\
\hline
\end{tabular}

Interpretation of the principal components

The construct loadings on the two components show that the interpretation of the two components was unchanged for all four interviews.

In each interview, all twelve constructs had their largest loadings with the same signs on the first component. The common theme of these constructs was the effectiveness of the project management policies and activities. The constructs specifically referred to the policies related to the communication, motivation, and technology of the project and activities related to the importance, programme quality, and progress of the project. At the four interviews, all the constructs also had some loadings on the second component. These constructs were divided into two groups with opposite signs of loadings. The first group consisted of constructs related to the effectiveness of critical project management policies and activities. The critical policies and activities were the ones that directly affected the project progress. Constructs with considerably large loadings in this group specifically referred to the policies and activities related to the importance, communication, and programme quality of the project $(\mathrm{C} 4, \mathrm{C} 6, \mathrm{C} 2)$. The second group consisted of constructs related to the effectiveness of the supporting policies and activities. The supporting policies and activities were the ones which indirectly affected the project progress. Constructs with considerably large loadings in this group referred to the policies and activities related to the technology, software training, and infrastructure of the programme $(\mathrm{C} 3, \mathrm{C} 7, \mathrm{C} 9)$. The second component, therefore, was interpreted as the gap between the critical and the supporting policies and activities.

In summary, in each of the four interviews, Tai's appreciation of the APSC project could be explained by two components. The first component referred to his appreciation of the effectiveness of the overall project management policies and activities. The second component 
referred to his appreciation of the gap between the critical and the supporting policies and activities.

\section{Changes in Tai's standards for and appreciation of the APSC project}

This section presents the changes in Tai's standards for and appreciation of the APSC project over the four interviews. These changes were identified by the changes in the loadings of Tai's appreciation of the APSC project, the ideal and less than ideal projects on the two components. These changes are presented in Table 5.4 and illustrated in Figure 5.4 below.

Table 5.4 Loadings of Tai's appreciation of the ideal project, the less than ideal project and the APSC project on the two components in the four interviews

\begin{tabular}{|l|l|l|l|l|l|l|l|l|}
\hline Interview & \multicolumn{2}{l|}{$T 1$} & \multicolumn{2}{l}{$T 2$} & \multicolumn{2}{l|}{$T 3$} & \multicolumn{2}{l|}{ T4 } \\
\hline Component & 1 & 2 & 1 & 2 & 1 & 2 & 1 & 2 \\
\hline Ideal project & -2.848 & -0.191 & -2.29 & -0.246 & -2.166 & -0.322 & -1.886 & -0.241 \\
\hline $\begin{array}{l}\text { Less than } \\
\text { ideal project }\end{array}$ & 4.044 & -0.882 & 4.624 & -0.672 & 4.758 & -0.477 & 5.039 & -0.258 \\
\hline $\begin{array}{l}\text { APSC } \\
\text { project }\end{array}$ & -0.253 & -0.679 & -0.881 & -0.581 & -0.851 & -0.513 & -1.233 & -0.25 \\
\hline Gap & 2.595 & & 1.409 & & 1.315 & & 0.653 & \\
\hline
\end{tabular}

Figure 5.4 Changes in Tai's appreciation of the APSC project over the four interviews

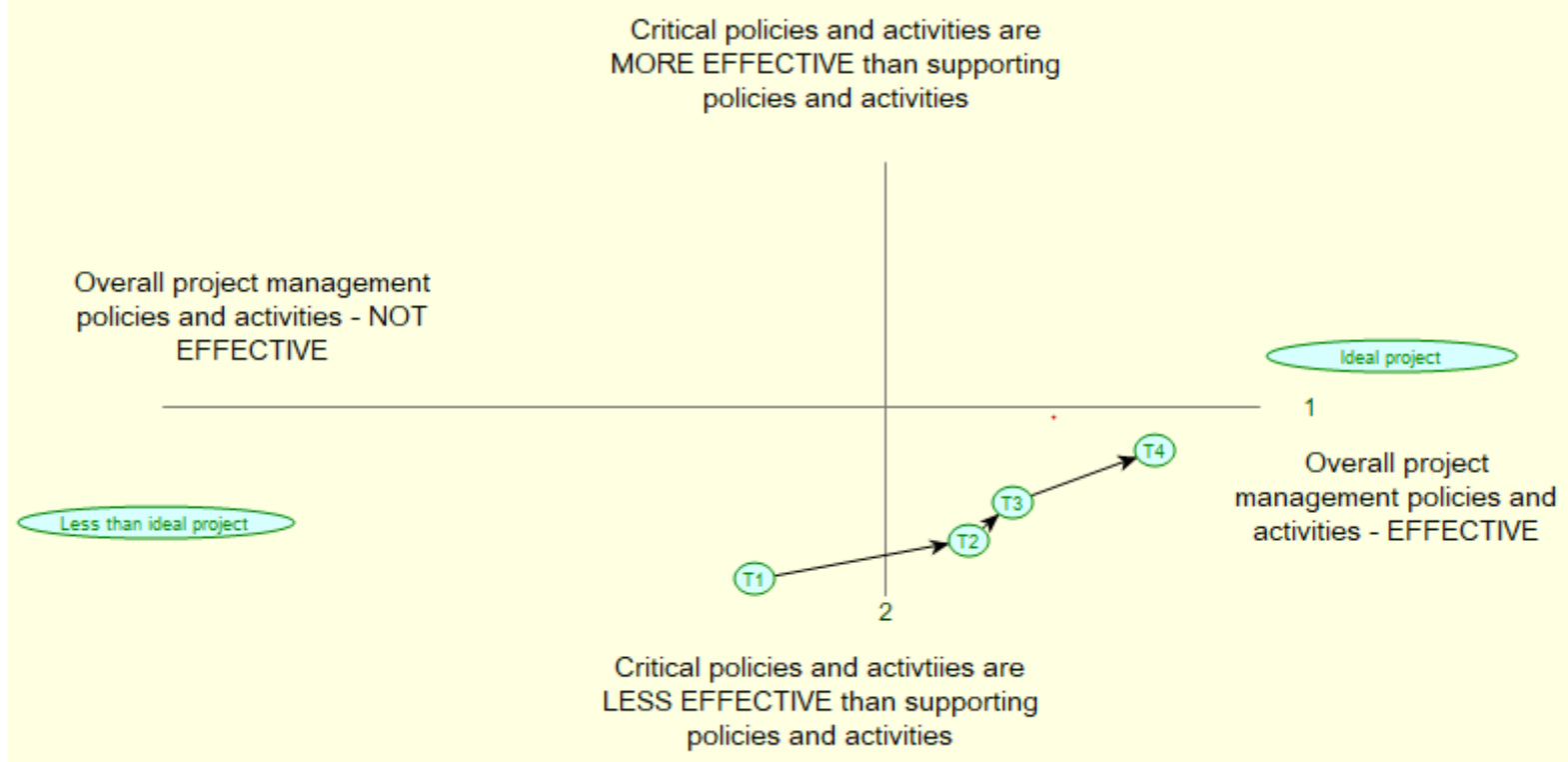

Because over the four interviews, Tai's appreciation of the APSC project had loadings on the two components, these components represented his standards for the project. Between these components, Tai's appreciation of the ideal and less than ideal projects had its largest 
loadings on the first component and very small loadings on the second component. The loadings on the first component also had opposite signs. The first component, therefore, represented his primary standards, and the second component represented his secondary standard for the project. The positions of the ideal and less than ideal projects on the PrinGrid map also indicate this. They are opposite regarding the horizontal axis and do not differ much regarding the vertical axis. Because the interpretation of the two components did not change over the four interviews, Tai's standards also did not change. Specifically, his primary standards were that the overall management policies and activities must be effective. His secondary standard was that the effectiveness of the supporting policies and activities must match the effectiveness of the critical ones.

The table and the map also show the changes in Tai's appreciation of the APSC project over the four interviews. At the first interview, his appreciation of the project (T1) is in the lower-left quadrant of the map. This indicates that Tai thought that the overall project management policies and activities were not effective (component 1), and that the critical policies and activities were not as good as the supporting ones (component 2). In the interviews after that, Tai's appreciation of the project became more positive. The gap between the loadings of Tai's appreciation of the APSC project and the ideal project on the first component kept decreasing. It decreased from 2.595 at $\mathrm{T} 1$ to 1.409 at $\mathrm{T} 2,1.315$ at $\mathrm{T} 3$, and 0.653 at $\mathrm{T} 4$. The loadings of his appreciation on the second component also kept decreasing from 0.679 to 0.581 , then to 0.513 , and finally to 0.25 . These changes, which are illustrated by the arrows T1T2, $\mathrm{T} 2 \mathrm{~T} 3$, and T3T4, indicate that Tai found the overall project management policies and activities increasingly more effective over time (component 1). He also thought that the critical policies and activities had improved and better matched the supporting ones (component 2). Arrow T2T3 is also shorter than arrows T1T2 and T3T4. This indicates that the improvement of Tai's appreciation from the second to the third interview was less than the improvements of his appreciation in the other interviews.

In summary, over the four interviews, while Tai's standards for the APSC project did not change, his appreciation of the project improved. Tai deemed the overall project management policies and activities to be better at each interview. Tai's perceptions of the critical policies and activities also improved and better matched the inherently good supporting ones. 


\subsubsection{Changes in Tai's Appreciation of the APSC Project through the Lens of Vickers' Concept}

The above section has presented the changes in Tai's standards and appreciation regarding the APSC project. This section builds on these findings to describe the changes in his appreciation of the project, as influenced by his standards and perceptions of related events and ideas, by using Vickers' concept of appreciative systems.

At the first interview (T1), based on his previous experience, Tai's primary standards were that the overall project management policies and activities must be effective. His secondary standard was that the effectiveness of the critical policies and activities should match the effectiveness of the supporting ones. Following these standards, Tai generally had a bad appreciation of the project. He thought that the overall project management policies and activities were not effective (the primary standards). The critical policies and activities, for instance, the communication and compensation policies, were not as good as the supporting ones, such as the infrastructure or technology policies (the secondary standard). Thus, regarding the project communication, Tai said:

We are still unclear about this project, do not know what our roles are, what our tasks are, and when to complete.

Between $\mathrm{T} 1$ and $\mathrm{T} 2$, as the representative of the faculty team, Tai had a meeting with the university leaders. In this meeting, he learned about their determination to obtain the accreditation by September of that year:

The project has been done for nearly two years, and the progress is not good, so the top leaders become nervous.

In line with the university's determination to complete the project, Tai noticed more activities and policies of the university to boost the project. The Research and Development Department organised the last external assessment of student learning outcomes, and this assessment had good results. The Department also involved more lecturers, "even the new lectures" in the assessment. The compensation policies for these lecturers were also very clear. Tai himself was also clearer about his tasks:

Now the project is restarting, the criteria for the teaching in the next trimester are more specified; my tasks are also clearer.

Altogether, Tai's appreciation of the effectiveness of the critical policies and activities of the project improved. In addition to these critical policies and activities, Tai also learned that the whole university was going to apply new ISO software which would "provide an electronic 
platform for many activities." Tai thought that this platform would help improve the "technology for the programme" and in the end would help the APSC project. Tai, therefore, believed that the supporting policies and activities for the project had improved. Altogether, compared to the first interview, Tai had a better appreciation of the overall project management policies and activities (the primary standards) at the second interview. Tai also thought that the critical policies and activities better matched the supporting ones (the secondary standard).

Between T2 and T3, Tai was only aware of the progress of his tasks and did not receive any other information about the APSC project. During this time, Tai focused on preparing the teaching plan for the new trimester. This preparation made him more confident in the qualifications of the lecturers:

I think this summer trimester, the faculty qualifications will improve. I am very sure that we can achieve that. I confirm.

Tai, therefore, thought that the critical policies and activities related to the programme quality had improved and better matched the supporting ones (the secondary standard). His appreciation of the overall project management policies and activities also improved (the primary standards).

Between T3 and T4, due to his position in the project, Tai attended the review meeting of the trimester. He also worked with the Research and Development Head. From these interactions, Tai learned the following:

The university has met $90 \%$ of the accreditation standards, and the remaining $10 \%$ is related to the conference participation of the faculty members.

As the leader of the faculty team, Tai knew that the faculty members already had plans to fulfil this $10 \%$. Because of this, Tai thought that the programme quality had improved. He also learned from the university leaders and the Research and Development Head that the accreditation would be done for Hanoi campus only and not include Ho Chi Minh campus anymore. Moreover, he and the Research and Development Head would also be in charge of the project. They would "attend the annual international conference of the accreditation in the United States to promote the relationship with the accreditation body." When the Head was transferred to a new position, Tai would be "the main person in charge of the project and work directly with the top leaders." These learnings put together made Tai think that the critical policies and activities of the project had improved. Finally, Tai had also learned that the university's application of the ISO software made "good progress" and believed that this 
application would help improve the programme quality. Due to this new information, Tai thought that the overall management policies and activities of the project had improved (the primary standards) and that the critical policies and activities also better matched the supporting ones (the secondary standard).

In summary, over the four interviews, with his initial standards, Tai learned different things related to the APSC project. While these learnings did not change his standards, they changed his appreciation regarding the project in a positive way. Over the four interviews, Tai's appreciation of the effectiveness of the overall management policies and activities improved. He also thought that the critical policies and activities better matched the inherently good supporting ones.

\subsection{Changes in Khiem's Appreciation of the APSC Project}

\subsubsection{Participant Background}

Khiem was a member of the APSC accreditation project at NUE University. Because FXN University started its accreditation project before NUE University, project members from NUE University, including Khiem, often contacted project members from FXN University for advice. With encouragement from the accreditation body, the two project teams had also been collaborating in organising seminars to promote the accreditation in Vietnam.

\subsubsection{Changes in Khiem's Standards for and Appreciation of the APSC Project}

Number of principal components

Because of logistical reasons, Khiem was only interviewed three times. The interviews were done at the time points of T1, T2 and T3. In the first interview, Khiem identified eleven constructs, and these constructs did not change in the remaining interviews. The principal component analysis of Khiem's repertory grids (see Appendix M) identified that one component could explain from $94 \%$ to $95 \%$ of his appreciation of the APSC project in the three interviews.

\section{Interpretation of the principal component}

In the three interviews, all the constructs in Khiem's repertory grids had the largest loadings with the same signs on the principal component. These constructs could be divided into three groups. The first group consisted of constructs related to the accreditation benefits to the university. These constructs specifically referred to the accreditation benefits to the university and the learnings of the project members (C4, C7, C8). The second group consisted of constructs related to the university resources and characteristics regarding the accreditation. 
These constructs specifically referred to the data management, the academic staff, the support of the related departments and other human and financial resources (C11, C5, C6, C1, C3). The third group consisted of constructs related to the management of the project complexity. These constructs specifically referred to the control of the work complexity and the guidance of the accreditation $(\mathrm{C} 2, \mathrm{C} 9, \mathrm{C} 10)$. Because the available resources and characteristics affected the ways in which the project complexity was managed, the component was interpreted as the accreditation benefits to the university and the management of the project complexity.

Changes in Khiem's standards for and appreciation of the project

This section presents the changes in Khiem's standards for and appreciation of the APSC project over the three interviews. These changes were identified based on the changes in the loadings of Khiem's appreciation of the APSC project, the ideal, and less than ideal projects on the principal component. These changes are presented in Table 5.5 and illustrated in Figure 5.5 below.

Table 5.5 Loadings of Khiem's appreciation of the ideal project, the less than ideal project, and the APSC project on the component

\begin{tabular}{|l|l|l|l|}
\hline Interview & $T 1$ & $T 2$ & $T 3$ \\
\hline Ideal project & -1.422 & -1.391 & -1.42 \\
\hline Less than ideal project & 5.202 & 5.235 & 5.204 \\
\hline APSC project & -0.751 & -0.819 & -0.758 \\
\hline Gap & 0.671 & 0.572 & 0.662 \\
\hline
\end{tabular}

Figure 5.5 Changes in Khiem's appreciation of the APSC project over the three interviews

Accreditation benefits and management of project complexity -

GOOD
Accreditation benefits

and management of project complexity - BAD

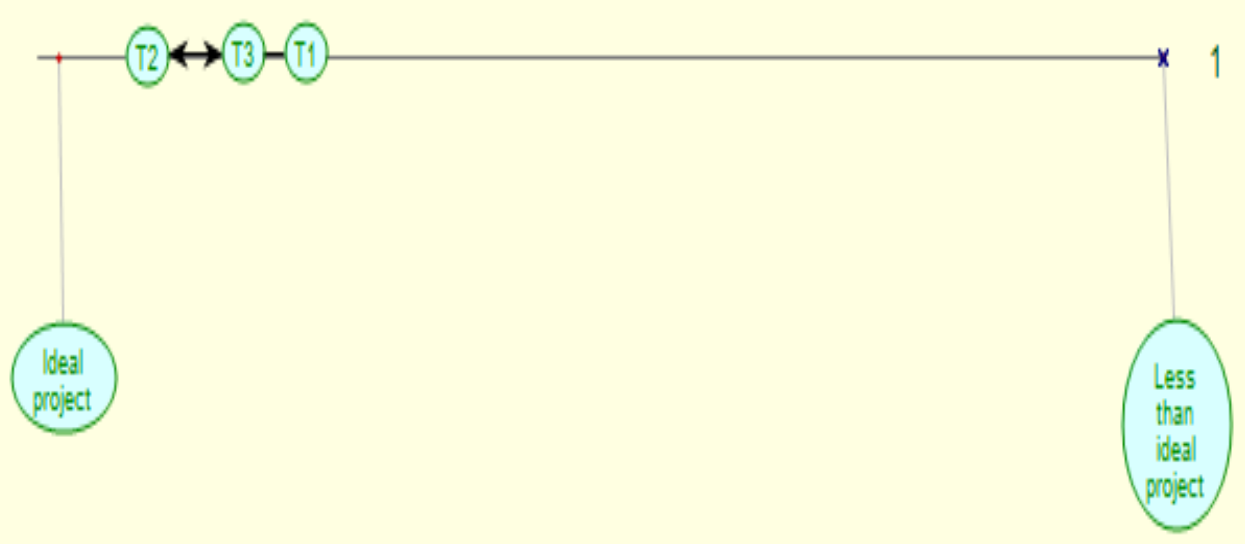

The table shows that Khiem's appreciation of the APSC project had loadings on the component. The component, therefore, represented his standards for the project. Khiem's 
appreciation of the ideal and less than ideal projects also had its largest loadings with opposite signs on the principal component. This means that the component could differentiate Khiem's appreciation of the ideal project from his appreciation of the less than ideal project and therefore represented his primary standards for the APSC project. The opposite positions of the ideal and less than ideal projects on the PrinGrid map also indicate this. Based on the interpretation of the component, Khiem's primary standards were that the accreditation must benefit the university and the management of the project complexity must be good.

The table and the map also show the changes in Khiem's appreciation of the APSC project over the three interviews. At the first interview, the gap between the loadings of Khiem's appreciation of the APSC project and the ideal project on the component was small (0.671). This is in line with the closeness between point (T1) and the ideal project on the map. In other words, at the first interview, Khiem thought that the project benefited the university and the management of the project complexity was good. The gap between the loadings of Khiem's appreciation of the APSC project and the ideal project on the component then decreased slightly to 0.572 at the second interview, followed by a small increase to 0.662 at the third interview. These changes, which are illustrated by arrows T1T2 and T2T3, indicated that Khiem's appreciation of the project improved slightly in the second interview, and worsened slightly in the third interview.

In summary, over the three interviews, Khiem did not change his standards for the APSC project at FXN University but changed his appreciation of the project. Khiem thought that the accreditation benefits and the management of the project complexity improved slightly from $\mathrm{T} 1$ to T2, but his impression worsened slightly again at T3.

\subsubsection{Changes in Khiem's Appreciation of the APSC Project through the Lens of Vickers'}

\section{Concept}

The above section has identified the changes in Khiem's standards and appreciation regarding the APSC project. This section uses these findings to describe the changes in his appreciation of the project, as influenced by his standards and perceptions of related events and ideas by using Vickers' concept of appreciative systems.

At the first interview (T1), based on his experience, his position in his own APSC project, and his contact with FXN University, Khiem had standards and appreciation regarding the APSC project at FXN University. His primary standards were that the project must benefit the university and the management of the project complexity must be good. Following these standards, Khiem had a generally good appreciation of the project. 
From the first to the second interview (from T1 to T2), because Khiem's university and FXN University used the same external assessment provider, he could see on the provider's website that "FXN University just organised an assessment of student learning outcomes." Khiem also learned that the accreditation body planned to conduct a site visit to his university. Because his university and FXN University were the only universities that were applying for the accreditation in Vietnam at that time, Khiem thought that FXN University also made good progress.

I think the panel will try to visit the two universities at the same time. Because FXN University has done this accreditation for a long time, I guess they are ready now.

Between T2 and T3, Khiem learned from an informal chat with the project administrator at FXN University about difficulties with the faculty qualifications:

She says everything is quite good, except for the faculty qualifications. I think because they are a private university, they can have that difficulty.

Khiem's appreciation of the project, therefore, slightly worsened.

In summary, over the data collection period, with his initial primary standards, Khiem received information about the APSC project at FXN University. This information did not change his standards but changed his appreciation of the project. Khiem's assessment of the accreditation benefits and the management of the project complexity improved slightly from the first to the second interview and decreased from the second to the third interview.

\subsection{Changes in Rafat's Appreciation of the APSC Project}

\subsubsection{Participant Background}

Rafat was the regional chair of the accreditation body. He was a university lecturer in India and had worked for many years as an accreditation mentor. During the data collection period, Rafat often contacted Tung, the project manager, regarding the project progress and the promotion of the accreditation in Vietnam.

\subsubsection{Changes in Rafat's Standards for and Appreciation of the APSC Project}

Number of principal components

Due to logistical reasons, Rafat was only interviewed twice at the two time points of T1 and T4. At his first interview, he came up with sixteen constructs, and these constructs did not change in his second interview. The principal component analysis of Rafat's repertory grids (see Appendix N) identified that over the two interviews, two components could explain about 95\% of his appreciation of the APSC project. The components explained about $88 \%$ and from 
$6 \%$ to $7 \%$ of his appreciation, respectively. The percentage variances explained by the components are presented in Table 5.6 below.

Table 5.6 Percentage variances explained by the two components over the two interviews Rafat - APSC project

\begin{tabular}{|l|c|c|c|c|}
\hline Interview & \multicolumn{2}{|c|}{$T 1$} & \multicolumn{2}{c|}{ T4 } \\
\hline Component & 1 & 2 & 1 & 2 \\
\hline Variance \% & 88.6 & 6.48 & 88.39 & 7.24 \\
\hline Cumulative variance \% & \multicolumn{3}{r|}{95.08} & 95.63 \\
\hline
\end{tabular}

Interpretation of the components

The construct loadings on the two components showed that the two components could be interpreted in the same way over the two interviews.

Over the two interviews, all sixteen constructs had their largest loadings with the same signs on the first component. These constructs could be divided into two groups. The first group consisted of constructs related to the university characteristics. These constructs specifically referred to the faculty qualifications, research, teaching, working culture, infrastructure, reputation, and internationalisation of the university, and the gap between the university characteristics and accreditation standards $(\mathrm{C} 1, \mathrm{C} 3, \mathrm{C} 4, \mathrm{C} 6, \mathrm{C} 7, \mathrm{C} 5, \mathrm{C} 14, \mathrm{C} 2, \mathrm{C} 11)$. The second group consisted of constructs related to the determination of the university to get the accreditation. These constructs specifically referred to the management systems and the decision making of the university and its interests and efforts to do the project $(\mathrm{C} 9, \mathrm{C} 12, \mathrm{C} 13$, $\mathrm{C} 8, \mathrm{C} 10, \mathrm{C} 15, \mathrm{C} 16)$. The first component, therefore, could be interpreted as the characteristics and determination of the university regarding the accreditation. While all sixteen constructs had their largest loadings on the first component, only two constructs had considerably large loadings with the same signs on the second component. These constructs referred to whether the university worked with their full potential (C8) and whether the university must work hard to fill the gap with the accreditation (C11). The second component, therefore, could be interpreted as the extent to which the university needed to work hard to obtain the accreditation.

In summary, for the two interviews, Rafat's appreciation of the APSC project at FXN University could be explained by two components. The first component referred to the characteristics and determination of the university regarding the accreditation. The second component referred to the extent to which the university needed to work hard to get the accreditation. 
Rafat's standards for and appreciation of the project

This section presents the changes in Rafat's standards for and appreciation of the APSC project. These changes were identified by the changes in the loadings of Rafat's appreciation of the APSC project, the ideal, and less than ideal projects on the two components. These changes are presented in Table 5.7 and illustrated on the PrinGrid map in Figure 5.6 below.

Table 5.7 Loadings of Rafat's appreciation of the ideal project, the less than ideal project, and the APSC project on the two components over the two interviews

\begin{tabular}{|c|c|c|c|c|}
\hline Interview & $T 1$ & & $T 4$ & \\
\hline Component & 1 & 2 & 1 & 2 \\
\hline Ideal project & -2.301 & -0.868 & -2.301 & -0.853 \\
\hline Less than ideal project & 5.678 & -0.448 & 5.678 & -0.468 \\
\hline APSC project & -0.172 & 1.153 & -0.17 & 1.235 \\
\hline Gap & 2.129 & 2.021 & 2.131 & 2.088 \\
\hline
\end{tabular}

Figure 5.6 Changes in Rafat's appreciation of the APSC project at FXN University over the two interviews

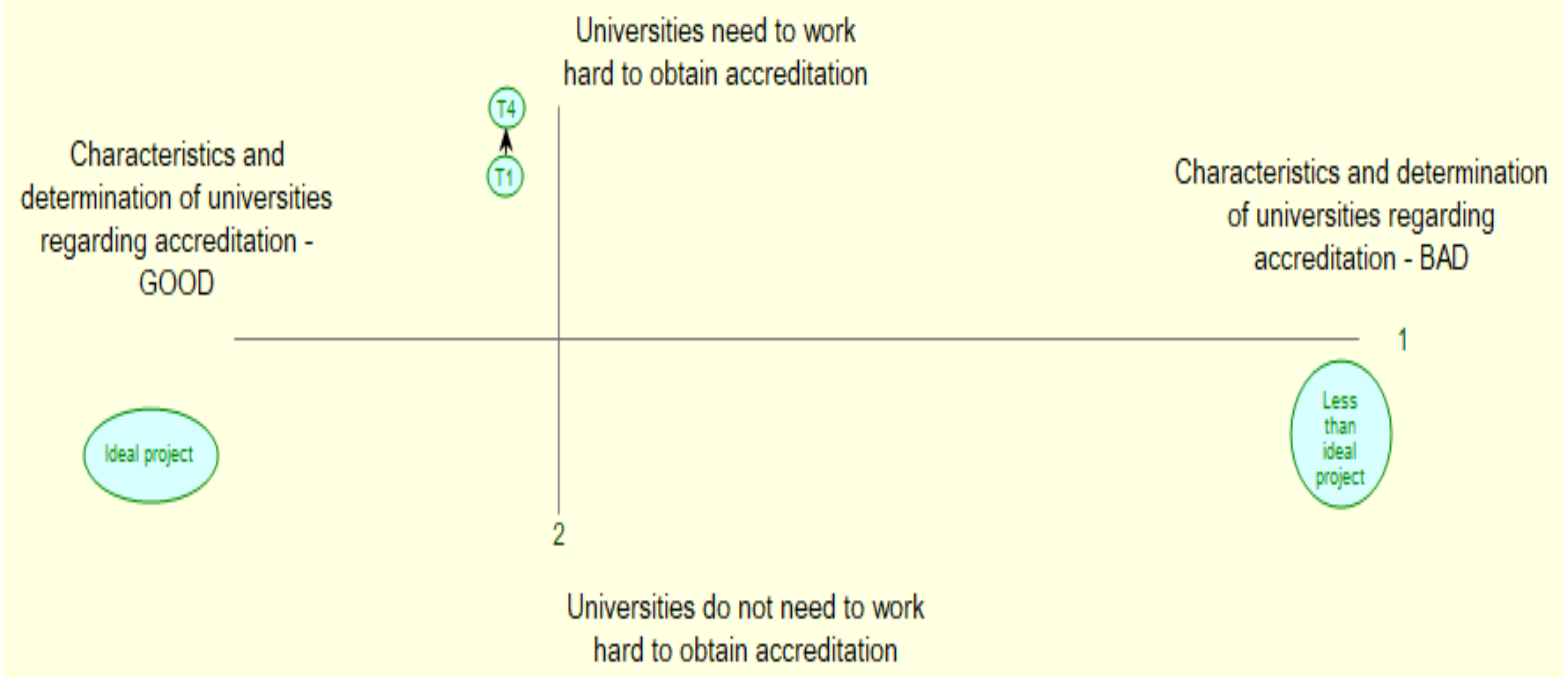

Because Rafat's appreciation of the APSC project at FXN University had loadings on the two components, these components represented his standards for the project. Between these components, Rafat's appreciation of the ideal and less than ideal projects had the largest loadings on the first component and very small loadings on the second component. The loadings on the first component also had opposite signs. The first component, therefore, could differentiate Rafat's appreciation of the ideal from the less than ideal project, but the second component could not differentiate this appreciation. The positions of the ideal and the less than ideal projects on the PrinGrid map also indicate this. They are opposite regarding the horizontal 
axis and do not differ much with regard to their positioning in relation to the vertical axis. The first component, therefore, represented Rafat's primary standards, and the second component represented his secondary standard for the APSC project. Following the interpretation of the components, at the first interviews, Rafat's primary standards were that the characteristics and determination of the university regarding the accreditation must be good. His secondary standard was that the extent to which the university needed to work to get the accreditation must be moderate. Because the interpretation of the components was unchanged over the two interviews, Rafat's standards for the project were also unchanged.

The table and the map also show the changes in Rafat's appreciation of the APSC project over the two interviews. On the map, Rafat's appreciation of the project at the two interviews, T1 and T4, is in the upper-left quadrant. This indicates that at both interviews, Rafat thought that the characteristics and determination of FXN University regarding the accreditation were relatively good. He also thought that the university needed to work hard to get the accreditation. The gap between the loadings of Rafat's appreciation of the APSC project and the ideal project on the first component increased slightly from 2.129 to 2.131 between T1 and T4. Meanwhile, the loading of his appreciation on the second component increased from 1.153 to 1.235 . These changes, which are illustrated by arrow T1T4, indicate that his appreciation had minor changes related to the second component. Specifically, in comparison to his appreciation at T1, at T4, Rafat thought that the university must work a little harder to obtain the accreditation.

In summary, over the two interviews, Rafat's standards for the project did not change. However, his appreciation changed in that Rafat thought that FXN University needed to work a little harder to get the accreditation.

\subsubsection{Changes in Rafat's Appreciation of the APSC Project through the Lens of Vickers'}

\section{Concept}

The above section has identified the changes in Rafat's standards for and appreciation of the APSC project. This section uses these findings to describe the changes in his appreciation of the project, as influenced by his standards and perceptions of related events and ideas by using Vickers' concept of appreciative systems.

At the first interview (T1), with his experience, his position as the regional chair of the accreditation body and his involvement in the APSC project at FXN University, Rafat's primary standards for the project were that the characteristics and determination of the university regarding the accreditation must be good. His secondary standard was that the 
university only needed to work moderately hard to get the accreditation. Following these standards, Rafat thought that the characteristics and determination of FXN University were generally good (the primary standards). However, he thought that the university must work relatively hard to fill the gap between its characteristics and the accreditation standards (the secondary standard).

Between T1 andT4, Rafat learned from Tung, the project manager, that while the university had hired "some faculty members with a $\mathrm{PhD}$," it would take more time to complete the project because of "schedule and mentor issues." These learnings did not change Rafat's general appreciation of the project (the primary standards). However, they caused him to believe that the university must work harder to obtain the accreditation (the secondary standard):

The fact that they're taking a little more time indicates that they need to work hard. Because if others are the same, the project progress must have been better, this is my guess, but I am not sure $100 \%$.

In summary, as a representative of the accreditation body, Rafat had standards for the APSC project at FXN University. During the data collection period, Rafat received information about the project from the project manager. This information made Rafat think that the university needed to work harder to obtain the accreditation.

\subsection{Chapter Summary}

This chapter has presented the changes in the appreciation of four participants about the APSC case, as influenced by their standards and perceptions of events and ideas. Following the same presentation format, the next chapter presents the data analysis and findings of the ABDC case. 


\section{Chapter 6 ABCD Case}

\subsection{Overview of the Chapter}

Following the presentation format of chapters four and five, this chapter presents the data analysis and findings of the ABCD case. The chapter begins with a project overview. This is followed by the presentation of the changes in three participants' appreciation of the project, as influenced by their standards and perceptions of related events and ideas.

\subsection{Overview of the ABCD Accreditation Project}

The objective of the ABCD accreditation project at NZV University, New Zealand, was to renew the accreditation of a training programme. During the data collection period, the project focused on developing the self-study report and organising the site visit of the panel. The project stakeholders included the committee members, university leader, representatives of the related departments, students, external writer, employers, alumni, and the accreditation panel. From this group of stakeholders, three were selected to participate in this study. They were Amish, the project manager; Nikki, the project administrator; and Sumy, the data collector and analyst. The interviews were conducted before and after four monthly meetings of the committee. While the manager and data analyst participated in eight interviews, the administrator was only available for six interviews before and after three meetings. The time points of the interviews and the participants at each time point are illustrated in Figure 6.1 below.

Figure 6.1 Time points of the interviews

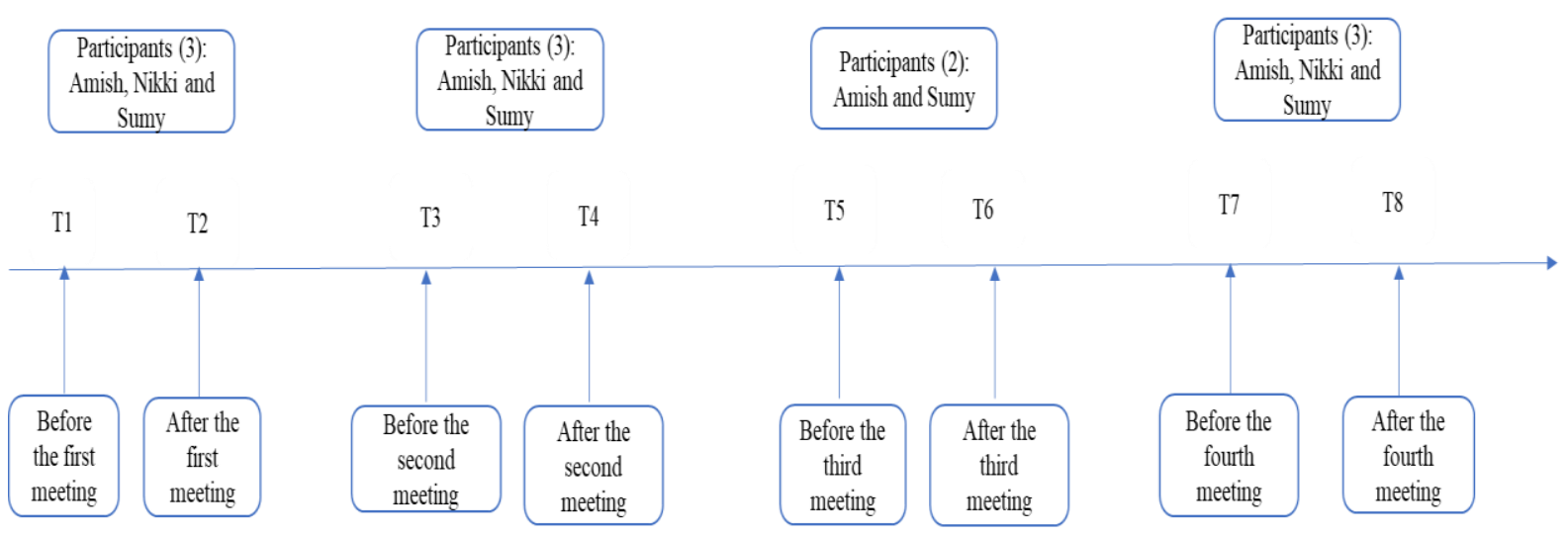




\subsection{Changes in Amish' Appreciation of the ABCD Project}

\subsubsection{Participant Background}

Amish was the project manager. He had a background in engineering and management and had worked at the university for more than fifteen years. Amish had also been the former director of the target programme of the ABCD project. Because of this, he had great insights into the programme and the university.

\subsubsection{Changes in Amish's Standards for and Appreciation of the ABCD Project}

Number of principal components

Amish participated in eight interviews. In the first interview, he identified twenty-two constructs, and these constructs were kept the same in the remaining interviews. The principal component analysis (see Appendix O) identified that two components could explain Amish's appreciation of the ABCD project over the eight interviews. These components, respectively, explained from $85 \%$ to $91 \%$ and from $4 \%$ to $9 \%$ of the appreciation. The percentage variances explained by the components are presented in Table 6.1 below.

Table 6.1 Percentage variances explained by two principal components during eight interviews - Amish - ABCD project

\begin{tabular}{|c|c|c|c|c|c|c|c|c|}
\hline Interview & \multicolumn{2}{|l|}{$T 1$} & \multicolumn{2}{|l|}{$T 2$} & \multicolumn{2}{|l|}{$T 3$} & \multicolumn{2}{|l|}{ T4 } \\
\hline Component & 1 & 2 & 1 & 2 & 1 & 2 & 1 & 2 \\
\hline Variance $\%$ & 90.04 & 5.67 & 90.99 & 4.36 & 89.87 & 3.85 & 90.31 & 4.76 \\
\hline Cumulative variance $\%$ & \multicolumn{2}{|l|}{95.71} & \multicolumn{2}{|l|}{95.36} & \multicolumn{2}{|l|}{93.72} & \multicolumn{2}{|l|}{95.07} \\
\hline Interview & \multicolumn{2}{|l|}{$T 5$} & \multicolumn{2}{|l|}{ T6 } & \multicolumn{2}{|l|}{$T 7$} & \multicolumn{2}{|l|}{$T 8$} \\
\hline Component & 1 & 2 & 1 & 2 & 1 & 2 & 1 & 2 \\
\hline Variance $\%$ & 89.68 & 5.03 & 88.76 & 5.24 & 86.84 & 7.26 & 85.4 & 8.72 \\
\hline Cumulative variance $\%$ & \multicolumn{2}{|l|}{94.71} & \multicolumn{2}{|l|}{94} & \multicolumn{2}{|l|}{94.11} & \multicolumn{2}{|l|}{94.12} \\
\hline
\end{tabular}

Interpretation of the principal components

The construct loadings on the components indicate that while the interpretation of the first component did not change over the eight interviews, the interpretation of the second component changed at the sixth interview.

Over the eight interviews, all constructs had their largest loadings with the same signs on the first component. These constructs could be divided into three groups. The first group consisted of constructs related to the university resources for the accreditation. These constructs specifically referred to data availability, funding, networks, and experience of the university (C15, C16, C20, C13, C10, C19, C6, C9, C2, C11, C22). The second group consisted 
of constructs related to the commitment of the committee members and related people. These constructs specifically referred to the commitment of the committee members, report writer, project manager, academic and professional staff, and university leader (C12, C14, C8, C21, $\mathrm{C} 1, \mathrm{C} 5, \mathrm{C} 7, \mathrm{C} 4, \mathrm{C} 3, \mathrm{C} 6, \mathrm{C} 17, \mathrm{C} 18)$. The first component, therefore, could be interpreted as the university resources and commitment to gaining the accreditation.

The interpretation of the second component changed in the sixth interview. From the first to the fifth interview, all constructs had some loadings on it. These constructs could be divided into two groups with opposite signs of loadings. The first group consisted of constructs related to the university resources. Constructs with considerably large loadings in this group were the experience of the committee members (C9) and the accreditation networks of the university $(\mathrm{C} 10)$. The second group consisted of constructs related to the university commitment. Constructs with considerable large loadings in this group were the commitment of the programme director and university leader (C5, C3). The second component, therefore, could be interpreted as the gap between the university resources and commitment.

From the sixth interview, even though all the constructs still had some loadings on the second component, their division into two groups with opposite signs of loadings changed. The first group consisted of constructs related to the overall resources of the university and the commitment of the committee members and top leader. Constructs with considerable large loadings in this group specifically referred to the university characteristics (C15), the university experience in project management $(\mathrm{C} 19)$, and the involvement and directions of the leader $(\mathrm{C} 3$, $\mathrm{C} 17, \mathrm{C} 18)$. The second group consisted of constructs related to the commitment of programme director and related departments. Constructs with considerably large loadings in this group were the administrative support and data availability of the university (C4, C16) and the commitment of the programme director (C5). The second component, therefore, could be interpreted as the gap between two groups of aspects of the ABCD project. The first group consisted of the commitment of the programme director and related departments, and the second group consisted of the resources of the university and the commitment of the committee members and top leader.

In summary, over the eight interviews, Amish's appreciation of the ABCD project could be explained by two components. The first component did not change over the eight interviews and referred to his appreciation of the university resources and commitment to achieving the accreditation. Meanwhile, the second component changed in the sixth interview. 
From the first to the fifth interview, it referred to the gap between the university resources and commitment. From the sixth interview onwards, it referred to the gap between two other groups of aspects of the project. The first group consisted of the commitment of the programme director and related departments. The second group consisted of the resources of the university and the commitment of the committee members and top leader.

\section{Changes in Amish's standards for and appreciation of the ABCD project}

This section presents the changes in Amish's standards for and appreciation of the ABCD project. These changes were identified based on the changes in the loadings of Amish's appreciation of the $\mathrm{ABCD}$ project, the ideal and less than ideal projects on the two components (Table 6.2). Because the second component changed at the sixth interview, the changes in Amish's appreciation over the eight interviews are illustrated by two PrinGrid maps. The maps present the changes from the first to the fifth interview (Figure 6.2) and from the sixth to the eighth interview (Figure 6.3), respectively.

Table 6.2 Loadings of Amish's appreciation of the ideal and less than ideal projects and the $A B C D$ project on the two principal components over the eight interviews

\begin{tabular}{|c|c|c|c|c|c|c|c|c|}
\hline Interview & \multicolumn{2}{|l|}{$T 1$} & \multicolumn{2}{|l|}{$T 2$} & \multicolumn{2}{|l|}{$T 3$} & \multicolumn{2}{|l|}{$T 4$} \\
\hline Component & 1 & 2 & 1 & 2 & 1 & 2 & 1 & 2 \\
\hline Ideal project & -3.927 & 0.387 & -3.832 & 0.48 & -3.891 & 0.412 & -3.89 & 0.275 \\
\hline $\begin{array}{l}\text { Less than } \\
\text { ideal project }\end{array}$ & 5.375 & -0.584 & 5.467 & -0.658 & 5.415 & -0.675 & 5.414 & -0.611 \\
\hline $\mathrm{ABCD}$ project & -0.445 & -1.196 & -0.902 & -0.72 & -0.622 & -0.495 & -0.643 & -1.023 \\
\hline Gap & 3.482 & -1.583 & 2.93 & -1.2 & 3.269 & -0.907 & 3.247 & -1.298 \\
\hline Interview & \multicolumn{2}{|l|}{$T 5$} & \multicolumn{2}{|l|}{ T6 } & \multicolumn{2}{|l|}{$T 7$} & \multicolumn{2}{|l|}{$T 8$} \\
\hline Component & 1 & 2 & 1 & 2 & 1 & 2 & 1 & 2 \\
\hline Ideal project & -3.728 & -0.35 & -3.776 & -0.137 & -3.725 & -0.251 & -3.745 & -0.347 \\
\hline $\begin{array}{l}\text { Less than } \\
\text { ideal project }\end{array}$ & 5.565 & 0.615 & 5.529 & 0.198 & 5.577 & 0.079 & 5.558 & -0.041 \\
\hline $\begin{array}{l}\mathrm{ABCD} \\
\text { project }\end{array}$ & -1.462 & 1.292 & -1.241 & 1.624 & -1.514 & 2.008 & -1.402 & 2.238 \\
\hline Gap & 2.266 & 1.642 & 2.535 & 1.761 & 2.211 & 2.259 & 2.343 & 2.585 \\
\hline
\end{tabular}


Figure 6.2 Changes in Amish's appreciation of the ABCD project, ideal and less than ideal projects from the first to the fifth interview

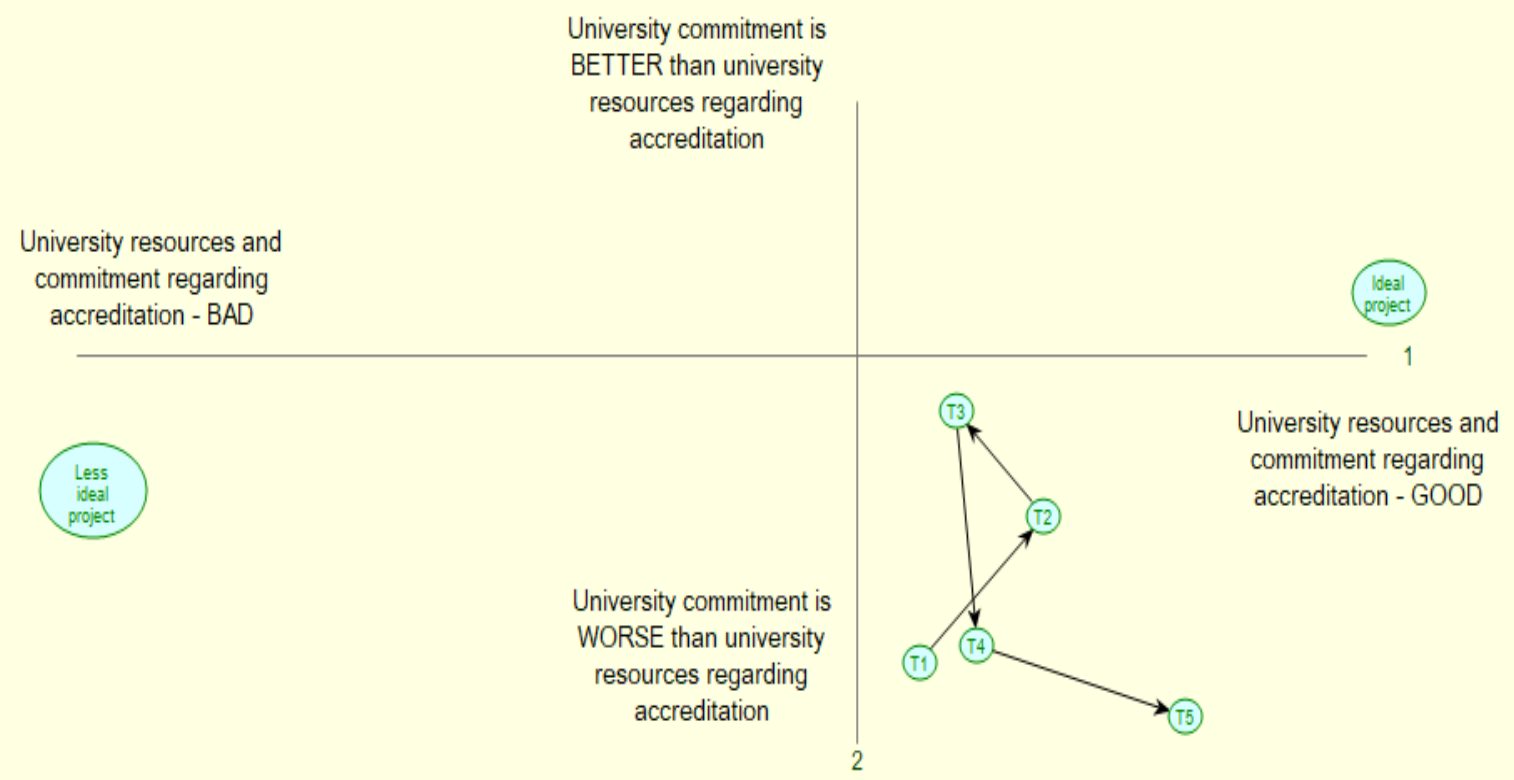

Figure 6.3 Changes in Amish's appreciation of the ABCD project, ideal, and less than ideal projects from the sixth to the eighth interview

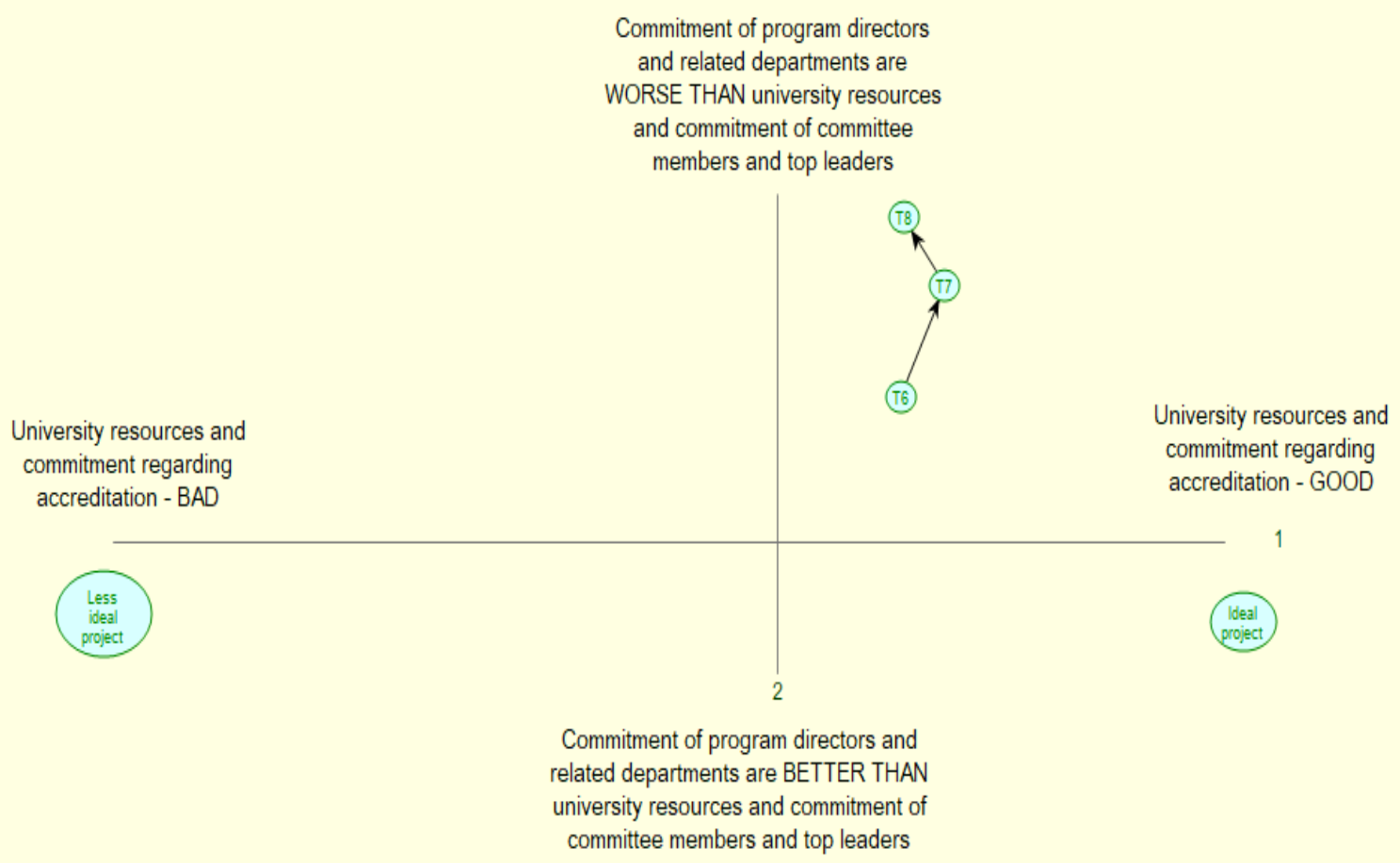

Because over the eight interviews, Amish's appreciation of the ABCD project had loadings on the two components, these components represented his standards for the project. A comparison of these components shows that Amish's appreciation of the ideal and less than ideal projects had their largest loadings on the first component and very small loadings on the 
second component. The loadings on the first component also had opposite signs. The positions of the ideal and less than ideal projects on the PrinGrid maps also indicate this. They are opposite regarding the horizontal axes and do not differ much regarding the vertical axes. This means that the first component could differentiate Amish's appreciation of the ideal project from the less than ideal project, while the second component just represented his concern about the project. The first component, therefore, represented Amish's primary standards, and the second component represented his secondary standard for the ACBD project.

Following the interpretation of the components, at T1, Amish's primary standards were that the university resources and commitment must be good. His secondary standard was that the commitment of the university must match its resources. Because the interpretation of the first component did not change over the eight time points, Amish's primary standards also did not change. Meanwhile, because the interpretation of the second component changed from T6, his secondary standard also changed from this time point for the remainder of the study. The new standard was the match between two groups of aspects of the project. The first group consisted of the commitment of the programme director and the related departments. The second group consisted of the resources of the university and the commitment of the committee members and the top leader.

The table and the maps above also show the changes in Amish's appreciation of the $\mathrm{ABCD}$ project. The maps show all eight data points in the same quadrants of the maps, which indicates that over the eight interviews, Amish thought that the overall resources and commitment of the university were good (component 1). However, from the first to the fifth interview, he generally believed that the university commitment did not match its resources. In the remaining interviews, he thought that the commitment of the programme director and related departments did not match the university resources and the commitment of the committee and university leader (component 2). Overall, the changes in Amish's appreciation could be described in three stages: from the first to the fifth interview, the fifth to the sixth interview, and the sixth to the eighth interview.

From the first to the fifth interview, Amish's appreciation of the ABCD changed in different ways. From T1 to T2, the direction of arrow T1T2 indicates that the appreciation became more positive. Amish thought that the university resources and commitment had improved (component 1) and the university commitment better matched its resources (component 2). From the second to the third interview, the direction of arrow T2T3 indicates 
that while the university commitment also better matched its resources (component 2), the overall university resources and commitment worsened (component 1). Arrow T3T4 indicates that from the third to the fourth interview, Amish thought that the overall university resources and commitment did not change much (component 1) but that the university commitment less matched its resources (component 2). Finally, arrow T4T5 indicates that from the fourth to the fifth interview, while Amish thought that the overall university resources and commitment had improved (component 1), his appreciation of the match between the university commitment and its resources diminished, resulting in a bigger gap between them (component 2).

Amish's appreciation at T5 and T6 is presented on two separate maps. During this time, the gap between the loadings of Amish's appreciation of the ABCD project and the ideal project on the first component increased from 2.266 to 2.535. This increase indicated that Amish's appreciation of the university resources and commitment worsened (component 1). The interpretation of the second component changed at T6. This indicated that Amish changed his concern about the project. At T5, he was concerned about the gap between the university resources and commitment. At T6, this concern changed to the gap between two groups of different aspects of the project. The first group consisted of the commitment of the programme director and the related departments. The second group consisted of overall university resources and the commitment of the committee members and university leader. From T5 to T6, the loading of Amish's appreciation of the ABCD project on the second component also increased from 1.292 to 1.624. This indicated that Amish thought that the first group of aspects was worse than the second group or that there was a gap between them. This gap was also larger than the gap related to the overall university commitment and resources at T5.

The changes in Amish's appreciation from T6 to T8 are illustrated by arrows T6T7 and T7T8 in Figure 6.3. The arrows show that regarding component 1, Amish's appreciation of the university resources and commitment improved from T6 to T7 and diminished from T7 to T8. Regarding component 2 , he thought that the commitment of the programme director and the related departments less matched the resources of the university and the commitment of the committee members and the top leader.

In summary, over the eight interviews, while Amish's primary standards for the ABCD project did not change, his secondary standard changed at the sixth interview. Along with these changes, his appreciation of the project also changed. These changes focused on the overall university resources and commitment regarding the project. From the first to the fifth interview, 
Amish was also concerned about the gap between the resources and commitment. From the sixth to the eighth interview, this concern changed to the gap between two groups of other aspects of the project. The first group consisted of the commitment of the programme director and the related departments. The second group consisted of the university resources and the commitment of the committee members and the top leader.

\subsubsection{Changes in Amish's Appreciation of the ABCD Project through the Lens of Vickers'}

\section{Concept}

The above section has presented the changes in Amish's standards for and appreciation of the $A B C D$ project over the eight interviews. This section used these findings to describe the changes in his appreciation of the project, as influenced by his standards and perceptions of related events and ideas, by applying Vickers' concept of appreciative systems.

\section{Before the first meeting (T1)}

Before the first meeting, based on his experience with the project to date, Amish's primary standards for the $\mathrm{ABCD}$ project were that the university resources and commitment to the accreditation must be good. His secondary standard was that the university commitment must match its resources. Following these standards, Amish thought the overall university resources and commitment were good (the primary standards). However, the university commitment did not match its resources (the secondary standard).

From before to after the first meeting (T1 to T2)

Between T1 to T2, as the project manager, Amish chaired the committee meeting and was aware that the project had a new data collector. He also learned that more data was available at one campus of the university. At the meeting, the committee members also showed their experience and knowledge about accreditation:

Somebody has brought a survey, which was done for a previous accreditation, and we can use this survey for this project. Some committee members also gave more information about the management systems of the university.

However, Amish saw that the committee members were not clear about the expectations of the panel. These learnings, therefore, affected his appreciation of the university resources.

At the meeting, Amish also found out that the top leader, programme director and committee members were more committed: 
The top leader has solved two project issues and became more committed.

The programme director showed up for the first time, and the committee members had a more positive discussion about report writing.

However, there were "some fights" at the beginning of the meeting. These learnings affected Amish's appreciation of the university commitment to the project.

Overall, from T1 to T2, Amish learned things that affected his appreciation of the university resources and commitment in both positive and negative ways. Overall, the learnings resulted in a positive impact on Amish's appreciation of the project as he generally thought that the university resources and commitment had improved (the primary standards). The commitment also better matched the resources (the secondary standard).

From after the first meeting to before the second meeting (T2 to T3)

Between T2 to T3, Amish noticed that the data collector was working very hard and learned that the project could use the materials of a previous accreditation. Amish also had more information about the impact of the accreditation networks and was, therefore, more confident:

I learned that the university did not participate in any conference of the accreditation in the last few years, but this did not affect the effectiveness of the $A B C D$ project.

However, Amish saw that some committee members did not know much about project management. These learnings affected his appreciation of the university resources.

Besides the resources, Amish saw that the related departments supported the project more than they had done previously. The academic staff was also more committed and spent "a lot of extra time to complete the job." The report writer also worked hard, and based on Amish's feedback was able to improve the report:

I have worked with $P$ [the report writer], and the things have been improved, gave him the feedback, and he improved, the report improved.

However, Amish saw that the university leader did not engage in the project and did not solve some of the project issues. There were also complaints about the quality of the programme director's work. The complaints made Amish think that the director had a low commitment to both his everyday tasks and the project. These learnings combined affected Amish's appreciation of the university commitment.

Overall, between T2 to T3, Amish learned things that affected his appreciation of the university resources and commitment in both positive and negative ways. With more negative effects of the learnings, Amish thought that the university resources for and commitment to the 
accreditation project had worsened (the primary standards). However, he also believed that the commitment better matched the resources (the secondary standard).

\section{From before to after the second meeting (T3 to T4)}

During this time, Amish chaired the second committee meeting. At the meeting, he was aware that the first draft of the self-study report was ready, and the committee members were "clearer about what the panel asked for." The committee members also had more experience in accreditation and project management:

We have two new senior members with a lot of experience in accreditation.

The committee members also demonstrate their experience in managing projects.

These observations made Amish have a better appreciation of the university resources for the project.

Along with the resources, Amish also learned things related to the commitment. On the positive side, he learned that the top leader "agreed with the timeline of the project," and "the committee members were very constructive, listened to each other during the meeting". On the negative side, Amish saw that the committee members pointed out "some gaps" in the selfstudy report and that the writer "did not complete a few sessions." One member of the administrative group also did not finish his work as he had promised, and the data analyst could not collect enough data for the survey:

There are only fourteen responses, and we need at least fifteen, the last time it was more than one hundred.

Overall, from T2 to T3, Amish learned things that affected his appreciation of the university resources and commitment in both positive and negative ways. In the end, his general appreciation of the project did not change much (the primary standards). However, he believed the university commitment better matched its resources (the secondary standard).

From after the second meeting to before the third meeting (T4 to T5)

In the time between T4 and T5, Amish saw that the project submitted the self-study report and prepared for the site visit of the panel. Amish, in general, was "very happy with the report." In a positive development regarding the university resources, Amish realised that the committee members knew the panel members "through networks." With the project progress, the committee members were also clearer about the "systems and processes" of the university and the project management. Amish, therefore, was more confident in the experience and 
knowledge of the university. However, on the negative side, he acknowledged the issues related to data availability:

Data still have problems, I don't say all the data are not available, but there were few cases because somebody didn't keep the records.

In parallel with the university resources, Amish also learned things related to the university commitment. He saw that the administrative group worked very hard on "weekends and evenings" to complete their tasks, and all committee members, especially the top leader, were more committed:

$J$ (the top leader) has discussed with the project team about the panel and their expectations. He had strong leadership, and if something he has not done, it must be because they were beyond his control.

However, the programme director was not committed:

The programme director is the major problem. Nothing changes. That is the problem, is still the problem.

In summary, in this period, Amish learned things that affected his appreciation of the project in both positive and negative ways. With the positive effects of these learnings outweighing the negative ones, Amish generally thought that the university resources and commitment had improved (the primary standards). However, there was a gap in his appreciation of the commitment and resources, which likely suggests that he considered the match between these two aspects had lessened (the secondary standard).

From before to after the third meeting (T5 to T6)

During this time, Amish chaired the third committee meeting, which was to prepare for the site visit of the panel. At the meeting, Amish saw that while most of the committee members "demonstrated their experience and discussed the previous accreditations," some, including the programme director, showed that they lacked experience and were not clear about what the panel asked for:

The programme director is very new, just a few weeks. In the last meeting, the first time he came to the meeting, and he must meet the panel. He did not know much about the university processes.

These learnings, therefore, affected Amish's appreciation of the university resources.

Regarding the university commitment, Amish was very confident about his commitment and that of the administrative group. The commitment of the top leader and the academic staff was also good: 
In the meeting, all committee members agreed that the top leader was doing a good job, and more and more staff members from related departments were committed and came to help the project.

However, Amish found it "difficult" to know about the commitment of the related departments and had a big concern about that of the programme director. Other committee members also shared this concern with him. Due to this concern, Amish changed his secondary standard to the match between two groups of other aspects of the project. The first group consisted of the commitment of the programme director and the related departments. The second group consisted of the university resources and the commitment of the committee members and the top leader. Following this standard, Amish thought that the first group of aspects did not match the second one. The gap between them was also larger than the gap between the university resources and commitment at T5. Meanwhile, regarding the primary standards, Amish thought that the overall university resources and commitment had worsened.

From after the third meeting to before the fourth meeting (T6 to T7)

During this time, the accreditation panel visited the university, and Amish unofficially learned about the good accreditation result. This result made him believe more in the good reputation of the university:

We have the best result because of the university name, mainly because of that. Because of our name, our university, we have the best result for the accreditation. That is the only reason why we have such a good result.

Amish also acknowledged that the experience of the committee had helped a lot. However, while the data collector did "a fantastic job", some data were "still very difficult to collect." These learnings affected Amish's appreciation of university resources in both positive and negative ways.

Regarding the university commitment, Amish saw that the commitment of the top leader and most of the academic staff was very good:

The panel said that the top leader is the best. The top leader is fantastic. He was the main person to meet the panel. The academic staff was very committed, about sixteen people came to meet the panel, very big commitment.

However, some academic staff, especially the programme director, was not committed:

About $10 \%$ of the academics did not know what the panel asked for, and the panel mentioned that some new people [the programme director] did not know about the programme. This is really the problem. 
In summary, between T6 and T7, Amish learned things that affected his appreciation of the university resources and commitment in both positive and negative ways. The positive effects of these learnings mostly outweighed the negative ones. Thus, at T7, Amish thought that the university resources and commitment had improved (the primary standards). However, the commitment of the programme director did not match the resources of the university and the commitment of other related people as well as it did at T6 (the secondary standard).

\section{From before and after the fourth meeting (T7 to T8)}

During this time, Amish chaired the last committee meeting, which was to review the implementation of the project. In the meeting, he saw that the committee discussed the data issues:

It [the data] was an issue that we identified in that, uhm, yeah, that day, was not collected properly. Yeah. Sumy [the data analyst] really helped. If Sumy had not been there, we would have trouble, this problem also, it has been discussed in the last meeting.

The committee was also concerned about the elimination of the data analyst position and worried about future human resources. They also emphasised the importance and necessity of attending more accreditation conferences to develop accreditation networks:

That came out in the meeting. Networking is extremely important.

All these learnings affected Amish's appreciation of the university resources in negative ways. Meanwhile, regarding the university commitment, Amish saw that the committee members were "really committed" to the meeting. They also had very good feedback on the commitment of the top leader. However, the programme director was still not committed. He kept asking "the types of questions which show[ed] he [didn't] know." Overall, what Amish learned in the time from $\mathrm{T} 7$ to $\mathrm{T} 8$ affected his appreciation of the project in both positive and negative ways. In this case, the negative effect outweighed the positive ones. This meant that Amish thought that the university resources and commitment had worsened (the primary standards). The commitment of the programme director also did not match the good resources of the university and the good commitment of the other related people as well as before (the secondary standard).

In summary, during the data collection period, with his initial standards and appreciation regarding the $\mathrm{ABCD}$ project, Amish learned different events and ideas related to the project. These learnings changed his standards and appreciation regarding the university resources and the commitment of related people. 


\subsection{Changes in Nikki's Appreciation of the ABCD Project}

\subsubsection{Participant Background}

Nikki was the project administrator. She had worked at the university for five years and had been involved in other accreditation processes before the ABCD project.

\subsubsection{Changes in Nikki's Standards for and Appreciation of the ABCD Project}

Number of principal components

Due to logistical reasons, Nikki was only available for six interviews. Her interviews took place before and after the first, second, and fourth meetings. In the first interview, Nikki identified eighteen constructs and these constructs remained the same for all her interviews. The principal component analysis (see Appendix P) identified that two components could explain Nikki's appreciation of the $\mathrm{ABCD}$ project over the six interviews. These components, respectively, explained from $79 \%$ to $84 \%$, and about $9 \%$ of her appreciation. The percentage variances explained by each component are described in Table 6.3 below.

Table 6.3 Percentage variances explained by two principal components over six interviews Nikki - ABCD project

\begin{tabular}{|c|c|c|c|c|c|c|}
\hline Interview & \multicolumn{2}{|l|}{$T 1$} & \multicolumn{2}{|l|}{$T 2$} & \multicolumn{2}{|l|}{$T 3$} \\
\hline Component & 1 & 2 & 1 & 2 & 1 & 2 \\
\hline Variance $\%$ & 79.34 & 9.69 & 83.22 & 7.8 & 80.04 & 9.73 \\
\hline Cumulative variance $\%$ & 79.34 & 89.03 & 83.22 & 91.02 & 80.04 & 89.77 \\
\hline Interview & \multicolumn{2}{|l|}{$T 4$} & \multicolumn{2}{|l|}{$T 7$} & \multicolumn{2}{|l|}{$T 8$} \\
\hline Component & 1 & 2 & 1 & 2 & 1 & 2 \\
\hline Variance $\%$ & 82.84 & 9.02 & 84.77 & 8.9 & 83.9 & 9.14 \\
\hline Cumulative variance $\%$ & 82.84 & 91.86 & 84.77 & 93.68 & 83.9 & 93.04 \\
\hline
\end{tabular}

Interpretation of the principal components

The construct loadings on the components show that while the interpretation of the first component was unchanged over the six interviews, the interpretation of the second component changed. The second component was interpreted in one way in the first and third interviews and in another way in the other interviews.

Over the six interviews, all constructs had their largest loadings or considerably large loadings with the same signs on the first component. These constructs could be divided into two groups. The first group consisted of constructs related to the university resources for accreditation. These constructs specifically referred to the resources, knowledge and experience of the university, the quality of the academic and administrative staff, and the 
programme quality $(\mathrm{C} 12, \mathrm{C} 9, \mathrm{C} 13, \mathrm{C} 15, \mathrm{C} 16, \mathrm{C} 8, \mathrm{C} 5)$. The second group consisted of constructs related to the university efforts to do the projects. These constructs specifically referred to the desire and investment of the university, the involvement of the top leader, project members and related people, the project management, and the project results $(\mathrm{C} 17, \mathrm{C} 18, \mathrm{C} 3$, $\mathrm{C} 10, \mathrm{C} 2, \mathrm{C} 6, \mathrm{C} 14, \mathrm{C} 11, \mathrm{C} 1, \mathrm{C} 4, \mathrm{C} 7)$. The first component, therefore, could be interpreted as the university resources and efforts towards accreditation.

Regarding the second component, the same constructs had considerably large loadings on the component in the first and third interviews. These constructs could be divided into two groups with opposite signs of loadings. The first group consisted of constructs related to the internal resources and efforts of the committee. Examples of these constructs were the number, experience, and knowledge of the committee members (C13, C9, C5), and the commitment of the committee members and report writers $(\mathrm{C} 10, \mathrm{C} 6)$. The second group consisted of constructs related to the ability of the committee to mobilise the external resources and efforts. Examples of these constructs were the project management and time management of the committees $(\mathrm{C} 16, \mathrm{C} 4)$, the involvement of the top leader and programme director $(\mathrm{C} 3, \mathrm{C} 2)$, and the quality of the programme (C8). The second component, therefore, could be interpreted as the gap between the internal resources and efforts of the committee and its ability to mobilise the external resources and efforts.

In the second, fourth, fifth, and sixth interviews, other constructs had loadings on the second component. These constructs could be divided into two groups with opposite signs of loadings. The first group consisted of constructs related to the university's desire for the accreditation. Among these constructs, $\mathrm{C} 17$, which referred to whether the university really wanted accreditation, had its largest loading on the component. The second group consisted of constructs related to the university resources and efforts for accreditation. Examples of these constructs were the project management knowledge of the university (C16) and the programme quality (C8). The second component, therefore, was interpreted as the gap between the university resources and efforts and its desire for accreditation.

In summary, over her six interviews, Nikki's appreciation of the ABCD project could be described by two components. The first component referred to her appreciation of the overall university resources and efforts and was unchanged over the six interviews. The second component, meanwhile, changed. In the first and third interviews, it referred to the gap between the internal and external resources and efforts made by the university. In the other interviews, 
it referred to the gap between the university resources and efforts and its desire for the accreditation.

\section{Changes in Nikki's standards for and appreciation of the ABCD project}

This section presents the changes in Nikki's standards and appreciation regarding the ABCD project. These changes were identified from the changes in the loadings of Nikki's appreciation of the ABCD project, and the ideal and less than ideal projects on the two components (Table 6.4). Because of the changes in the interpretation of the second component, Nikki's appreciation of these projects over the six interviews is illustrated by four PrinGrid maps. These maps (Figure 6.4 to 6.7 ) describe Nikki's appreciation at T1, T2, T3, and from T4 to T8, respectively.

Table 6.4 Loadings of Nikki's appreciation of the ideal and less than ideal projects and the $A B C D$ project on the two components

\begin{tabular}{|l|l|l|l|l|l|l|}
\hline Interviews & \multicolumn{2}{l}{$T 1$} & \multicolumn{2}{l}{$T 2$} & \multicolumn{2}{l|}{ T3 } \\
\hline Component & 1 & 2 & 1 & 2 & 1 & 2 \\
\hline Ideal project & -2.791 & -0.228 & -2.722 & -0.271 & -2.745 & -0.012 \\
\hline Less than ideal project & 5.65 & -0.161 & 5.727 & 0.376 & 5.704 & -0.118 \\
\hline ABCD project & 0.503 & 1.842 & -0.148 & -0.446 & -0.029 & 1.935 \\
\hline Gap & 3.294 & 2.07 & 2.574 & -0.175 & 2.716 & 1.947 \\
\hline Interviews & $T 4$ & \multicolumn{7}{l}{$T 7$} & $T 8$ & \\
\hline Component & 1 & 2 & 1 & 2 & 1 & 2 \\
\hline Ideal project & -2.656 & -0.161 & -2.591 & -0.116 & -2.61 & -0.105 \\
\hline Less than ideal project & 5.8 & 0.394 & 5.865 & 0.374 & 5.845 & 0.371 \\
\hline ABCD project & -0.51 & -1.077 & -0.828 & -0.837 & -0.732 & -0.926 \\
\hline Gap & 2.146 & -0.916 & 1.763 & -0.721 & 1.878 & -0.905 \\
\hline
\end{tabular}


Figure 6.4 Nikki's appreciation of the ABCD project, and the ideal and less than ideal projects at the first interview (T1)

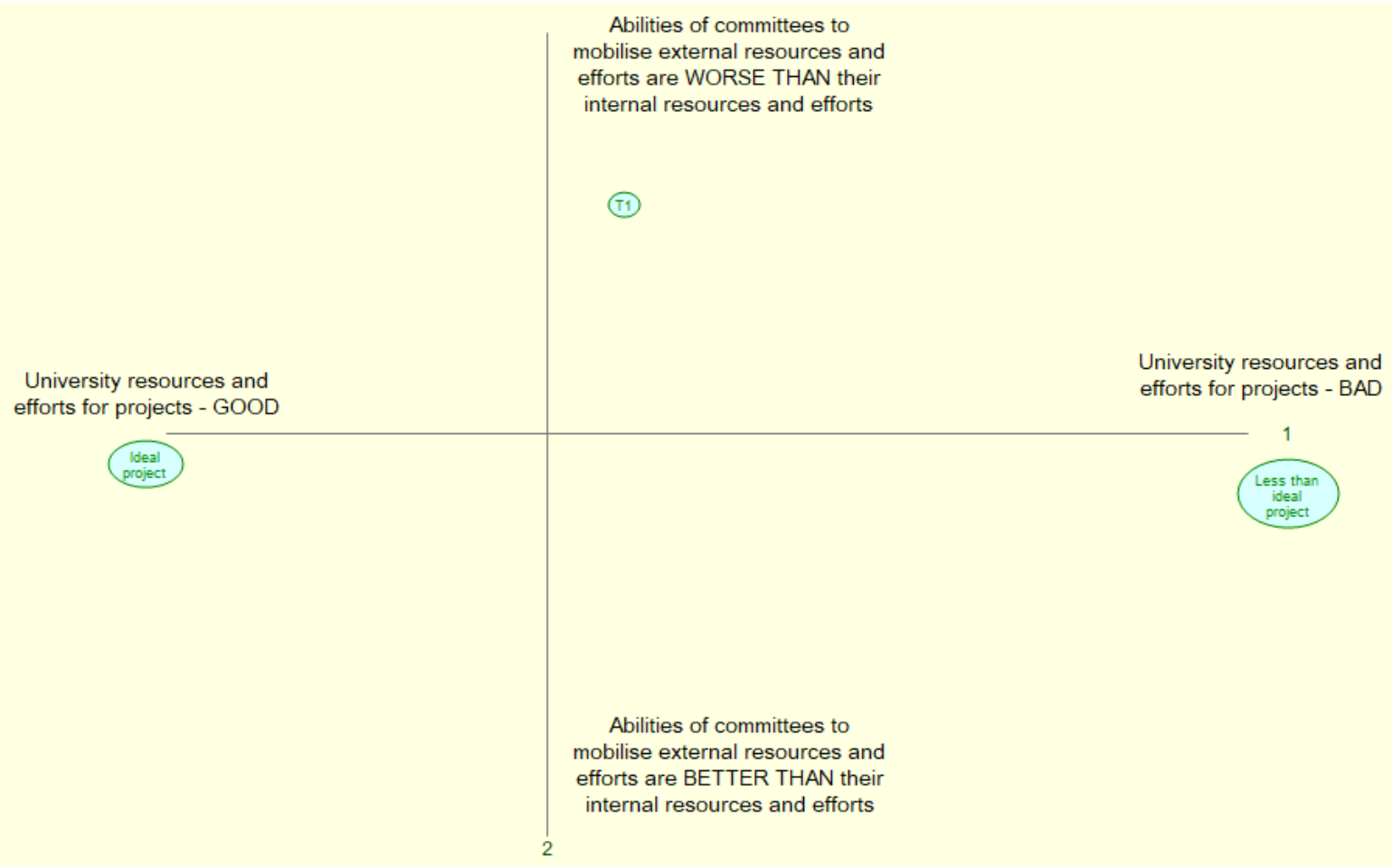

Figure 6.5 Nikki's appreciation of the ABCD project, and the ideal and less than ideal projects at the second interview (T2)

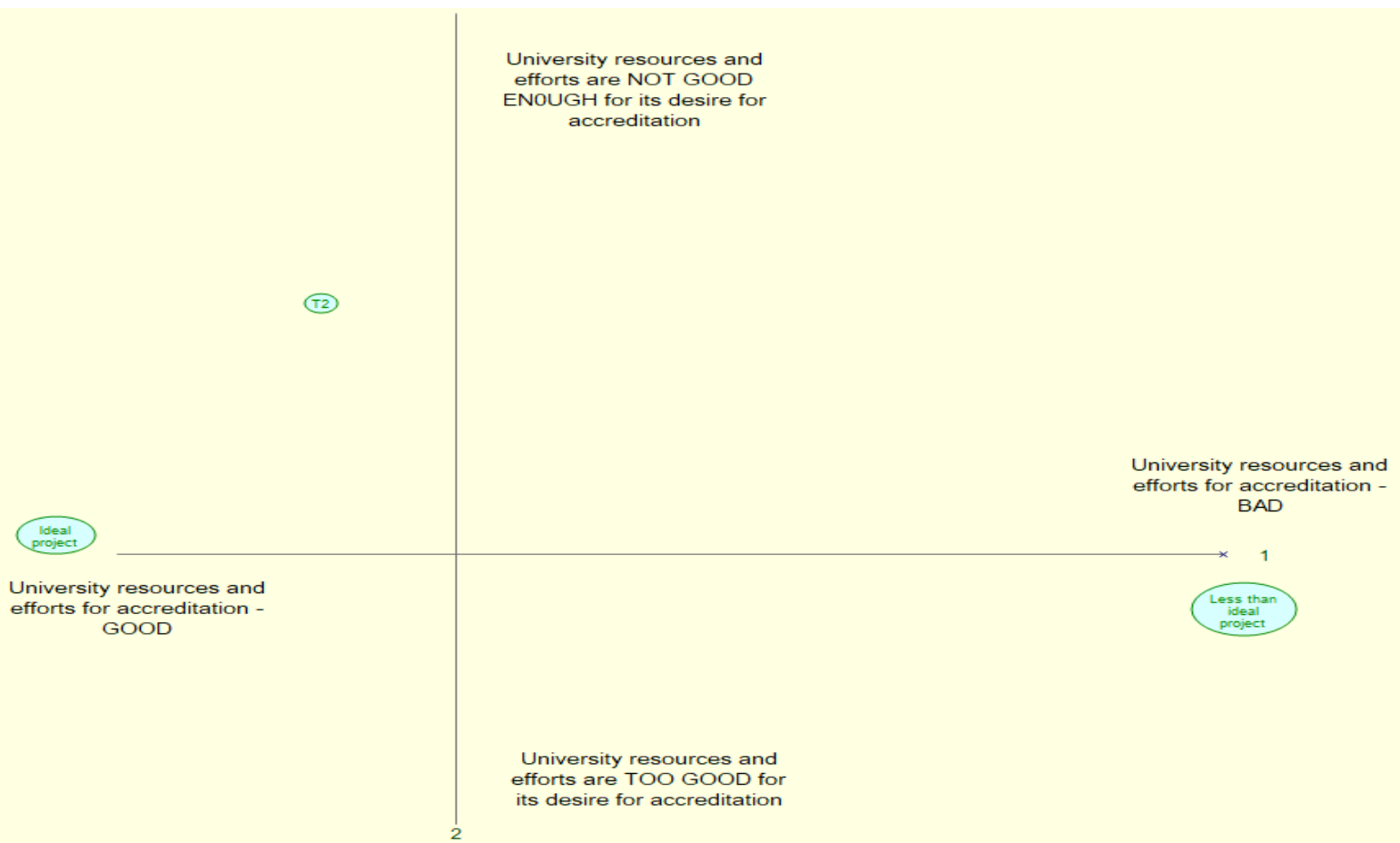


Figure 6.6 Nikki's appreciation of the ABCD project, and the ideal and less than ideal projects at the third interview (T3)

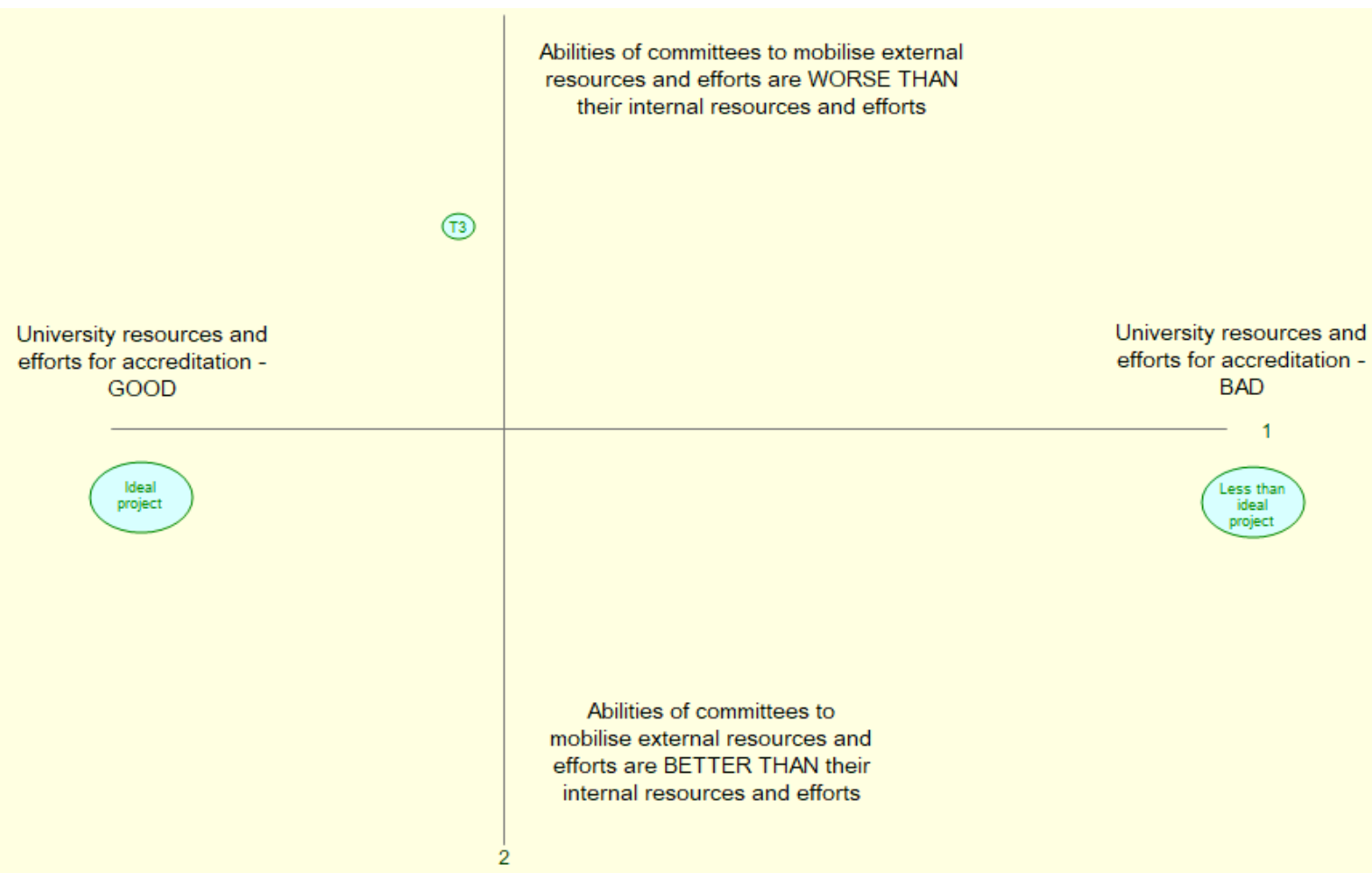

Figure 6.7 Changes in Nikki's appreciation of the ABCD project, and the ideal and less than ideal projects from the fourth (T4) to the sixth interview (T8)

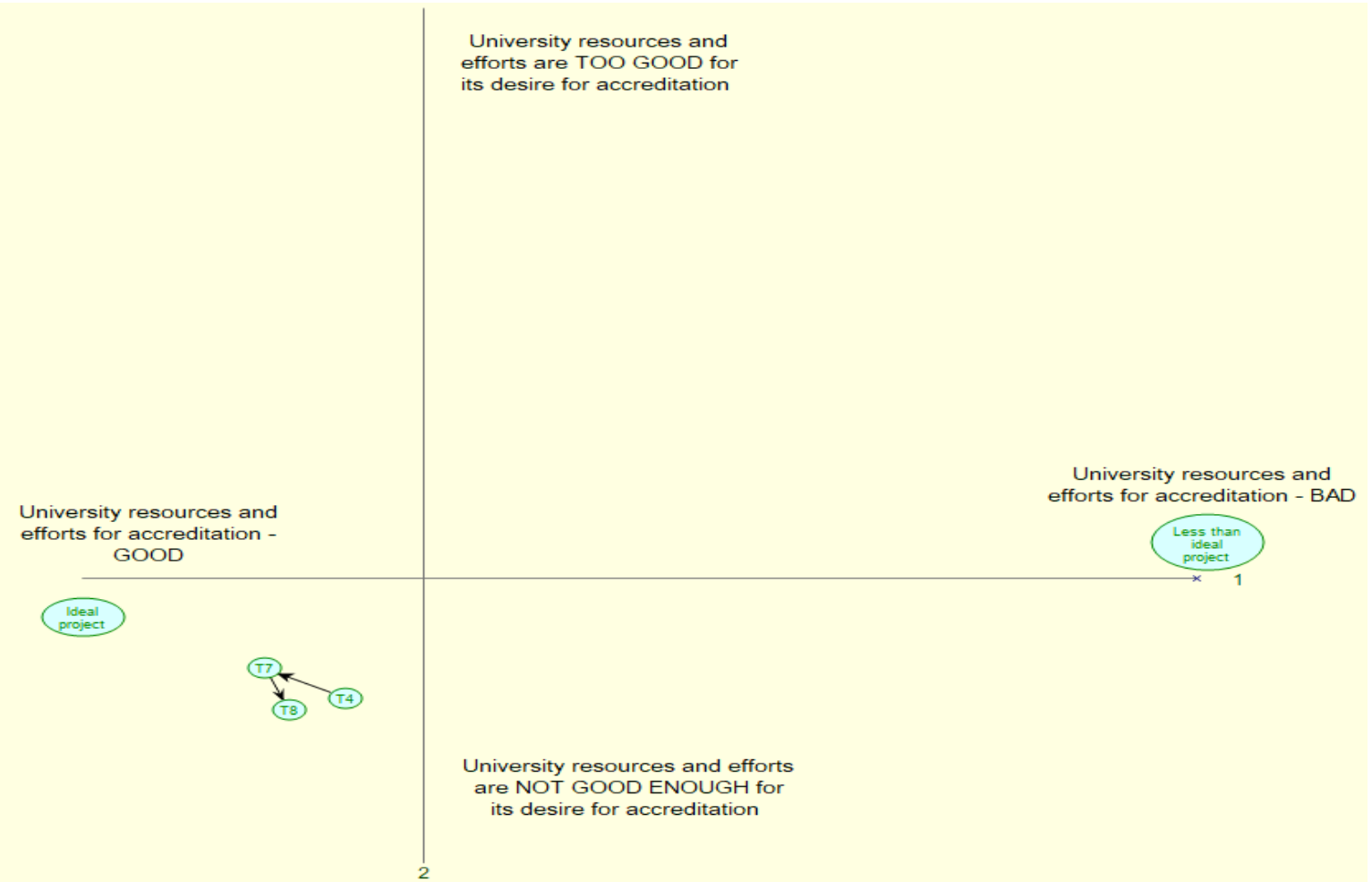


Because over the six interviews, Nikki's appreciation of the ABCD project had loadings on the two components, these components represented her standards for the project. A comparison of these components shows that Nikki's appreciation of the ideal and less than ideal projects had the largest loadings on the first component and very small loadings on the second component. The loadings on the first component also had opposite signs. This means that the first component could differentiate between Nikki's appreciation of the ideal and the less than ideal project. The second component, meanwhile, only referred to Nikki's concern about the project. The positions of the ideal and less than ideal projects on the PrinGrid maps also indicate this. They are opposite regarding the horizontal axes and do not differ much regarding the vertical axes. The first component, therefore, represented Nikki's primary standards, and the second component represented her secondary standard for the project. Based on the interpretation of the components, Nikki's primary standards were unchanged throughout the interviews and were that the university resources and efforts for the accreditation must be good. Nikki's secondary standard, meanwhile, changed. At T1 and T3, the standard was that the ability of the committee to mobilise external resources and efforts must match its internal resources and efforts. At the other interviews, the standard was that the university resources and efforts must match its desire for the accreditation.

The table and the maps also show the changes in Nikki's appreciation of the ABCD project over the six interviews. At T1, the gap between the loadings of Nikki's appreciation of the ABCD project and the ideal project on the first component was large (3.294). The loading of her appreciation of the ABCD project on the second component was also large (2.07). These loadings, which are illustrated by point (T1) in the upper right quadrant of Figure 6.4, indicate that Nikki had a generally bad appreciation of the ABCD project. She thought that the university resources and efforts were not good (component 1) and the committee's ability to mobilise external resources and efforts did not match its internal resources and efforts (component 2).

From T1 to T4, the gap between the loadings of Nikki's appreciation of the ABCD project and the ideal project changed for the first component. It decreased to 2.574 at T2, increased to 2.716 at $\mathrm{T} 3$, and finally decreased to 2.146 at T4. These changes indicate that Nikki's appreciation of the university resources and efforts improved at T2 and T4 but worsened at T3. The second component had two different interpretations over the course of the interviews, one at T1 and T3 and a different one at T2 and T4. This indicates that Nikki's concern changed during this time. At T1 and T3, Nikki was concerned about the gap between 
the internal and external resources and efforts of the committee. At T2 and T4, she was concerned about the gap between the university resources and efforts and its desire. The loadings of Nikki's appreciation of the ABCD project on the second component also changed over these time points. It was 1.842 at $\mathrm{T} 1$, decreased to 0.446 at $\mathrm{T} 2$, increased to 1.935 at $\mathrm{T} 3$, and finally decreased to 1.077 at $\mathrm{T} 4$. These loadings, which are illustrated by points $\mathrm{T} 1$ to $\mathrm{T} 4$ in Figures 6.4 to 6.7, indicate that at T1 and T3, Nikki thought that the committee's ability to mobilise the external resources and efforts did not match its internal resources and efforts. At $\mathrm{T} 2$ and T4, she thought that the university resources and efforts did not match its desire. In other words, at all four time points, Nikki thought that there were gaps between different aspects of the project. The changes in the loadings also indicate that the gaps at T2 and T4 were smaller than the gaps at $\mathrm{T} 1$ and $\mathrm{T} 3$, respectively.

Nikki continued changing her appreciation of the ABCD project from her fourth to fifth interview (T4 to T7) and from her fifth to sixth interview (T7 to T8). From T4 to T7, the gap between the loadings of Nikki's appreciation of the ABCD project and the ideal project on the first component decreased from 2.146 to 1.763 . The loading of her appreciation of the ABCD project on the second component decreased from 1.077 to 0.837 . These changes, which are illustrated by arrow T4T7 in Figure 6.8, indicate that Nikki's appreciation of the overall university resources and efforts improved, and the university resources and efforts better matched its desire. Arrow T7T8 suggests that from T7 to T8, Nikki thought that the university resources and efforts worsened, and the university resources and efforts less matched its desire.

In summary, over the six time points, Nikki's primary standards regarding the overall university resources and efforts for the ABCD project were the same. Her secondary standard, meanwhile, changed between two gaps. The first gap was between the internal and external resources and efforts of the committee. The second gap was between the university resources and efforts and its desire for the accreditation. Along with these standards, Nikki's appreciation of the project changed in both positive and negative ways.

\subsubsection{Changes in Nikki's Appreciation of the ABCD Project through the Lens of Vickers' Concept}

The above section has presented the changes in Nikki's standards for and appreciation of the $\mathrm{ABCD}$ project. This section uses these findings to describe the changes in her appreciation of the project, as influenced by her standards and perceptions of related events and ideas, by applying Vickers' concept of appreciative systems. 


\section{Before the first meeting (T1)}

Before the first meeting, based on her experience, Nikki's primary standards for the ABCD project were that the university resources and efforts must be good. The secondary standard was that the abilities of the committee to mobilise the external resources and efforts must match its internal resources and efforts. Following these standards, Nikki had a generally bad appreciation of the project. She thought that the university resources and efforts were not good (the primary standards). She also thought that the ability of the committee to mobilise the external resources and efforts did not match its internal resources and efforts (the secondary standard). In other words, there was a gap between the internal and external resources and efforts of the committee.

From before to after the first meeting (T1 to T2)

Between T1 and T2, Nikki attended the first committee meeting. In the meeting, the project manager introduced the project plan. Even though Nikki thought that this introduction was "a little bit late," she believed that the plan would help ensure that the project was more "on track" and involved more related people. Nikki also saw that the programme director was more committed:

The programme director, who had never attended the monthly meetings of the committee before, attended the meeting for the first time and was $100 \%$ committed.

Nikki, therefore, thought that the committee was better at mobilising the external resources and efforts.

Meanwhile, regarding its internal resources and efforts, Nikki saw that the majority of the committee members were committed and "willing to do what they need[ed] to do to get the job done." They also demonstrated more knowledge of the accreditation application process:

We have a marketing person who really knows about the stuff, you know, she demonstrated that more and more at the meeting. It is just a good reminder that yeah, we do have people who know this stuff.

However, Nikki also saw that there were disputes at the beginning of the meeting. Furthermore, one committee member was absent without apology:

One of the committee members who said and who actually asked for the meeting to be at the time that it was, didn't come and also didn't give their apology. 
Altogether, Nikki learned things that affected her appreciation of the university resources and efforts in both positive and negative ways, with the positive effects outweighing the negative ones. Thus, she thought that the university resources and efforts had improved (the primary standards). As a result of her greater appreciation of the involvement of people outside the committee, Nikki also thought that the gap between the committee's internal resources and efforts and its ability to mobilise the external resources and efforts diminished and not a concern anymore. Instead, she was concerned about the match between the university resources and effort and its desire for the accreditation. This also became her new secondary standard for the project. Following this standard, Nikki thought that the university resources and effort were not good enough for its desire; or, in other words, that there was a gap between them. However, this gap was smaller compared to the gap related to the external and internal resources and efforts at $\mathrm{T} 1$.

From after the first meeting to before the second meeting (T2 to T3)

After the first meeting but before the second meeting, Nikki had a brief meeting with the project manager, writer, and data analyst and learned that the project was "sticking to the timeline." Nikki was also aware that the project had two new "very knowledgeable" members. Nikki, therefore, thought that the internal resources and efforts of the committee had improved. Meanwhile, regarding the external resources and efforts, she saw that the programme director became more active and organised a meeting as he had promised:

Because I am the project administrator, he has sent me an email asking about organising the meeting, so, I know he has done it.

However, from Nikki's perspective, the top leader did not seem to be involved in Nikki's other daily work as much as he was expected to. Nikki, therefore, thought that the top leader was also not involved much in the project:

As far as I know, there's been no involvement, don't have any information about his involvement.

Altogether, from T2 to T3, Nikki's learnings affected her standards and appreciation regarding the $\mathrm{ABCD}$ project. In this case, her learnings resulted in a more negative appreciation of the involvement of people outside the committee such as the university leader and the programme director, and Nikki was more concerned about the match between the ability of the committee to mobilise the external resources and efforts and its internal resources and efforts. This again became her secondary standard for the project. Following this standard, Nikki thought that the external resources and efforts did not match the internal ones; or in other words, 
there was a gap between them. In comparison to the gap related to the desire of the university at T2, this gap was also larger. Regarding her primary standards, Nikki also thought that the overall university resources and efforts had worsened.

From before to after the second meeting (T3 to T4)

Between T3 and T4, Nikki participated in the second committee meeting, which was to "review the self-audit report" and "talk about the surveys." Nikki saw that the members had "a really good turnaround":

In the meeting, all the committee members were committed and focused on reviewing and giving feedback.

The programme director also attended the meeting and "seemed very involved and committed." From her daily work, Nikki doubted her previously negative appreciation of the top leader:

I actually don't know. Maybe because I was getting used to the way of working of the new top leader, moving from the old one who was very open and the new one. I don't know. He could be involved with the project manager, and there could be discussions.

Nikki, therefore, thought that the committee's ability to mobilise the external resources and efforts improved and not a concern anymore. Instead, she was again concerned about the match between the university resources and efforts and its desire for the accreditation. This again became her secondary standard for the project. Following this standard, Nikki thought that the university resources and efforts did not match its desire or that there was a gap between them. However, in comparison to the gap related to the internal and external resources and efforts at T3, this gap was smaller. Regarding the primary standards, Nikki also thought that the overall university resources and efforts for the project had improved.

From after the second meeting to before the fourth meeting (T4 to T7)

After the second meeting but before the fourth meeting, Nikki had become aware that the panel visit was completed and had learned about the good accreditation result. The result made Nikki more confident in the reputation of the university and the program quality:

In my opinion, I only, I think because we got good results because they [the panel] certainly appreciated that we are a really good school. Besides, I suppose I just have to concede that the programme quality is going better then.

Nikki also believed that the project management knowledge of the committee was better. However, she realised the limitations of human resources: 
The project analyst must work a lot of overtime, more hours than she would normally do. This indicates that the project needed the project analyst more and the project was more "under-resourced."

Moreover, Nikki had learned at this point that the accreditation was expensive and that "the money situation" was not good. All these learnings affected Nikki's appreciation of the university resources in both positive and negative ways.

Meanwhile, regarding the university commitment, Nikki saw the determination of the administrative group and the committee members to obtain the accreditation. The project manager and the university leader were especially committed to the project:

The project manager was outstanding. He was trying to do this on an aeroplane most of the time, I think. He kept being awesome. And $K$ [the university leader] was $110 \%$ engaged and committed. I do have full faith in him. He's a smart man, and the panel was really impressed by him.

However, the commitment of the programme director was still a problem:

He did not show his total commitment to the process and asked many questions which showed that he did not understand the programme.

Overall, in the period between T4 and T7, Nikki learned things that affected her appreciation of the university resources and efforts in both positive and negative ways. In this case, the positive effects outweighed the negative ones. Thus, Nikki thought that the university resources and efforts had improved (the primary standards) and better matched its desire for the accreditation (the secondary standard).

From before to after the fourth meeting (T7 to T8)

Between T7 and T8, Nikki attended the review meeting of the project and saw that the committee discussed issues related to the behaviour of the programme director:

The committee has strongly pointed out that having two people for the position of the programme director and no clear responsibilities between them made it confused.

Nikki herself also saw that the programme director "did not take the panel's recommendations seriously" in the meeting. Nikki, therefore, thought that the overall university resources and efforts had worsened slightly (the primary standards) and less matched its desire for the accreditation (the secondary standard).

In summary, during the data collection period, Nikki's learned things that affected her standards and appreciation regarding the ABCD project. Her primary standards focused on the overall university resources and efforts and did not change. Her secondary standard, 
meanwhile, changed. In the first and third interviews, it referred to the match between the internal and external resources and efforts of the committee. In the remaining interviews, it was the match between the university resources and efforts and its desire for the accreditation. Following these standards, Nikki's appreciation of the ABCD project changed in both positive and negative ways.

\subsection{Changes in Sumy's Appreciation of the ABCD Project}

\subsubsection{Participant Background}

Sumy was a part-time data collector and analyst for the ABCD project. She had an educational background in information technology and had worked for big companies in the United States, the United Kingdom, and India. Sumy's work on the ABCD project was her first job in a New Zealand organisation and her first project on accreditation. At the time of the interviews, she was also a master student at NZV University.

\subsubsection{Changes in Sumy's Standards for and Appreciation of the ABCD Project}

Number of principal components

Sumy participated in eight interviews. In the first interview, she identified ten constructs for the repertory grid. She added two more new constructs at the second interview. While the constructs did not change at the third interview, Sumy found that one construct was not relevant anymore and removed it from the grid at the fourth interview. The constructs were then unchanged in the remaining interviews. The principal component analysis (see Appendix Q) identified that two principal components could explain Sumy's appreciation of the ABCD project over the eight interviews. The components explained from 88 to $92 \%$ and from $5 \%$ to $6 \%$ of the appreciation, respectively. The percentage variances explained by each component are presented in Table 6.5 below. Because Sumy's repertory grid did not change from the second to the third interview and from the seventh to eighth interview, these interviews are described in the same boxes in the table. 
Table 6.5 Percentage variances explained by two principal components over the eight interviews - Sumy - ABCD project

\begin{tabular}{|c|c|c|c|c|c|c|}
\hline Interview & \multicolumn{2}{|l|}{$T 1$} & \multicolumn{2}{|c|}{$T 2 \& T 3$} & \multicolumn{2}{|l|}{$T 4$} \\
\hline Component & 1 & 2 & 1 & 2 & 1 & 2 \\
\hline Variance $\%$ & 91.79 & 6.27 & 88.02 & 6.51 & 91.09 & 5.05 \\
\hline Cumulative variance $\%$ & \multicolumn{2}{|l|}{98.06} & \multicolumn{2}{|l|}{94.53} & \multicolumn{2}{|l|}{96.14} \\
\hline Interview & \multicolumn{2}{|l|}{$T 5$} & \multicolumn{2}{|l|}{ T6 } & \multicolumn{2}{|c|}{$T 7 \& T 8$} \\
\hline Component & 1 & 2 & 1 & 2 & 1 & 2 \\
\hline Variance $\%$ & 90.08 & 5.92 & 90.21 & 5.64 & 90.62 & 5.04 \\
\hline Cumulative variance $\%$ & \multicolumn{2}{|l|}{96.01} & \multicolumn{2}{|l|}{95.85} & \multicolumn{2}{|l|}{95.66} \\
\hline
\end{tabular}

\section{Interpretation of the principal components}

The construct loadings on the two components show that the interpretation of the two components had minor changes over the eight interviews.

Over the eight interviews, all the constructs had their largest loadings with the same signs on the first component. At the first interview, the constructs could be divided into two groups. The first group consisted of constructs related to the university capabilities regarding accreditation. These constructs specifically referred to the data storage, people, and other characteristics of the university $(\mathrm{C} 4, \mathrm{C} 5, \mathrm{C} 7, \mathrm{C} 8, \mathrm{C} 10)$. The second group consisted of constructs related to the capabilities of the committee. These constructs specifically referred to the experience, abilities and knowledge of the committee members and chair $(\mathrm{C} 1, \mathrm{C} 2, \mathrm{C} 6, \mathrm{C} 9$, C3). The first component, therefore, could be interpreted as the capabilities of the university and committee regarding the accreditation. At the second and third interviews, Sumy had two new constructs which referred to the project plans and timeline $(\mathrm{C} 11, \mathrm{C} 12)$. They also had their largest loadings on the first component, and the loadings had the same signs as the existing constructs. The first component, therefore, was interpreted just like it was in the first interview, except that now the interpretation also included time management. From the fourth interview onwards, Sumy removed construct C9, which referred to the consolidation of different accreditations. The interpretation of the first component, therefore, was the same as it was in the second and third interviews, but without the consolidation of different accreditations.

The interpretation of the second component also had minor changes at the second and third interviews. At the first interview, all the constructs had some loadings on this component. These constructs could be divided into two groups with opposite signs of loadings. The first group consisted of constructs related to the ability of the committees to consolidate different accreditations. Constructs with considerably large loadings in this group specifically referred 
to the experience in accreditation (C6) and the ability to consolidate different accreditations of the committee members (C9). The second group consisted of constructs related to the other capabilities of the university and committee. Constructs with considerably large loadings in this group referred to the quality of the students and academic staff, the knowledge of the committee about the panel, and the project management abilities of the committee chair $(\mathrm{C} 7$, $\mathrm{C} 8, \mathrm{C} 2, \mathrm{C} 3$ ). The second component, therefore, could be interpreted as the gap between the capability to consolidate different accreditations and other capabilities of the committee and university. At the second and third interviews, the two new constructs also had considerably large loadings on the second component. Their loadings had the same signs as the loadings of the constructs regarding the other capabilities of the university. The interpretation of the second component, therefore, was the same as it was in the first interview, except that the other capabilities category now also included time management.

The interpretation of the second component changed significantly at the fourth interview when Sumy removed the construct regarding the consolidation of different accreditations (C9). From this interview onwards, the constructs with considerably large loadings on the second component could be divided into two groups with opposite signs of loadings. The first group consisted of constructs referring to the project plans and timelines $(\mathrm{C} 11, \mathrm{C} 12)$. The second group consisted of constructs referring to the university characteristics and the abilities of the committee members. These constructs specifically referred to the knowledge of the committee, the project management skills of the committee chair, and the human resources for the project $(\mathrm{C} 2, \mathrm{C} 3, \mathrm{C} 5)$. The second component, therefore, could be interpreted as the gap between the time management and other capabilities of the committee.

\section{Changes in Sumy's standards for and appreciation of the ABCD project}

This section presents the changes in Sumy's standards for and appreciation of the ABCD project. These changes were identified from the changes in the loadings of Sumy's appreciation of the ABCD project, and the ideal and less than ideal projects on the two components (Table 6.6). These changes are presented on two PrinGrid maps because the interpretation of the second component changed significantly at the fourth interview for the remainder of the data collection. The first map (Figure 6.8) presents the changes from the first to the third interview. The second map (Figure 6.9) presents the changes from the fourth to the eighth interview. In the first map, the minor changes in the interpretation of the two components at the second and third interviews are presented in brackets. The table and the maps are presented below. 
Table 6.6 Loadings of Sumy's appreciation of the ideal project and less than ideal projects and the ABCD project on the two components

\begin{tabular}{|c|c|c|c|c|c|c|}
\hline Interview & \multicolumn{2}{|l|}{$T 1$} & \multicolumn{2}{|c|}{$T 2 \& T 3$} & \multicolumn{2}{|l|}{$T 4$} \\
\hline Component & 1 & 2 & 1 & 2 & 1 & 2 \\
\hline Ideal project & -2.762 & -0.005 & -3.267 & 0.256 & -2.987 & -0.498 \\
\hline Less than ideal project & 3.529 & -0.556 & 3.614 & & 3.619 & -0.435 \\
\hline $\mathrm{ABCD}$ project & -1.245 & -0.44 & -0.955 & -0.792 & -1.388 & -0.654 \\
\hline Gap & 1.517 & & 2.312 & - & 1.599 & \\
\hline Interview & \multicolumn{2}{|l|}{$T 5$} & \multicolumn{2}{|l|}{ T6 } & \multicolumn{2}{|l|}{$T 7 \& T 8$} \\
\hline Component & 1 & 2 & 1 & 2 & 1 & 2 \\
\hline Ideal project & -2.943 & -0.354 & -2.914 & -0.322 & -2.883 & -0.35 \\
\hline Less than ideal project & 3.663 & -0.436 & 3.689 & -0.455 & 3.717 & -0.458 \\
\hline $\mathrm{ABCD}$ project & -1.655 & -0.889 & -1.789 & -0.875 & -1.919 & -0.78 \\
\hline Gap & 1.288 & & 1.125 & & 0.964 & \\
\hline
\end{tabular}

Figure 6.8 Changes in Sumy's appreciation of the ABCD project, and the ideal and less than ideal projects from the first to the third interview

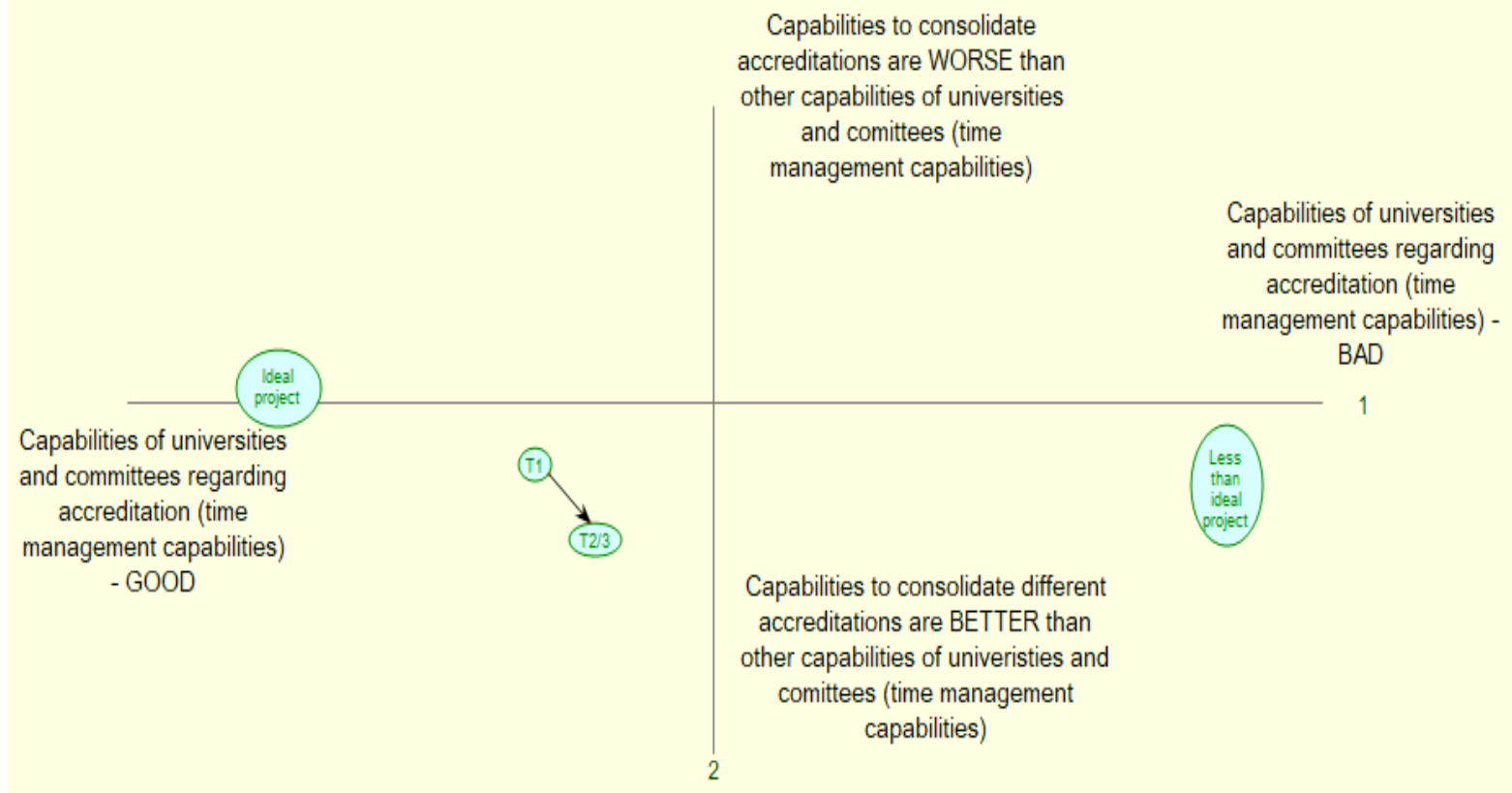


Figure 6.9 Changes in Sumy's appreciation of the ABCD project, and the ideal and less than ideal projects from the fourth to the eighth interview

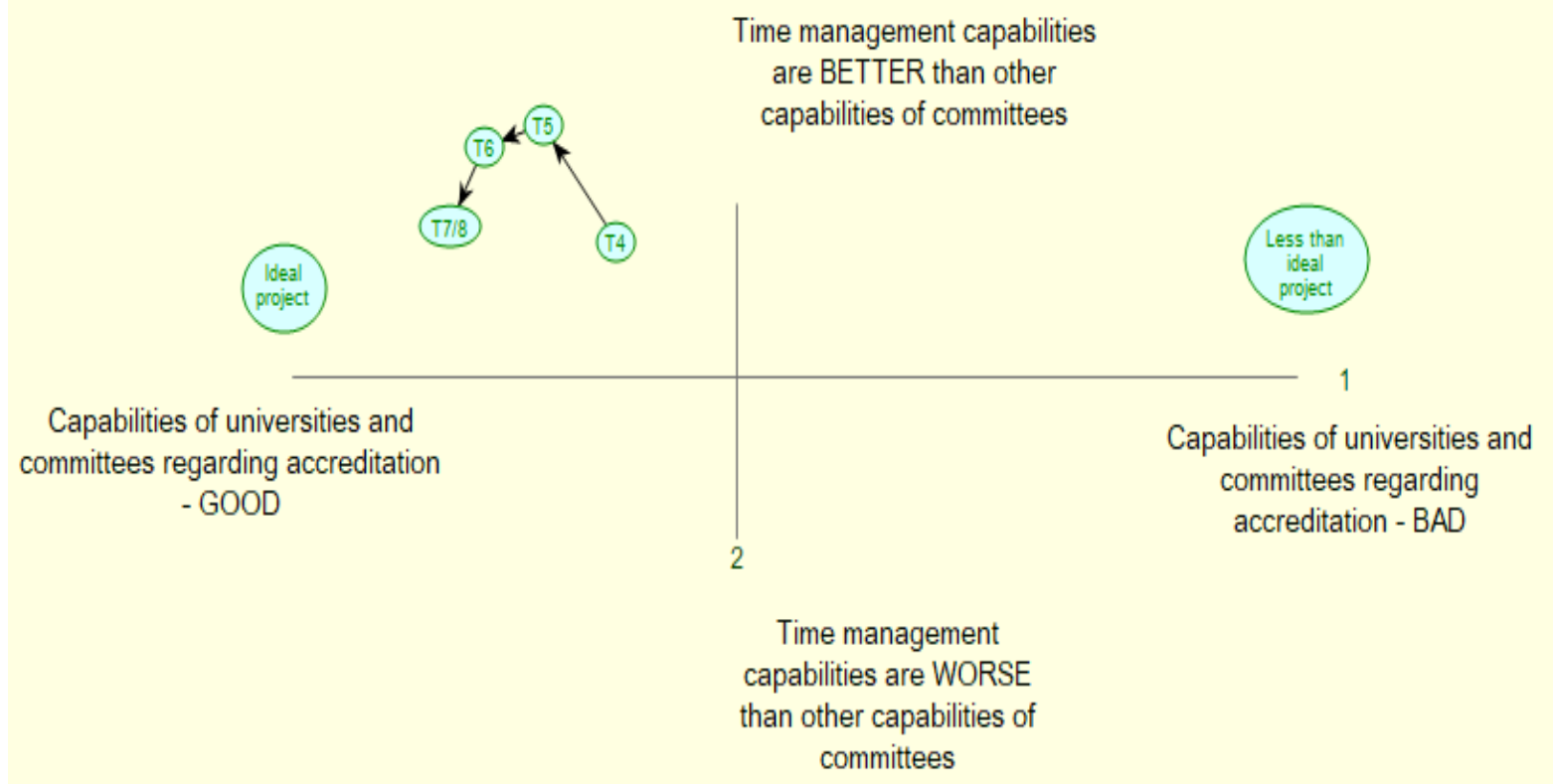

Because over the eight interviews, Sumy's appreciation of the ABCD project had loadings on the two components, these components represented her standards for the project. A comparison of these components shows that Sumy's appreciations of the ideal and less than ideal projects had their largest loadings on the first component and very small loadings on the second component. The loadings on the first component also had opposite signs. The first component, therefore, could distinguish Sumy's appreciation of the ideal project from her appreciation of the less than ideal project. Meanwhile, the second component just referred to her concern about the project. The positions of the ideal and less than ideal projects on the PrinGrid maps also indicate this. They are opposite regarding the horizontal axes and do not differ much regarding the vertical axes. The first component, therefore, represented Sumy's primary standards, and the second component represented her secondary standard for the project.

Following the interpretation of the components, Sumy's primary and secondary standards changed over the eight interviews. At T1, her primary standards were that the capabilities of the university and the committee regarding the accreditation must be good. At the second and third interviews, the standards included the project time management. From the fourth interview, the standards did not include the capabilities of consolidating different accreditations. Meanwhile, following the interpretation of the second component, at T1, 
Sumy's secondary standard was that the efforts made towards consolidating different accreditation must match other efforts undertaken by the university and committee At the second and third interviews, this standard was the same, except that the other efforts also included time management. From the fourth interview onwards, the standard changed to that the time management must match other capabilities of the committee.

The table and the PrinGrid maps also show the changes in Sumy's appreciation of the ABCD project over the eight time points. At T1, the gap between the loadings of Sumy's appreciation of the ABCD project and the ideal project on the first component was moderate (1.517). The loading of her appreciation of the ABCD project on the second component was small (-0.44). These loadings, which are illustrated by point (T1) in the lower quadrant of Figure 6.9, indicate that at the first interview, Sumy thought that the overall capabilities of university and committee regarding the accreditation were generally good. The capabilities to consolidate different accreditations were slightly better than other capabilities of the university and committee. From T1 to T2/3, the gap between the loadings of Sumy's appreciation of the $\mathrm{ABCD}$ project and the ideal project on the first component increased from 1.517 to 2.312. The loadings of Sumy's appreciation of the ABCD project on the second component increased from 0.44 to 0.792 . These changes, which are illustrated by arrow T1T2/3, indicate that Sumy's appreciation of the overall capabilities of the university and the committee worsened. The changes in the interpretation of the first component also indicate that in addition to the previous capabilities, at T2/3, Sumy also evaluated the time management. Similarly, regarding the second component, Sumy not only added the time management capabilities to the other capabilities of the committee but also thought that these other capabilities less matched the capabilities to consolidate different accreditations.

From T3 to T4, the gap between the loadings of Sumy's appreciation of the ABCD project and the ideal project on the first component decreased from 2.312 to 1.599 . This indicates that Sumy thought that the overall capabilities of the university and the committee had improved. At this point, these capabilities did not include the consolidation of different accreditations. Meanwhile, the changes in the interpretation of the second component indicate that Sumy changed her concern. At T3, she was concerned about the gap between the consolidation of different accreditations and the other capabilities of the university. At T4, her concern was about the gap between the time management and the other capabilities of the university. The loading of her appreciation of the ABCD project on the second component also decreased from 0.792 to 0.654 . This indicates that at T4, Sumy thought that the time 
management was better than the other capabilities; or in other words, there was a gap between them. However, in comparison to the gap related to the consolidation of different accreditations at T3, this gap was smaller.

The changes in Sumy's appreciation from T4 to T8 are illustrated by arrows T4T5, T5T6, and T6T7/8 in Figure 6.9. These arrows show that the changes in the appreciation regarding component 1 were positive, and the changes regarding component 2 were both positive and negative. Regarding component 1, over these interviews, Sumy thought that the overall capabilities of the university and the committee had improved. Regarding component 2, from T4 to T5, Sumy thought that the other capabilities did not match the good time management capabilities as well as before. In the remaining interviews, she thought that the other capabilities better matched the time management capabilities.

In summary, the principal component analysis shows that Sumy had standards for the capabilities of the university and committee. During the data collection period, Sumy changed these standards. Along with these changes, her appreciation of the project changed in both positive and negative ways.

\subsubsection{Changes in Sumy's Appreciation of the ABCD Project through the Lens of Vickers'}

\section{Concept}

The above section has identified the changes in Sumy's standards for and appreciation of the $\mathrm{ABCD}$ project. This section uses these findings to describe the changes in her appreciation of the project, as influenced by her standards and perceptions of related events and ideas, by applying Vickers' concept of appreciative systems.

\section{Before the first meeting (T1)}

In the first interview, based on her previous work experience and her little involvement in the project, Sumy's primary standards for the ABCD project were that the overall capabilities of the university and committee regarding the accreditation must be good. Her secondary standard was that the capabilities of consolidating different accreditations must match other capabilities. Following these standards, Sumy thought that the overall capabilities of the university and the committee were generally good (the primary standards). However, because Sumy knew that the university had obtained many accreditations before, she believed that its capabilities to consolidate different accreditations were slightly better than other capabilities, such as the data storage systems, student quality and project management (the secondary standard). 
From before to after the first meeting (T1 to T2)

During this time, Sumy attended the first meeting of the committee. In this meeting, the chair introduced the project plan and discussed it with the committee members. These events affected her thinking:

Yeah, this thing really strikes me, why are you doing it now, why don't you do it before, if the timeline was introduced earlier, the project could have been managed better.

This comment shows how Sumy realised that the plan and timeline were critical to the project and came up with new standards for the time management capabilities of the committee. These new standards were added to both her primary and secondary standards. The late introduction of the plan also made Sumy think that the time management in the ABCD project was not very good.

At the beginning of the meeting, Sumy also saw a small dispute among the committee members and therefore thought that the project management was worse:

The dispute was not on the agenda, and it had nothing to do with the supposed discussion.

Sumy also saw that the committee members were not clear about the expectations of the panel. They did not know whether the panel was interested in the future or the past of the target programme:

Since they have done it before, I have thought that they know people, and they know what they are doing, but here, after sitting at the meeting, I have a feeling that they don't know what they are doing

In addition to that, Sumy learned that there was "a vacuum of leadership" in the target programme. These learnings made Sumy's appreciation of the overall capabilities of the university and committee, including the time management capabilities, worsen (the primary standards). She also thought that other capabilities of the university and the committee, including the time management capabilities, were less demonstrated and less matched its capabilities to consolidate different accreditations than they were at T1 (the secondary standard). In other words, there was a gap between the capabilities to consolidate different accreditations and the other capabilities.

From after the first meeting to before the second meeting (T2 to T3)

During this time, Sumy did not learn anything related to the ABCD project and did not change her standards and appreciation regarding the project. 
From before to after the second meeting (T3 to T4)

During this time, Sumy attended the second meeting, which was to review the first draft of the self-study report and discuss issues related to the surveys and the curriculum vitae of the lecturers. Sumy learned that the committee did not consolidate different accreditations and just worked specifically on the ABCD accreditation. Sumy, therefore, thought that the consolidation of different accreditations was not a standard for the project anymore. Her secondary standard also changed to the match between the time management and other capabilities of the committee and university. Along with these standards, Sumy saw that the project plan and timeline were clearer:

Everybody like knows you're doing this, you're doing that, and they consolidate stuff and all the actions according to the plan are completed.

Sumy also believed that the project had filled all the necessary roles:

They have $P$ [the report writer] writing the report, I am collecting the data, and as we are going with the timeline, we have a strict deadline... In fact, we finish before that. It is going as planned because the university has the resources.

With these learnings, Sumy thought that the overall capabilities of the university and the committee had improved (the primary standards). The time management capabilities were also better than other capabilities of the committee and university. In other words, she perceived a gap between these capabilities (the secondary standard). However, in comparison to the gap related to the consolidation of different accreditations, this gap was smaller.

From after the second meeting to before the third meeting (T4 to T5)

During this time, Sumy was involved in the preparation of the base room. She saw that all the necessary resources for the accreditation were ready:

They have everything, from the infrastructure, budget, to people. Yes, it increases, yes, my perception of them of having the resources, having the capacities has increased.

Sumy also saw that the work was completed "on and even before the schedule." These observations made her believe that the capabilities of the university and the committee had improved (the primary standards). The time management capabilities were also clearly demonstrated and therefore, more exceeded the other capabilities of the committee and university (the secondary standard). 
From before to after the third meeting (T5 to T6)

During this time, Sumy attended the third committee meeting and learned that the chair had submitted the self-study report. Sumy also saw that the committee members very much engaged in discussing the preparation for the panel visit.

They really know what they're doing. Even they had some differences, the kind of discussion that followed shows that they all had read things. Everyone was on the same page, worked in harmony and was sticking to the work.

Sumy, therefore, thought that the overall capabilities of the committee and university had improved (the primary standards). Other capabilities of the committee, such as its knowledge, were also more clearly demonstrated and therefore better matched the time management capabilities (the secondary standard).

From after the third meeting to before the fourth meeting (T6 to T7)

In the time after the third meeting but before the fourth meeting, Sumy had become aware that the panel visited the university. Sumy was only involved a little in the set-up of the base room and did not meet the panel directly. However, with the base room preparation, Sumy could see that the committee knew a lot about the accreditation:

They knew what they were doing, how the room should be set up, how the things should be presented, even from the simple things.

Because of this, Sumy thought that the overall capabilities of the committee and university had improved (the primary standards). Other capabilities of the committee, such as its knowledge of the accreditation, were also more demonstrated and better matched the time management capabilities than they were at T6 (the secondary standard).

From before to after the fourth meeting (T7 to T8)

During this time, Sumy attended the review meeting of the project but did not learn anything new. The meeting just confirmed her existing standards and appreciation regarding the project.

In summary, over the eight time points, starting with her initial standards and appreciation, Sumy learned things related to the ABCD project. These learnings changed her standards for the project. Her primary standards focused on the overall capabilities of the committee and university and did not change; however, her secondary standard changed. From the first to the third interview, it referred to the gap between the capabilities to consolidate different accreditations and other capabilities of the university and committee. In the remaining interviews, it was the gap between the time management capabilities and other capabilities. 
Following these standards, Sumy's appreciation of the project changed in both positive and negative ways.

\subsection{Chapter Summary}

This chapter has presented the changes in the three participants' appreciation of the ABCD projects, as influenced by their standards and perceptions of related events and ideas. These changes, along with the changes in the participants' appreciation in the two cases in chapter four and five, will be discussed in the next chapter. 


\section{Chapter 7 Discussion}

\subsection{Overview of the Chapter}

Chapter four, five, and six have applied components of Vickers' concept of appreciative systems to describe the changes in the participant perceptions of projects. Of the 47 interviews conducted in this research, there were only two interviews (Sumy's third and eighth interviews in the $\mathrm{ABCD}$ case) in which the participant reported no substantive change in her appreciation of the project. All remaining interviews highlighted events and ideas that led to changes in the participants' standards and project appreciation. These changes, therefore, indicate the dynamics of stakeholder perception of projects. Moreover, the components of Vickers' concept of appreciative systems also indicate different aspects of stakeholder perception. Stakeholder standards refer to their perception of the significance of facts in projects. Stakeholder perceptions of events and ideas refer to their observations and the information or abstract ideas that they learn. Stakeholder appreciation refers to their judgments about the state of projects (see section 3.4.1). Based on these components, this chapter identifies the nature and common themes of the dynamics of stakeholder appreciation of the projects and their drivers. Specifically, the chapter begins with a discussion of the nature and dynamics of stakeholder perceptions of events and ideas and continues with similar discussions of their standards and appreciation of their projects. Based on these discussions, a conceptual framework to describe the overall nature and dynamics of stakeholders' appreciation of projects, as influenced by their individual characteristics, is proposed at the end of the chapter.

\subsection{Nature and Dynamics of the Perceptions of Related Events and Ideas}

\subsubsection{Stakeholder Networks as Sources of the Events and Ideas}

It seems clear that how individual stakeholders perceive and respond to events and ideas that pertain to their projects will depend upon many factors. However, the specific roles that the stakeholders occupy throughout the course of the projects, the roles that they play in the wider organisations, and the informal communication that they are involved in and have access to would seem to be particularly important. Examples of these follow.

Roles of stakeholders in projects

A good example of how the roles of stakeholders in their projects can impact upon their perception of events and ideas was when Thu, the MTEO project manager, met with the 
accreditation panel during their preliminary visit to the university between T1 and T2. During this visit, Thu received information regarding the panel members' assessment of the university. However, Hung, the employer representative of the project, was not privy to these insights and only received general information about the project from the interview guidelines during this period. Similar differences can be observed in the information received by Tai and Rafat in the APSC and by Amish and Nikki in the ABCD cases. Over the four interviews, Tai, as a project member, learned a wide range of information about the project, including the results of the external assessment of the student quality, how committed the leaders were, and the fact that the leaders decided to re-submit the self-report. Meanwhile, Rafat, the regional chair of the accreditation association, only learned that the university had employed several new faculty members with a $\mathrm{PhD}$, and it would take them more time to complete the project. With regard to the $\mathrm{ABCD}$ case, Amish, as the project manager, worked with the self-study report writer and learned about the quality of the report and the competence of the writer between T3 to T4. At the same time, Nikki, as the project administrator, did not know about the report but exchanged emails with the programme director and learned that he had organised a small meeting for the project. Overall, these examples illustrate that the specific roles of stakeholders in projects affect the events and ideas that they are susceptible to.

\section{Roles of stakeholders in wider organisations}

The roles of stakeholders in their wider organisations also affect the events and ideas that they are susceptible to. For example, Nga was not much involved in the MTEO case between T3 and T4 because the official visit of the panel was completed. However, as a member of the International Collaboration Department, she still learned about the new policies of the university to improve its operation according to the accreditation standards. She also learned about the recruitment of a senior member to the Quality Assurance Department, which was in charge of accreditation. This information made Nga think that the university was serious about the accreditation. In another example, between T3 and T4, Amish, in his role at the university that was not directly related to his position as the project manager of the ABCD case, heard complaints about the daily work of the programme director. These complaints made him think that the director was as uncommitted to the target project as he was in his daily work. In the same case, Nikki, the project administrator, noticed that the university leader did not involve himself much in her daily work. Because of this, she thought that the leader also did not involve himself much in the project. The APSC case also features an example of stakeholders learning information through other roles that affect their perception of the project. In this case, through 
his position as a vice-rector, Tung was involved in a panel visit of another accreditation organisation between T1 and T2. During this visit, he received feedback on the general faculty qualifications of the university, which affected his appreciation of the faculty qualifications of the target programme in the APSC project.

\section{Informal communication by stakeholders}

Individual stakeholders also learn events and ideas related to their projects from their informal communication. For example, in the MTEO case, between her second and third interviews, Nga learned from the Facebook page of one Department Head about the panel's negative comments about the university's data storage system. In the same case, between T3 and T4, Hung, an employer representative, learned about the good results of the project from his friend, who was working at the university. In the APSC case, Khiem, a member of an accreditation project at another university, learned about the difficulties of the project at FXN University from an informal chat with the project administrator between $\mathrm{T} 2$ and $\mathrm{T} 3$.

In summary, the above discussion shows that individual stakeholders learn events and ideas related to their projects from multiples sources in their complex social networks. This indicates the multiple roles that each individual stakeholder can occupy and the complex interactions that stakeholders have from these roles. These multiple roles have been described by many scholars, such as Jansson (2005), Post et al. (2002), Pouloudi et al. (2016), and Winn (2001). The complex interactions of stakeholders have also been described in many theoretical and empirical studies. Examples of theoretical studies that address the complexity of stakeholder interactions include Key (1999), Fassin (2008), and Ackermann and Eden (2011) in the stakeholder literature, and Eskerod, Huemann, and Savage (2015) in the project management literature. Among the empirical studies that have examined this issue are Ziervogel and Downing (2004) in the stakeholder literature, and Mead (2001) and Papadopoulos and Merali (2009) in the project management literature. The above discussion also shows that during project implementation, stakeholder interactions in their social networks are susceptible to changes. This finding is in line with the dynamics of stakeholder networks that have previously been observed in studies on organisations in general (e.g., Frost, 1995; South et al., 2015) and in specific projects (e.g., Kreiner, 1995; Missonier \& Loufrani-Fedida, 2014; Papadopoulos \& Merali, 2008). Overall, this research provides an empirical illustration of stakeholder networks and their dynamics and offers an approach to explain how these networks affect stakeholder perception. 


\subsubsection{Dynamics of the Perceptions of Related Events and Ideas}

From their networks, individual stakeholders can have access or no access to events and ideas related to projects. Along with their existing standards and appreciation, stakeholders can also find accessible events and ideas relevant and irrelevant to projects.

\section{Access and no access}

The social networks of individual stakeholders affect whether they have access to events and ideas. This impact of the social networks can be seen in the example of Thu, the project manager of the MTEO case, Nga, the member of the International Collaboration Department, and Hung, the employer representative on the project. Due to her managerial role, Thu had access to detailed feedback from the panel and information relating to the commitment of the university leaders throughout the data collection period. Meanwhile, due to her supporting role in the project, her role in the International Collaboration Department, and her informal communication, Nga had access to the general feedback of the panel, the information relating to the commitment of the university departments, and the information relating to the project management techniques. However, Hung's social networks included his role as an employer representative and his informal communication and as a result, he only had access to the interview guidelines, the things that happened in his interviews and the general results of the project.

Similar to the MTEO case, in the APSC case, the social networks of the participants affected their access to the events and ideas related to the project. Thus, as Tung's social network included his role as the project manager, he had access to detailed information about the plan and progress of the project as well as the responses of the accreditation association. Meanwhile, due to their role as external stakeholders, Khiem and Rafat could only guess about the progress and difficulties of the project. In another example from the ABCD case, the project manager, Amish had access to information about all aspects of the project through his position and his social network, from the report writing and data collection to the feedback and recommendations of the panel. Meanwhile, the social network of Sumy, who worked as a data collector and analyst on the project, gave Sumy access to a substantial amount of information on the data but little information on other aspects of the project.

Overall, the social network of each individual stakeholder affects their access to the events and ideas related to their project. Because these networks reflect the interactions among stakeholders, they also create the fluxes of events and ideas. Figure 3.2 in the methodology 
chapter, which illustrates the dynamics of stakeholder perception through the lens of Vickers' concept, therefore, can be updated to describe the dynamics of stakeholder access to events and ideas in Figure 7.1 as follows.

Figure 7.1 Dynamics of stakeholder access to events and ideas

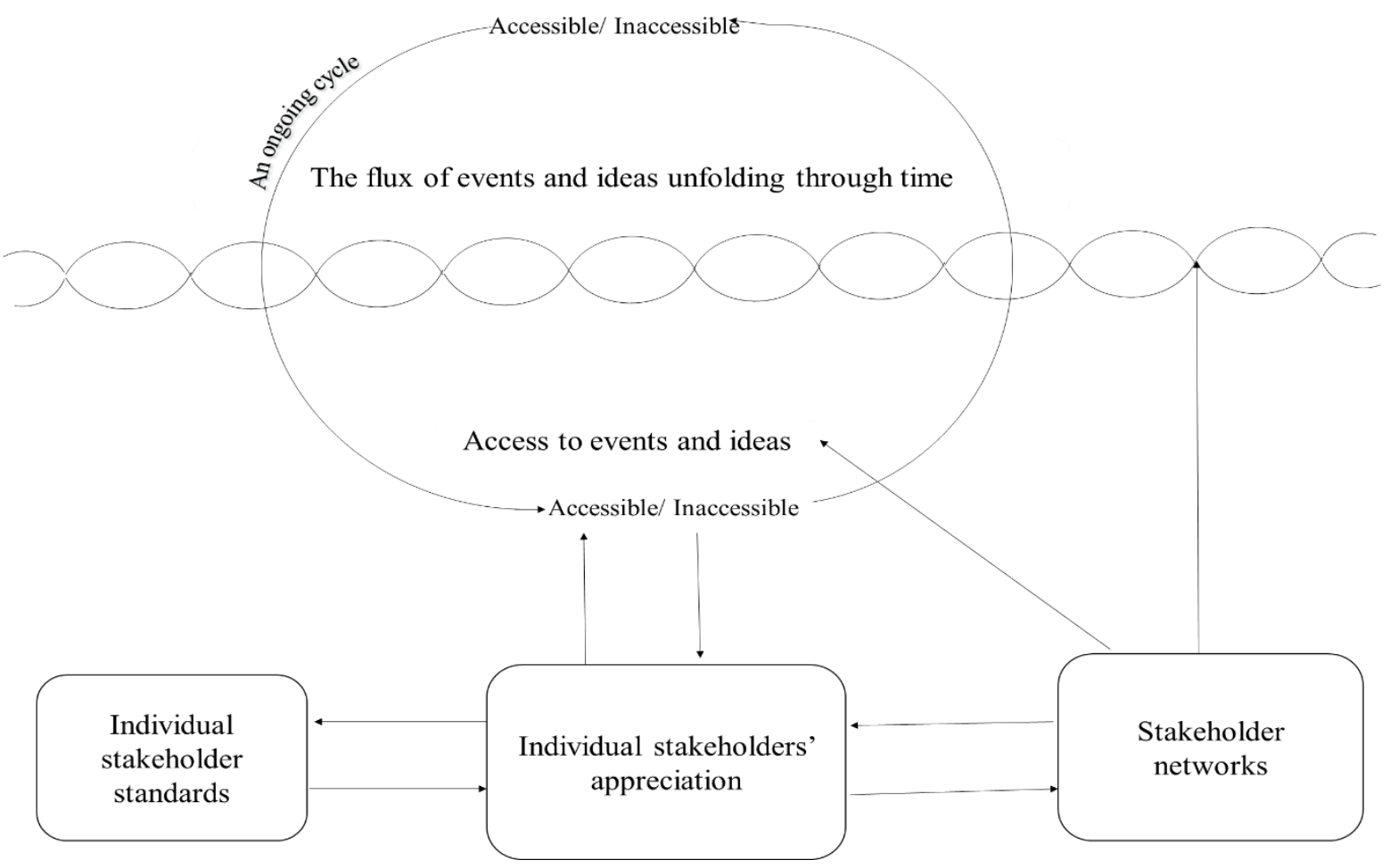

In comparison to Figure 3.2, this figure replaces stakeholder actions with stakeholder networks and describes the dynamics related to stakeholder access to the events and ideas. The abovementioned effects of stakeholder networks on the fluxes of events and ideas and stakeholder access to the events and ideas are illustrated by the arrows between them.

\section{Relevance and irrelevance}

Along with their existing standards and appreciation regarding projects, individual stakeholders may find each accessible event and idea relevant or irrelevant to the projects. For example, in the APSC case, while both Tung and Tai were aware of the application of the new ISO software and the development of online training at the university, they had different perceptions of the relevance of this application and development to the project. While Tai thought that it was related to the technology of the target programme, Tung did not. In the MTEO case, both Thu and Nga were aware of the management techniques used in the project. 
However, while Nga thought that these techniques were relevant to the project, Thu did not. Similarly, in the ABCD case, while the three participants attended the same monthly committee meetings and had access to similar information in the meetings, they had different perceptions of the relevance of the information related to the programme director. While Amish and Nikki thought that the performance and commitment of the director were especially relevant to the project, Sumy did not, and instead, she paid attention to the commitment and performance of all the committee members.

The changes in the standards and appreciation of individual stakeholders regarding projects also affect the events and ideas that they find relevant to projects. For example, in the ABCD case, Sumy had developed new standards for the committee's capabilities in planning and time management by her second interview. She, therefore, paid attention to these capabilities and included them in her appreciation of the project following this interview. Nga from the MTEO case had also developed new standards for the strict requirements of the panel and the project implementation steps since her third interview. She, therefore, paid attention to these aspects and included them in her appreciation of the project since this interview. Relevance and irrelevance, therefore, can be described as the ways in which individual stakeholders learn events and ideas.

In summary, during project implementation, depending on their networks, individual stakeholders have access or no access to particular events and ideas. They also find the events and ideas relevant or irrelevant to specific projects. Studies such as Aaltonen et al. (2008) in the project management literature and Pouloudi et al. (2016) and Ziervogel and Downing (2004) in the stakeholder literature have shown that stakeholders receive information from their networks or this information affects their perception. Studies have also described in detail the ways in which stakeholders receive information from the networks. For example, Sloan and Oliver (2013) categorise the different processes in which stakeholders learn about critical incidents as asking, disclosing, opening, and valuing. The current study adds to the existing knowledge by providing a way to explain how individual stakeholders learn information from their networks and how this information impacts their perception. In addition, building on Vickers' (1965) and Checkland's (2000) general observation, by explaining the impact of stakeholder networks on stakeholder perception, this research clarifies and provides evidence for the relationship between individual actions and perception of events and ideas as the relationship between stakeholder actions and networks, between stakeholder networks and the 
fluxes of events and ideas, and between stakeholder networks and their access to the events and ideas.

\subsection{Nature and Dynamics of Standards of Individual Stakeholders}

\subsubsection{Nature of Individual Stakeholders' Perception of Ideal and Less than Ideal Projects}

As explained in the methodology and data analysis as well as the findings chapters, stakeholders' standards for projects are identified by the principal component analysis of their repertory grids, under the consideration of their thinking about the ideal and less than ideal projects. The nature of the standards, therefore, depends on the nature of stakeholder thinking about the ideal and less than ideal projects. Meanwhile, the constructs and ratings that stakeholders have for ideal and less than ideal projects in repertory grids provide information about stakeholder thinking about how stakeholder interactions in the target projects should be. For example, in the MTEO case, these constructs and ratings in Thu's repertory grid at T1 reflected her expectations that the project stakeholders were the accreditation association, the university leaders, the project members, and all university departments. She also thought that these stakeholders should have particular interactions such as the panel's requirements must be strict, the accreditation association must issue many reference materials, the university leaders must care about the project, and the university's evidence documents must be good. Following the network approaches, Thu's thinking of how the stakeholder interactions in the MTEO project should be at $\mathrm{T} 1$ is described in Figure 7.2 as follows.

Figure 7.2 Thu's thinking of how the stakeholder interactions in the MTEO project should be at $T 1$

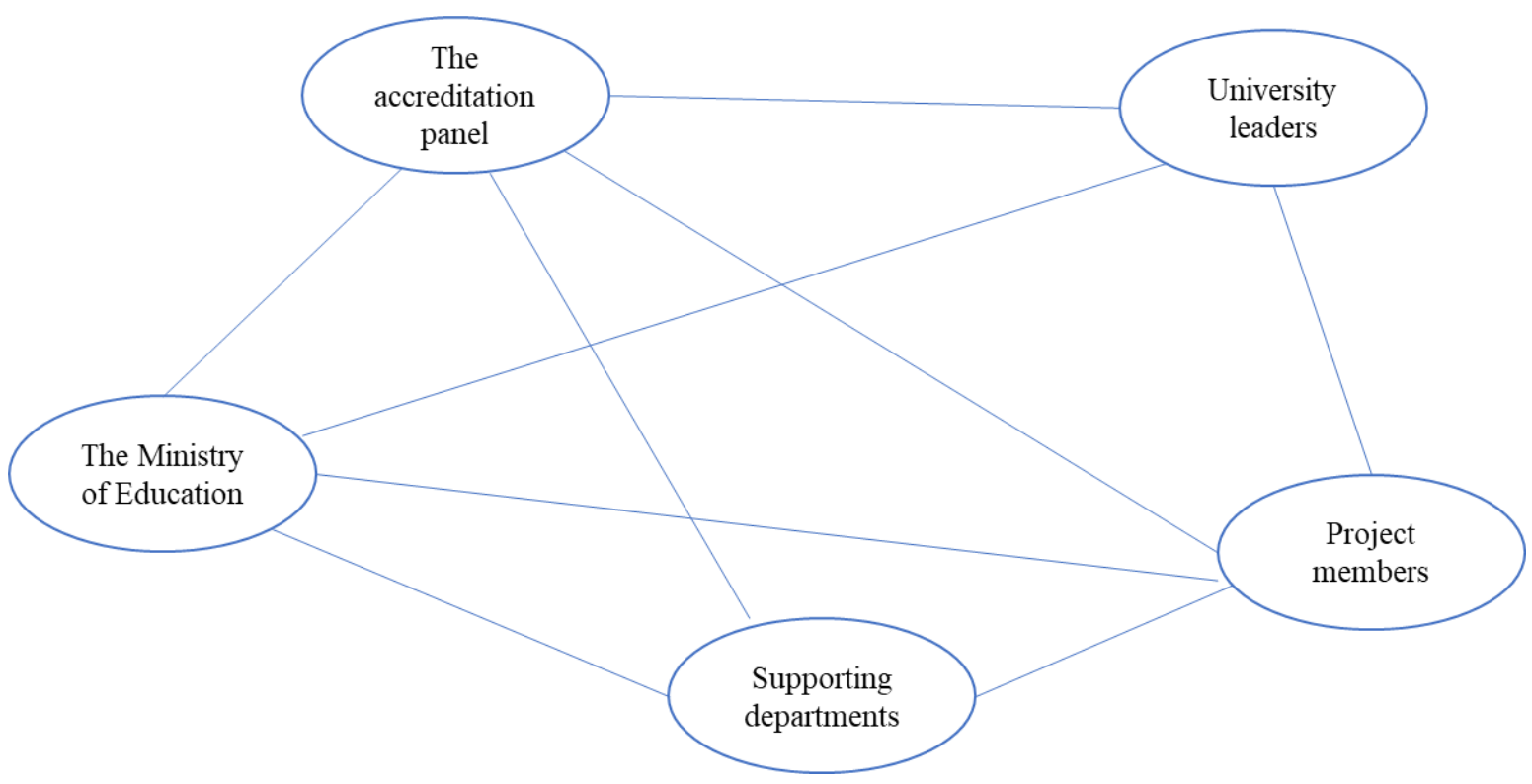


In the above figure, the network represents Thu's thinking of how the stakeholder interactions in the MTEO project should be. The circles represent the project stakeholders, and the lines represent their interactions. Thu's network at T1 differed from Hung's at the same time. Thus, his constructs and ratings suggested that he expected the project stakeholders to be the university community, project members, and government. He also thought that these stakeholders should have interactions such as the project must benefit the university and community, the project results must be clear and objective, and the governmental policies should moderately support the project. Hung's expectation of the stakeholder interactions at T1 is illustrated in Figure 7.3 as follows.

Figure 7.3 Hung's thinking of how the stakeholder interactions in the MTEO project should be at $T 1$

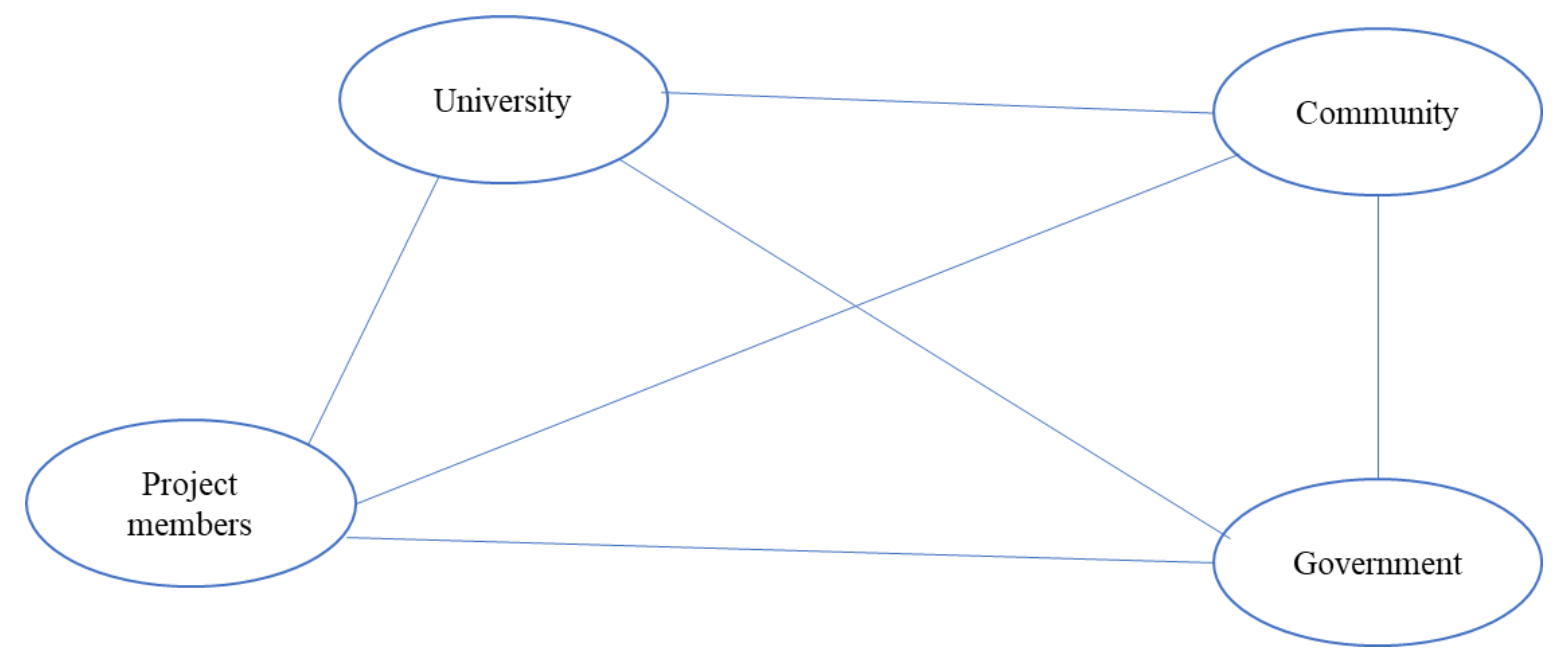

In summary, the thinking of individual stakeholders about the ideal and less than ideal projects, as presented in their repertory grids, indicates their expectation of how the stakeholder interactions in the target projects should be. Following this expectation, they appreciate the stakeholder interactions in the target projects. The next sections identify the nature and dynamics of the standards that individual stakeholders use to appreciate their projects.

\subsubsection{Nature of Individual Stakeholders'Standards}

Along with stakeholder expectation of how stakeholder interactions in projects should be, their standards for projects at a particular time point are their standards for stakeholder interactions in projects. Primary standards refer to the interactions that are important to projects and decide whether projects are good or bad. Secondary standards refer to the interactions that are not important but should be considered in projects. For example, Thu's primary standards in the MTEO case at T1 were that the university characteristics and activities regarding the 
accreditation must be good. Her secondary standard was that the characteristics of the university and the accreditation must match each other. These standards are illustrated in Figure 7.4 as follows.

Figure 7.4 Thu's primary and secondary standards for stakeholder interactions in the MTEO project at $T 1$

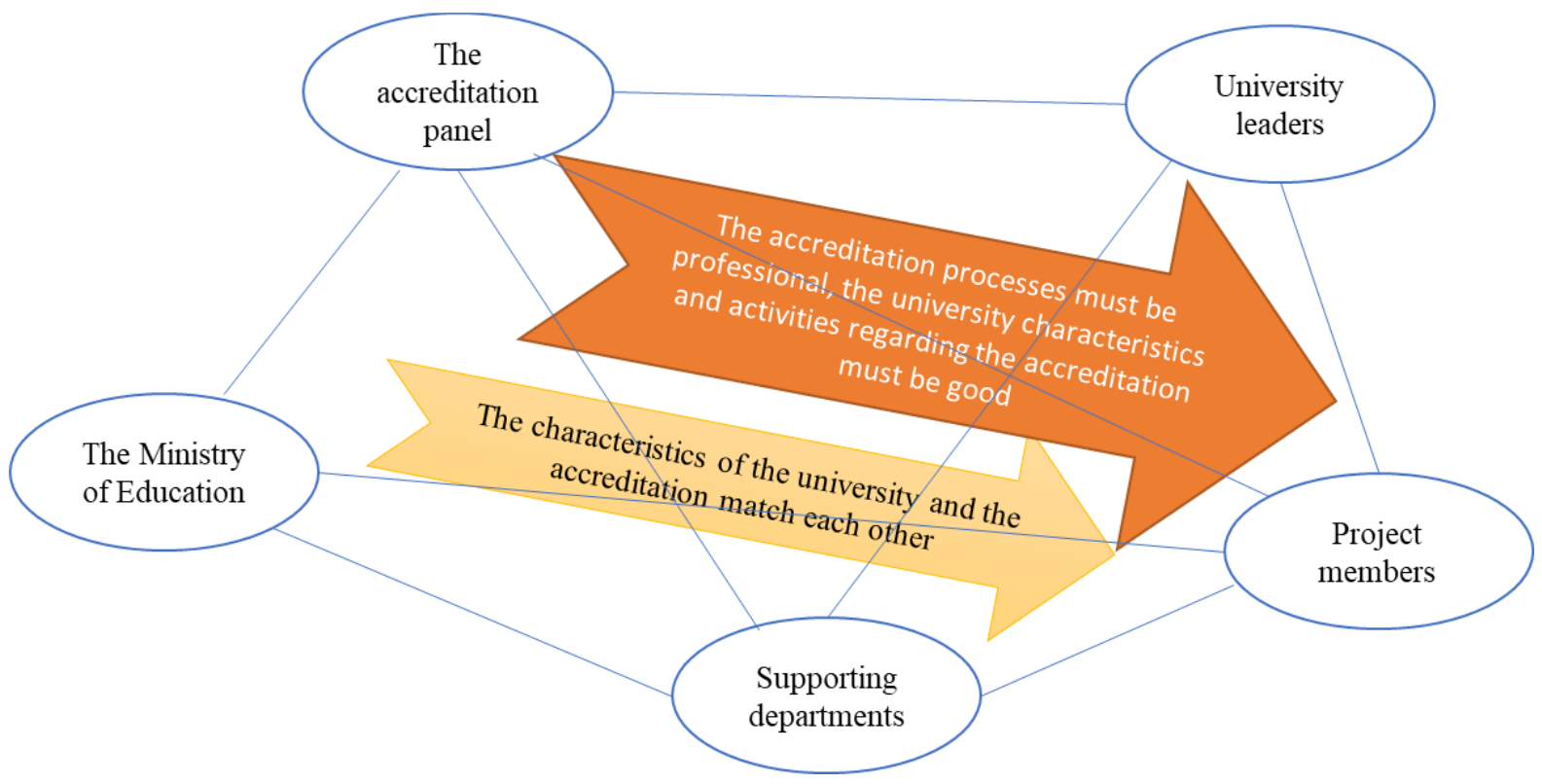

The network in the above figure describes Thu's expectation of how stakeholder interactions in the MTEO project should be based on the explanation provided in section 7.3.1. The arrows describe her standards for these interactions or her thinking of the importance of the interactions. The orange arrow describes the primary standards, and the brown arrow describes the secondary standard. Similar to Thu, Hung also had three standards for the MTEO project at T1. His primary standards were that the project must benefit the university and community and that the project implementation must be easy and good. One of his secondary standards was that the human resources of the project must match its importance. Other secondary standards were that the objectivity of the project results and the governmental support must be moderate. Hung's primary and secondary standards are illustrated in Figure 7.5 by the orange and brown arrows, respectively, as follows. 


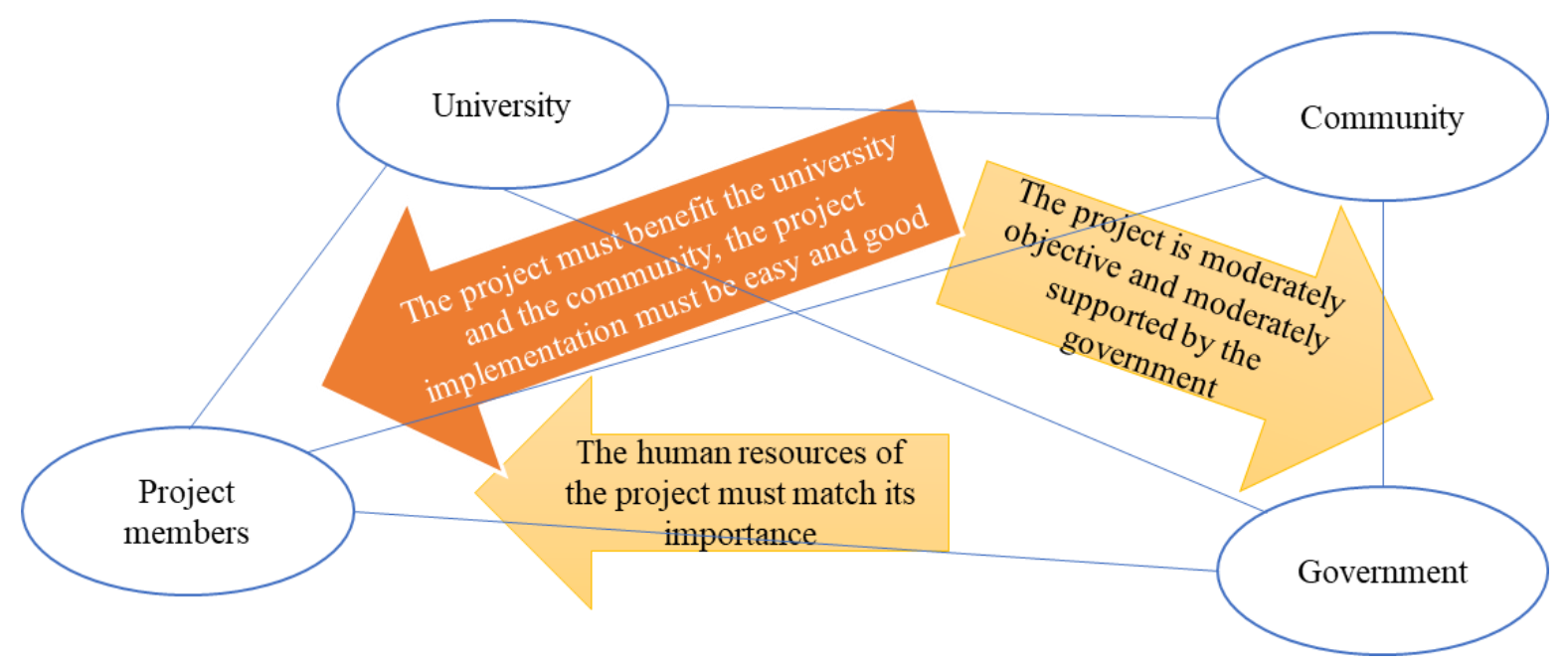

Similar to Hung and Thu, other participants' primary and secondary standards for their projects at a specific time point can also be interpreted as their standards for the stakeholder interactions in the projects. This interpretation provides a way to describe the individual characteristics of stakeholders that affect the dynamics of their perception. The stakeholder management and project management literature have described different characteristics of individual stakeholders that affect their perception and interaction. These characteristics are the interests of stakeholders (Argenti, 1997; Coombs \& Gilley, 2005; Hjorts $\varnothing$ et al., 2005; Jansson, 2005), their objectives and priorities (Pouloudi \& Whitley, 1997; Wolfe \& Putler, 2002), privacy concerns (Fedorowicz et al., 2010), values (Agle et al., 1999; Introna \& Pouloudi, 1999; Pouloudi, Currie, \& Whitley, 2016), identity awareness (York et al., 2016); care about fairness (Bridoux \& Stoelhorst, 2014), "relational models" (Bridoux \& Stoelhorst, 2016, p. 230) and beliefs (Aaltonen, 2011). However, very few studies describe the individual characteristics of stakeholders that affect the dynamics of their perceptions, as identified in this study. Based on this identification, the characteristics of stakeholders that affect their interaction and perception can be described as their standards for stakeholder interactions related to aspects of projects. For example, concerns about fairness of stakeholders (Bridoux \& Stoelhorst, 2014) can be described as their standards for stakeholder interactions related to the fairness in projects. The privacy concerns of individual stakeholders (Fedorowicz et al., 2010) can be described as their standards for the stakeholder interactions related to privacy protection in projects.

In summary, this section has identified that individual stakeholders' standards for projects can be interpreted as their standards for stakeholder interactions in projects. This 
identification provides a way to describe the characteristics of individual stakeholders that affect stakeholder dynamics. This identification also provides a way to understand other characteristics of individual stakeholders that have been identified as affecting stakeholder interaction and perception by existing studies.

\subsubsection{Dynamics of Individual Stakeholders' Standards}

The data presented in this study showed that during project implementation, individual stakeholders' standards for stakeholder interactions in projects can stay the same or change.

\section{Stay the same}

The primary and secondary standards of each individual stakeholder for stakeholder interactions in projects can stay the same during project implementation. For example, in the MTEO case, Thu's primary and secondary standards remained unchanged over the four interviews. The primary standards were that the accreditation must be professional and that the

university characteristics and activities regarding the accreditation must be good. The secondary standard was that the university characteristics must match the professionalism of the accreditation processes. The standards of Khiem and Rafat from the APSC case also did not change during the data collection period. Khiem's primary standards were that the accreditation must benefit the university and the management of the project complexity must be good. Rafat's primary standards were that the characteristics and determination of the university regarding the accreditation must be good and his secondary standard was that the extent to which the university needed to work to obtain the accreditation should be moderate. Finally, in the ABCD case, Amish's standards for the project also remained the same from T1 to T5. His primary standards were that the resources and commitment of the university regarding the accreditation must be good. His secondary one was that the commitment of the university must match its resources for the accreditation.

\section{Change}

It is also possible, however, that individual stakeholders' standards for projects change during project implementation. These changes can happen even when their expectation of stakeholder interactions in projects is unchanged. For example, between T5 and T6, Amish had the same expectation of who the stakeholders of the $\mathrm{ABCD}$ project should be and how they should interact. Even though both this expectation and Amish's primary standard were unchanged, his secondary standard changed. At T5, the secondary standard was the gap between the resources and commitment of the university regarding the accreditation. At T6, however, it was the gap 
between two groups of other aspects of the project: the commitment of the program director and the related departments; and the resources of the university and the commitment of the committee members and the top leader. The same dynamic pattern can be observed with regard to Nikki's standards for the ABDC project. From T1 to T4, she had the same expectation of who the stakeholders of the project should be and how they should interact. However, while her primary standards stayed the same, her secondary one changed. At T1 and T3, her secondary standard was the gap between the internal resources and efforts of the committee, and its abilities to mobilise the external resources and efforts. At T2 and T4, the secondary standard was the gap between the university's desire and its resources and efforts for the accreditation. These changes in Nikki's standards are illustrated in Figure 7.6 as follows. 
Figure 7.6 Changes in Nikki's standards for the ABCD project between T1 and T2, while her expectation of the overall stakeholder interactions remained the same
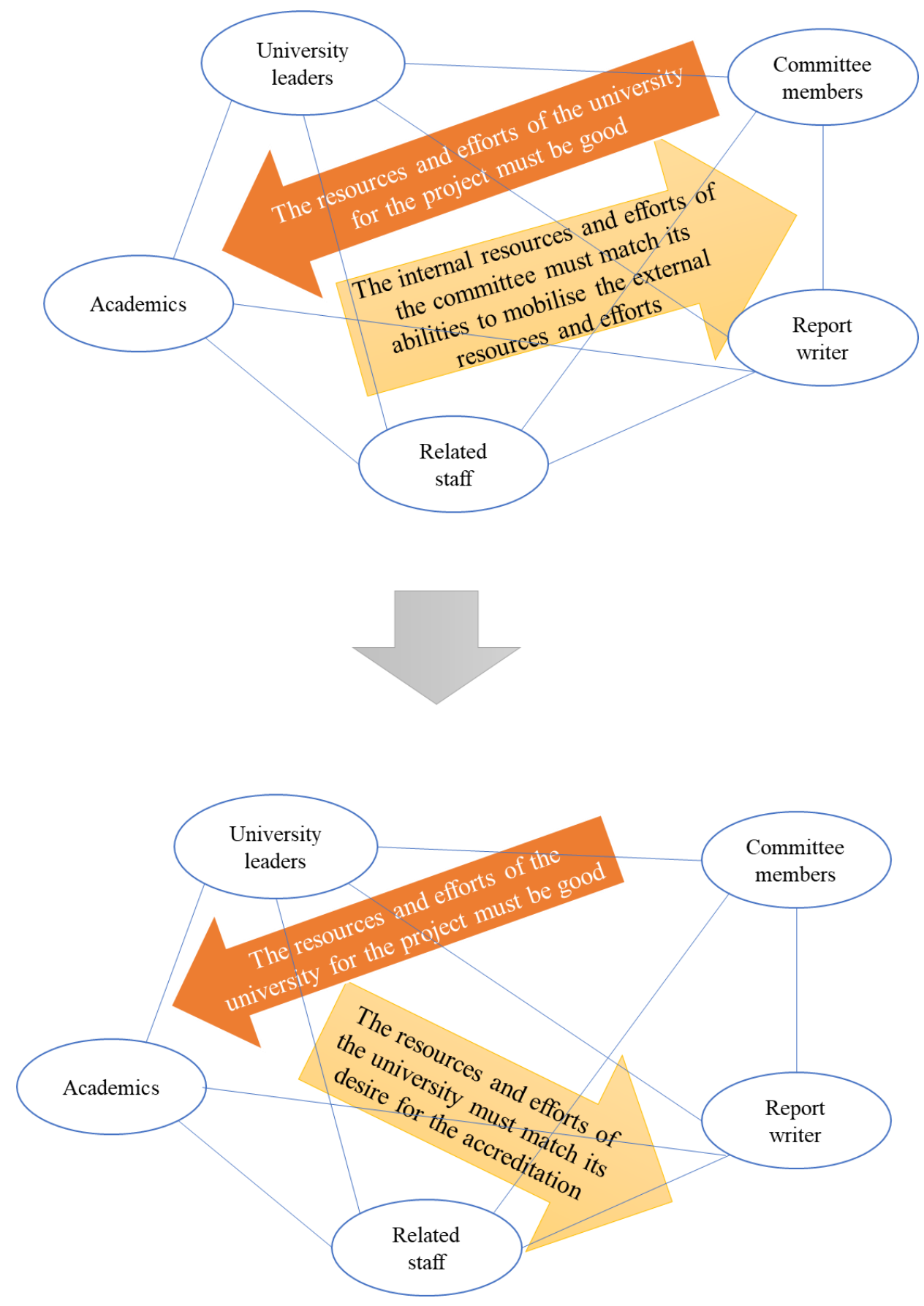

The stakeholder network in the figure describes Nikki's expectation of stakeholder interactions in the project, which is the same between $\mathrm{T} 1$ and $\mathrm{T} 2$. The orange arrow, which describes her primary standards, stays the same, but the brown arrow, which describes her secondary standard, changes. 
Individual stakeholders' standards for projects can also change when their expectations of stakeholder interactions in the project change. For example, in the MTEO case, Nga's expectation of the stakeholder interactions in the project increased and included the panel's strict requirements and the suitable project implementation steps at her second interview (T2). Along with these changes, her primary standards also changed to include these new interactions. Another example of this pattern can be found in the APSC project data. At his second interview, Tung's expectation of the overall stakeholder interactions in the project changed to include the faculty qualifications. Along with these changes, both his primary and secondary standards changed and included these new interactions.

Sumy from the ABCD case is another participant who changed her standards while maintaining her expectations for stakeholder interaction. Thus, between her first and second interviews, Sumy's expectations of the stakeholder interactions in the project increased to also include the committee's capabilities in planning and time management. Along with these changes, her primary and secondary standards also changed to include these new stakeholder interactions. Furthermore, between her third and fourth interviews, Sumy's expectation of the overall project stakeholder interactions decreased to exclude the consolidation of different accreditations. Along with these changes, her primary standards changed, too, so that they no longer included this consolidation. Her secondary standard, meanwhile, changed from the gap between the capabilities to consolidate different accreditations and other capabilities of the university and committee to the gap between the capabilities in time management and planning and other capabilities of the committee. The changes in Sumy's standards between her third and fourth interviews are illustrated in Figure 7.7 as follows. 
Figure 7.7 Changes in Sumy's standards for the ABCD project between T3 and T4, while her expectation of the overall stakeholder interactions changed
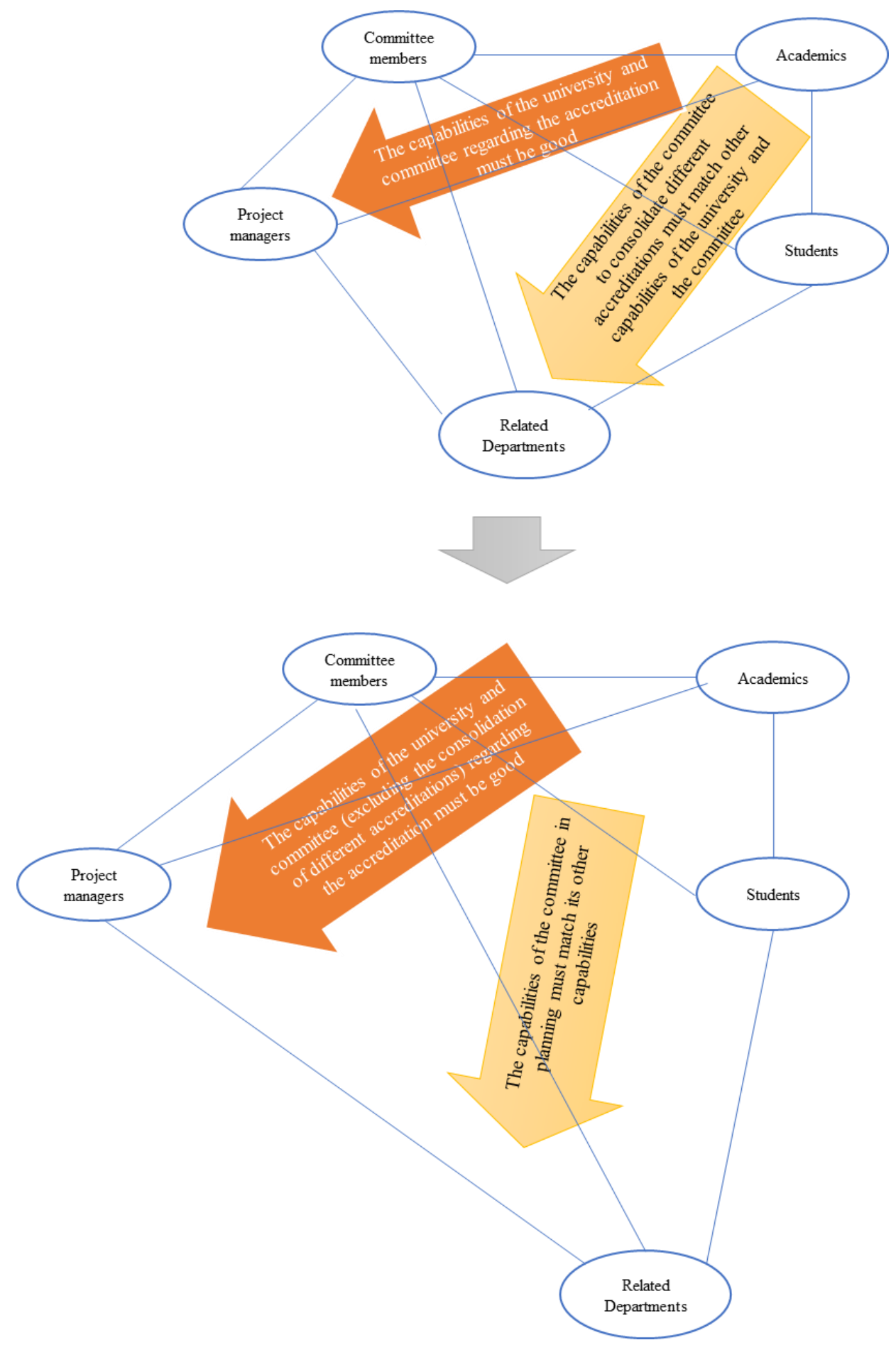

The stakeholder network as illustrated in the figure describes how Sumy's expectations of the overall stakeholder interactions in the project change. Along with this change, Sumy's primary and secondary standards, captured by the orange and brown arrows, respectively, also change. 
In summary, individual stakeholders' standards for stakeholder interactions in projects are dynamic during project implementation. The standards can stay the same or change. These dynamics provide more understanding of the dynamics of individual characteristics of stakeholders. Various studies have described the dynamics of stakeholder interaction, such as stakeholder composition (Pouloudi \& Whitley, 1997) or stakeholder participation (Green \& Hunton-Clarke, 2003). Studies have also described the impact of individual characteristics of stakeholders on stakeholder perceptions and interactions (e.g., Aaltonen, 2011; Bridoux \& Stoelhorst, 2014, 2016; York et al., 2016). However, not many studies have yet described the dynamics of individual characteristics of stakeholders. As section 7.3.2 has discussed that individual stakeholders' standards for stakeholder interactions can provide more understanding of other characteristics of individual stakeholders, the dynamics of these standards provide a way to describe the dynamics of these characteristics.

\subsection{Nature and Dynamics of Appreciation of Individual Stakeholders}

\subsubsection{Nature of Appreciation of Individual Stakeholders}

The appreciation of individual stakeholders regarding their projects can be interpreted as the stakeholder interactions that stakeholders focus on and their levels of concern about these interactions. Following their standards for projects, each individual stakeholder focuses on particular stakeholder interactions in projects. These focal interactions can be primary or secondary, depending on whether the standards are primary or secondary. For example, in the APSC case, following his three standards, the primary focal interactions of Hung were the benefits, ease and implementation of the project. His secondary focal interactions were the gap between the project's human resources and its importance, the objectivity of the project results and the governmental support of the project. Another example would be Nikki from the ABCD case. In her first interview, following her standards, Nikki's primary focal interactions were the university resources and efforts for the accreditation. Her secondary focal interaction was the gap between the internal resources and efforts of the committee and its abilities to mobilise the external resources and efforts.

Individual stakeholders' appreciation of these focal interactions marks their levels of concern about the interactions. Because the primary focal interactions decide whether the projects are ideal or less than ideal, the appreciation of these interactions indicates how ideal projects are. If stakeholders think that the interactions are good, they will have low levels of 
concern about the interactions. If stakeholders think that the interactions are not good, they will have high levels of concern about the interactions. Meanwhile, the appreciation of the secondary focal interactions indicates whether these interactions are noticeable or unnoticeable in projects. If stakeholders think that these interactions are noticeable, they will have high levels of concern about the interactions. If stakeholders think that these interactions are not noticeable, they will have low levels of concern about the interactions. Overall, individual stakeholders' appreciation of projects is identified as their levels of concern about their focal interactions in projects.

An example of this pattern is Hung's appreciation of the MTEO case. In his first interview, regarding his primary focal interactions, Hung appreciated that the benefits, ease, and implementation of the project were relatively good. Because of this, he had a low level of concern about these interactions. Meanwhile, regarding the first secondary interactions that he focused on, Hung appreciated that the gap between the human resources and the importance of the project was relatively large and noticeable in the project. Regarding the other secondary interactions that he focused on, Hung thought that the project was not very objective and not supported by governmental policies. He, therefore, thought that these interactions were noticeable because they were different from his expectation of the moderate levels of the objectivity and governmental support of a "normal" project. Hung, therefore, had high levels of concern about all these secondary interactions. Hung's levels of concern about the MTEO project at T1 are illustrated in Figure 7.8 as follows. 


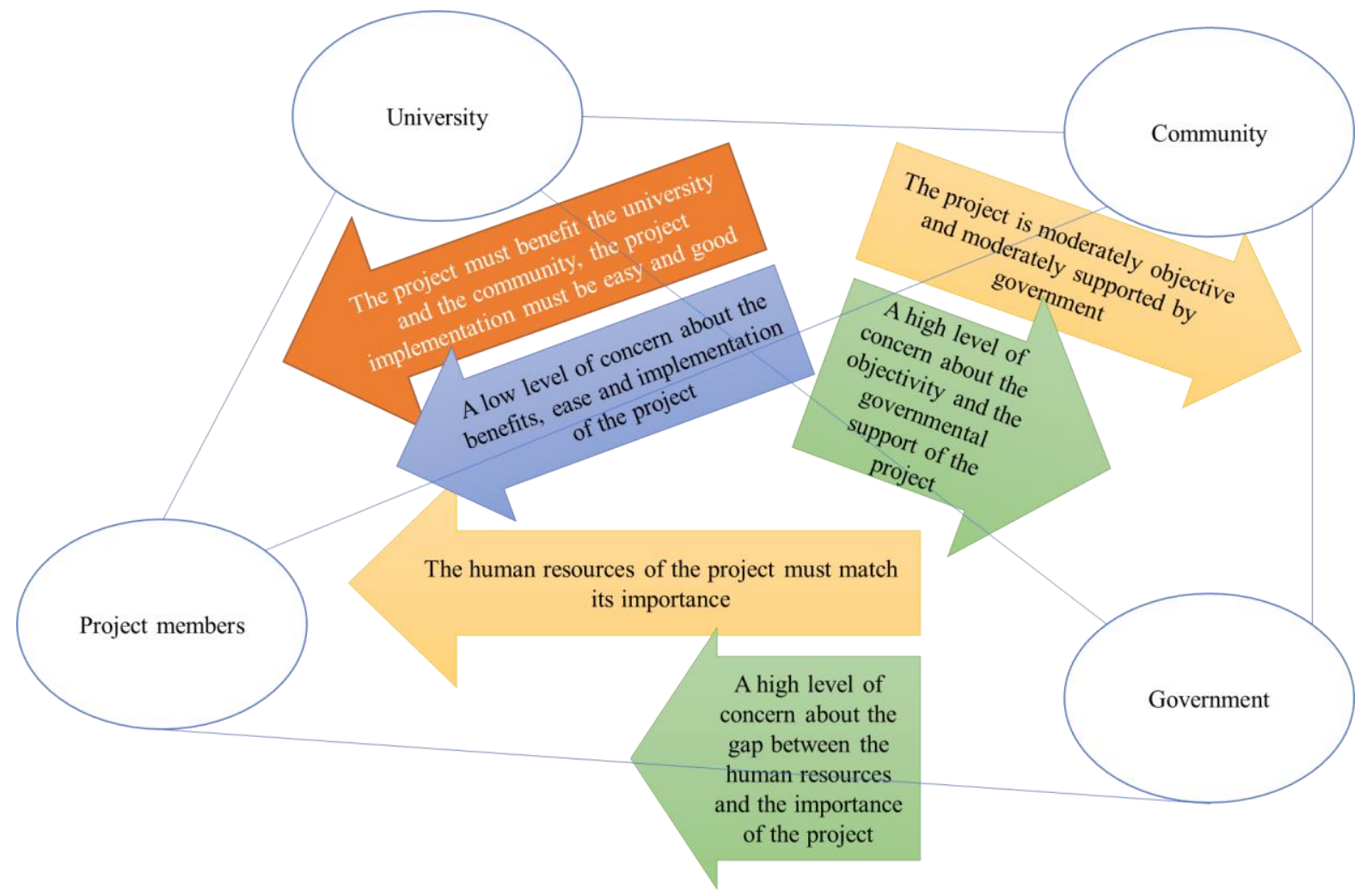

The figure is an extension of Figure 7.5 provided in section 7.3.2. Thus, as previously discussed, Hung's primary and secondary standards for stakeholder interactions in the MTEO project are described by the orange and brown arrows, respectively. His levels of concern about these interactions are described by the blue and green arrows, respectively. The parallels between the concern arrows and the standard arrows illustrate that the standards determined the focal stakeholder interactions. The differences between the lengths of the concern and the standard arrows represent Hung's levels of concern. The larger the differences, the higher the levels of concern and vice versa.

Another example of this pattern is displayed by Nikki from the ABCD case. At her first interview, Nikki had particular levels of concern about her focal interactions. Regarding the primary ones, she thought that the university resources and efforts for the project were not very good. Regarding the secondary ones, she thought that the gap between the internal resources and efforts of the committee and its abilities to mobilise the external resources was large and noticeable. Because of this, Nikki had high levels of concern about both her primary and secondary focal interactions. In the same way as presented here for Hung and Nikki, other participants' appreciation of their projects at particular time points was also identified as their levels of concern about the stakeholder interactions that they focused on. 
This observation regarding how stakeholders' appreciation is formed provides greater insights into the dynamics of their perception, as influenced by their individual characteristics. Research has described the dynamics of the perception of stakeholders without considering stakeholders' individual characteristics. For example, in the stakeholder literature, Eesley and Lenox (2006) and Fassin (2010) described the dynamics of the perception of focal organisations about stakeholder salience. In the project management literature, Liu and Chiu (2016), Cuppen et al. (2015), Besson and Rowe (2001), and Aaltonen et al. (2008) described the dynamics of stakeholder perception about project risks, what should be done to projects, project visions, and stakeholder salience, respectively. In line with these existing studies, this research offers a method to describe the dynamics of stakeholder perception that also reflects their individual characteristics. Thus, the dynamics of stakeholder perception can be described as the dynamics of their appreciation of stakeholder interactions related to particular aspects of projects. For example, the dynamics of stakeholder perception of project risks (Liu \& Chiu, 2016) can be described as the dynamics of stakeholders' appreciation of stakeholder interactions related to project risks. The dynamics of stakeholder perception of project visions (Besson \& Rowe, 2001) can be described as the dynamics of stakeholders' appreciation of stakeholder interactions related to project visions.

In summary, the appreciation of projects by individual stakeholders is identified as their focal interactions and levels of concern about these interactions. This identification provides a deeper understanding of the dynamics of stakeholder perception, as influenced by their individual characteristics.

\subsubsection{Dynamics of Appreciation by Individual Stakeholders}

The dynamics of individual stakeholders' appreciation about projects are created by the dynamics of their focal interactions and their levels of concern about these interactions. Following the dynamics of stakeholders' standards for projects, the focal interactions of stakeholders can stay the same, or change during project implementation. Stakeholders' levels of concern about these interactions, meanwhile, can stay the same, decrease, or increase. Following the discussion of the standards in section 7.3.3, this section focuses on the dynamics of the levels of concern.

\section{Stay the same}

During project implementation, the levels of concern of individual stakeholders about their focal interactions in projects can stay the same. This can happen when stakeholders learn no 
event or idea related to projects, or when the events and ideas that they learn just confirm their existing levels of concern. For example, Sumy (ABCD case) did not receive information related to the project between $\mathrm{T} 2$ and $\mathrm{T} 3$ and did not change her levels of concern about the project in that time. Between T7 and T8, Sumy also attended the last meeting of the accreditation committee and received information relating to the project. However, this information did not change her appreciation of the project. Sumy still thought that the capabilities of the university and committee were good and that the gap between the planning capabilities and other capabilities of the committee was small. Her levels of concern about these interactions in the project, therefore, did not change.

\section{Decrease}

During project implementation, the levels of concern of individual stakeholders about their focal interactions can decrease. This can happen when the focal interactions stay the same. For example, while Amish from the ABCD case focused on the same primary and secondary interactions, his levels of concern about them decreased between T1 and T2. Regarding the primary ones, Amish thought that the resources and efforts of the university for the project improved. Regarding the secondary one, he thought that the gap between the commitment and resources of the university diminished. A decrease in concern can also be observed in the data from Tung from the APSC case from T2 to T3 and T3 to T4 while his focal interactions stayed the same. Regarding the primary ones, Tung thought that the transparency of the accreditation and the university efforts to do the project improved. Regarding the secondary one, he thought that the gap between the research and faculty qualifications of the university and the accreditation standards diminished.

The levels of concern of individual stakeholders about their focal interactions can also decrease when those focal interactions change. Thus, the secondary interactions that Nikki from the ABCD case focused on changed between T1 and T2. At T1, Nikki focused on the gap between the internal resources and efforts of the committee and its abilities to mobilise the external resources and efforts. At T2, she focused on the gap between the resources and efforts of the university and its desire for the accreditation. Along with these changes, Nikki thought that the gap related to the internal and external resources and efforts at T1 was larger and more noticeable than the gap related to the desire at T2. Because of this, Nikki's level of concern about the secondary interactions at T2 was lower than it was at T1. Nga from the MTEO case provides another example for a decrease in concern in conjunction with a change in focal interactions. Thus, between T2 and T3, Nga included the panel's requirements and the project 
implementation steps in her primary focal interactions, which was the project effectiveness and efficiency. Along with these changes, she thought that the project effectiveness and efficiency improved. Her level of concern about these aspects of the project, therefore, decreased.

\section{Increase}

During project implementation, individual stakeholders' levels of concern about the focal interactions can increase. This can happen when the focal interactions stayed the same. For example, in the ABCD case, Amish's focal interactions, both the primary and secondary, were unchanged between T7 and T8. However, regarding the primary ones, Amish thought that the resources and commitment of the university regarding the accreditation had worsened. Regarding the secondary one, he thought that the gap between the resources and commitment of the university at T8 was larger and more noticeable than it was at T7. Amish's level of concern about these both interactions, therefore, increased. In another example, the primary interactions that Khiem focused on in the APSC case, namely the benefits of the accreditation and the management of the project complexity, stayed the same between T2 and T4. However, because Khiem thought these aspects of the project had worsened during this time, his level of concern about them increased.

The levels of concern of individual stakeholders about their focal interactions can also increase when these focal interactions change. For example, with more understanding of the project, Hung from the MTEO case realised that the governmental support was important and changed it from the secondary to primary focal interactions between T2 and T3. Regarding the new primary interactions, Hung thought that the benefits, ease, and implementation of the project had worsened. Regarding the new secondary ones, he thought that the project objectivity decreased, and the issue related to it became more noticeable than it had been before. Hung's levels of concern about both these interactions, therefore, increased. Another example of this type of increase in concern can be seen in the case of Tung from the APSC case at his second interview. At this interview, Tung added the faculty qualifications to both his primary and secondary focal interactions. Along with these additions, regarding the new primary interactions, Tung thought that the transparency of the accreditation and the university efforts to do the project had worsened. Regarding the secondary interactions, he thought that the gap between the research and faculty qualifications of the university and the accreditation standards had widened. Because of this, his levels of concern about both the focal interactions increased. 
This section has shown that individual stakeholders' appreciation of stakeholder interactions in projects can change in two ways. In the first, the focal interactions of individual stakeholders can stay the same or change. In the second, the levels of concern of individual stakeholders about these interactions can stay the same, increase, or decrease. These two ways are in line with the ways changes in stakeholder perception are described in the existing literature. Specifically, the existing literature has shown that the content of stakeholder perception can change. Thus, research has noted changes with regard to stakeholders' stances on or expectations of projects (Pan, 2005), their thinking about what should be done to a project (Cuppen et al., 2015), and their perceptions of projection visions (Besson \& Rowe, 2001). These content changes are in line with the changes in the focal interactions of stakeholders. The existing literature also shows that stakeholder perception can have changes in their levels. For example, Fassin (2010), Eesley and Lenox (2006), and Aaltonen et al. (2008) demonstrated that the perception of focal organisations or projects about stakeholder salience may increase or decrease. Liu and Chiu (2016) found that stakeholder perception of project risks may also increase or decrease. These level changes are in line with the changes in the levels of concern of stakeholders, which have been identified in this research. Overall, the dynamics of stakeholders' appreciation of projects give more insights into the dynamics of stakeholder perception, which have been described in the existing stakeholder and project management literature.

\subsection{Proposed Framework of the Dynamics of Individual Stakeholders' Appreciation}

\subsubsection{Framework of the Dynamics of Individual Stakeholders' Appreciation}

As explained in the methodology chapter, this research applies Vickers' concept of appreciative systems to study the dynamics of stakeholders' appreciation of projects, as influenced by their standards and perceptions of events and ideas. In the above sections, the nature and common themes of these dynamics have been identified. Based on this identification, a framework to describe the dynamics of individual stakeholders' appreciation of projects and their drivers is proposed. For the purpose of presentation, the framework is presented in two figures. Figure 7.9 describes it in general, and Figure 7.10 describes it in detail. 
Figure 7.9 Dynamic relationships between individual stakeholders' appreciation of projects and their drivers

The flux of events and ideas unfolding through time

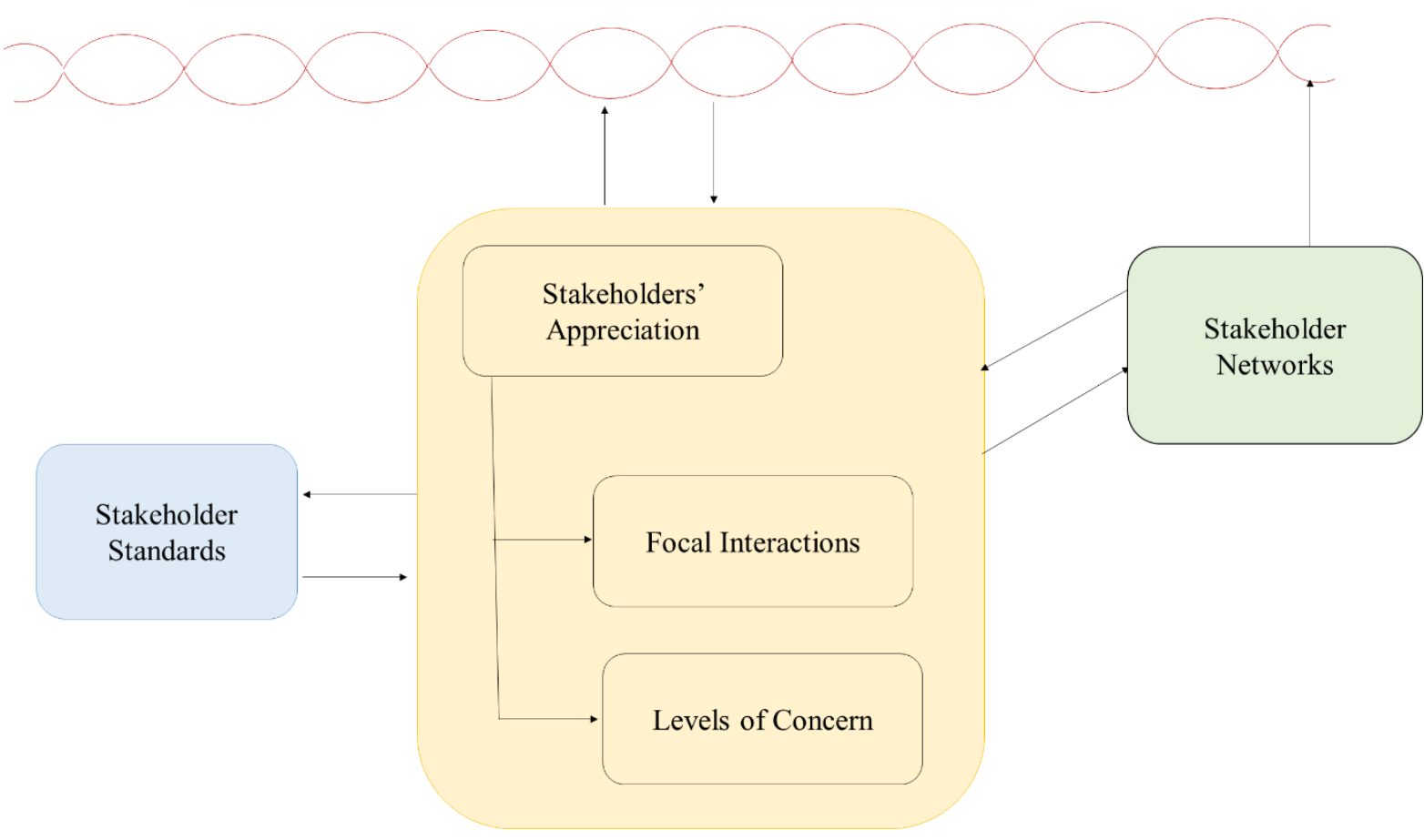

The above framework was developed from the framework presented in section 7.2.2 (Figure 7.1) to include descriptions that capture the appreciation of individual stakeholders. Specifically, stakeholders' appreciation of projects consists of their focal interactions and levels of concern about these interactions. There are dynamic relationships between this appreciation, the standards of stakeholders for projects, stakeholder networks, and the flux of events and ideas. These dynamic relationships explain how the standards and networks of stakeholders are the drivers of the changes in stakeholders' appreciation of projects. These dynamic relationships are illustrated in detail in Figure 7.10 as follows. 
Figure 7.10 Dynamics of individual stakeholders' appreciation of projects and their drivers

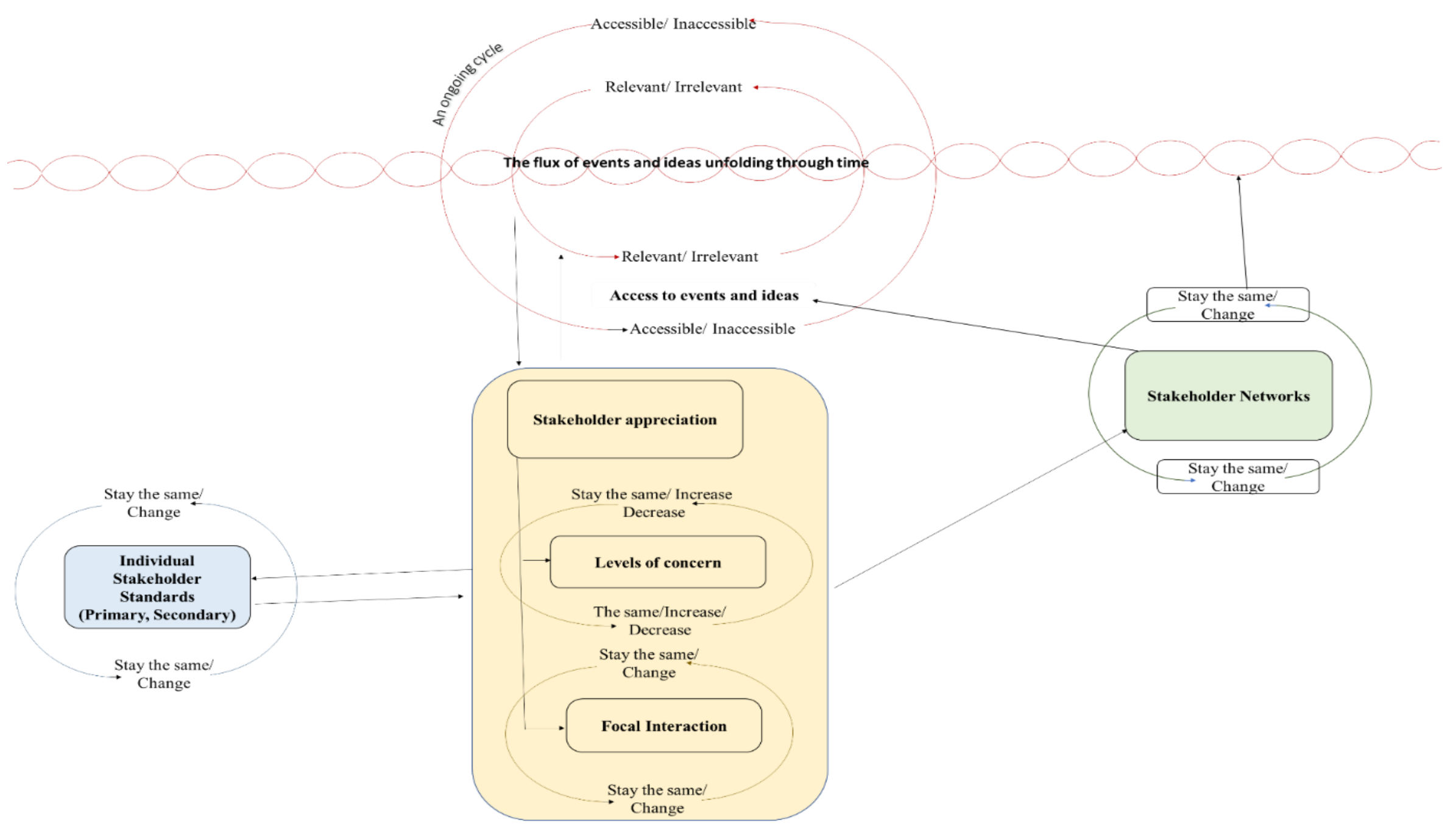


As illustrated in the above figure, at each time point, with their perceptions of events and ideas related to projects, stakeholders have standards for stakeholder interactions in projects. These standards can be primary or secondary. Primary standards are important and decide whether projects are ideal or less than ideal. Secondary standards are not important but should be considered in projects. Following these standards, stakeholders have primary and secondary focal interactions in projects and have different levels of concern about these interactions. If stakeholders think that these interactions are good or unnoticeable, they will have low levels of concern about them. If stakeholders think that these interactions are not good or noticeable, they will have high levels of concern about them.

During project implementation, stakeholder networks, including the interactions related to their roles in their projects and wider organisations and their other informal communication, can stay the same or change. On the one hand, these networks create fluxes of events and ideas. On the other hand, they affect stakeholder access to events and ideas. For each accessible event and idea, stakeholders may deem them relevant or irrelevant to their projects. Along with these perceptions of events and ideas, the standards of stakeholders for the interactions in projects can stay the same or change. The focal interactions of stakeholders in projects can also stay the same or change. Stakeholders' levels of concern about these interactions can also stay the same, increase, or decrease. With the new standards and appreciation, stakeholders will engage in new interactions in their social networks, learn new events and ideas, and have different dynamics in their standards and appreciation. Overall, the framework explains the dynamics of individual stakeholders' appreciation of projects, as influenced by their networks and standards.

\subsubsection{An Example of the Application of the Framework}

In this section, the changes in the appreciation of one participant regarding their project are described by using the proposed framework. This participant is Tung, the project manager in the APSC case. At his first interview (T1), with his experience, Tung's primary standards for the project were that the accreditation must be transparent and that the university efforts must be good. His secondary standard was that the research activities must match the accreditation standards. Following these standards, at T1, Tung primarily focused on the transparency of the accreditation and the university efforts to do the project and secondarily focused on the gap between the research activities and the accreditation standards. Tung specifically thought that the accreditation was very transparent, the university efforts were not very good, and the gap 
between the research activities and the accreditation standards was large. Because of this, Tung had high levels of concern about both his primary and secondary focal interactions.

Between T1 and T2, as the manager of the project and the vice-rector of the university, Tung's social network changed. He engaged in different interactions, such as participating in the panel visit by another accreditation organisation and communicating with the project administrator. Tung, therefore, had access to particular events and ideas and found particular events and ideas relevant to the project. Examples of these relevant events and ideas were the external assessment of the student quality, the increase in the involvement of the supporting departments, and the comments of the panel from another accreditation organisation on the qualifications of the faculty. These events and ideas made Tung realise the importance of the faculty qualifications and added it to both his primary and secondary standards. Along with these standard changes, Tung also changed his focal interactions in the MTEO project. Specifically, he primarily focused on the transparency of the accreditation and the university efforts to do the project, including the faculty qualifications. He secondarily focused on the gap between the research and faculty qualifications and the accreditation standards. Because Tung thought that the overall university efforts decreased and the gap between the research and faculty qualifications and the accreditation standards enlarged, his levels of concern about these primary and secondary interactions increased.

Between T2 and T3, with his position in the project, Tung engaged in different interactions in his social network such as interacting with the project administrator and the regional chair of the accreditation body. From this interaction, Tung had access to different events and ideas and found particular events and ideas relevant to the project. For example, Tung saw that the university leaders' attention to the project and the faculty qualifications improved. Tung also realised that the project progress was slow and the accreditation representative lacked enthusiasm for the university. Along with these events and ideas, Tung did not change his primary and secondary standards for the project and therefore focused on the same stakeholder interactions. However, his levels of concern about these interactions changed. Regarding the primary interactions, Tung thought that the transparency of the accreditation and the university efforts improved. Regarding the secondary interactions, he thought that the gap between the research and faculty qualifications of the university and the accreditation standards diminished. Because of this, his levels of concern about these both interactions decreased. 
Between T3 and T4, as the vice-rector of the university, Tung continued engaging in different interactions in his social network. He attended the review meeting of the trimester, had access to different events and ideas in the meeting, and found particular events and ideas relevant to the project. For example, Tung learned about the improvements in the teaching activities and faculty qualifications, and the plans to re-submit the self-report and attend the accreditation conferences. Along with these events and ideas, Tung did not change his primary and secondary standards for the project and therefore focused on the same stakeholder interactions. However, his levels of concern about these interactions changed. Regarding the primary interactions, Tung thought that the university efforts to do the project improved. Regarding the secondary interactions, he thought that the gap between the research and faculty qualifications of the university and the accreditation standards diminished. Because of this, his levels of concern about these both interactions decreased.

Similar to Tung, the changes in other participants' appreciation of their projects can be described by applying the proposed framework. The framework, therefore, provides a way to describe the dynamics of stakeholders' appreciation of projects, as influenced by their social networks and individual characteristics.

\subsubsection{Discussion of the Framework as the Main Research Findings}

The framework illustrates the impact of the standards and social networks of individual stakeholders on the dynamics of their appreciation of projects. While the social networks reflect stakeholders' present interactions, the standards are based on their distinctive life and work experience and reflect their individual characteristics. Some of these aspects have been discussed previously in different related fields. For instance, Pouloudi et al. (2016) and Sloan and Oliver (2013) in the stakeholder literature and Aaltonen et al. (2008) and Pan (2005) in the project management literature have explored the influence of stakeholder networks on the dynamics of stakeholder perception. Studies such as Agle et al. (1999), Coombs and Gilley (2005), Fedorowicz et al. (2010), and Weitzner and Deutsch (2015) in the stakeholder literature and Aaltonen (2011), Ojiako et al. (2014), and Tukiainen et al. (2010) in the project management literature have described the effects of individual characteristics of stakeholders on their perception. However, very few studies describe how the individual characteristics that reflect the experience of stakeholders interact with their present interaction and affect the changes in their perception. Explaining this effect, therefore, is the biggest contribution of this research. 
By addressing the impact of these individual characteristics of stakeholders on the changes in their perception and thinking, the research empirically describes the complex ways in which the perception of individual stakeholders about their projects change. Stakeholders can focus on the same stakeholder interactions in projects or change the interactions that they focus on. Their levels of concern about these interactions can stay the same, increase, or decrease. The drivers of these changes, which are the standards and social networks of stakeholders, can also stay the same or change. This complexity is in line with the complexity caused by the dynamics, complex interaction and individual differences of stakeholders that scholars in the stakeholder literature such as Beaulieu and Pasquero (2002), Johnson et al. (2008), Pouloudi et al. (2016), and Solaimani et al. (2013) have theoretically argued. This complexity is also in line with the complexity of the thinking of project stakeholders that scholars in the project management literature such as Eskerod and Larsen (2018), Kreiner (1995), and Padalkar and Gopinath (2016) have theoretically argued. This research, therefore, provides an empirical illustration of these theoretical arguments.

By describing the impact of stakeholders' standards on the changes in their perception, the framework provides a way to explain the impact of individual characteristics of stakeholder on their perception. Many existing studies, such as Agle et al. (1999) in the stakeholder literature and Mazur et al. (2014) and Tukiainen et al. (2010) in the project management literature, have shown and provided their own explanation of these effects. According to the proposed framework, these effects can be explained as the impacts of stakeholders' standards for stakeholder interaction in projects on stakeholders' appreciation of particular aspects of projects. For example, Agle et al. (1999) found that the values of CEOs impact their perception of stakeholder salience. Following the proposed framework, the values of CEOs can be described as their standards for stakeholder interactions. These standards affect the stakeholder interactions that CEOs pay attention to and their levels of concern about these interactions. These together affect CEOs' perception of stakeholder salience. Tukiainen et al.'s (2010) finding can also be explained using the proposed framework. Thus, Tukiainen et al. (2010) found that the cultural values of project managers affect their perception of unexpected events of projects. Following the framework, the cultural values can be described as project managers' standards for stakeholder interactions in projects. These standards affect the interactions related to projects that project managers expect and pay attention to. These expectations, therefore, affect their perception of the unexpected events of projects. In addition, because the framework 
shows the dynamics of stakeholders' standards, it also provides more insights into the dynamics of individual characteristics of stakeholders.

The research also provides a way to explain the impact of stakeholder networks on the changes in their perception. Existing studies such as Aaltonen et al. (2008), Pan (2005) in the project management literature and Pouloudi et al. (2016) and Sloan and Oliver (2013) in the stakeholder literature have described and provided their own explanation of the impact of stakeholder networks on the changes in stakeholder perception. In line with these existing studies, the proposed framework in this research can provide another way to explain their effects. Specifically, the impact of stakeholder networks on their perception can be described as the impact of stakeholder networks on their access to the events and ideas and their perception of the relevance of the events and ideas. These perceptions, in turn, affect stakeholders' appreciation of stakeholder interactions in projects. For example, Aaltonen et al. (2008) found that stakeholder interactions, such as withholding resources or creating coalitions, can affect project teams' perception of stakeholder salience. Following the proposed framework, these effects can be described as the impact of the events and ideas related to withholding resources and creating coalitions that each project team member has access to from their social networks. These accessible events and ideas, in turn, affect the team members' perception of the relevance of the events and ideas and their appreciation of the interactions related to stakeholder salience. Sloan and Oliver's (2013) finding that critical emotional incidents can affect the trust between stakeholders can also be explained using the proposed framework. Thus, the effects can be described as the impact of the critical emotional incidents that stakeholders may have access to through their social networks and found relevant on their appreciation of the interactions related to the trust among stakeholders.

The framework can be used as an initial step to study the impact of individual characteristics of stakeholders on stakeholder interaction as well as the dynamics between different stakeholder interactions. Bosse and Coughlan (2016), Bridoux and Stoelhorst (2014), and York et al. (2016) in the stakeholder literature and Aaltonen (2011) and Purvis et al. (2015) in the project management literature have described the impact of individual characteristics of stakeholders on their interaction. Studies, such as Mahon et al. (2004) and Dorobantu et al. (2016) in the stakeholder literature, and Aaltonen and Sivonen (2009) and Butt et al. (2016) in the project management literature, have described the relationship between different stakeholder interactions. The proposed framework, meanwhile, explains that the interactions 
of stakeholders in their social networks and their individual characteristics can lead to changes in their perception of projects. Because the changes in perception, in turn, can lead to the changes in interaction, this framework can be used as an initial step to explain the effects of the individual characteristics of stakeholders on stakeholder interaction or the effects between different stakeholder interactions at individual levels.

Finally, the research demonstrates that Vickers' concept of appreciative systems, combined with the Repertory Grid Technique, can provide more insights into the dynamics of stakeholder perception of projects. Vickers' concept of appreciative systems has been applied to analyse the changes in stakeholder perception in different studies (e.g., Blackmore, 2005; Cohen-Blankshtain \& Nijkamp, 2004; Regev et al., 2011). Different methods have also been used to collect and analyse data on the components of Vickers' appreciative systems. These methods include a quantitative survey in Cohen-Blankshtain and Nijkamp (2004) and a qualitative case study in Regev et al. (2011). The Repertory Grid Technique has also been widely applied in research to study the changes in the perception of individuals (e.g., Bezzi, 1999; Large, 1976). However, very few studies use the Repertory Grid Technique to collect and analyse data on components of Vickers' appreciative systems. The current study has shown that the combination of Vickers' concept and the Repertory Grid Technique provides more insights into the dynamics of individual stakeholders' perception of projects in particular and the dynamics of stakeholder involvement in general.

In summary, the research has identified the dynamics of stakeholder appreciation of projects and the influence of individual stakeholders' standards and networks on these dynamics. The research, therefore, gives more insights into the impact of individual characteristics of stakeholders on the dynamics of their perception in particular and the complexity of stakeholder thinking and behaviour in general. Based on the findings, the study also suggests ways to study the impact of individual characteristics of stakeholders on stakeholder interactions and the dynamic relationship between these different interactions. Finally, the research demonstrates that a combination of Vickers' concept and the Repertory Grid Technique increases our understanding of the dynamics of project stakeholders.

\subsection{Chapter Summary}

This chapter has proposed a framework to describe the dynamics of stakeholders' appreciation of their projects, as influenced by their individual characteristics. While the framework is in line with the findings of existing studies that focus on the dynamics of stakeholder perception, 
it especially provides a greater understanding of the impact of individual characteristics of stakeholders on these dynamics. Based on that, it provides further insights into the complexity and changing nature of stakeholder perception and thinking at individual levels. Following this, the conclusions of the research will be presented in the next chapter. 


\section{Chapter 8 Conclusions}

\subsection{Overview of the Chapter}

Chapter seven has proposed a framework to describe the dynamics of stakeholders' appreciation of projects, as influenced by their individual characteristics. This chapter provides a conclusion to this thesis, by first revisiting the research objective and then continuing with the theoretical contributions, practical implications and methodological lessons. The chapter ends with the research limitations and recommendations for future research.

\subsection{Research Objective Revisited}

The objective of this research has been to explore the dynamics of individual stakeholders in projects. The review of the literature of stakeholder management and stakeholders in projects has identified that these dynamics can be described as the dynamics of stakeholder perception of projects, as influenced by their individual characteristics. Through the lens of Vickers' concept of appreciative systems, these dynamics are then described as the dynamics of stakeholders' appreciation of projects, as influenced by their standards and perceptions of events and ideas. Following these descriptions, empirical data from ten stakeholders in three university accreditation projects have been collected and analysed by using the Repertory Grid Technique. Based on this analysis, the nature and common themes of the changes in stakeholders' appreciation, standards, and perceptions of event and ideas have been identified. Following this identification, a framework to describe the dynamics of stakeholders' appreciation of projects and their drivers, has been proposed. The dynamics and drivers are summarised as follows.

The dynamics of individual stakeholders' appreciation of projects consist of the dynamics of their focal interactions and levels of concern about these interactions. During project implementation, stakeholders can focus on the same interactions in projects or change the interactions that they focus on. These interactions can be primary and secondary. The primary interactions are important and decide whether projects are ideal or less ideal. The secondary interactions are not important but noticeable in projects. The levels of concern of stakeholders about these interactions can stay the same, increase or decrease.

The drivers of these dynamics are the changes in the standards and social networks of individual stakeholders. From their experience and background, or in other words, their existing perceptions of events and ideas related to projects, each individual stakeholder has 
standards for stakeholder interactions in projects. These standards can be primary and secondary. Primary standards are important and determine whether projects are ideal or less than ideal. Secondary standards are not important but refer to the stakeholder interactions that should be considered. These standards affect the stakeholder interactions that individual stakeholders focus on. During project implementation, these standards can change or stay the same and affect the focal interactions of stakeholders. The social networks of stakeholders, including their roles in projects and wider organisations and other informal communication, can also affect their appreciation. From their social networks, stakeholders will have access to different events and ideas and find particular events and ideas relevant to projects. These events and ideas, along with the standards, will affect stakeholders' appreciation of projects. During project implementation, the social networks of stakeholders can stay the same or change. Along with the changes in their social networks, stakeholders can learn new events and ideas relevant to projects and change their standards and appreciation of projects. Overall, both the current social networks of stakeholders and their standards, which reflect their experience and background, are the drivers of the dynamics of stakeholders' appreciation of projects.

\subsection{Theoretical Contributions}

\subsubsection{Stakeholder Management Literature}

The biggest contribution of this research to the stakeholder management literature is providing further understanding of the dynamics of stakeholders at individual levels. In other words, the research provides further understanding of stakeholder dynamics, as influenced by their individual characteristics, which are linked to their experience and background. Studies have described the dynamics of stakeholders both theoretically (Freeman, 1984; Mitchell et al., 1997) and empirically (Agle et al., 1999; Pouloudi \& Whitley, 1997). Studies have described the influences of the present social networks of stakeholders on these dynamics (Crilly et al., 2012; Dorobantu et al., 2016). Studies have also described the impacts of stakeholders' individual characteristics, which are linked to their experience and background, on their thinking and behaviour (Bosse and Coughlan, 2016; v. Werder, 2011). However, very few studies focus on the impacts of these individual characteristics on the dynamics of stakeholder thinking and behaviour. This research gives more insights into these impacts by showing that the standards of individual stakeholders, which are products of their past experience and background, can interact with the present social networks of stakeholders and affect the dynamics of their appreciation of projects. By describing these impacts, the research also 
provides a way to explain the impacts of different individual characteristics of stakeholders on their thinking and behaviour, which have been described by existing studies such as Bosse and Coughlan (2016) and v. Werder (2011). Overall, the research emphasises the importance of studying stakeholders at individual levels or in other words, under the consideration of their individual characteristics.

By describing the complex ways in which stakeholders' appreciation of projects change, as influenced by their standards and social networks, the research provides a way to describe the complexity of stakeholder thinking and behaviour at individual levels. This complexity is in line with the dynamics, social networks and individual differences of stakeholders that have been argued theoretically by scholars such as Beaulieu and Pasquero (2002), Johnson et al. (2008), Pouloudi et al. (2016) and Solaimani et al. (2013). Given the fact that this complexity has not been empirically studied much, this research provides an empirical illustration of it. The research, therefore, addresses the limitations related to oversimplifying stakeholders and their involvement. Scholars such as Mainardes et al. (2011) have theoretically pointed out these limitations. The research also enriches the stakeholder management literature. Scholars such as Mitchell (2012) and Pedrini and Ferri (2019) have ardently called for this enrichment.

In addition to the above main contributions, the research has supplementary contributions in explaining the influence of the social networks of stakeholders on their dynamics. The importance of studying these social networks is not new and has been emphasised by many scholars such as Ackermann and Eden (2011), Fassin (2008), Pouloudi and Whitley (1997), and Rowley (1997). Many scholars have also described and explained the impacts of these networks on the dynamics of stakeholder empirically (e.g., Crilly et al., 2012; Dorobantu et al., 2016). This research is in line with the existing research in explaining these impacts. However, it goes further to explain how these networks interact with the individual characteristics of stakeholders and affect stakeholder dynamics. Overall, the research provides a deeper understanding of the impacts of stakeholders' social networks on their dynamics and emphasises the importance of studying stakeholders in their networks.

Finally, the research proposes a method, which is the combination of Vickers' concept of appreciative systems and the Repertory Grid Technique, to analyse stakeholders. Given the scarcity of applications of Vickers' concept in empirical studies (Blunden, 1994; Checkland, 2000; Williams, 2005), this research provides an empirical application of it. The research also 
shows that the Repertory Grid Technique can be applied to collect and analyse data on components of Vickers' concept.

\subsubsection{Project Management Literature}

In line with the contributions in the stakeholder management literature, the biggest contribution of this research to the project management literature is providing further understanding of the dynamics of project stakeholders at individual levels. In other words, the research provides further understanding of the dynamics of project stakeholders, as influenced by their individual characteristics, which are linked to their experience and background. Studies have described and explained the dynamics of stakeholders in projects (e.g., Boonstra, 2006; Olander \& Landin, 2005) and the influence of stakeholder networks on these dynamics (e.g., Aaltonen \& Sivonen, 2009; Williams et al., 2015). The impacts of the individual characteristics of project stakeholders on their thinking and behaviour have also been described (e.g., Tukiainen et al., 2010; Valerdi \& Majchrzak, 2003). However, very few studies explain the impacts of the individual characteristics of project stakeholders on their dynamics. This study has provided a deeper understanding of these impacts. By describing these impacts, the research also provides a way to explain how different individual characteristics of project stakeholders affect their thinking and behaviour, which have been described by existing studies such as Mazur et al. (2014) and Tukiainen et al. (2010). Overall, the research also emphasises the importance of studying project stakeholders at individual levels or in other words, under the consideration of their individual characteristics.

By describing the complex ways in which stakeholders' appreciation of projects change, as influenced by their standards and social networks, the research provides an empirical demonstration of the complexity of the project environment. This complexity is in line with the complexity that has been theoretically described by scholars such as Engwall (2003), Eskerod, Huemann, and Savage (2015), Eskerod and Larsen (2018), and Kreiner (1995). With the focus on the complexity, the research is also in line with existing empirical research that explores the complexity and uncertainty of project phenomena (e.g., Azim et al., 2010; Perminova et al., 2008; Thamhain, 2013). The research, therefore, is an answer to Padalkar and Gopinath's (2016) call for more studies of the "non-deterministic" perspective of projects.

In addition to the above main contributions, the research provides one more empirical illustration of the dynamics and social networks of projects. The dynamic state of projects is not new and has been described both theoretically (e.g., Collyer \& Warren, 2009; Jepsen \& 
Eskerod, 2009; Mok et al., 2015) and empirically (Boonstra, 2006, Liu \& Chiu, 2016). However, overall, studies of this state are still limited in comparison to studies of the static situation (Aaltonen et al., 2015). This research, therefore, provides one more empirical illustration of project dynamics. On a similar theme, the social networks of projects have been described by many studies theoretically (Eskerod, Huemann, \& Savage, 2015; Yang et al., 2011) and empirically (Mead, 2001; Papadopoulos \& Merali, 2009). The impacts of these networks on stakeholder dynamics have also been described theoretically (Mok et al., 2015; Yang et al., 2011) and empirically (Aaltonen \& Sivonen, 2009; Butt et al., 2016). This research is in line with the existing research and indicates that projects are not centres of their networks. Each project stakeholder has their own set of stakeholders, and these sets of stakeholders can affect them more than other direct stakeholders of projects. The research, therefore, emphasises the importance of studying projects and their dynamics under the consideration of their networks.

Finally, the research provides a method, which is a combination of Vickers' concept of appreciative systems and the Repertory Grid Technique, to analyse stakeholders in the specific context of projects. While a number of project management studies have applied the Repertory Grid Technique (e.g., Napier et al., 2009; Wagner et al., 2015), very few have applied Vickers' concept, and fewer applied a combination of both. The use of this combination, therefore, provides, an empirical application of Vickers' concept in the context of projects. This combination offers a new lens into both projects and project stakeholders.

In summary, the biggest contributions of this research to both the stakeholder management and project management literature are providing more insights into the dynamics of stakeholders at individual levels, or in other words, the dynamics of stakeholders, as influenced by their individual characteristics, which are linked to their experience and background. With these insights, the research helps to explain the complexity of stakeholder thinking and behaviour. The research also provides a way to explain the impacts of stakeholder networks on their dynamics as well as emphasise the dynamic nature and social networks of projects. Finally, the research proposes a new stakeholder analysis method.

\subsection{Practical Implications}

This research provides project managers with a deeper understanding of the dynamics of stakeholders at individual levels. This understanding helps the managers develop stakeholder management strategies. 
Project managers can learn that individual stakeholders change their appreciation of projects during project implementation. Individual stakeholders can focus on the same stakeholder interactions or change the interactions that they focus on. Their levels of concern about these interactions can remain the same, decrease, or increase. The managers, therefore, may realise that frequent communication with stakeholders is necessary to understand their concerns. Based on this understanding, the mangers may develop strategies to deal with stakeholders.

Project managers should get to know stakeholders as distinctive individuals to understand the drivers of the changes in their appreciation of projects. Project managers should get information about not only the present interactions of stakeholders in their social networks but also their past experience and background. Based on this knowledge, project managers may identify stakeholders' standards for projects and how these standards interact with things that stakeholders learn from their social networks and affect their appreciation of projects. With a deeper understanding of the drivers of the changes in stakeholders' appreciation of projects, project managers may develop suitable strategies to address these drivers and influence stakeholders' appreciation.

Project managers can use the combination of Vickers' concept of appreciative systems and the Repertory Grid Technique as used in this research, or shortened versions of it, to collect and analyse data about the changes in stakeholder perception of projects. Specifically, project managers can interview stakeholders over time directly, as the researcher has done in this research, to collect data about their thinking and perception. The managers can also pre-select parts of the elements and constructs of the repertory grids to develop questionnaires to survey stakeholders. Project managers can apply all or parts of the data analysis as used in this research and identify different aspects of the changes in stakeholder perception of projects. Examples of these aspects are whether the focal interactions of stakeholders change, whether their levels of concern about the interactions change in positive or negative ways, and how the social networks, experience and background of stakeholders affect these changes. Knowledge of these aspects helps project managers evaluate the effectiveness of those stakeholder management strategies that are currently in use and prepare for future approaches.

\subsection{Methodological Lessons}

In addition to the theoretical contributions and empirical implications, the research also provides methodological lessons which benefit future researchers in similar studies. 
The research shows that the Repertory Grid Technique and Vickers' concept of appreciative systems can supplement each other in identifying the changes in individual perception. By applying Vickers' concept, the changes in stakeholder perception of projects could be described as the changes in their appreciation of projects, as influenced by their perceptions of related events and ideas as well as their standards. Among these changes, the changes in the standards and appreciation could be identified by using the principal component analysis of the Repertory Grid Technique. These changes were also triangulated with their perceptions of events and ideas as described through the lens of Vickers' concept. Overall, the combination of Vickers' concept and the Repertory Grid Technique helped to identify the changes in individual stakeholders' perceptions of projects and the drivers of those changes in a rigorous way.

The research also shows that the Repertory Grid Technique had the potential to elicit information from the participants with limited biases caused by the interviewers and, therefore, explore how the experience and background of the participants affected their thinking. However, the use of the technique could be time-consuming and tiresome for the participants. The first interviews, which used six elemental cards and repeated elicitation questions, often lasted from one and a half to two hours and, in many cases, led to participant fatigue. Jankowicz (2005) and Fransella et al. (2004) have previously discussed these problems as the disadvantages of the technique. Being aware of these disadvantages, the researcher has tried different ways to mitigate them. For example, the interview guidelines were sent to the participants, and some of the elements were selected prior to the interviews. Refreshments were also provided. However, despite these mitigation efforts, the interviews were still long and tiresome for some participants. Future researchers, therefore, should consider the disadvantages of the technique. For example, they should ensure that the potential participants understand and are willing to attend the interviews in advance or prepare ways to decrease the participant fatigue during the interviews.

To study the changes in stakeholder thinking over time, the research methodology required multiple interviews with each participant. The planned number of interviews was four for each participant for the MTEO and the APSC cases and eight for the ABCD case. These plans substantially increased the imposition on and required commitment of the participants, which increased the likelihood that some of them might withdraw from the study mid-way. The researcher, therefore, tried different strategies to increase participant commitment. For 
example, the researcher selected participants who were interested in the research and formally committed to the interviews. The researcher actively developed personal relationships with the participants to gain their support. Face-to-face, instead of virtual interviews, were also employed to maintain their commitment. In line with cultural customs, participants associated with the cases in Vietnam also received gifts and tokens of appreciation to further maintain interest. While these strategies did help to engage the participants, there were still three of them whom the researcher could not interview as many times as planned. Future researchers should be aware of this challenge when applying similar data collection plans.

Finally, the research shows the necessity of having pilot interviews. The application of the Repertory Grid Technique required the researcher to have a good understanding of the technique. It also required the researcher to be flexible. For example, the researcher had to decide the order of the elements to discuss or the best time to ask the laddering questions. Furthermore, the participants might not be familiar with the technique and become confused or even annoyed during the interviews, so the researcher must develop strategies to appease them and navigate their frustration. Having pilot interviews, therefore, benefits future researchers in practising the technique and preparing for possible issues that may arise.

\subsection{Research Limitations}

Along with the theoretical contributions, practical implications, and methodological lessons, the research also has limitations. The first limitation is related to the use of Vickers' concept of appreciative systems and the Repertory Grid Technique to explore the dynamics of stakeholder perception of projects, and the influence of their individual characteristics on the dynamics. While the concept and technique were suitable to study these dynamics and influence, they limited the insights that could be gained. For example, through the lens of Vickers' concept, stakeholder characteristics, which are linked to their experience and background, are described specifically as their standards. The dynamics of stakeholder perception of projects are described specifically as the dynamics of their appreciation of projects. While these descriptions offer a wide range of individual characteristics of stakeholders and their perceptions, other concepts and theories can also be used to describe and explore them.

Another limitation lies in the qualitative approach of the research. Both the Repertory Grid Technique and Vickers' concept focused on producing qualitative descriptions of the changes in individual stakeholders' perception of projects, and the impacts of stakeholders' 
standards and social networks on these changes. This qualitative approach was suitable to explore the complexities and subtleties of the changes. This approach has inherent limitations, such as the limited generalisability to a large population and potential researcher biases (Cavana et al., 2001). However, the data collection and analysis methods used in this research, as presented in sections 3.7.3 and 4.2, helped to reduce these limitations

The next limitation is related to the approach that was chosen to explore the dynamics of stakeholder perception. The dynamics of situations can be studied as continuous, periodic, and punctuated (Windsor, 2010). In this research, the dynamics were studied periodically by comparing data that were collected at time points about one month apart. Given the speed of the changes in the project cases, these time intervals were suitable (Windsor, 2010). However, future research can follow other approaches to study stakeholder dynamics.

The selection of the participants in the accreditation projects is another limitation. These participants were selected because of the researcher's good access to the projects (Yin, 2018). The stakeholders of accreditation projects are not only diverse in their background and individual characteristics but also required to interact regularly. The projects, thereby, provide suitable context to demonstrate the impacts of individual characteristics of stakeholders on their dynamics. Given the nature of the research topic, the research results can also be transferred to projects in other fields. However, similar empirical studies in other fields can potentially provide more insights into the dynamics of stakeholder perception in those projects.

Another limitation of this research is the chosen data collection period of four to six months for each case. This period was chosen because this time frame was suitable to capture the changes in the stakeholders' appreciation, given the speed of change in the accreditation projects. Moreover, because this was a $\mathrm{PhD}$ study with three cases, this period allowed the research to organise data collection and other research activities. However, this period might limit the insights that could be gained. Other insights can be obtained by examining stakeholder dynamics in much shorter or longer time frames, such as days or years.

The final limitation is related to the cultural aspects of the three cases. The cases were set in two countries with potential cultural differences, namely New Zealand and Vietnam. The cultural differences presumably affect the individual characteristics of stakeholders. However, this research compared each individual stakeholder's appreciation of their projects with their own appreciation between different time points. The cultural differences, therefore, were 
embedded in the individual characteristics themselves and did not affect the comparison. However, future research can explore the potential impacts of these differences.

\subsection{Recommendations for Future Research}

There are a number of potential directions for future research. Future research can explore the dynamics of other aspects of stakeholder involvement in projects at individual levels or in other words, as influenced by their individual characteristics. This research specifically focuses on the dynamics of stakeholders' appreciation of projects and has found a framework to describe these dynamics, as influenced by the individual characteristics of stakeholders. Following these findings, future research can explore how individual characteristics of stakeholders impact the dynamics of other aspects of their involvement, such as their influence, power, and salience.

Scope for future research also exists in applying other theoretical approaches to explore the dynamics of stakeholder perception and the influence of their individual characteristics on these dynamics. This research specifically used Vickers' concept of appreciative systems to explore these dynamics and influence. Future research can use other concepts and theories to explore them. Quantitative approaches, instead of qualitative ones, can also be applied. Following these approaches, data are collected by surveying a large number of stakeholders in order to gain quantifiable data.

Future research could also apply other approaches to study stakeholder dynamics. This research focused on the periodic dynamics of stakeholder perception. Data on the changes in the perception and the drivers of these changes were collected and measured monthly within periods of four to six months. Given the speed of change in the accreditation projects, these periods and intervals were suitable. However, future research may focus on other dynamics, such as continuous or punctuated dynamics. It could also focus on much longer or shorter periods, such as days or years, in other types of projects.

This research has proposed a framework to describe the dynamics of individual stakeholders' appreciation of the whole project, as influenced by their individual characteristics. The existing literature is abundant with research that describes the changes in stakeholder perception of specific aspects of projects, such as project visions (Besson \& Rowe, 2001) and stakeholder salience (Aaltonen et al., 2008). The proposed framework, therefore, can be applied to identify the dynamics of stakeholders' appreciation of these aspects of projects, and how individual characteristics of stakeholders affect these dynamics. In other 
words, the framework can be used to describe how stakeholders' standards for different aspects of projects can interact with the events and ideas that stakeholders learn from their social networks and affect their appreciation of these aspects. In addition, as the changes in stakeholder perception can potentially lead to changes in their interaction, the framework can also be used as an initial step to explore the dynamic relationship between different stakeholder interactions, as influenced by their individual characteristics. 


\section{References}

AACSB International (2020, September 3). Who We are. https://www.aacsb.edu/about Aaltonen, K. (2011). Project stakeholder analysis as an environmental interpretation process. International Journal of Project Management, 29(2), 165-183. https://doi.org/10.1016/j.ijproman.2010.02.001

Aaltonen, K., Jaakko, K., \& Tuomas, O. (2008). Stakeholder salience in global projects. International Journal of Project Management, 26(5), 509-516.

Aaltonen, K., \& Kujala, J. (2010). A project lifecycle perspective on stakeholder influence strategies in global projects. Scandinavian Journal of Management, 26(4), 381-397.

Aaltonen, K., \& Kujala, J. (2016). Towards an improved understanding of project stakeholder landscapes. International Journal of Project Management, 34(8), 1537-1552.

Aaltonen, K., Kujala, J., Havela, L., \& Savage, G. (2015). Stakeholder Dynamics During the Project Front-End: The Case of Nuclear Waste Repository Projects. Project Management Journal, 46(6), 15-41.

Aaltonen, K., \& Sivonen, R. (2009). Response strategies to stakeholder pressures in global projects. International Journal of Project Management, 27(2), 131-141.

Ackermann, F., \& Eden, C. (2011). Strategic management of stakeholders: Theory and practice. Long Range Planning, 44(3), 179-196.

Afreen, S., \& Kumar, S. (2016). Between a rock and a hard place: The dynamics of stakeholder interactions influencing corporate sustainability practices. Sustainability Accounting, Management and Policy Journal, 7(3), 350-375.

Agle, B. R., Mitchell, R. K., \& Sonnenfeld, J. A. (1999). Who matters to Ceos? An investigation of stakeholder attributes and salience, corporate performance, and Ceo values. Academy of Management Journal, 42(5), 507-525. 
Akpinar, M., \& Vincze, Z. (2016). The dynamics of coopetition: A stakeholder view of the German automotive industry. Industrial Marketing Management, 57, 53-63.

Alani, R. A., \& Ilusanya, G. (2008). Accreditation outcomes, quality of and access to university education in Nigeria. Quality Assurance in Education, 16(3), 301-312.

Alghamdi, F. A. M. (2016). Understanding the change processes resulting from accreditation of colleges in Saudi Arabia [Doctoral thesis, University of Wollongong, Australia]. University of Wollongong thesis collection 1954-2016. https://ro.uow.edu.au/theses/4760/

AMBA (2020, September 3). About Us. https://www.associationofmbas.com/about-us/

Andersen, E. S., Grude, K. V., \& Haug, T. (2009). Goal directed project management: Effective techniques and strategies. Kogan Page Publishers.

Antonacopoulou, E. P., \& Meric, J. (2005). A critique of stake-holder theory: Management science or a sophisticated ideology of control? Corporate Governance: The International Journal of Business in Society, 5(2), 22-33.

Antony, J., Lizarelli, F. L., Fernandes, M. M., Dempsey, M., Brennan, A., \& McFarlane, J. (2019). A study into the reasons for process improvement project failures: Results from a pilot survey. International Journal of Quality \& Reliability Management, 36(10), 1699-1720. https://doi.org/10.1108/IJQRM-03-2019-0093

Aragonés-Beltrán, P., García-Melón, M., \& Montesinos-Valera, J. (2017). How to assess stakeholders' influence in project management? A proposal based on the Analytic Network Process. International Journal of Project Management, 35(3), 451-462.

Argenti, J. (1997). Stakeholders: The case against. Long Range Planning, 30(3), 442-445.

Azim, S., Gale, A., Lawlor-Wright, T., Kirkham, R., Khan, A., \& Alam, M. (2010). The importance of soft skills in complex projects. International Journal of Managing Projects in Business, 3(3), 387-401. 
Baggio, R., \& Cooper, C. (2010). Knowledge transfer in a tourism destination: The effects of a network structure. The Service Industries Journal, 30(10), 1757-1771.

Bakker, R. M. (2010). Taking Stock of Temporary Organizational Forms: A Systematic Review and Research Agenda. International Journal of Management Reviews, 12(4), 466-486. https://doi.org/10.1111/j.1468-2370.2010.00281.x

Baron, D. P. (1995). Integrated strategy: Market and nonmarket components. California Management Review, 37(2), 47-65.

Beaulieu, S., \& Pasquero, J. (2002). Reintroducing stakeholder dynamics in stakeholder thinking: A negotiated-order perspective. The Journal of Corporate Citizenship, Summer 2002, 53-69. https://www.jstor.org/stable/jcorpciti.6.53?seq=1

Berger, P. L., \& Luckmann, T. (1991). The social construction of reality: A treatise in the sociology of knowledge. Penguin UK.

Beringer, C., Jonas, D., \& Gemünden, H. G. (2012). Establishing project portfolio management: An exploratory analysis of the influence of internal stakeholders' interactions. Project Management Journal, 43(6), 16-32.

Besson, P., \& Rowe, F. (2001). ERP project dynamics and enacted dialogue: Perceived understanding, perceived leeway, and the nature of task-related conflicts. $A C M$ SIGMIS Database: The DATABASE for Advances in Information Systems, 32(4), 4766.

Bezzi, A. (1999). What is this thing called geoscience? Epistemological dimensions elicited with the repertory grid and their implications for scientific literacy. Science Education, 83(6), 675-700.

Blackmore, C. (2005). Learning to appreciate learning systems for environmental decision making: A ‘work-in-progress' perspective. Systems Research and Behavioral Science: 
The Official Journal of the International Federation for Systems Research, 22(4), $329-341$.

Blom, R., Davenport, L. D., \& Bowe, B. J. (2012). Reputation cycles: The value of accreditation for undergraduate journalism programs. Journalism \& Mass Communication Educator, 67(4), 392-406.

Blunden, M. (1994). Vickers and postliberalism. American Behavioral Scientist, 38(1), 1125.

Boonstra, A. (2006). Interpreting an ERP-implementation project from a stakeholder perspective. International Journal of Project Management, 24(1), 38-52.

Boonstra, A., Boddy, D., \& Bell, S. (2008). Stakeholder management in IOS projects: Analysis of an attempt to implement an electronic patient file. European Journal of Information Systems, 17(2), 100-111. https://doi.org/10.1057/ejis.2008.2

Bosse, D. A., \& Coughlan, R. (2016). Stakeholder Relationship Bonds. Journal of Management Studies, 53(7), 1197-1222.

Bourne, L. (2008). SRMM®: Stakeholder relationship management maturity [PowerPoint Slides]. Project Management Institute.

Bourne, L., \& Walker, D. H. (2005). Visualising and mapping stakeholder influence. Management Decision, 43(5), 649-660.

Bridoux, F., \& Stoelhorst, J. W. (2014). Microfoundations for stakeholder theory: Managing stakeholders with heterogeneous motives. Strategic Management Journal, 35(1), 107125.

Bridoux, F., \& Stoelhorst, J. W. (2016). Stakeholder relationships and social welfare: A behavioral theory of contributions to joint value creation. Academy of Management Review, 41(2), 229-251. 
Brocklesby, J. (2007). The theoretical underpinnings of soft systems methodologyComparing the work of Geoffrey Vickers and Humberto Maturana. Systems Research and Behavioral Science: The Official Journal of the International Federation for Systems Research, 24(2), 157-168.

Brugha, R., \& Varvasovszky, Z. (2000). Stakeholder analysis: A review. Health Policy and Planning, 15(3), 239-246.

Bryson, J. M. (2004). What to do when Stakeholders matter: Stakeholder Identification and Analysis Techniques. Public Management Review, 6(1), 21-53. https://doi.org/10.1080/14719030410001675722

Butt, A., Naaranoja, M., \& Savolainen, J. (2016). Project change stakeholder communication. International Journal of Project Management, 34(8), 1579-1595.

Campbell, J. T., Campbell, T. C., Sirmon, D. G., Bierman, L., \& Tuggle, C. S. (2012). Shareholder influence over director nomination via proxy access: Implications for agency conflict and stakeholder value. Strategic Management Journal, 33(12), 14311451.

Carrington, P. J., Scott, J., \& Wasserman, S. (2005). Models and methods in social network analysis. Cambridge University Press.

Carter, S. M. (2006). The interaction of top management group, stakeholder, and situational factors on certain corporate reputation management activities. Journal of Management Studies, 43(5), 1145-1176.

Catalano, A. S., Lyons-White, J., Mills, M. M., \& Knight, A. T. (2019). Learning from published project failures in conservation. Biological Conservation, 238, 108-223.

Cavana, R. Y., Delahaye, B. L., \& Sekaran, U. (2001). Applied business research: Qualitative and quantitative methods. John Wiley \& Sons Australia. http://eprints.qut.edu.au/10523/ 
Chase, L. C., Siemer, W. F., \& Decker, D. J. (2002). Designing stakeholder involvement strategies to resolve wildlife management controversies. Wildlife Society Bulletin, 30(3), 937-950.

Checkland, P. (1994). Systems theory and management thinking. American Behavioral Scientist, 38(1), 75-91.

Checkland, P. (2000). Soft systems methodology: A thirty year retrospective. Systems Research and Behavioral Science, 17(S1), S11-S58.

Checkland, P. B., \& Casar, A. (1986). Vickers' concept of an appreciative system: A systemic account. Journal of Applied Systems Analysis, 13(3), 3-17.

Christensen, C. M., Anthony, S. D., Berstell, G., \& Nitterhouse, D. (2007). Finding the right job for your product. MIT Sloan Management Review, 48(3), 38.

Clarkson, M. E. (1995). A stakeholder framework for analyzing and evaluating corporate social performance. Academy of Management Review, 20(1), 92-117.

Cleland, D. I. (1986). Measuring Success: The owner's viewpoint. Proceedings of the 18th Annual Seminar/Symposium (Montreal/Canada), 6-12.

Clifton, D., \& Amran, A. (2011). The stakeholder approach: A sustainability perspective. Journal of Business Ethics, 98(1), 121-136.

Cohen-Blankshtain, G., \& Nijkamp, P. (2004). The appreciative system of urban ICT policies: An analysis of perceptions of urban policy makers. Growth and Change, 35(2), 166-197.

Collyer, S., \& Warren, C. M. J. (2009). Project management approaches for dynamic environments. International Journal of Project Management, 27(4), 355-364. https://doi.org/10.1016/j.ijproman.2008.04.004 
Coombs, J. E., \& Gilley, K. M. (2005). Stakeholder management as a predictor of CEO compensation: Main effects and interactions with financial performance. Strategic Management Journal, 26(9), 827-840.

Crilly, D., Zollo, M., \& Hansen, M. T. (2012). Faking it or muddling through? Understanding decoupling in response to stakeholder pressures. Academy of Management Journal, 55(6), 1429-1448.

Cuppen, E. (2016). 15 Stakeholder Analysis. In P. Van der Duin (Ed.), Foresight in Organizations: Methods and Tools (pp.208-214). Routledge

Cuppen, E., Bosch-Rekveldt, M. G., Pikaar, E., \& Mehos, D. C. (2016). F in large-scale energy infrastructure projects: Revealing perspectives using Q methodology. International Journal of Project Management, 34(7), 1347-1359.

Cuppen, E., Brunsting, S., Pesch, U., \& Feenstra, Y. (2015). How stakeholder interactions can reduce space for moral considerations in decision making: A contested CCS project in the Netherlands. Environment and Planning A, 47(9), 1963-1978.

Curtis, A. M., Wells, T. M., Higbee, T., \& Lowry, P. B. (2008). An overview and tutorial of the repertory grid technique in information systems research. Communications of the Association for Information Systems (CAIS), 23(3), 37-62.

Davies, J., \& Mabin, V. J. (2001). Knowledge management and the framing of information: A contribution to OR/MS practice and pedagogy. Taylor \& Francis.

De Luque, M. S., Washburn, N. T., Waldman, D. A., \& House, R. J. (2008). Unrequited profit: How stakeholder and economic values relate to subordinates' perceptions of leadership and firm performance. Administrative Science Quarterly, 53(4), 626-654.

Donaldson, T., \& Preston, L. E. (1995). The stakeholder theory of the corporation: Concepts, evidence, and implications. Academy of Management Review, 20(1), 65-91. 
Dorobantu, S., Henisz, W. J., \& Nartey, L. (2016). Not all sparks light a fire: Stakeholder and shareholder reactions to critical events in contested markets. Administrative Science Quarterly, 62(3), 561-597.

Easterby-Smith, M., Thorpe, R., Jackson, P., \& Lowe, A. (2008). Management Research. SAGE.

Eesley, C., \& Lenox, M. J. (2006). Firm responses to secondary stakeholder action. Strategic Management Journal, 27(8), 765-781.

EFMD Global (2020, September 3). About Us. https://www.efmdglobal.org/about-efmdglobal/\#

Eisenhardt, K. M. (1989). Building theories from case study research. (Special Forum on Theory Building). Academy of Management Review, 14(4), 532-550. https://doi.org/10.2307/258557

El Akremi, A., Gond, J. P., Swaen, V., De Roeck, K., \& Igalens, J. (2015). How do employees perceive corporate responsibility? Development and validation of a multidimensional corporate stakeholder responsibility scale. Journal of Management, 44(2), 619-657.

Elias, A. A. (2004). Analysing the dynamics of stakeholders in the Wellington Transmission Gully Project: A systems thinking and modelling approach to environmental conflict management [Doctoral thesis, Victoria University of Wellington, New Zealand]. The Library of Victoria University of Wellington.

Elias, A.A. (2008). Group model building: Energy efficiency in New Zealand's residential sector. Proceedings of the 6th Annual Australian and New Zealand Academy of Management Operations Management Symposium, Gold Coast, Australia, 1-11.

Elias, A. A. (2012). A system dynamics model for stakeholder analysis in environmental conflicts. Journal of Environmental Planning and Management, 55(3), 387-406. 
Elias, A. A. (2016). Analysing the stakes of stakeholders in research and development project management: A systems approach. R\&D Management, 46(4), 749-760. https://doi.org/10.1111/radm.12122

Elias, A. A. (2017). Systems thinking and modelling for stakeholder management. IIM Kozhikode Society \& Management Review, 6(2), 123-131.

Elias, A. A. (2019). Strategy development through stakeholder involvement: A New Zealand study. Global Journal of Flexible Systems Management, 20(4), 313-322.

Elias, A. A. (2021). Kerala's Innovations and Flexibility for Covid-19 Recovery: Storytelling using Systems Thinking. Global Journal of Flexible Systems Management, 22, 33-43.

Elias, A. A., Cavana, R. Y., \& Jackson, L. S. (2000). Linking Stakeholder Literature and System Dynamics: Opportunities for Research. Proceedings of the International Conference on Systems Thinking in Management, Geelong, Australia, 174-179

Elias, A. A., Cavana, R. Y., \& Jackson, L. S. (2001). Stakeholder analysis to enrich the systems thinking and modelling methodology. Proceedings of the 19th International Conference of the Systems Dynamics Society, 52-70.

Elias, A. A., \& Davis, D. (2018). Analysing public sector continuous improvement: a systems approach. International Journal of Public Sector Management, 31(1), 2-13.

Elias, A.A., Jackson, L. S., \& Cavana, R. Y. (2004). Changing positions and interests of stakeholders in environmental conflict: A New Zealand transport infrastructure case. Asia Pacific Viewpoint, 45(1), 87-104. https://doi.org/10.1111/j.14678376.2004.00229.x

Elias, A.A., \& Zwikael, O. (2007). Stakeholder participation in project management: A New Zealand study. Proceedings of the 5th Annual Australian and New Zealand Academy of Management Operations Management Symposium, Melbourne. 
Elliott, C. J., \& Goh, S. C. (2013). Does accreditation promote organizational learning? A multiple case study of Canadian university business schools. Journal of Management Development, 32(7), 737-755.

Engelbrecht, J., \& Thomas, A. (2017). Changes in stakeholder dynamics and salience during a mining disaster. South African Journal of Business Management, 48(4), 71-81.

Engwall, M. (2003). The resource allocation syndrome: The prime challenge of multi-project management? International Journal of Project Management, 21(6), 403-409.

Eskerod, P., Huemann, M., \& Ringhofer, C. (2015). Stakeholder inclusiveness: Enriching project management with general stakeholder theory. Project Management Journal, $46(6), 42-53$.

Eskerod, P., Huemann, M., \& Savage, G. (2015). Project stakeholder management—Past and present. Project Management Journal, 46(6), 6-14.

Eskerod, P., \& Larsen, T. (2018). Advancing project stakeholder analysis by the concept 'shadows of the context'. International Journal of Project Management, 36(1), 161169.

Eskerod, P., \& Vaagaasar, A. L. (2014). Stakeholder management strategies and practices during a project course. Project Management Journal, 45(5), 71-85.

Fassin, Y. (2008). Imperfections and shortcomings of the stakeholder model's graphical representation. Journal of Business Ethics, 80(4), 879-888.

Fassin, Y. (2010). A dynamic perspective in Freeman's stakeholder model. Journal of Business Ethics, 96(1), 39-49.

Fedorowicz, J., Gogan, J. L., \& Culnan, M. J. (2010). Barriers to Interorganisational Information Sharing in e-Government: A Stakeholder Analysis. The Information Society, 26(5), 315-329. https://doi.org/10.1080/01972243.2010.511556 
Ferrer, E., \& Grimm, K. J. (2012). Issues in collecting longitudinal data. In H. E. Cooper, P. M. Camic, D. L. Long, A. T. Panter, D. E. Rindskopf, \& K. J. Sher (Eds.). APA handbook of research methods in psychology, Vol 2: Research designs: Quantitative, qualitative, neuropsychological, and biological (pp.275-290). American Psychological Association

Fielder, K. (1982). Causal schemata: Review and criticism of research on a popular construct. Journal of Personality and Social Psychology, 42(6), 1001-1013.

Fottler, M. D., Blair, J. D., Whitehead, C. J., Laus, M. D., \& Savage, G. T. (1989). Assessing key stakeholders: Who matters to hospitals and why. Journal of Healthcare Management, 34(4), 525-546.

Fransella, F., Bell, R., \& Bannister, D. (2004). A manual for repertory grid technique. John Wiley \& Sons.

https://books.google.co.nz/books?hl=en\&lr=\&id=GPnqDPZhDKAC\&oi=fnd\&pg=PR $5 \& \mathrm{dq}=$ repertory+grid\&ots=q4qo2gFeZ3\&sig=N_Iu_NeFfgRe0pM3v9R2HZAYjNw

Freeman, R. E. (1984). Strategic Management: A stakeholder approach. Boston: Pitman.

Freeman, R.E., \& Evan, W. M. (1990). Corporate governance: A stakeholder interpretation. The Journal of Behavioral Economics, 19(4), 337-359. https://doi.org/10.1016/00905720(90)90022-Y

Freeman, R. E. \& Phillips, R. A. (2002). Stakeholder theory: A libertarian defense. Business Ethics Quarterly, 12(03), 331-349.

Freeman, R. E., Wicks, A. C., \& Parmar, B. (2004). Stakeholder theory and "the corporate objective revisited". Organization Science, 15(3), 364-369.

Friedman, A. L., \& Miles, S. (2002). Developing stakeholder theory. Journal of Management Studies, 39(1), 1-21. 
Frost, F. (1995). The use of stakeholder analysis to understand ethical and moral issues in the primary resource sector. Journal of Business Ethics, 14(8), 653-661. https://doi.org/10.1007/BF00871346

Gaines. R. N. \& Shaw L. G. M. (2018a). Rep Plus conceptual representation software: Managing the Rep Plus Web Server and its Scripts - including WebGrid. Retrieved July 1, 2019, from: http://pages.cpsc.ucalgary.ca/ gaines/Manuals/RepServe.pdf

Gaines, B. R. \& Shaw, L. G. (2018b). Rep Plus conceptual representation software: RepGrid Manual, managing and analysing collections of grids. Retrieved July 1, 2019 from: http://pages.cpsc.ucalgary.ca/ gaines/Manuals/RepGrids.pdf

Gaines, B. R. \& Shaw, L. G. (2018c). Rep Plus conceptual representation software: RepGrids Manual, managing and analysing collections of grids. Retrieved July 1, 2019 from: http://pages.cpsc.ucalgary.ca/ gaines/Manuals/RepGrids.pdf

Gaines. B. R. \& Shaw L. G. M. (2018d). Rep Plus conceptual representation software: RepNet Manual, managing editing and scritpting the Rep Plus Graphics System. Retrieved July 1, 2019, from: http://pages.cpsc.ucalgary.ca/ gaines/Manuals/RepNet.pdf

Gällstedt, M. (2003). Working conditions in projects: Perceptions of stress and motivation among project team members and project managers. International Journal of Project Management, 21(6), 449-455.

Gattiker, T. F., \& Carter, C. R. (2010). Understanding project champions' ability to gain intra-organizational commitment for environmental projects. Journal of Operations Management, 28(1), 72-85. 
Geraldi, J. G., Lee-Kelley, L., \& Kutsch, E. (2010). The Titanic sunk, so what? Project manager response to unexpected events. International Journal of Project Management, 28(6), 547-558.

Geraldi, J., Maylor, H., \& Williams, T. (2011). Now, let's make it really complex (complicated) A systematic review of the complexities of projects. International Journal of Operations \& Production Management, 31(9), 966-990.

Gergen, K. J. (1985). The social constructionist movement in modern psychology. American Psychologist, 40(3), 266-275.

Gergen, K. J. (2009). Relational being: Beyond self and community. Oxford University Press. https://books.google.co.nz/books?hl=en\&lr=\&id=SweMLEe6TpgC\&oi=fnd\&pg=PP1 $\& \mathrm{dq}=$ Gergen,+ relational+being $+\&$ ots $=\_\mathrm{mLx} 6 \mathrm{~J} 4 \mathrm{k} 0 \mathrm{p} \& \operatorname{sig}=-$ leBwXU9sbfIzNt5zhF0JAvwDTI

Gergen, K. J. (2015). An Invitation to Social Construction (3rd ed.). SAGE Publications Ltd. Giaglis, G. M., Klein, S., \& O’ Keefe, R. M. (2002). The role of intermediaries in electronic marketplaces: Developing a contingency model. Information Systems Journal, 12(3), 231-246. https://doi.org/10.1046/j.1365-2575.2002.00123.x

Green, A. O., \& Hunton-Clarke, L. (2003). A typology of stakeholder participation for company environmental decision-making. Business Strategy and the Environment, 12(5), 292-299.

Guba, E. G., \& Lincoln, Y. S. (1994). Competing paradigms in qualitative research. In N. K. Denzin, \& Y. S. Lincoln (Eds.), Handbook of Qualitative Research, (pp.105-117). Thousand Oaks: Sage Publications.

Gupta, S. K., Gunasekaran, A., Antony, J., Gupta, S., Bag, S., \& Roubaud, D. (2019). Systematic literature review of project failures: Current trends and scope for future research. Computers \& Industrial Engineering, 127, 274-285. 
Hall, M., Millo, Y., \& Barman, E. (2015). Who and What Really Counts? Stakeholder Prioritization and Accounting for Social Value. Journal of Management Studies, 52(7), 907-934.

Harrison, J. S., \& Wicks, A. C. (2013). Stakeholder theory, value, and firm performance. Business Ethics Quarterly, 23(01), 97-124.

Hazelton, J. A., Windhorst, K., \& Amezaga, J. M. (2013). Forest based biomass for energy in Uganda: Stakeholder dynamics in feedstock production. Biomass and Bioenergy, 59, 100-115. https://doi.org/10.1016/j.biombioe.2013.04.014

Henisz, W. J., Dorobantu, S., \& Nartey, L. J. (2014). Spinning gold: The financial returns to stakeholder engagement. Strategic Management Journal, 35(12), 1727-1748.

Herriott, R. E., \& Firestone, W. A. (1983). Multisite qualitative policy research: Optimizing description and generalizability. Educational Researcher, 12(2), 14-19.

Heugens, P. P., \& Van Oosterhout, H. J. (2002). The confines of stakeholder management: Evidence from the Dutch manufacturing sector. Journal of Business Ethics, 40(4), $387-403$.

Hillman, A. J., \& Keim, G. D. (2001). Shareholder value, stakeholder management, and social issues: What's the bottom line? Strategic Management Journal, 22(2), 125139.

Hjortsø, C., Christensen, S., \& Tarp, P. (2005). Rapid stakeholder and conflict assessment for natural resource management using cognitive mapping: The case of Damdoi Forest Enterprise, Vietnam. Agriculture and Human Values, 22(2), 149-167. https://doi.org/10.1007/s10460-004-8275-z

Hughes, D. L., Dwivedi, Y. K., Rana, N. P., \& Simintiras, A. C. (2016). Information systems project failure-analysis of causal links using interpretive structural modelling. Production Planning \& Control, 27(16), 1313-1333. 
Project Management Institute (2013). A Guide to the Project Management Body of Knowledge (PMBOK® Guide)-Fifth Edition (5th ed.). Project Management Institute. Project Management Institute (2017). A guide to the Project Management Body of Knowledge (PMBOK Guide) (6th ed.). Project Management Institute.

Jankowicz, D. (2005). The easy guide to repertory grids. John Wiley \& Sons.

Jansson, E. (2005). The stakeholder model: The influence of the ownership and governance structures. Journal of Business Ethics, 56(1), 1-13.

Jenkins, P. D. (2011). Redesigning community colleges for completion: Lessons from research on high-performance organizations (Assessment of Evidence Series). Columbia University, Community College Research Center.

Jepsen, A. L., \& Eskerod, P. (2009). Stakeholder analysis in projects: Challenges in using current guidelines in the real world. International Journal of Project Management, 27(4), 335-343.

Johnson, G., Scholes, K., \& Whittington, R. (2008). Exploring corporate strategy: Text \& cases. Pearson education.

Kaptein, M. (2008). Developing a measure of unethical behavior in the workplace: A stakeholder perspective. Journal of Management, 34(5), 978-1008.

Kelly, G. (2001). The Psychology of Personal Constructs: Volume One: Theory and Personality. Florence: Taylor and Francis. (Original work published 1955)

Key, S. (1999). Toward a new theory of the firm: A critique of stakeholder "theory". Management Decision, 37(4), 317-328.

Khurram, S., \& Petit, S. C. (2017). Investigating the Dynamics of Stakeholder Salience: What Happens When the Institutional Change Process Unfolds? Journal of Business Ethics, 143(3), 485-515. https://doi.org/10.1007/s10551-015-2768-0 
Knemeyer, A. M., Ponzurick, T. G., \& Logar, C. M. (2002). A qualitative examination of factors affecting reverse logistics systems for end-of-life computers. International Journal of Physical Distribution \& Logistics Management, 32(6), 455-479.

Kreiner, K. (1995). In search of relevance: Project management in drifting environments. Scandinavian Journal of Management, 11(4), 335-346.

Lamberg, J. A., Pajunen, K., Parvinen, P., \& Savage, G. T. (2008). Stakeholder management and path dependence in organizational transitions. Management Decision, 46(6), 846863.

Lange, E., \& Hehl-Lange, S. (2005). Combining a participatory planning approach with a virtual landscape model for the siting of wind turbines. Journal of Environmental Planning and Management, 48(6), 833-852. https://doi.org/10.1080/09640560500294277

Länsiluoto, A., Järvenpää, M., \& Krumwiede, K. (2013). Conflicting interests but filtered key targets: Stakeholder and resource-dependency analyses at a University of Applied Sciences. Management Accounting Research, 24(3), 228-245.

Large, R. G. (1976). The use of the role construct repertory grid in studying changes during psychotherapy. Australian \& New Zealand Journal of Psychiatry, 10(4), 315-320.

Littau, P., Jujagiri, N. J., \& Adlbrecht, G. (2010). 25 years of stakeholder theory in project management literature (1984-2009). Project Management Journal, 41(4), 17-29.

Laumann, E. O., Marsden, P. V., \& Prensky, D. (1989). The boundary specification problem in network analysis. In Freeman, L. C. (Eds). Research methods in social network analysis (pp.61-87). Routledge.

Leach, W. D., Pelkey, N. W., \& Sabatier, P. A. (2002). Stakeholder partnerships as collaborative policymaking: Evaluation criteria applied to watershed management in 
California and Washington. Journal of Policy Analysis and Management: The Journal of the Association for Public Policy Analysis and Management, 21(4), 645-670.

Lépineux, F. (2005). Stakeholder theory, society and social cohesion. Corporate Governance: The International Journal of Business in Society.

Lim, S. L., Quercia, D., \& Finkelstein, A. (2010). StakeNet: Using social networks to analyse the stakeholders of large-scale software projects. Proceedings of the 32Nd ACM/IEEE International Conference on Software Engineering-Volume 1, 295-304.

Liu, J. Y. C., \& Chiu, G. C. T. (2016). Influence of Project Partnering on Stakeholder Role Ambiguity and Project Manager Risk Perception in Information System Projects. Project Management Journal, 47(6), 94-110. https://doi.org/10.1177/875697281604700607

Maani, K., \& Cavana, R. Y. (2007). Systems thinking, system dynamics: Managing change and complexity. Prentice Hall.

Mahon, J. F., Heugens, P. P. M. A. R., \& Lamertz, K. (2004). Social networks and nonmarket strategy. Journal of Public Affairs, 4(2), 170-189. https://doi.org/10.1002/pa.179

Mainardes, E. W., Alves, H., \& Raposo, M. (2011). Stakeholder theory: Issues to resolve. Management Decision, 49(2), 226-252.

Marsden, P. V. (2005). Recent developments in network measurement. In P. J. Carrington, J. Scott, \& S. Wasserman (Eds.), Models and methods in social network analysis (Vol. 28) (pp.8-19). Cambridge University Press.

Marshall, R. S., Akoorie, M. E., Hamann, R., \& Sinha, P. (2010). Environmental practices in the wine industry: An empirical application of the theory of reasoned action and stakeholder theory in the United States and New Zealand. Journal of World Business, 45(4), 405-414. 
Martini, U., \& Buffa, F. (2015). Local networks, stakeholder dynamics and sustainability in tourism. Opportunities and limits in the light of stakeholder theory and SNA. Sinergie Italian Journal of Management, 33(Jan-Apr), 113-130.

Mazur, A., Pisarski, A., Chang, A., \& Ashkanasy, N. M. (2014). Rating defence major project success: The role of personal attributes and stakeholder relationships. International Journal of Project Management, 32(6), 944-957.

McKenna, A., \& Metcalfe, M. (2013). The linguistic turn in project conceptualization. International Journal of Project Management, 31(8), 1154-1162.

Mead, S. P. (2001). Using social network analysis to visualize project teams. Project Management Journal, 32(4), 32-38.

Medina, A., \& Francis, A. J. (2015). What are the characteristics that software development project team members associate with a good project manager? Project Management Journal, 46(5), 81-93.

Memon, P. A., \& Wilson, G. A. (2007). Contesting governance of indigenous forests in New Zealand: The case of the West Coast Forest Accord. Journal of Environmental Planning and Management, 50(6), 745-764. https://doi.org/10.1080/09640560701608705

Mendelow, A. (1991). Stakeholder Mapping: The Power Interest Matrix. Proceedings of 2 nd International Conference on Information Systems, Cambridge, MA, 5(2), 61.

Metcalfe, M., \& Sastrowardoyo, S. (2013). Complex project conceptualisation and argument mapping. International Journal of Project Management, 31(8), 1129-1138.

Miller, K., McAdam, M., \& McAdam, R. (2014). The changing university business model: A stakeholder perspective. $R \& D$ Management, 44(3), 265-287. 
Missonier, S., \& Loufrani-Fedida, S. (2014). Stakeholder analysis and engagement in projects: From stakeholder relational perspective to stakeholder relational ontology. International Journal of Project Management, 32(7), 1108-1122.

Mitchell, R. K. (2012). Book review: Stakeholder theory: Impact and prospects. Organization Studies, 33(10), 1407-1411.

Mitchell, R. K., Agle, B. R., \& Wood, D. J. (1997). Toward a theory of stakeholder identification and salience: Defining the principle of who and what really counts. Academy of Management Review, 22(4), 853-886.

Mitchell, R. K., Weaver, G. R., Agle, B. R., Bailey, A. D., \& Carlson, J. (2016). Stakeholder agency and social welfare: Pluralism and decision making in the multi-objective corporation. Academy of Management Review, 41(2), 252-275.

Modig, N. (2007). A continuum of organizations formed to carry out projects: Temporary and stationary organization forms. International Journal of Project Management, 25(8), 807-814. https://doi.org/10.1016/j.ijproman.2007.03.008

Mok, K. Y., Shen, G. Q., \& Yang, J. (2015). Stakeholder management studies in mega construction projects: A review and future directions. International Journal of Project Management, 33(2), 446-457.

Mok, K. Y., Shen, G. Q., Yang, R. J., \& Li, C. Z. (2017). Investigating key challenges in major public engineering projects by a network-theory based analysis of stakeholder concerns: A case study. International Journal of Project Management, 35(1), 78-94.

Morgan, G. (1980). Paradigms, metaphors, and puzzle solving in organization theory. Administrative Science Quarterly, 25(4), 605-622.

Morris, P. W. (1994). The management of projects. Thomas Telford.

Moutinho, L., \& Vargas-Sanchez, A. (2018). Strategic Management in Tourism, CABI Tourism Texts. Cabi. 
Mukherjee, S. (2019). How stakeholder engagement affects IT projects. International Journal of Innovative Research in Science, Engineering and Technology, 8(3), 3516-3518.

Napier, N. P., Keil, M., \& Tan, F. B. (2009). IT project managers' construction of successful project management practice: A repertory grid investigation. Information Systems Journal, 19(3), 255-282.

Nguyen, H. C., Evers, C., \& Marshall, S. (2017). Accreditation of Viet Nam's higher education: Achievements and challenges after a dozen years of development. Quality Assurance in Education, 25(4), 475-488.

Ojiako, U., Chipulu, M., Gardiner, P., Williams, T., Mota, C., Maguire, S., Shou, Y., \& Stamati, T. (2014). Effect of project role, age and gender differences on the formation and revision of project decision judgements. International Journal of Project Management, 32(4), 556-567. https://doi.org/10.1016/j.ijproman.2013.09.001

Olander, S. (2007). Stakeholder impact analysis in construction project management. Construction Management and Economics, 25(3), 277-287.

Olander, S., \& Landin, A. (2005). Evaluation of stakeholder influence in the implementation of construction projects. International Journal of Project Management, 23(4), 321328.

Onsman, A. (2010). Dismantling the perceived barriers to the implementation of national higher education accreditation guidelines in the Kingdom of Saudi Arabia. Journal of Higher Education Policy and Management, 32(5), 511-519.

Orlikowski, W. J., \& Gash, D. C. (1994). Technological frames: Making sense of information technology in organizations. ACM Transactions on Information Systems (TOIS), 12(2), 174-207. 
Padalkar, M., \& Gopinath, S. (2016). Six decades of project management research: Thematic trends and future opportunities. International Journal of Project Management, 34(7), $1305-1321$.

Pajunen, K. (2006). Stakeholder influences in organizational survival. Journal of Management Studies, 43(6), 1261-1288.

Pan, G. S. (2005). Information systems project abandonment: A stakeholder analysis. International Journal of Information Management, 25(2), 173-184.

Pankratz, O., Basten, D., Pansini, F., Terzieva, M., Morabito, V., \& Anaya, L. A. (2014). Ladder to success-eliciting project managers' perceptions of IS project success criteria. International Journal of Information Systems and Project Management, 2(2), $5-24$.

Papadopoulos, T., \& Merali, Y. (2008). Stakeholder network dynamics and emergent trajectories of Lean implementation projects: A study in the UK National Health Service. Public Money and Management, 28(1), 41-48.

Papadopoulos, T., \& Merali, Y. (2009). Stakeholder dynamics and the implementation of process innovations: The case of Lean thinking in a UK NHS Hospital Trust. International Journal of Healthcare Technology and Management, 10(4/5), 303-324.

Parmar, B. L., Freeman, R. E., Harrison, J. S., Wicks, A. C., Purnell, L., \& De Colle, S. (2010). Stakeholder theory: The state of the art. The Academy of Management Annals, $4(1), 403-445$.

Pedrini, M., \& Ferri, L. M. (2019). Stakeholder management: A systematic literature review. Corporate Governance: The International Journal of Business in Society, 19(1), 4459.

Perminova, O., Gustafsson, M., \& Wikström, K. (2008). Defining uncertainty in projects-a new perspective. International Journal of Project Management, 26(1), 73-79. 
Phillips, R. (2004). Ethics and a manager's obligations under stakeholder theory. Ivey Business Journal, 68(4), 1-4.

Post, J. E., Preston, L. E., \& Sachs, S. (2002). Managing the extended enterprise: The new stakeholder view. California Management Review, 45(1), 6-28.

Postema, T., Groen, A., \& Krabbendam, K. (2012). A model to evaluate stakeholder dynamics during innovation implementation. International Journal of Innovation Management, 16(5), 1250025-1-1250025-20. https://doi.org/10.1142/S136391961200385X

Pouloudi, A., \& Whitley, E. A. (1997). Stakeholder identification in inter-organizational systems: Gaining insights for drug use management systems. European Journal of Information Systems, 6(1), 1-14. https://doi.org/10.1057/palgrave.ejis.3000252

Pouloudi, N., Currie, W., \& Whitley, E. A. (2016). Entangled stakeholder roles and perceptions in health information systems: A longitudinal study of the UK NHS N3 network. Journal of the Association for Information Systems, 17(2), 107-161.

Purvis, R. L., Zagenczyk, T. J., \& McCray, G. E. (2015). What's in it for me? Using expectancy theory and climate to explain stakeholder participation, its direction and intensity. International Journal of Project Management, 33(1), 3-14.

Ramirez, R. (1999). Stakeholder analysis and conflict management. In D. Buckles (Ed.), Cultivating Peace: Conflict and Collaboration in Natural Resource Management, (pp.101-126). Idrc

Reger, R. K. (1990). The repertory grid technique for eliciting the content and structure of cognitive constructive systems. In A.S. Huff (Ed.), Mapping Strategic Thought (pp. 301 - 309). John Wiley \& Sons. 
Regev, G., Hayard, O., \& Wegmann, A. (2011). Service systems and value modeling from an appreciative system perspective. In M. Snene, J. Ralyté, \& J. H. Morin (Eds.), Exploring Services Science (pp.146-157). Springer Berlin Heidelberg.

Richardson, G. P., \& Andersen, D. F. (2010). Stakeholder dynamics. Proceedings of the 28th International Conference of the System Dynamics Society, 1-19.

Ripolles Meliá, M., Blesa Pérez, A., \& Roig Dobón, S. (2010). The influence of innovation orientation on the internationalisation of SMEs in the service sector. The Service Industries Journal, 30(5), 777-791.

Romero, E. J. (2008). AACSB accreditation: Addressing faculty concerns. Academy of Management Learning \& Education, 7(2), 245-255.

Rowley, T. I., \& Moldoveanu, M. (2003). When will stakeholder groups act? An interest-and identity-based model of stakeholder group mobilization. Academy of Management Review, 28(2), 204-219.

Rowley, T. J. (1997). Moving beyond dyadic ties: A network theory of stakeholder influences. Academy of Management Review, 22(4), 887-910.

Russo, A., \& Perrini, F. (2010). Investigating stakeholder theory and social capital: CSR in large firms and SMEs. Journal of Business Ethics, 91(2), 207-221.

Saunders, M. (2007). Research methods for business students (4th ed.). Financial Times/Prentice Hall.

Savage, G. T., Dunkin, J. W., \& Ford, D. M. (2004). Responding to a crisis: A stakeholder analysis of community health organizations. Journal of Health and Human Services Administration, 26(4), 383-414.

Schneider, M. (2002). A stakeholder model of organizational leadership. Organization Science, 13(2), 209-220. 
Scott, J., \& Carrington, P. (2014). The SAGE Handbook of Social Network Analysis. SAGE Publications Ltd. https://doi.org/10.4135/9781446294413

Senge, P. M. (1997). The fifth discipline. Measuring Business Excellence, 1(3), 46-51.

Serravalle, F., Ferraris, A., Vrontis, D., Thrassou, A., \& Christofi, M. (2019). Augmented reality in the tourism industry: A multi-stakeholder analysis of museums. Tourism Management Perspectives, 32, 100549.

Sewell, K. W., Adams-Webber, J., Mitterer, J., \& Cromwell, R. L. (1992). Computerized repertory grids: Review of the literature. International Journal of Personal Construct Psychology, 5(1), 1-23.

Shotter, J. (1997). The social construction of our inner selves. Journal of Constructivist Psychology, 10(1), 7-24.

Slater, P. (1976). Explorations of Intrapersonal Space: Volume I. John Wiley \& Sons.

Slater, P. (1977). The Measurement of Intrapersonal Space by Grid Technique: Slater, P. Dimensions of intrapersonal space (Vol. 2). Wiley.

Sloan, P., \& Oliver, D. (2013). Building trust in multi-stakeholder partnerships: Critical emotional incidents and practices of engagement. Organization Studies, 34(12), $1835-1868$.

Solaimani, S., Guldemond, N., \& Bouwman, H. (2013). Dynamic stakeholder interaction analysis: Innovative smart living design cases. Electronic Markets, 23(4), 317-328.

Soste, L., Wang, Q. J., Robertson, D., Chaffe, R., Handley, S., \& Wei, Y. (2015). Engendering stakeholder ownership in scenario planning. Technological Forecasting and Social Change, 91, 250-263.

South, A. J., Levitt, R. E., \& Dewulf, G. (2015). Dynamic stakeholder networks and the governance of PPPs. Proceedings of the 2nd International Conference on PublicPrivate Partnerships, 26-29. 
Spangenberg, J. H., Heong, K. L., Klotzbücher, A., Klotzbücher, T., Nguyen, Q. A., Tekken, V., Truong, D. T., Türke, M., \& Settele, J. (2018). Doing what with whom? Stakeholder analysis in a large transdisciplinary research project in South-East Asia. Paddy and Water Environment, 16(2), 321-337.

Stjerne, I. S., \& Svejenova, S. (2016). Connecting temporary and permanent organizing: Tensions and boundary work in sequential film projects. Organization Studies, 37(12), 1771-1792.

Sundaram, A. K., \& Inkpen, A. C. (2004). Stakeholder theory and "The corporate objective revisited": A reply. Organization Science, 15(3), 370-371.

Svendsen, A. C., \& Laberge, M. (2005). Convening stakeholder networks: A new way of thinking, being and engaging. Journal of Corporate Citizenship, Autumn 2005 (19), 91-104.

Tan, F. B., \& Hunter, M. G. (2002). The repertory grid technique: A method for the study of cognition in information systems. MIS Quarterly, 26(1), 39-57.

Tantalo, C., \& Priem, R. L. (2014). Value creation through stakeholder synergy. Strategic Management Journal, 37(2), 314-329.

Thamhain, H. (2013). Managing risks in complex projects. Project Management Journal, 44(2), 20-35.

Tukiainen, S., Aaltonen, K., \& Murtonen, M. (2010). Coping with an unexpected event: Project managers' contrasting sensemaking in a stakeholder conflict in China. International Journal of Managing Projects in Business, 3(3), 526-543.

Turkulainen, V., Aaltonen, K., \& Lohikoski, P. (2015). Managing Project Stakeholder Communication: The Qstock Festival Case. Project Management Journal, 46(6), 74 91. 
Turner, J. R., \& Müller, R. (2003). On the nature of the project as a temporary organization. International Journal of Project Management, 21(1), 1-8.

Turner, R. J., Huemann, M., Anbari, F. T., \& Bredillet, C. N. (2010). Perspectives on projects. Routledge.

Tuuli, M. M., \& Rowlinson, S. (2010). What empowers individuals and teams in project settings? A critical incident analysis. Engineering, Construction and Architectural Management, 17(1), 9-20.

Un, C. A., \& Montoro-Sanchez, A. (2010). Public funding for product, process and organisational innovation in service industries. The Service Industries Journal, 30(1), $133-147$.

University of Calgary (2018). Rep Plus V1.1R [Computer software]. https://pages.cpsc.ucalgary.ca/ gaines/repplus/blog/files/079ebab90d30f7434265affac d362682-15.html

v. Werder, A. (2011). Corporate governance and stakeholder opportunism. Organization Science, 22(5), 1345-1358.

Valerdi, R., \& Majchrzak, A. (2003). Individual players in a team sport: Stakeholder change in commitment in ISD projects. AMCIS 2003 Proceedings, 1389-1393.

Vallaster, C., \& Von Wallpach, S. (2013). An online discursive inquiry into the social dynamics of multi-stakeholder brand meaning co-creation. Journal of Business Research, 66(9), 1505-1515. https://doi.org/10.1016/j.jbusres.2012.09.012

van Offenbeek, M. A., \& Vos, J. F. (2016). An integrative framework for managing project issues across stakeholder groups. International Journal of Project Management, 34(1), 44-57. 
Vandekerckhove, W., \& Dentchev, N. (2005). A Network Perspective on Stakeholder Management: Facilitating Entrepreneurs in the Discovery of Opportunities. Journal of Business Ethics, 60(3), 221-232. https://doi.org/10.1007/s10551-005-0130-7

Vickers, G. (1970). Freedom in a Rocking Boat. London: Allan Lane.

Vickers, G. (1995). The art of judgment: a study of policy making. Thousand Oaks: Sage Publishers. (Original work published 1965)

Vickers, G. (1984). The Vickers Papers. HarperCollins.

Vickers, S. G. (1987). Policymaking, Communication, and Social Learning: Essays of Sir Geoffrey Vickers (G. B. Adams, J., Forester, \& B. L., Catron, Eds.). Transaction Books. New Brunswick NJ.

Vlăsceanu, L., Grünberg, L., \& Pârlea, D. (2004). Quality assurance and accreditation: A glossary of basic terms and definitions. Unesco-Cepes Bucharest.

Wagner, H., Pankratz, O., Mellis, W., \& Basten, D. (2015). Effort of EAI projects: A repertory grid investigation of influencing factors. Project Management Journal, 46(5), 62-80.

Wang, W., Liu, W., \& Mingers, J. (2015). A systemic method for organisational stakeholder identification and analysis using Soft Systems Methodology (SSM). European Journal of Operational Research, 246(2), 562-574.

Wasserman, S., \& Galaskiewicz, J. (1994). Advances in social network analysis research in the social and behavioral sciences. SAGE.

Waxenberger, B., \& Spence, L. J. (2003). Reinterpretation of a metaphor: From stakes to claims. Strategic Change, 12(5), 239-249.

Weick, K. E., Sutcliffe, K. M., \& Obstfeld, D. (2005). Organizing and the process of sensemaking. Organization Science, 16(4), 409-421. 
Weitzner, D., \& Deutsch, Y. (2015). Understanding Motivation and Social Influence in Stakeholder Prioritization. Organization Studies, 36(10), 1337-1360.

Wiersma, W. (2009). Research methods in education: An introduction (9th ed.). Pearson/Allyn and Bacon.

Williams, G. (2005). Geoffrey Vickers: Philosopher of responsibility. Systems Research and Behavioral Science: The Official Journal of the International Federation for Systems Research, 22(4), 291-298.

Williams, N. L., Ferdinand, N., \& Pasian, B. (2015). Online Stakeholder Interactions in the Early Stage of a Megaproject. Project Management Journal, 46(6), 92-110.

Windsor, D. (2010). The role of dynamics in stakeholder thinking. Journal of Business Ethics, 96(1), 79.

Winn, M. I. (2001). Building stakeholder theory with a decision modeling methodology. Business \& Society, 40(2), 133-166.

Winter, M., Smith, C., Morris, P., \& Cicmil, S. (2006). Directions for future research in project management: The main findings of a UK government-funded research network. International journal of project management, 24(8), 638-649.

Wolfe, R. A., \& Putler, D. S. (2002). How tight are the ties that bind stakeholder groups? Organization Science, 13(1), 64-80.

Wolsink, M., \& Breukers, S. (2010). Contrasting the core beliefs regarding the effective implementation of wind power. An international study of stakeholder perspectives. Journal of Environmental Planning and Management, 53(5), 535-558. https://doi.org/10.1080/09640561003633581

Yang, C. C., \& Yeh, C. H. (2014). Application of System Dynamics in Environmental Risk Management of Project Management for External Stakeholders. Systemic Practice and Action Research, 27(3), 211-225. https://doi.org/10.1007/s11213-013-9283-y 
Yang, J., Shen, G. Q., Ho, M., Drew, D. S., \& Xue, X. (2011). Stakeholder management in construction: An empirical study to address research gaps in previous studies. International Journal of Project Management, 29(7), 900-910.

Yang, R. J., Wang, Y., \& Jin, X.-H. (2014). Stakeholders' attributes, behaviors, and decisionmaking strategies in construction projects: Importance and correlations in practice. Project Management Journal, 45(3), 74-90.

Yang, R. J., Zou, P. X., \& Wang, J. (2016). Modelling stakeholder-associated risk networks in green building projects. International Journal of Project Management, 34(1), 6681.

Yang, R. J. (2014). An investigation of stakeholder analysis in urban development projects: Empirical or rationalistic perspectives. International Journal of Project Management, 32(5), 838-849.

Yin, R. K. (2018). Case Study Research and Applications: design and methods. Sage publications.

York, J. G., O’Neil, I., \& Sarasvathy, S. D. (2016). Exploring environmental entrepreneurship: Identity coupling, venture goals, and stakeholder incentives. Journal of Management Studies, 53(5), 695-737.

Ziervogel, G., \& Downing, T. (2004). Stakeholder Networks: Improving Seasonal Climate Forecasts. Climatic Change, 65(1), 73-101.

\section{https://doi.org/10.1023/B:CLIM.0000037492.18679.9e}

Zwikael, O., Elias, A. A., \& Ahn, M. (2012). Stakeholder collaboration and engagement in virtual Projects. International Journal of Networking and Virtual Organisations, 10 (2): 117-136. 


\section{Appendixes}

Appendix A: Interview guides in English

Appendix B: The grid sheet in English

Appendix C: Interview guides in Vietnamese

Appendix D: The grid sheet in Vietnamese

Appendix E: Evidence of ethical approval

Appendix F: Information sheets and consent forms for supervisors in English and Vietnamese

Appendix G: Information sheets and consent forms for participants in English and

Vietnamese

Appendix H: Results of the principal component analysis of Thu's repertory grids - MTEO case

Appendix I: Results of the principal component analysis of Nga's repertory grids - MTEO

case

Appendix J: Results of the principal component analysis of Hung's repertory grids - MTEO case

Appendix K: Results of the principal component analysis of Tung's repertory grids - APSC case

Appendix L: Results of the principal component analysis of Tai's repertory grids - APSC case

Appendix M: Results of the principal component analysis of Khiem's repertory grids - APSC case

Appendix N: Results of the principal component analysis of Rafat's repertory grids -APSC case

Appendix O: Results of the principal component analysis of Amish's repertory grids - ABCD case

Appendix P: Results of the principal component analysis of Nikki's repertory grids - ABCD case

Appendix Q: Results of the principal component analysis of Sumy's repertory grids - ABCD case 


\section{Appendix A: Interview Guides in English}

\section{AN EXPLORATION OF THE DYNAMICS OF STAKEHOLDER PERCEPTION OF PROJECTS}

\section{DATA COLLECTION GUIDELINES}

Data are collected from interviewing stakeholders of accreditation projects. For each participant, there are four interviews. In the first interviews, the Repertory Grid Technique is applied to create the repertory grids for the participants. In the second, third and fourth interviews, these grids are re-rated, and a number of open questions are also used.

\section{GUIDELINES FOR THE FIRST INTERVIEWS}

\section{AN EXPLANATION OF THE STEPS OF INTERVIEWS USING THE REPERTORY GRID TECHNIQUES}

The first interviews start with questions about the participant background. The main sections of the interviews then consist of five steps. In step 1, the participants and the researcher agree on the interview topics. In step 2, the participants identify the elements or the objects of the interviews. In step 3, the participants identify characteristics or constructs of the objects. In step 4, the participants rate the objects (the elements) according to the characteristics (the constructs). In step 5, the participants review their answers and ratings. The detailed explanations of the steps and their examples are presented below.

\section{Background information}

How long have you been working? What is your relationship to the project? How do you affect, or are you affected by the project?

\section{Main section}

2.1. Step 1: Agreeing on the topic.

The researcher explains to the participants the interview topics, which are the participant perception of their accreditation projects. 


\subsection{Step 2: Eliciting the elements.}

The researcher shows the participants six small cards with texts on them. The texts are "Ideal project", "Less than ideal project," "The target accreditation project," and the names of three other projects that the participants have worked on and find most relevant to their perception of the target projects. An illustration of the six cards is presented below.

Figure A1 An illustration of the element cards
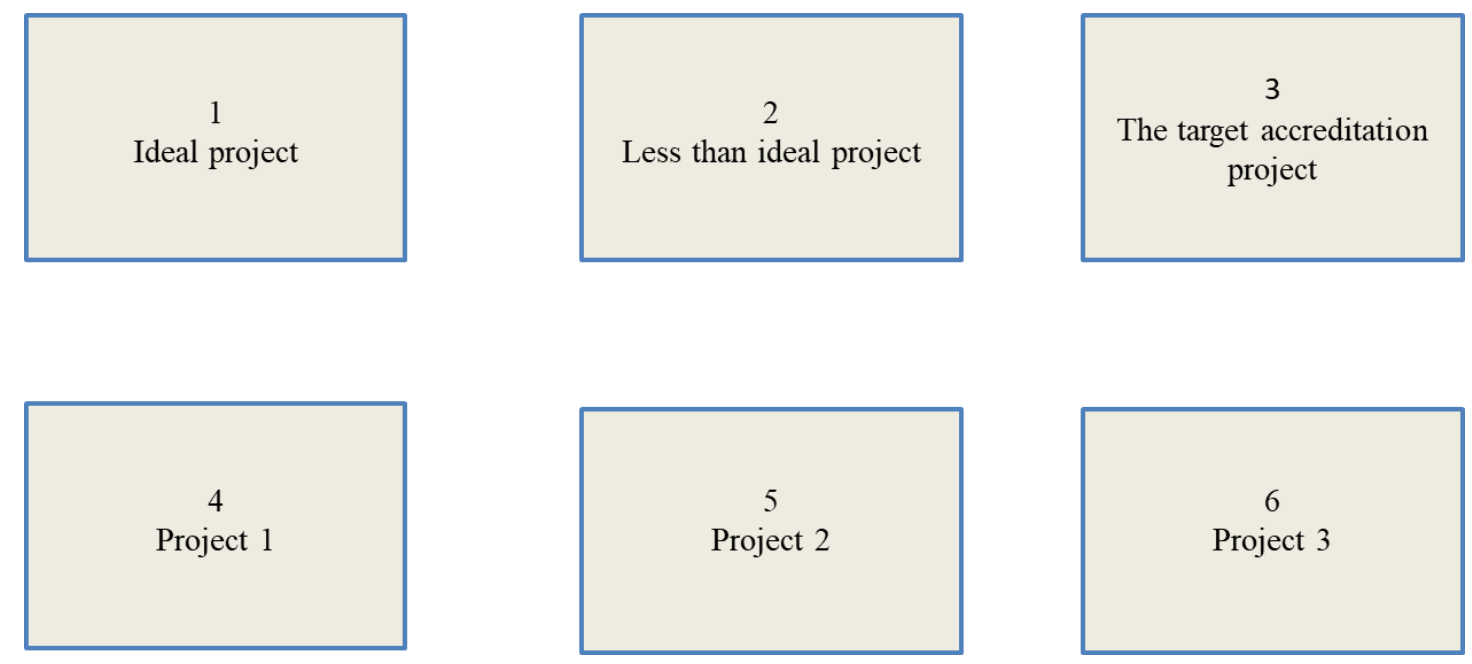

These six projects are written at the top of the grid sheets. The top of a grid sheet is illustrated below.

Table Al An illustration of the top of a grid sheet

\begin{tabular}{|c|c|c|c|c|c|c|c|c|}
\hline & & $\begin{array}{c}\text { Element } \\
1\end{array}$ & $\begin{array}{c}\text { Element } \\
2\end{array}$ & $\begin{array}{c}\text { Element } \\
\mathbf{3} \\
\end{array}$ & $\begin{array}{c}\text { Element } \\
4\end{array}$ & $\begin{array}{c}\text { Element } \\
5\end{array}$ & $\begin{array}{c}\text { Element } \\
6\end{array}$ & \\
\hline $\begin{array}{l}\mathbf{N} \\
\mathbf{0}\end{array}$ & $\begin{array}{c}\text { Constructs } \\
\text { (1) }\end{array}$ & $\begin{array}{c}\text { Ideal } \\
\text { project }\end{array}$ & $\begin{array}{l}\text { Less } \\
\text { than } \\
\text { ideal } \\
\text { project }\end{array}$ & $\begin{array}{c}\text { The } \\
\text { target } \\
\text { project }\end{array}$ & $\begin{array}{c}\text { Project } \\
1\end{array}$ & $\begin{array}{c}\text { Project } \\
2\end{array}$ & $\begin{array}{c}\text { Project } \\
3\end{array}$ & $\begin{array}{l}\text { Opposite constructs } \\
\text { (5) }\end{array}$ \\
\hline 1 & & & & & & & & \\
\hline
\end{tabular}

\subsection{Step 3: Eliciting the constructs}

The researcher chooses a set of any three cards and asks the participants: "Which two of these are the same in some ways, and different from the third? " An illustration of the set of three cards is presented below. 
Figure A2 An illustration of three cards for the triadic elicitation

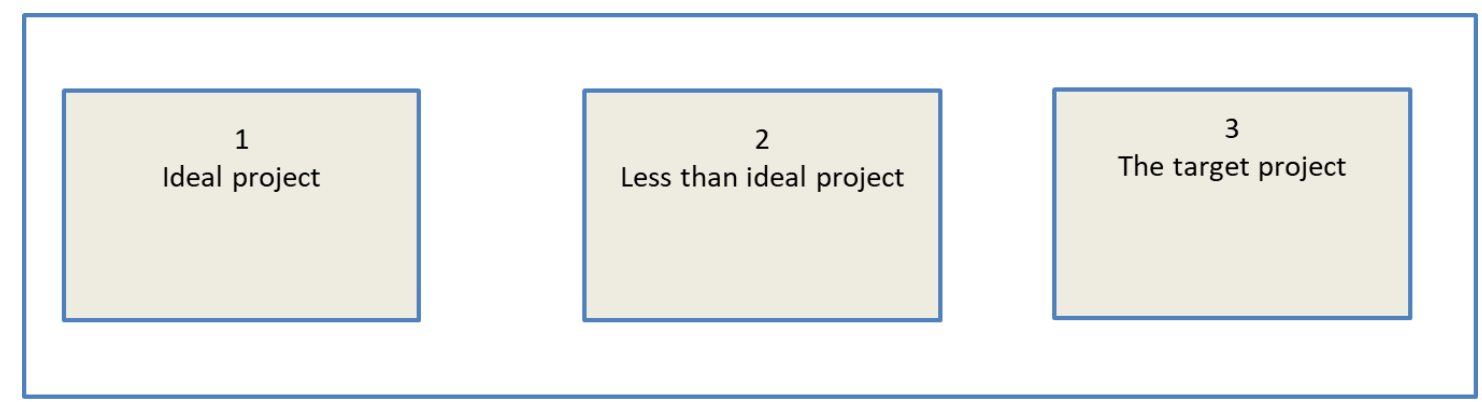

When the participants decide the characteristics, the characteristics are written down on the grid sheets as the constructs. An example of the answers in a grid sheet is presented below. Table A 2 An illustration of the answers on a grid sheet

\begin{tabular}{|c|c|c|c|c|c|c|c|c|}
\hline & & Element & Element & Element & Element & Element & Element \\
$\mathbf{1}$ & $\mathbf{2}$ & $\mathbf{3}$ & $\mathbf{4}$ & $\mathbf{5}$ & \\
\cline { 2 - 8 } $\mathbf{N}$ & $\begin{array}{c}\text { Constructs } \\
\mathbf{( 1 )}\end{array}$ & $\begin{array}{c}\text { Ideal } \\
\text { project }\end{array}$ & $\begin{array}{c}\text { Less } \\
\text { than } \\
\text { ideal } \\
\text { project }\end{array}$ & $\begin{array}{c}\text { The } \\
\text { target } \\
\text { project }\end{array}$ & $\begin{array}{c}\text { Project } \\
1\end{array}$ & $\begin{array}{c}\text { Project } \\
2\end{array}$ & $\begin{array}{c}\text { Project } \\
3\end{array}$ & Opposite constructs \\
& & & & & & & (5) \\
1 & $\begin{array}{c}\text { Being on } \\
\text { time }\end{array}$ & & & & & & & Being late \\
\hline
\end{tabular}

The question of "Which two of these are the same in some way, and different from the third?" can be asked many times for the same set of three cards to elicit as many constructs as possible. Other sets of three cards are then used similarly until no new constructs come up. When the meanings of constructs are not clear, laddering questions can be asked to clarify the meanings. Examples of these questions are:

- What do you mean by...?

- What sort of thing do you have in mind when you say...?

- Can you give me an example of the one and the other?

- Why does...make the project management different?

The answers to these questions will replace the originally unclear answers in the grid sheets.

2.4. Step 4: Rating the elements

When all the constructs have been elicited, the participants rate the element projects according to the constructs. The scales are from " 1 " to " 5 " or from " 1 " to "7" and the same for 
participants within the same cases. "1" defines the words on the left of the Grids and " 5 " or "7" defines the words on the right of the Grids. The participants can choose numbers from "1" to " 5 " or " 1 " to " 7 " to say which end of the scales the projects are nearest to. An example of a grid sheet with the 5-point scale is presented below:

Figure A3 An example of a grid with the 5-point scale rating (Source: Jankowicz, 2005)

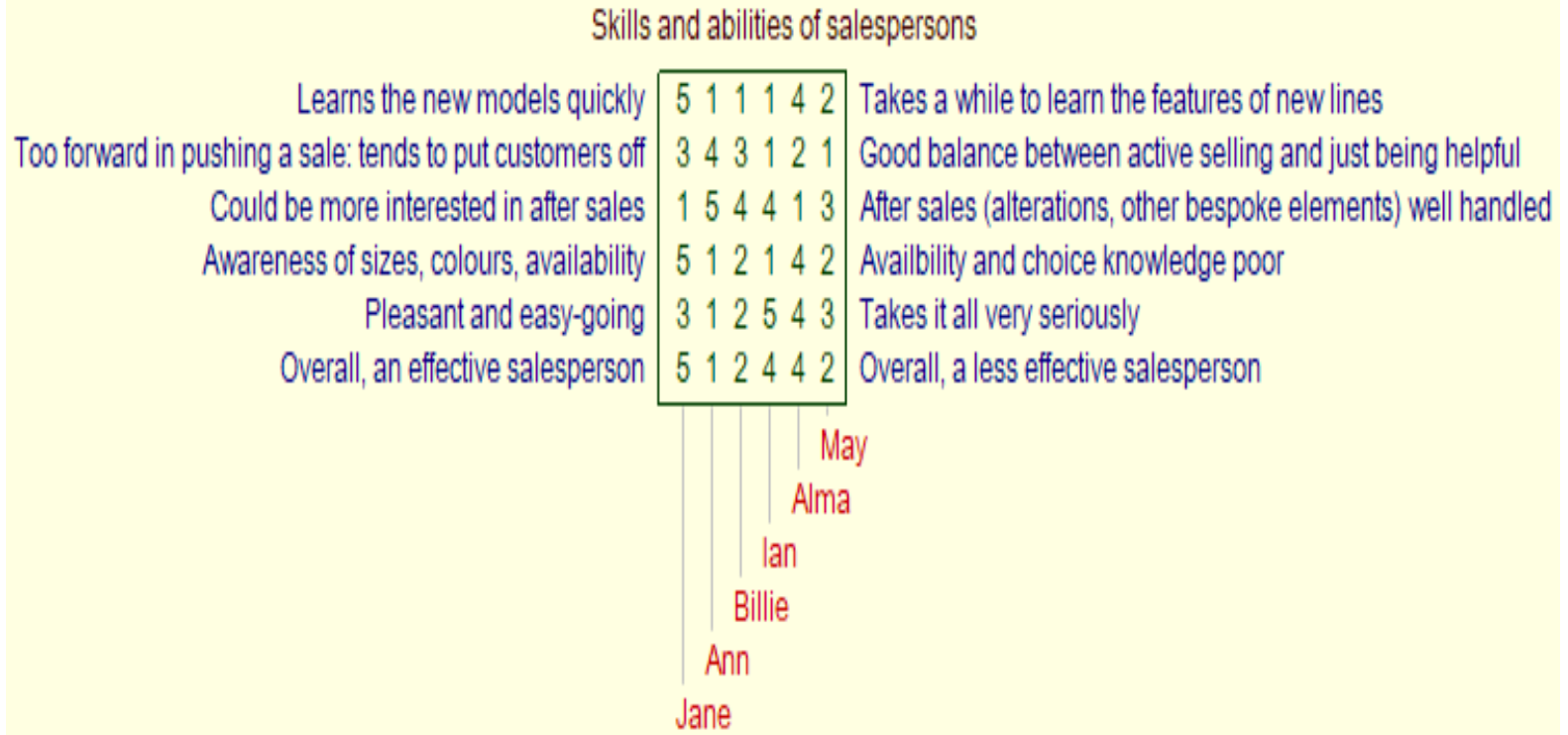

2.5. Step 5: Reviewing the elicited constructs and ratings.

When the grids and the ratings are completed, the participants review the overall content of the grids.

\section{GUIDELINES FOR THE SECOND, THIRD AND FOURTH INTERVIEWS}

These interviews are to capture the changes in the participant perception of their projects at the time of the interviews and the reasons for those changes. To avoid the biases caused by the grids, the interviews begin first with general questions:

1. How can you describe the ideal and less than ideal projects now? Is there any change in comparison to the previous interviews? What are the reasons for these changes? What has happened since the last interviews that make you change your thinking?

2. Can you please tell me what has happened in the target project since the last interview?

3. Do you have any concern about the projects now?

After that, the researcher shows the participants the grid sheets that record their answers in the previous interviews. The researcher asks the participants whether they want to modify the 
constructs and ratings in the Grids. The participants can also leave the ratings blank if they do not find the constructs relevant anymore. The participants are also asked to explain the reasons for the changes they made to the grids:

4. Do you want to add new constructs to the grid? What are they? Why do you want to add these constructs?

5. Is any construct not relevant anymore? Do you want to remove it from the grid? Why is that?

6. Why do your ratings change? Why do you leave the rating blank? Why do you keep the ratings the same? What has happened, and how does it affect your re-ratings? 


\section{Appendix B: The Grid Sheet in English}

\section{GRID SHEET}

Topic: Perception of the....... accreditation project

Hello, I am Van Thi La, a PhD student from the School of Management, Victoria University of Wellington. Thank you for agreeing to participate in this interview. This interview is the first part of my data collection plan which consists of four interviews. The goal of this interview is to understand your thinking about the...... project. The steps of this interview are based on the Repertory Grid Technique. The results of the interview will be filled out in the grid sheet below. Please be aware that I am not trying to find correct answers; I am trying to get your free opinion on the topic. This interview is expected to last from one and a half to two hours and will be audio recorded.

Name:

Phone:

Email:

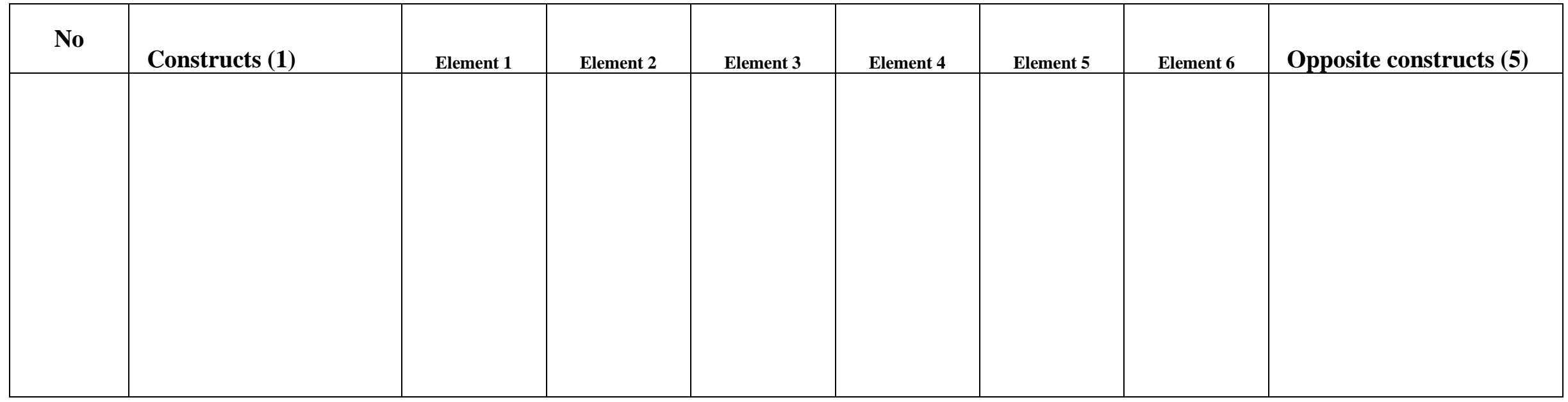




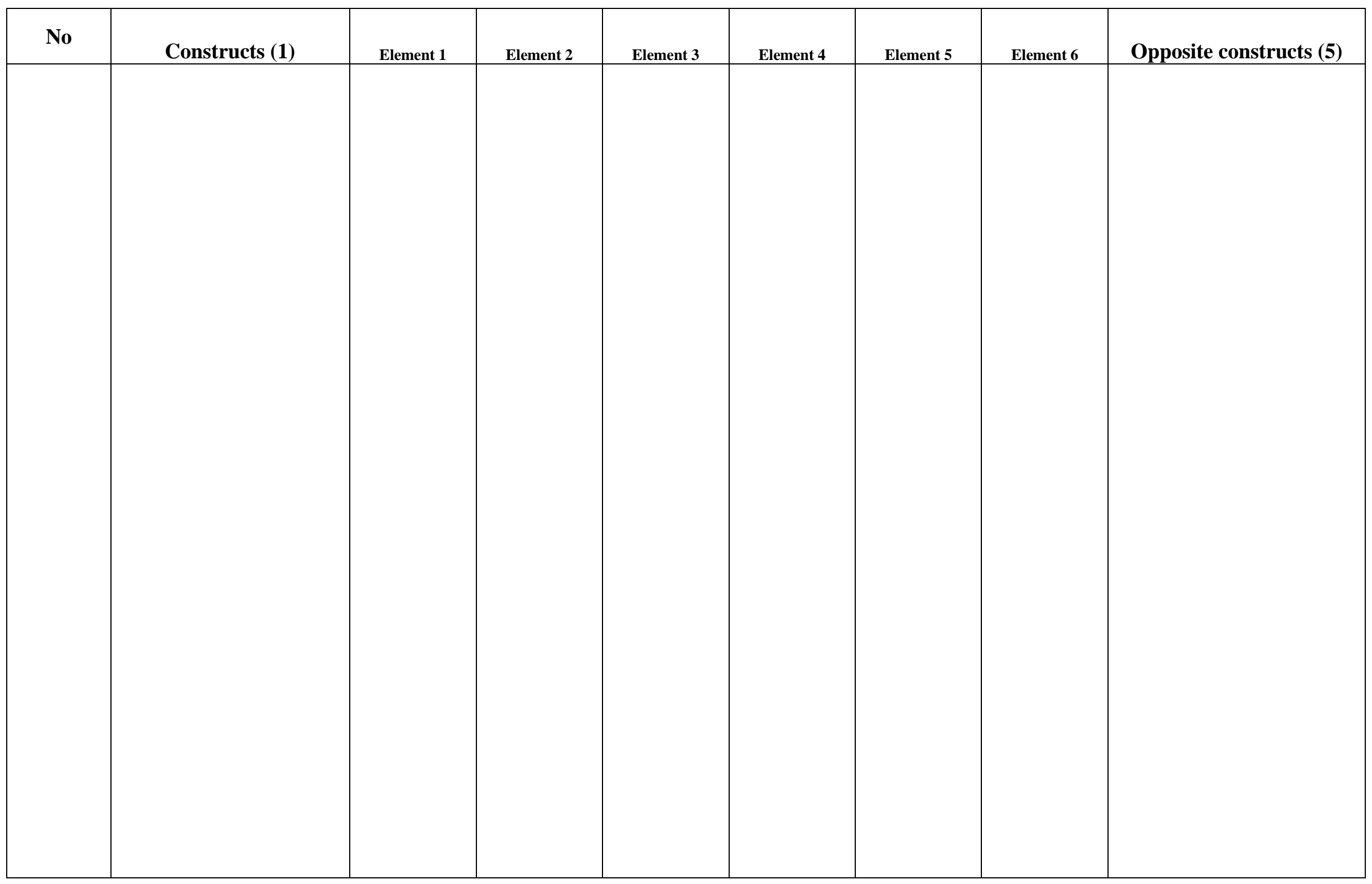

240 


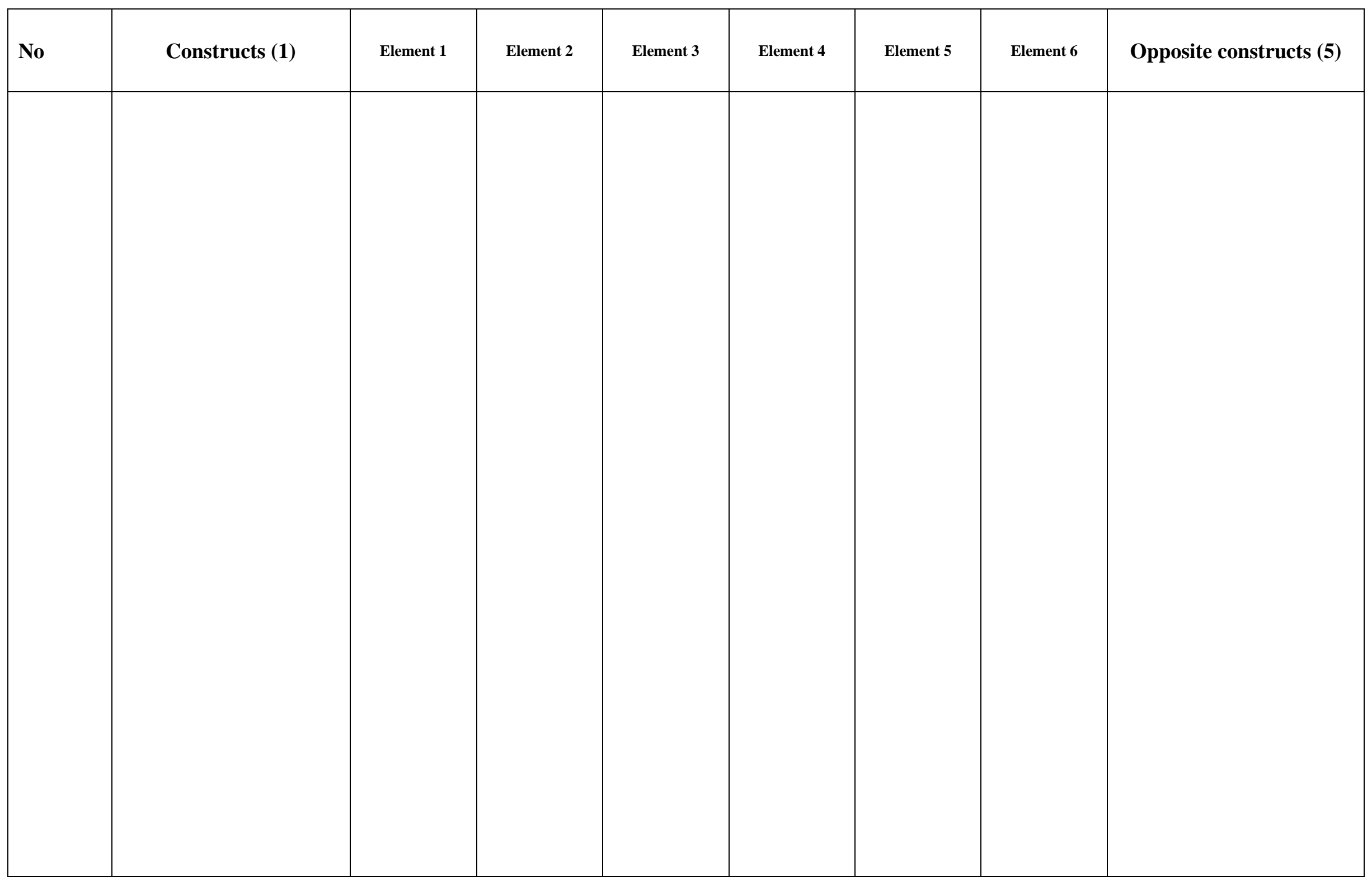

241 


\section{Appendix C: Interview Guides in Vietnamese}

\section{NGHIÊN CÚU SỤ THAY ĐỔI NHẬN THỨC CỦA CÁC BÊE LIÊN QUAN VỀ DỤ ÁN}

\section{HƯớNG DÃ̃N CÂU HỎI PHỎNG VẤN}

Số liệu được thu thập bằng phỏng vấn những người liên quan đến dự án kiểm định....tại trường Đại học...... Mỗi đối tượng phỏng vấn được phỏng vấn định kỳ trong bốn đến năm tháng. Mỗi lần phỏng vấn kéo dài khoảng từ 30 phút đến 2 tiếng. Trong lần phỏng vấn đầu tiên, phương pháp Lưới thông tin được sử dụng để xây dựng các bảng miêu tả nhận thức của người được phỏng vấn về dự án. Trong các lần phỏng vấn tiếp sau, người phỏng vấn thay đổi nội dung và xếp hạng của những bảng này và trả lời một số câu hỏi mở liên quan.

\section{HƯớNG DẪN CHO PHỎNG VẤN LẦN THỨ NHẤT}

\section{GIẢI THÍCH BƯỚC PHỎNG VÂN SỬ DỤNG PHƯƠNG PHÁP LƯỚI THÔNG TIN}

Phỏng vấn bắt đầu với những câu hỏi về kinh nghiệm, vị trí và vai trò của người được phỏng vấn trong dự án kiểm định. Phần chính của phỏng vấn bao gồm năm bước. Ở bước 01 , người phỏng vấn và nghiên cứu viên sẽ thống nhất về chủ đề phỏng vấn. Ở bước 02 , người được phỏng vấn sẽ xác định các yếu tố hay các dự án sẽ được thảo luận trong phỏng vấn. Ở bước 03 , người được phỏng vấn sẽ xác định các đặc điểm của các yếu tố này. Ở bước 04 , người được phỏng vấn sẽ xếp hạng các yếu tố theo các đặc điểm này. Ở bước 05 , người được phỏng vấn sẽ xem xét lại các câu trả lời và xếp hạng của họ. Mỗi bước được giải thích chi tiết cùng ví dụ minh hoạ dưới đây.

\section{Thông tin chung về người được phỏng vấn}

Xin Anh/Chị nói qua về kinh nghiệm làm việc của Anh/Chị?

Mối liên hệ của Anh/Chị với dụ án kiểm định.....ở truờng Đại học....?Anh huởng của Anh/Chị đến dụ án cũng như dụ án ảnh hưởng đến Anh/Chị như thế nào?

\section{Phần chính}

2.1. Bước 1: Thống nhất chủ đề phỏng vấn. 
Nghiên cứu viên giải thích cho người được phỏng vấn về chủ đề của cuộc phỏng vấn. Chủ đề đó là: "Nhận thức của Anh/Chị về dự án kiểm định .....của trường Đại học ....."

2.2. Bước 02: Xác định các yếu tố.

Nghiên cứu viên đưa cho người được phỏng vấn 06 thẻ giấy. Trên các thẻ giấy lần lượt ghi: "Dự án lý tưởng”, "Dự án không lý tưởng”, "Dự án kiểm định mục tiêu của nghiên cứu”, và 03 dự án khác người được phỏng vấn đã từng có kinh nghiệm và thấy liên quan nhiều nhất đến nhận thức của họ đối với dự án mục tiêu. Minh hoạ của 06 thẻ giấy này như sau.
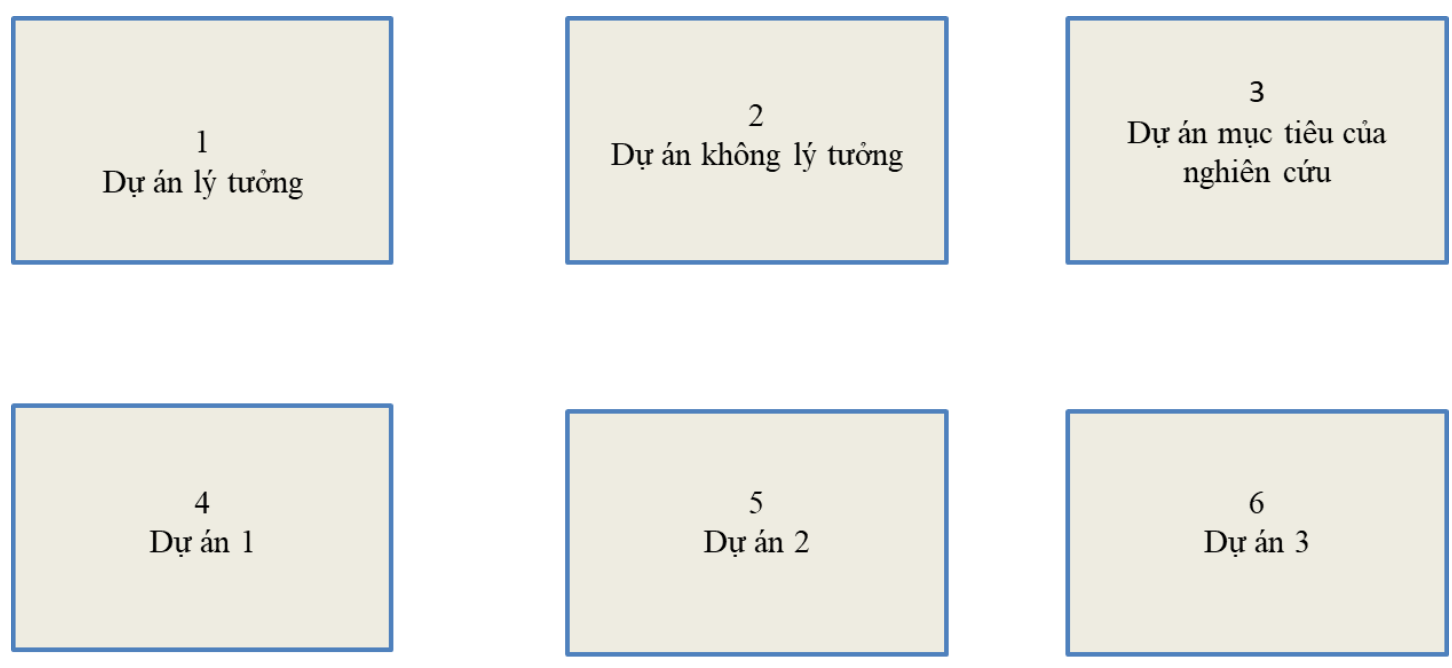

Sáu dự án này sẽ được ghi ở hàng trên cùng của Bảng lưới. Minh hoạ của dòng trên cùng của một Bảng lưới như sau:

\begin{tabular}{|c|c|c|c|c|c|c|c|}
\hline & $\begin{array}{c}\text { Yếu tố } \\
1\end{array}$ & $\begin{array}{c}\text { Yếu tố } \\
2\end{array}$ & $\begin{array}{c}\text { Yếu tố } \\
3\end{array}$ & $\begin{array}{c}\text { Yếu tố } \\
4\end{array}$ & $\begin{array}{c}\text { Yếu tố } \\
5\end{array}$ & $\begin{array}{c}\text { Yếu tố } \\
6\end{array}$ & \\
\hline Đặc điểm (1) & $\begin{array}{l}\text { Dự án } \\
\text { lý } \\
\text { tưởng }\end{array}$ & $\begin{array}{l}\text { Dự án } \\
\text { kém lý } \\
\text { tưởng }\end{array}$ & $\begin{array}{l}\text { Dụ̣ án } \\
\text { mục } \\
\text { tiêu }\end{array}$ & Dự án 1 & Dự án 2 & Dụ án 3 & $\begin{array}{l}\text { Đặc điểm đối } \\
\text { lập (5) }\end{array}$ \\
\hline & & & & & & & \\
\hline & & & & & & & \\
\hline
\end{tabular}

\subsection{Bước 3: Xác định các đặc điểm của dự án}

Mục đích của bước này là xác định nhận thức của người được phỏng vấn về các đặc điểm của dự án. Nghiên cứu viên sẽ chọn bất cứ 03 thẻ giấy nào và hỏi người được phỏng vấn: "Hãy nêu một đặc điểm khiến 02 dự án bất kỳ trong 03 dự án dưới đây giống nhau, và khác biệt với dự án thứ 03 ?” Ví dụ của một bộ ba thẻ giấy được minh hoạ dưới đây. 


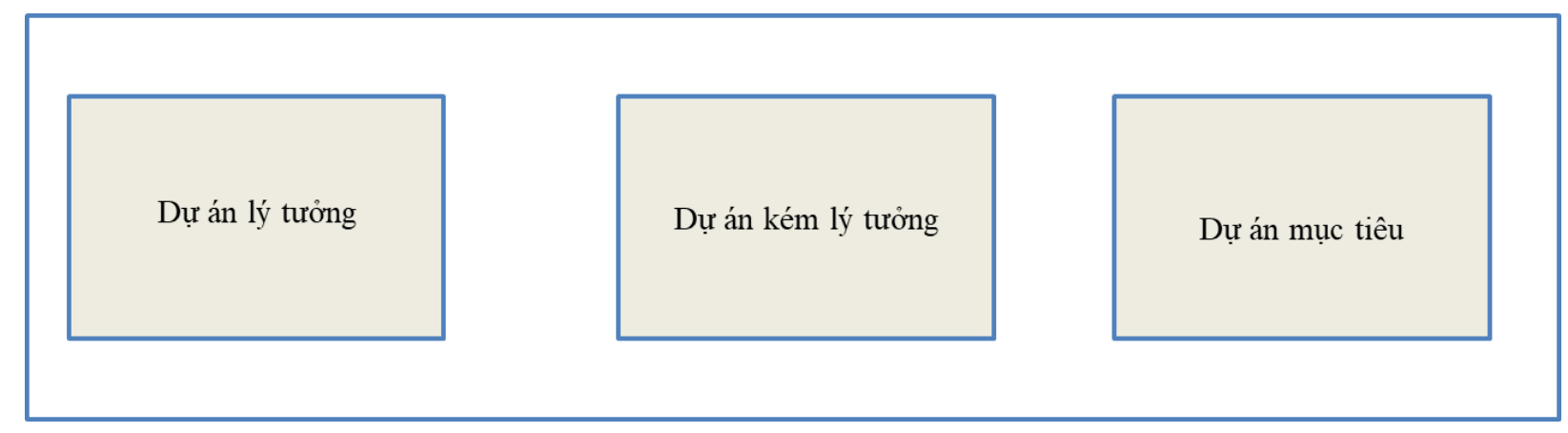

Khi người được phỏng vấn đã xác định được một đặc điểm, đặc điểm này sẽ được viết xuống Bảng Lưới. Ví dụ về câu trả lời ghi trong một Bảng Lưới được minh hoạ dưới đây.

\begin{tabular}{|c|c|c|c|c|c|c|c|}
\hline & $\begin{array}{c}\text { Yếu tố } \\
1\end{array}$ & $\begin{array}{c}\text { Yếu tố } \\
2\end{array}$ & $\begin{array}{c}\text { Yếu tố } \\
3\end{array}$ & $\begin{array}{c}\text { Yếu tố } \\
4\end{array}$ & $\begin{array}{c}\text { Yếu tố } \\
5\end{array}$ & $\begin{array}{c}\text { Yếu tố } \\
6\end{array}$ & \\
\hline Đặc điểm (1) & $\begin{array}{l}\text { Dự án lý } \\
\text { tướng }\end{array}$ & $\begin{array}{l}\text { Dự án lý } \\
\text { tưởng }\end{array}$ & $\begin{array}{c}\text { Dự án } \\
\text { mục tiêu }\end{array}$ & Dự án 1 & Dự án 2 & Dự án 3 & $\begin{array}{c}\text { Đặc điểm đối } \\
\text { lập (5) }\end{array}$ \\
\hline $\begin{array}{c}\text { Hoàn thành đúng } \\
\text { thời hạn }\end{array}$ & & & & & Bị trễ \\
\hline
\end{tabular}

Câu hỏi: "Hãy nêu một đặc điểm khiến 02 dự án bất kỳ trong 03 dự án dưới đây giống nhau, và khác biệt với dự án thứ 03?” sẽ được hỏi nhiều lần cho cùng một bộ 03 thẻ giấy để có thể xác định càng nhiều đặc điểm càng tốt. Các bộ 03 thẻ giấy khác sẽ được hỏi tương tự cho đến khi không có đặc điểm mới nào xuất hiên. Nếu một đặc điểm nào đó không rõ ràng, người được phỏng vấn có thể đặt thêm câu hỏi để làm rõ các đặc điểm. Ví dụ các câu hỏi là:

- Đặc điểm này có nghĩa là gì?

- $\quad$ Anh chị nghĩ gì khi nói về đặc điểm này?

- Anh chị có thể cho một ví dụ về đặc điểm này?

- Tại sao đặc điểm này làm cho các dự án khác biệt ?

2.7. Bước 4: Xếp hạng các yếu tố.

Khi các đặc điểm đã được xác định, người được phỏng vấn sẽ xếp hạng các yếu tố (dự án) theo các đặc điểm này. Thang xếp hạng từ " 1 " đến " 5 " hoặc "7" sẽ được sử dụng. "1" là cho những đặc điểm ở phía bên trái của Bảng lưới và " 5 " hoặc "7" là cho những đặc điểm phía bên phải của Bảng lưới. 
2.8. Bước 5: Xem xét lại các đặc điểm và xếp hạng.

Sau khi Bảng lưới và xếp hạng đã hoàn thành, người được phỏng vấn sẽ xem xét, kiểm tra lại toàn bộ nội dung của Bảng lưới.

\section{HƯớNG DÃN PHỎNG VẤN LẦN THÚ’ 02, THÚ̉ 03 VÀ THÚ’ 04}

Sau lần phỏng vấn đầu tiên, những lần phỏng vấn tiếp sau tập trung vào nắm bắt những thay đổi trong nhận thức của người được phỏng vấn về dự án. Phỏng vấn bắt đầu bằng những câu hỏi tổng quan như:

1. Anh/Chị có thể miêu tả quan điểm của Anh/Chị về dự án kiểm định tốt và không tốt ở thời điểm hiện tại? Liệu có thay đổi nào trong nhận thức của Anh/Chị so với lần phỏng vấn trước? Nguyên nhân của những thay đổi đó là gì? Điều gì đã xảy ra từ lần phỏng vấn trước khiến Anh/Chị thay đổi quan điểm?

2. Anh/Chị có thể cho biết điều gì đã xảy ra từ lần phỏng vấn trước? Bao gồm tất cả những hoạt động liên quan đến dự án và những hoạt động không liên quan đến dự án.

3. Anh/Chị có quan tâm nào cho dự án tại thời điểm hiện tại?

Sau đó, người được phỏng vấn sẽ hỏi liệu họ có muốn thay đổi nội dung và xếp hạng của Bảng lưới cho phù hợp với suy nghĩ hiện tại của họ về dứ án:

1. Có đặc điểm nào Anh/Chị không thấy phù hợp nữa và muốn bỏ ra khỏi Bảng lưới? Anh/Chị có thể giải thích lý do?

2. Anh/Chị có muốn thêm đặc điểm mới vào trong Bảng? Những đặc điểm mới đó là gì? Tại sao Anh/Chị lại muốn thêm những đặc điểm đó?

3. Tại sao xếp hạng của Anh/Chị thay đổi? Điều gì đã xảy ra ảnh hưởng đến xếp hạng của Anh/Chị? Tại sao Anh/Chị muốn để trống xếp hạng? Tại sao Anh/Chị không thay đổi xếp hạng? 


\section{Appendix D: The Grid Sheet in Vietnamese}

\section{BẢNG LƯỚI THÔNG TIN}

Chủ đề: Suy nghĩ về dự án kiểm định.......

Tôi là La Thị Vân, nghiên cứu sinh tại Truờng Quản lý, Đại học Victoria, Wellington, New Zealand. Cảm ơn vì đã tham gia vào cuộc phỏng vấn này. Phỏng vấn này là phỏng vấn đầu tiên trong kế hoạch phỏng vấn bao gồm bốn cuộc phỏng vấn. Mục đích của phỏng vấn này là để hiểu suy nghĩ của bạn về dụ án kiểm định......ở truờng đại học...... Nhũ̃ng buớc trong phỏng vấn này dụa trên Kỹ Thuật Bảng Luới. Kết quả phỏng vấn sẽ được điền vào bảng duơói đây. Mục đỉch của phỏng vấn không phải để tìm câu trả lời đúng, mà để xác định quan điểm của bạn về chủ đề phỏng vấn. Cuộc phỏng vấn sẽ kéo dài từ một tiếng ruỡi đến hai tiếng và được ghi âm.

Tên:

Điện thoại: $\quad$ Email:

\begin{tabular}{|c|c|c|c|c|c|c|c|c|}
\hline Stt & Đặc điểm (1) & Yếu tố 1 & Yếu tố 2 & Yếu tô 3 & Yếu tố 4 & Yếu tố 5 & Yếu tố 6 & Đặc điểm đối lập (5) \\
\hline & & & & & & & & \\
\end{tabular}




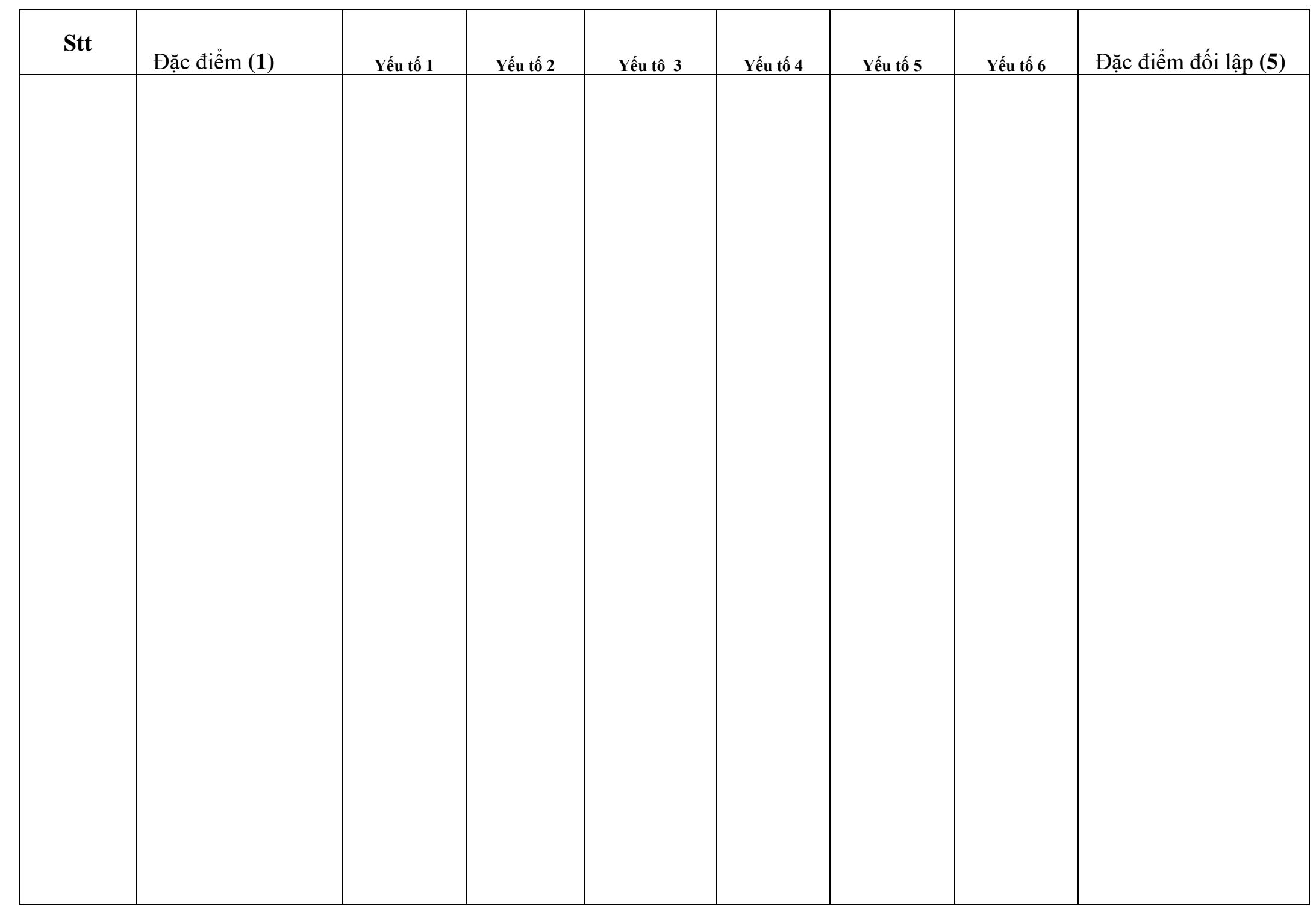




\section{Appendix E: Evidence of Ethical Approval}

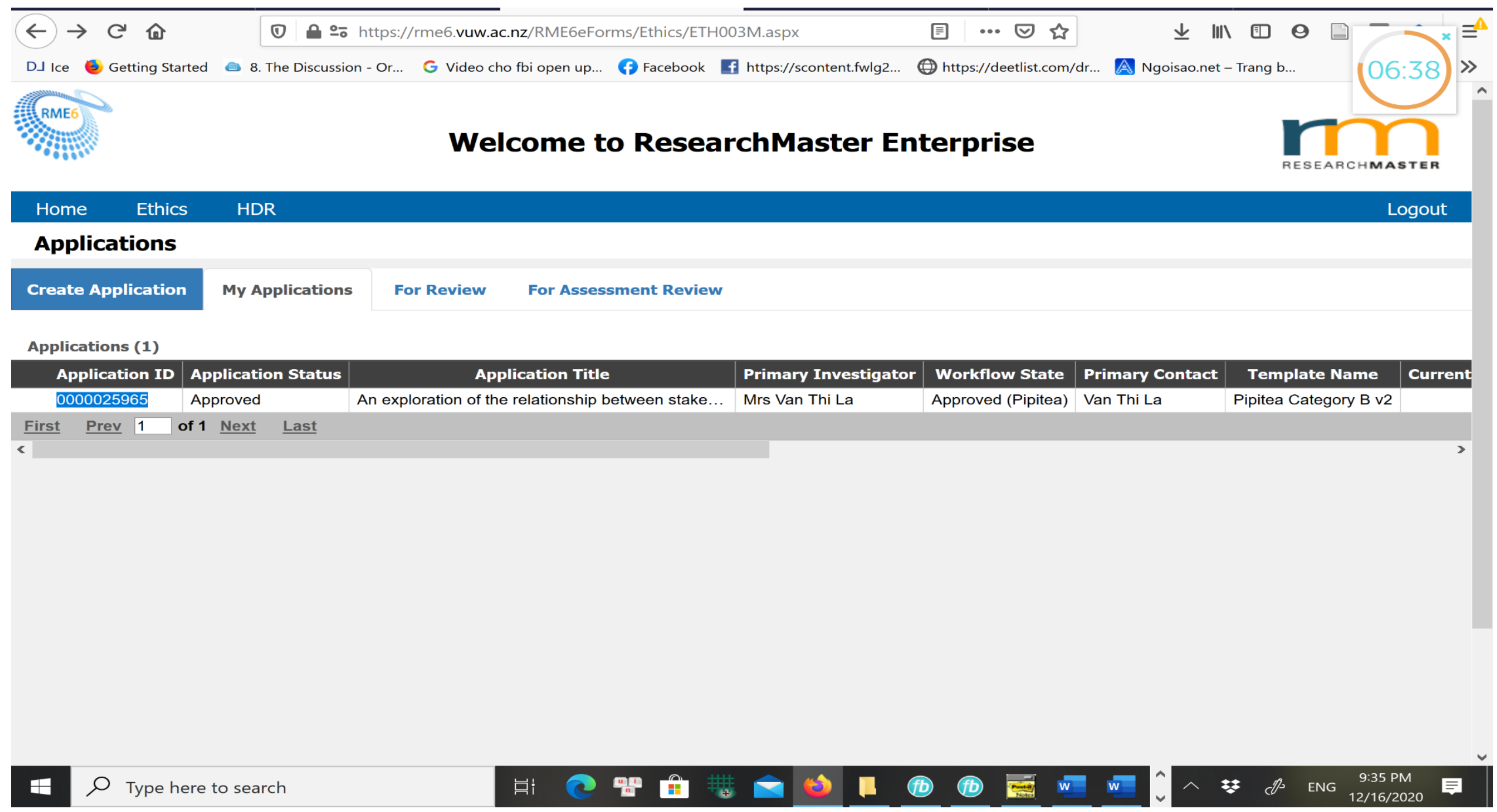




\section{Appendix F: Information Sheets and Consent Forms for Supervisors in English and Vietnamese}

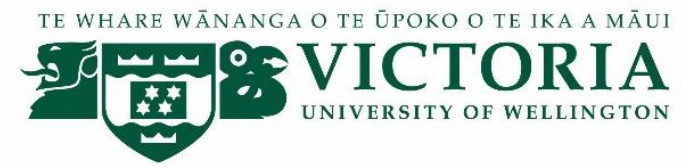

\section{NGHIÊN CÚU VỀ MỐI QUAN HẸ GIŨA TUUONG TÁC CỦA CÁC B̂EN LIÊN QUAN VÀ NHẬN THÜC CỦA HO VỂ DỦ ÁN \\ AN EXPLORATION OF THE RELATIONSHIP BETWEEN STAKEHOLDERS INTERACTION AND THEIR PERCEPTION OF PROJECTS}

\section{THƯ MÒ̀I CHO QUẢN LÝ CỦA NGƯờI ĐƯởC PHỎNG VẤN INFORMATION SHEET FOR SUPERVISORS}

Xin hãy đọc thông tin dưới đây trước khi quyết định cho phép hoặc không cho phép nhân viên của Anh/Chị tham gia nghiên cứu này. Xin cảm ơn rất nhiều nếu Anh/Chị cho phép nhân viên của Anh/Chị tham gia. Nếu Anh/Chị không cho phép nhân viên của Anh/Chị tham gia được, cảm ơn vì đã xem xét thư mời này.

Please read this information before deciding whether or not to let your staff members participate in this research. If you decide to let them participate, thank you. If you decide not to let them participate, thank you for considering this request.

Tôi là ai?

Who am I?

Tên tôi là La Thị Vân, nghiên cứu sinh tại Trường Quản Lý, Đại học Victoria, New Zealand. Nghiên cứu này để phục vụ cho luận án tiến sĩ của tôi

My name is Van Thi La, and I am a Doctoral student in the School of Management at Victoria University of Wellington. This research project contributes to my PhD thesis.

Mục đích của nghiên cứu này là gì?

What is the aim of the project?

Nghiên cứu này tập trung vào khám phá và phân tích tương tác giữa các đối tượng liên quan trong một dự án ảnh hưởng đến nhận thức của họ về dự án như thế nào trong một giai đoạn của dự án. Nghiên cứu này đã được đồng ý bởi Hội đồng Đạo đức Đại học Victoria, Wellington [Mã đơn tham vấn trên Research-Master: 0000025965]. 
This project focuses on exploring and analysing how interactions between individual stakeholders change their perceptions of projects during project implementation.

This research has been approved by the Victoria University of Wellington Human Ethics Committee [Research-Master application reference number: 0000025965]

\section{Anh/Chị có thể giúp bằng cách nào?}

\section{How can you help?}

Anh/Chị được gửi thư mời này bởi vì Anh/Chị là quản lý liên quan đến dự án kiểm định .... của Đại học ..... Nếu Anh/Chị đồng ý để nhân viên của mình liên quan đến dự án .... tham

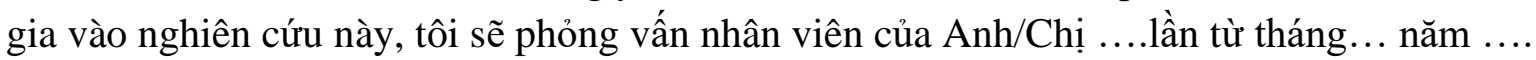
đến tháng .....năm ..... Nhân viên của Anh/Chị sẽ được hỏi về nhận thức của họ về dự án. Mỗi phỏng vấn sẽ kéo dài khoảng 60 phút. Tôi sẽ ghi âm phỏng vấn với sự cho phép của nhân viên Anh/Chị và chép lại phỏng vấn sau đó. Anh/Chị có thể quyết định cho nhân viên của mình rút khỏi nghiên cứu bằng cách liên lạc với tôi 02 tuần sau khi nhân viên của Anh/Chị nhận được tóm tắt của phỏng vấn. Nếu Anh/Chị quyết định rút khỏi nghiên cứu, toàn bộ thông tin anh chị cung cấp sẽ bị huỷ hoặc chuyển lại cho nhân viên Anh/Chị.

You have been given this information because you are the supervisor of departments or organisations which are stakeholders of the.....accreditation project, in.... University. If you agree to let your staff members take part in this research, I will interview them .... times from ...... to .... I will ask them about their perception of the project. Each interview will take about 60 minutes. I will audio record the interviews with their permission and write it up later. You can decide that your staff members withdraw from the study by contacting me at any time within 2 weeks after they receive the summaries of the interviews. If you withdraw, the information your staff members provided will be destroyed and returned to them.

\section{Điều gì sẽ xảy ra với tên tổ chức của Anh/Chị, danh tính của nhân viên Anh/Chị và thông tin họ cung cấp?}

What will happen to your organisation's name, your staff members' identities and the information they give?

Nghiên cứu này là bảo mật. Những nghiên cứu viên dưới đây sẽ biết về tên tổ chức của Anh/Chị, danh tính của người được phỏng vấn nhưng những dữ liệu nghiên cứu sẽ được xử lý để danh tính của tổ chức Anh/Chị và người được phỏng vấn sẽ không được tiết lộ trong bất cứ báo cáo, thuyết trình hay văn bản nào. Tuy nhiên, người được phỏng vấn cần biết rằng đồng nghiệp của họ trong tổ chức có thể biết về sự tham gia của họ trong nghiên cứu. Chỉ có tôi và giáo viên hướng dẫn của tôi sẽ đọc bản chép lại phỏng vấn của nhân viên Anh/Chị. Bản chép lại của phỏng vấn, tóm tắt và ghi âm sẽ được giữ an toàn và huỷ vào ngày 01 tháng 09 năm 2022. 
This research is confidential. The researchers named below will be aware of your organisation's name and the participants' identities. The participants' identities might also be obvious to other colleagues in your organisation. Howver, the data will be combined, and your organisation's name as well the participants' identities will not be revealed in any reports, presentations, or public documentation.

$r$

Only my supervisors and I will read the notes or transcripts of the interviews. The interview transcripts, summaries and any recordings will be kept securely and destroyed by the $1^{\text {st }}$ of September 2022.

\section{Nghiên cứu này phục vụ cho mục đích gì}

\section{What will the project produce?}

Thông tin từ nghiên cứu này sẽ được sử dụng trong luận văn tiến sĩ của tôi.

The information from this research will be used in my PhD dissertation

\section{Nếu nhận lời mời phỏng vấn này, quyền lợi của Anh/Chị sẽ là gì?}

If you accept this invitation, what are your rights as the supervisor of the participants?

Anh/Chị không phải nhận lời mời phỏng vấn này nếu Anh/Chị không muốn. Nếu Anh/Chị quyết định để nhân viên của Anh/Chị tham gia, Anh/Chị có những quyền sau:

- yêu cầu nhân viên của Anh/Chị rút khỏi nghiên cứu bất cứ lúc nào trong vòng 2 tuần sau khi nhận được tóm tắt phỏng vấn.

- hỏi câu hỏi bất kỳ về nghiên cứu ở bất cứ thời điểm nào.

You do not have to accept this request if you don't want to. If you do decide to let your staff members participate, you have the right to:

- $\quad$ ask your staff members to withdraw from the study any time within two weeks after they receive the summaries of the interviews.

- $\quad$ ask any question about the study at any time.

\section{Nếu Anh/Chị có bất kỳ câu hỏi hay vấn đề gì, Anh/Chị có thể liên hệ ai? If you have any questions or problems, who can you contact?}

Nếu Anh/Chị có bất kỳ câu hỏi nào, bây giờ hoặc trong tương lai, anh chị có thể liên hệ:

\section{Sinh viên:}

Tên: Van Thi La

Email address: VanThi.La@vuw.ac.nz

\section{Giáo viên hướng dẫn:}

Tên: AProf. Arun Elias

Vị trí: Phó chủ nhiệm khoa Trường Kinh

Doanh Victoria

Trường: Trường Quản lý 
Điện thoại: 044635736

Arun.elias@vuw.ac.nz

If you have any questions, either now or in the future, please feel free to contact:

Student:

Name: Van Thi La

Email address: VanThi.La@vuw.ac.nz

\section{Supervisor:}

Name: AProf. Arun Elias

Role: Associate Dean

School: School of Management

Phone: 044635736

Arun.elias@vuw.ac.nz

\section{Thông tin về Hội đồng đạo đức}

\section{Human Ethics Committee information}

Nếu Anh/Chị có bất cứ quan tâm nào đến vấn đề đạo đức của nghiên cứu này, Anh/Chị có thể liện hệ Uỷ viên Hội đồng Đạo đức trường Đại học Victoria: Dr. Loveridge. Email judith.loveridge@vuw.ac.nz or telephone +64-4-463 6028.

\section{Human Ethics Committee information}

If you have any concerns about the ethical conduct of the research you may contact the Victoria University HEC Convenor: Dr Judith Loveridge. Email judith.loveridge@ vuw.ac.nz or telephone +64-4-463 6028 . 


\title{
NGHIÊN CÚU VỀ MỐI QUAN Hệ GIỬA TƯƠNG TÁC CỦA CÁC BÊN LIÊN QUAN VÀ NHẬN THỨC CỦA HỌ VỀ DƯ ÁN AN EXPLORATION OF THE RELATIONSHIP BETWEEN STAKEHOLDERS INTERACTION AND THEIR PERCEPTION OF PROJECTS
}

\author{
THƯ ĐỒNG Ý CỦA QUẢN LÝ \\ CONSENT TO INTERVIEW FOR SUPERVISORS
}

Thư này sẽ được giữ trong 4 năm

This consent form will be held for 4 years.

Ngiên cứu viên: La Thị Vân, Trường Quản lý, Đại học Victoria, Wellington Researcher: Van Thi La, School of Management, Victoria University of Wellington.

- Tôi đã đọc Thư mời và đã hiểu về dự án nghiên cứu. Tôi hiểu rằng tôi có thể hỏi các câu hỏi ở bất cứ thời điểm nào.

• Tồi đồng ý để nhân viên của mình tham gia vào phỏng vấn có ghi âm.

- I have read the Information Sheet, and the project has been explained to me. My questions have been answered to my satisfaction. I understand that I can ask further questions at any time.

- I agree to let my staff members take part in the audio-recorded interviews.

Tôi hiểu rằng:

- Tôi có thể quyết định để nhân viên của tôi rút khỏi ngiên cứu ở bất cứ thời điểm nào trong vòng 02 tuần sau khi họ nhận được tóm tắt của phỏng vấn. Bất cứ thông tin nào nhân viên của tôi cung cấp sẽ được trả lại cho họ hoặc bị huỷ.

• Toàn bộ thông tin được cung cấp sẽ bị huỷ trước ngày 01 tháng 09 năm 2022.

- B Bất cứ thông tin nào nhân viên của tôi cung cấp sẽ được nghiên cứu viên và giáo viên hướng dẫn giữ bảo mật.

- K Kết quả của ngiên cứu sẽ được sử dụng cho một luận văn tiến sĩ.

- Các thành viên khác trong dự án kiểm định .... của trường đại học ....và các đồng nghiệp khác trong tổ chức của tôi có thể biết được sự tham gia của nhân viên của tôi vào nghiên cứu. Tuy nhiên, nhân viên của tôi sẽ được dùng tên giả trong báo cáo ngiên cứu và danh tính của họ sẽ không bị tiết lộ với người đọc ngiên cứu. Tên của tổ chức của tôi 
sẽ không được sử dụng trọng báo cáo, cũng như sẽ không có thông tin nào có thể nhận diện tổ chức của tôi.

\section{I understand that:}

- I can decide that my staff members withdraw from this study at any point within two weeks after they receive the summaries of the interviews. Any information my staff members have provided will be returned to them or destroyed.

- $\quad$ The information provided will be destroyed by 01/Sep/2022.

- $\quad$ Any information my staff members provide will be kept confidential to the researcher and supervisors.

- I understand that the results will be used for a PhD dissertation.

- I understand that the participation of my staff members in the interviews can be known by members of the ..... accreditation project and other colleagues in my organisation. However, they will be referred to by pseudonyms in reports, and they will not be identifiable to wider research audiences. Our organisation's name will not be used in reports, nor will any information that would identify us.

Chữ ký/ Signature:

Tên/ Name:

Vị trí/ Position:

Ngày/ Date:

Chi tiết liên lạc/ Contact details (Email): 


\title{
Appendix G: Information Sheets and Consent Forms for
}

\section{Participants in English and Vietnamese}

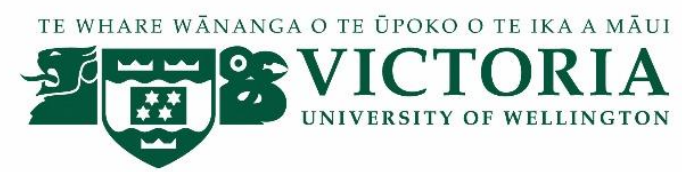

\section{NGHIÊN CÚU VỀ MỐI QUAN HÊ GIƯ⿱A TUUONG TÁC CỦA CÁC B̂EN LIÊN QUAN VÀ NHÂAN THÚC CỦA HO VỂ DỦ ÁN \\ AN EXPLORATION OF THE RELATIONSHIP BETWEEN STAKEHOLDERS INTERACTION AND THEIR PERCEPTION OF PROJECTS}

\author{
THƯ MỜI THAM GIA PHỎNG VẦN \\ INFORMATION SHEET FOR INTERVIEW PARTICIPANTS
}

Kính mời Anh/Chị tham gia vào ngiên cứu dưới đây. Xin hãy đọc thông tin dưới đấy trước khi quyết định tham gia. Xin cảm ơn rất nhiều nếu Anh/Chị tham gia,. Nếu Anh/Chị không tham gia, cảm ơn vì đã xem xét thư mời này.

You are invited to take part in this research. Please read this information before deciding whether or not to take part. If you decide to participate, thank you. If you decide not to participate, thank you for considering this request.

\section{Tôi là ai?}

\section{Who am I?}

Tên tôi là La Thị Vân, nghiên cứu sinh tại Trường Quản Lý, Đại học Victoria, New Zealand. Ngiên cứu này để phục vụ cho luận án tiến sĩ của tôi.

My name is Van Thi La and I am a Doctoral student in the School of Management at Victoria University of Wellington. This research project contributes to my PhD thesis.

Mục đích của ngiên cứu là gì?

\section{What is the aim of the project?}

Nghiên cứu này tập trung xác định và phân tich mối quan hệ giữa tương tác của các đối tượng liên quan và nhận thức của họ về dự án trong một giai đoạn của dự án. Nghiên cứu này đã được đồng ý bởi Hội đồng Đạo đức Đại học Victoria, Wellington.

This project focuses on exploring and analysing how interactions between individual stakeholders change their perceptions of projects during project implementation.

This research has been approved by the Victoria University of Wellington Human Ethics Committee [Research-Master application reference number: 0000025965] 


\section{Anh/Chị có thể giúp bằng cách nào?}

\section{How can you help?}

Anh/Chị được mời tham gia ngiên cứu này bởi vì Anh/Chị liên quan đến dự án kiểm định .... của Đại học .... Nếu Anh/Chị tham gia, tôi sẽ phỏng vấn Anh/Chị .... lần từ tháng.... năm ....đến tháng.... Năm..... Anh/Chị sẽ được hỏi về nhận thức của Anh/Chị về dự án. Mỗi phỏng vấn sẽ kéo dài khoảng 60 phút. Tôi sẽ ghi âm phỏng vấn với sự cho phép của anh chị và viết lại sau đó. Anh/Chị có thể chọn không trả lời bất cứ câu hỏi nào hoặc dừng phỏng vấn bất cứ thời điểm nào, không cần đưa ra lý do. Anh/Chị có thể rút khỏi nghiên cứu bằng cách liên lạc với tôi ở bất cứ thời điểm nào 02 tuần sau khi nhận được tóm tắt của phỏng vấn. Nếu anh chị rút khỏi nghiêm cứu, toàn bộ thông tin anh chị cung cấp sẽ bị huỷ hoặc chuyển lại cho anh chị.

You have been invited to participate because you are a stakeholder of the ..... accreditation project in ...... University. If you agree to take part, I will interview you four times from ..... to ...... I will ask you about your perception of the project. Each interview will take from one to two hours. I will audio record the interviews with your permission and write it up later. You can choose to not answer any question or stop the interviews at any time, without giving a reason. You can withdraw from the study by contacting me at any time within 2 weeks after receiving the summaries of the interviews. If you withdraw, the information you provided will be destroyed or returned to you.

\section{Điều gì sẽ xảy ra với danh tính của Anh/Chị và thông tin Anh/Chị cung cấp?} What will happen to your identity and the information you give?

Nghiên cứu này sẽ bảo mật thông tin. Những ngiên cứu viên dưới đây sẽ biết về danh tính của anh chị những dự liệu nghiên cứu sẽ được xử lý để danh tính của Anh/Chị sẽ không được tiết lộ trong bất cứ báo cáo, thuyết trình hay văn bản nào. Tuy nhiên, $\mathrm{Anh} / \mathrm{Chị} \mathrm{cần} \mathrm{biết} \mathrm{rằng}$ đồng nghiệp của Anh/Chị trong tổ chức có thể biết về sự tham gia của Anh/Chị trong nghiên cứu

Chỉ có tôi và giáo sư hướng dẫn của tôi sẽ đọc bản chép lại phỏng vấn của Anh/Chị. Bản chép lại của phỏng vấn, tóm tắt và ghi âm sẽ được giữ an toàn và huỷ vào ngày 01 tháng 09 năm 2022.

This research is confidential. This means that the researchers named below will be aware of your identity, but the research data will be combined, and your identity will not be revealed in any reports, presentations, or public documentation. However, you should be aware that your identity might be obvious to other colleagues in your organisation.

Only my supervisors and I will read the notes or transcripts of the interviews. The interview transcripts, summaries and any recordings will be kept securely and destroyed by the $1^{\text {st }}$ of September 2022. 


\section{Nghiên cứu này phục vụ cho mục đích gì}

\section{What will the project produce?}

Thông tin từ nghiên cứu này sẽ được sử dụng trong luận văn tiến sĩ của tôi.

The information from my research will be used in my PhD dissertation

\section{Nếu bạn nhận lời mò̀i phỏng vấn này, quyền lọi của Anh/Chị sẽ là gì? \\ If you accept this invitation, what are your rights as a research participant?}

Anh/Chị không phải nhận lời mời phỏng vấn này nếu Anh/Chị không muốn. Nếu Anh/Chị quyết định tham gia, Anh/Chị có những quyền sau:

- $\quad$ không trả lời câu hỏi bất kỳ;

- yêu cầu tắt máy ghi âm bất cứ thời điểm nào trong cuộc phỏng vấn.

- rút khỏi ngiên cứu bất cứ lúc nào trong vong 2 tuần sau khi nhận được tóm tắt phỏng vấn.

- hỏi câu hỏi bất kỳ về ngiên cứu ở bất cứ thời điểm nào.

- đọc lại và nhận xét vào tóm tắt của phỏng vấn.

- có thể đọc bất cứ báo cáo nào của nghiên cứu bằng cách email nghiên cứu viên và yêu cầu một bản copy của báo cáo.

You do not have to accept this invitation if you don't want to. If you do decide to participate, you have the right to:

- $\quad$ choose not to answer any question;

- $\quad$ ask for the recorder to be turned off at any time during the interviews;

- withdraw from the study any time within 2 weeks after receiving the summaries of your interviews;

- $\quad$ ask any question about the study at any time;

- $\quad$ read over and comment on a written summary of your interviews;

- $\quad$ be able to read any report of this research by emailing the researcher to request a copy.

\section{Nếu Anh/Chị có bất kỳ câu hỏi hay vấn đề gì, Anh/Chị có thể liên hệ ai?} If you have any questions or problems, who can you contact?

Nếu Anh/Chị có bất kỳ câu hỏi nào, bây giờ hoặc trong tương lại, anh chị có thể liên hệ:

\section{Sinh viên:}

Tên: Van Thi La

Địa chỉ Email: address:

VanThi.La@vuw.ac.nz

\section{Giáo viên hướng dẫn:}

Tên: Phó giáo sư. Arun Elias

Vị trí: Phó chủ nhiệm khoa Trường Kinh Doanh Victoria

Trường: Trường Quản lý

Điện thoại: 044635736

Arun.elias@vuw.ac.nz

If you have any questions, either now or in the future, please feel free to contact: 
Student:

Name: Van Thi La

Email address: VanThi.La@vuw.ac.nz
Supervisor:

Name: AProf. Arun Elias

Role: Associate Dean

School: School of Management

Phone: 044635736

Arun.elias@vuw.ac.nz

\section{Thông tin về Hội đồng đạo đức}

\section{Human Ethics Committee information}

Nếu Anh/Chị có bất cứ quan tâm nào đến vấn đề đạo đức của nghiên cứu này, Anh/Chị có thể liện hệ Uỷ viên Hội động Đạo đức trường Đại học Victoria: Tiến sĩ. Loveridge. Email judith.loveridge@vuw.ac.nz or telephone +64-4-463 6028.

If you have any concerns about the ethical conduct of the research you may contact the Victoria University HEC Convenor: Dr Judith Loveridge. Email judith.loveridge@vuw.ac.nz or telephone +64-4-4636028. 


\section{NGHIÊN CÚU VỀ MỐI QUAN Hệ GIỬA TƯƠNG TÁC CỦA CÁC BÊN LIÊN QUAN VÀ NHẬN THỨC CỦA HỌ VỀ DƯ ÁN \\ AN EXPLORATION OF THE RELATIONSHIP BETWEEN STAKEHOLDERS INTERACTION AND THEIR PERCEPTION OF PROJECTS}

\section{THU' ĐỒNG Ý THAM GIA PHỎNG VẤN CONSENT TO INTERVIEW}

Thư này sẽ được giữ trong 4 năm

This consent form will be held for 4 years.

Ngiên cứu viên: La Thị Vân, Trường Quản lý, Đại học Victoria, Wellington Researcher: Van Thi La, School of Management, Victoria University of Wellington.

- Tôi đã đọc Thư mời tham gia phỏng vấn và đã hiểu về dự án nghiên cứu. Tôi hiểu rằng tôi có thể hỏi các câu hỏi ở bất cứ thời điểm nào.

• Tôi đồng ý tham gia vào phỏng vấn có ghi âm.

- I have read the Information Sheet and the project has been explained to me. My questions have been answered to my satisfaction. I understand that I can ask further questions at any time.

- I agree to take part in audio recorded interviews.

Tôi hiểu rằng:

- Tôi có thể quyết định rút khỏi ngiên cứu ở bất cứ thời điểm nào trong vòng 02 tuần sau khi nhận được tóm tắt của phỏng vấn. Bất cứ thông tin nào tôi cung cấp sẽ được trả lại hoặc bị huỷ.

• Toàn bộ thông tin được cung cấp sẽ bị huỷ trước ngày 01 tháng 09 năm 2022.

- Bất cứ thông tin nào tôi cung cấp sẽ được nghiên cứu viên và giáo viên hướng dẫn giữ bảo mật.

• K Kết quả của ngiên cứu sẽ được sử dụng cho một luận văn tiến sĩ.

- Các thành viên khác trong dự án kiểm định ......của trường đại học ...... và các đồng nghiệp khác trong tổ chức của tôi có thể biết được sự tham gia của tôi. Tuy nhiên, tôi sẽ được dùng tên giả trong báo cáo ngiên cứu và danh tính của tôi sẽ không bị tiết lộ với công chúng. Tên của tổ chức của tôi sẽ không được sử dụng trọng báo cáo, cũng như sẽ không có thông tin nào có thể nhận diện tổ chức của tôi. 
I understand that:

- I may withdraw from this study at any point within two weeks after receiving the summary of the interviews and any information that I have provided will be returned to me or destroyed.

- $\quad$ The identifiable information I have provided will be destroyed by 01/Sep/2022.

- $\quad$ Any information I provide will be kept confidential to the researcher and the supervisors.

- I understand that the results will be used for a PhD dissertation.

- $\quad$ I understand that my participation in the interviews can be known by members of the ...... accreditation project and colleagues in my organisation. However, I will be referred to by pseudonyms in reports, and will not be identifiable to wider research audiences.

- I would like to receive summaries of my interviews.

Yes No

-

- I would like to receive summaries of the research results and have Yes No added my email address below.

$\square \square$

Chữ ký/ Signature of participant:

Tên/ Name of participant:

Ngày/ Date:

Địa chỉ liên hệ/ Contact details: 


\section{Appendix H: Results of the Principal Component Analysis of Thu's Repertory Grids - MTEO Case}

Table HI Percentage variance explained by each component - Thu (critical numbers are highlighted)

\begin{tabular}{|c|c|c|c|c|c|c|c|c|c|c|c|c|}
\hline & \multicolumn{6}{|c|}{ The first interview } & \multicolumn{6}{|c|}{ The second interview } \\
\hline & 1 & 2 & 3 & 4 & 5 & 6 & 1 & 2 & 3 & 4 & 5 & 6 \\
\hline Variance $\%$ & 75.65 & 13.51 & 5.16 & 4.42 & 1.25 & 0 & 75.83 & 13.28 & 5.61 & 4.17 & 1.11 & 0 \\
\hline Frontier estimate $\%$ & 40.83 & 24.17 & 15.83 & 10.28 & 6.11 & 2.78 & 40.83 & 24.17 & 15.83 & 10.28 & 6.11 & 2.78 \\
\hline \multirow[t]{3}{*}{ Cumulative variance $\%$} & 75.65 & 89.17 & 94.32 & 98.75 & 100 & 100 & 75.83 & 89.11 & 94.72 & 98.89 & 100 & 100 \\
\hline & \multicolumn{6}{|c|}{ The third interview } & \multicolumn{6}{|c|}{ The fourth interview } \\
\hline & 1 & 2 & 3 & 4 & 5 & 6 & 1 & 2 & 3 & 4 & 5 & 6 \\
\hline Variance \% & 77.12 & 11.36 & 6.28 & 3.97 & 1.27 & 0 & 76.74 & 10.79 & 6.38 & 3.94 & 2.15 & 76.74 \\
\hline Frontier estimate $\%$ & 40.83 & 24.17 & 15.83 & 10.28 & 6.11 & 2.78 & 40.83 & 24.17 & 15.83 & 10.28 & 6.11 & 40.83 \\
\hline Cumulative variance $\%$ & 77.12 & 88.48 & 94.76 & 98.73 & 100 & 100 & 76.74 & 87.53 & 93.91 & 97.85 & 100 & 76.74 \\
\hline
\end{tabular}

Table H2 Loadings of constructs on the components - Thu (Green: large loadings on component 1, blue and yellow: loadings on component 2 with opposite signs)

\begin{tabular}{|c|c|c|c|c|c|c|c|c|c|c|c|c|c|c|c|c|c|c|c|c|}
\hline $\begin{array}{l}\text { Constru } \\
\text { cts }\end{array}$ & \multicolumn{5}{|c|}{ The first interview } & \multicolumn{5}{|c|}{ The second interview } & \multicolumn{5}{|c|}{ The third interview } & \multicolumn{5}{|c|}{ The fourth interview } \\
\hline & 1 & 2 & 3 & 4 & 5 & 1 & 2 & 3 & 4 & 5 & 1 & 2 & 3 & 4 & 5 & 1 & 2 & 3 & 4 & 5 \\
\hline 1 & $\begin{array}{r}1.54 \\
5\end{array}$ & $\begin{array}{r}- \\
0.67 \\
8\end{array}$ & $\begin{array}{r}- \\
0.09 \\
7\end{array}$ & $\begin{array}{r}- \\
0.01\end{array}$ & 0.35 & 1.54 & $\begin{array}{r}- \\
0.69\end{array}$ & $\begin{array}{r}- \\
0.09\end{array}$ & 0.08 & 0.32 & 1.61 & $\begin{array}{r}- \\
0.70\end{array}$ & 0.05 & 0.06 & 0.38 & 1.60 & 0.11 & 0.12 & 0.28 & 0.28 \\
\hline 2 & $\begin{array}{r}- \\
1.08 \\
1\end{array}$ & $\begin{array}{r}0.86 \\
6\end{array}$ & $\begin{array}{r}- \\
0.77 \\
4\end{array}$ & $\begin{array}{r}0.12 \\
5\end{array}$ & $\begin{array}{r}- \\
0.05 \\
6\end{array}$ & $\begin{array}{r}- \\
0.99 \\
4\end{array}$ & $\begin{array}{r}0.98 \\
9\end{array}$ & $\begin{array}{r}- \\
0.94 \\
2\end{array}$ & $\begin{array}{r}0.15 \\
9\end{array}$ & $\begin{array}{r}0.09 \\
1\end{array}$ & $\begin{array}{r}- \\
0.80 \\
5\end{array}$ & $\begin{array}{r}0.10 \\
7\end{array}$ & $\begin{array}{r}- \\
1.20 \\
2\end{array}$ & $\begin{array}{r}0.16 \\
8\end{array}$ & $\begin{array}{r}- \\
0.12 \\
5\end{array}$ & $\begin{array}{r}- \\
0.85 \\
5\end{array}$ & $\begin{array}{r}- \\
0.06 \\
4\end{array}$ & $\begin{array}{r}- \\
1.17 \\
6\end{array}$ & 0.01 & $\begin{array}{r}- \\
0.16 \\
9\end{array}$ \\
\hline 3 & $\begin{array}{r}- \\
1.57 \\
9\end{array}$ & $\begin{array}{r}- \\
0.63 \\
3\end{array}$ & $\begin{array}{r}- \\
0.28 \\
1\end{array}$ & $\begin{array}{r} \\
0.36 \\
5\end{array}$ & $\begin{array}{r}- \\
0.06 \\
1\end{array}$ & $\begin{array}{r}- \\
1.51 \\
4\end{array}$ & $\begin{array}{r}- \\
0.50 \\
3\end{array}$ & $\begin{array}{r}- \\
0.38 \\
1\end{array}$ & $\begin{array}{r}- \\
0.25 \\
2\end{array}$ & $\begin{array}{r}0.07 \\
4\end{array}$ & $\begin{array}{r}- \\
1.60 \\
9\end{array}$ & $\begin{array}{r}- \\
0.48 \\
2\end{array}$ & 0.25 & $0.39^{-}$ & $\begin{array}{r}0.27 \\
6\end{array}$ & $\begin{array}{r}- \\
1.59 \\
2\end{array}$ & $\begin{array}{r}- \\
0.46 \\
7\end{array}$ & $0.1 \overline{3}$ & $\begin{array}{r}- \\
0.05 \\
4\end{array}$ & $\begin{array}{r}0.58 \\
3\end{array}$ \\
\hline
\end{tabular}




\begin{tabular}{|c|c|c|c|c|c|c|c|c|c|c|c|c|c|c|c|c|c|c|c|c|}
\hline 4 & $\begin{array}{r}- \\
1.56 \\
7\end{array}$ & $\begin{array}{r}- \\
0.57 \\
6\end{array}$ & $\begin{array}{r}- \\
0.19 \\
3\end{array}$ & $\begin{array}{r}- \\
0.05 \\
5\end{array}$ & $\begin{array}{r}0.39 \\
4\end{array}$ & $\begin{array}{r}- \\
1.56 \\
6\end{array}$ & $\begin{array}{r}- \\
0.56 \\
6\end{array}$ & $\begin{array}{r}- \\
0.22 \\
1\end{array}$ & $\begin{array}{r}- \\
0.11 \\
4\end{array}$ & $\begin{array}{r}0.38 \\
5\end{array}$ & $\begin{array}{r}- \\
1.57\end{array}$ & $\begin{array}{r}- \\
0.60 \\
7\end{array}$ & $\begin{array}{r}- \\
0.13 \\
5\end{array}$ & $\begin{array}{r}- \\
0.14 \\
6\end{array}$ & $\begin{array}{r}0.32 \\
8 \\
\end{array}$ & $\begin{array}{r}- \\
1.55 \\
4\end{array}$ & $\begin{array}{r}- \\
0.55 \\
4\end{array}$ & 0.01 & $\begin{array}{r}0.18 \\
6\end{array}$ & $\begin{array}{r}0.47 \\
6\end{array}$ \\
\hline & 1.47 & 1.19 & - & 0.25 & 0.35 & 1.45 & 1.20 & 0.43 & 0.19 & 0.37 & 1.39 & 1.03 & 0.72 & 0.21 & 0.56 & - & 1.00 & 0.87 & 0.41 & 0.36 \\
\hline 5 & 2 & 6 & 0.37 & 8 & 5 & 3 & 1 & 7 & 7 & 6 & 7 & 9 & 6 & 6 & 6 & 1.36 & 3 & 8 & 2 & 3 \\
\hline 6 & $\begin{array}{r}1.53 \\
1 \\
\end{array}$ & 0.03 & $\begin{array}{r}0.12 \\
3 \\
\end{array}$ & $\begin{array}{r}- \\
0.04 \\
9 \\
\end{array}$ & $\begin{array}{r}- \\
0.15 \\
9 \\
\end{array}$ & 1.6 & $\begin{array}{r}0.12 \\
7 \\
\end{array}$ & $\begin{array}{r}- \\
0.00 \\
1\end{array}$ & $\begin{array}{r}0.12 \\
9 \\
\end{array}$ & $\begin{array}{r}- \\
0.00 \\
3 \\
\end{array}$ & 1.78 & $\begin{array}{r}0.08 \\
1\end{array}$ & $\begin{array}{r}- \\
0.20 \\
8\end{array}$ & $\begin{array}{r}0.32 \\
9\end{array}$ & $\begin{array}{r}- \\
0.07 \\
3\end{array}$ & $\begin{array}{r}1.56 \\
2\end{array}$ & $\begin{array}{r}0.22 \\
8\end{array}$ & $\begin{array}{r}- \\
0.16 \\
9\end{array}$ & $\begin{array}{r}0.34 \\
9\end{array}$ & $\begin{array}{r}0.43 \\
8\end{array}$ \\
\hline 7 & $\begin{array}{r}1.64 \\
5 \\
\end{array}$ & $\begin{array}{r}0.64 \\
4 \\
\end{array}$ & $\begin{array}{r}- \\
0.23 \\
5\end{array}$ & $\begin{array}{r}0.16 \\
1 \\
\end{array}$ & $\begin{array}{r}0.01 \\
2 \\
\end{array}$ & $\begin{array}{r}1.65 \\
4 \\
\end{array}$ & $\begin{array}{r}0.63 \\
2 \\
\end{array}$ & $\begin{array}{r}- \\
0.23 \\
7\end{array}$ & 0.11 & 0 & $\begin{array}{r}1.67 \\
8 \\
\end{array}$ & $\begin{array}{r}0.53 \\
5 \\
\end{array}$ & $\begin{array}{r}- \\
0.30 \\
4 \\
\end{array}$ & $\begin{array}{r}0.06 \\
3 \\
\end{array}$ & $\begin{array}{r}0.06 \\
3 \\
\end{array}$ & $\begin{array}{r}1.67 \\
5 \\
\end{array}$ & $\begin{array}{r}0.43 \\
5 \\
\end{array}$ & $\begin{array}{r}- \\
0.43 \\
5 \\
\end{array}$ & $\begin{array}{r}- \\
0.02 \\
5 \\
\end{array}$ & $\begin{array}{r}- \\
0.13 \\
3 \\
\end{array}$ \\
\hline 8 & $\begin{array}{r}1.38 \\
1 \\
\end{array}$ & $\begin{array}{r}1.05 \\
3 \\
\end{array}$ & $\begin{array}{r}- \\
0.16 \\
2 \\
\end{array}$ & $\begin{array}{r}- \\
0.40 \\
9 \\
\end{array}$ & $\begin{array}{r}0.22 \\
1 \\
\end{array}$ & $\begin{array}{r}1.38 \\
7 \\
\end{array}$ & $\begin{array}{r}1.05 \\
1 \\
\end{array}$ & $\begin{array}{r}- \\
0.06 \\
1\end{array}$ & $\begin{array}{r}- \\
0.43 \\
6 \\
\end{array}$ & $\begin{array}{r}0.19 \\
1 \\
\end{array}$ & 1.49 & $\begin{array}{r}0.94 \\
8 \\
\end{array}$ & $\begin{array}{r}- \\
0.22 \\
2 \\
\end{array}$ & 0.33 & $\begin{array}{r}0.01 \\
2 \\
\end{array}$ & $\begin{array}{r}1.29 \\
6 \\
\end{array}$ & $\begin{array}{r}0.75 \\
8 \\
\end{array}$ & $\begin{array}{r}0.15 \\
9 \\
\end{array}$ & $\begin{array}{r}- \\
0.49 \\
2\end{array}$ & $\begin{array}{r}0.13 \\
2 \\
\end{array}$ \\
\hline 9 & $\begin{array}{r}1.51 \\
3 \\
\end{array}$ & $\begin{array}{r}0.42 \\
1 \\
\end{array}$ & $\begin{array}{r}- \\
0.00 \\
3\end{array}$ & $\begin{array}{r}- \\
0.22 \\
1 \\
\end{array}$ & 0.31 & $\begin{array}{r}1.58 \\
2 \\
\end{array}$ & $\begin{array}{r}0.52 \\
6 \\
\end{array}$ & $\begin{array}{r}- \\
0.08 \\
\end{array}$ & $\begin{array}{r}- \\
0.04 \\
6\end{array}$ & $\begin{array}{r}- \\
0.16 \\
5 \\
\end{array}$ & $\begin{array}{r}1.67 \\
8 \\
\end{array}$ & $\begin{array}{r}0.53 \\
5 \\
\end{array}$ & $\begin{array}{r}- \\
0.30 \\
4 \\
\end{array}$ & $\begin{array}{r}0.06 \\
3 \\
\end{array}$ & $\begin{array}{r}0.06 \\
3 \\
\end{array}$ & $\begin{array}{r}1.71 \\
3 \\
\end{array}$ & $\begin{array}{r}0.34 \\
8 \\
\end{array}$ & $\begin{array}{r}- \\
0.31 \\
5 \\
\end{array}$ & $\begin{array}{r}0.21 \\
4 \\
\end{array}$ & 0.24 \\
\hline 10 & $\begin{array}{r}1.59 \\
8 \\
\end{array}$ & $\begin{array}{r}- \\
0.62 \\
4 \\
\end{array}$ & -0.3 & $\begin{array}{r}- \\
0.13 \\
3 \\
\end{array}$ & $\begin{array}{r}0.06 \\
1 \\
\end{array}$ & $\begin{array}{r}1.59 \\
5 \\
\end{array}$ & $\begin{array}{r}- \\
0.62 \\
9 \\
\end{array}$ & $\begin{array}{r}- \\
0.25 \\
7 \\
\end{array}$ & $\begin{array}{r}- \\
0.22 \\
1 \\
\end{array}$ & $\begin{array}{r}0.01 \\
2 \\
\end{array}$ & $\begin{array}{r}1.57 \\
9 \\
\end{array}$ & $\begin{array}{r}- \\
0.66 \\
8 \\
\end{array}$ & $\begin{array}{r}- \\
0.04 \\
2 \\
\end{array}$ & $\begin{array}{r}- \\
0.32 \\
3 \\
\end{array}$ & $\begin{array}{r}- \\
0.10 \\
8 \\
\end{array}$ & $\begin{array}{r}1.55 \\
8 \\
\end{array}$ & $\begin{array}{r}- \\
0.73 \\
7 \\
\end{array}$ & $\begin{array}{r}0.03 \\
5 \\
\end{array}$ & $\begin{array}{r}- \\
0.29 \\
1\end{array}$ & $\begin{array}{r}0.02 \\
2 \\
\end{array}$ \\
\hline 11 & $\begin{array}{r}1.48 \\
8 \\
\end{array}$ & $\begin{array}{r}1.26 \\
2 \\
\end{array}$ & $\begin{array}{r}- \\
0.16 \\
9\end{array}$ & $\begin{array}{r}- \\
0.25 \\
4 \\
\end{array}$ & $\begin{array}{r}- \\
0.15 \\
7 \\
\end{array}$ & $\begin{array}{r}1.49 \\
5 \\
\end{array}$ & 1.26 & $\begin{array}{r}- \\
0.07 \\
8 \\
\end{array}$ & $\begin{array}{r}- \\
0.25 \\
8\end{array}$ & $\begin{array}{r}- \\
0.17 \\
6 \\
\end{array}$ & $\begin{array}{r}1.51 \\
6 \\
\end{array}$ & $\begin{array}{r}1.22 \\
6 \\
\end{array}$ & $\begin{array}{r}- \\
0.25 \\
6 \\
\end{array}$ & $\begin{array}{r}- \\
0.23 \\
7 \\
\end{array}$ & $\begin{array}{r}- \\
0.05 \\
6 \\
\end{array}$ & $\begin{array}{r}1.52 \\
7 \\
\end{array}$ & $\begin{array}{r}1.09 \\
9 \\
\end{array}$ & $\begin{array}{r}- \\
0.47 \\
3 \\
\end{array}$ & $\begin{array}{r}- \\
0.39 \\
9\end{array}$ & $\begin{array}{r}- \\
0.04 \\
8 \\
\end{array}$ \\
\hline 12 & $\begin{array}{r}- \\
1.41 \\
7\end{array}$ & $\begin{array}{r}- \\
0.93 \\
5 \\
\end{array}$ & 0 & $\begin{array}{r}0.18 \\
6 \\
\end{array}$ & $\begin{array}{r}- \\
0.25 \\
5 \\
\end{array}$ & $\begin{array}{r}- \\
1.42 \\
4\end{array}$ & $\begin{array}{r}- \\
0.92 \\
5\end{array}$ & $\begin{array}{r}- \\
0.05\end{array}$ & 0.19 & $\begin{array}{r}- \\
0.24 \\
4\end{array}$ & $\begin{array}{r}- \\
1.35 \\
3 \\
\end{array}$ & $\begin{array}{r}- \\
0.83 \\
3 \\
\end{array}$ & $\begin{array}{r}- \\
0.12 \\
4 \\
\end{array}$ & $\begin{array}{r}0.32 \\
9 \\
\end{array}$ & $\begin{array}{r}- \\
0.13 \\
4 \\
\end{array}$ & $\begin{array}{r}- \\
1.42 \\
5\end{array}$ & $\begin{array}{r}- \\
0.70 \\
1 \\
\end{array}$ & $\begin{array}{r}- \\
0.07 \\
9 \\
\end{array}$ & $\begin{array}{r}0.05 \\
2 \\
\end{array}$ & $\begin{array}{r}- \\
0.08 \\
1 \\
\end{array}$ \\
\hline 13 & $\begin{array}{r}- \\
1.77 \\
3 \\
\end{array}$ & $\begin{array}{r}0.86 \\
8 \\
\end{array}$ & $\begin{array}{r}- \\
0.06 \\
9\end{array}$ & $\begin{array}{r}- \\
0.72 \\
9 \\
\end{array}$ & $\begin{array}{r}- \\
0.32 \\
3 \\
\end{array}$ & $\begin{array}{r}- \\
1.77 \\
7\end{array}$ & 0.9 & $\begin{array}{r}0.08 \\
1 \\
\end{array}$ & $\begin{array}{r}- \\
0.67 \\
1\end{array}$ & $\begin{array}{r}- \\
0.33 \\
5\end{array}$ & $\begin{array}{r}- \\
1.78 \\
1 \\
\end{array}$ & $\begin{array}{r}1.00 \\
4 \\
\end{array}$ & $\begin{array}{r}- \\
0.12 \\
3 \\
\end{array}$ & $\begin{array}{r}- \\
0.53 \\
6 \\
\end{array}$ & $\begin{array}{r}- \\
0.23 \\
6 \\
\end{array}$ & $\begin{array}{r}- \\
1.76 \\
3 \\
\end{array}$ & $\begin{array}{r}0.96 \\
8 \\
\end{array}$ & $\begin{array}{r}- \\
0.19 \\
7 \\
\end{array}$ & $\begin{array}{r}- \\
0.62 \\
8 \\
\end{array}$ & $\begin{array}{r}0.24 \\
5 \\
\end{array}$ \\
\hline 14 & $\begin{array}{r}- \\
1.50 \\
2\end{array}$ & $\begin{array}{r}0.96 \\
6\end{array}$ & $\begin{array}{r}- \\
0.09 \\
3\end{array}$ & $\begin{array}{r}0.29 \\
1\end{array}$ & $\begin{array}{r}0.22 \\
7\end{array}$ & $\begin{array}{r}- \\
1.56\end{array}$ & $\begin{array}{r}0.86 \\
2\end{array}$ & $\begin{array}{r}- \\
0.01 \\
1\end{array}$ & $\begin{array}{r}0.13 \\
1\end{array}$ & $\begin{array}{r}0.09 \\
9\end{array}$ & $\begin{array}{r}- \\
1.70 \\
3\end{array}$ & $\begin{array}{r}0.75 \\
3\end{array}$ & $\begin{array}{r}0.10 \\
6\end{array}$ & $\begin{array}{r}- \\
0.04 \\
9\end{array}$ & $\begin{array}{r}- \\
0.13 \\
2\end{array}$ & $\begin{array}{r}- \\
1.68 \\
7\end{array}$ & $\begin{array}{r}0.79 \\
4\end{array}$ & $\begin{array}{r}0.04 \\
4\end{array}$ & $\begin{array}{r}- \\
0.14 \\
9\end{array}$ & $\begin{array}{r}0.02 \\
9\end{array}$ \\
\hline
\end{tabular}




\begin{tabular}{|c|c|c|c|c|c|c|c|c|c|c|c|c|c|c|c|c|c|c|c|c|}
\hline 15 & $\begin{array}{r}1.59 \\
8\end{array}$ & $\begin{array}{r}0.14 \\
1\end{array}$ & $\begin{array}{r}0.00 \\
8\end{array}$ & $\begin{array}{r}0.14 \\
2\end{array}$ & $\begin{array}{r}0.00 \\
2\end{array}$ & $\begin{array}{r}1.52 \\
8\end{array}$ & 0.02 & $\begin{array}{r}0.15 \\
7\end{array}$ & $\begin{array}{r}- \\
0.02 \\
7\end{array}$ & $\begin{array}{r}- \\
0.16 \\
7\end{array}$ & $\begin{array}{r}1.51 \\
9\end{array}$ & $\begin{array}{r}0.05 \\
1\end{array}$ & $\begin{array}{r}0.20 \\
3\end{array}$ & $\begin{array}{r}- \\
0.04 \\
1\end{array}$ & $\begin{array}{r}- \\
0.19 \\
1\end{array}$ & $\begin{array}{r}1.44 \\
4\end{array}$ & $\begin{array}{r}0.09 \\
4\end{array}$ & $\begin{array}{r}0.19 \\
7\end{array}$ & $\begin{array}{r}- \\
0.11 \\
8\end{array}$ & $\begin{array}{r}0.04 \\
7\end{array}$ \\
\hline 16 & $\begin{array}{r}1.56 \\
2\end{array}$ & $\begin{array}{r}- \\
0.50 \\
5\end{array}$ & $\begin{array}{r}- \\
0.46 \\
2\end{array}$ & $\begin{array}{r}- \\
0.35 \\
7\end{array}$ & $\begin{array}{r}0.02 \\
8\end{array}$ & $\begin{array}{r}1.55 \\
9\end{array}$ & $\begin{array}{r}- \\
0.50 \\
3\end{array}$ & $\begin{array}{r}- \\
0.36 \\
9\end{array}$ & $\begin{array}{r}- \\
0.46 \\
6\end{array}$ & 0.04 & 1.54 & $\begin{array}{r}- \\
0.54 \\
3\end{array}$ & $\begin{array}{r}- \\
0.15 \\
7\end{array}$ & $\begin{array}{r}- \\
0.56 \\
6\end{array}$ & 0.16 & 1.52 & $\begin{array}{r}- \\
0.65\end{array}$ & $\begin{array}{r}- \\
0.08 \\
5\end{array}$ & $\begin{array}{r}- \\
0.53\end{array}$ & $\begin{array}{r}0.12 \\
9\end{array}$ \\
\hline 17 & 1.7 & $\begin{array}{r}0.03 \\
4\end{array}$ & $\begin{array}{r}- \\
0.34 \\
7\end{array}$ & $\begin{array}{r}0.16 \\
1\end{array}$ & $\begin{array}{r}- \\
0.01 \\
3\end{array}$ & $\begin{array}{r}1.70 \\
5\end{array}$ & $\begin{array}{r}0.02 \\
4\end{array}$ & $\begin{array}{r}- \\
0.35 \\
1\end{array}$ & $\begin{array}{r}0.07 \\
6\end{array}$ & $\begin{array}{r}- \\
0.04 \\
1\end{array}$ & $\begin{array}{r}1.71 \\
6\end{array}$ & $\begin{array}{r}- \\
0.07 \\
2\end{array}$ & $\begin{array}{r}- \\
0.28 \\
9\end{array}$ & $\begin{array}{r}- \\
0.00 \\
8\end{array}$ & $\begin{array}{r}- \\
0.05 \\
7\end{array}$ & $\begin{array}{r}1.56 \\
5\end{array}$ & $\begin{array}{r}- \\
0.02 \\
5\end{array}$ & $\begin{array}{r}- \\
0.24 \\
8\end{array}$ & $\begin{array}{r}0.04 \\
2\end{array}$ & 0.39 \\
\hline 18 & $\begin{array}{r}1.56 \\
2 \\
\end{array}$ & $\begin{array}{r}- \\
0.50 \\
5\end{array}$ & $\begin{array}{r}- \\
0.46 \\
2\end{array}$ & $\begin{array}{r}- \\
0.35 \\
7\end{array}$ & $\begin{array}{r}0.02 \\
8 \\
\end{array}$ & $\begin{array}{r}1.55 \\
9 \\
\end{array}$ & $\begin{array}{r}- \\
0.50 \\
3\end{array}$ & $\begin{array}{r}- \\
0.36 \\
9\end{array}$ & $\begin{array}{r}- \\
0.46 \\
6\end{array}$ & 0.04 & $\begin{array}{r}1.62 \\
6 \\
\end{array}$ & $\begin{array}{r}- \\
0.50 \\
3\end{array}$ & $\begin{array}{r}- \\
0.33 \\
6\end{array}$ & $\begin{array}{r}- \\
0.43 \\
9\end{array}$ & $\begin{array}{r}0.02 \\
7 \\
\end{array}$ & $\begin{array}{r}1.61 \\
1\end{array}$ & $\begin{array}{r}- \\
0.61 \\
9\end{array}$ & $\begin{array}{r}- \\
0.28 \\
5\end{array}$ & $\begin{array}{r}- \\
0.33 \\
1\end{array}$ & $\begin{array}{r}0.18 \\
6 \\
\end{array}$ \\
\hline 19 & $\begin{array}{r}- \\
0.97 \\
9\end{array}$ & $\begin{array}{r}- \\
0.36 \\
9\end{array}$ & $\begin{array}{r}- \\
1.38 \\
7\end{array}$ & $\begin{array}{r}0.50 \\
5\end{array}$ & $\begin{array}{r}- \\
0.24 \\
7\end{array}$ & $\begin{array}{r}- \\
0.96 \\
5\end{array}$ & 0.35 & $\begin{array}{r}- \\
1.46 \\
1\end{array}$ & $\begin{array}{r}0.23 \\
8\end{array}$ & $\begin{array}{r}- \\
0.29 \\
9\end{array}$ & $\begin{array}{r}- \\
0.92 \\
1\end{array}$ & $\begin{array}{r}- \\
0.59 \\
9\end{array}$ & $\begin{array}{r}- \\
1.42 \\
3\end{array}$ & $\begin{array}{r}0.11 \\
6\end{array}$ & $\begin{array}{r}- \\
0.29 \\
5\end{array}$ & $\begin{array}{r}- \\
1.00 \\
3\end{array}$ & $\begin{array}{r}- \\
0.80 \\
5\end{array}$ & $\begin{array}{r}- \\
1.27\end{array}$ & $\begin{array}{r}- \\
0.06 \\
8\end{array}$ & $\begin{array}{r}- \\
0.24 \\
4\end{array}$ \\
\hline 20 & $\begin{array}{r}1.59 \\
8\end{array}$ & $\begin{array}{r}- \\
0.62 \\
4\end{array}$ & -0.3 & $\begin{array}{r}- \\
0.13 \\
3\end{array}$ & $\begin{array}{r}0.06 \\
1\end{array}$ & $\begin{array}{r}1.66 \\
7\end{array}$ & $\begin{array}{r}- \\
0.52 \\
2\end{array}$ & $\begin{array}{r}- \\
0.41 \\
5\end{array}$ & $\begin{array}{r}- \\
0.06 \\
5\end{array}$ & $\begin{array}{r}0.17 \\
7\end{array}$ & $\begin{array}{r}1.66 \\
5\end{array}$ & $\begin{array}{r}- \\
0.62 \\
8\end{array}$ & $\begin{array}{r}- \\
0.22 \\
1\end{array}$ & $\begin{array}{r}- \\
0.19 \\
5\end{array}$ & 0.08 & $\begin{array}{r}1.64 \\
9\end{array}$ & $\begin{array}{r}- \\
0.70 \\
6\end{array}$ & $\begin{array}{r}- \\
0.16 \\
5\end{array}$ & $\begin{array}{r}- \\
0.09 \\
1\end{array}$ & $\begin{array}{r}0.07 \\
8\end{array}$ \\
\hline 21 & 1.49 & $\begin{array}{r}- \\
0.16 \\
8\end{array}$ & $\begin{array}{r}- \\
0.38 \\
5\end{array}$ & $\begin{array}{r}- \\
0.40 \\
9\end{array}$ & $\begin{array}{r}0.17 \\
1\end{array}$ & $\begin{array}{r}1.48 \\
9\end{array}$ & $\begin{array}{r}- \\
0.16 \\
7\end{array}$ & $\begin{array}{r}- \\
0.28 \\
8\end{array}$ & $\begin{array}{r}- \\
0.50 \\
3\end{array}$ & $\begin{array}{r}0.10 \\
9\end{array}$ & $\begin{array}{r}1.56 \\
3\end{array}$ & $\begin{array}{r}- \\
0.17 \\
4\end{array}$ & $\begin{array}{r}- \\
0.31 \\
8\end{array}$ & $\begin{array}{r}- \\
0.46 \\
1\end{array}$ & $\begin{array}{r}0.21 \\
6\end{array}$ & $\begin{array}{r}1.56 \\
9\end{array}$ & $\begin{array}{r}- \\
0.27 \\
9\end{array}$ & $\begin{array}{r}- \\
0.32 \\
7\end{array}$ & $\begin{array}{r}- \\
0.26 \\
4\end{array}$ & $\begin{array}{r}0.34 \\
2\end{array}$ \\
\hline 22 & $\begin{array}{r}1.53 \\
1\end{array}$ & 0.03 & $\begin{array}{r}0.12 \\
3\end{array}$ & $\begin{array}{r}- \\
0.04 \\
9\end{array}$ & $\begin{array}{r}- \\
0.15 \\
9\end{array}$ & 1.6 & $\begin{array}{r}0.12 \\
7\end{array}$ & $\begin{array}{r}- \\
0.00 \\
1\end{array}$ & $\begin{array}{r}0.12 \\
9\end{array}$ & $\begin{array}{r}- \\
0.00 \\
3\end{array}$ & $\begin{array}{r}1.60 \\
5\end{array}$ & $\begin{array}{r}0.09 \\
1\end{array}$ & $\begin{array}{r}0.02 \\
4\end{array}$ & $\begin{array}{r}0.08 \\
6\end{array}$ & $\begin{array}{r}- \\
0.00 \\
4\end{array}$ & $\begin{array}{r}1.59 \\
9\end{array}$ & $\begin{array}{r}0.05 \\
1\end{array}$ & $\begin{array}{r}- \\
0.03 \\
8\end{array}$ & $\begin{array}{r}0.01 \\
5\end{array}$ & $\begin{array}{r}- \\
0.17 \\
5\end{array}$ \\
\hline 23 & $\begin{array}{r}1.71 \\
8\end{array}$ & $\begin{array}{r}0.30 \\
7\end{array}$ & $\begin{array}{r}- \\
0.31 \\
2\end{array}$ & $\begin{array}{r}0.21 \\
3\end{array}$ & 0.13 & $\begin{array}{r}1.72 \\
4\end{array}$ & $\begin{array}{r}0.29 \\
6\end{array}$ & $\begin{array}{r}- \\
0.31 \\
8\end{array}$ & $\begin{array}{r}0.14 \\
6\end{array}$ & 0.15 & $\begin{array}{r}1.74 \\
2\end{array}$ & $\begin{array}{r}0.20 \\
7\end{array}$ & $\begin{array}{r}- \\
0.32 \\
3\end{array}$ & $\begin{array}{r}0.08 \\
5\end{array}$ & $\begin{array}{r}- \\
0.12 \\
5\end{array}$ & $\begin{array}{r}1.71 \\
7\end{array}$ & $\begin{array}{r}0.09 \\
5\end{array}$ & $\begin{array}{r}- \\
0.39 \\
4\end{array}$ & $\begin{array}{r}- \\
0.09 \\
2\end{array}$ & $\begin{array}{r}- \\
0.28 \\
9\end{array}$ \\
\hline 24 & $\begin{array}{r}- \\
1.66 \\
4\end{array}$ & $\begin{array}{r}0.25 \\
3\end{array}$ & $\begin{array}{r}0.10 \\
8\end{array}$ & $\begin{array}{r}- \\
0.33 \\
3\end{array}$ & $\begin{array}{r}- \\
0.16 \\
4\end{array}$ & $\begin{array}{r}- \\
1.67 \\
2\end{array}$ & $\begin{array}{r}- \\
0.23 \\
4\end{array}$ & $\begin{array}{r}0.15 \\
8\end{array}$ & $\begin{array}{r}- \\
0.28 \\
4\end{array}$ & $\begin{array}{r}- \\
0.16 \\
1\end{array}$ & $\begin{array}{r}- \\
1.69 \\
1\end{array}$ & $\begin{array}{r}- \\
0.13 \\
1\end{array}$ & $\begin{array}{r}0.15 \\
6\end{array}$ & $\begin{array}{r}- \\
0.21 \\
3\end{array}$ & $\begin{array}{r}- \\
0.18 \\
4\end{array}$ & $\begin{array}{r}1.62 \\
6\end{array}$ & $\begin{array}{r}- \\
0.15 \\
5\end{array}$ & $\begin{array}{r}0.20 \\
4\end{array}$ & $\begin{array}{r}- \\
0.28 \\
2\end{array}$ & 0.16 \\
\hline 25 & 1.88 & $\begin{array}{r}- \\
0.10 \\
6\end{array}$ & $\begin{array}{r}- \\
0.36 \\
9\end{array}$ & $\begin{array}{r}- \\
1.15 \\
9\end{array}$ & $\begin{array}{r}0.11 \\
4\end{array}$ & $\begin{array}{r}- \\
1.88 \\
9\end{array}$ & $\begin{array}{r}- \\
0.06 \\
5\end{array}$ & $\begin{array}{r}- \\
0.15 \\
9\end{array}$ & $\begin{array}{r}- \\
1.19 \\
9\end{array}$ & $\begin{array}{r}0.04 \\
8\end{array}$ & $\begin{array}{r}- \\
1.92 \\
2\end{array}$ & $\begin{array}{r}0.01 \\
7\end{array}$ & $\begin{array}{r}- \\
0.10 \\
3\end{array}$ & $\begin{array}{r}- \\
1.15 \\
4\end{array}$ & $\begin{array}{r}- \\
0.01 \\
5\end{array}$ & $\begin{array}{r}- \\
1.69 \\
9\end{array}$ & $\begin{array}{r}- \\
0.23 \\
4\end{array}$ & $\begin{array}{r}- \\
0.11 \\
7\end{array}$ & $\begin{array}{r}- \\
1.06 \\
7\end{array}$ & $\begin{array}{r}0.00 \\
7\end{array}$ \\
\hline
\end{tabular}




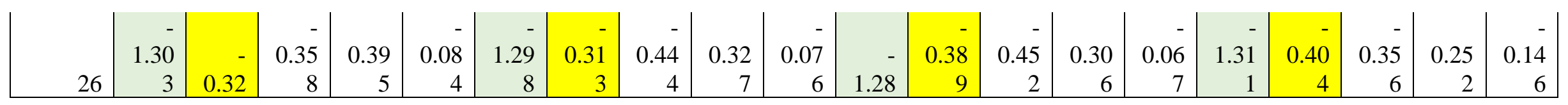

Table H3 Loadings of the elements on the components over the four interviews - Thu

\begin{tabular}{|c|c|c|c|c|c|c|c|c|c|c|c|}
\hline \multirow{2}{*}{ No. } & \multirow{2}{*}{ Elements } & \multicolumn{5}{|c|}{ The first interview } & \multicolumn{5}{|c|}{ The second interview } \\
\hline & & 1 & 2 & 3 & 4 & 5 & 1 & 2 & 3 & 4 & 5 \\
\hline 1 & Ideal project & -3.837 & -0.612 & -1.419 & 0.478 & -0.125 & -3.798 & -0.576 & -1.564 & 0.22 & -0.142 \\
\hline 2 & Less than ideal project & 6.241 & -0.456 & -0.757 & -0.369 & 0.078 & 6.272 & -0.445 & -0.682 & -0.513 & 0.034 \\
\hline 3 & MTEO project & -1.557 & -1.108 & 0.711 & -1.085 & -0.488 & -1.696 & -1.056 & 1.011 & -0.861 & -0.47 \\
\hline 4 & Project 1 & 0.85 & -1.178 & 0.992 & 1.271 & 0.102 & 0.866 & -1.245 & 0.715 & 1.358 & 0.151 \\
\hline 5 & Project 2 & -2.112 & 0.641 & 0.259 & -0.59 & 0.788 & -2.089 & 0.626 & 0.314 & -0.595 & 0.738 \\
\hline 6 & Project 3 & 0.415 & 2.714 & 0.213 & 0.294 & -0.356 & 0.444 & 2.695 & 0.206 & 0.391 & -0.311 \\
\hline \multirow{2}{*}{ No. } & \multirow{2}{*}{ Elements } & \multicolumn{5}{|c|}{ The third interview } & \multicolumn{5}{|c|}{ The fourth interview } \\
\hline & & 1 & 2 & 3 & 4 & 5 & 1 & 2 & 3 & 4 & 5 \\
\hline 1 & Ideal project & -3.688 & -0.92 & -1.626 & 0.105 & -0.151 & -3.921 & -1.18 & -1.431 & -0.06 & -0.159 \\
\hline 2 & Less than ideal project & 6.356 & -0.579 & -0.515 & -0.621 & -0.013 & 6.146 & -0.78 & -0.457 & -0.505 & 0.192 \\
\hline 3 & MTEO project & -2.072 & -0.37 & 1.23 & -0.693 & -0.577 & -2.139 & -0.268 & 1.354 & -1.063 & -0.222 \\
\hline 4 & Project 1 & 0.927 & -1.155 & 0.787 & 1.33 & 0.161 & 0.891 & -0.766 & 0.815 & 1.274 & -0.422 \\
\hline 5 & Project 2 & -2.14 & 0.461 & 0.357 & -0.631 & 0.791 & -1.509 & 0.646 & 0.235 & 0.356 & 1.091 \\
\hline 6 & Project 3 & 0.616 & 2.564 & -0.232 & 0.51 & -0.21 & 0.532 & 2.347 & -0.516 & -0.002 & -0.48 \\
\hline
\end{tabular}




\section{Appendix I: Results of the Principal Component Analysis of Nga's Repertory Grids - MTEO Case}

Table II Percentage variances explained by principal components - Nga - MTEO case

\begin{tabular}{|c|c|c|c|c|c|c|c|c|c|c|c|c|}
\hline & \multicolumn{6}{|c|}{ The first interview } & \multicolumn{6}{|c|}{ The second interview } \\
\hline & 1 & 2 & 3 & 4 & 5 & 6 & 1 & 2 & 3 & 4 & 5 & 6 \\
\hline Variance $\%$ & 83.95 & 9.74 & 4.36 & 1.13 & 0.82 & 0 & 83.89 & 9.69 & 4.5 & 1.23 & 0.69 & 0 \\
\hline Frontier estimate $\%$ & 40.83 & 24.17 & 15.83 & 10.28 & 6.11 & 2.78 & 40.83 & 24.17 & 15.83 & 10.28 & 6.11 & 2.78 \\
\hline \multirow[t]{3}{*}{ Cumulative variance\% } & 83.95 & 93.69 & 98.05 & 99.18 & 100 & 100 & 83.89 & 93.59 & 98.08 & 99.31 & 100 & 100 \\
\hline & \multicolumn{6}{|c|}{ The third interview } & \multicolumn{6}{|c|}{ The third interview } \\
\hline & 1 & 2 & 3 & 4 & 5 & 6 & 1 & 2 & 3 & 4 & 5 & 6 \\
\hline Variance $\%$ & 82.89 & 9.51 & 5.76 & 1.41 & 0.43 & 0 & 82.54 & 9.48 & 5.86 & 1.82 & 0.3 & 0 \\
\hline Frontier estimate $\%$ & 40.83 & 24.17 & 15.83 & 10.28 & 6.11 & 2.78 & 40.83 & 24.17 & 15.83 & 10.28 & 6.11 & 2.78 \\
\hline Cumulative variance $\%$ & 82.89 & 92.4 & 98.16 & 99.57 & 100 & 100 & 82.54 & 92.02 & 97.88 & 99.7 & 100 & 100 \\
\hline
\end{tabular}


Table I2 Loadings of constructs on components - Nga-MTEO case (Green: loadings on component 1, Yellow: loadings on component 2)

\begin{tabular}{|c|c|c|c|c|c|c|c|c|c|c|c|c|c|c|c|c|}
\hline & \multicolumn{4}{|c|}{ The first interview } & \multicolumn{4}{|c|}{ The second interview } & \multicolumn{4}{|c|}{ The third interview } & \multicolumn{4}{|c|}{ The fourth interview } \\
\hline & 1 & 2 & 3 & 4 & 1 & 2 & 3 & 4 & 1 & 2 & 3 & 4 & 1 & 2 & 3 & 4 \\
\hline 1 & 1.726 & -0.086 & 0.111 & 0.029 & 1.667 & -0.02 & -0.021 & -0.169 & 1.724 & -0.057 & 0.121 & 0.158 & 1.797 & -0.04 & 0.151 & 0.067 \\
\hline 2 & 1.666 & -0.525 & -0.303 & -0.02 & 1.608 & -0.464 & -0.432 & -0.2 & 1.619 & -0.38 & -0.499 & -0.026 & 1.668 & -0.422 & -0.39 & 0.069 \\
\hline 3 & 1.68 & -0.279 & -0.166 & 0.314 & 1.677 & -0.285 & -0.139 & 0.348 & 1.687 & -0.235 & -0.087 & 0.224 & 1.772 & -0.243 & -0.069 & 0.021 \\
\hline 4 & 1.793 & -0.08 & 0.172 & 0.085 & 1.79 & -0.085 & 0.189 & 0.101 & 1.794 & -0.044 & 0.194 & 0.02 & 1.797 & -0.04 & 0.151 & 0.067 \\
\hline 5 & -1.733 & 519 & 242 & -0.036 & -1.732 & 0.529 & 222 & -0.07 & -1.747 & 0.461 & 0.244 & -0.053 & -1.746 & 0.446 & 0.289 & 0.025 \\
\hline 6 & -1.696 & & -0.179 & 0.152 & -1.696 & -0.203 & -0.184 & 0.153 & -1.817 & -0.165 & -0.414 & -0.004 & -1.823 & -0.163 & -0.372 & -0.112 \\
\hline 7 & 1.546 & -0.375 & -0.581 & -0.432 & 1.552 & -0.395 & -0.579 & -0.386 & 1.619 & & & -0.026 & 1.528 & -0.342 & -0.617 & -0.001 \\
\hline 8 & 756 & & & 0.054 & 1752 & 0.134 & 0331 & & 1817 & & 0414 & 0.004 & 1.823 & 0.163 & & 0.112 \\
\hline 9 & 763 & -0.299 & -0.035 & 0.06 & 1.761 & -0.30 & -0.016 & 0.085 & 1.77 & -0.2 & -0.025 & 0.037 & 1.772 & -0.243 & -0.0 & 0.021 \\
\hline 10 & 853 & & 0.586 & 0.134 & 1.849 & & & & 1.84 & & & -0.012 & & & & 0.158 \\
\hline 11 & 852 & & 862 & -0.0 & 1.8 & & 33 & & & & 889 & -0.261 & 1.839 & 68 & 915 & -0.002 \\
\hline 12 & 1.733 & -0.519 & -0.242 & 0.036 & 1.732 & -0.5 & -0.222 & 0.07 & 1.747 & -0.4 & -0.244 & 0.053 & 1.746 & -0.446 & -0.289 & -0.025 \\
\hline 13 & -1.823 & & & & & & & & & & & & & & & -0.112 \\
\hline 14 & 1.733 & -0.519 & -0.242 & 0.036 & 1.732 & -0.529 & -0.222 & 0.07 & 1.747 & -0.461 & -0.244 & 0.053 & 1.746 & -0.446 & -0.289 & -0.025 \\
\hline 15 & 1.756 & 0.134 & 0.318 & 0.054 & 1.752 & 0.134 & 0.331 & 0.032 & 1.817 & & 0.414 & 0.004 & 1.823 & & & 0.112 \\
\hline 16 & & & & & & & & & & & & & & & & 0.393 \\
\hline 17 & -0.847 & -1.27 & 0.719 & -0.455 & -0.853 & -1.264 & 0.713 & -0.472 & -0.685 & -1.388 & 0.756 & -0.466 & -0.59 & -1.398 & 0.935 & -0.393 \\
\hline 18 & -1.162 & -1.216 & 0.267 & & -1.173 & -1.202 & 0.282 & 0.231 & -1.075 & -1.364 & 0.425 & 0.37 & -1.221 & -1.405 & 0.082 & 0.795 \\
\hline 19 & -1.672 & & & & -1.679 & & & & & & & 0.629 & & -0.249 & 0.2 & 0.686 \\
\hline 20 & 1.732 & -0.005 & 0.033 & -0.121 & 1.735 & -0.016 & 0.042 & -0.085 & 1.794 & -0.044 & 0.194 & 0.02 & 1.797 & -0.04 & 0.151 & 0.067 \\
\hline 21 & 1.696 & & & & & & & -0.153 & & & & & 1.823 & 0.163 & 0.372 & 0.112 \\
\hline 22 & 1.733 & -0.519 & -0.242 & 0.036 & 1.732 & -0.529 & -0.222 & 0.07 & 1.747 & -0.461 & -0.244 & 0.053 & 1.746 & -0.446 & -0.289 & -0.025 \\
\hline 23 & 1.68 & -0.279 & -0.166 & 0.314 & 1.677 & -0.285 & -0.139 & 0.348 & 1.77 & -0.252 & -0.025 & 0.037 & 1.772 & -0.243 & -0.069 & 0.021 \\
\hline 24 & & -0.519 & -0.242 & & & -0.529 & -0.222 & & & -0.461 & -0.244 & 0.053 & 1.746 & -0.446 & -0.289 & -0.025 \\
\hline 25 & 1.629 & 0.203 & 0.119 & -0.208 & 1.628 & 0.199 & 0.12 & -0.238 & 1.747 & 0.151 & 0.34 & 0.141 & 1.823 & 0.163 & 0.372 & 0.112 \\
\hline 26 & & & & & & & & & 1.677 & -0.474 & -0.317 & 0.19 & 1.667 & -0.462 & -0.4 & 0.157 \\
\hline 27 & & & & & & & & & 1.501 & -0.192 & -0.863 & -0.459 & 1.545 & -0.269 & -0.634 & -0.345 \\
\hline
\end{tabular}


Table I3 Loadings of elements on components - Nga - MTEO case

\begin{tabular}{|c|c|c|c|c|c|c|c|c|c|}
\hline \multirow[b]{2}{*}{ No. } & \multirow[b]{2}{*}{ Elements } & \multicolumn{4}{|c|}{ The first interview (T1) } & \multicolumn{4}{|c|}{ The second interview (T2) } \\
\hline & & 1 & 2 & 3 & 4 & 1 & 2 & 3 & 4 \\
\hline 1 & An ideal project & -2.701 & -1.878 & 0.922 & -0.066 & -2.728 & -1.857 & 0.942 & -0.059 \\
\hline 2 & A less than ideal project & 7.165 & -0.73 & -0.228 & 0.017 & 7.141 & -0.741 & -0.212 & 0.035 \\
\hline 3 & MTEO project & -1.495 & 0.635 & -0.785 & -0.593 & -1.38 & 0.576 & -0.842 & -0.556 \\
\hline 4 & Project 1 & -1.653 & -0.052 & -0.343 & -0.161 & -1.678 & -0.027 & -0.362 & -0.252 \\
\hline 5 & Project 2 & 0.743 & 1.854 & 1.171 & 0.071 & 0.725 & 1.868 & 1.175 & 0.046 \\
\hline 6 & Project 3 & -2.058 & 0.171 & -0.737 & 0.732 & -2.079 & 0.182 & -0.701 & 0.786 \\
\hline \multirow[b]{2}{*}{ No. } & \multicolumn{5}{|c|}{ The third interview (T3) } & \multicolumn{4}{|c|}{ The fourth interview (T4) } \\
\hline & Elements & 1 & 2 & 3 & 4 & 1 & 2 & 3 & 4 \\
\hline 1 & An ideal project & -2.663 & -2.016 & 0.946 & -0.341 & -2.576 & -2.068 & 1.087 & -0.255 \\
\hline 2 & A less than ideal project & 7.562 & -0.675 & -0.279 & 0.03 & 7.619 & -0.66 & -0.336 & -0.016 \\
\hline 3 & MTEO project & -1.524 & 0.826 & -1.246 & -0.733 & -1.594 & 0.856 & -0.817 & -0.976 \\
\hline 4 & Project 1 & -1.811 & -0.117 & -0.501 & 0.465 & -2.077 & -0.14 & -0.77 & 0.706 \\
\hline 5 & Project 2 & 0.604 & 1.833 & 1.501 & -0.055 & 0.67 & 1.8 & 1.537 & 0.177 \\
\hline 6 & Project 3 & -2.168 & 0.149 & -0.422 & 0.634 & -2.043 & 0.212 & -0.701 & 0.363 \\
\hline
\end{tabular}




\section{Appendix J: Results of the Principal Component Analysis of Hung's Repertory Grids - MTEO Case}

Table J1 Percentage variances explained by principal components - Hung - MTEO case

\begin{tabular}{|c|c|c|c|c|c|c|c|c|c|c|c|c|}
\hline & \multicolumn{6}{|c|}{ The first interview (T1) } & \multicolumn{6}{|c|}{ The second interview (T2) } \\
\hline Components & 1 & 2 & 3 & 4 & 5 & 6 & 1 & 2 & 3 & 4 & 5 & 6 \\
\hline Variance \% & 58.24 & 16.56 & 14.91 & 8.63 & 1.67 & 0 & 64.96 & 16.04 & 9.58 & 7.07 & 2.35 & 0 \\
\hline Frontier estimate $\%$ & 40.83 & 24.17 & 15.83 & 10.28 & 6.11 & 2.78 & 40.83 & 24.17 & 15.83 & 10.28 & 6.11 & 2.78 \\
\hline \multirow[t]{2}{*}{ Cumulative variance $\%$} & 58.24 & 74.8 & 89.7 & 98.33 & 100 & 100 & 64.96 & 81 & 90.58 & 97.65 & 100 & 100 \\
\hline & \multicolumn{6}{|c|}{ The third interview (T3) } & \multicolumn{6}{|c|}{ The fourth interview (T4) } \\
\hline Components & 1 & 2 & 3 & 4 & 5 & 6 & 1 & 2 & 3 & 4 & 5 & 6 \\
\hline Variance $\%$ & 62.35 & 16.09 & 10.45 & 8.78 & 2.34 & 0 & 65.8 & 15.64 & 11.2 & 5.11 & 2.25 & 0 \\
\hline Frontier estimate $\%$ & 40.83 & 24.17 & 15.83 & 10.28 & 6.11 & 2.78 & 40.83 & 24.17 & 15.83 & 10.28 & 6.11 & 2.78 \\
\hline Cumulative variance $\%$ & 62.35 & 78.44 & 88.88 & 97.66 & 100 & 100 & 65.8 & 81.44 & 92.63 & 97.75 & 100 & 100 \\
\hline
\end{tabular}

Table J2 Loadings of constructs on components - Hung - MTEO case

\begin{tabular}{|c|c|c|c|c|c|c|c|c|c|c|c|c|c|c|c|c|c|c|c|c|}
\hline \multirow{2}{*}{$\begin{array}{l}\mathrm{N} \\
\mathrm{o}\end{array}$} & \multicolumn{5}{|c|}{ The first interview } & \multicolumn{5}{|c|}{ The second interview } & \multicolumn{5}{|c|}{ The third interview } & \multicolumn{5}{|c|}{ The fourth interview } \\
\hline & 1 & 2 & 3 & 4 & 5 & 1 & 2 & 3 & 4 & 5 & 1 & 2 & 3 & 4 & 5 & 1 & 2 & 3 & 4 & 5 \\
\hline 1 & 1.579 & 0.126 & $0.322^{-}$ & $\begin{array}{r}- \\
0.574\end{array}$ & -0.31 & 1.532 & 0.193 & 0.173 & $\begin{array}{r}- \\
0.652 \\
\end{array}$ & $\begin{array}{r}- \\
0.379 \\
\end{array}$ & 1.424 & 0.033 & -0.6 & $\begin{array}{r}- \\
0.705 \\
\end{array}$ & $\begin{array}{r}- \\
0.388 \\
\end{array}$ & 1.384 & 0.069 & $0.867^{-}$ & $\begin{array}{r}- \\
0.425\end{array}$ & 0.43 \\
\hline 2 & 1.182 & 0.052 & $\begin{array}{r}- \\
0.925\end{array}$ & 0.528 & $\begin{array}{r}- \\
0.668\end{array}$ & 1.064 & 0.193 & 0.947 & -0.15 & $\begin{array}{r}- \\
0.761\end{array}$ & 1.09 & $\begin{array}{r}- \\
0.037\end{array}$ & $\begin{array}{r}- \\
0.857\end{array}$ & 0.487 & $\begin{array}{r}- \\
0.712\end{array}$ & 1.023 & $\begin{array}{r}- \\
0.105\end{array}$ & -0.78 & 0.803 & 0.597 \\
\hline 3 & 1.111 & $\begin{array}{r}- \\
0.614 \\
\end{array}$ & $\begin{array}{r}- \\
1.242\end{array}$ & $\begin{array}{r}- \\
0.328 \\
\end{array}$ & 0.134 & 1.068 & $\begin{array}{r}- \\
0.429 \\
\end{array}$ & $\begin{array}{r}- \\
0.617 \\
\end{array}$ & $\begin{array}{r}- \\
0.902 \\
\end{array}$ & 0.139 & 0.833 & $\begin{array}{r}- \\
0.796 \\
\end{array}$ & $\begin{array}{r}- \\
1.357 \\
\end{array}$ & $\begin{array}{r}- \\
0.321\end{array}$ & 0.077 & 0.761 & $\begin{array}{r}- \\
0.836 \\
\end{array}$ & $\begin{array}{r}- \\
1.412^{-}\end{array}$ & $\begin{array}{r}- \\
0.059 \\
\end{array}$ & $\begin{array}{r}- \\
0.053 \\
\end{array}$ \\
\hline 4 & $\begin{array}{r}- \\
1.135\end{array}$ & 0.404 & 1.173 & 0.122 & $\begin{array}{r}- \\
0.372\end{array}$ & $\begin{array}{r}- \\
0.894\end{array}$ & 0.22 & 0.924 & 1.128 & $\begin{array}{r}- \\
0.085\end{array}$ & $\begin{array}{r}- \\
0.911\end{array}$ & 0.565 & 1.319 & 0.109 & $\begin{array}{r}- \\
0.283 \\
\end{array}$ & $\begin{array}{r}- \\
0.819\end{array}$ & 0.625 & 1.347 & $\begin{array}{r}- \\
0.137\end{array}$ & 0.293 \\
\hline 5 & 1.58 & -0.33 & 0.888 & 0.603 & -0.34 & 1.711 & 0.441 & 0.082 & 0.735 & $\begin{array}{r}- \\
0.448 \\
\end{array}$ & 1.702 & $\begin{array}{r}- \\
0.319 \\
\end{array}$ & 0.647 & 0.448 & $\begin{array}{r}- \\
0.504\end{array}$ & 1.777 & $\begin{array}{r}- \\
0.205\end{array}$ & 0.617 & 0.365 & 0.398 \\
\hline 6 & $\begin{array}{r}- \\
1.616\end{array}$ & $\begin{array}{r}- \\
0.926\end{array}$ & $\begin{array}{r}- \\
0.003\end{array}$ & $\begin{array}{r}- \\
0.596\end{array}$ & $0.212^{-}$ & -1.78 & $\begin{array}{r}- \\
0.914\end{array}$ & 0.377 & $\begin{array}{r}- \\
0.439\end{array}$ & $\begin{array}{r}- \\
0.183 \\
\end{array}$ & -1.79 & $\begin{array}{r}- \\
0.923 \\
\end{array}$ & 0.137 & $\begin{array}{r}- \\
0.534\end{array}$ & -0.1 & $\begin{array}{r}- \\
1.697 \\
\end{array}$ & $\begin{array}{r}- \\
0.937 \\
\end{array}$ & 0.211 & -0.71 & 0.252 \\
\hline
\end{tabular}




\begin{tabular}{|c|c|c|c|c|c|c|c|c|c|c|c|c|c|c|c|c|c|c|c|c|}
\hline 7 & $1.711^{-}$ & $\begin{array}{r}- \\
0.795 \\
\end{array}$ & $\begin{array}{r}- \\
0.165 \\
\end{array}$ & $\begin{array}{r}- \\
0.188^{-} \\
\end{array}$ & $0.156^{-}$ & $1.742^{-}$ & $\begin{array}{r}- \\
0.777 \\
\end{array}$ & 0.103 & $0.015^{-}$ & 0.017 & $\begin{array}{r}- \\
1.755^{-} \\
\end{array}$ & $\begin{array}{r}- \\
0.721 \\
\end{array}$ & 0.216 & $0.035^{-}$ & 0.033 & $\begin{array}{r}- \\
1.998 \\
\end{array}$ & $\begin{array}{r}- \\
0.648 \\
\end{array}$ & $0.039^{-}$ & 0.35 & $0.002^{-}$ \\
\hline 8 & 1.807 & $0.664^{-}$ & $0.327^{-}$ & 0.22 & $\begin{array}{r}- \\
0.099 \\
\end{array}$ & -1.83 & 0.631 & $\begin{array}{r}- \\
0.276 \\
\end{array}$ & 0.201 & 0.067 & -1.84 & $\begin{array}{r}- \\
0.578 \\
\end{array}$ & 0.065 & 0.378 & 0.047 & $\begin{array}{r}- \\
1.791 \\
\end{array}$ & $\begin{array}{r}- \\
0.707\end{array}$ & 0.351 & 0.197 & $0.025^{-}$ \\
\hline 9 & $1.205^{-}$ & 1.217 & $0.404^{-}$ & $0.81 \overline{6}^{-}$ & $0.126^{-}$ & $1.294^{-}$ & 1.263 & 0.505 & 0.503 & 0.025 & $1.395^{-}$ & 1.137 & $0.544^{-}$ & $\begin{array}{r}- \\
0.877 \\
\end{array}$ & -0.11 & $1.494^{-}$ & 1.092 & $\begin{array}{r}- \\
0.689 \\
\end{array}$ & -0.6 & 0.239 \\
\hline 10 & 1.705 & $0.227^{-}$ & 0.544 & -0.41 & 0.063 & 1.866 & $0.288^{-}$ & 0.595 & $0.064^{-}$ & 0.057 & 1.854 & $0.233^{-}$ & 0.482 & $\begin{array}{r}- \\
0.437\end{array}$ & 0.098 & 1.864 & $0.094^{-}$ & 0.325 & $0.236^{-}$ & -0.05 \\
\hline 11 & 1.679 & 0.991 & $0.292^{-}$ & $\begin{array}{r}- \\
0.068 \\
\end{array}$ & 0.185 & 1.863 & 1.023 & $\begin{array}{r}- \\
0.024 \\
\end{array}$ & 0.029 & 0.272 & 1.876 & 0.982 & -0.22 & $\begin{array}{r}- \\
0.041 \\
\end{array}$ & 0.247 & 1.998 & 0.648 & 0.039 & -0.35 & 0.002 \\
\hline 12 & 0.883 & -0.18 & $\begin{array}{r}- \\
1.449 \\
\end{array}$ & 1.042 & 0.054 & 1.279 & $\begin{array}{r}- \\
0.016 \\
\end{array}$ & $\begin{array}{r}- \\
1.318^{-}\end{array}$ & 0.674 & 0.516 & 1.277 & $\begin{array}{r}- \\
0.013 \\
\end{array}$ & $\begin{array}{r}- \\
0.387 \\
\end{array}$ & 1.453 & 0.449 & 1.431 & $\begin{array}{r}- \\
0.297 \\
\end{array}$ & -0.04 & 0.575 & $\begin{array}{r}- \\
0.539 \\
\end{array}$ \\
\hline 13 & 1.731 & $0.009^{-}$ & $\begin{array}{r}- \\
0.367 \\
\end{array}$ & 0.52 & 0.157 & 1.958 & 0.033 & $\begin{array}{r}- \\
0.691\end{array}$ & 0.011 & 0.025 & 1.951 & $0.029^{-}$ & $0.517^{-}$ & 0.491 & 0.004 & 1.986 & 0.012 & 0.605 & 0.151 & $0.194^{-}$ \\
\hline 14 & 1.568 & 0.939 & $0.577^{-}$ & 0.482 & 0.135 & 1.728 & 0.999 & $0.60 \overline{6}^{-}$ & 0.154 & 0.126 & 1.705 & 0.93 & $0.605^{-}$ & 0.455 & 0.213 & 1.769 & 0.778 & $0.492^{-}$ & 0.391 & 0.139 \\
\hline 15 & 1.221 & $\begin{array}{r}- \\
1.565 \\
\end{array}$ & -0.33 & $0.694^{-}$ & $\begin{array}{r}- \\
0.077\end{array}$ & 1.543 & $1.509^{-}$ & 0.413 & $0.261^{-}$ & 0.29 & 1.27 & $1.572^{-}$ & 0.073 & $0.516^{-}$ & 0.18 & 1.372 & $1.422^{-}$ & $\begin{array}{r}- \\
0.077\end{array}$ & $\begin{array}{r}- \\
0.682 \\
\end{array}$ & -0.11 \\
\hline 16 & 1.778 & 0.421 & 0.806 & 0.326 & 0.019 & 1.92 & 0.314 & 0.231 & 0.492 & $0.102^{-}$ & 1.919 & 0.405 & 0.443 & 0.156 & $0.16^{-}$ & 1.921 & 0.532 & 0.277 & 0.187 & 0.075 \\
\hline 17 & 0.253 & 1.239 & -0.71 & $\begin{array}{r}- \\
0.872 \\
\end{array}$ & -0.16 & 0.436 & 1.32 & 0.518 & $\begin{array}{r}- \\
0.369 \\
\end{array}$ & 0.226 & 0.573 & 1.338 & 0.056 & $\begin{array}{r}- \\
0.604 \\
\end{array}$ & 0.328 & 0.411 & 1.555 & $\begin{array}{r}- \\
0.398 \\
\end{array}$ & -0.27 & 0.402 \\
\hline
\end{tabular}


Table J3 Loadings of elements on components - Hung - MTEO case

\begin{tabular}{|c|c|c|c|c|c|c|c|c|c|c|c|}
\hline & & \multicolumn{5}{|c|}{ The first interview } & \multicolumn{5}{|c|}{ The second interview } \\
\hline & & 1 & 2 & 3 & 4 & 5 & 1 & 2 & 3 & 4 & 5 \\
\hline 1 & An ideal project & -3.917 & 0.423 & 0.421 & -0.503 & 0.573 & -3.625 & 0.315 & 0.646 & 0.018 & 0.807 \\
\hline 2 & A less than ideal project & 3.93 & -0.512 & -0.671 & 0.088 & 0.578 & 4.184 & -0.407 & -0.772 & -0.638 & 0.552 \\
\hline 3 & MTEO project & -1.06 & -0.296 & -2.294 & -0.597 & -0.396 & -2.44 & 0.081 & -0.777 & -1.32 & -0.547 \\
\hline 4 & Project 1 & -1.098 & -0.456 & 0.193 & 1.998 & -0.156 & -0.806 & -0.519 & -1.307 & 1.486 & -0.163 \\
\hline 5 & Project 2 & 1.53 & 2.554 & 0.682 & -0.163 & -0.272 & 1.783 & 2.444 & 0.71 & 0.34 & -0.301 \\
\hline \multirow[t]{3}{*}{6} & Project 3 & 0.614 & -1.712 & 1.668 & -0.823 & -0.327 & 0.905 & -1.914 & 1.499 & 0.114 & -0.347 \\
\hline & & \multicolumn{5}{|c|}{ The third interview } & \multicolumn{5}{|c|}{ The fourth interview } \\
\hline & & 1 & 2 & 3 & 4 & 5 & 1 & 2 & 3 & 4 & 5 \\
\hline 1 & An ideal project & -3.647 & 0.612 & 0.56 & -0.306 & 0.779 & -3.767 & 0.584 & 0.536 & -0.467 & -0.714 \\
\hline 2 & A less than ideal project & 4.113 & -0.628 & -0.863 & 0.146 & 0.626 & 3.974 & -0.635 & -0.962 & 0.124 & -0.638 \\
\hline 3 & MTEO project & -2.298 & -0.568 & -1.798 & -0.606 & -0.435 & -2.572 & -0.723 & -1.821 & -0.115 & 0.473 \\
\hline 4 & Project 1 & -0.812 & -0.284 & 0.325 & 2.053 & -0.27 & -0.746 & -0.55 & 0.957 & 1.457 & 0.13 \\
\hline 5 & Project 2 & 1.83 & 2.533 & 0.273 & -0.387 & -0.375 & 1.656 & 2.604 & -0.016 & -0.053 & 0.356 \\
\hline 6 & Project 3 & 0.814 & -1.666 & 1.503 & -0.9 & -0.325 & 1.455 & -1.279 & 1.306 & -0.945 & 0.392 \\
\hline
\end{tabular}




\section{Appendix K: Results of the Principal Component Analysis of Tung's Repertory Grids - APSC Case}

Table K1 List of constructs - Tung - APSC case

\begin{tabular}{|r|l|}
\hline No & Constructs \\
\hline 1 & Clear strategic planning and leadership to achieve accreditation \\
\hline 2 & Effective objective-based management \\
\hline 3 & Training programme's characteristics fit accreditation standards. \\
\hline 4 & Faculty qualifications are not good \\
\hline 5 & Good research \\
\hline 6 & Students are good at English \\
\hline 7 & Clear and specific accreditation standards \\
\hline 8 & University's operation is suitable to accreditation standards \\
\hline 9 & Projects progress well \\
\hline 10 & Projects are done in internationally standardised ways \\
\hline 11 & Leaders are determined to obtain accreditation \\
\hline 12 & Suitable resources \\
\hline 13 & Programmes use English \\
\hline 14 & Universities do projects by themselves, really make changes to universities \\
\hline 15 & Programme structures follow international standards \\
\hline 16 & Standards for training programmes are not strict \\
\hline 17 & Implementers can trust the transparency of the accreditation and want to invest in it \\
\hline 18 & No support of related departments \\
\hline 19 & Rigorous accreditation processes make implementers trust the transparency of the accreditation \\
\hline 20 & No guidance and training for project implementers \\
\hline 21 & Pressure for accreditation is not much, which is good for organisations \\
\hline 22 & High internal motivation leads to high investment in accreditation \\
\hline 23 & Faculty qualifications must improve immediately \\
\hline 24 & Faculty qualifications are not good enough for the changes in universities \\
\hline & \\
\hline 1
\end{tabular}


Table K2 Percentage variances explained by principal components - Tung - APSC case

\begin{tabular}{|c|c|c|c|c|c|c|c|c|c|c|c|c|}
\hline Interviews & \multicolumn{6}{|c|}{ The first interview } & \multicolumn{6}{|c|}{ The second interview } \\
\hline Components & 1 & 2 & 3 & 4 & 5 & 6 & 1 & 2 & 3 & 4 & 5 & 6 \\
\hline Variance $\%$ & 88.28 & 5.42 & 4.45 & 1.44 & 0.41 & 0 & 83.69 & 8.56 & 5.26 & 1.44 & 1.05 & 0 \\
\hline Frontier estimate $\%$ & 40.83 & 24.17 & 15.83 & 10.28 & 6.11 & 2.78 & 40.83 & 24.17 & 15.83 & 10.28 & 6.11 & 2.78 \\
\hline Cumulative variance $\%$ & 88.28 & 93.7 & 98.15 & 99.59 & 100 & 100 & 83.69 & 92.25 & 97.51 & 98.95 & 100 & 100 \\
\hline Interviews & \multicolumn{6}{|c|}{ The third interview } & \multicolumn{6}{|c|}{ The fourth interiew } \\
\hline Components & 1 & 2 & 3 & 4 & 5 & 6 & 1 & 2 & 3 & 4 & 5 & 6 \\
\hline Variance $\%$ & 83.84 & 7.95 & 5.45 & 1.79 & 0.97 & 0 & 84.95 & 7.13 & 5.4 & 1.59 & 0.94 & 0 \\
\hline Frontier estimate $\%$ & 40.83 & 24.17 & 15.83 & 10.28 & 6.11 & 2.78 & 40.83 & 24.17 & 15.83 & 10.28 & 6.11 & 2.78 \\
\hline Cumulative variance \% & 83.84 & 91.79 & 97.24 & 99.03 & 100 & 100 & 84.95 & 92.08 & 97.48 & 99.06 & 100 & 100 \\
\hline
\end{tabular}


Table K3 Loadings of constructs on components - Tung - APSC case (Green: loadings on the first component, blue and yellow: loadings on the second component with opposite signs)

\begin{tabular}{|c|c|c|c|c|c|c|c|c|c|c|c|c|c|c|c|c|c|}
\hline \multirow[t]{2}{*}{ Constructs } & \multicolumn{4}{|c|}{ The first interview } & \multicolumn{5}{|c|}{ The second interview } & \multicolumn{4}{|c|}{ The third interview } & \multicolumn{4}{|c|}{ The fourth interview } \\
\hline & 1 & 2 & 3 & 4 & 1 & 2 & 3 & 4 & 5 & 1 & 2 & 3 & 4 & 1 & 2 & 3 & 4 \\
\hline 1 & 1.738 & 0.095 & 0 & 0.079 & 1.733 & 0.11 & 0.025 & -0.083 & 0.119 & 1.768 & -0.104 & 0.095 & 0.058 & 1.771 & 0.057 & 0.093 & 0.014 \\
\hline 2 & 1.738 & -0.169 & -0.099 & 0.438 & 1.723 & -0.112 & -0.027 & -0.012 & 0.526 & 1.729 & -0.069 & -0.022 & 0.115 & 1.733 & 0.026 & -0.036 & 0.012 \\
\hline 3 & 1.801 & -0.178 & 0.229 & -0.039 & 1.804 & -0.147 & 0.233 & 0.044 & -0.014 & 1.808 & -0.14 & 0.211 & 0.002 & 1.81 & 0.088 & 0.221 & 0.016 \\
\hline 4 & -1.576 & -0.511 & 0.291 & 0.085 & -1.566 & -0.579 & 0.312 & 0.028 & -0.044 & -1.556 & -0.63 & 0.267 & -0.014 & -1.547 & 0.6 & 0.362 & 0.054 \\
\hline 5 & -1.614 & -0.904 & 0.361 & 0.045 & -1.6 & -0.845 & 0.337 & 0.409 & 0.02 & -1.582 & -0.913 & 0.296 & 0.36 & -1.609 & 0.63 & 0.319 & 0.333 \\
\hline 6 & 1.801 & -0.178 & 0.229 & -0.039 & 1.804 & -0.147 & 0.233 & 0.044 & -0.014 & 1.808 & -0.14 & 0.211 & 0.002 & 1.81 & 0.088 & 0.221 & 0.016 \\
\hline 7 & 1.801 & -0.178 & 0.229 & -0.039 & 1.804 & -0.147 & 0.233 & 0.044 & -0.014 & 1.808 & -0.14 & 0.211 & 0.002 & 1.81 & 0.088 & 0.221 & 0.016 \\
\hline 8 & 1.769 & -0.173 & 0.065 & 0.199 & 1.764 & -0.129 & 0.103 & 0.016 & 0.256 & 1.768 & -0.104 & 0.095 & 0.058 & 1.771 & 0.057 & 0.093 & 0.014 \\
\hline 9 & 1.614 & 0.904 & -0.361 & -0.045 & 1.671 & 0.588 & -0.129 & -0.282 & -0.154 & 1.621 & 0.877 & -0.179 & -0.416 & 1.648 & -0.599 & -0.191 & -0.33 \\
\hline 10 & 1.738 & -0.169 & -0.099 & 0.438 & 1.764 & -0.129 & 0.103 & 0.016 & 0.256 & 1.768 & -0.104 & 0.095 & 0.058 & 1.771 & 0.057 & 0.093 & 0.014 \\
\hline 11 & 1.77 & 0.09 & 0.164 & -0.16 & 1.774 & 0.092 & 0.155 & -0.055 & -0.151 & 1.808 & -0.14 & 0.211 & 0.002 & 1.81 & 0.088 & 0.221 & 0.016 \\
\hline 12 & 1.77 & 0.09 & 0.164 & -0.16 & 1.774 & 0.092 & 0.155 & -0.055 & -0.151 & 1.771 & 0.106 & 0.143 & -0.117 & 1.769 & -0.13 & 0.126 & -0.098 \\
\hline 13 & 1.801 & -0.178 & 0.229 & -0.039 & 1.804 & -0.147 & 0.233 & 0.044 & -0.014 & 1.808 & -0.14 & 0.211 & 0.002 & 1.81 & 0.088 & 0.221 & 0.016 \\
\hline 14 & 1.801 & -0.178 & 0.229 & -0.039 & 1.804 & -0.147 & 0.233 & 0.044 & -0.014 & 1.808 & -0.14 & 0.211 & 0.002 & 1.81 & 0.088 & 0.221 & 0.016 \\
\hline 15 & 1.769 & -0.173 & 0.065 & 0.199 & 1.764 & -0.129 & 0.103 & 0.016 & 0.256 & 1.768 & -0.104 & 0.095 & 0.058 & 1.771 & 0.057 & 0.093 & 0.014 \\
\hline 16 & -1.23 & 1.111 & 0.327 & 0.367 & -1.257 & 0.903 & 0.424 & -0.688 & 0.09 & -1.272 & 0.874 & 0.466 & -0.632 & -1.283 & -0.877 & 0.367 & -0.706 \\
\hline 17 & 1.801 & -0.178 & 0.229 & -0.039 & 1.804 & -0.147 & 0.233 & 0.044 & -0.014 & 1.808 & -0.14 & 0.211 & 0.002 & 1.81 & 0.088 & 0.221 & 0.016 \\
\hline 18 & -1.67 & -0.234 & -0.102 & 0.442 & -1.774 & -0.092 & -0.155 & 0.055 & 0.151 & -1.771 & -0.106 & -0.143 & 0.117 & -1.769 & 0.13 & -0.126 & 0.098 \\
\hline 19 & 1.801 & -0.178 & 0.229 & -0.039 & 1.804 & -0.147 & 0.233 & 0.044 & -0.014 & 1.808 & -0.14 & 0.211 & 0.002 & 1.81 & 0.088 & 0.221 & 0.016 \\
\hline 20 & -1.707 & -0.099 & 0.164 & -0.317 & -1.774 & -0.092 & -0.155 & 0.055 & 0.151 & -1.771 & -0.106 & -0.143 & 0.117 & -1.769 & 0.13 & -0.126 & 0.098 \\
\hline 21 & 0.75 & -0.464 & -1.486 & -0.056 & 0.763 & -0.558 & -1.438 & -0.25 & 0.118 & 0.726 & -0.16 & -1.56 & -0.238 & 0.722 & 0.393 & -1.513 & -0.298 \\
\hline 22 & 1.639 & 0.238 & -0.062 & -0.203 & 1.678 & 0.304 & 0.025 & 0.128 & -0.36 & 1.708 & 0.068 & 0.103 & 0.138 & 1.707 & -0.101 & 0.084 & 0.18 \\
\hline 23 & & & & & 1.177 & -1.531 & -0.503 & -0.305 & -0.271 & 1.193 & -1.454 & -0.671 & -0.381 & 1.206 & 1.552 & -0.441 & -0.272 \\
\hline 24 & & & & & -1.2 & -1.25 & 0.963 & -0.465 & 0.043 & -1.214 & -1.079 & 0.757 & -0.655 & -1.202 & 1.012 & 0.937 & -0.548 \\
\hline
\end{tabular}


Figure K1 PrinGrid map - Tung - the first interview - the APSC project

C19. Rigorous accreditation processes make implementers trust the transparency of the accreditation

$$
\text { C13. Programmes use English }
$$

C17. Implementers can trust the transparency of the accreditation and want to invest in it

C14. Universities do projects by themselve, really makes changes to universities

$$
\text { C6. Students are good at English }
$$

C7. Clear and specific accreditation standards

C21. Pressure for accreditation is not much, which is good for organisations

C3. Training programmes' characteristics fit accreditation standards

C15. Program structures followinternational standards

C10. Projects are done in internationally standardised ways

C8. University's operation is suitable to accreditation standards.

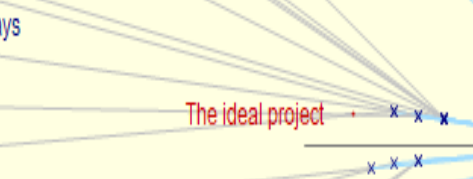

C16. Standards for training programmes are strict

C9. Projects do not progress well

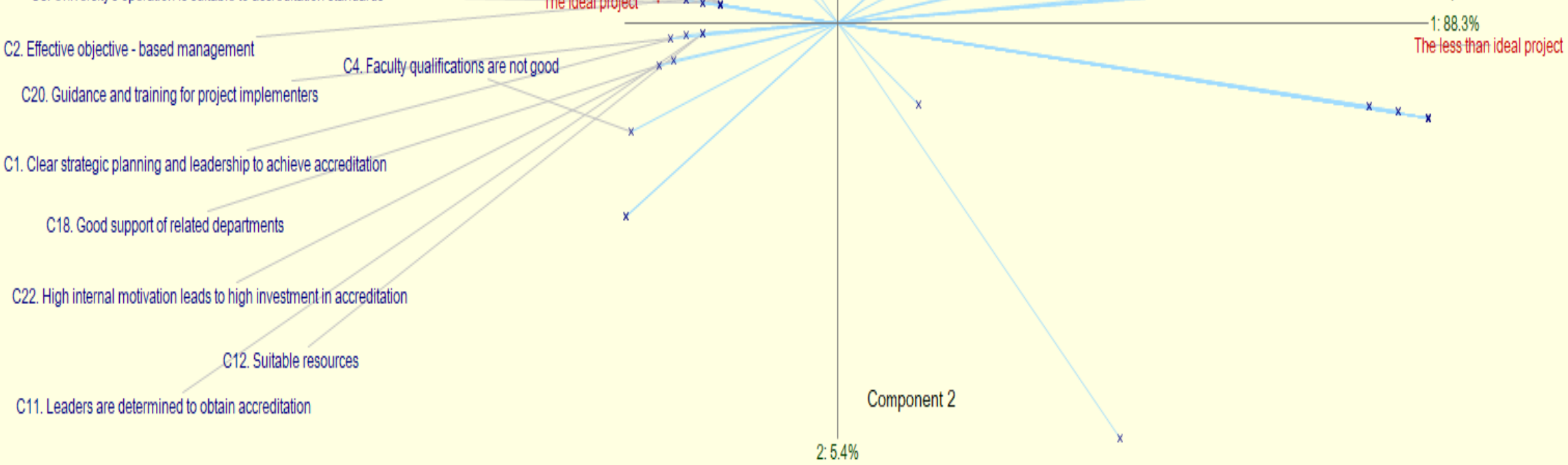


Figure K2 PrinGid Map - Tung - the second interview - the APSC project

C19. Rigorous accreditation processes make implementers trust the transparency of the accreditation

C17. Implementers can trust the tranparency of the accreditation and want to invest in it

C14. Universities do projects by themselves, really makes changes to universities

C21. Pressure for accreditation is not much, which is good for organisations

C7. Clear and specific accreditation standards C6. Students are good at English

C15. Program structure follows international standards

C8. University operations are suitable to accreditation standards

C13. Programmes use English

C2. Effective objective-based management-

$-\infty$

C23. Faculty qualifications must improve immediately

C16. Standards for training programmes are strict
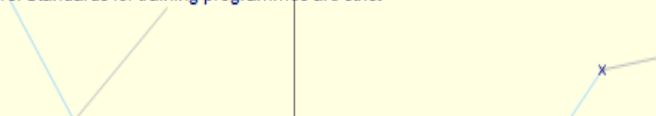
C5. Research activities are not good C4. Faculty qualifications are not good

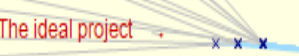

C10. Projects are done in an internationally standardised ways

C1. Clear strategic planning and leadership to achieve accreditation

C11. Leaders are determined to obtain accreditation

C22. High internal motivation leads to high investment in accreditation

C12. Suitable resources

C18. There is support of related departments

C20. There are guidance and training for project implementers

Component

$-1: 83.7 \%$

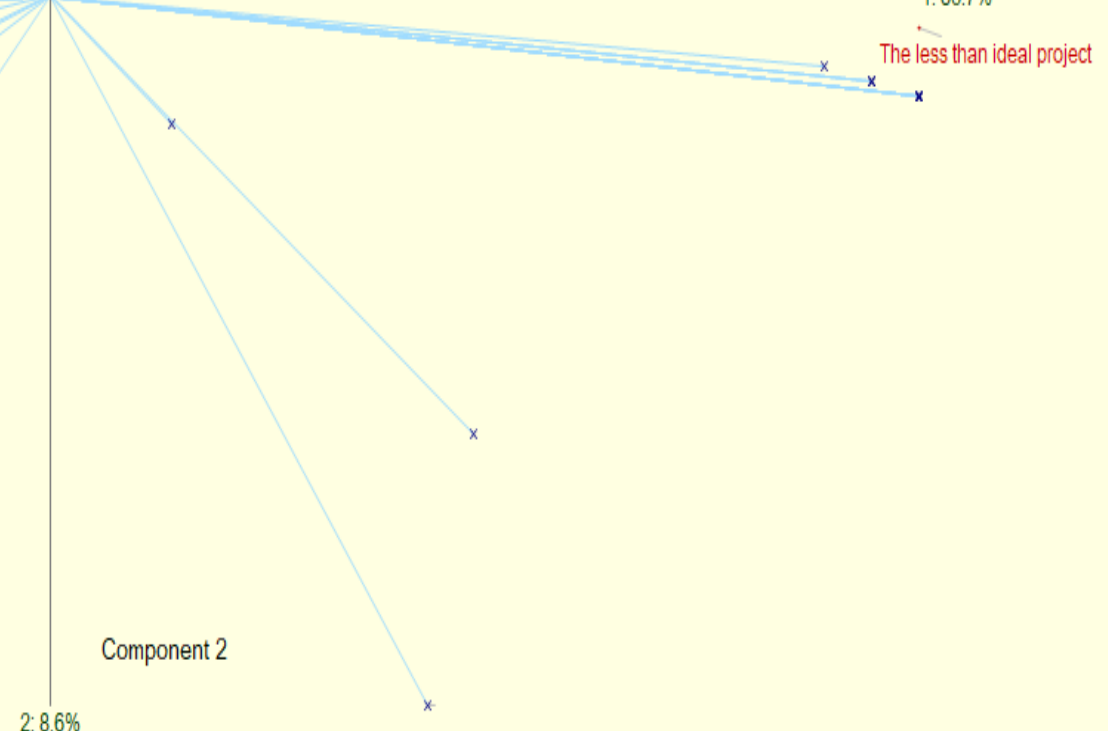


Table K4 Loadings of elements on principal components - Tung - APSC case

\begin{tabular}{|c|c|c|c|c|c|c|c|c|c|}
\hline Interviews & \multicolumn{4}{|c|}{ The first interview } & \multicolumn{5}{|c|}{ The second interview } \\
\hline Components & 1 & 2 & 3 & 4 & 1 & 2 & 3 & 4 & 5 \\
\hline Ideal Project & -2.174 & 0.174 & 1.429 & 0.313 & -2.33 & 0.174 & 1.733 & -0.062 & 0.137 \\
\hline Less than ideal project & 7.114 & -0.174 & 0.203 & -0.02 & 7.345 & -0.191 & 0.237 & 0.023 & -0.007 \\
\hline APSC project & -0.73 & 1.574 & -0.349 & -0.365 & -0.752 & 1.869 & -0.473 & -0.318 & -0.373 \\
\hline Project 1 & -1.829 & -0.869 & -0.43 & -0.162 & -1.699 & -1.777 & -0.377 & -0.458 & -0.297 \\
\hline Project 2 & -0.746 & 0.027 & -0.872 & 0.723 & -0.99 & 0.138 & -0.796 & -0.09 & 0.739 \\
\hline Project 3 & -1.634 & -0.732 & 0.019 & -0.488 & -1.574 & -0.213 & -0.324 & 0.905 & -0.198 \\
\hline Interviews & \multicolumn{4}{|c|}{ The third interview } & \multicolumn{4}{|c|}{ The fourth interview } & \\
\hline Components & 1 & 2 & 3 & 4 & 1 & 2 & 3 & 4 & \\
\hline Ideal Project & -2.297 & -0.013 & 1.776 & -0.092 & -2.275 & -0.275 & 1.745 & -0.081 & \\
\hline Less than ideal project & 7.374 & -0.175 & 0.22 & 0.001 & 7.396 & 0.105 & 0.228 & 0.009 & \\
\hline APSC project & -0.9 & 1.849 & -0.427 & -0.424 & -1.009 & -1.553 & -0.585 & -0.385 & \\
\hline Project 1 & -1.67 & -1.646 & -0.593 & -0.599 & -1.646 & 1.737 & -0.329 & -0.47 & \\
\hline Project 2 & -0.966 & 0.267 & -0.728 & 0.202 & -0.944 & -0.221 & -0.794 & -0.008 & \\
\hline Project 3 & -1.541 & -0.282 & -0.248 & 0.912 & -1.521 & 0.207 & -0.265 & 0.934 & \\
\hline
\end{tabular}




\section{Appendix L: Results of the Principal Component Analysis of Tai's Repertory Grids - APSC Case}

Table L1 List of constructs - Tai - APSC case

\begin{tabular}{|r|l|}
\hline No. & Constructs \\
\hline 1 & Projects do not progress well and do not follow plans \\
\hline 2 & Projects do not meet required criteria \\
\hline 3 & Infrastructure meets projects' requirements \\
\hline 4 & Projects are very important and help to create revenue and growth for organisations \\
\hline 5 & Management policies are not comprehensive and detailed, and do not specify project members' tasks and plans \\
\hline 6 & No communication channel, project members are not clear about tasks and do not contribute 100\% of their abilities \\
\hline 7 & Projects use software in quality management \\
\hline 8 & Good activities to increase project members' cohesiveness \\
\hline 9 & No technology training for project members \\
\hline 10 & Unclear renumeration and management policies \\
\hline 11 & Projects are piloted, tested, assessed and applied in bigger scope \\
\hline 12 & Leaders have good awareness and management \\
\hline
\end{tabular}

Table L2 Percentage variances explained by principal components - Tai - APSC case

\begin{tabular}{|c|c|c|c|c|c|c|c|c|c|c|c|c|}
\hline Interviews & \multicolumn{6}{|c|}{ The first interview } & \multicolumn{6}{|c|}{ The second interview } \\
\hline Components & 1 & 2 & 3 & 4 & 5 & 6 & 1 & 2 & 3 & 4 & 5 & 6 \\
\hline Variance $\%$ & 84.46 & 8.61 & 5.23 & 1.7 & 0 & 0 & 86.89 & 7.81 & 3.35 & 1.95 & 0 & 0 \\
\hline Frontier estimate $\%$ & 40.83 & 24.17 & 15.83 & 10.28 & 6.11 & 2.78 & 40.83 & 24.17 & 15.83 & 10.28 & 6.11 & 2.78 \\
\hline Cumulative variance & 84.46 & 93.07 & 98.3 & 100 & 100 & 100 & 86.89 & 94.7 & 98.05 & 100 & 100 & 100 \\
\hline Interviews & \multicolumn{6}{|c|}{ The third interview } & \multicolumn{6}{|c|}{ The fourth interview } \\
\hline Components & 1 & 2 & 3 & 4 & 5 & 6 & 1 & 2 & 3 & 4 & 5 & 6 \\
\hline Variance $\%$ & 85.98 & 8.21 & 3.37 & 2.45 & 0 & 0 & 89.66 & 6.5 & 2.84 & 1 & 0 & 0 \\
\hline Frontier estimate $\%$ & 40.83 & 24.17 & 15.83 & 10.28 & 6.11 & 2.78 & 40.83 & 24.17 & 15.83 & 10.28 & 6.11 & 2.78 \\
\hline Cumulative variance & 85.98 & 94.18 & 97.55 & 100 & 100 & 100 & 89.66 & 96.16 & 99 & 100 & 100 & 100 \\
\hline
\end{tabular}


Table L3 Loadings of constructs on components - Tai-APSC case (Green: loadings on the first component, blue and yellow: loadings on the second component with opposite signs)

\begin{tabular}{|c|c|c|c|c|c|c|c|c|c|c|c|c|c|c|c|c|}
\hline \multirow[t]{2}{*}{ No. } & \multicolumn{4}{|c|}{ The first interview } & \multicolumn{4}{|c|}{ The second interview } & \multicolumn{4}{|c|}{ The third interview } & \multicolumn{4}{|c|}{ The fourth interview } \\
\hline & 1 & 2 & 3 & 4 & 1 & 2 & 3 & 4 & 1 & 2 & 3 & 4 & 1 & 2 & 3 & 4 \\
\hline 1 & -1.529 & 0.583 & 0.135 & 0.252 & -1.612 & 0.448 & 0.486 & -0.032 & -1.586 & 0.424 & 0.289 & -0.188 & -1.655 & 0.171 & 0.213 & -0.138 \\
\hline 2 & -1.553 & 0.488 & 0.127 & -0.036 & -1.586 & 0.548 & -0.043 & 0.125 & -1.669 & 0.524 & 0.051 & 0.373 & -1.724 & 0.421 & 0.187 & 0.146 \\
\hline 3 & 1.796 & 0.437 & -0.611 & 0.368 & 1.78 & 0.562 & 0.656 & -0.101 & 1.759 & 0.692 & 0.55 & -0.224 & 1.722 & 0.303 & 0.489 & -0.197 \\
\hline 4 & 1.367 & -0.934 & -0.752 & -0.168 & 1.589 & -0.771 & 0.005 & -0.465 & 1.661 & -0.538 & -0.354 & -0.401 & 1.728 & -0.328 & -0.476 & -0.122 \\
\hline 5 & -1.739 & 0.042 & -0.499 & -0.241 & -1.583 & 0.326 & -0.081 & -0.216 & -1.595 & 0.41 & -0.013 & -0.216 & -1.655 & 0.171 & 0.213 & -0.138 \\
\hline 6 & -1.51 & 0.847 & -0.706 & 0.059 & -1.598 & 0.387 & 0.203 & -0.124 & -1.595 & 0.41 & -0.013 & -0.216 & -1.655 & 0.171 & 0.213 & -0.138 \\
\hline 7 & 1.911 & 0.597 & -0.011 & -0.291 & 1.898 & 0.814 & -0.559 & -0.129 & 1.699 & 0.847 & -0.515 & -0.02 & 1.738 & 1.184 & -0.314 & -0.129 \\
\hline 8 & 1.684 & -0.05 & -0.083 & 0.458 & 1.619 & -0.154 & 0.489 & 0.058 & 1.662 & -0.285 & 0.699 & 0 & 1.716 & -0.605 & 0.391 & -0.193 \\
\hline 9 & -1.911 & -0.597 & 0.011 & 0.291 & -1.821 & -0.602 & 0.156 & 0.036 & -1.692 & -0.567 & 0.136 & 0.008 & -1.655 & -0.342 & -0.141 & -0.112 \\
\hline 10 & -1.646 & 0.265 & -0.186 & -0.139 & -1.583 & 0.326 & -0.081 & -0.216 & -1.595 & 0.41 & -0.013 & -0.216 & -1.651 & 0.263 & -0.076 & -0.115 \\
\hline 11 & 1.906 & 0.453 & 0.552 & -0.067 & 1.643 & 0.169 & -0.002 & 0.555 & 1.602 & 0.388 & 0.005 & 0.566 & 1.581 & 0.196 & 0.371 & 0.374 \\
\hline 12 & 1.841 & 0.47 & -0.316 & -0.105 & 1.809 & 0.441 & 0.09 & -0.284 & 1.726 & 0.37 & 0.021 & -0.296 & 1.724 & 0.092 & 0.167 & -0.172 \\
\hline
\end{tabular}


Figure L1 PrinGrid map - Tai - the first interview - the APSC project

C4. Projects are very important and help to create revenue and growth for organisations

C6. Clear communication channels and plans make project members contribute $100 \%$ of their abilities

C1. Projects progress well and follow plans

\begin{abstract}
C10. Clear renumeration and management policies
C2. Projects meet required criteria

C8. Good activities to increase project members' cohesiveness

C5. Management policies are comprehensive and detailed, and specify project members' tasks and plans
\end{abstract}

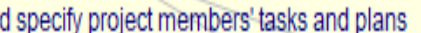

Component 2

2. $8.6 \%$

\section{C9. No technology training for project members}

C7. Projects do not use software in quality management

C12. Leaders do not pay much attention to projects

C3. Infrastructure does not meet project requirements

$x$

11. Projects are not piloted, tested, assessed and applied in bigger scope

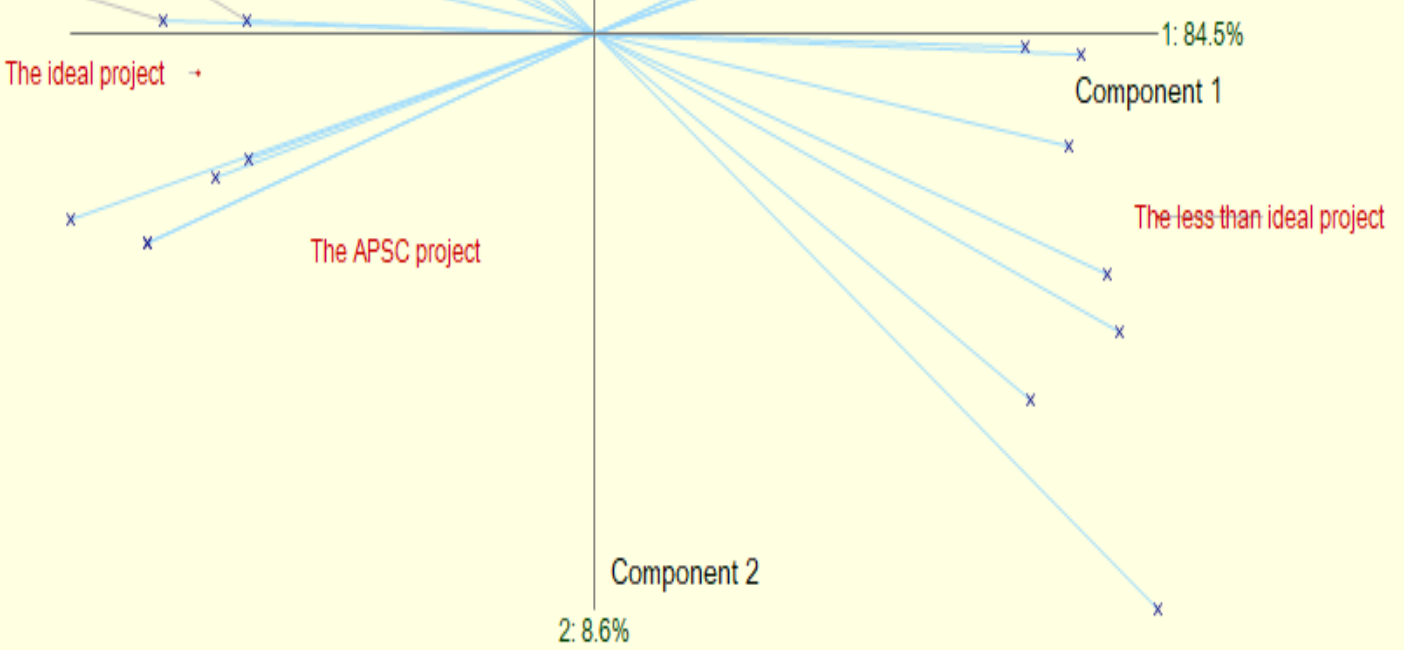


Table L4 Loadings of elements on principal components - Tai - APSC case

\begin{tabular}{|c|c|c|c|c|c|c|c|c|}
\hline Interviews & \multicolumn{4}{|c|}{ The first interview } & \multicolumn{4}{|c|}{ The second interview } \\
\hline Components & 1 & 2 & 3 & 4 & 1 & 2 & 3 & 4 \\
\hline Ideal project & -2.848 & -0.191 & -0.414 & -0.093 & -2.29 & -0.246 & -0.066 & -0.344 \\
\hline Less than ideal project & 4.044 & -0.882 & -0.554 & -0.071 & 4.624 & -0.672 & 0.003 & -0.203 \\
\hline APSC project & -0.253 & -0.679 & 1.226 & -0.08 & -0.881 & -0.581 & -0.428 & 0.653 \\
\hline Project 1 & 1.482 & 1.403 & 0.118 & -0.387 & 1.089 & 1.426 & -0.413 & -0.002 \\
\hline Project 2 & -2.848 & -0.191 & -0.414 & -0.093 & -2.29 & -0.246 & -0.066 & -0.344 \\
\hline Project 3 & 0.423 & 0.539 & 0.038 & 0.725 & -0.252 & 0.318 & 0.972 & 0.24 \\
\hline Interviews & \multicolumn{4}{|c|}{ The third interview } & \multicolumn{4}{|c|}{ The fourth interview } \\
\hline Components & 1 & 2 & 3 & 4 & 1 & 2 & 3 & 4 \\
\hline Ideal project & -2.166 & -0.322 & -0.147 & -0.363 & -1.886 & -0.241 & -0.286 & -0.222 \\
\hline Less than ideal project & 4.758 & -0.477 & -0.201 & -0.194 & 5.039 & -0.258 & -0.247 & -0.038 \\
\hline APSC project & -0.851 & -0.513 & -0.275 & 0.791 & -1.233 & -0.25 & -0.183 & 0.528 \\
\hline Project 1 & 0.272 & 1.56 & -0.261 & 0.047 & 0.035 & 1.425 & 0.101 & -0.003 \\
\hline Project 2 & -2.166 & -0.322 & -0.147 & -0.363 & -1.886 & -0.241 & -0.286 & -0.222 \\
\hline Project 3 & 0.152 & 0.074 & 1.03 & 0.082 & -0.069 & -0.435 & 0.9 & -0.044 \\
\hline
\end{tabular}




\section{Appendix M: Results of the Principal Component Analysis of Khiem's Repertory Grids - APSC Case}

Table M1 List of constructs - Khiem - APSC case

\begin{tabular}{|r|l|}
\hline No. & Constructs \\
\hline 1 & Payment procedures related to accreditation fees are fast \\
\hline 2 & Control of work complexity is good \\
\hline 3 & Accreditation projects have full-time staff \\
\hline 4 & Projects benefit organisations \\
\hline 5 & Academic staff does not have good academic expertise \\
\hline 6 & Related departments have old-fashioned thinking and do not support projects \\
\hline 7 & Projects are based on real demands of society \\
\hline 8 & Project members can learn much from projects \\
\hline 9 & Project implementation follows specific procedures \\
\hline 10 & No reference material \\
\hline 11 & Standardised data lead to good project results \\
\hline
\end{tabular}

Table M2 Percentage variances explained by principal components - Khiem - APSC case

\begin{tabular}{|c|c|c|c|c|c|c|c|c|c|c|c|c|c|c|c|c|c|c|}
\hline Interviews & \multicolumn{6}{|c|}{ The first interview } & \multicolumn{6}{|c|}{ The second interview } & \multicolumn{6}{|c|}{ The third interview } \\
\hline Components & 1 & 2 & 3 & 4 & 5 & 6 & 1 & 2 & 3 & 4 & 5 & 6 & 1 & 2 & 3 & 4 & 5 & 6 \\
\hline Variance $\%$ & $\begin{array}{r}94.6 \\
7 \\
\end{array}$ & 2.68 & 1.73 & 0.58 & $\begin{array}{r}0.3 \\
3 \\
\end{array}$ & 0 & $\begin{array}{r}95.4 \\
2 \\
\end{array}$ & 2.21 & 1.51 & 0.5 & $\begin{array}{r}0.3 \\
5 \\
\end{array}$ & 0 & $\begin{array}{r}94.3 \\
9 \\
\end{array}$ & 3.02 & 1.36 & 0.88 & $\begin{array}{r}0.3 \\
4 \\
\end{array}$ & 0 \\
\hline $\begin{array}{l}\text { Frontier estimate } \\
\%\end{array}$ & $\begin{array}{r}40.8 \\
3 \\
\end{array}$ & $\begin{array}{r}24.1 \\
7 \\
\end{array}$ & $\begin{array}{r}15.8 \\
3 \\
\end{array}$ & $\begin{array}{r}10.2 \\
8 \\
\end{array}$ & $\begin{array}{r}6.1 \\
1 \\
\end{array}$ & $\begin{array}{r}2.7 \\
8 \\
\end{array}$ & $\begin{array}{r}40.8 \\
3 \\
\end{array}$ & $\begin{array}{r}24.1 \\
7 \\
\end{array}$ & $\begin{array}{r}15.8 \\
3 \\
\end{array}$ & $\begin{array}{r}10.2 \\
8 \\
\end{array}$ & $\begin{array}{r}6.1 \\
1 \\
\end{array}$ & $\begin{array}{r}2.7 \\
8 \\
\end{array}$ & $\begin{array}{r}40.8 \\
3 \\
\end{array}$ & $\begin{array}{r}24.1 \\
7 \\
\end{array}$ & $\begin{array}{r}15.8 \\
3 \\
\end{array}$ & $\begin{array}{r}10.2 \\
8 \\
\end{array}$ & $\begin{array}{r}6.1 \\
1 \\
\end{array}$ & $\begin{array}{r}2.7 \\
8 \\
\end{array}$ \\
\hline $\begin{array}{l}\text { Cumulative } \\
\text { variance }\end{array}$ & $\begin{array}{r}94.6 \\
7\end{array}$ & $\begin{array}{r}97.3 \\
5\end{array}$ & $\begin{array}{r}99.0 \\
8\end{array}$ & $\begin{array}{r}99.6 \\
7\end{array}$ & 100 & 100 & $\begin{array}{r}95.4 \\
2\end{array}$ & $\begin{array}{r}97.6 \\
4\end{array}$ & $\begin{array}{r}99.1 \\
5\end{array}$ & $\begin{array}{r}99.6 \\
5\end{array}$ & 100 & 100 & $\begin{array}{r}94.3 \\
9 \\
\end{array}$ & $\begin{array}{r}97.4 \\
2\end{array}$ & $\begin{array}{r}98.7 \\
7\end{array}$ & $\begin{array}{r}99.6 \\
6\end{array}$ & 100 & 100 \\
\hline
\end{tabular}


Table M3 Loadings of constructs on components - Khiem - APSC case

\begin{tabular}{|c|c|c|c|c|c|c|c|c|c|}
\hline \multirow[t]{2}{*}{ Constructs } & \multicolumn{3}{|c|}{ The first interview } & \multicolumn{3}{|c|}{ The second interview } & \multicolumn{3}{|c|}{ The third interview } \\
\hline & 1 & 2 & 3 & 1 & 2 & 3 & 1 & 2 & 3 \\
\hline 1 & 1.747 & -0.263 & 0.277 & 1.744 & 0.047 & 0.41 & 1.747 & -0.017 & 0.445 \\
\hline 2 & 1.547 & 0.504 & -0.043 & 1.544 & -0.501 & -0.246 & 1.514 & 0.468 & 0.109 \\
\hline 3 & 1.603 & -0.622 & 0.152 & 1.64 & 0.531 & 0.093 & 1.636 & -0.486 & 0.108 \\
\hline 4 & 1.811 & -0.044 & -0.228 & 1.814 & 0.107 & -0.158 & 1.811 & -0.17 & -0.132 \\
\hline 5 & -1.691 & -0.329 & -0.225 & -1.684 & 0.361 & -0.074 & -1.647 & -0.622 & 0.276 \\
\hline 6 & -1.811 & 0.044 & 0.228 & -1.814 & -0.107 & 0.158 & -1.811 & 0.17 & 0.132 \\
\hline 7 & 1.811 & -0.044 & -0.228 & 1.814 & 0.107 & -0.158 & 1.811 & -0.17 & -0.132 \\
\hline 8 & 1.811 & -0.044 & -0.228 & 1.814 & 0.107 & -0.158 & 1.811 & -0.17 & -0.132 \\
\hline 9 & 1.811 & -0.044 & -0.228 & 1.814 & 0.107 & -0.158 & 1.811 & -0.17 & -0.132 \\
\hline 10 & -1.703 & 0.022 & -0.377 & -1.696 & 0.172 & -0.384 & -1.703 & -0.222 & -0.3 \\
\hline 11 & 1.691 & 0.329 & 0.225 & 1.732 & -0.142 & 0.1 & 1.735 & 0.145 & 0.012 \\
\hline
\end{tabular}


Figure M1PrinGrid map - Khiem - the first interview - the APSC project

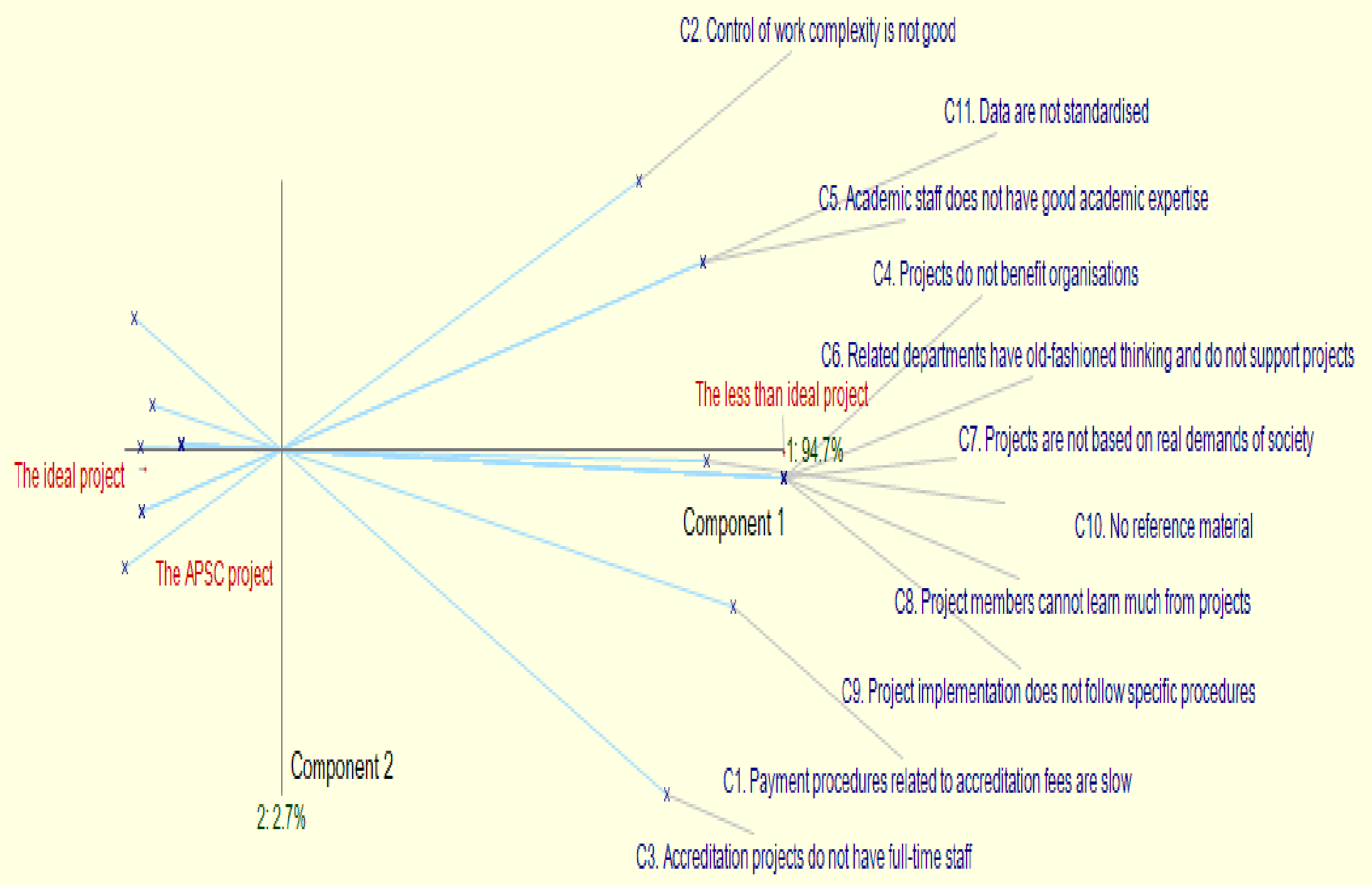


Table M4 Loadings of elements on components - Khiem - APSC case

\begin{tabular}{|c|c|c|c|c|c|c|c|c|c|}
\hline Interviews & \multicolumn{3}{|c|}{ The first interview } & \multicolumn{3}{|c|}{ The second interview } & \multicolumn{3}{|c|}{ The third interview } \\
\hline Components & 1 & 2 & 3 & 1 & 2 & 3 & 1 & 2 & 3 \\
\hline Ideal project & -1.422 & -0.093 & -0.276 & -1.391 & 0.196 & -0.121 & -1.42 & -0.291 & -0.154 \\
\hline Less than ideal project & 5.202 & -0.021 & -0.089 & 5.235 & 0.047 & -0.057 & 5.204 & -0.087 & -0.046 \\
\hline APSC project & -0.751 & 0.699 & 0.234 & -0.819 & -0.578 & -0.057 & -0.758 & 0.736 & -0.298 \\
\hline Project 1 & -0.55 & -0.318 & 0.589 & -0.612 & -0.079 & 0.619 & -0.553 & 0.236 & 0.596 \\
\hline Project 2 & -1.243 & 0.254 & -0.313 & -1.212 & -0.184 & -0.347 & -1.244 & 0.012 & -0.049 \\
\hline Project 3 & -1.236 & -0.522 & -0.145 & -1.201 & 0.598 & -0.036 & -1.23 & -0.606 & -0.05 \\
\hline
\end{tabular}




\section{Appendix N: Results of the Principal Component Analysis of Rafat's Repertory Grids -APSC Case}

Table N1 List of constructs - Rafat - APSC case

\begin{tabular}{|r|l|}
\hline No. & Constructs \\
\hline 1 & Adequate proportion of PhD faculty \\
\hline 2 & Not enough internationalisation \\
\hline 3 & Enough research outputs \\
\hline 4 & Classes are conducted regularly \\
\hline 5 & Faculty is young and enthusiastic \\
\hline 6 & Universities have learning culture \\
\hline 7 & Infrastructure is very good \\
\hline 8 & Working currently at only 25\% of their potential \\
\hline 9 & Very good management systems \\
\hline 10 & Work very fast and will complete the accreditation very fast \\
\hline 11 & Universities must work hard to fill the gap to get the accreditation \\
\hline 12 & Universities have fast decision making \\
\hline 13 & Top leaders are highly interested in accreditation \\
\hline 14 & Universities are well thought in their own location \\
\hline 15 & Faculty works very hard to finish accreditation early \\
\hline 16 & Universities provide much support for faculties' PhD study \\
\hline
\end{tabular}

Table N2 Percentage variances explained by principal components

\begin{tabular}{|c|c|c|c|c|c|c|c|c|c|c|c|c|}
\hline Interviews & \multicolumn{6}{|c|}{ The first interview } & \multicolumn{6}{|c|}{ The second interview } \\
\hline Components & 1 & 2 & 3 & 4 & 5 & 6 & 1 & 2 & 3 & 4 & 5 & 6 \\
\hline Variance $\%$ & 88.6 & 6.48 & 2.85 & 1.46 & 0.61 & 0 & 88.39 & 7.24 & 2.84 & 0.94 & 0.59 & 0 \\
\hline Frontier estimate $\%$ & 40.83 & 24.17 & 15.83 & 10.28 & 6.11 & 2.78 & 40.83 & 24.17 & 15.83 & 10.28 & 6.11 & 2.78 \\
\hline Cumulative variance $\%$ & 88.6 & 95.08 & 97.93 & 99.39 & 100 & 100 & 88.39 & 95.63 & 98.47 & 99.41 & 100 & 100 \\
\hline
\end{tabular}


Table N3 Loadings of constructs on components - Rafat-APSC case (Green: loadings on the first component, blue and yellow: loadings on the second component with opposite signs)

\begin{tabular}{|c|c|c|c|c|c|c|c|}
\hline \multirow[t]{2}{*}{ Constructs } & \multicolumn{4}{|c|}{ The first interview } & \multicolumn{3}{|c|}{ The second interview } \\
\hline & 1 & 2 & 3 & 4 & 1 & 2 & 3 \\
\hline 1 & 1.629 & 0.362 & 0.209 & -0.112 & 1.638 & 0.137 & 0.208 \\
\hline 2 & -1.414 & -0.369 & 0.153 & 0.655 & -1.423 & -0.145 & 0.192 \\
\hline 3 & 1.337 & 0.326 & 0.554 & 0.05 & 1.337 & 0.285 & 0.585 \\
\hline 4 & 1.75 & -0.51 & -0.077 & -0.066 & 1.749 & -0.504 & -0.111 \\
\hline 5 & 1.75 & -0.51 & -0.077 & -0.066 & 1.749 & -0.504 & -0.111 \\
\hline 6 & 1.75 & -0.51 & -0.077 & -0.066 & 1.749 & -0.504 & -0.111 \\
\hline 7 & 1.721 & -0.451 & 0.161 & 0.109 & 1.721 & -0.457 & 0.148 \\
\hline 8 & -1.584 & -0.679 & 0.001 & 0.151 & -1.584 & -0.685 & -0.008 \\
\hline 9 & 1.676 & -0.135 & -0.049 & 0.07 & 1.676 & -0.131 & -0.049 \\
\hline 10 & 1.594 & 0.28 & -0.479 & 0.172 & 1.585 & 0.521 & -0.443 \\
\hline 11 & -1.519 & -0.835 & -0.018 & -0.164 & -1.51 & -1.058 & -0.07 \\
\hline 12 & 1.639 & -0.037 & -0.269 & 0.211 & 1.639 & -0.027 & -0.243 \\
\hline 13 & 1.75 & -0.51 & -0.077 & -0.066 & 1.749 & -0.504 & -0.111 \\
\hline 14 & 1.584 & -0.299 & 0.454 & -0.059 & 1.584 & -0.325 & 0.433 \\
\hline 15 & 1.594 & 0.28 & -0.479 & 0.172 & 1.594 & 0.299 & -0.44 \\
\hline 16 & 1.61 & 0.202 & 0.438 & 0.242 & 1.61 & 0.184 & 0.466 \\
\hline
\end{tabular}


Figure N1 PrinGrid Map - Rafat - the first interview - the APSC project

C13. Top leaders are highly interested in accreditation

C6. Universities have learning culture

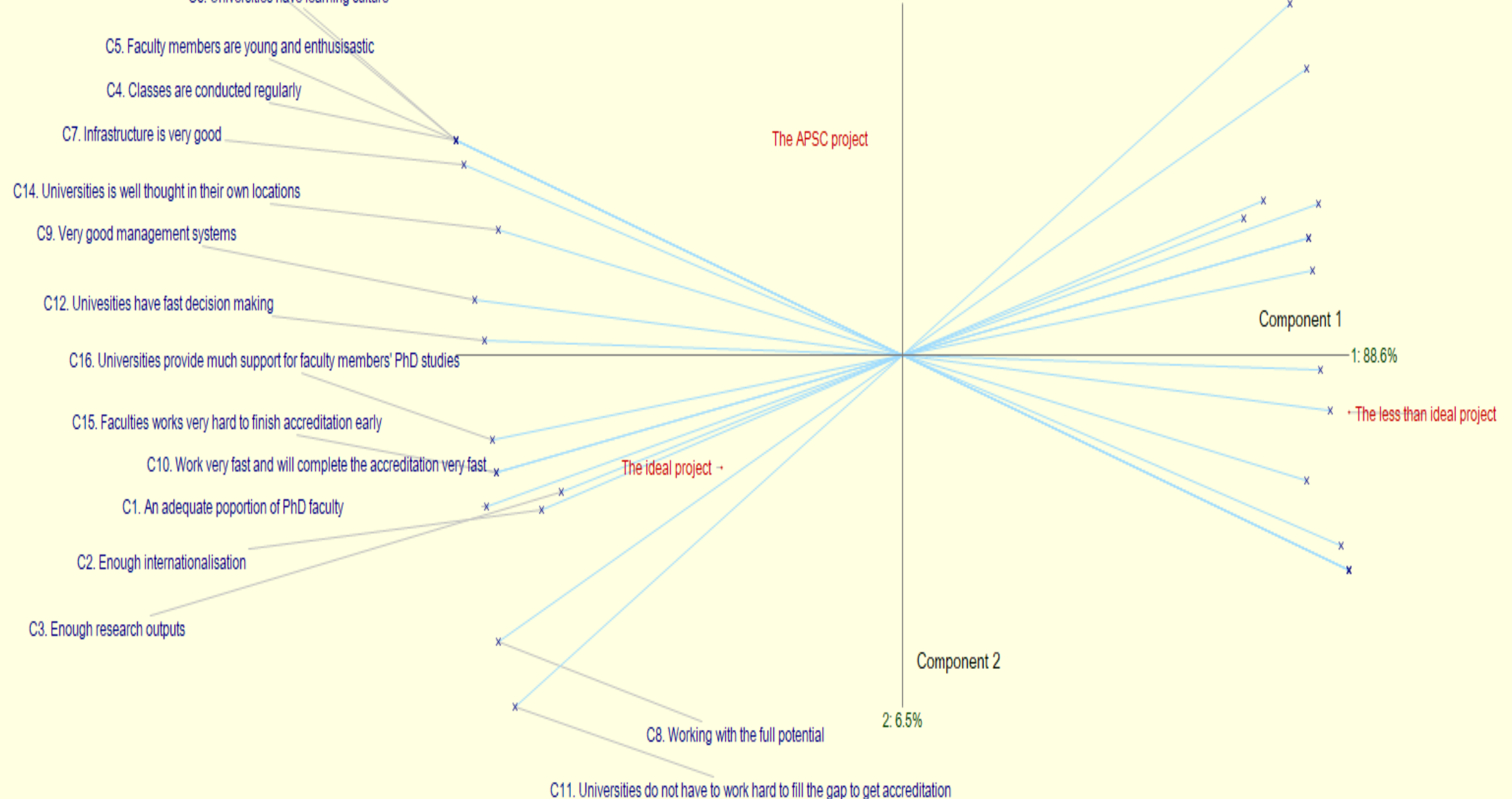

C11. Universities do not have to work hard to fill the gap to get accreditition 
Table N4 Loadings of elements on components - Rafat - APSC case

\begin{tabular}{|l|r|r|r|r|r|r|r|}
\hline Interviews & \multicolumn{4}{|c|}{ The first interview } & \multicolumn{3}{|c|}{ The second interview } \\
\hline Components & 1 & 2 & 3 & 4 & 1 & 2 \\
\hline Ideal project & -2.301 & -0.868 & -0.207 & 0.097 & -2.301 & -0.853 & -0.251 \\
\hline Less than ideal project & 5.678 & -0.448 & -0.045 & -0.028 & 5.678 & -0.468 & -0.065 \\
\hline APSC project & -0.172 & 1.153 & 0.033 & -0.447 & -0.17 & 1.235 & -0.008 \\
\hline Project 1 & -0.557 & 0.309 & 0.835 & 0.438 & -0.558 & 0.261 & 0.904 \\
\hline Project 2 & -0.71 & 0.515 & -0.768 & 0.351 & -0.712 & 0.582 & -0.679 \\
\hline Project 3 & -1.937 & -0.661 & 0.152 & -0.411 & -1.937 & -0.757 & 0.099 \\
\hline
\end{tabular}




\section{Appendix O: Results of the Principal Component Analysis of Amish's Repertory Grids - ABCD Case}

Table O1 List of constructs - Amish-ABCD case

\begin{tabular}{|r|l|}
\hline No. & Constructs \\
\hline 1 & Bad report quality \\
\hline 2 & No good understanding of the processes and systems of universities \\
\hline 3 & Bad leadership of Deans \\
\hline 4 & Weak administrative support \\
\hline 5 & Leadership is committed (e.g., permanent programme directors) \\
\hline 6 & Established experienced Deans \\
\hline 7 & Committed staff (academically and professionally working in projects) \\
\hline 8 & Projects are internally done \\
\hline 9 & Committee members have experience in doing projects \\
\hline 10 & Good accreditation networks \\
\hline 11 & Learnings from other accreditation projects \\
\hline 12 & Good accreditation committees \\
\hline 13 & Ample funding for projects \\
\hline 14 & Committees are clear about panels' expectations \\
\hline 15 & Given universities' characteristics, the chance to get accreditation is low \\
\hline 16 & Data are not available \\
\hline 17 & No direction from top leaders (e.g., Deans) \\
\hline 18 & High involvement of Deans \\
\hline 19 & Universities have less experience in managing accreditation projects \\
\hline 20 & Resources for accreditation is high, the chance for success is high \\
\hline 21 & Not expert writers \\
\hline 22 & Projects cannot use previous accreditation work \\
\hline
\end{tabular}


Table O2 Percentage variances explained by principal components - Amish - ABCD case

\begin{tabular}{|c|c|c|c|c|c|c|c|c|c|c|c|c|c|c|c|c|c|c|}
\hline Interviews & \multicolumn{6}{|c|}{ The first interview (T1) } & \multicolumn{6}{|c|}{ The second interview (T2) } & \multicolumn{6}{|c|}{ The third interview (T3) } \\
\hline Components & 1 & 2 & 3 & 4 & 5 & 6 & 1 & 2 & 3 & 4 & 5 & 6 & 1 & 2 & 3 & 4 & 5 & 6 \\
\hline & 90.0 & & & & 0.6 & & 90.9 & & & & 0.9 & & 89.8 & & & & 0.9 & \\
\hline Variance $\%$ & 4 & 5.67 & 2.3 & 1.35 & 4 & 0 & 9 & 4.36 & 2.14 & 1.56 & 4 & 0 & 7 & 3.85 & 3.42 & 1.95 & 1 & 0 \\
\hline & 40.8 & 24.1 & 15.8 & 10.2 & 6.1 & 2.7 & 40.8 & 24.1 & 15.8 & 10.2 & 6.1 & 2.7 & 40.8 & 24.1 & 15.8 & 10.2 & 6.1 & 2.7 \\
\hline Frontier estimate $\%$ & 3 & 7 & 3 & 8 & 1 & 8 & 3 & 7 & 3 & 8 & 1 & 8 & 3 & 7 & 3 & 8 & 1 & 8 \\
\hline Cumulative variance & 90.0 & 95.7 & & 99.3 & & & 90.9 & 95.3 & & 99.0 & & & 89.8 & 93.7 & 97.1 & 99.0 & & \\
\hline$\%$ & 4 & 1 & 98 & 6 & 100 & 100 & 9 & 6 & 97.5 & 6 & 100 & 100 & 7 & 2 & 4 & 9 & 100 & 100 \\
\hline Interviews & \multicolumn{6}{|c|}{ The fourth interview (T4) } & \multicolumn{6}{|c|}{ The fifth interview (T5) } & \multicolumn{6}{|c|}{ The sixth interview (T6) } \\
\hline Components & 1 & 2 & 3 & 4 & 5 & 6 & 1 & 2 & 3 & 4 & 5 & 6 & 1 & 2 & 3 & 4 & 5 & 6 \\
\hline & 90.3 & & & & 0.7 & & 89.6 & & & & 0.6 & & 88.7 & & & & 0.7 & \\
\hline Variance $\%$ & 1 & 4.76 & 2.87 & 1.35 & 1 & 0 & 8 & 5.03 & 2.98 & 1.62 & 9 & 0 & 6 & 5.24 & 3.64 & 1.59 & 6 & 0 \\
\hline & 40.8 & 24.1 & 15.8 & 10.2 & 6.1 & 2.7 & 40.8 & 24.1 & 15.8 & 10.2 & 6.1 & 2.7 & 40.8 & 24.1 & 15.8 & 10.2 & 6.1 & 2.7 \\
\hline Frontier estimate $\%$ & 3 & 7 & 3 & 8 & 1 & 8 & 3 & 7 & 3 & 8 & 1 & 8 & 3 & 7 & 3 & 8 & 1 & 8 \\
\hline Cumulative variance & 90.3 & 95.0 & 97.9 & 99.2 & & & 89.6 & 94.7 & 97.6 & 99.3 & & & 88.7 & & 97.6 & 99.2 & & \\
\hline$\%$ & 1 & 7 & 4 & 9 & 100 & 100 & 8 & 1 & 9 & 1 & 100 & 100 & 6 & 94 & 4 & 4 & 100 & 100 \\
\hline Interviews & \multicolumn{6}{|c|}{ The seventh interview (T7) } & \multicolumn{6}{|c|}{ The eighth interview (T8) } & & & & & & \\
\hline Components & 1 & 2 & 3 & 4 & 5 & 6 & 1 & 2 & 3 & 4 & 5 & 6 & & & & & & \\
\hline & 86.8 & & & & 0.7 & & & & & & 0.8 & & & & & & & \\
\hline Variance $\%$ & 4 & 7.26 & 3.55 & 1.63 & 1 & 0 & 85.4 & 8.72 & 3.51 & 1.56 & 1 & 0 & & & & & & \\
\hline & 40.8 & 24.1 & 15.8 & 10.2 & 6.1 & 2.7 & 40.8 & 24.1 & 15.8 & 10.2 & 6.1 & 2.7 & & & & & & \\
\hline Frontier estimate $\%$ & 3 & 7 & 3 & 8 & 1 & 8 & 3 & 7 & 3 & 8 & 1 & 8 & & & & & & \\
\hline Cumulative variance & 86.8 & 94.1 & 97.6 & 99.2 & & & & 94.1 & 97.6 & 99.1 & & & & & & & & \\
\hline$\%$ & 4 & 1 & 6 & 9 & 100 & 100 & 85.4 & 2 & 3 & 9 & 100 & 100 & & & & & & \\
\hline
\end{tabular}


Table O3 Loadings of constructs on components - Amish-ABCD case (Green: loadings on the first component, blue and yellow: loadings on the second component with opposite signs)

\begin{tabular}{|c|c|c|c|c|c|c|c|c|c|c|c|c|c|c|c|c|}
\hline \multirow[t]{2}{*}{ Constructs } & \multicolumn{4}{|c|}{ The first interview } & \multicolumn{4}{|c|}{ The second interview } & \multicolumn{4}{|c|}{ The third interview } & \multicolumn{4}{|c|}{ The fourth interview } \\
\hline & 1 & 2 & 3 & 4 & 1 & 2 & 3 & 4 & 1 & 2 & 3 & 4 & 1 & 2 & 3 & 4 \\
\hline 1 & -1.717 & 0.063 & 0.177 & -0.225 & -1.753 & -0.028 & 0.113 & 0.328 & -1.77 & -0.144 & 0.568 & 0.28 & -1.717 & 0.119 & 0.134 & 0.236 \\
\hline 2 & -1.968 & -0.412 & 0.265 & -0.062 & -1.934 & -0.258 & 0.41 & -0.319 & -1.917 & -0.264 & -0.634 & 0.14 & -1.97 & -0.368 & 0.309 & 0.13 \\
\hline 3 & -1.224 & 0.727 & -0.341 & -0.083 & -1.243 & 0.691 & -0.296 & 0.193 & -1.218 & 0.82 & -0.021 & -0.092 & -1.244 & 0.551 & -0.525 & 0.057 \\
\hline 4 & -1.573 & 0.502 & 0.243 & -0.168 & -1.587 & 0.363 & 0.258 & 0.206 & -1.618 & 0.219 & 0.319 & 0.35 & -1.563 & 0.551 & 0.204 & 0.161 \\
\hline 5 & 1.666 & -0.687 & -0.348 & -0.521 & 1.652 & -0.414 & -0.296 & 0.262 & 1.646 & -0.445 & 0.762 & -0.1 & 1.644 & -0.697 & -0.445 & 0.495 \\
\hline 6 & 1.474 & -0.252 & 0.253 & -0.08 & 1.5 & -0.184 & 0.292 & 0.008 & 1.47 & -0.297 & 0.089 & 0.232 & 1.497 & -0.064 & 0.35 & 0.05 \\
\hline 7 & 1.368 & -0.288 & 0.407 & 0.14 & 1.371 & -0.437 & 0.294 & -0.093 & 1.397 & -0.357 & -0.512 & 0.162 & 1.343 & -0.496 & 0.28 & 0.125 \\
\hline 8 & 1.493 & -0.05 & 0.363 & 0.058 & 1.5 & -0.184 & 0.292 & 0.008 & 1.47 & -0.297 & 0.089 & 0.232 & 1.443 & -0.44 & 0.036 & 0.306 \\
\hline 9 & 2.006 & 0.815 & -0.044 & 0.339 & 2.048 & 0.673 & 0.031 & -0.349 & 1.943 & 0.365 & 0.35 & -0.14 & 1.97 & 0.368 & -0.309 & -0.13 \\
\hline 10 & 2.117 & 0.832 & -0.038 & -0.212 & 2.151 & 0.761 & 0.141 & -0.104 & 2.077 & 0.543 & 0.297 & 0.11 & 2.078 & 0.41 & -0.393 & 0.398 \\
\hline 11 & 1.954 & 0.191 & -0.215 & -0.407 & 2.022 & 0.507 & 0.143 & -0.205 & 2.004 & 0.483 & -0.304 & 0.04 & 2.006 & 0.543 & 0.009 & 0.088 \\
\hline 12 & 1.493 & -0.05 & 0.363 & 0.058 & 1.462 & -0.322 & 0.145 & 0.23 & 1.497 & -0.196 & -0.195 & 0.232 & 1.524 & 0.124 & 0.507 & -0.079 \\
\hline 13 & 1.474 & -0.252 & 0.253 & -0.08 & 1.5 & -0.184 & 0.292 & 0.008 & 1.497 & -0.196 & -0.195 & 0.232 & 1.497 & -0.064 & 0.35 & 0.05 \\
\hline 14 & 1.744 & 0.424 & 0.275 & -0.105 & 1.718 & 0.184 & 0.142 & 0.432 & 1.749 & 0.328 & -0.127 & 0.372 & 1.777 & 0.611 & 0.332 & 0.027 \\
\hline 15 & -1.717 & 0.063 & 0.177 & -0.225 & -1.715 & 0.11 & 0.26 & 0.105 & -1.717 & 0.058 & 0.001 & 0.28 & -1.717 & 0.119 & 0.134 & 0.236 \\
\hline 16 & -1.699 & 0.265 & 0.287 & -0.087 & -1.715 & 0.11 & 0.26 & 0.105 & -1.744 & -0.043 & 0.285 & 0.28 & -1.717 & 0.119 & 0.134 & 0.236 \\
\hline 17 & -1.699 & 0.265 & 0.287 & -0.087 & -1.715 & 0.11 & 0.26 & 0.105 & -1.717 & 0.058 & 0.001 & 0.28 & -1.717 & 0.119 & 0.134 & 0.236 \\
\hline 18 & 1.349 & -0.489 & & 0.001 & & -0.437 & 0.294 & -0.093 & 1.344 & -0.559 & & 0.162 & 1.37 & & 0.437 & -0.004 \\
\hline 19 & -1.744 & -0.424 & -0.275 & 0.105 & -1.718 & -0.184 & -0.142 & -0.432 & -1.723 & -0.227 & -0.157 & -0.372 & -1.75 & -0.423 & -0.175 & -0.156 \\
\hline 20 & 1.699 & -0.265 & -0.287 & 0.087 & 1.677 & -0.249 & -0.407 & 0.118 & 1.691 & -0.159 & 0.282 & -0.28 & 1.69 & -0.307 & -0.291 & -0.108 \\
\hline 21 & -1.717 & 0.063 & 0.177 & -0.225 & -1.715 & 0.11 & 0.26 & 0.105 & -1.744 & -0.043 & 0.285 & 0.28 & -1.717 & 0.119 & 0.134 & 0.236 \\
\hline \multirow[t]{2}{*}{22} & -1.725 & -0.222 & -0.164 & 0.243 & -1.756 & -0.323 & -0.289 & -0.209 & -1.749 & -0.328 & 0.127 & -0.372 & -1.75 & -0.423 & -0.175 & -0.156 \\
\hline & \multicolumn{4}{|c|}{ The fifth interview } & \multicolumn{4}{|c|}{ The sixth interview } & \multicolumn{4}{|c|}{ The seventh interview } & \multicolumn{4}{|c|}{ The eighth interview } \\
\hline Constructs & 1 & 2 & 3 & 4 & 1 & 2 & 3 & 4 & 1 & 2 & 3 & 4 & 1 & 2 & 3 & 4 \\
\hline 1 & -1.824 & 0.203 & 0.141 & 0.394 & -1.812 & 0.318 & 0.09 & 0.394 & -1.827 & 0.303 & 0.052 & 0.348 & -1.821 & 0.333 & 0.046 & 0.356 \\
\hline
\end{tabular}




\begin{tabular}{|c|c|c|c|c|c|c|c|c|c|c|c|c|c|c|c|c|}
\hline 2 & -2.088 & 0.61 & -0.156 & 0.239 & -2.021 & 0.244 & -0.435 & 0.216 & -2.027 & 0.118 & -0.464 & 0.198 & -2.024 & 0.122 & -0.467 & 0.208 \\
\hline 3 & -1.297 & -0.328 & 0.7 & 0.072 & -1.345 & 0.376 & 0.829 & 0.055 & -1.363 & 0.46 & 0.762 & -0.026 & -1.356 & 0.515 & 0.743 & -0.028 \\
\hline 4 & -1.571 & -0.453 & -0.006 & 0.294 & -1.526 & -0.625 & 0.216 & 0.212 & -1.506 & -0.614 & 0.319 & 0.251 & -1.515 & -0.584 & 0.344 & 0.245 \\
\hline 5 & 1.541 & 0.946 & 0.484 & 0.323 & 1.519 & 1.294 & 0.022 & 0.345 & 1.408 & 1.645 & -0.181 & 0.266 & 1.432 & 1.598 & -0.236 & 0.258 \\
\hline 6 & 1.5 & 0.149 & -0.255 & 0.172 & 1.501 & -0.023 & -0.264 & 0.199 & 1.5 & 0.014 & -0.248 & 0.224 & 1.56 & -0.304 & -0.23 & 0.176 \\
\hline 7 & 1.307 & 0.579 & -0.255 & 0.183 & 1.371 & 0.081 & -0.507 & 0.149 & 1.432 & -0.223 & -0.505 & 0.125 & 1.487 & -0.556 & -0.481 & 0.076 \\
\hline 8 & 1.439 & 0.375 & -0.107 & 0.261 & 1.449 & 0.257 & -0.225 & 0.277 & 1.5 & 0.014 & -0.248 & 0.224 & 1.501 & -0.012 & -0.242 & 0.229 \\
\hline 9 & 1.966 & -0.156 & 0.452 & -0.062 & 1.917 & 0.315 & 0.513 & -0.062 & 1.964 & 0.17 & 0.461 & -0.149 & 1.907 & 0.462 & 0.443 & -0.103 \\
\hline 10 & 2.119 & -0.344 & 0.485 & 0.289 & 2.117 & -0.135 & 0.595 & 0.186 & 2.117 & 0.046 & 0.609 & 0.171 & 2.176 & -0.277 & 0.622 & 0.084 \\
\hline 11 & 2.048 & -0.368 & 0.188 & 0.123 & 2.039 & -0.311 & 0.313 & 0.059 & 2.049 & -0.191 & 0.352 & 0.072 & 2.045 & -0.236 & 0.36 & 0.037 \\
\hline 12 & 1.561 & -0.078 & -0.403 & 0.083 & 1.553 & -0.302 & -0.303 & 0.122 & 1.563 & -0.275 & -0.246 & 0.175 & 1.501 & -0.012 & -0.242 & 0.229 \\
\hline 13 & 1.5 & 0.149 & -0.255 & 0.172 & 1.501 & -0.023 & -0.264 & 0.199 & 1.5 & 0.014 & -0.248 & 0.224 & 1.501 & -0.012 & -0.242 & 0.229 \\
\hline 14 & 1.885 & -0.711 & -0.254 & 0.149 & 1.762 & -0.229 & 0.222 & 0.3 & 1.7 & 0.199 & 0.268 & 0.374 & 1.704 & 0.198 & 0.27 & 0.376 \\
\hline 15 & -1.763 & -0.023 & -0.007 & 0.305 & -1.76 & 0.038 & 0.051 & 0.317 & -1.827 & 0.303 & 0.052 & 0.348 & -1.821 & 0.333 & 0.046 & 0.356 \\
\hline 16 & -1.703 & -0.25 & -0.155 & 0.217 & -1.708 & -0.242 & 0.012 & 0.239 & -1.638 & -0.563 & 0.06 & 0.201 & -1.587 & -0.835 & 0.094 & 0.145 \\
\hline 17 & -1.763 & -0.023 & -0.007 & 0.305 & -1.76 & 0.038 & 0.051 & 0.317 & -1.827 & 0.303 & 0.052 & 0.348 & -1.821 & 0.333 & 0.046 & 0.356 \\
\hline 18 & 1.429 & 0.125 & -0.552 & 0.006 & 1.423 & -0.199 & -0.547 & 0.072 & 1.495 & -0.512 & -0.503 & 0.076 & 1.487 & -0.556 & -0.481 & 0.076 \\
\hline 19 & -1.824 & 0.484 & 0.106 & -0.238 & -1.814 & 0.509 & -0.183 & -0.223 & -1.889 & 0.667 & -0.275 & -0.227 & -1.88 & 0.678 & -0.306 & -0.218 \\
\hline 20 & 1.642 & 0.477 & 0.303 & -0.128 & 1.657 & 0.522 & 0.027 & -0.162 & 1.638 & 0.563 & -0.06 & -0.201 & 1.587 & 0.835 & -0.094 & -0.145 \\
\hline 21 & -1.703 & -0.25 & -0.155 & 0.217 & -1.708 & -0.242 & 0.012 & 0.239 & -1.701 & -0.274 & 0.057 & 0.25 & -1.704 & -0.251 & 0.07 & 0.25 \\
\hline 22 & -1.763 & 0.258 & -0.042 & -0.327 & -1.814 & 0.509 & -0.183 & -0.223 & -1.826 & 0.378 & -0.273 & -0.276 & -1.763 & 0.094 & -0.282 & -0.324 \\
\hline
\end{tabular}


Figure O1 PrinGrid map - Amish - the first interview - the ABCD project

C20. Resources for accreditition are high, the chance for success is high

\section{C7. Committed stat (academically and professionally working in projects)}

C5. Leadership is committed (e.g. permanentprogramme directors)

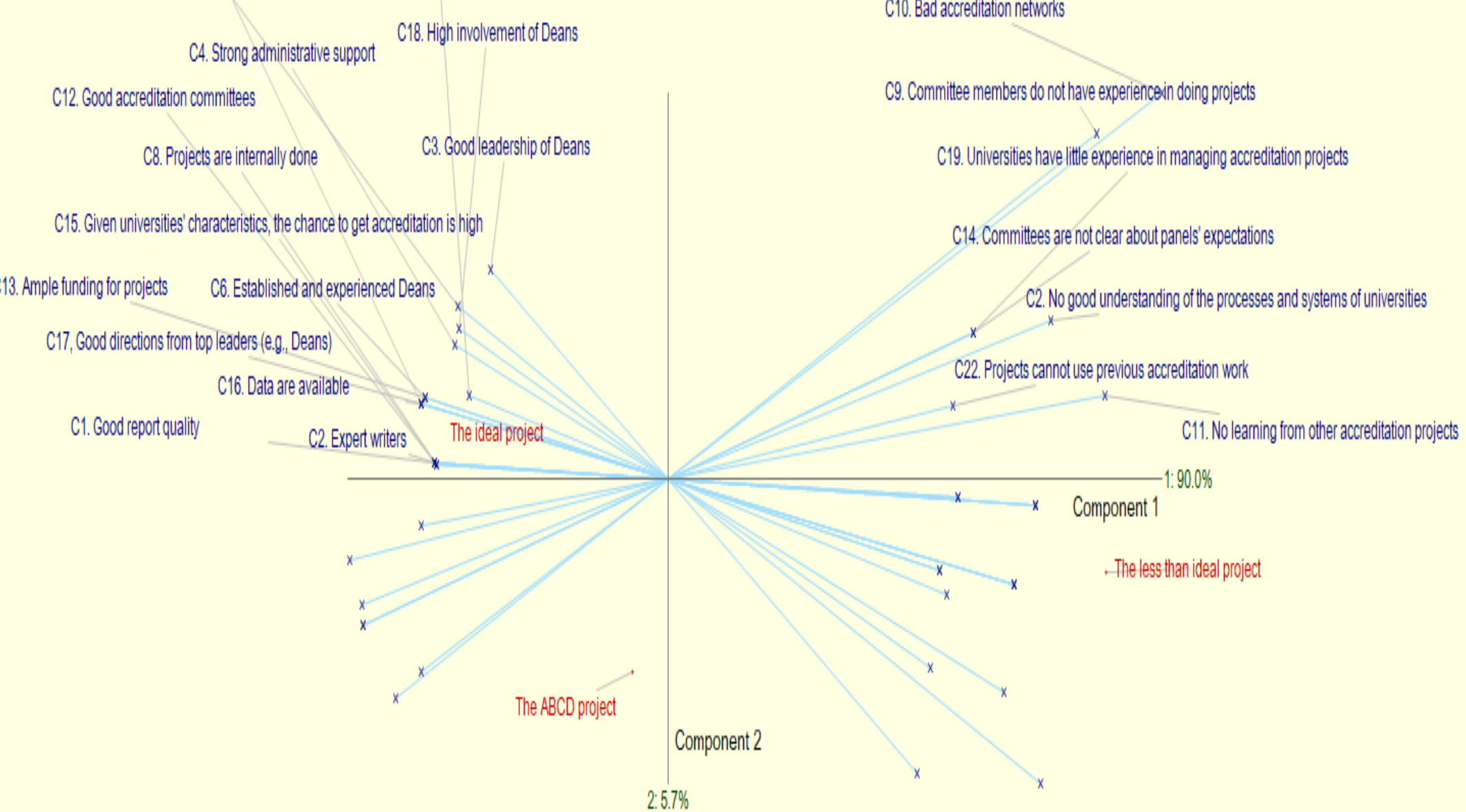


Figure O1 PrinGrid map - Amish - the sixth interview - the ABCD project

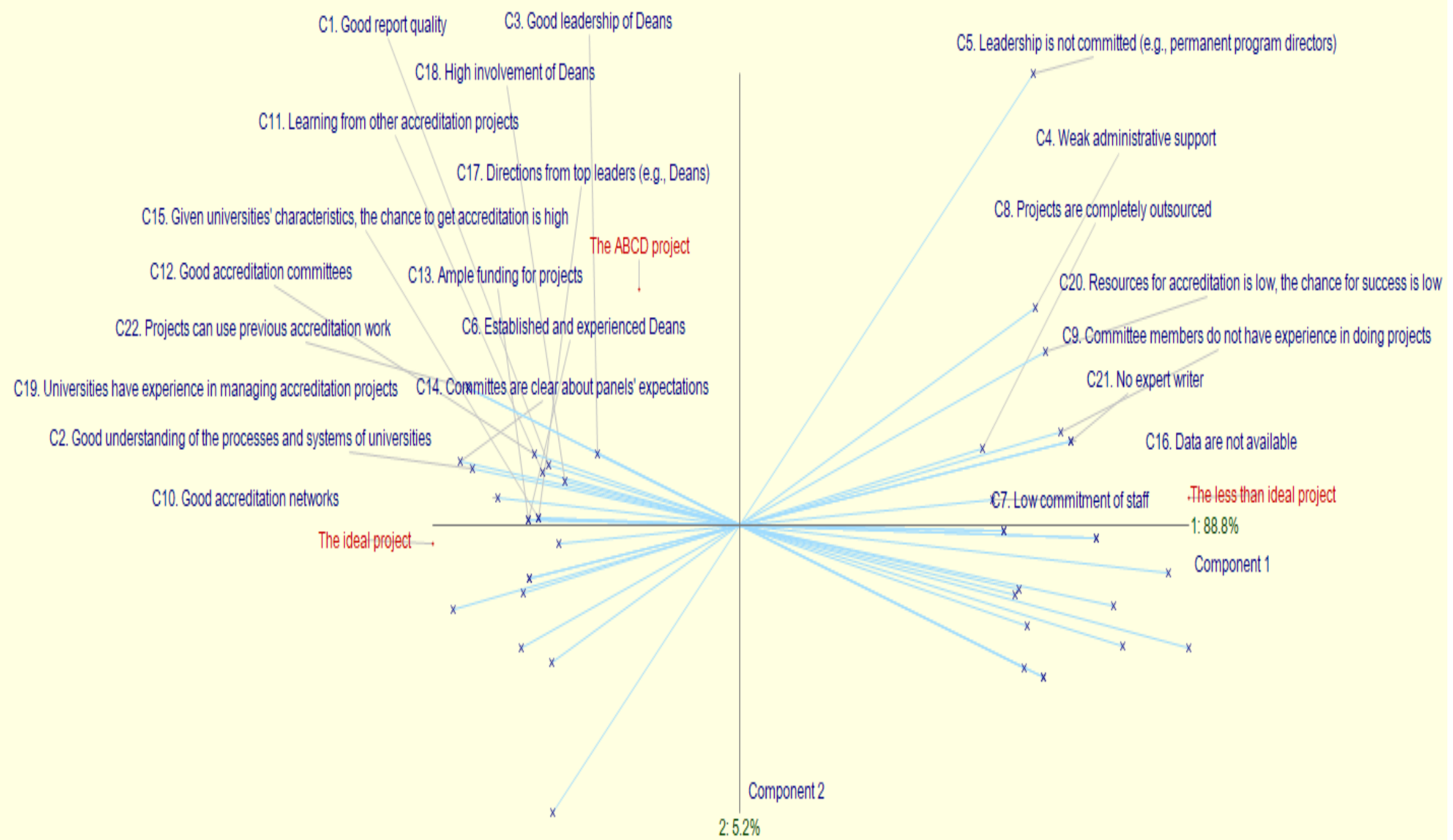


Table O4 Loadings of elements on principal components - Amish - ABCD case

\begin{tabular}{|c|c|c|c|c|c|c|c|c|c|c|c|c|}
\hline Interviews & \multicolumn{4}{|c|}{ The first interview } & \multicolumn{4}{|c|}{ The second interview } & \multicolumn{4}{|c|}{ The third interview } \\
\hline Components & 1 & 2 & 3 & 4 & 1 & 2 & 3 & 4 & 1 & 2 & 3 & 4 \\
\hline Ideal project & -3.927 & 0.387 & -0.566 & -0.053 & -3.832 & 0.48 & -0.355 & -0.433 & -3.891 & 0.412 & -0.123 & -0.521 \\
\hline Less than ideal project & 5.375 & -0.584 & 0.143 & 0.134 & 5.467 & -0.658 & 0.092 & -0.367 & 5.415 & -0.675 & -0.365 & -0.18 \\
\hline $\mathrm{ABCD}$ project & -0.445 & -1.196 & -0.417 & -0.401 & -0.902 & -0.72 & -0.536 & 0.693 & -0.622 & -0.495 & 1.309 & 0 \\
\hline Project 1 & 2.958 & 1.406 & -0.167 & -0.236 & 3.049 & 1.318 & -0.006 & 0.313 & 2.984 & 1.283 & 0.157 & 0.244 \\
\hline Project 2 & -1.312 & -0.05 & -0.012 & 0.797 & -1.223 & -0.228 & -0.2 & -0.381 & -1.273 & -0.187 & -0.53 & -0.435 \\
\hline Project 3 & -2.649 & 0.037 & 1.019 & -0.241 & -2.558 & -0.192 & 1.006 & 0.176 & -2.614 & -0.338 & -0.447 & 0.892 \\
\hline Interviews & \multicolumn{4}{|c|}{ The fourth interview } & \multicolumn{4}{|c|}{ The fifth interview } & \multicolumn{4}{|c|}{ The sixth interview } \\
\hline Components & 1 & 2 & 3 & 4 & 1 & 2 & 3 & 4 & 1 & 2 & 3 & 4 \\
\hline Ideal project & -3.89 & 0.275 & -0.48 & -0.09 & -3.728 & -0.35 & 0.399 & -0.312 & -3.776 & -0.137 & 0.36 & -0.364 \\
\hline Less than ideal project & 5.414 & -0.611 & 0.314 & -0.086 & 5.565 & 0.615 & -0.406 & -0.142 & 5.529 & 0.198 & -0.729 & -0.103 \\
\hline $\mathrm{ABCD}$ project & -0.643 & -1.023 & -0.663 & 0.372 & -1.462 & 1.292 & 0.65 & 0.287 & -1.241 & 1.624 & 0.189 & 0.247 \\
\hline Project 1 & 2.997 & 1.323 & -0.369 & 0.154 & 3.17 & -1.156 & 0.652 & 0.25 & 3.113 & -0.599 & 1.178 & 0.161 \\
\hline Project 2 & -1.272 & -0.114 & 0.177 & -0.765 & -1.106 & -0.111 & -0.397 & -0.71 & -1.149 & -0.318 & -0.387 & -0.645 \\
\hline Project 3 & -2.606 & 0.15 & 1.021 & 0.414 & -2.439 & -0.289 & -0.898 & 0.628 & -2.477 & -0.768 & -0.611 & 0.703 \\
\hline Interviews & \multicolumn{4}{|c|}{ The seventh interview } & \multicolumn{4}{|c|}{ The eighth interview } & & & & \\
\hline Components & 1 & 2 & 3 & 4 & 1 & 2 & 3 & 4 & & & & \\
\hline Ideal project & -3.725 & -0.251 & 0.334 & -0.402 & -3.745 & -0.347 & 0.323 & -0.433 & & & & \\
\hline Less than ideal project & 5.577 & 0.079 & -0.766 & -0.099 & 5.558 & -0.041 & -0.771 & -0.109 & & & & \\
\hline $\mathrm{ABCD}$ project & -1.514 & 2.008 & -0.012 & 0.162 & -1.402 & 2.238 & -0.058 & 0.171 & & & & \\
\hline Project 1 & 3.164 & -0.36 & 1.262 & 0.166 & 3.137 & -0.312 & 1.275 & 0.154 & & & & \\
\hline Project 2 & -1.088 & -0.572 & -0.353 & -0.61 & -1.116 & -0.528 & -0.349 & -0.559 & & & & \\
\hline Project 3 & -2.414 & -0.904 & -0.465 & 0.782 & -2.432 & -1.01 & -0.42 & 0.776 & & & & \\
\hline
\end{tabular}




\section{Appendix P: Results of the Principal Component Analysis of Nikki's Repertory Grids - ABCD Case}

Table P1 List of constructs - Nikki - ABCD case

\begin{tabular}{|r|l|}
\hline No. & Constructs \\
\hline 1 & No project plan \\
\hline 2 & No involvement of key people (e.g., MBA Directors) \\
\hline 3 & No involvement of top leadership (e.g., Deans) \\
\hline 4 & Bad project timeline \\
\hline 5 & Understaffed (e.g., part-time people for data analysis) \\
\hline 6 & Report writers with less understanding of universities \\
\hline 7 & Little possibility of getting the best results for accreditation (e.g., recredited for five years) \\
\hline 8 & Programme quality is not good \\
\hline 9 & Committee Chairs have much knowledge of programmes \\
\hline 10 & Great accreditation committee members \\
\hline 11 & Chairs' communication is ineffective \\
\hline 12 & Universities do not have resources at high levels \\
\hline 13 & Having people with experience and knowledge of accreditation \\
\hline 14 & Dedicated staff \\
\hline 15 & Universities with high quality of staff, enough staff \\
\hline 16 & Universities have experts in project management \\
\hline 17 & Universities do not really want accreditation \\
\hline 18 & Less investment in accreditation \\
\hline
\end{tabular}


Table P2 Percentage variances explained by principal components - Nikki-ABCD case

\begin{tabular}{|c|c|c|c|c|c|c|c|c|c|c|c|c|c|c|c|c|c|c|}
\hline Interviews & \multicolumn{6}{|c|}{ The first interview (T1) } & \multicolumn{6}{|c|}{ The second interview (T2) } & \multicolumn{6}{|c|}{ The third interview (T3) } \\
\hline Components & 1 & 2 & 3 & 4 & 5 & 6 & 1 & 2 & 3 & 4 & 5 & 6 & 1 & 2 & 3 & 4 & 5 & 6 \\
\hline Variance $\%$ & $\begin{array}{r}79.3 \\
4 \\
\end{array}$ & 9.69 & 8.95 & 1.45 & $\begin{array}{r}0.5 \\
7 \\
\end{array}$ & 0 & $\begin{array}{r}83.2 \\
2 \\
\end{array}$ & 7.8 & 6.75 & 1.67 & $\begin{array}{r}0.5 \\
6 \\
\end{array}$ & 0 & $\begin{array}{r}80.0 \\
4 \\
\end{array}$ & 9.73 & 7.41 & 2.39 & $\begin{array}{r}0.4 \\
3 \\
\end{array}$ & 0 \\
\hline Frontier estimate $\%$ & $\begin{array}{r}40.8 \\
3\end{array}$ & $\begin{array}{r}24.1 \\
7\end{array}$ & $\begin{array}{r}15.8 \\
3\end{array}$ & $\begin{array}{r}10.2 \\
8\end{array}$ & $\begin{array}{r}6.1 \\
1\end{array}$ & $\begin{array}{r}2.7 \\
8\end{array}$ & $\begin{array}{r}40.8 \\
3\end{array}$ & $\begin{array}{r}24.1 \\
7\end{array}$ & $\begin{array}{r}15.8 \\
3\end{array}$ & $\begin{array}{r}10.2 \\
8\end{array}$ & $\begin{array}{r}6.1 \\
1\end{array}$ & $\begin{array}{r}2.7 \\
8\end{array}$ & $\begin{array}{r}40.8 \\
3\end{array}$ & $\begin{array}{r}24.1 \\
7\end{array}$ & $\begin{array}{r}15.8 \\
3\end{array}$ & $\begin{array}{r}10.2 \\
8\end{array}$ & $\begin{array}{r}6.1 \\
1\end{array}$ & $\begin{array}{r}2.7 \\
8\end{array}$ \\
\hline $\begin{array}{l}\text { Cumulative } \\
\text { variance }\end{array}$ & $\begin{array}{r}79.3 \\
4 \\
\end{array}$ & $\begin{array}{r}89.0 \\
3 \\
\end{array}$ & $\begin{array}{r}97.9 \\
8 \\
\end{array}$ & $\begin{array}{r}99.4 \\
3 \\
\end{array}$ & 100 & 100 & $\begin{array}{r}83.2 \\
2 \\
\end{array}$ & $\begin{array}{r}91.0 \\
2 \\
\end{array}$ & $\begin{array}{r}97.7 \\
7 \\
\end{array}$ & $\begin{array}{r}99.4 \\
4 \\
\end{array}$ & 100 & 100 & $\begin{array}{r}80.0 \\
4 \\
\end{array}$ & $\begin{array}{r}89.7 \\
7 \\
\end{array}$ & $\begin{array}{r}97.1 \\
9 \\
\end{array}$ & $\begin{array}{r}99.5 \\
7 \\
\end{array}$ & 100 & 100 \\
\hline Interviews & \multicolumn{6}{|c|}{ The fourth interview (T4) } & \multicolumn{6}{|c|}{ The fifth interview (T7) } & \multicolumn{6}{|c|}{ The sixth interview (T8) } \\
\hline Components & 1 & 2 & 3 & 4 & 5 & 6 & 1 & 2 & 3 & 4 & 5 & 6 & 1 & 2 & 3 & 4 & 5 & 6 \\
\hline Variance $\%$ & $\begin{array}{r}82.8 \\
4\end{array}$ & 9.02 & 5 & 2.7 & $\begin{array}{r}0.4 \\
4\end{array}$ & 0 & $\begin{array}{r}84.7 \\
7\end{array}$ & 8.9 & 4.5 & 1.15 & $\begin{array}{r}0.6 \\
7\end{array}$ & 0 & 83.9 & 9.14 & 4.86 & 1.47 & $\begin{array}{r}0.6 \\
4\end{array}$ & 0 \\
\hline Frontier estimate $\%$ & $\begin{array}{r}40.8 \\
3 \\
\end{array}$ & $\begin{array}{r}24.1 \\
7 \\
\end{array}$ & $\begin{array}{r}15.8 \\
3 \\
\end{array}$ & $\begin{array}{r}10.2 \\
8 \\
\end{array}$ & $\begin{array}{r}6.1 \\
1 \\
\end{array}$ & $\begin{array}{r}2.7 \\
8 \\
\end{array}$ & $\begin{array}{r}40.8 \\
3 \\
\end{array}$ & $\begin{array}{r}24.1 \\
7 \\
\end{array}$ & $\begin{array}{r}15.8 \\
3 \\
\end{array}$ & $\begin{array}{r}10.2 \\
8 \\
\end{array}$ & $\begin{array}{r}6.1 \\
1 \\
\end{array}$ & $\begin{array}{r}2.7 \\
8 \\
\end{array}$ & $\begin{array}{r}40.8 \\
3 \\
\end{array}$ & $\begin{array}{r}24.1 \\
7 \\
\end{array}$ & $\begin{array}{r}15.8 \\
3 \\
\end{array}$ & $\begin{array}{r}10.2 \\
8 \\
\end{array}$ & $\begin{array}{r}6.1 \\
1 \\
\end{array}$ & $\begin{array}{r}2.7 \\
8 \\
\end{array}$ \\
\hline $\begin{array}{l}\text { Cumulative } \\
\text { variance }\end{array}$ & $\begin{array}{r}82.8 \\
4 \\
\end{array}$ & $\begin{array}{r}91.8 \\
6 \\
\end{array}$ & $\begin{array}{r}96.8 \\
6 \\
\end{array}$ & $\begin{array}{r}99.5 \\
6 \\
\end{array}$ & 100 & 100 & $\begin{array}{r}84.7 \\
7 \\
\end{array}$ & $\begin{array}{r}93.6 \\
8 \\
\end{array}$ & $\begin{array}{r}98.1 \\
8\end{array}$ & $\begin{array}{r}99.3 \\
3 \\
\end{array}$ & 100 & 100 & 83.9 & $\begin{array}{r}93.0 \\
4 \\
\end{array}$ & $\begin{array}{r}97.8 \\
9 \\
\end{array}$ & $\begin{array}{r}99.3 \\
6 \\
\end{array}$ & 100 & 100 \\
\hline
\end{tabular}

Table P3 Loadings of constructs on components - Nikki - ABCD case (Green: loadings on the first component, blue and yellow: loadings on the second component with opposite signs)

\begin{tabular}{|c|c|c|c|c|c|c|c|c|c|c|c|c|}
\hline \multirow{3}{*}{ Construct } & \multicolumn{12}{|c|}{ Components } \\
\hline & \multicolumn{4}{|c|}{ The first interview (T1) } & \multicolumn{4}{|c|}{ The second interview (T2) } & \multicolumn{4}{|c|}{ The third interview (T3) } \\
\hline & 1 & 2 & 3 & 4 & 1 & 2 & 3 & 4 & 1 & 2 & 3 & 4 \\
\hline 1 & -1.816 & -0.43 & 0.375 & 0.469 & -1.615 & -0.284 & -0.306 & 0.223 & -1.612 & 0.334 & -0.188 & 0.296 \\
\hline 2 & -1.599 & -0.423 & 0.14 & -0.265 & -1.595 & 0.079 & 0.21 & -0.332 & -1.594 & -0.099 & 0.027 & -0.391 \\
\hline 3 & -1.602 & -1.096 & -0.038 & -0.184 & -1.526 & 0.102 & 1.218 & 0.09 & -1.542 & -1.55 & -0.28 & -0.064 \\
\hline 4 & -1.358 & -0.795 & -0.089 & -0.409 & -1.585 & 0.186 & 0.531 & -0.045 & -1.594 & -0.099 & 0.027 & -0.391 \\
\hline 5 & -1.743 & 0.334 & 0.223 & 0.063 & -1.643 & 0.269 & -0.155 & -0.181 & -1.64 & 0.136 & 0.31 & -0.156 \\
\hline 6 & -1.466 & 0.344 & -0.081 & -0.285 & -1.501 & -0.143 & -0.224 & -0.143 & -1.496 & 0.307 & -0.065 & -0.079 \\
\hline
\end{tabular}




\begin{tabular}{|c|c|c|c|c|c|c|c|c|c|c|c|c|}
\hline 7 & -1.78 & -0.048 & 0.299 & 0.266 & -1.746 & 0.235 & 0.084 & 0.471 & -1.752 & -0.647 & 0.172 & 0.595 \\
\hline 8 & -1.746 & -0.34 & 0.046 & 0.144 & -1.746 & 0.235 & 0.084 & 0.471 & -1.754 & -0.242 & 0.18 & 0.408 \\
\hline 9 & 1.707 & -0.716 & -0.148 & 0.14 & 1.768 & -0.022 & 0.558 & 0.102 & 1.758 & -0.569 & -0.195 & -0.032 \\
\hline 10 & 1.575 & -0.81 & 0.251 & 0.079 & 1.501 & 0.143 & 0.224 & 0.143 & 1.498 & -0.712 & 0.057 & 0.267 \\
\hline 11 & -1.743 & 0.334 & 0.223 & 0.063 & -1.757 & 0.128 & -0.237 & 0.184 & -1.756 & 0.163 & 0.188 & 0.22 \\
\hline 12 & -1.743 & 0.334 & 0.223 & 0.063 & -1.643 & 0.269 & -0.155 & -0.181 & -1.64 & 0.136 & 0.31 & -0.156 \\
\hline 13 & 1.61 & -1.101 & -0.003 & -0.043 & 1.674 & 0.201 & 0.992 & -0.087 & 1.66 & -0.974 & -0.104 & -0.344 \\
\hline 14 & 1.746 & 0.34 & -0.046 & -0.144 & 1.595 & -0.079 & -0.21 & 0.332 & 1.594 & 0.099 & -0.027 & 0.391 \\
\hline 15 & 1.597 & -0.251 & -0.318 & 0.345 & 1.643 & -0.269 & 0.155 & 0.181 & 1.64 & -0.136 & -0.31 & 0.156 \\
\hline 16 & 1.67 & 0.514 & -0.469 & -0.061 & 1.632 & -0.376 & -0.166 & -0.105 & 1.638 & 0.27 & -0.302 & -0.032 \\
\hline 17 & -1.15 & 0.124 & -1.878 & 0.061 & -1.094 & -1.903 & 0.218 & 0.038 & -1.089 & 0.378 & -1.881 & 0.054 \\
\hline 18 & -1.449 & -0.444 & -1.005 & 0.143 & -1.662 & -0.252 & 0.494 & -0.116 & -1.662 & -0.711 & -0.387 & -0.064 \\
\hline Constructs & \multicolumn{4}{|c|}{ The fourth interview (T4) } & \multicolumn{4}{|c|}{ The fifth interview (T7) } & \multicolumn{4}{|c|}{ The sixth interview (T8) } \\
\hline & 1 & 2 & 3 & 4 & 1 & 2 & 3 & 4 & 1 & 2 & 3 & 4 \\
\hline 1 & -1.622 & -0.242 & -0.21 & 0.318 & -1.683 & -0.393 & -0.452 & -0.114 & -1.683 & -0.433 & -0.425 & -0.022 \\
\hline 2 & -1.633 & -0.134 & 0.028 & -0.55 & -1.591 & 0.131 & 0.388 & -0.087 & -1.53 & 0.378 & 0.552 & 0.186 \\
\hline 3 & -1.506 & 0.207 & 0.942 & -0.086 & -1.595 & -0.231 & 0.641 & -0.439 & -1.595 & -0.201 & 0.591 & -0.508 \\
\hline 4 & -1.596 & 0.104 & 0.276 & -0.275 & -1.591 & 0.131 & 0.388 & -0.087 & -1.591 & 0.158 & 0.36 & -0.151 \\
\hline 5 & -1.648 & 0.238 & -0.143 & -0.189 & -1.587 & 0.493 & 0.136 & 0.264 & -1.587 & 0.517 & 0.129 & 0.205 \\
\hline 6 & -1.513 & -0.162 & -0.142 & -0.062 & -1.518 & -0.102 & -0.095 & -0.025 & -1.519 & -0.11 & -0.087 & -0.026 \\
\hline 7 & -1.72 & 0.396 & 0.038 & 0.467 & -1.752 & 0.202 & -0.22 & 0.175 & -1.752 & 0.194 & -0.209 & 0.208 \\
\hline 8 & -1.72 & 0.396 & 0.038 & 0.467 & -1.752 & 0.202 & -0.22 & 0.175 & -1.752 & 0.194 & -0.209 & 0.208 \\
\hline 9 & 1.794 & 0.079 & 0.457 & 0.084 & 1.812 & -0.015 & 0.377 & 0.204 & 1.812 & 0.026 & 0.401 & 0.129 \\
\hline 10 & 1.55 & 0.4 & 0.39 & 0.337 & 1.518 & 0.102 & 0.095 & 0.025 & 1.579 & 0.33 & 0.279 & 0.363 \\
\hline 11 & -1.72 & 0.396 & 0.038 & 0.467 & -1.752 & 0.202 & -0.22 & 0.175 & -1.752 & 0.194 & -0.209 & 0.208 \\
\hline 12 & -1.648 & 0.238 & -0.143 & -0.189 & -1.587 & 0.493 & 0.136 & 0.264 & -1.587 & 0.517 & 0.129 & 0.205 \\
\hline 13 & 1.711 & 0.345 & 0.876 & -0.129 & 1.739 & 0.218 & 0.861 & 0.142 & 1.74 & 0.294 & 0.848 & 0.004 \\
\hline 14 & 1.596 & -0.104 & -0.276 & 0.275 & 1.591 & -0.131 & -0.388 & 0.087 & 1.591 & -0.158 & -0.36 & 0.151 \\
\hline 15 & 1.648 & -0.238 & 0.143 & 0.189 & 1.647 & -0.306 & 0.021 & 0.114 & 1.647 & -0.297 & 0.063 & 0.132 \\
\hline 16 & 1.611 & -0.476 & -0.105 & -0.087 & 1.647 & -0.306 & 0.021 & 0.114 & 1.647 & -0.297 & 0.063 & 0.132 \\
\hline
\end{tabular}




\begin{tabular}{|c|c|c|c|c|c|c|c|c|c|c|c|c|}
\hline 17 & -1.149 & -1.949 & 0.38 & 0.25 & -1.183 & -1.965 & 0.144 & 0.207 & -1.184 & -1.931 & 0.347 & 0.26 \\
\hline 18 & -1.652 & -0.11 & 0.627 & 0.019 & -1.639 & -0.147 & 0.598 & 0.229 & -1.639 & -0.083 & 0.637 & 0.171 \\
\hline
\end{tabular}


Figure P1 PrinGrid map - Nikki - the first interview - the ABCD project

C9. Committee Chairs have much knowledge of programmes

C13. There are people with experience and knowledge of accreditation

C10. Great accreditation committee members C11. Chairs' communication is effective

C12. Universities have resources at high levels

C6. Report witers with much understanding of universities

C15. Universities with high quality of staff, enough staff

C5. Adequate staff

C17. Universities really want accreditation

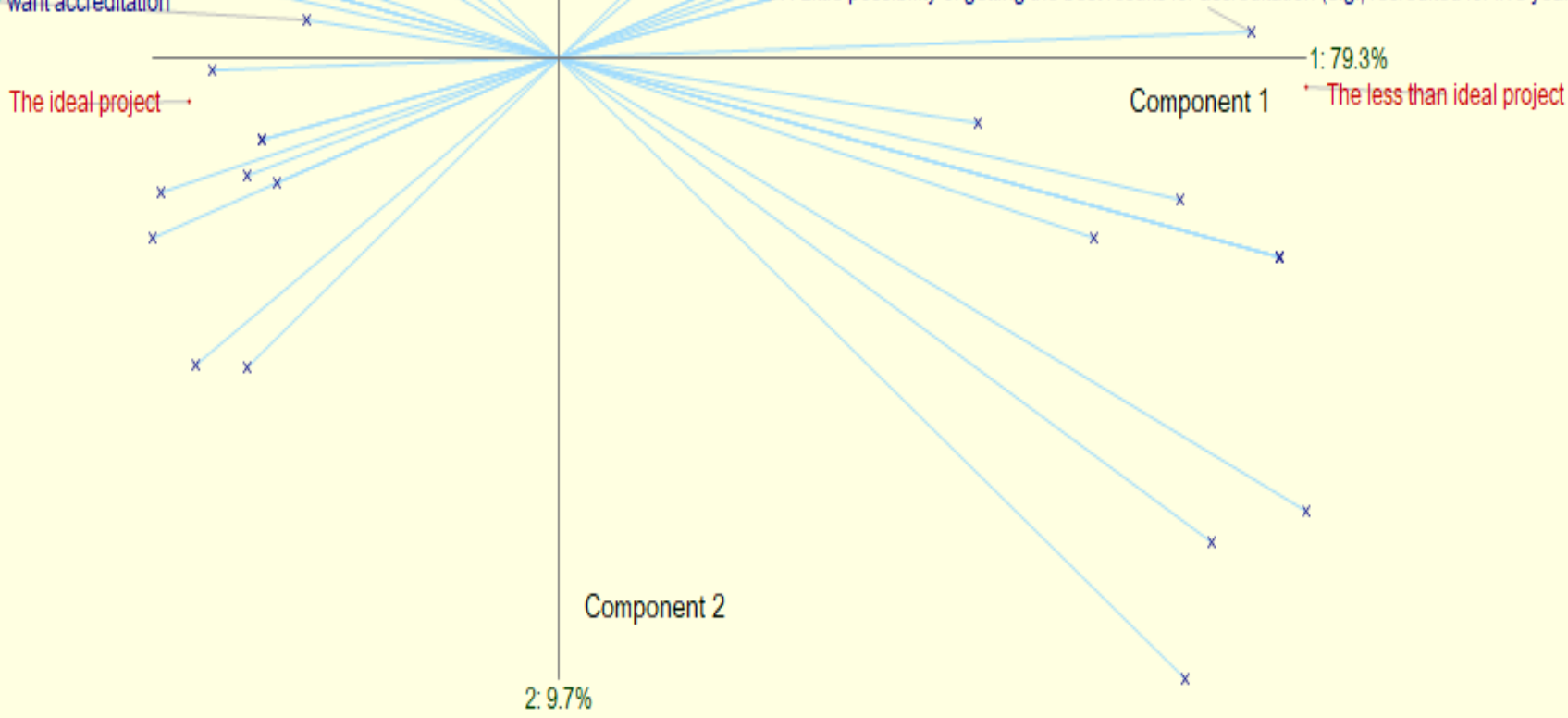

C3. No involvement of top leadership (eg. Deans)

C2. No involvement of key people (e.g., MBA Directors)

$$
\text { C4. Bad project timeline C18. Less investment in accreditation }
$$

The ABCD project $\quad C 16$. Universities do not have experts in project management

C1. No project plan C14. Staff is not dedicated

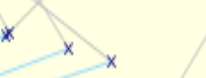

\section{C8. Programme quality is not good}

7. Little possibility of getting the best results for accreditation (e.g., recredited for five years) 
Figure P2 PrinGrid map - Nikki - the second interview - the ABCD project

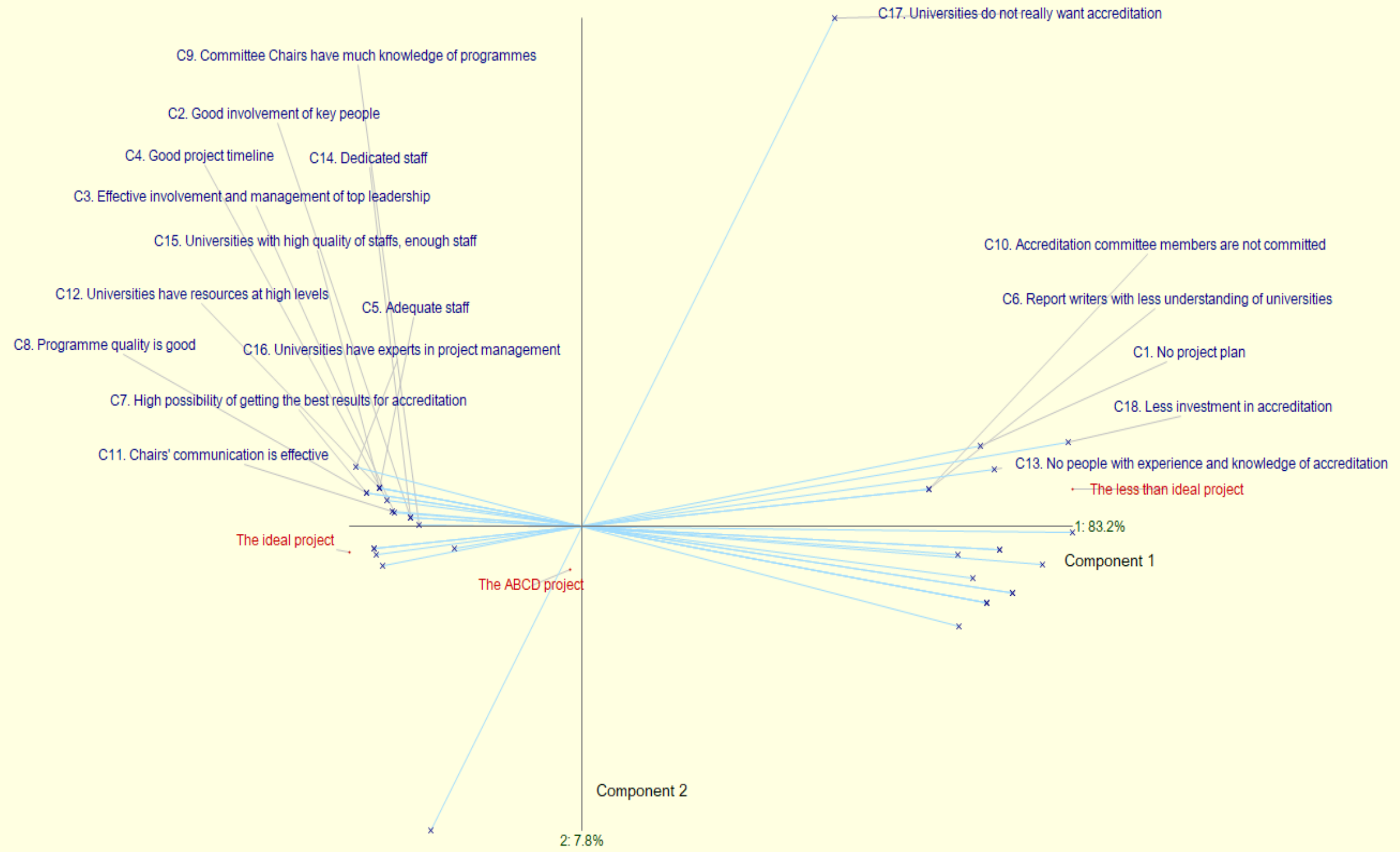


Table P4 Loadings of elements on components - Nikki - ABCD case

\begin{tabular}{|c|c|c|c|c|c|c|c|c|c|c|c|c|}
\hline Interviews & \multicolumn{4}{|c|}{ The first interview } & \multicolumn{4}{|c|}{ The second interview } & \multicolumn{4}{|c|}{ The third interview } \\
\hline Components & 1 & 2 & 3 & 4 & 1 & 2 & 3 & 4 & 1 & 2 & 3 & 4 \\
\hline Ideal project & -2.791 & -0.228 & -0.301 & -0.22 & -2.722 & -0.271 & 0.045 & 0.017 & -2.745 & -0.012 & -0.292 & 0.035 \\
\hline Less than ideal project & 5.65 & -0.161 & 0.416 & 0.18 & 5.727 & 0.376 & -0.169 & 0.195 & 5.704 & -0.118 & 0.387 & 0.26 \\
\hline ABCD project & 0.503 & 1.842 & -0.35 & -0.379 & -0.148 & -0.446 & -1.252 & -0.555 & -0.029 & 1.935 & 0.032 & -0.444 \\
\hline Project 1 & 0.469 & -1.404 & -1.174 & -0.229 & 0.65 & -0.797 & 1.426 & -0.292 & 0.624 & -1.121 & -1.182 & -0.558 \\
\hline Project 2 & -2.019 & 0.4 & -0.437 & 0.762 & -1.564 & -0.591 & -0.319 & 0.709 & -1.585 & 0.133 & -0.51 & 0.888 \\
\hline Project 3 & -1.812 & -0.45 & 1.846 & -0.114 & -1.943 & 1.73 & 0.269 & -0.075 & -1.969 & -0.816 & 1.564 & -0.181 \\
\hline Interviews & \multicolumn{4}{|c|}{ The fourth interview (T4) } & \multicolumn{4}{|c|}{ The fifth interview (T7) } & \multicolumn{4}{|c|}{ The sixth interview (T8) } \\
\hline Components & 1 & 2 & 3 & 4 & 1 & 2 & 3 & 4 & 1 & 2 & 3 & 4 \\
\hline Ideal project & -2.656 & -0.161 & -0.029 & 0.109 & -2.591 & -0.116 & -0.055 & 0.183 & -2.61 & -0.105 & -0.004 & 0.233 \\
\hline Less than ideal project & 5.8 & 0.394 & -0.32 & 0.159 & 5.865 & 0.374 & -0.351 & 0.06 & 5.845 & 0.371 & -0.352 & 0.094 \\
\hline $\mathrm{ABCD}$ project & -0.51 & -1.077 & -0.835 & -0.683 & -0.828 & -0.837 & -0.499 & -0.607 & -0.732 & -0.926 & -0.596 & -0.602 \\
\hline Project 1 & 0.718 & -0.609 & 1.412 & -0.214 & 0.777 & -0.782 & 1.303 & 0.044 & 0.76 & -0.688 & 1.372 & -0.022 \\
\hline Project 2 & -1.495 & -0.362 & -0.227 & 0.942 & -1.442 & -0.463 & -0.635 & 0.464 & -1.461 & -0.489 & -0.556 & 0.484 \\
\hline Project 3 & -1.856 & 1.814 & 0 & -0.314 & -1.781 & 1.823 & 0.237 & -0.143 & -1.802 & 1.837 & 0.136 & -0.188 \\
\hline
\end{tabular}




\section{Appendix Q: Results of the Principal Component Analysis of Sumy's Repertory Grids - ABCD Case}

Table Q1 List of constructs - Sumy - ABCD case

\begin{tabular}{|r|l|}
\hline No. & Constructs \\
\hline 1 & Committee members are experienced in accreditations \\
\hline 2 & Committee members know what panels look for \\
\hline 3 & Project managers are mindful of time, agenda and manage things well \\
\hline 4 & No streamlined process to store data and retrieve data \\
\hline 5 & Universities have necessary people to do accreditation projects \\
\hline 6 & Committees have experience from other accreditation projects \\
\hline 7 & University characteristics are not good enough for accreditation \\
\hline 8 & Good professors and teaching staff \\
\hline 9 & Project teams can consolidate different accreditations \\
\hline 10 & As universities are very good, accreditation will be renewed easily \\
\hline 11 & Bad plans \\
\hline 12 & Clear timelines \\
\hline
\end{tabular}

Table Q2 Percentage variances explained by principal components - Sumy - ABCD case

\begin{tabular}{|c|c|c|c|c|c|c|c|c|c|c|c|c|c|c|c|c|c|c|}
\hline Interviews & \multicolumn{6}{|c|}{ The first interview (T1) } & \multicolumn{6}{|c|}{ The second and third interview (T2 \& T3) } & \multicolumn{6}{|c|}{ The fourth interview (T4) } \\
\hline Components & 1 & 2 & 3 & 4 & 5 & 6 & 1 & 2 & 3 & 4 & 5 & 6 & 1 & 2 & 3 & 4 & 5 & 6 \\
\hline & 91.7 & & & & 0.1 & & 88.0 & & & & 0.2 & & 91.0 & & & & 0.1 & \\
\hline Variance $\%$ & 9 & 6.27 & 1.07 & 0.75 & 3 & 0 & 2 & 6.51 & 3.53 & 1.67 & 7 & 0 & 9 & 5.05 & 2.99 & 0.7 & 7 & 0 \\
\hline & 40.8 & 24.1 & 15.8 & 10.2 & 6.1 & 2.7 & 40.8 & 24.1 & 15.8 & 10.2 & 6.1 & 2.7 & 40.8 & 24.1 & 15.8 & 10.2 & 6.1 & 2.7 \\
\hline Frontier estimate $\%$ & 3 & 7 & 3 & 8 & 1 & 8 & 3 & 7 & 3 & 8 & 1 & 8 & 3 & 7 & 3 & 8 & 1 & 8 \\
\hline Cumulative & 91.7 & 98.0 & 99.1 & 99.8 & & & 88.0 & 94.5 & 98.0 & 99.7 & & & 91.0 & 96.1 & 99.1 & 99.8 & & \\
\hline variance & 9 & 6 & 2 & 7 & 100 & 100 & 2 & 3 & 6 & 3 & 100 & 100 & 9 & 4 & 3 & 3 & 100 & 100 \\
\hline Interviews & \multicolumn{6}{|c|}{ The fifth interview (T5) } & \multicolumn{6}{|c|}{ The sixth interview (T6) } & \multicolumn{6}{|c|}{$\begin{array}{l}\text { The seventh and eighth interview (T7 \& } \\
\text { T8) }\end{array}$} \\
\hline
\end{tabular}




\begin{tabular}{|c|c|c|c|c|c|c|c|c|c|c|c|c|c|c|c|c|c|c|}
\hline Components & 1 & 2 & 3 & 4 & 5 & 6 & 1 & 2 & 3 & 4 & 5 & 6 & 1 & 2 & 3 & 4 & 5 & 6 \\
\hline & 90.0 & & & & & & 90.2 & & & & 0.3 & & 90.6 & & & & 0.3 & \\
\hline Variance $\%$ & 8 & 5.92 & 2.77 & 0.92 & 0.3 & 0 & 1 & 5.64 & 2.93 & 0.88 & 4 & 0 & 2 & 5.04 & 2.92 & 1.09 & 3 & 0 \\
\hline & 40.8 & 24.1 & 15.8 & 10.2 & 6.1 & 2.7 & 40.8 & 24.1 & 15.8 & 10.2 & 6.1 & 2.7 & 40.8 & 24.1 & 15.8 & 10.2 & 6.1 & 2.7 \\
\hline Frontier estimate $\%$ & 3 & 7 & 3 & 8 & 1 & 8 & 3 & 7 & 3 & 8 & 1 & 8 & 3 & 7 & 3 & 8 & 1 & 8 \\
\hline Cumulative & 90.0 & 96.0 & 98.7 & & & & 90.2 & 95.8 & 98.7 & 99.6 & & & 90.6 & 95.6 & 98.5 & 99.6 & & \\
\hline variance & 8 & 1 & 8 & 99.7 & 100 & 100 & 1 & 5 & 8 & 6 & 100 & 100 & 2 & 6 & 8 & 7 & 100 & 100 \\
\hline
\end{tabular}

Table Q3 Loadings of constructs on components - Sumy - ABCD case (Green: loadings on the first component, blue and yellow: loadings on the second component with opposite signs)

\begin{tabular}{|c|c|c|c|c|c|c|c|c|c|c|}
\hline \multirow[t]{2}{*}{ Constructs } & \multicolumn{3}{|c|}{ The first interview (T1) } & \multicolumn{4}{|c|}{ The second and the third interview (T2 \& $\mathrm{T} 3$ ) } & \multicolumn{3}{|c|}{ The fourth interview (T4) } \\
\hline & 1 & 2 & 3 & 1 & 2 & 3 & 4 & 1 & 2 & 3 \\
\hline 1 & 1.811 & 0.08 & 0.192 & 1.797 & 0.176 & -0.227 & 0.077 & 1.779 & -0.217 & 0.296 \\
\hline 2 & 1.795 & -0.338 & 0.098 & 1.789 & -0.207 & -0.349 & -0.095 & 1.789 & -0.413 & -0.037 \\
\hline 3 & 1.795 & -0.338 & 0.098 & 1.71 & -0.45 & -0.353 & 0.274 & 1.789 & -0.413 & -0.037 \\
\hline 4 & -1.51 & 0.195 & -0.273 & -1.541 & 0.211 & -0.082 & -0.228 & -1.511 & 0.161 & 0.132 \\
\hline 5 & 1.795 & -0.338 & 0.098 & 1.71 & -0.45 & -0.353 & 0.274 & 1.789 & -0.413 & -0.037 \\
\hline 6 & 2.153 & 0.691 & -0.27 & 2.104 & 0.85 & -0.041 & -0.239 & 2.112 & 0.308 & 0.804 \\
\hline 7 & -1.604 & 0.467 & 0.168 & -1.613 & 0.351 & 0.037 & 0.312 & -1.64 & 0.117 & 0.338 \\
\hline 8 & 1.604 & -0.467 & -0.168 & 1.613 & -0.351 & -0.037 & -0.312 & 1.64 & -0.117 & -0.338 \\
\hline 9 & 2.06 & 0.962 & 0.172 & 2.032 & 0.99 & 0.077 & 0.302 & & & \\
\hline 10 & 1.837 & -0.003 & -0.282 & 1.84 & 0.081 & 0.145 & -0.259 & 1.854 & 0.168 & 0.042 \\
\hline 11 & & & & -1.584 & 0.306 & -0.453 & 0.108 & -1.706 & -0.464 & 0.259 \\
\hline 12 & & & & -1.336 & 0.309 & -0.884 & -0.215 & -1.547 & -0.956 & 0.226 \\
\hline Constructs & \multicolumn{3}{|c|}{ The fifth interview (T5) } & \multicolumn{3}{|c|}{ The sixth interview (T6) } & \multicolumn{4}{|c|}{ The seventh and eighth interview (T7 \& T8) } \\
\hline & 1 & 2 & 3 & 1 & 2 & 3 & 1 & 2 & 3 & 4 \\
\hline 1 & 1.771 & -0.279 & 0.292 & 1.918 & 0.017 & 0.402 & 1.922 & -0.03 & 0.39 & -0.055 \\
\hline 2 & 1.776 & -0.464 & -0.043 & 1.768 & -0.497 & -0.014 & 1.922 & -0.243 & 0.093 & -0.27 \\
\hline 3 & 1.776 & -0.464 & -0.043 & 1.768 & -0.497 & -0.014 & 1.761 & -0.52 & -0.047 & 0.023 \\
\hline
\end{tabular}




\begin{tabular}{|c|c|c|c|c|c|c|c|c|c|c|}
\hline 4 & -1.49 & 0.277 & 0.123 & -1.478 & 0.299 & 0.162 & -1.466 & 0.298 & 0.201 & -0.441 \\
\hline 5 & 1.916 & -0.169 & 0.06 & 1.919 & -0.201 & 0.104 & 1.922 & -0.243 & 0.093 & -0.27 \\
\hline 6 & 2.132 & 0.277 & 0.766 & 2.141 & 0.3 & 0.717 & 2.147 & 0.248 & 0.707 & 0.18 \\
\hline 7 & -1.635 & 0.167 & 0.355 & -1.631 & 0.222 & 0.341 & -1.626 & 0.234 & 0.359 & 0.067 \\
\hline 8 & 1.635 & -0.167 & -0.355 & 1.631 & -0.222 & -0.341 & 1.626 & -0.234 & -0.359 & -0.067 \\
\hline 9 & & & & & & & & & & \\
\hline 10 & 1.855 & 0.093 & 0.017 & 1.854 & 0.061 & -0.025 & 1.852 & 0.044 & -0.042 & 0.168 \\
\hline 11 & -1.715 & -0.39 & 0.296 & -1.716 & -0.336 & 0.352 & -1.717 & -0.33 & 0.354 & -0.078 \\
\hline 12 & -1.71 & -1.168 & 0.171 & -1.728 & -1.124 & 0.263 & -1.743 & -1.106 & 0.228 & 0.089 \\
\hline
\end{tabular}


Figure Q1 PrinGrid map - Sumy - the first interview - the ABCD case

C7. University characteristics are good enough for accreditation

\section{C8. Good professors and teaching staff}

C5. Universities have neccessary people to do acceeditation projects

$$
\text { C. Project managers are minditu of time, agenda and manage things well }
$$

\section{C2. Commrittee members know what panels look for}

C10. As universitiles are very good acceditailon will be renewed easily

C4. Streaminilned processes to stoce and retrieve dala

\section{C9. Project tearms cannot consolidate different accreditations}

C6. Comminitees do not have experience fiom onther acceditation projects

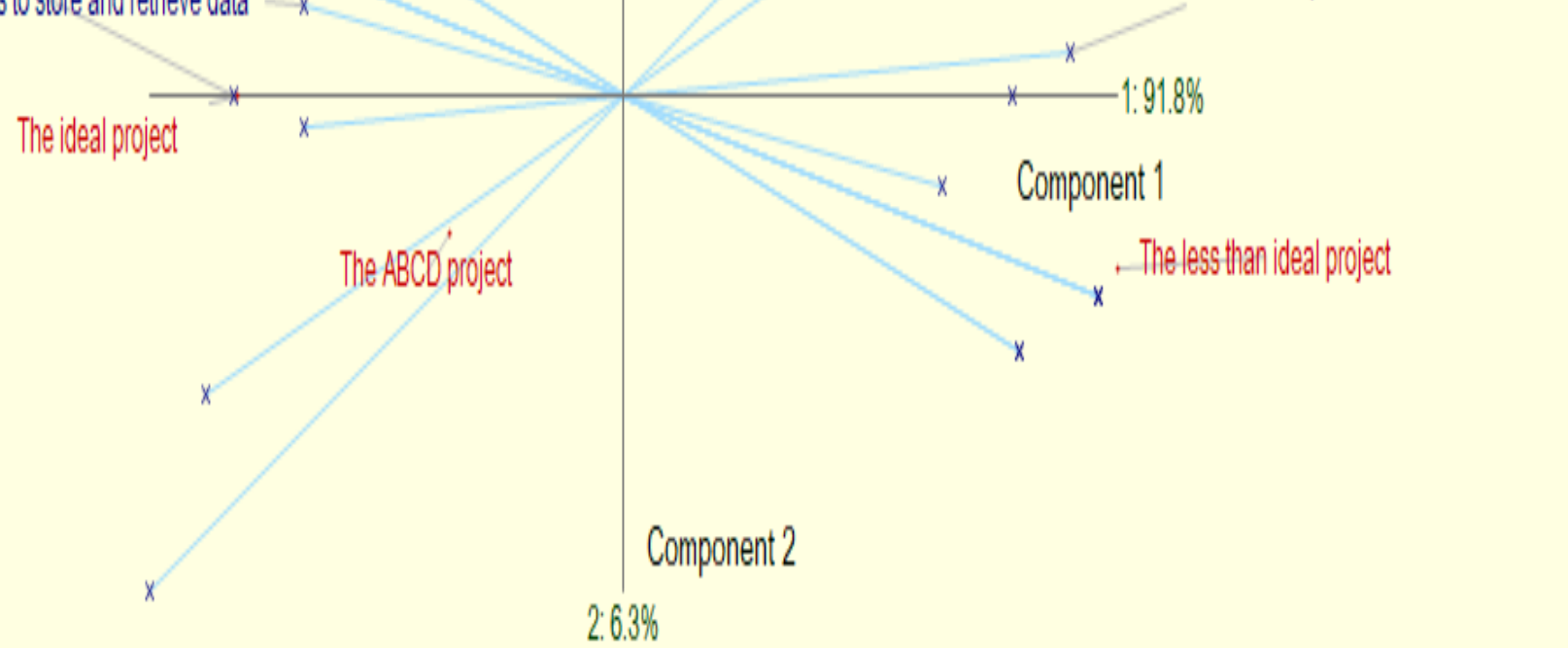


Figure Q2 PrinGrid map - Sumy - the second \& third interviews - the ABCD case

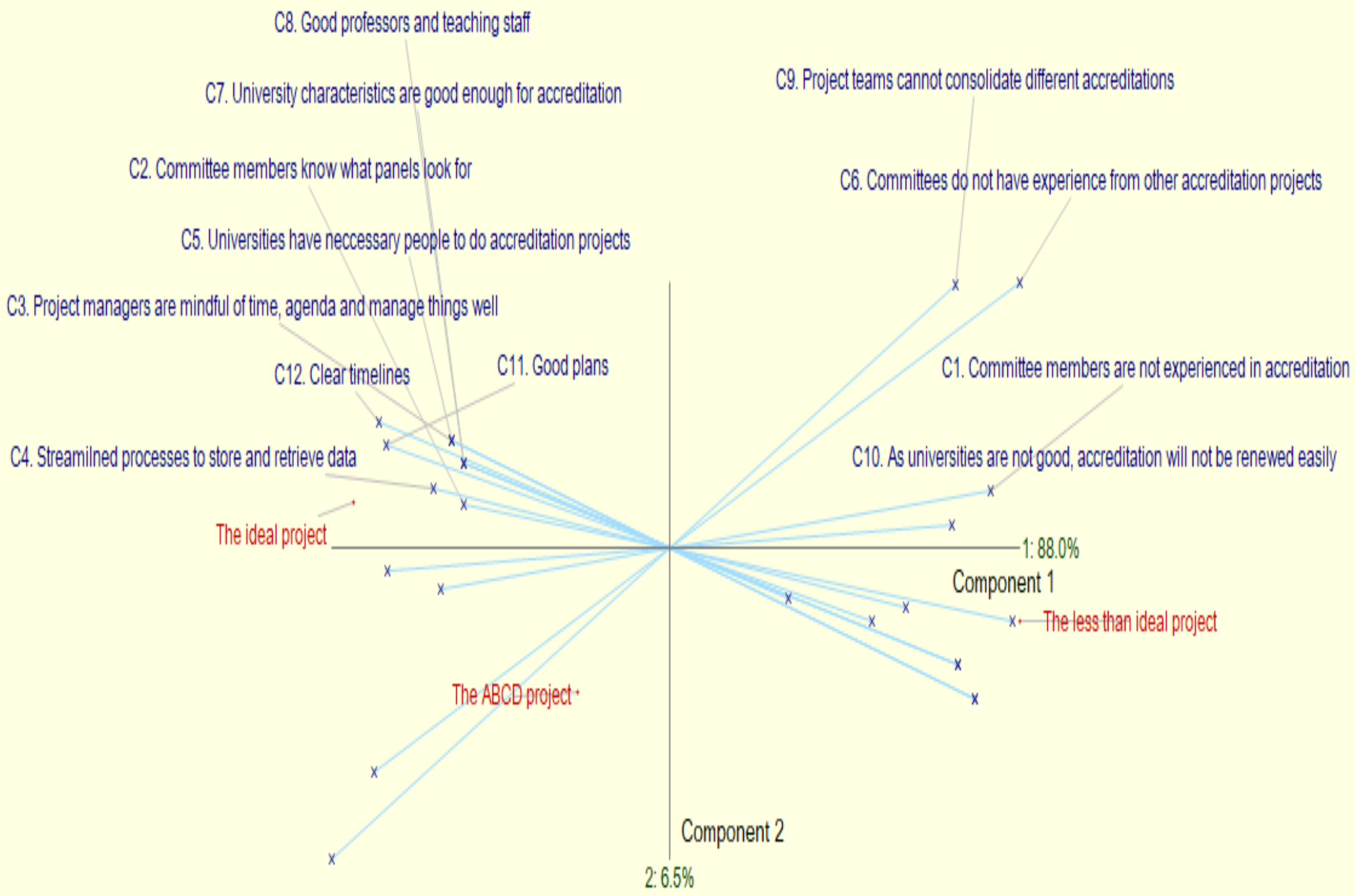


Figure Q3 PrinGrid map - Sumy - the fourth interview - the ABCD case

C5. Universities have neccessany people to do accreditiation projects

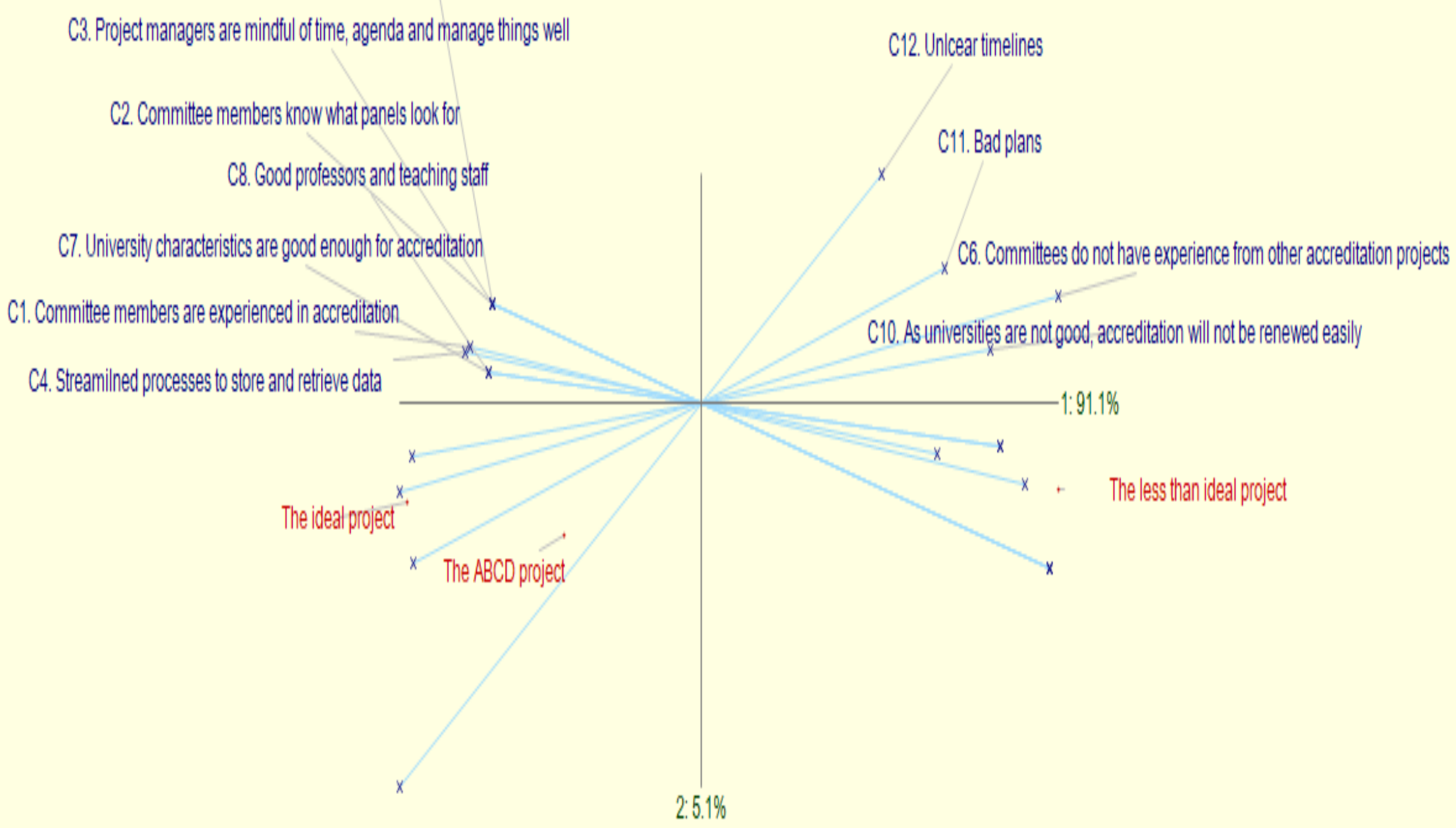


Table Q4 Loadings of elements on components - Sumy - ABCD case

\begin{tabular}{|c|c|c|c|c|c|c|c|c|c|c|}
\hline Interviews & \multicolumn{3}{|c|}{ The first interview (T1) } & \multicolumn{4}{|c|}{ The second and third interview (T2 \& T3) } & \multicolumn{3}{|c|}{ The fourth interview (T4) } \\
\hline Components & 1 & 2 & 3 & 1 & 2 & 3 & 4 & 1 & 2 & 3 \\
\hline Ideal Project & -2.762 & -0.005 & -0.047 & -3.267 & 0.256 & -0.791 & -0.223 & -2.987 & -0.498 & 0.303 \\
\hline Less than ideal project & 3.529 & -0.556 & 0.086 & 3.614 & -0.404 & -0.388 & -0.116 & 3.619 & -0.435 & -0.2 \\
\hline $\mathrm{ABCD}$ project & -1.245 & -0.44 & 0.428 & -0.955 & -0.792 & -0.009 & 0.611 & -1.388 & -0.654 & -0.268 \\
\hline Project 1 & 2.486 & 0.135 & -0.256 & 2.634 & 0.16 & 0.142 & -0.197 & 2.59 & 0.243 & 0.097 \\
\hline Project 2 & -2.188 & -0.384 & -0.327 & -2.12 & -0.471 & 0.751 & -0.361 & -1.72 & 0.808 & -0.632 \\
\hline Project 3 & 0.18 & 1.249 & 0.116 & 0.096 & 1.251 & 0.295 & 0.285 & -0.114 & 0.536 & 0.701 \\
\hline Interviews & \multicolumn{3}{|c|}{ The fifth interview (T5) } & \multicolumn{3}{|c|}{ The sixth interview (T6) } & \multicolumn{4}{|c|}{ The seventh and eighth interview (T7 \& T8) } \\
\hline Components & 1 & 2 & 3 & 1 & 2 & 3 & 1 & 2 & 3 & 4 \\
\hline Ideal Project & -2.943 & -0.354 & 0.288 & -2.914 & -0.322 & 0.408 & -2.883 & -0.35 & 0.429 & -0.395 \\
\hline Less than ideal project & 3.663 & -0.436 & -0.199 & 3.689 & -0.455 & -0.133 & 3.717 & -0.458 & -0.14 & -0.178 \\
\hline $\mathrm{ABCD}$ project & -1.655 & -0.889 & -0.211 & -1.789 & -0.875 & -0.253 & -1.919 & -0.78 & -0.299 & 0.383 \\
\hline Project 1 & 2.642 & 0.224 & 0.076 & 2.666 & 0.193 & 0.039 & 2.692 & 0.182 & 0.041 & 0.025 \\
\hline Project 2 & -1.652 & 0.895 & -0.644 & -1.63 & 0.814 & -0.697 & -1.608 & 0.805 & -0.668 & -0.117 \\
\hline Project 3 & -0.056 & 0.56 & 0.69 & -0.022 & 0.645 & 0.635 & 0.001 & 0.6 & 0.638 & 0.281 \\
\hline
\end{tabular}

